Supporting Information

\title{
Rhodium-Catalyzed Regiodivergent Synthesis of
}

\section{Alkylboronates via Deoxygenative Hydroboration of Aryl Ketones: Mechanism and Origin of Selectivities}

Bing Zhang, ${ }^{+, *}$ Xin $\mathrm{Xu},{ }^{\neq, *}$ Lei Tao, ${ }^{+}$Zhenyang Lin, ${ }^{\ddagger, *}$ and Wanxiang Zhao ${ }^{+, *}$

${ }^{+}$State Key Laboratory of Chemo/Biosensing and Chemometrics, College of Chemistry and Chemical Engineering, Hunan University, Changsha, Hunan 410082, P. R. China

‡Department of Chemistry, The Hong Kong University of Science and Technology, 999077 Clear Water Bay, Kowloon, Hong Kong, P. R. China

\section{Table of Contents}

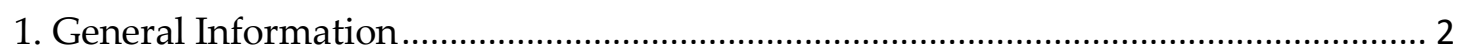

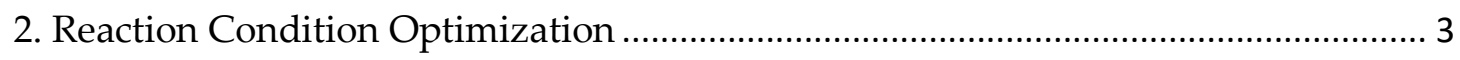

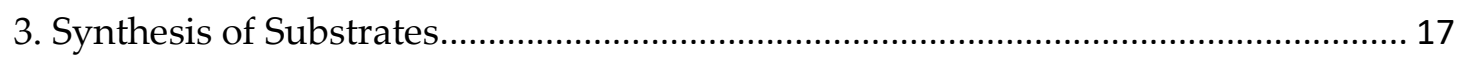

4. General Procedures for Synthesis of Alkylboronates................................................. 20

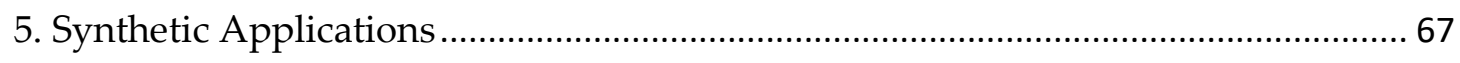

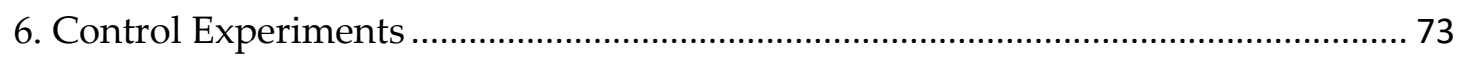

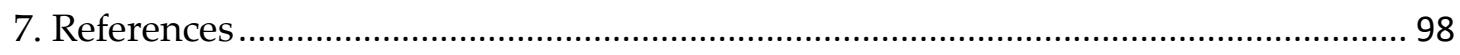

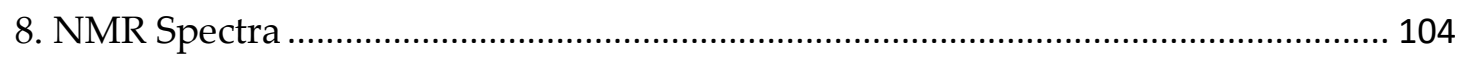

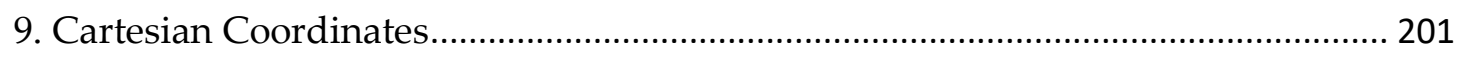




\section{General Information}

Unless otherwise noted, all reactions were conducted in oven-dried flasks or vials with a magnetic stirrer under nitrogen atmosphere. Solvents were purified under nitrogen using a solvent purification system. Analytical thin layer chromatography (TLC) was performed using silica gel plates. Visualisation was by ultraviolet fluorescence, and/or phosphomolybdic acid, and/or $\mathrm{KMnO}_{4}$. Flash column chromatography was performed using EM Science (200-300 mesh) silica gel.

${ }^{1} \mathrm{H}$-Nuclear Magnetic Resonance $\left({ }^{1} \mathrm{H} \mathrm{NMR}\right)$ and ${ }^{13} \mathrm{C}$ Nuclear Magnetic Resonance $\left({ }^{13} \mathrm{C}\right.$ NMR) spectra were recorded on Bruker $400 \mathrm{MHz}$ at $20{ }^{\circ} \mathrm{C}$ with $\mathrm{CDCl}_{3}$ as solvent, and were reported in ppm referenced to tetramethylsilane $(\delta$ $=0$ ppm for $\left.{ }^{1} \mathrm{H}-\mathrm{NMR}\right)$ and deuteriochloroform ( $\delta=77.16$ ppm for $\left.{ }^{13} \mathrm{C}-\mathrm{NMR}\right)$. The data are reported as follows: chemical shift (ppm), multiplicity (s = singlet, $\mathrm{d}=$ doublet, $\mathrm{dd}=$ doublet of doublet, $\mathrm{t}=$ triplet, $\mathrm{m}=$ multiplet, $\mathrm{m}_{\mathrm{c}}=$ centered multiplet, br $=$ broad), coupling constant $J(\mathrm{~Hz})$, and integration. High resolution mass spectra were recorded on a Bruker Maxis System using Electron Spray Ionization (ESI). IR spectra were collected on a Spectrum QATR$S$ from SHIMADZU and reported in unit of $\mathrm{cm}^{-1}$.

Ligands, bases, bis(pinacolate)diboron (B2pin2), and pinacolborane (HBpin) were all purchased from Energy Chemicals Inc. or Macklin Inc. and used as received. $[\mathrm{RhCl}(\mathrm{cod})]_{2}$ was prepared according to the literature procedure and all characterization data are in accordance with the literature ${ }^{[1]}$. 


\section{Reaction Condition Optimization}

\subsection{Synthesis of linear alkylboronates from ketones}

Table S1. Solvent evaluation ${ }^{a}$

\begin{tabular}{|c|c|c|}
\hline${ }^{2}{ }_{12}$ & $\begin{array}{c}{[\mathrm{RhCl}(\mathrm{cod})]_{2}(2 \mathrm{~mol} \%)} \\
\mathrm{P}\left({ }^{n} \mathrm{Bu}\right)_{3}(12 \mathrm{~mol} \%) \\
\mathrm{B}_{2} \operatorname{pin}_{2}(1.8 \text { eq. }), \mathrm{MeOH}(1.0 \text { eq. }) \\
\text { LiOAC }(50 \mathrm{~mol} \%), \mathrm{S}(2.0 \mathrm{~mL}), 70^{\circ} \mathrm{C}, 12 \mathrm{~h}\end{array}$ & $2 a$ \\
\hline Entry & Solvent $(2.0 \mathrm{~mL})$ & Yield $(\%)^{b}$ \\
\hline 1 & THF & 36 \\
\hline 2 & DCE & NR \\
\hline 3 & $\mathrm{CH}_{3} \mathrm{CN}$ & 27 \\
\hline 4 & 1,4-dioxane & 42 \\
\hline 5 & toluene & 48 \\
\hline 6 & СуH & 46 \\
\hline 7 & CPME & 49 \\
\hline 8 & hexane & 60 \\
\hline 9 & MTBE & 52 \\
\hline 10 & DMF & 37 \\
\hline 11 & 2-Me-THF & 44 \\
\hline 12 & DME & 20 \\
\hline 13 & IPE & 63 \\
\hline
\end{tabular}

${ }^{a}$ Conditions : 1a $(0.3 \mathrm{mmol}),[\mathrm{Rh}(\mathrm{cod}) \mathrm{Cl}]_{2}(2 \mathrm{~mol} \%), \mathrm{P}\left({ }^{n} \mathrm{Bu}\right) 3(12 \mathrm{~mol} \%), \mathrm{LiOAc}(50 \mathrm{~mol} \%)$, $\mathrm{B}_{2}$ pin 2 (1.8 equiv), $\mathrm{MeOH}$ (1.0 equiv), Solvent $(2.0 \mathrm{~mL}), 70{ }^{\circ} \mathrm{C}, 12 \mathrm{~h} .{ }^{b}$ Yields were determined by ${ }^{1} \mathrm{H}$ NMR analysis using $\mathrm{CH}_{2} \mathrm{Br}_{2}$ as an internal standard. $\mathrm{DCE}=1$,2-dichloroethane, $\mathrm{CyH}$ = cyclohexane, $\mathrm{CPME}=$ methoxycyclopentane, $\mathrm{MTBE}=2$-methoxy-2-methylpropane, $\mathrm{DME}=1,2$-dimethoxyethane, IPE $=2$-isopropoxypropane. 
Table S2. Base evaluation ${ }^{a}$

\begin{tabular}{|c|c|c|}
\hline Ph & $\begin{array}{c}{[\mathrm{RhCl}(\mathrm{cod})]_{2}(2 \mathrm{~mol} \%)} \\
\mathrm{P}\left({ }^{n} \mathrm{Bu}\right)_{3}(12 \mathrm{~mol} \%) \\
\mathrm{B}_{2} \operatorname{pin}_{2}(1.8 \text { eq. }), \mathrm{MeOH}(1.0 \text { eq. }) \\
\text { base }(50 \mathrm{~mol} \%), \text { IPE }(2.0 \mathrm{~mL}), 70^{\circ} \mathrm{C}, 12 \mathrm{~h}\end{array}$ & $\widetilde{2 a}^{B p i}$ \\
\hline Entry & base $(50 \mathrm{mmol} \%)$ & Yield $(\%)^{b}$ \\
\hline 1 & LiOAc & 64 \\
\hline 2 & HCOOLi & 44 \\
\hline 3 & $\mathrm{Li}_{2} \mathrm{CO}_{3}$ & 74 \\
\hline 4 & $\mathrm{Li}_{3} \mathrm{PO}_{4}$ & 63 \\
\hline 5 & $\mathrm{NaOAC}$ & 79 \\
\hline 6 & $\mathrm{HCOONa}$ & 80 \\
\hline 7 & $\mathrm{Na}_{2} \mathrm{CO}_{3}$ & 73 \\
\hline 8 & PhCOONa & 50 \\
\hline 9 & $\mathrm{PhONa}$ & 61 \\
\hline 10 & $\mathrm{CF}_{3} \mathrm{COONa}$ & 47 \\
\hline 11 & KF & 70 \\
\hline 12 & HCOOK & 82 \\
\hline 13 & $\mathrm{~K}_{3} \mathrm{PO}_{4}$ & 57 \\
\hline 14 & $\mathrm{NaOPiv} \cdot \mathrm{H}_{2} \mathrm{O}$ & 46 \\
\hline 15 & MeOK & ND \\
\hline 16 & ${ }^{t} \mathrm{BuOK}$ & trace \\
\hline 17 & PhCOOK & 67 \\
\hline 18 & $\mathrm{CF}_{3} \mathrm{COOK}$ & 61 \\
\hline 19 & KOAc & 65 \\
\hline 20 & $\mathrm{~K}_{2} \mathrm{CO}_{3}$ & 70 \\
\hline 21 & CsF & 73 \\
\hline 22 & $\mathrm{Cs}_{2} \mathrm{CO}_{3}$ & 50 \\
\hline 23 & CsOAc & 46 \\
\hline 24 & DMAP & 63 \\
\hline
\end{tabular}

${ }^{a}$ Conditions : 1a $(0.3 \mathrm{mmol}),[\mathrm{Rh}(\mathrm{cod}) \mathrm{Cl}]_{2}(2 \mathrm{~mol} \%), \mathrm{P}\left({ }^{n} \mathrm{Bu}\right)_{3}(12 \mathrm{~mol} \%)$, Base (50 mol\%), $\mathrm{B}_{2}$ pin 2 (1.8 equiv), $\mathrm{MeOH}$ (1.0 equiv), IPE (2.0 mL), $70{ }^{\circ} \mathrm{C}, 12 \mathrm{~h} .{ }^{b}$ Yields were determined by ${ }^{1} \mathrm{H}$ NMR analysis using $\mathrm{CH}_{2} \mathrm{Br}_{2}$ as an internal standard. 
Table S3. Base-loading evaluation ${ }^{a}$

\begin{tabular}{|c|c|c|}
\hline Ph & $\begin{array}{c}{[\mathrm{RhCl}(\mathrm{cod})]_{2}(2 \mathrm{~mol} \%)} \\
\mathrm{P}\left({ }^{n} \mathrm{Bu}\right)_{3}(12 \mathrm{~mol} \%) \\
\mathrm{B}_{2} \mathrm{pin}_{2}(1.8 \mathrm{eq} .), \mathrm{MeOH}(1.0 \text { eq. }) \\
\mathrm{HCOOK}(\mathrm{x} \mathrm{mol} \%) . \mathrm{PE}(2.0 \mathrm{~mL}) .70^{\circ} \mathrm{C} .12 \mathrm{~h}\end{array}$ & 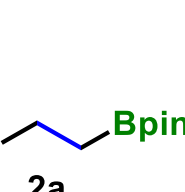 \\
\hline Entry & $\operatorname{HCOOK}(\mathrm{x} \mathrm{mol} \%)$ & Yield $(\%)^{b}$ \\
\hline 1 & 0 & NR \\
\hline 2 & $10 \mathrm{~mol} \%$ & 68 \\
\hline 3 & $20 \mathrm{~mol} \%$ & 72 \\
\hline 4 & $50 \mathrm{~mol} \%$ & 82 \\
\hline 5 & $100 \mathrm{~mol} \%$ & 78 \\
\hline
\end{tabular}

${ }^{a}$ Conditions : 1a $(0.3 \mathrm{mmol}),[\mathrm{Rh}(\operatorname{cod}) \mathrm{Cl}]_{2}(2 \mathrm{~mol} \%), \mathrm{P}\left({ }^{n} \mathrm{Bu}\right)_{3}(12 \mathrm{~mol} \%), \mathrm{HCOOK}(\mathrm{x} \mathrm{mol} \%)$, $\mathrm{B}_{2}$ pinz (1.8 equiv), $\mathrm{MeOH}\left(1.0\right.$ equiv), IPE $(2.0 \mathrm{~mL}), 70{ }^{\circ} \mathrm{C}, 12 \mathrm{~h} .{ }^{b}$ Yields were determined by ${ }^{1} \mathrm{H}$ NMR analysis using $\mathrm{CH}_{2} \mathrm{Br}_{2}$ as an internal standard. 
Table S4. Ligand evaluation ${ }^{a}$

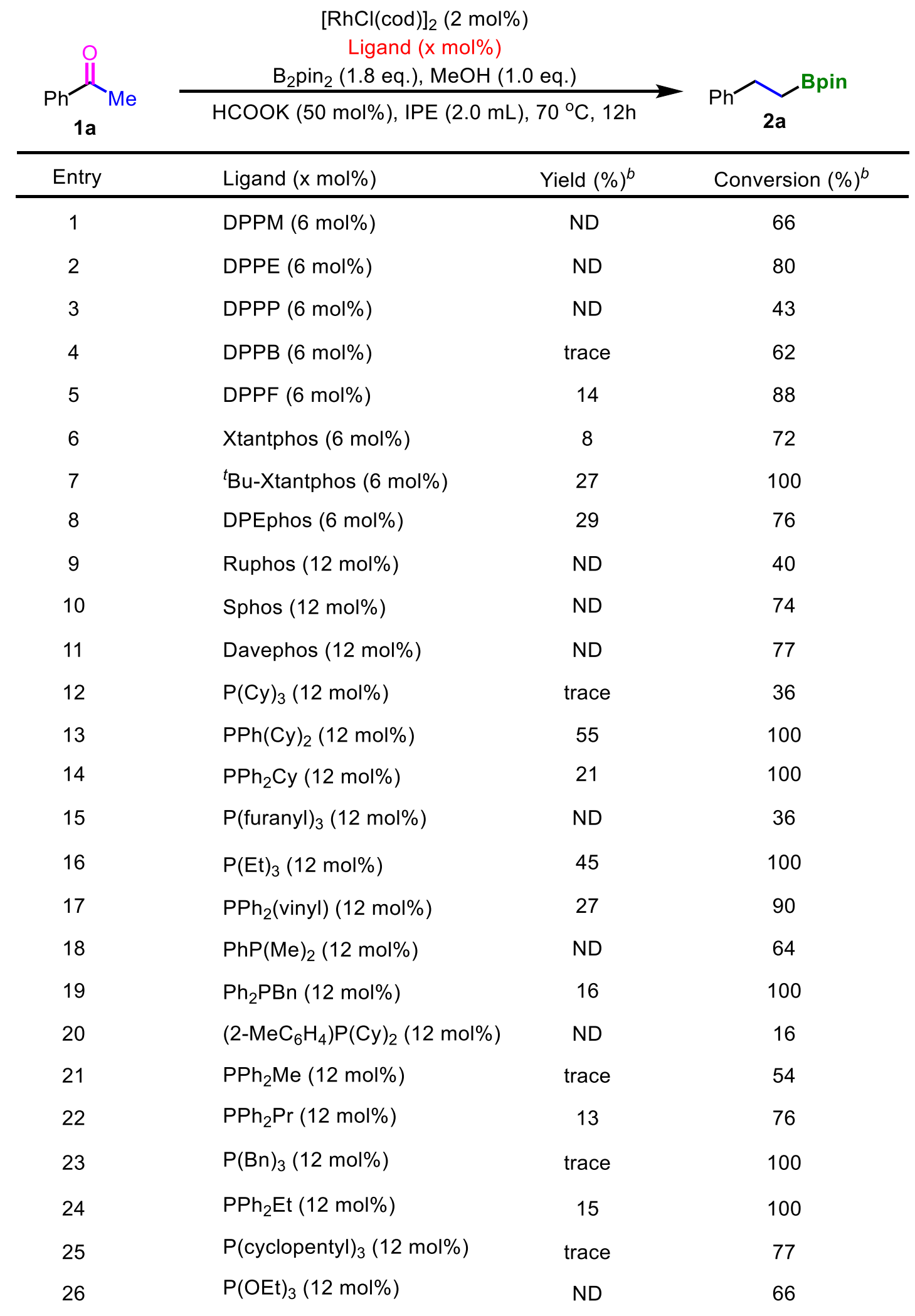

${ }^{a}$ Conditions : 1a $(0.3 \mathrm{mmol}),[\mathrm{Rh}(\mathrm{cod}) \mathrm{Cl}]_{2}(2 \mathrm{~mol} \%)$, Ligand (x mol\%), HCOOK (50 mol\%), B2pin2 (1.8 equiv), $\mathrm{MeOH}$ (1.0 equiv), IPE $(2.0 \mathrm{~mL}), 70{ }^{\circ} \mathrm{C}, 12 \mathrm{~h} .{ }^{b}$ Yields and conversions were determined by ${ }^{1} \mathrm{H}$ NMR analysis using $\mathrm{CH}_{2} \mathrm{Br}_{2}$ as an internal standard. 
Table S5. [H]-source evaluation ${ }^{a}$

\begin{tabular}{|c|c|c|}
\hline , & $\begin{array}{c}{[\mathrm{RhCl}(\mathrm{cod})]_{2}(2 \mathrm{~mol} \%)} \\
\mathrm{P}\left({ }^{n} \mathrm{Bu}\right)_{3}(12 \mathrm{~mol} \%) \\
\mathrm{B}_{2} \mathrm{pin}_{2}(1.8 \text { eq. }),[\mathrm{H}](1.0 \text { eq. }) \\
\mathrm{HCOOK}(50 \mathrm{~mol} \%)\left(\mathrm{PF}(20 \mathrm{ml}) 70^{\circ} \mathrm{C} \quad 12 \mathrm{~h}\right.\end{array}$ & D \\
\hline $1 \mathrm{a}$ & $\mathrm{HCOOK}(50 \mathrm{~mol} \%), \operatorname{IPE}(2.0 \mathrm{~mL}), 70^{\circ} \mathrm{C}, 12 \mathrm{~h}$ & $2 a$ \\
\hline Entry & {$[\mathrm{H}]$ source (1.0 eq.) } & Yield $(\%)^{b}$ \\
\hline 1 & $\mathrm{MeOH}$ & 79 \\
\hline 2 & 'PrOH & $85(73)$ \\
\hline 3 & ${ }^{t} \mathrm{BuOH}$ & 80 \\
\hline 4 & $\mathrm{PhCOOH}$ & trace \\
\hline 5 & $\mathrm{H}_{2} \mathrm{O}$ & trace \\
\hline 6 & HBpin & 34 \\
\hline $7^{c}$ & HBpin & 78 \\
\hline $8^{d}$ & HBpin & 43 \\
\hline
\end{tabular}

aConditions : 1a (0.3 mmol), [Rh(cod)Cl $]_{2}(2 \mathrm{~mol} \%), \mathrm{P}\left({ }^{n} \mathrm{Bu}\right)_{3}(12 \mathrm{~mol} \%), \operatorname{HCOOK}(50 \mathrm{~mol} \%)$, B2pinz (1.8 equiv), $[\mathrm{H}]$ (1.0 equiv), IPE $(2.0 \mathrm{~mL}), 70{ }^{\circ} \mathrm{C}, 12 \mathrm{~h} .{ }^{b}$ Yields were determined by ${ }^{1} \mathrm{H}$ NMR analysis using $\mathrm{CH}_{2} \mathrm{Br}_{2}$ as an internal standard, Isolated yield in parentheses. ${ }^{c} \mathrm{~B}_{2}$ pin2 (1.2 eq.). ${ }^{d}$ No B2pin2 and HBpin (1.8 eq.).

Table S6. ${ }^{i} \mathrm{PrOH}$ loading evaluation ${ }^{a}$

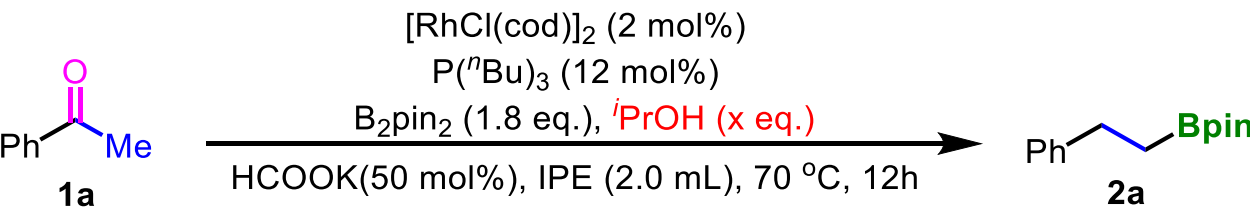

\begin{tabular}{ccc}
\hline Entry & ${ }^{i} \mathrm{PrOH}(\mathrm{x}$ eq. $)$ & Yield $(\%)^{b}$ \\
\hline 1 & 0 & 40 \\
2 & 0.5 & 65 \\
3 & 0.8 & 79 \\
4 & 1.0 & 85 \\
5 & 1.5 & 45 \\
\hline
\end{tabular}

${ }^{a}$ Conditions : 1a $(0.3 \mathrm{mmol}),[\mathrm{Rh}(\mathrm{cod}) \mathrm{Cl}]_{2}(2 \mathrm{~mol} \%), \mathrm{P}\left({ }^{n} \mathrm{Bu}\right)_{3}(12 \mathrm{~mol} \%), \operatorname{HCOOK}(50 \mathrm{~mol} \%)$, B 2 pin 2 (1.8 equiv), ${ }^{i} \mathrm{PrOH}$ (x equiv), IPE $(2.0 \mathrm{~mL}), 70{ }^{\circ} \mathrm{C}, 12 \mathrm{~h} .{ }^{b}$ Yields were determined by ${ }^{1} \mathrm{H}$ NMR analysis using $\mathrm{CH}_{2} \mathrm{Br}_{2}$ as an internal standard, Isolated yield in parentheses. 
Table S7. Reaction temperature evaluation ${ }^{a}$

\begin{tabular}{|c|c|c|}
\hline & $\begin{array}{c}{[\mathrm{RhCl}(\mathrm{cod})]_{2}(2 \mathrm{~mol} \%)} \\
\mathrm{P}\left({ }^{n} \mathrm{Bu}\right)_{3}(12 \mathrm{~mol} \%) \\
\mathrm{B}_{2} \mathrm{pin}_{2}(1.8 \text { eq. }),{ }^{i} \mathrm{PrOH}(1.0 \text { eq. }) \\
\end{array}$ & \\
\hline $1 \mathrm{a}$ & MCOUn (JU mOT\%), IRE (2.0 mL), I, ILM & $\mathbf{2 a}$ \\
\hline Entry & $\mathrm{T}\left(\mathrm{x}^{\circ} \mathrm{C}\right)$ & Yield $(\%)^{b}$ \\
\hline 1 & $50^{\circ} \mathrm{C}$ & 58 \\
\hline 2 & $60^{\circ} \mathrm{C}$ & 68 \\
\hline 3 & $80^{\circ} \mathrm{C}$ & 82 \\
\hline 4 & $90^{\circ} \mathrm{C}$ & 76 \\
\hline
\end{tabular}

${ }^{a}$ Conditions : 1a $(0.3 \mathrm{mmol}),[\mathrm{Rh}(\mathrm{cod}) \mathrm{Cl}]_{2}(2 \mathrm{~mol} \%), \mathrm{P}\left({ }^{(} \mathrm{Bu}\right)_{3}(12 \mathrm{~mol} \%), \operatorname{HCOOK}(50 \mathrm{~mol} \%)$, $\mathrm{B}_{2}$ pin2 (1.8 equiv), ${ }^{i} \mathrm{PrOH}\left(1.0\right.$ equiv), IPE $(2.0 \mathrm{~mL}), \mathrm{T}{ }^{\circ} \mathrm{C}, 12 \mathrm{~h} .{ }^{b}$ Yields were determined by ${ }^{1} \mathrm{H}$ NMR analysis using $\mathrm{CH}_{2} \mathrm{Br}_{2}$ as an internal standard. 


\subsection{Synthesis of branched alkylboronates from ketones}

Table S8. Base evaluation ${ }^{a}$

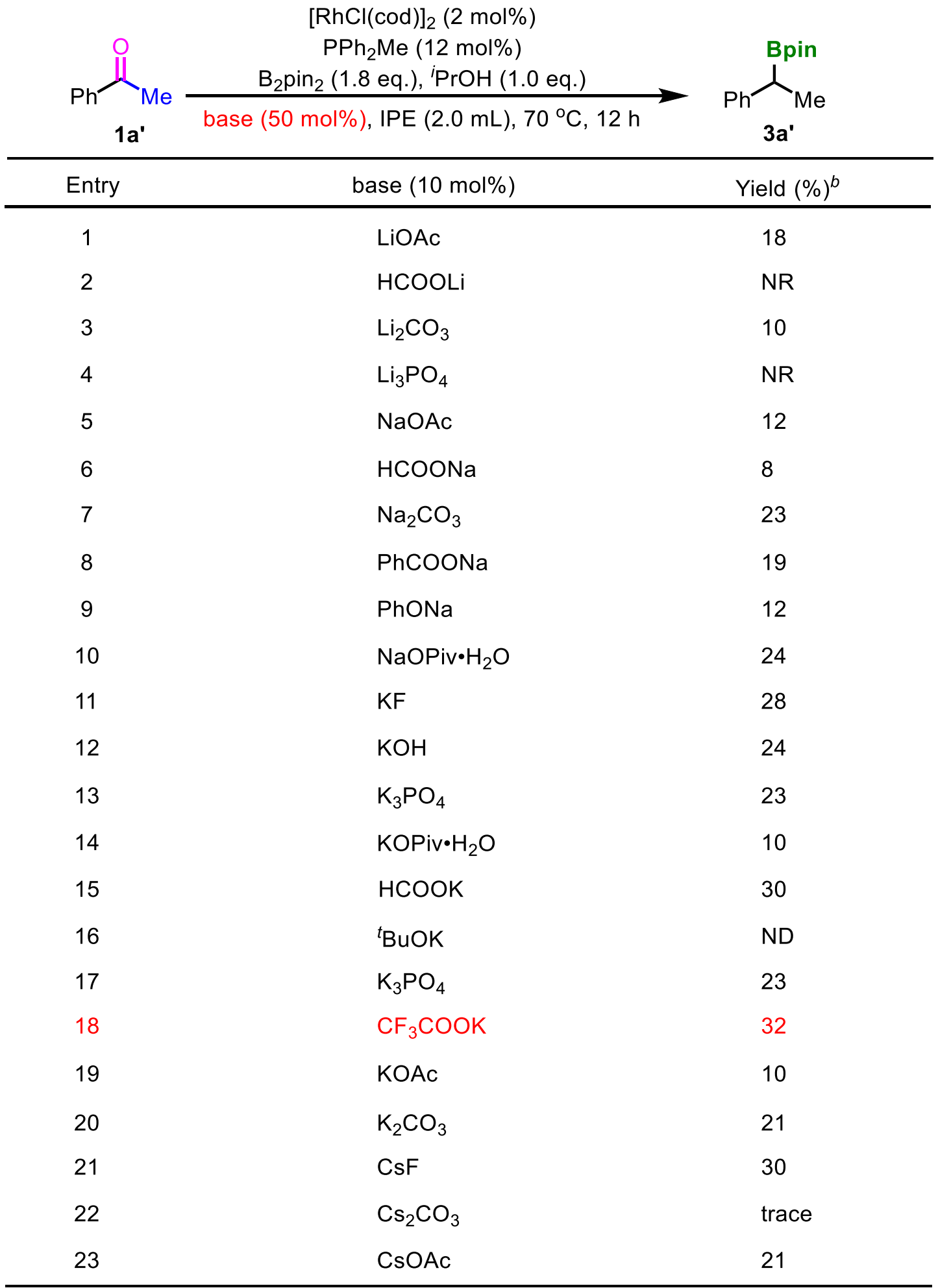

aConditions : 1a' (0.3 mmol), [Rh(cod)Cl]2 (2 mol\%), PPh $2 \mathrm{Me}(12 \mathrm{~mol} \%)$, Base (50 mol\%), B2pinz (1.8 equiv), ${ }^{i} \operatorname{PrOH}\left(1.0\right.$ equiv), IPE $(2.0 \mathrm{~mL}), 70{ }^{\circ} \mathrm{C}, 12 \mathrm{~h} .{ }^{b}$ Yields were determined by GC analysis using dodecane as the internal standard. 
Table S9. Base-loading evaluation ${ }^{a}$

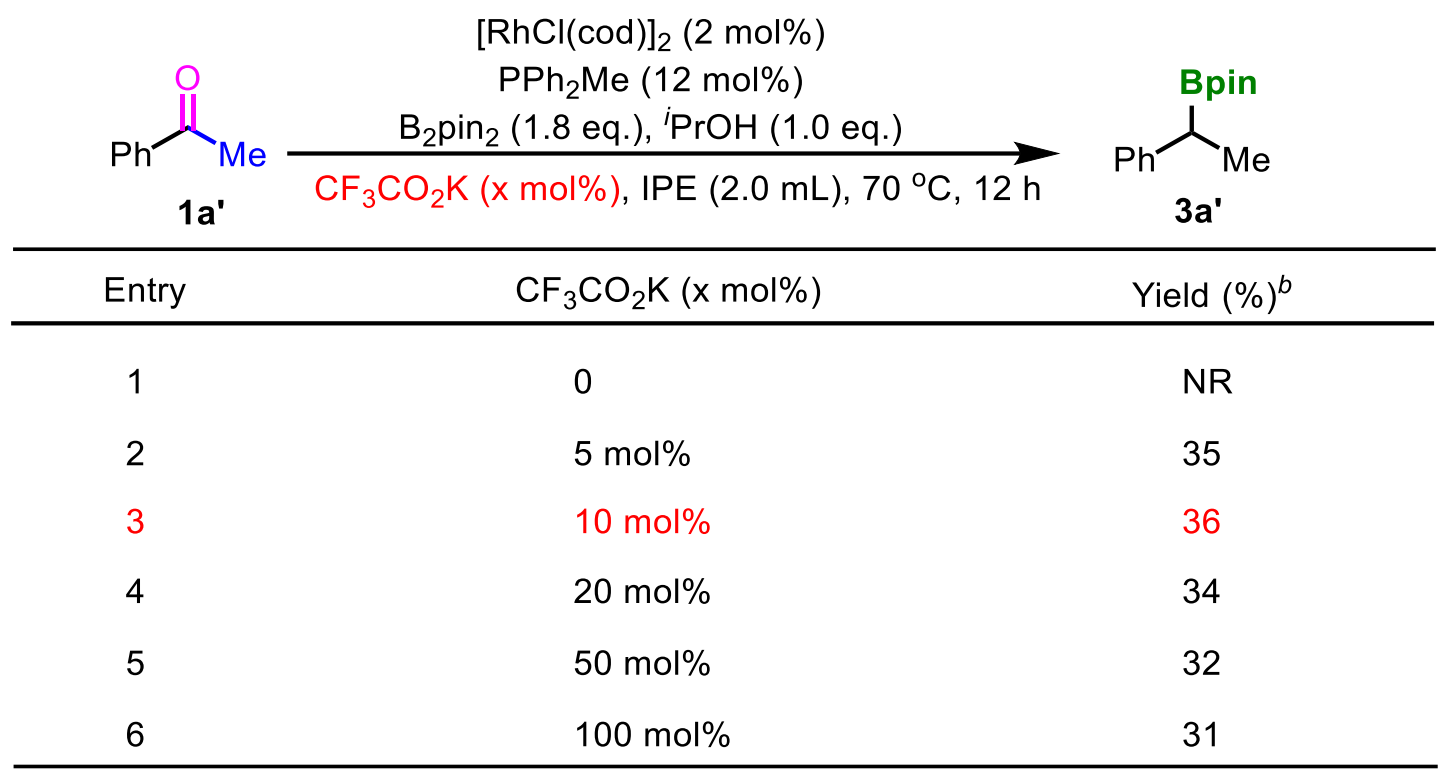

${ }^{a}$ Conditions : 1a' (0.3 mmol), [ $\left.\mathrm{Rh}(\mathrm{cod}) \mathrm{Cl}\right]_{2}(2 \mathrm{~mol} \%), \mathrm{PPh}_{2} \mathrm{Me}(12 \mathrm{~mol} \%), \mathrm{CF}_{3} \mathrm{CO}_{2} \mathrm{~K}(\mathrm{x} \mathrm{mol} \%)$, $\mathrm{B}_{2}$ pin 2 (1.8 equiv), ${ }^{i} \mathrm{PrOH}$ (1.0 equiv), IPE $(2.0 \mathrm{~mL}), 70{ }^{\circ} \mathrm{C}, 12 \mathrm{~h} .{ }^{b}$ Yields were determined by GC analysis using dodecane as the internal standard. 
Table S10. Solvent evaluation ${ }^{a}$

\begin{tabular}{|c|c|c|}
\hline $\mathrm{Ph}_{1 \mathrm{a}^{\prime}}$ & $\begin{array}{c}{[\mathrm{RhCl}(\mathrm{cod})]_{2}(2 \mathrm{~mol} \%)} \\
\mathrm{PPh}{ }_{2} \mathrm{Me}(12 \mathrm{~mol} \%) \\
\mathrm{B}_{2} \mathrm{pin}_{2}(1.8 \mathrm{eq} .),{ }^{,} \mathrm{PrOH}(1.0 \text { eq. }) \\
\mathrm{CF}_{3} \mathrm{COOK}(10 \mathrm{~mol} \%), \mathrm{S}(2.0 \mathrm{~mL}), 70^{\circ} \mathrm{C}, 12 \mathrm{~h}\end{array}$ & $\overbrace{3 a^{\prime}}^{B p i n}$ \\
\hline Entry & Solvent $(2.0 \mathrm{~mL})$ & Yield $(\%)^{b}$ \\
\hline 1 & THF & 33 \\
\hline 2 & DCE & 6 \\
\hline 3 & 1,4-dioxane & 9 \\
\hline 4 & СуH & 31 \\
\hline 5 & CPME & 21 \\
\hline 6 & MTBE & 22 \\
\hline 7 & 2-Me-THF & 25 \\
\hline 8 & DME & ND \\
\hline 9 & IPE & 33 \\
\hline 10 & $\mathrm{CH}_{3} \mathrm{CN}$ & ND \\
\hline 11 & Hexane & 33 \\
\hline 12 & ${ }^{\text {nOctane }}$ & 33 \\
\hline 13 & Benzene & 14 \\
\hline 14 & THF:IPE = 1:4 & 49 \\
\hline 16 & THF:hex $=1: 4$ & 60 \\
\hline 15 & THF:CyH = 1:4 & 65 \\
\hline 17 & $\mathrm{THF}: \mathrm{CyH}=4: 1$ & 32 \\
\hline $18^{c}$ & $\mathrm{THF}: \mathrm{CyH}=1: 4$ & 65 \\
\hline
\end{tabular}

${ }^{a}$ Conditions : 1a' (0.3 mmol), [Rh(cod)Cl]2 (2 mol\%), $\mathrm{PPh} 2 \mathrm{Me}(12 \mathrm{~mol} \%), \mathrm{CF}_{3} \mathrm{CO}_{2} \mathrm{~K}(10$ mol\%), B2pinz (1.8 equiv), $\operatorname{PrOH}\left(1.0\right.$ equiv), Solvent $(2.0 \mathrm{~mL}), 70{ }^{\circ} \mathrm{C}, 12 \mathrm{~h} .{ }^{b}$ Yields were determined by GC analysis using dodecane as the internal standard. 'Room temperature, $24 \mathrm{~h}$. $\mathrm{DCE}=1,2$-dichloroethane, $\mathrm{CyH}=$ cyclohexane, $\mathrm{CPME}=$ methoxycyclopentane, $\mathrm{MTBE}$ =2-methoxy-2-methylpropane, $\mathrm{DME}=1$,2-dimethoxyethane, $\mathrm{IPE}=2$-isopropoxypropane. 
Table S11. Ligand evaluation ${ }^{a}$

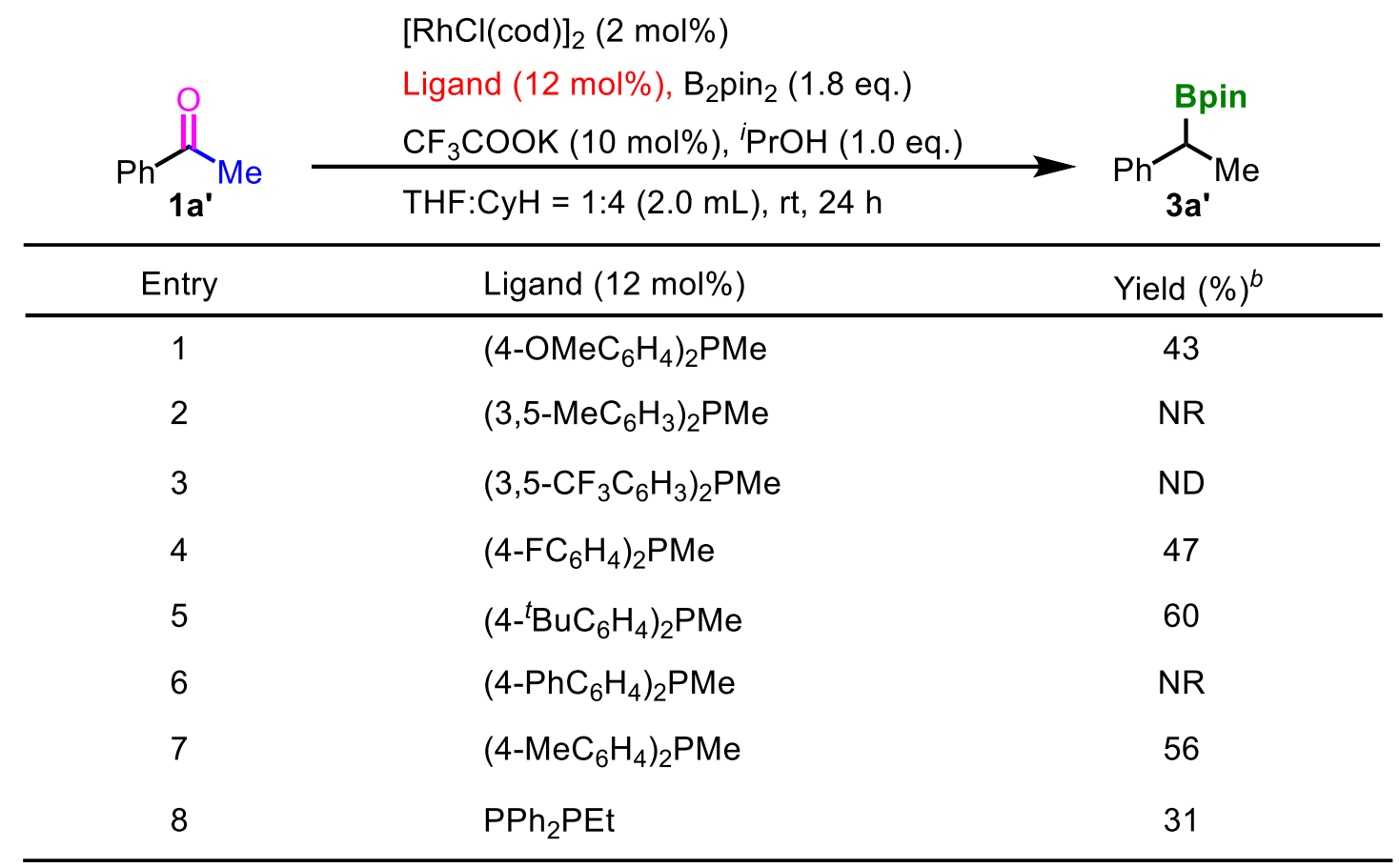

aConditions : 1a' (0.3 mmol), [Rh(cod)Cl]2 (2 mol\%), Ligand (12 mol\%), $\mathrm{CF}_{3} \mathrm{COOK}(10$ mol\%), B2pin2 (1.8 equiv), ${ }^{i} \mathrm{PrOH}$ (1.0 equiv), THF:CyH = 1:4 (2.0 mL), rt, 24 h. ${ }^{b}$ Yields were determined by GC analysis using dodecane as the internal standard.

\section{Table S12. [H]-source evaluation ${ }^{a}$}

\begin{tabular}{|c|c|c|}
\hline & {$[\mathrm{RhCl}(\mathrm{cod})]_{2}(2 \mathrm{~mol} \%)$} & \\
\hline & $\mathrm{PPh}_{2} \mathrm{Me}\left(12 \mathrm{~mol}^{2}\right), \mathrm{B}_{2} \mathrm{pin}_{2}$ (1.8 eq.) & Bpin \\
\hline & $\mathrm{CF}_{3} \mathrm{COOK}(10 \mathrm{~mol} \%),[\mathrm{H}]$ (1.0 eq.) & \\
\hline $1 a^{\prime}$ & $\mathrm{THF}: \mathrm{CyH}=1: 4(2.0 \mathrm{~mL}), \mathrm{rt}, 24 \mathrm{~h}$ & $3 a^{\prime}$ \\
\hline Entry & {$[\mathrm{H}]$ source (1.0 eq.) } & Yield $(\%)^{b}$ \\
\hline 1 & $\mathrm{MeOH}$ & 46 \\
\hline 2 & ${ }^{i} \mathrm{PrOH}$ & 65 \\
\hline 3 & ${ }^{t} \mathrm{BuOH}$ & 60 \\
\hline 4 & HFIP & 40 \\
\hline 5 & HBpin & 68 \\
\hline 6 & - & 20 \\
\hline
\end{tabular}

${ }^{a}$ Conditions : 1a' (0.3 mmol), [Rh(cod)Cl] 2 (2 mol\%), PPh $2 \mathrm{Me}(12 \mathrm{~mol} \%), \mathrm{CF}_{3} \mathrm{COOK}(10$ mol\%), B2pinz (1.8 equiv), [H] (1.0 equiv), THF:CyH = 1:4 (2.0 mL), rt, 24 h. ${ }^{b}$ Yields were determined by GC analysis using dodecane as the internal standard. 
Table S13. B2pin2-loading evaluation ${ }^{a}$

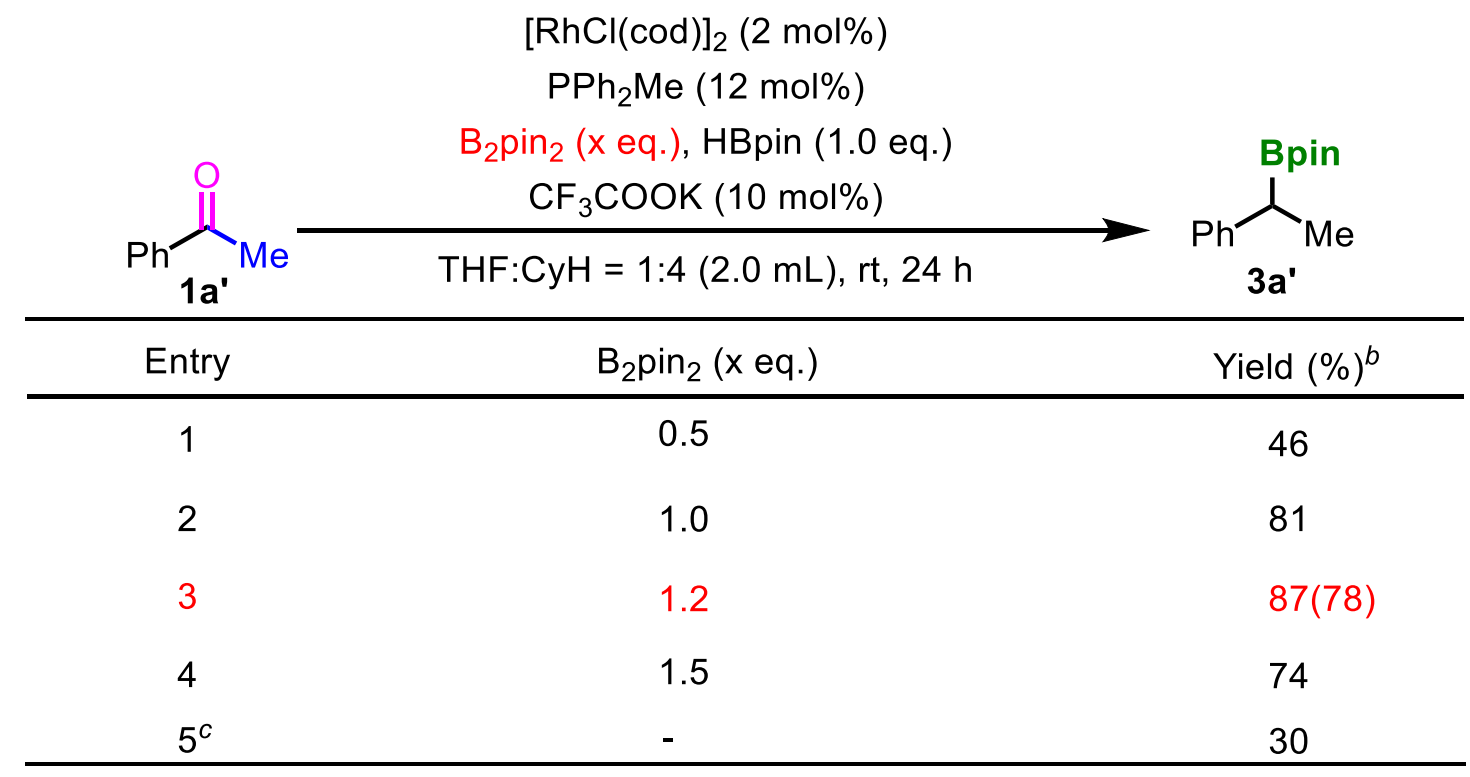

${ }^{a}$ Conditions : 1a' (0.3 mmol), [Rh(cod)Cl]2 (2 mol\%), PPh $2 \mathrm{Me}(12 \mathrm{~mol} \%), \mathrm{CF}_{3} \mathrm{COOK}(10$ mol\%), B2pin2 (x equiv), HBpin (1.0 equiv), THF:CyH = 1:4 $(2.0 \mathrm{~mL}), \mathrm{rt}, 24 \mathrm{~h} .{ }^{b}$ Yields were determined by GC analysis using dodecane as the internal standard, isolated yield in parentheses. ${ }^{c} \mathrm{HB}$ pin (2.2 eq.), no B2pin2.

Table S14. HBpin-loading evaluation ${ }^{a}$

\begin{tabular}{|c|c|c|}
\hline & $\begin{array}{c}{[\mathrm{RhCl}(\mathrm{cod})]_{2}(2 \mathrm{~mol} \%)} \\
\mathrm{PPh}_{2} \mathrm{Me}(12 \mathrm{~mol} \%) \\
\mathrm{B}_{2} \mathrm{pin}_{2}(1.2 \text { eq. }), \mathrm{HBpin}(\mathrm{x} \text { eq. }) \\
\mathrm{CF}_{3} \mathrm{COOK}(10 \mathrm{~mol} \%)\end{array}$ & ${ }^{\text {Bpin }}$ \\
\hline $\mathrm{Ph}^{\prime} \mathrm{a}^{\prime}$ & THF:CyH = 1:4 (2.0 mL), rt, $24 \mathrm{~h}$ & $3 \mathbf{a}^{\prime}$ \\
\hline Entry & HBpin (x eq.) & Yield $(\%)^{b}$ \\
\hline 1 & 2.0 & 76 \\
\hline 2 & 1.5 & 76 \\
\hline 3 & 1.2 & 84 \\
\hline 4 & 1.0 & $87(78)$ \\
\hline 5 & 0.5 & 42 \\
\hline
\end{tabular}

aConditions : 1a' $(0.3 \mathrm{mmol}),[\mathrm{Rh}(\mathrm{cod}) \mathrm{Cl}]_{2}(2 \mathrm{~mol} \%), \mathrm{PPh}_{2} \mathrm{Me}(12 \mathrm{~mol} \%), \mathrm{CF}_{3} \mathrm{COOK}(10$ mol\%), B2pinz (1.2 equiv), HBpin (x equiv), THF:CyH = 1:4 $(2.0 \mathrm{~mL}), \mathrm{rt}, 24 \mathrm{~h} .{ }^{b}$ Yields were determined by GC analysis using dodecane as the internal standard, isolated yield in parentheses. 


\subsection{Synthesis of 1,1,2-triboronates from ketones}

\section{Table S15. Solvent evaluation ${ }^{a}$}

\begin{tabular}{|c|c|c|}
\hline & $\begin{array}{c}{[\mathrm{RhCl}(\mathrm{cod})]_{2}(2 \mathrm{~mol} \%)} \\
\mathrm{P}\left({ }^{n} \mathrm{Bu}\right)_{3}(12 \mathrm{~mol} \%) \\
\mathrm{B}_{2} \operatorname{pin}_{2}(4.0 \text { eq. }),{ }^{i} \operatorname{PrOH}(1.0 \text { eq. }) \\
\end{array}$ & Ppir $^{\text {Bpin }}$ \\
\hline & $\mathrm{NaOAc}(50 \mathrm{~mol} \%), \mathrm{S}(2.0 \mathrm{~mL}), 70^{\circ} \mathrm{C}, 12 \mathrm{~h}$ & 4a Bpin \\
\hline Entry & Solvent $(2.0 \mathrm{~mL})$ & Yield $(\%)^{b}$ \\
\hline 1 & THF & NR \\
\hline 2 & СуH & 55 \\
\hline 3 & hexane & 59 \\
\hline 4 & CPME & 40 \\
\hline 5 & IPE & 46 \\
\hline 6 & MTBE & 21 \\
\hline 7 & DME & 15 \\
\hline
\end{tabular}

${ }^{a}$ Conditions : 1a $(0.3 \mathrm{mmol}),[\mathrm{Rh}(\mathrm{cod}) \mathrm{Cl}]_{2}(2 \mathrm{~mol} \%), \mathrm{P}\left({ }^{n} \mathrm{Bu}\right) 3(12 \mathrm{~mol} \%), \mathrm{NaOAc}(50 \mathrm{~mol} \%)$, B2pin2 (4.0 equiv), ${ }^{i} \mathrm{PrOH}$ (1.0 equiv), Solvent $(2.0 \mathrm{~mL}), 70^{\circ} \mathrm{C}, 12 \mathrm{~h} .{ }^{a}$ Yields were determined by GC analysis using dodecane as the internal standard. $\mathrm{CyH}=$ cyclohexane, $\mathrm{CPME}=$ methoxycyclopentane, $\mathrm{MTBE}=2$-methoxy-2-methylpropane, $\mathrm{DME}=1$,2-dimethoxyethane, $\mathrm{IPE}=2$-isopropoxypropane.

\section{Table S16. Base evaluation ${ }^{a}$}

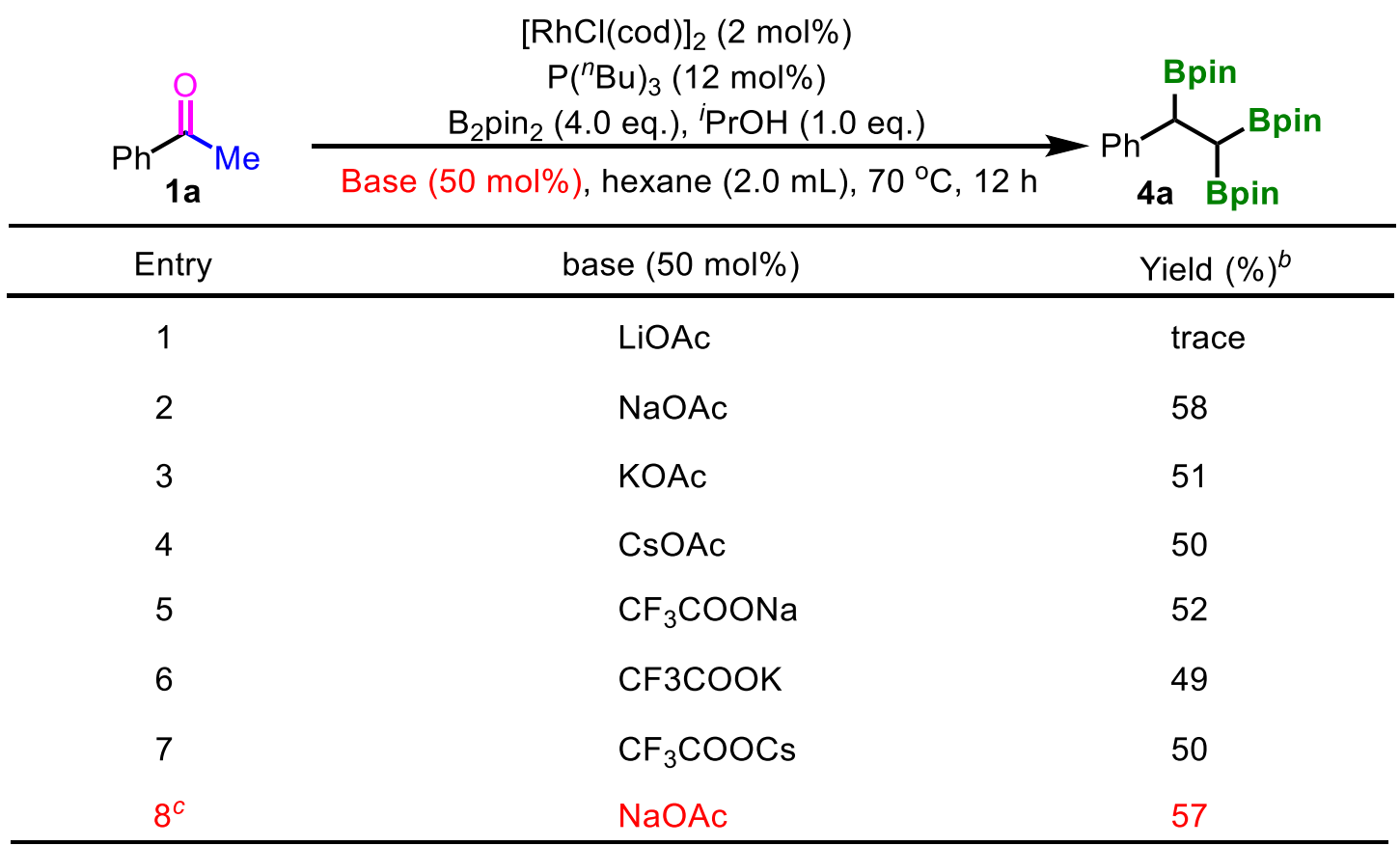


${ }^{a}$ Conditions : 1a $(0.3 \mathrm{mmol}),[\mathrm{Rh}(\mathrm{cod}) \mathrm{Cl}]_{2}(2 \mathrm{~mol} \%), \mathrm{P}\left({ }^{n} \mathrm{Bu}\right) 3(12 \mathrm{~mol} \%), \mathrm{NaOAc}(50 \mathrm{~mol} \%)$, B 2 pin 2 (x equiv), $\mathrm{PrOH}$ (1.0 equiv), hexane $(2.0 \mathrm{~mL}), 70{ }^{\circ} \mathrm{C}, 12 \mathrm{~h} .{ }^{b}$ Yields were determined by GC analysis using dodecane as the internal standard. ${ }^{c} \mathrm{No}{ }^{i} \mathrm{PrOH}$.

Table S17. B2pin2-loading evaluation ${ }^{a}$

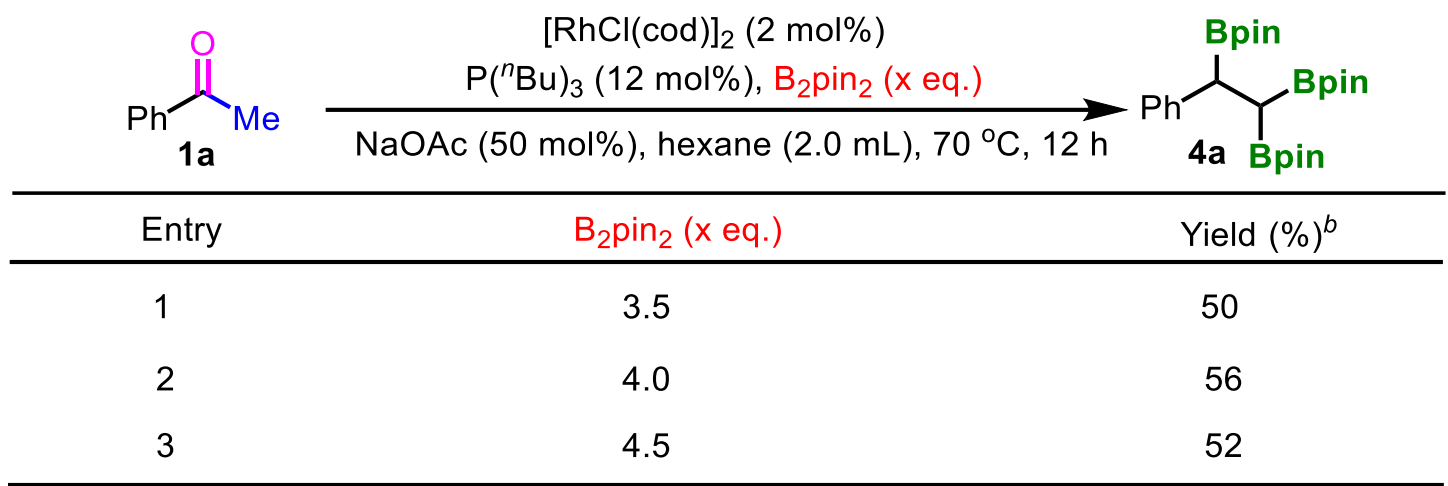

${ }^{a}$ Conditions : 1a $(0.3 \mathrm{mmol}),[\mathrm{Rh}(\mathrm{cod}) \mathrm{Cl}]_{2}(2 \mathrm{~mol} \%), \mathrm{P}\left({ }^{n} \mathrm{Bu}\right) 3(12 \mathrm{~mol} \%), \mathrm{NaOAc}(50 \mathrm{~mol} \%)$, $\mathrm{B}_{2}$ pinz (x equiv), hexane $(2.0 \mathrm{~mL}), 70^{\circ} \mathrm{C}, 12 \mathrm{~h} .{ }^{a}$ Yields were determined by GC analysis using dodecane as the internal standard.

Table S18. Base-loading evaluation ${ }^{a}$

\begin{tabular}{|c|c|c|}
\hline ha & $\begin{array}{c}{[\mathrm{RhCl}(\mathrm{cod})]_{2}\left(2 \mathrm{~mol}^{\%}\right)} \\
\mathrm{P}\left({ }^{n} \mathrm{Bu}\right)_{3}(12 \mathrm{~mol} \%), \mathrm{B}_{2} \mathrm{pin}_{2}(4.0 \text { eq. }) \\
\text { NaOAc }(x \mathrm{~mol} \%), \text { hexane }(2.0 \mathrm{~mL}), 70^{\circ} \mathrm{C}, 12 \mathrm{~h}\end{array}$ & $\overbrace{4 a}^{B p i n} B p$ \\
\hline Entry & $\mathrm{NaOAc}(\mathrm{x} \mathrm{mol} \%)$ & Yield $(\%)^{b}$ \\
\hline 1 & 0 & NR \\
\hline 2 & $10 \mathrm{~mol} \%$ & 34 \\
\hline 3 & $50 \mathrm{~mol} \%$ & 56 \\
\hline 4 & $100 \mathrm{~mol} \%$ & 60 \\
\hline 5 & $150 \mathrm{~mol} \%$ & 65 \\
\hline 6 & $200 \mathrm{~mol} \%$ & 64 \\
\hline
\end{tabular}

${ }^{a}$ Conditions : 1a $(0.3 \mathrm{mmol}),[\mathrm{Rh}(\mathrm{cod}) \mathrm{Cl}]_{2}(2 \mathrm{~mol} \%), \mathrm{P}\left({ }^{n} \mathrm{Bu}\right)_{3}(12 \mathrm{~mol} \%), \mathrm{NaOAc}\left(\mathrm{x} \mathrm{mol}^{2}\right)$, B2pin2 (4.0 equiv), hexane $(2.0 \mathrm{~mL}), 70{ }^{\circ} \mathrm{C}, 12 \mathrm{~h}$. ${ }^{a}$ Yields were determined by GC analysis using dodecane as the internal standard. 
Table S19. Solvent-loading evaluation ${ }^{a}$

\begin{tabular}{|c|c|c|}
\hline $\mathrm{Ph}_{1}$ & $\begin{array}{l}{[\mathrm{RhCl}(\mathrm{cod})]_{2}(2 \mathrm{~mol} \%)} \\
\mathrm{P}\left({ }^{n} \mathrm{Bu}\right)_{3}(12 \mathrm{~mol} \%) \\
\mathrm{B}_{2} \mathrm{pin}_{2}(4.0 \text { eq. }), \mathrm{NaOAc}(150 \mathrm{~mol} \%) \\
\text { hexane }(\mathrm{x} \mathrm{mL}), 70^{\circ} \mathrm{C}, 12 \mathrm{~h}\end{array}$ & $\underbrace{\text { Bpin }}_{\text {Bpin }}$ \\
\hline Entry & hexane $(x \mathrm{~mL})$ & Yield $(\%)^{b}$ \\
\hline 1 & $1.0 \mathrm{~mL}$ & 62 \\
\hline 2 & $2.0 \mathrm{~mL}$ & 66 \\
\hline 3 & $3.0 \mathrm{~mL}$ & $72(66)$ \\
\hline
\end{tabular}

${ }^{a}$ Conditions : 1a $(0.3 \mathrm{mmol}),[\mathrm{Rh}(\mathrm{cod}) \mathrm{Cl}]_{2}(2 \mathrm{~mol} \%), \mathrm{P}\left({ }^{n} \mathrm{Bu}\right) 3(12 \mathrm{~mol} \%), \mathrm{NaOAc}(150 \mathrm{~mol} \%)$, B2pinz (4.0 equiv), hexane $(x \mathrm{~mL}), 70{ }^{\circ} \mathrm{C}, 12 \mathrm{~h}$. ${ }^{a}$ Yields were determined by GC analysis using dodecane as the internal standard, isolated yield in parentheses.

Table S20. Reaction temperature evaluation ${ }^{a}$

\begin{tabular}{|c|c|c|}
\hline $\mathrm{Ph}_{1}$ & $\begin{array}{l}{[\mathrm{RhCl}(\mathrm{cod})]_{2}(2 \mathrm{~mol} \%)} \\
\mathrm{P}\left({ }^{n} \mathrm{Bu}\right)_{3}(12 \mathrm{~mol} \%) \\
\mathrm{B}_{2} \mathrm{pin}_{2}(4.0 \mathrm{eq} .), \mathrm{NaOAc}(150 \mathrm{~mol} \%) \\
\text { hexane }(2 \mathrm{~mL}), \mathrm{T}, 12 \mathrm{~h}\end{array}$ & $\overbrace{4 a}^{B p i n} B$ Bpin \\
\hline Entry & $\mathrm{T}\left(\mathrm{x}^{\circ} \mathrm{C}\right)$ & Yield $(\%)^{b}$ \\
\hline 1 & 50 & 60 \\
\hline 2 & 70 & 72 \\
\hline 3 & 90 & 57 \\
\hline
\end{tabular}

${ }^{a}$ Conditions : 1a (0.3 mmol), [Rh(cod)Cl] 2 (2 mol\%), $\mathrm{P}\left({ }^{n} \mathrm{Bu}\right) 3$ (12 mol\%), NaOAc (150 mol\%), $\mathrm{B}_{2}$ pin 2 (4.0 equiv), hexane $(3 \mathrm{~mL}), \mathrm{T}^{\circ} \mathrm{C}, 12 \mathrm{~h}$. ${ }^{a}$ Yields were determined by GC analysis using dodecane as the internal standard, isolated yield in parentheses. 


\section{Synthesis of Substrates}

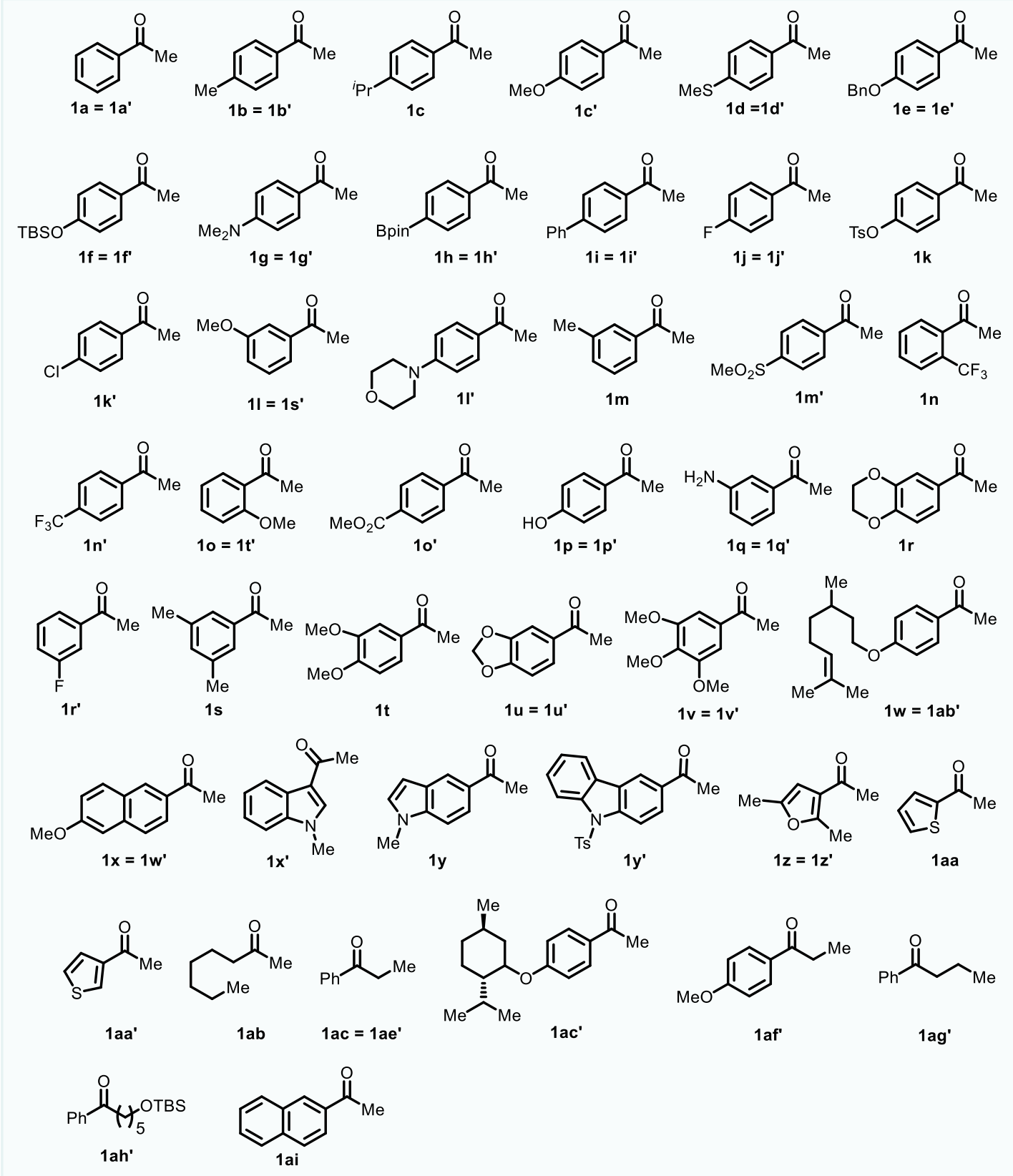

Ketones 1a-1d, 1g, 1i-ij, 1k'-1v', 1l-1v, 1x, 1z-1ac, 1z'-1aa', 1ae'-1ag', 1ai were purchased from Energy Chemicals Inc. and used as received. Ketones $\mathbf{1} \mathbf{e}^{[2]}, \mathbf{1} \mathbf{f}^{[2]}$, $1 \mathbf{h}^{[2]}, \mathbf{1} \mathbf{k}^{[3]}, \mathbf{1} \mathbf{w}^{[2]}, \mathbf{1} \mathbf{y}^{[2]}, \mathbf{1} \mathbf{x}^{[2]}, \mathbf{1} \mathbf{y}^{[5]}, \mathbf{1 a c}{ }^{[4]}, \mathbf{1} \mathbf{a h}^{[2]}$ were prepared according to the reported procedure. 

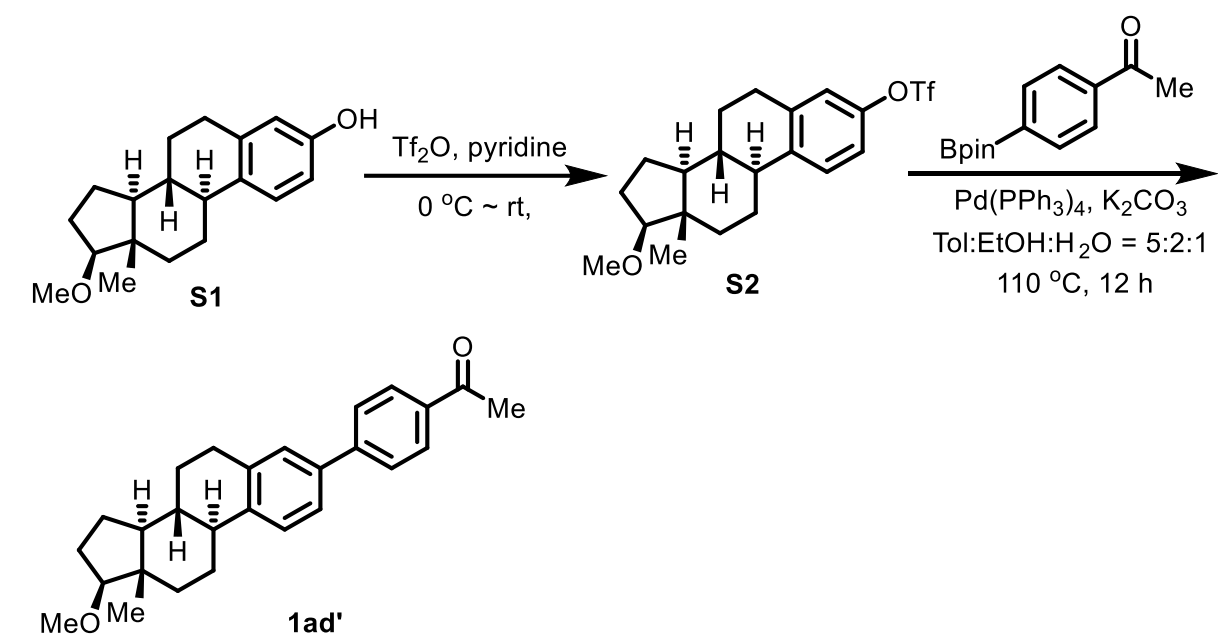

A flame-dried flask was successively charged with estradiol S1 (1.0 eq, 3.0 $\mathrm{mmol})$, pyridine $(2.0 \mathrm{eq}, 6.0 \mathrm{mmol})$, and dry DCM $(20 \mathrm{~mL})$, followed by the addition of a solution of triflic anhydride dropwise (1.2 eq, $3.6 \mathrm{mmol}$ ) in DCM $(5 \mathrm{~mL})$ at $0^{\circ} \mathrm{C}$. The solution was then warmed up to room temperature and stirred for 12 hours. The reaction mixture was quenched with $\mathrm{H}_{2} \mathrm{O}(50 \mathrm{~mL})$, and was extracted with EtOAc, dried with $\mathrm{Na}_{2} \mathrm{SO}_{4}$, filtered and concentrated. The residue was purified by silica gel flash chromatography to afford the product S2 (1.07 g, 86\% yield).

To a solution of $\mathbf{S 2}$ (1.0 eq, $2.0 \mathrm{mmol})$, 1-(4-(4,4,5,5-tetramethyl-1,3,2dioxaborolan-2-yl)phenyl)ethan-1-one 1 h (1.0 eq, $2.0 \mathrm{mmol}), \mathrm{Pd}\left(\mathrm{PPh}_{3}\right)_{4}(5 \mathrm{~mol} \%$, $0.1 \mathrm{mmol}), \mathrm{K}_{2} \mathrm{CO}_{3}(3.5 \mathrm{eq}, 7.0 \mathrm{mmol})$ in toluene $(12.5 \mathrm{~mL})$ was added $\mathrm{EtOH}(5.0$ $\mathrm{mL})$ and $\mathrm{H}_{2} \mathrm{O}(2.5 \mathrm{~mL})$. The resulting mixture was then heated up to $110{ }^{\circ} \mathrm{C}$ and stirred for 12 hours. After cooled to room temperature, the mixture was quenched with saturated $\mathrm{NH}_{4} \mathrm{Cl}$, extracted with EtOAc, dried with $\mathrm{Na}_{2} \mathrm{SO}_{4}$, filtered and concentrated. The residue was purified by silica gel flash chromatography to afford 1ad' as a white solid in 78\% yield (605 mg, petroleum ether: $\mathrm{EtOAc}=20: 1)$.

$\mathbf{R}_{f}=0.45$ (petroleum ether : EtOAc $=5: 1$ ).

${ }^{1} \mathrm{H}$ NMR (400 MHz, $\left.\mathrm{CDCl}_{3}\right) \delta 8.01(\mathrm{~d}, J=8.1 \mathrm{~Hz}, 2 \mathrm{H}), 7.67(\mathrm{~d}, J=8.4 \mathrm{~Hz}, 2 \mathrm{H})$, $7.44-7.38(\mathrm{~m}, 2 \mathrm{H}), 7.36(\mathrm{~s}, 1 \mathrm{H}), 3.39(\mathrm{~s}, 3 \mathrm{H}), 3.34(\mathrm{t}, J=8.3 \mathrm{~Hz}, 1 \mathrm{H}), 2.99-2.91$ $(\mathrm{m}, 2 \mathrm{H}), 2.63(\mathrm{~s}, 3 \mathrm{H}), 2.41-2.25(\mathrm{~m}, 2 \mathrm{H}), 2.15-2.04(\mathrm{~m}, 2 \mathrm{H}), 1.98-1.90(\mathrm{~m}, 1 \mathrm{H})$, $1.77-1.69(\mathrm{~m}, 1 \mathrm{H}), 1.60-1.34(\mathrm{~m}, 6 \mathrm{H}), 1.30-1.20(\mathrm{~m}, 1 \mathrm{H}), 0.81(\mathrm{~s}, 3 \mathrm{H})$. 
${ }^{13} \mathrm{C}$ NMR (100 MHz, $\left.\mathrm{CDCl}_{3}\right) \delta$ 197.9, 145.8, 140.9, 137.5, 137.2, 135.7, 129.0, 127.9, 127.1, 126.2, 124.6, 90.8, 58.0, 50.5, 44.5, 43.3, 38.4, 38.1, 29.8, 27.8, 27.3, 26.8, 26.4, 23.2, 11.7.

HRMS ( $\mathrm{APCl}^{+}$) calcd for $\mathrm{C}_{27} \mathrm{H}_{33} \mathrm{O}_{2}{ }^{+}[\mathrm{M}+\mathrm{H}]^{+}: 389.2475$, found: 389.2480.

IR (neat, cm ${ }^{-1}$ ): 2926, 2862, 1678, 1602, 1356, 1264, 1107, 961, 823, 594.

\section{Synthesis of ligand $\left({ }^{t} \mathrm{BuC}_{6} \mathrm{H}_{4}\right)_{2} \mathrm{PMe}$}

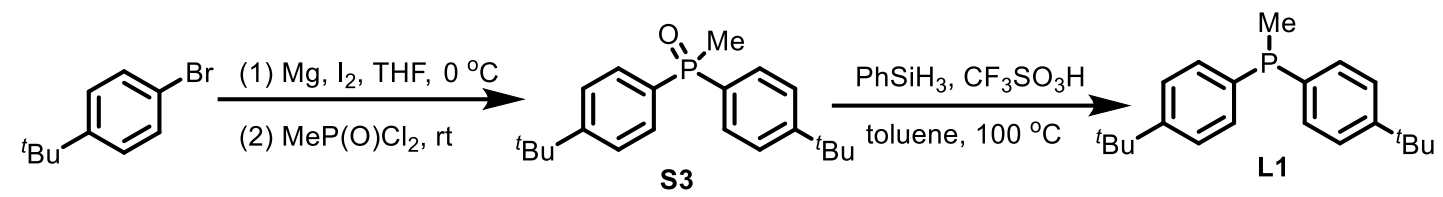

To a solution of magnesium turnings (395 $\mathrm{mg}, 16.5 \mathrm{mmol}$ ) and a catalytic amount of $\mathrm{I}_{2}$ in anhydrous THF $(10 \mathrm{~mL})$ at $0{ }^{\circ} \mathrm{C}$ was added 1-bromo-4-tertbutylbenzene ( $3.5 \mathrm{~g}, 16.5 \mathrm{mmol})$ dropwise. The solution was then warmed up to $40{ }^{\circ} \mathrm{C}$ and stirred for 2 hours. After cooled to room temperature, methylphosphonic dichloride $(665 \mathrm{mg}, 5.0 \mathrm{mmol})$ in dry THF $(10 \mathrm{~mL})$ was added dropwise and stirred for an additional 2 hours. The reaction mixture was quenched with $1 \mathrm{M} \mathrm{HCl}(15 \mathrm{~mL})$, extracted with EtOAc, dried with $\mathrm{Na}_{2} \mathrm{SO}_{4}$, filtered and concentrated. The residue was purified by silica gel flash chromatography to afford phosphine oxide S3 as a white solid in $85 \%$ yield (1.42 g, DCM: $\mathrm{MeOH}=20: 1)$.

$\mathbf{R}_{f}=0.35$ (DCM: $\left.\mathrm{MeOH}=20: 1\right)$.

${ }^{1} \mathrm{H}$ NMR (400 MHz, $\left.\mathrm{CDCl}_{3}\right) \delta 7.68-7.62(\mathrm{~m}, 4 \mathrm{H}), 7.50-7.44(\mathrm{~m}, 4 \mathrm{H}), 1.98(\mathrm{~d}, J$ $=13.1 \mathrm{~Hz}, 3 \mathrm{H}), 1.31(\mathrm{~s}, 18 \mathrm{H})$.

${ }^{13} \mathrm{C}$ NMR (100 MHz, $\left.\mathrm{CDCl}_{3}\right) \delta 155.1$ (d, $\left.J=2.8 \mathrm{~Hz}\right), 131.5,130.4(\mathrm{~d}, J=10.1 \mathrm{~Hz})$, $125.6(\mathrm{~d}, J=12.1 \mathrm{~Hz}), 35.0,31.2,16.9(\mathrm{~d}, J=73.6 \mathrm{~Hz})$.

31P NMR (162 MHz, $\left.\mathrm{CDCl}_{3}\right) \delta 29.7$.

HRMS (ESI ${ }^{+}$) calcd for $\mathrm{C}_{21} \mathrm{H}_{29} \mathrm{OPNa}^{+}[\mathrm{M}+\mathrm{Na}]^{+}:$351.1848, found: 351.1844 .

IR (neat, cm ${ }^{-1}$ ): 2972, 2856, 1657, 1574, 1377, 1321, 1269, 1134, 1023, 845, 726.

Following the literature procedure ${ }^{[6]}$, a flame-dried flask was charged with $\mathrm{CF}_{3} \mathrm{SO}_{3} \mathrm{H} \quad(0.3 \mathrm{mmol}, 15 \mathrm{~mol} \%)$ and toluene $(8.0 \mathrm{~mL})$. The methyldiphenylphosphine oxide $(656 \mathrm{mg}, 2.0 \mathrm{mmol})$ was added and the solution was stirred for 5 minutes at room temperature. After adding the $\mathrm{PhSiH}_{3}(864 \mathrm{mg}, 8 \mathrm{mmol})$, the reaction mixture was stirred for $24 \mathrm{~h}$ at $100{ }^{\circ} \mathrm{C}$. 
After cooled to room temperature, the mixture was quenched with saturated $\mathrm{NH}_{4} \mathrm{Cl}$, extracted with EtOAc, dried with $\mathrm{Na}_{2} \mathrm{SO}_{4}$, filtered and concentrated. The residue was purified by silica gel flash chromatography to afford $\left({ }^{t} \mathrm{BuC}_{6} \mathrm{H}_{4}\right)_{2} \mathrm{PMe} \mathbf{L 1}$ as a white solid in $74 \%$ yield $(461 \mathrm{mg}$, petroleum ether: $\mathrm{EtOAc}=30: 1)$.

$\mathbf{R}_{f}=0.45$ (petroleum ether : EtOAc $=20: 1$ ).

${ }^{1} \mathrm{H}$ NMR (400 MHz, $\left.\mathrm{CDCl}_{3}\right) 7.37$ (d, J=4.3 Hz, 8H), $1.62(\mathrm{~d}, J=3.3 \mathrm{~Hz}, 3 \mathrm{H}), 1.31$ (s, 18H).

${ }^{13} \mathrm{C}$ NMR (100 MHz, $\left.\mathrm{CDCl}_{3}\right) \delta$ 151.4, 136.7 (d, J = 9.7 Hz), 132.0 (d, J = 16.7 Hz), $125.5(\mathrm{~d}, J=6.5 \mathrm{~Hz}), 34.7,31.4,12.8(\mathrm{~d}, J=13.0 \mathrm{~Hz})$.

${ }^{31 P}$ NMR (162 MHz, $\left.\mathrm{CDCl}_{3}\right) \delta-29.8$.

Spectral data matched those previously reported. ${ }^{[7]}$

\section{General Procedures for Synthesis of Alkylboronates}

\section{General procedure for the synthesis of linear alkylboronates}

\section{General Procedure 1 (GP1)}

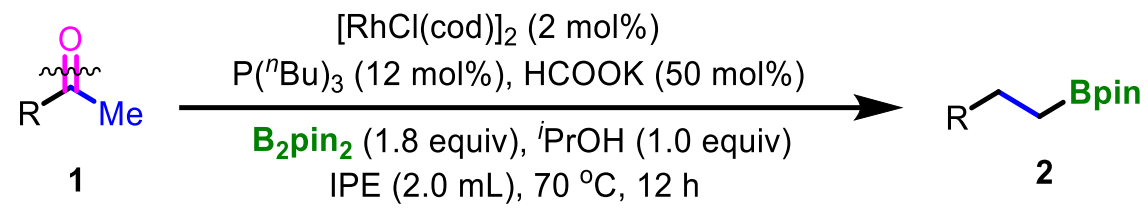

In a glove box, to an oven-dried $10-\mathrm{mL}$ vial were added $[\mathrm{RhCl}(\mathrm{cod})]_{2}(4.9$ $\mathrm{mg}, 0.01 \mathrm{mmol}), \mathrm{P}\left({ }^{n} \mathrm{Bu}\right)_{3}(12.1 \mathrm{mg}, 0.06 \mathrm{mmol})$, and IPE $(1.0 \mathrm{~mL})$. The resulting solution was stirred for $30 \mathrm{~min}$. Then, HCOOK (21.0 mg, $0.25 \mathrm{mmol}), \mathrm{B}_{2}$ pin 2 (228.6 mg, $0.90 \mathrm{mmol})$, and ${ }^{i} \mathrm{PrOH}(38.1 \mathrm{mg}, 0.50 \mathrm{mmol})$ were added, and the resulting mixture was stirred for another $30 \mathrm{~min}$. Ketone $\mathbf{1}$ in IPE (1.0 mL) was added. The reaction mixture was sealed, removed from the glovebox and stirred at $70{ }^{\circ} \mathrm{C}$ for the duration of time indicated. After cooled to room temperature, the mixture was concentrated under vacuo and purified by silica gel chromatography using petroleum ether : EtOAc as the eluent to afford the desired products 2 .

\section{General Procedure 2 (GP2)}




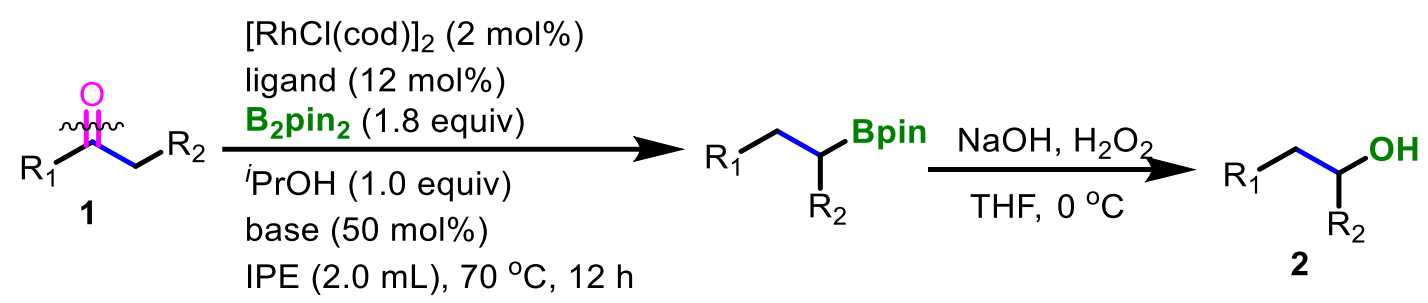

In a glove box, to an oven-dried $10-\mathrm{mL}$ vial were added $[\mathrm{RhCl}(\operatorname{cod})]_{2}(4.9$ $\mathrm{mg}, 0.01 \mathrm{mmol})$, ligand $(0.06 \mathrm{mmol})$, and IPE $(1.0 \mathrm{~mL})$. The resulting solution was stirred for $30 \mathrm{~min}$. Then, Base $(0.25 \mathrm{mmol}), \mathrm{B}_{2}$ pin $2(228.6 \mathrm{mg}, 0.90 \mathrm{mmol})$, and ${ }^{\mathrm{P} O \mathrm{PH}}(38.1 \mathrm{mg}, 0.50 \mathrm{mmol})$ were added, and the resulting mixture was stirred for another $30 \mathrm{~min}$. Ketone $\mathbf{1}$ in IPE $(1.0 \mathrm{~mL})$ was added. The reaction mixture was sealed, removed from the glovebox and stirred at $70{ }^{\circ} \mathrm{C}$ for the duration of time indicated. After cooled to room temperature, the reaction mixture was concentrated in vacuo, to the stirred solution of the crude residue in THF $(1.0 \mathrm{~mL})$ was added $\mathrm{NaOH}\left(1.5\right.$ equiv, $3.0 \mathrm{M}$ ) and $\mathrm{H}_{2} \mathrm{O}_{2}$ (1.5 equiv, 30\%) at $0{ }^{\circ} \mathrm{C}$. The reaction mixture was stirred for 0.5 hour, then extracted with EtOAc, dried with $\mathrm{Na}_{2} \mathrm{SO}_{4}$, filtered and concentrated. The residue was purified by silica gel chromatography using petroleum ether : EtOAc as the eluent to afford the desired products 2 .

\section{4,4,5,5-Tetramethyl-2-phenethyl-1,3,2-dioxaborolane (2a).}

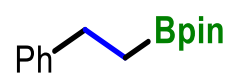

Compound 2a was prepared following the GP1 using acetophenone 1a (60.0 $\mathrm{mg}, 0.5 \mathrm{mmol})$, [RhCl(cod) $]_{2}(4.9 \mathrm{mg}, 0.01 \mathrm{mmol}), \mathrm{P}\left({ }^{n} \mathrm{Bu}\right)_{3}(12.1 \mathrm{mg}, 0.06 \mathrm{mmol})$, B2pine (228.6 mg, $0.90 \mathrm{mmol})$, $\mathrm{PrOH}(38.1 \mathrm{mg}, 0.50 \mathrm{mmol})$, HCOOK (21.0 mg, $0.25 \mathrm{mmol})$ and IPE $(2.0 \mathrm{~mL})$. The product was obtained as a colourless oil in $73 \%$ yield $(84.7 \mathrm{mg}$, eluent : petroleum ether : EtOAc $=40: 1)$. $\mathrm{R}_{f}=0.50$ (petroleum ether : $\mathrm{EtOAc}=20: 1$ ).

${ }^{1} \mathrm{H}$ NMR $\left(400 \mathrm{MHz}, \mathrm{CDCl}_{3}\right) \delta$ 8 $7.19-7.11(\mathrm{~m}, 4 \mathrm{H}), 7.11-7.02(\mathrm{~m}, 1 \mathrm{H}), 2.66(\mathrm{t}, J=$ $8.3 \mathrm{~Hz}, 2 \mathrm{H}), 1.13(\mathrm{~s}, 12 \mathrm{H}), 1.05(\mathrm{t}, J=8.3 \mathrm{~Hz}, 2 \mathrm{H})$.

${ }^{13} \mathrm{C}$ NMR (100 MHz, $\left.\mathrm{CDCl}_{3}\right) \delta$ 144.6, 128.3, 128.1, 125.6, 83.2, 30.1, 25.0. The carbon signal attached to B was not observed due to low intensity. Spectral data matched those previously reported. ${ }^{[8]}$ 
4,4,5,5-Tetramethyl-2-(4-methylphenethyl)-1,3,2-dioxaborolane (2b).<smiles>Cc1ccc(CCCc2ccccc2)cc1</smiles>

Compound $\mathbf{2} \mathbf{b}$ was prepared following the GP1 using 1-( $p$-tolyl)ethan-1-one $\mathbf{1 b}$ (67.0 mg, $0.5 \mathrm{mmol}),[\mathrm{RhCl}(\operatorname{cod})]_{2}(4.9 \mathrm{mg}, 0.01 \mathrm{mmol}), \mathrm{P}\left({ }^{n} \mathrm{Bu}\right) 3(12.1 \mathrm{mg}, 0.06$ $\mathrm{mmol}), B_{2}$ pin2 (228.6 mg, $\left.0.90 \mathrm{mmol}\right),{ }^{i} \mathrm{PrOH}$ (38.1 mg, $\left.0.50 \mathrm{mmol}\right), \mathrm{HCOOK}$ $(21.0 \mathrm{mg}, 0.25 \mathrm{mmol})$ and IPE $(2.0 \mathrm{~mL})$. The product was obtained as a colourless oil in $67 \%$ yield $(82.4 \mathrm{mg}$, eluent : petroleum ether : EtOAc $=40: 1)$.

$\mathrm{R}_{f}=0.51$ (petroleum ether $: \mathrm{EtOAc}=20: 1$ ).

${ }^{1} \mathrm{H}$ NMR (400 MHz, $\left.\mathrm{CDCl}_{3}\right) \delta 7.07-6.97(\mathrm{~m}, 4 \mathrm{H}), 2.64(\mathrm{t}, J=8.4 \mathrm{~Hz}, 2 \mathrm{H}), 2.24(\mathrm{~s}$, $3 \mathrm{H}), 1.16(\mathrm{~s}, 12 \mathrm{H}), 1.06(\mathrm{t}, J=8.5 \mathrm{~Hz}, 2 \mathrm{H})$.

${ }^{13} \mathrm{C}$ NMR (100 MHz, $\left.\mathrm{CDCl}_{3}\right) \delta 141.5,135.0,129.0,128.0,83.2,29.6,24.9,21.1$. The carbon signal attached to B was not observed due to low intensity.

Spectral data matched those previously reported. ${ }^{[8]}$

2-(4-Isopropylphenethyl)-4,4,5,5-tetramethyl-1,3,2-dioxaborolane (2c).<smiles>CCCc1ccc(CC[B]c2ccccc2)cc1</smiles>

Compound 2c was prepared following the GP1 using 1-(4isopropylphenyl)ethan-1-one $1 \mathrm{c}(81.0 \mathrm{mg}, 0.5 \mathrm{mmol})$, [ $\mathrm{RhCl}(\mathrm{cod})]_{2}(4.9 \mathrm{mg}, 0.01$ $\mathrm{mmol}), \mathrm{P}\left({ }^{n} \mathrm{Bu}\right){ }_{3}(12.1 \mathrm{mg}, 0.06 \mathrm{mmol}), \mathrm{B}_{2} \mathrm{pin}_{2}(228.6 \mathrm{mg}, 0.90 \mathrm{mmol}),{ }^{2} \mathrm{PrOH}(38.1$ $\mathrm{mg}, 0.50 \mathrm{mmol})$, HCOOK (21.0 mg, $0.25 \mathrm{mmol})$ and IPE (2.0 mL). The product was obtained as a colourless oil in $59 \%$ yield ( $80.8 \mathrm{mg}$, eluent : petroleum ether : $\mathrm{EtOAc}=40: 1)$.

$\mathbf{R}_{f}=0.56$ (petroleum ether : $\mathrm{EtOAC}=20: 1$ ).

${ }^{1} \mathrm{H}$ NMR (400 MHz, $\left.\mathrm{CDCl}_{3}\right) \delta 77.19-7.09(\mathrm{~m}, 4 \mathrm{H}), 2.93-2.82(\mathrm{~m}, 1 \mathrm{H}), 2.72(\mathrm{t}$, $J=7.4 \mathrm{~Hz}, 2 \mathrm{H}), 1.24(\mathrm{~d}, J=7.0 \mathrm{~Hz}, 6 \mathrm{H}), 1.23(\mathrm{~s}, 12 \mathrm{H}), 1.13(\mathrm{t}, J=7.4 \mathrm{~Hz}, 2 \mathrm{H})$.

${ }^{13} \mathrm{C}$ NMR (100 MHz, $\mathrm{CDCl}_{3}$ ) $\delta$ 146.1, 141.9, 128.0, 126.3, 83.2, 33.8, 29.6, 24.9, 24.2.

The carbon signal attached to $B$ was not observed due to low intensity.

HRMS (ESI') calcd for $\mathrm{C}_{17} \mathrm{H}_{27} \mathrm{BO}_{2} \mathrm{Na}^{+}[\mathrm{M}+\mathrm{Na}]^{+}:$297.1996, found: 297.1997.

IR (neat, cm$^{-1}$ ): 2963, 2931, 2872, 1683, 1377, 1327, 1141, 971, 835. 
<smiles>CSc1ccc(CCCc2ccccc2)cc1</smiles>

Compound 2d was prepared following the GP1 using 1-(4(methylthio)phenyl)ethan-1-one $1 \mathrm{~d}(83.0 \mathrm{mg}, 0.5 \mathrm{mmol})$, [ $\mathrm{RhCl}(\mathrm{cod})]_{2}(4.9 \mathrm{mg}$, $0.01 \mathrm{mmol}), \mathrm{P}\left({ }^{n} \mathrm{Bu}\right)_{3}(12.1 \mathrm{mg}, 0.06 \mathrm{mmol}), \mathrm{B}_{2} \mathrm{pin}_{2}(228.6 \mathrm{mg}, 0.90 \mathrm{mmol}),{ }^{\mathrm{P}} \mathrm{PrOH}$ (38.1 mg, $0.50 \mathrm{mmol})$, HCOOK (21.0 mg, $0.25 \mathrm{mmol})$ and IPE $(2.0 \mathrm{~mL})$. The product was obtained as a colourless oil in $73 \%$ yield $(101.5 \mathrm{mg}$, eluent : petroleum ether : $\mathrm{EtOAc}=40: 1)$.

$\mathbf{R}_{f}=0.46$ (petroleum ether : $\mathrm{EtOAc}=20: 1$ ).

${ }^{1} \mathrm{H}$ NMR (400 MHz, $\left.\mathrm{CDCl}_{3}\right) \delta 7.21-7.12(\mathrm{~m}, 4 \mathrm{H}), 2.70(\mathrm{t}, J=8.4 \mathrm{~Hz}, 2 \mathrm{H}), 2.45(\mathrm{~s}$, $3 \mathrm{H}), 1.22(\mathrm{~s}, 12 \mathrm{H}), 1.12(\mathrm{t}, J=8.3 \mathrm{~Hz}, 2 \mathrm{H})$.

${ }^{13} \mathrm{C}$ NMR (100 MHz, $\left.\mathrm{CDCl}_{3}\right) \delta 141.8,134.9,128.7,127.3,83.2,29.5,24.9,16.6$. The carbon signal attached to $B$ was not observed due to low intensity.

Spectral data matched those previously reported. ${ }^{[9]}$

2-(4-(Benzyloxy)phenethyl)-4,4,5,5-tetramethyl-1,3,2-dioxaborolane (2e).<smiles>c1ccc(CCCc2ccc(OCc3ccccc3)cc2)cc1</smiles>

Compound 2e was prepared following the GP1 using 1-(4(benzyloxy)phenyl)ethan-1-one $1 \mathrm{~d}(113.0 \mathrm{mg}, 0.5 \mathrm{mmol}),[\mathrm{RhCl}(\mathrm{cod})]_{2}(4.9 \mathrm{mg}$, $0.01 \mathrm{mmol}), \mathrm{P}\left({ }^{n} \mathrm{Bu}\right)_{3}(12.1 \mathrm{mg}, 0.06 \mathrm{mmol}), \mathrm{B}_{2} \mathrm{pin}_{2}(228.6 \mathrm{mg}, 0.90 \mathrm{mmol}),{ }^{i} \mathrm{PrOH}$ (38.1 mg, $0.50 \mathrm{mmol})$, HCOOK (21.0 mg, $0.25 \mathrm{mmol})$ and IPE $(2.0 \mathrm{~mL})$. The product was obtained as a colourless oil in $68 \%$ yield $(114.9 \mathrm{mg}$, eluent : petroleum ether : $\mathrm{EtOAc}=40: 1)$.

$\mathbf{R}_{f}=0.48$ (petroleum ether : $\mathrm{EtOAC}=10: 1$ ).

${ }^{1} \mathrm{H}$ NMR (400 MHz, $\left.\mathrm{CDCl}_{3}\right) \delta 7.47$ - 7.43 (m, 2H), 7.42 - 7.37 (m, 2H), 7.36 - 7.30 $(\mathrm{m}, 1 \mathrm{H}), 7.16(\mathrm{~d}, J=8.5 \mathrm{~Hz}, 2 \mathrm{H}), 6.91(\mathrm{~d}, J=8.6 \mathrm{~Hz}, 2 \mathrm{H}), 5.06(\mathrm{~s}, 2 \mathrm{H}), 2.73(\mathrm{t}, J=$ $8.2 \mathrm{~Hz}, 2 \mathrm{H}), 1.24(\mathrm{~s}, 12 \mathrm{H}), 1.15(\mathrm{t}, J=8.3 \mathrm{~Hz}, 2 \mathrm{H})$.

${ }^{13} \mathrm{C}$ NMR (100 MHz, $\mathrm{CDCl}_{3}$ ) $\delta$ 156.9, 137.4, 137.0, 129.0, 128.6, 127.9, 127.5, 114.7, $83.1,70.1,29.2,24.9$. The carbon signal attached to B was not observed due to low intensity. 
Spectral data matched those previously reported. ${ }^{[8]}$

tert-Butyldimethyl(4-(2-(4,4,5,5-tetramethyl-1,3,2-dioxaborolan2yl)ethyl)phenoxy)silane (2f).<smiles>CC(C)(C)Oc1ccc(CC[B]c2ccccc2)cc1</smiles>

Compound 2f was prepared following the GP1 using 1-(4-((tertbutyldimethylsilyl)oxy)phenyl)ethan-1-one $\quad$ If $(125.0 \quad \mathrm{mg}, \quad 0.5 \mathrm{mmol})$, [RhCl(cod)]2 (4.9 mg, $0.01 \mathrm{mmol}), \mathrm{P}\left({ }^{(} \mathrm{Bu}\right) 3$ (12.1 mg, $\left.0.06 \mathrm{mmol}\right), \mathrm{B}_{2} \mathrm{pin}_{2}(228.6$ $\mathrm{mg}, 0.90 \mathrm{mmol}),{ }^{i} \mathrm{PrOH}(38.1 \mathrm{mg}, 0.50 \mathrm{mmol}), \mathrm{HCOOK}(21.0 \mathrm{mg}, 0.25 \mathrm{mmol})$ and IPE $(2.0 \mathrm{~mL})$. The product was obtained as a colourless oil in $74 \%$ yield $(133.9 \mathrm{mg}$, eluent : petroleum ether : EtOAc $=40: 1)$.

$\mathbf{R}_{f}=0.49$ (petroleum ether : $\mathrm{EtOAc}=20: 1$ ).

${ }^{1} \mathrm{H}$ NMR (400 MHz, CDCl $)$ \& $7.09-7.03(\mathrm{~m}, 2 \mathrm{H}), 6.76-6.70(\mathrm{~m}, 2 \mathrm{H}), 2.68(\mathrm{t}, J=$ $8.0 \mathrm{~Hz}, 2 \mathrm{H}), 1.20(\mathrm{~s}, 12 \mathrm{H}), 1.11(\mathrm{t}, J=8.0 \mathrm{~Hz}, 2 \mathrm{H}), 0.97(\mathrm{~s}, 9 \mathrm{H}), 0.17(\mathrm{~s}, 6 \mathrm{H})$.

${ }^{13} \mathrm{C}$ NMR (100 MHz, $\mathrm{CDCl}_{3}$ ) $\delta$ 153.5, 137.2, 128.9, 119.8, 83.1, 29.2, 25.9, 24.9, 18.3, -4.3. The carbon signal attached to $\mathrm{B}$ was not observed due to low intensity.

HRMS (ESI ${ }^{+}$) calcd for $\mathrm{C}_{20} \mathrm{H}_{33} \mathrm{BO}_{3} \mathrm{SiNa}^{+}[\mathrm{M}+\mathrm{Na}]^{+}: 385.2341$, found: 385.2340 .

IR (neat, cm ${ }^{-1}$ ): 2937, 2857, 1612, 1506, 1370, 1321, 1259, 1156, 921, 838, 787.

$N, N$-Dimethyl-4-(2-(4,4,5,5-tetramethyl-1,3,2-dioxaborolan-2-yl)ethyl)aniline (2g).

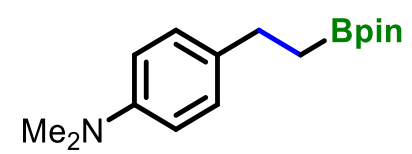

Compound $2 \mathrm{~g}$ was prepared following the GP1 using 1-(4(dimethylamino)phenyl)ethan-1-one $1 \mathbf{g}(81.5 \mathrm{mg}, 0.5 \mathrm{mmol}),[\mathrm{RhCl}(\mathrm{cod})]_{2}(4.9$ $\mathrm{mg}, 0.01 \mathrm{mmol}), \mathrm{P}\left({ }^{n} \mathrm{Bu}\right)_{3}(12.1 \mathrm{mg}, 0.06 \mathrm{mmol}), \mathrm{B}_{2} \mathrm{pin}_{2}(228.6 \mathrm{mg}, 0.90 \mathrm{mmol})$, iPrOH (38.1 mg, $0.50 \mathrm{mmol})$, HCOOK $(21.0 \mathrm{mg}, 0.25 \mathrm{mmol})$ and IPE $(2.0 \mathrm{~mL})$. The product was obtained as a colourless oil in $67 \%$ yield $(91.2 \mathrm{mg}$, eluent : petroleum ether: $\mathrm{EtOAc}=40: 1)$.

$\mathbf{R}_{f}=0.50$ (petroleum ether : EtOAc $=10: 1$ ).

${ }^{1} \mathrm{H}$ NMR (400 MHz, $\left.\mathrm{CDCl}_{3}\right) \delta 7.14-7.08(\mathrm{~m}, 2 \mathrm{H}), 6.74-6.68(\mathrm{~m}, 2 \mathrm{H}), 2.91(\mathrm{~s}$, 
$6 \mathrm{H}), 2.68(\mathrm{t}, J=8.3 \mathrm{~Hz}, 2 \mathrm{H}), 1.25(\mathrm{~s}, 12 \mathrm{H}), 1.12(\mathrm{t}, J=8.3 \mathrm{~Hz}, 2 \mathrm{H})$.

${ }^{13} \mathrm{C}$ NMR (100 MHz, $\left.\mathrm{CDCl}_{3}\right)$ 149.0, 133.1, 128.6, 113.2, 83.1, 41.1, 29.0, 24.9. The carbon signal attached to B was not observed due to low intensity.

Spectral data matched those previously reported..$^{[10]}$

\section{4,4,5,5-Tetramethyl-2-(4-(2-(4,4,5,5-tetramethyl-1,3,2-dioxaborolan-2- yl)ethyl)phenyl)-1,3,2-dioxaborolane (2h).}<smiles>Pc1ccccc1</smiles>

Compound $\mathbf{2 h}$ was prepared following the GP1 using 1-(4-(4,4,5,5-tetramethyl1,3,2-dioxaborolan-2-yl)phenyl)ethan-1-one $\quad \mathbf{1 h} \quad(123.0 \mathrm{mg}$, $0.5 \mathrm{mmol})$, $[\mathrm{RhCl}(\mathrm{cod})]_{2}(4.9 \mathrm{mg}, 0.01 \mathrm{mmol}), \mathrm{P}\left({ }^{n} \mathrm{Bu}\right)_{3}(12.1 \mathrm{mg}, 0.06 \mathrm{mmol}), \mathrm{B}_{2} \mathrm{pin}_{2}(228.6$ $\mathrm{mg}, 0.90 \mathrm{mmol}), \mathrm{PrOH}(38.1 \mathrm{mg}, 0.50 \mathrm{mmol})$, HCOOK (21.0 mg, $0.25 \mathrm{mmol}$ ) and IPE $(2.0 \mathrm{~mL})$. The product was obtained as a colourless oil in $51 \%$ yield (91.3 mg, eluent : petroleum ether : $\mathrm{EtOAc}=40: 1)$.

$\mathbf{R}_{f}=0.51$ (petroleum ether : EtOAc $=20: 1$ ).

${ }^{1} \mathrm{H}$ NMR (400 MHz, $\left.\mathrm{CDCl}_{3}\right) \delta 7.71(\mathrm{~d}, J=7.9 \mathrm{~Hz}, 2 \mathrm{H}), 7.23(\mathrm{~d}, J=7.7 \mathrm{~Hz}, 2 \mathrm{H})$, $2.75(\mathrm{t}, J=8.0 \mathrm{~Hz}, 2 \mathrm{H}), 1.33(\mathrm{~s}, 12 \mathrm{H}), 1.22(\mathrm{~s}, 12 \mathrm{H}), 1.13(\mathrm{t}, J=8.0 \mathrm{~Hz}, 2 \mathrm{H})$.

${ }^{13} \mathrm{C}$ NMR (100 MHz, $\left.\mathrm{CDCl}_{3}\right) \delta$ o 148.1, 134.9, 127.6, 83.7, 83.3, 30.3, 24.99, 24.95. The carbon signal attached to B was not observed due to low intensity. Spectral data matched those previously reported. ${ }^{[1]]}$

2-(2-([1,1'-Biphenyl]-4-yl)ethyl)-4,4,5,5-tetramethyl-1,3,2-dioxaborolane (2i).<smiles>c1ccc(CCc2ccc(-c3ccccc3)cc2)cc1</smiles>

Compound 2i was prepared following GP1 using 1-([1,1'-biphenyl]-4-yl)ethan1-one $1 \mathrm{i}$ (98.0 mg, $0.5 \mathrm{mmol})$, [ RhCl(cod) ]2 (4.9 mg, $0.01 \mathrm{mmol}), \mathrm{P}\left({ }^{n} \mathrm{Bu}\right)_{3}(12.1 \mathrm{mg}$, $0.06 \mathrm{mmol}$ ), B B $_{2} \mathrm{pin}_{2}$ (228.6 mg, $0.90 \mathrm{mmol}$ ), $\mathrm{PrOH}$ (38.1 mg, $0.50 \mathrm{mmol}$ ), HCOOK $(21.0 \mathrm{mg}, 0.25 \mathrm{mmol})$ and IPE $(2.0 \mathrm{~mL})$. The product was obtained as a colourless oil in $61 \%$ yield $(93.9 \mathrm{mg}$, eluent : petroleum ether : EtOAc $=40: 1)$.

$\mathbf{R}_{f}=0.55$ (petroleum ether : EtOAc $=20: 1$ ). 
${ }^{1} \mathrm{H}$ NMR (400 MHz, $\left.\mathrm{CDCl}_{3}\right) \delta 7.62-7.56(\mathrm{~m}, 2 \mathrm{H}), 7.54-7.48(\mathrm{~m}, 2 \mathrm{H}), 7.46-7.39$ (m, 2H), $7.35-7.27(\mathrm{~m}, 3 \mathrm{H}), 2.81(\mathrm{t}, J=8.2 \mathrm{~Hz}, 2 \mathrm{H}), 1.25-1.18(\mathrm{~m}, 14 \mathrm{H})$.

${ }^{13} \mathrm{C}$ NMR (100 MHz, $\left.\mathrm{CDCl}_{3}\right) \delta$ 143.7, 141.4, 138.6, 128.8, 128.6, 127.11, 127.08, 127.0, 83.3, 29.7, 25.0. The carbon signal attached to B was not observed due to low intensity.

Spectral data matched those previously reported. ${ }^{[8]}$

2-(4-Fluorophenethyl)-4,4,5,5-tetramethyl-1,3,2-dioxaborolane (2j).<smiles>Fc1ccc(CCBr)cc1</smiles>

Compound 2j was prepared following GP1 using 1-(4-fluorophenyl)ethan-1one $1 \mathbf{j}$ (69.0 mg, $0.5 \mathrm{mmol}),[\mathrm{RhCl}(\mathrm{cod})]_{2}(4.9 \mathrm{mg}, 0.01 \mathrm{mmol}), \mathrm{P}\left({ }^{n} \mathrm{Bu}\right) 3(12.1 \mathrm{mg}$, $0.06 \mathrm{mmol}), \mathrm{B}_{2}$ pin 2 (228.6 mg, $\left.0.90 \mathrm{mmol}\right),{ }^{i} \mathrm{PrOH}$ (38.1 mg, $\left.0.50 \mathrm{mmol}\right)$, HCOOK $(21.0 \mathrm{mg}, 0.25 \mathrm{mmol})$ and IPE $(2.0 \mathrm{~mL})$. The product was obtained as a colourless oil in $56 \%$ yield $(70.0 \mathrm{mg}$, eluent : petroleum ether : EtOAc $=40: 1)$.

$\mathbf{R}_{f}=0.62$ (petroleum ether : EtOAc $=20: 1$ ).

${ }^{1} \mathrm{H}$ NMR (400 MHz, $\left.\mathrm{CDCl}_{3}\right) \delta 7.28-7.19(\mathrm{~m}, 2 \mathrm{H}), 7.07-6.98(\mathrm{~m}, 2 \mathrm{H}), 2.80(\mathrm{t}, J=$ $8.1 \mathrm{~Hz}, 2 \mathrm{H}), 1.29(\mathrm{~s}, 12 \mathrm{H}), 1.20(\mathrm{t}, J=8.1 \mathrm{~Hz}, 2 \mathrm{H})$.

${ }^{13} \mathrm{C}$ NMR (100 MHz, $\left.\mathrm{CDCl}_{3}\right) \delta 161.2\left(J_{\mathrm{C}-\mathrm{F}}=242.7 \mathrm{~Hz}\right), 140.0$ (JC-F $\left.=3.2 \mathrm{~Hz}\right), 129.4$ $\left(J_{\mathrm{C}-\mathrm{F}}=7.7 \mathrm{~Hz}\right), 114.9\left(J_{\mathrm{C}-\mathrm{F}}=20.9 \mathrm{~Hz}\right), 83.2,29.3,24.9$. The carbon signal attached to $\mathrm{B}$ was not observed due to low intensity.

${ }^{19}$ F NMR (376 MHz, $\left.\mathrm{CDCl}_{3}\right)$ : $\delta$-118.4.

Spectral data matched those previously reported. ${ }^{[8]}$

4-(2-(4,4,5,5-Tetramethyl-1,3,2-dioxaborolan-2-yl)ethyl)phenyl 4methylbenzenesulfonate $(2 \mathrm{k})$.<smiles>CCCCOc1ccc(CCBr)cc1</smiles>

Compound 2k was prepared following GP1 using 4-acetylphenyl 4methylbenzenesulfonate 1k (145.0 mg, $0.5 \mathrm{mmol})$, [RhCl(cod)]2 (4.9 mg, 0.01 $\mathrm{mmol}), \mathrm{P}\left({ }^{n} \mathrm{Bu}\right) 3$ (12.1 mg, $\left.0.06 \mathrm{mmol}\right), \mathrm{B}_{2} \mathrm{pin}_{2}(228.6 \mathrm{mg}, 0.90 \mathrm{mmol}),{ }^{\mathrm{P}} \mathrm{PrOH}(38.1$ $\mathrm{mg}, 0.50 \mathrm{mmol})$, HCOOK (21.0 mg, $0.25 \mathrm{mmol})$ and IPE $(2.0 \mathrm{~mL})$. The product 
was obtained as a colourless oil in 53\% yield ( $106.5 \mathrm{mg}$, eluent : petroleum ether : $\mathrm{EtOAc}=20: 1)$.

$\mathbf{R}_{f}=0.65$ (petroleum ether $:$ EtOAc $=5: 1$ ).

${ }^{1} \mathrm{H}$ NMR (400 MHz, $\left.\mathrm{CDCl}_{3}\right)$ ठ $7.73-7.68(\mathrm{~m}, 2 \mathrm{H}), 7.33-7.28(\mathrm{~m}, 2 \mathrm{H}), 7.15-7.10$ $(\mathrm{m}, 2 \mathrm{H}), 6.89-6.84(\mathrm{~m}, 2 \mathrm{H}), 2.71(\mathrm{t}, J=8.1 \mathrm{~Hz}, 2 \mathrm{H}), 2.45(\mathrm{~s}, 3 \mathrm{H}), 1.20(\mathrm{~s}, 12 \mathrm{H})$, $1.11(\mathrm{t}, J=8.1 \mathrm{~Hz}, 2 \mathrm{H})$.

${ }^{13} \mathrm{C}$ NMR (100 MHz, $\left.\mathrm{CDCl}_{3}\right) \delta$ \& 147.6, 145.2, 143.4, 132.6, 129.8, 129.2, 128.6, 122.0, $83.3,29.4,24.9,21.8$. The carbon signal attached to B was not observed due to low intensity.

HRMS (ESI') calcd for $\mathrm{C}_{21} \mathrm{H}_{27} \mathrm{BO}_{5} \mathrm{SNa}^{+}[\mathrm{M}+\mathrm{Na}]^{+}: 425.1564$, found: 425.1564 .

IR (neat, cm-1): 2984, 2919, 1503, 1371, 1324, 1177, 1144, 865.

2-(3-Methoxyphenethyl)-4,4,5,5-tetramethyl-1,3,2-dioxaborolane (21).<smiles>COc1cccc(CCc2ccccc2)c1</smiles>

Compound 21 was prepared following GP1 using 1-(3-methoxyphenyl)ethan1-one 11 (75.0 mg, $0.5 \mathrm{mmol})$, [ RhCl(cod) ]2 (4.9 mg, $0.01 \mathrm{mmol}), \mathrm{P}\left({ }^{n} \mathrm{Bu}\right)_{3}(12.1 \mathrm{mg}$, $0.06 \mathrm{mmol}$ ), B2pin 2 (228.6 mg, $0.90 \mathrm{mmol}$ ), $\mathrm{PrOH}$ ( $38.1 \mathrm{mg}, 0.50 \mathrm{mmol})$, HCOOK $(21.0 \mathrm{mg}, 0.25 \mathrm{mmol})$ and IPE $(2.0 \mathrm{~mL})$. The product was obtained as a colourless oil in $61 \%$ yield $(79.9 \mathrm{mg}$, eluent : petroleum ether : EtOAc $=40: 1)$.

$\mathbf{R}_{f}=0.42$ (petroleum ether : EtOAc $=20: 1$ ).

${ }^{1} \mathrm{H}$ NMR (400 MHz, $\left.\mathrm{CDCl}_{3}\right)$ \& 7.21 - $7.14(\mathrm{~m}, 1 \mathrm{H}), 6.84-6.75(\mathrm{~m}, 2 \mathrm{H}), 6.73-6.67$ $(\mathrm{m}, 1 \mathrm{H}), 3.78(\mathrm{~s}, 3 \mathrm{H}), 2.72(\mathrm{t}, J=8.3 \mathrm{~Hz}, 2 \mathrm{H}), 1.23(\mathrm{~s}, 12 \mathrm{H}), 1.14(\mathrm{t}, J=8.3 \mathrm{~Hz}, 2 \mathrm{H})$. ${ }^{13} \mathrm{C}$ NMR (100 MHz, $\left.\mathrm{CDCl}_{3}\right) \delta$ 159.7, 146.2, 129.3, 120.5, 113.8, 111.1, 83.2, 55.2, $30.2,25.0$. The carbon signal attached to $B$ was not observed due to low intensity. Spectral data matched those previously reported. ${ }^{[8]}$

4,4,5,5-Tetramethyl-2-(3-methylphenethyl)-1,3,2-dioxaborolane (2m).<smiles>Cc1cccc(CCCc2ccccc2)c1</smiles>

Compound 2m was prepared following GP1 using 1-(m-tolyl)ethan-1-one $1 \mathbf{m}$ (67.0 mg, $0.5 \mathrm{mmol}),[\operatorname{RhCl}(\mathrm{cod})]_{2}(4.9 \mathrm{mg}, 0.01 \mathrm{mmol}), \mathrm{P}\left({ }^{n} \mathrm{Bu}\right)_{3}(12.1 \mathrm{mg}, 0.06$ 
mmol), B2pin2 (228.6 mg, $0.90 \mathrm{mmol}),{ }^{i} \mathrm{PrOH}$ (38.1 mg, $\left.0.50 \mathrm{mmol}\right)$, HCOOK $(21.0 \mathrm{mg}, 0.25 \mathrm{mmol})$ and IPE $(2.0 \mathrm{~mL})$. The product was obtained as a colourless oil in $65 \%$ yield $(80.0 \mathrm{mg}$, eluent : petroleum ether : EtOAc $=40: 1)$.

$\mathbf{R}_{f}=0.48$ (petroleum ether : EtOAc $=20: 1$ ).

${ }^{1} \mathrm{H}$ NMR (400 MHz, $\left.\mathrm{CDCl}_{3}\right) \delta 7.18-7.12(\mathrm{~m}, 1 \mathrm{H}), 7.06-6.94(\mathrm{~m}, 3 \mathrm{H}), 2.71(\mathrm{t}, J=$ $8.3 \mathrm{~Hz}, 2 \mathrm{H}), 2.31(\mathrm{~s}, 3 \mathrm{H}), 1.23(\mathrm{~s}, 12 \mathrm{H}), 1.13(\mathrm{t}, J=8.4 \mathrm{~Hz}, 2 \mathrm{H})$.

${ }^{13} \mathrm{C}$ NMR (100 MHz, $\left.\mathrm{CDCl}_{3}\right) \delta$ 144.5, 137.8, 129.0, 128.2, 126.3, 125.1, 83.2, 30.0, $25.0,21.6$. The carbon signal attached to B was not observed due to low intensity. Spectral data matched those previously reported. ${ }^{[12]}$

\section{4,4,5,5-Tetramethyl-2-(2-(trifluoromethyl)phenethyl)-1,3,2-dioxaborolane} (2n).<smiles>FC(F)(F)c1ccccc1CC[B]c1ccccc1</smiles>

Compound $2 n$ was prepared following GP1 using 1-(2(trifluoromethyl)phenyl)ethan-1-one 1 n $(94.0 \mathrm{mg}, 0.5 \mathrm{mmol}),[\mathrm{RhCl}(\mathrm{cod})]_{2}(4.9$ $\mathrm{mg}, 0.01 \mathrm{mmol}), \mathrm{P}\left({ }^{n} \mathrm{Bu}\right)_{3}(12.1 \mathrm{mg}, 0.06 \mathrm{mmol}), \mathrm{B}_{2}$ pin2 $(228.6 \mathrm{mg}, 0.90 \mathrm{mmol})$, ${ }^{i} \mathrm{PrOH}(38.1 \mathrm{mg}, 0.50 \mathrm{mmol}), \mathrm{HCOOK}(21.0 \mathrm{mg}, 0.25 \mathrm{mmol})$ and IPE $(2.0 \mathrm{~mL})$. The product was obtained as a colourless oil in $50 \%$ yield $(75.0 \mathrm{mg}$, eluent : petroleum ether : $\mathrm{EtOAc}=40: 1)$.

$\mathbf{R}_{f}=0.55$ (petroleum ether : $\mathrm{EtOAc}=20: 1$ ).

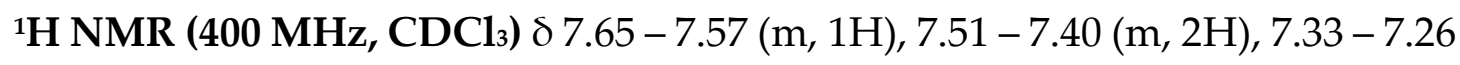
$(\mathrm{m}, 1 \mathrm{H}), 2.93(\mathrm{t}, J=8.3 \mathrm{~Hz}, 2 \mathrm{H}), 1.28(\mathrm{~s}, 12 \mathrm{H}), 1.17(\mathrm{t}, J=8.4 \mathrm{~Hz}, 2 \mathrm{H})$.

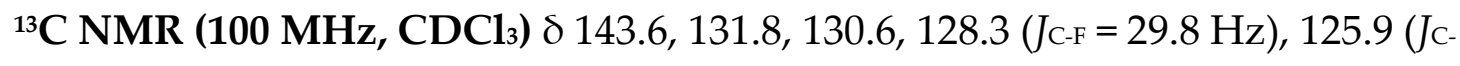
$\mathrm{F}=5.9 \mathrm{~Hz}), 125.7,124.8\left(J_{\mathrm{C}-\mathrm{F}}=272 \mathrm{~Hz}\right), 83.4,26.8\left(J_{\mathrm{C}-\mathrm{F}}=2.2 \mathrm{~Hz}\right), 25.0$. The carbon signal attached to B was not observed due to low intensity.

${ }^{19}$ F NMR (376 MHz, $\left.\mathrm{CDCl}_{3}\right)$ : $\delta-59.8$.

Spectral data matched those previously reported. ${ }^{[8]}$

2-(2-Methoxyphenethyl)-4,4,5,5-tetramethyl-1,3,2-dioxaborolane (2o)<smiles></smiles> 
Compound 2o was prepared following GP1 using 1-(2-methoxyphenyl)ethan1-one 10 (75.0 mg, $0.5 \mathrm{mmol})$, [ RhCl(cod)]2 (4.9 mg, $0.01 \mathrm{mmol}), \mathrm{P}\left({ }^{n} \mathrm{Bu}\right)_{3}(12.1$ $\mathrm{mg}, 0.06 \mathrm{mmol}), B_{2}$ pin $2(228.6 \mathrm{mg}, 0.90 \mathrm{mmol})$, PrOH (38.1 mg, $\left.0.50 \mathrm{mmol}\right)$, HCOOK ( $21.0 \mathrm{mg}, 0.25 \mathrm{mmol})$ and IPE $(2.0 \mathrm{~mL})$. The product was obtained as a colourless oil in $49 \%$ yield $(64.2 \mathrm{mg}$, eluent : petroleum ether : EtOAc $=40: 1$ ). $\mathbf{R}_{f}=0.45$ (petroleum ether : EtOAc $=20: 1$ ).

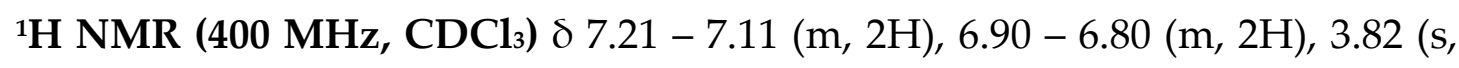
$3 \mathrm{H}), 2.74(\mathrm{t}, J=8.2 \mathrm{~Hz}, 2 \mathrm{H}), 1.23(\mathrm{~s}, 12 \mathrm{H}), 1.13(\mathrm{t}, J=8.2 \mathrm{~Hz}, 2 \mathrm{H})$.

${ }^{13} \mathrm{C}$ NMR (100 MHz, $\left.\mathrm{CDCl}_{3}\right) \delta$ 157.5, 132.9, 129.2, 126.8, 120.4, 110.2, 83.1, 55.3, 25.0, 24.5. The carbon signal attached to B was not observed due to low intensity. Spectral data matched those previously reported. ${ }^{[8]}$

\section{4-(2-(4,4,5,5-Tetramethyl-1,3,2-dioxaborolan-2-yl)ethyl)phenol (2p)}<smiles>Oc1ccc(CC[B][Ba])cc1</smiles>

Compound $2 p$ was prepared following the GP1 using 1-(4hydroxyphenyl)ethan-1-one $\mathbf{1 p}$ (40.8 mg, $0.3 \mathrm{mmol}$ ), [ $\mathrm{RhCl}(\mathrm{cod})]_{2}$ (3.0 mg, 0.006 $\mathrm{mmol}), \mathrm{P}\left({ }^{n} \mathrm{Bu}\right)_{3}(7.3 \mathrm{mg}, 0.036 \mathrm{mmol}), \mathrm{B}_{2} \mathrm{pin}_{2}(137.0 \mathrm{mg}, 0.54 \mathrm{mmol})$, HCOOK $(12.6 \mathrm{mg}, 0.15 \mathrm{mmol})$ and IPE $(2.0 \mathrm{~mL})$. The product was obtained as a colourless oil in $72 \%$ yield $(54.0 \mathrm{mg}$, eluent : petroleum ether : EtOAc $=5: 1)$.

$\mathbf{R}_{f}=0.77$ (petroleum ether : EtOAc $=1: 1$ ).

${ }^{1} \mathrm{H}$ NMR (400 MHz, $\left.\mathrm{CDCl}_{3}\right) \delta 7.11-7.03(\mathrm{~m}, 2 \mathrm{H}), 6.78-6.68(\mathrm{~m}, 2 \mathrm{H}), 5.10$ (s, $1 \mathrm{H}), 2.67(\mathrm{t}, J=8.1 \mathrm{~Hz}, 2 \mathrm{H}), 1.22(\mathrm{~s}, 12 \mathrm{H}), 1.11(\mathrm{t}, J=8.0 \mathrm{~Hz}, 2 \mathrm{H})$.

${ }^{13} \mathrm{C}$ NMR (100 MHz, $\mathrm{CDCl}_{3}$ ) $\delta$ 153.7, 136.7, 129.3, 115.2, 83.4, 29.2, 25.0. The carbon signal attached to $B$ was not observed due to low intensity. Spectral data matched those previously reported.[11]

3-(2-(4,4,5,5-Tetramethyl-1,3,2-dioxaborolan-2-yl)ethyl)aniline (2q)<smiles>Nc1cccc(CC[B]c2ccccc2)c1</smiles>

Compound $2 q$ was prepared following the GP1 using 1-(3- 
aminophenyl)ethan-1-one 1q (40.5 mg, $0.3 \mathrm{mmol})$, [RhCl(cod)]2 (3.0 mg, 0.006 $\mathrm{mmol}), \mathrm{P}\left({ }^{n} \mathrm{Bu}\right)_{3}(7.3 \mathrm{mg}, 0.036 \mathrm{mmol}), \mathrm{B}_{2} \mathrm{pin}_{2}(137.0 \mathrm{mg}, 0.54 \mathrm{mmol})$, HCOOK $(12.6 \mathrm{mg}, 0.15 \mathrm{mmol})$ and IPE $(2.0 \mathrm{~mL})$. The product was obtained as a colourless oil in $78 \%$ yield $(58.0 \mathrm{mg}$, eluent : petroleum ether : EtOAc $=5: 1)$.

$\mathbf{R}_{f}=0.65$ (petroleum ether : EtOAc $=2: 1$ ).

${ }^{1} \mathrm{H}$ NMR (400 MHz, $\left.\mathrm{CDCl}_{3}\right)$ ठ $7.09-7.00(\mathrm{~m}, 1 \mathrm{H}), 6.66-6.60(\mathrm{~m}, 1 \mathrm{H}), 6.58-6.54$ $(\mathrm{m}, 1 \mathrm{H}), 6.52-6.46(\mathrm{~m}, 1 \mathrm{H}), 3.41(\mathrm{~s}, 2 \mathrm{H}), 2.65(\mathrm{t}, J=8.0 \mathrm{~Hz}, 2 \mathrm{H}), 1.23(\mathrm{~s}, 12 \mathrm{H})$, $1.11(\mathrm{t}, J=8.1 \mathrm{~Hz}, 2 \mathrm{H})$.

${ }^{13}$ C NMR (100 MHz, CDCl $)$ ) $1146.4,146.0,129.3,118.7,115.1,112.7,83.3,30.1$, 25.0. The carbon signal attached to $B$ was not observed due to low intensity. Spectral data matched those previously reported..$^{[15]}$

\section{2-(2-(2,3-Dihydrobenzo[b][1,4]dioxin-6-yl)ethyl)-4,4,5,5-tetramethyl-1,3,2-} dioxaborolane (2r)<smiles>Brc1cccc(CCc2ccc3c(c2)OCCO3)c1</smiles>

Compound 2r was prepared following the GP1 using 1-(2,3dihydrobenzo[b][1,4]dioxin-6-yl)ethan-1-one $\quad$ 1r $(89.0 \quad \mathrm{mg}, \quad 0.5 \quad \mathrm{mmol})$, [RhCl(cod) $]_{2}(4.9 \mathrm{mg}, 0.01 \mathrm{mmol}), \mathrm{P}\left({ }^{n} \mathrm{Bu}\right)_{3}(12.1 \mathrm{mg}, 0.06 \mathrm{mmol}), \mathrm{B}_{2} \mathrm{pin}_{2}(228.6$ $\mathrm{mg}, 0.90 \mathrm{mmol}), \mathrm{PrOH}(38.1 \mathrm{mg}, 0.50 \mathrm{mmol})$, HCOOK (21.0 mg, $0.25 \mathrm{mmol}$ ) and IPE (2.0 mL). The product was obtained as a colourless oil in $65 \%$ yield ( 94.3 $\mathrm{mg}$, eluent : petroleum ether : $\mathrm{EtOAc}=40: 1)$.

$\mathbf{R}_{f}=0.43$ (petroleum ether : EtOAc $=20: 1$ ).

${ }^{1} \mathrm{H}$ NMR $\left(400 \mathrm{MHz}, \mathrm{CDCl}_{3}\right) \delta 6.82-6.64(\mathrm{~m}, 3 \mathrm{H}), 4.21(\mathrm{~s}, 4 \mathrm{H}), 2.64(\mathrm{t}, J=8.2 \mathrm{~Hz}$, $2 \mathrm{H}), 1.22(\mathrm{~s}, 12 \mathrm{H}), 1.09(\mathrm{t}, J=8.2 \mathrm{~Hz}, 2 \mathrm{H})$.

${ }^{13} \mathrm{C}$ NMR (100 MHz, $\left.\mathrm{CDCl}_{3}\right) \delta$ 143.2, 141.5, 138.0, 121.0, 116.9, 116.7, 83.2, 64.5, 64.4, 29.3, 24.9. The carbon signal attached to B was not observed due to low intensity.

HRMS (ESI ${ }^{+}$) calcd for $\mathrm{C}_{16} \mathrm{H}_{23} \mathrm{BO}_{4} \mathrm{Na}^{+}[\mathrm{M}+\mathrm{Na}]^{+}: 313.1582$, found: 313.1582 .

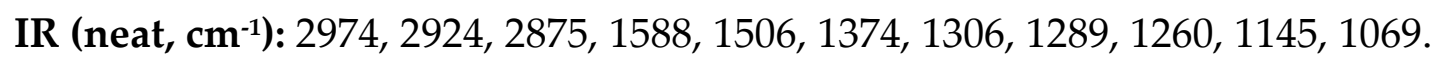

2-(3,5-Dimethylphenethyl)-4,4,5,5-tetramethyl-1,3,2-dioxaborolane (2s) 


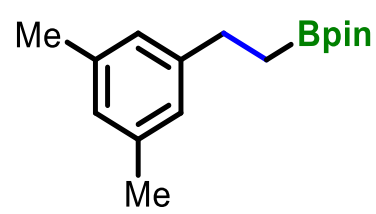

Compound 2s was prepared following the GP1 using 1-(3,5dimethylphenyl)ethan-1-one 1 s (74.0 mg, $0.5 \mathrm{mmol})$, [RhCl(cod)]2 (4.9 mg, 0.01 $\mathrm{mmol}), \mathrm{P}\left({ }^{n} \mathrm{Bu}\right)_{3}(12.1 \mathrm{mg}, 0.06 \mathrm{mmol}), \mathrm{B}_{2}$ pin 2 (228.6 mg, $\left.0.90 \mathrm{mmol}\right),{ }^{i} \mathrm{PrOH}(38.1$ $\mathrm{mg}$, $0.50 \mathrm{mmol})$, HCOOK $(21.0 \mathrm{mg}, 0.25 \mathrm{mmol})$ and IPE $(2.0 \mathrm{~mL})$. The product was obtained as a colourless oil in $68 \%$ yield $(88.4 \mathrm{mg}$, eluent : petroleum ether : EtOAc $=40: 1)$.

$\mathbf{R}_{f}=0.55$ (petroleum ether : $\mathrm{EtOAc}=20: 1$ ).

${ }^{1} \mathrm{H}$ NMR (400 MHz, CDCl 3$) \delta 6.86(\mathrm{~s}, 2 \mathrm{H}), 6.81(\mathrm{~s}, 1 \mathrm{H}), 2.70(\mathrm{t}, J=8.2,2 \mathrm{H}), 2.30$ $(\mathrm{s}, 6 \mathrm{H}), 1.25(\mathrm{~s}, 12 \mathrm{H}), 1.15(\mathrm{t}, J=8.2,2 \mathrm{H})$.

${ }^{13} \mathrm{C}$ NMR (100 MHz, $\left.\mathrm{CDCl}_{3}\right) \delta$ 144.5, 137.7, 127.2, 126.0, 83.1, 29.9, 24.9, 21.4. The carbon signal attached to $\mathrm{B}$ was not observed due to low intensity.

HRMS (ESI ${ }^{+}$) calcd for $\mathrm{C}_{16} \mathrm{H}_{25} \mathrm{BNaO}_{2}{ }^{+}[\mathrm{M}+\mathrm{Na}]^{+}:$283.1840, found: 283.1840 .

IR (neat, cm-1): 2975, 2919, 1950, 1609, 1457, 1370 1329, 1161, 967, 845.

\section{2-(3,4-Dimethoxyphenethyl)-4,4,5,5-tetramethyl-1,3,2-dioxaborolane (2t)}

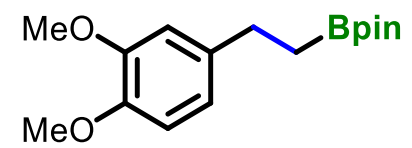

Compound 2t was prepared following the GP1 using 1-(3,4dimethoxyphenyl)ethan-1-one 1t $(90.0 \mathrm{mg}, 0.5 \mathrm{mmol}),[\mathrm{RhCl}(\operatorname{cod})]_{2}(4.9 \mathrm{mg}$, $0.01 \mathrm{mmol}), \mathrm{P}\left({ }^{(} \mathrm{Bu}\right)_{3}(12.1 \mathrm{mg}, 0.06 \mathrm{mmol}), \mathrm{B}_{2} \mathrm{pin}_{2}(228.6 \mathrm{mg}, 0.90 \mathrm{mmol})$, PrOH (38.1 mg, $0.50 \mathrm{mmol})$, HCOOK (21.0 mg, $0.25 \mathrm{mmol})$ and IPE $(2.0 \mathrm{~mL})$. The product was obtained as a colourless oil in $65 \%$ yield $(94.9 \mathrm{mg}$, eluent : petroleum ether : $\mathrm{EtOAc}=30: 1$ ).

$\mathbf{R}_{f}=0.45$ (petroleum ether : $\mathrm{EtOAc}=10: 1$ ).

${ }^{1} \mathrm{H}$ NMR (400 MHz, CDCl $) \delta 6.78-6.70(\mathrm{~m}, 3 \mathrm{H}), 3.84(\mathrm{~s}, 3 \mathrm{H}), 3.82(\mathrm{~s}, 3 \mathrm{H}), 2.67$ $(\mathrm{t}, J=8.0,2 \mathrm{H}), 1.20(\mathrm{~s}, 12 \mathrm{H}), 1.10(\mathrm{t}, J=8.0,2 \mathrm{H})$.

${ }^{13} \mathrm{C}$ NMR (100 MHz, $\left.\mathrm{CDCl}_{3}\right) \delta$ 148.7, 147.0, 137.2, 119.7, 111.6, 111.3, 83.1, 56.0, $55.8,29.6,24.9$. The carbon signal attached to $B$ was not observed due to low intensity.

Spectral data matched those previously reported. ${ }^{[13]}$ 

(2u)

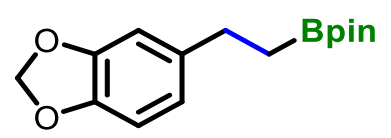

Compound $\mathbf{2} \mathbf{u}$ was prepared following the GP1 using 1-(benzo[d][1,3]dioxol5-yl)ethan-1-one $1 \mathbf{u}(82.0 \mathrm{mg}, 0.5 \mathrm{mmol})$, [RhCl(cod)]2 (4.9 mg, $0.01 \mathrm{mmol})$, $\mathrm{P}\left({ }^{n} \mathrm{Bu}\right)_{3}(12.1 \mathrm{mg}, 0.06 \mathrm{mmol}), \mathrm{B}_{2} \mathrm{pin}_{2}(228.6 \mathrm{mg}, 0.90 \mathrm{mmol}),{ }^{i} \mathrm{PrOH}(38.1 \mathrm{mg}, 0.50$ $\mathrm{mmol})$, HCOOK $(21.0 \mathrm{mg}, 0.25 \mathrm{mmol})$ and IPE $(2.0 \mathrm{~mL})$. The product was obtained as a colourless oil in $61 \%$ yield $(84.2 \mathrm{mg}$, eluent : petroleum ether : $\mathrm{EtOAc}=40: 1)$.

$\mathbf{R}_{f}=0.46$ (petroleum ether : $\mathrm{EtOAc}=20: 1$ ).

${ }^{1} \mathrm{H}$ NMR (400 MHz, $\left.\mathrm{CDCl}_{3}\right) \delta 6.75-6.62(\mathrm{~m}, 3 \mathrm{H}), 5.90(\mathrm{~s}, 2 \mathrm{H}), 2.66(\mathrm{t}, J=8.3 \mathrm{~Hz}$, 2H), $1.23(\mathrm{~s}, 12 \mathrm{H}), 1.09(\mathrm{t}, J=8.4 \mathrm{~Hz}, 2 \mathrm{H})$.

${ }^{13} \mathrm{C}$ NMR (100 MHz, $\mathrm{CDCl}_{3}$ ) $\delta$ 147.5, 145.4, 138.6, 120.7, 108.8, 108.1, 100.8, 83.3, $29.9,25.0$. The carbon signal attached to B was not observed due to low intensity. Spectral data matched those previously reported. ${ }^{[8]}$

4,4,5,5-Tetramethyl-2-(3,4,5-trimethoxyphenethyl)-1,3,2-dioxaborolane (2v)<smiles>COc1cc(CCCc2ccccc2)cc(OC)c1OC</smiles>

Compound 2v was prepared following the GP1 using 1-(3,4,5trimethoxyphenyl)ethan-1-one $1 \mathbf{v}(105.0 \mathrm{mg}, 0.5 \mathrm{mmol}),[\mathrm{RhCl}(\mathrm{cod})]_{2}(4.9 \mathrm{mg}$, $0.01 \mathrm{mmol}), \mathrm{P}\left({ }^{n} \mathrm{Bu}\right)_{3}(12.1 \mathrm{mg}, 0.06 \mathrm{mmol}), \mathrm{B}_{2} \mathrm{pin}_{2}(228.6 \mathrm{mg}, 0.90 \mathrm{mmol}),{ }^{i} \mathrm{PrOH}$ (38.1 mg, $0.50 \mathrm{mmol})$, HCOOK (21.0 mg, $0.25 \mathrm{mmol})$ and IPE (2.0 mL). The product was obtained as a colourless oil in $67 \%$ yield $(107.9 \mathrm{mg}$, eluent : petroleum ether : $\mathrm{EtOAc}=20: 1)$.

$\mathbf{R}_{f}=0.55$ (petroleum ether $: \mathrm{EtOAc}=5: 1$ ).

${ }^{1} \mathrm{H}$ NMR (400 MHz, $\left.\mathrm{CDCl}_{3}\right) \delta 6.44(\mathrm{~s}, 2 \mathrm{H}), 3.83(\mathrm{~s}, 6 \mathrm{H}), 3.80(\mathrm{~s}, 3 \mathrm{H}), 2.69(\mathrm{t}, J=$ $8.0 \mathrm{~Hz}, 2 \mathrm{H}), 1.22(\mathrm{~s}, 12 \mathrm{H}), 1.13(\mathrm{t}, J=8.0 \mathrm{~Hz}, 2 \mathrm{H})$.

${ }^{13} \mathrm{C}$ NMR (100 MHz, $\left.\mathrm{CDCl}_{3}\right) \delta$ 153.1, 140.4, 136.0, 105.0, 83.2, 61.0, 56.1, 30.5, 25.0.

The carbon signal attached to B was not observed due to low intensity. 
Spectral data matched those previously reported. ${ }^{[8]}$

2-(4-((3,7-Dimethyloct-6-en-1-yl)oxy)phenethyl)-4,4,5,5-tetramethyl-1,3,2dioxaborolane $(2 \mathrm{w})$<smiles>CC(C)=CCCC(C)CCOc1ccc(CCCc2ccccc2)cc1</smiles>

Compound 2w was prepared following the GP1 using 1-(4-((3,7-dimethyloct6-en-1-yl)oxy)phenyl)ethan-1-one $\mathbf{1 w}$ (145.0 mg, $0.5 \mathrm{mmol})$, [ $\mathrm{RhCl}(\mathrm{cod})]_{2}$ (4.9 $\mathrm{mg}, 0.01 \mathrm{mmol}), \mathrm{P}\left({ }^{n} \mathrm{Bu}\right)_{3}(12.1 \mathrm{mg}, 0.06 \mathrm{mmol}), \mathrm{B}_{2} \mathrm{pin}_{2}(228.6 \mathrm{mg}, 0.90 \mathrm{mmol})$, iPrOH (38.1 mg, $0.50 \mathrm{mmol})$, HCOOK $(21.0 \mathrm{mg}, 0.25 \mathrm{mmol})$ and IPE $(2.0 \mathrm{~mL})$. The product was obtained as a colourless oil in 54\% yield (104.2 mg, eluent : petroleum ether : $\mathrm{EtOAc}=40: 1)$.

$\mathbf{R}_{f}=0.45$ (petroleum ether : EtOAc $=20: 1$ ).

${ }^{1} \mathrm{H}$ NMR (400 MHz, $\left.\mathrm{CDCl}_{3}\right)$ \& $7.14(\mathrm{~d}, J=8.6 \mathrm{~Hz}, 2 \mathrm{H}), 6.86-6.80(\mathrm{~m}, 2 \mathrm{H}), 5.13$ $(\mathrm{t}, J=7.4 \mathrm{~Hz}, 1 \mathrm{H}), 4.04-3.92(\mathrm{~m}, 2 \mathrm{H}), 2.70(\mathrm{t}, J=8.2 \mathrm{~Hz}, 2 \mathrm{H}), 2.11-1.94(\mathrm{~m}, 2 \mathrm{H})$, $1.89-1.79(\mathrm{~m}, 1 \mathrm{H}), 1.77-1.66(\mathrm{~m}, 4 \mathrm{H}), 1.66-1.58(\mathrm{~m}, 4 \mathrm{H}), 1.47-1.37(\mathrm{~m}, 1 \mathrm{H})$, $1.24(\mathrm{~s}, 13 \mathrm{H}), 1.14(\mathrm{t}, J=8.2 \mathrm{~Hz}, 2 \mathrm{H}), 0.97(\mathrm{~d}, J=6.7 \mathrm{~Hz}, 3 \mathrm{H})$.

${ }^{13} \mathrm{C}$ NMR (100 MHz, $\left.\mathrm{CDCl}_{3}\right) \delta$ \& 157.2, 136.5, 131.4, 128.9, 124.9, 114.4, 83.2, 66.4, $37.3,36.4,29.7,29.2,25.9,25.6,25.0,19.7,17.8$. The carbon signal attached to $B$ was not observed due to low intensity.

HRMS (ESI') calcd for $\mathrm{C}_{24} \mathrm{H}_{39} \mathrm{BO}_{3} \mathrm{Na}^{+}[\mathrm{M}+\mathrm{Na}]^{+}$: 409.2884, found: 409.2884 .

IR (neat, cm-1): 2963, 2922, 2866, 1606, 1515, 1380, 1321, 1253, 1153, 976, 835.

2-(2-(6-Methoxynaphthalen-2-yl)ethyl)-4,4,5,5-tetramethyl-1,3,2dioxaborolane $(2 x)$

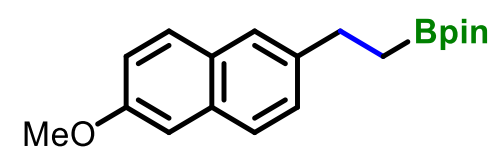

Compound $2 \mathrm{x}$ was prepared following the GP1 using 1-(6methoxynaphthalen-2-yl)ethan-1-one $1 \mathbf{x}$ (100.0 mg, $0.5 \mathrm{mmol}),[\mathrm{RhCl}(\mathrm{cod})]_{2}(4.9$ $\mathrm{mg}, 0.01 \mathrm{mmol}), \mathrm{P}\left({ }^{n} \mathrm{Bu}\right)_{3}(12.1 \mathrm{mg}, 0.06 \mathrm{mmol}), \mathrm{B}_{2} \mathrm{pin}_{2}$ (228.6 mg, $0.90 \mathrm{mmol}$ ), 
iPrOH (38.1 mg, $0.50 \mathrm{mmol})$, HCOOK (21.0 mg, $0.25 \mathrm{mmol})$ and IPE (2.0 mL). The product was obtained as a colourless oil in $43 \%$ yield $(60.6 \mathrm{mg}$, eluent : petroleum ether : $\mathrm{EtOAc}=40: 1)$.

$\mathbf{R}_{f}=0.47$ (petroleum ether : EtOAc $=20: 1$ ).

${ }^{1} \mathrm{H}$ NMR (400 MHz, $\left.\mathrm{CDCl}_{3}\right)$ \& $7.69-7.63(\mathrm{~m}, 2 \mathrm{H}), 7.59(\mathrm{~s}, 1 \mathrm{H}), 7.34(\mathrm{~d}, J=8.3 \mathrm{~Hz}$, 1H), $7.14-7.09(\mathrm{~m}, 2 \mathrm{H}), 3.91(\mathrm{~s}, 3 \mathrm{H}), 2.90(\mathrm{t}, J=8.1 \mathrm{~Hz}, 2 \mathrm{H}), 1.28-1.21(\mathrm{~m}, 14 \mathrm{H})$. ${ }^{13} \mathrm{C}$ NMR (100 MHz, $\left.\mathrm{CDCl}_{3}\right) \delta$ 157.1, 139.8, 133.0, 129.2, 129.0, 127.9, 126.7, 125.7, 118.6, 105.8, 83.2, 55.4, 30.0, 25.0. The carbon signal attached to B was not observed due to low intensity.

HRMS (ESI') calcd for $\mathrm{C}_{19} \mathrm{H}_{25} \mathrm{BO}_{3} \mathrm{Na}^{+}[\mathrm{M}+\mathrm{Na}]^{+}: 335.1789$, found: 335.1789 .

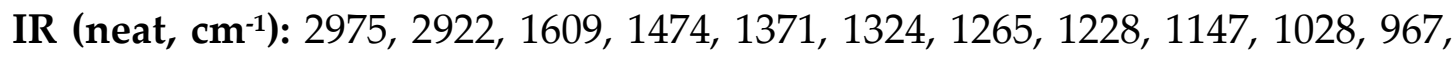
855 .

1-Methyl-5-(2-(4,4,5,5-tetramethyl-1,3,2-dioxaborolan-2-yl)ethyl)-1H-indole (2y)

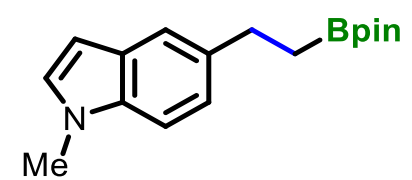

Compound 2y was prepared following the GP1 using 1-(1-methyl-1H-indol-5yl)ethan-1-one 1y (86.5 mg, $0.5 \mathrm{mmol})$, [RhCl(cod) ]2 $(4.9 \mathrm{mg}, 0.01 \mathrm{mmol}), \mathrm{P}\left({ }^{n} \mathrm{Bu}\right)_{3}$ (12.1 mg, $0.06 \mathrm{mmol}$ ), B2pin 2 (228.6 mg, $0.90 \mathrm{mmol}$ ), $\operatorname{PrOH}(38.1 \mathrm{mg}, 0.50 \mathrm{mmol}$ ), HCOOK $(21.0 \mathrm{mg}, 0.25 \mathrm{mmol})$ and IPE $(2.0 \mathrm{~mL})$. The product was obtained as a colourless oil in $42 \%$ yield $(60.0 \mathrm{mg}$, eluent : petroleum ether : EtOAc $=40: 1)$. $\mathbf{R}_{f}=0.46$ (petroleum ether : EtOAc $=10: 1$ ).

${ }^{1} \mathrm{H}$ NMR (400 MHz, $\left.\mathrm{CDCl}_{3}\right)$ \& $7.52(\mathrm{~s}, 1 \mathrm{H}), 7.31-7.26(\mathrm{~m}, 1 \mathrm{H}), 7.23-7.14(\mathrm{~m}$, $1 \mathrm{H}), 7.06(\mathrm{~d}, J=3.0 \mathrm{~Hz}, 1 \mathrm{H}), 6.47(\mathrm{~d}, J=2.9 \mathrm{~Hz}, 1 \mathrm{H}), 3.81(\mathrm{~s}, 3 \mathrm{H}), 2.92(\mathrm{t}, J=8.3$ $\mathrm{Hz}, 2 \mathrm{H}), 1.31-1.27(\mathrm{~m}, 14 \mathrm{H})$.

${ }^{13} \mathrm{C}$ NMR (100 MHz, CDCl $) \delta$ d 135.5, 135.4, 128.8, 128.7, 122.4, 119.5, 108.9, 100.6, $83.1,32.9,30.2,25.0$. The carbon signal attached to B was not observed due to low intensity.

HRMS (ESI') calcd for $\mathrm{C}_{17} \mathrm{H}_{24} \mathrm{BNO}_{2} \mathrm{Na}^{+}[\mathrm{M}+\mathrm{Na}]^{+}: 308.1792$, found: 308.1792 .

IR (neat, (cm-1): 2975, 2937, 1489, 1371, 1318, 1244, 1141, 965, 850, 797, 726. 


\section{2-(2,5-Dimethylfuran-3-yl)ethan-1-ol (2z)}

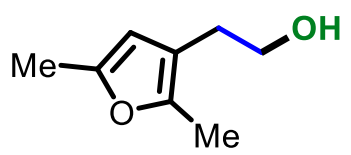

Compound $2 \mathbf{z}$ was prepared following the GP2 using 1-(2,5-dimethylfuran-3yl)ethan-1-one $\mathbf{1 z}(41.4 \mathrm{mg}, 0.3 \mathrm{mmol})$, [RhCl(cod)]2 (3.0 mg, $0.006 \mathrm{mmol})$, $\mathrm{P}\left({ }^{n} \mathrm{Bu}\right)_{3}(7.3 \mathrm{mg}, 0.036 \mathrm{mmol}), \mathrm{B}_{2}$ pin2 $(137.0 \mathrm{mg}, 0.54 \mathrm{mmol})$, HCOOK (12.6 mg, $0.15 \mathrm{mmol}),{ }^{\mathrm{i}} \mathrm{PrOH}(22.9 \mathrm{mg}, 0.30 \mathrm{mmol})$ and IPE $(2.0 \mathrm{~mL})$. The product was obtained as a colourless oil in $62 \%$ yield $(26.0 \mathrm{mg}$, eluent : petroleum ether : $\mathrm{EtOAc}=3: 1)$.

$\mathbf{R}_{f}=0.46$ (petroleum ether $:$ EtOAc $=1: 1$ ).

${ }^{1}$ H NMR (400 MHz, $\left.\mathrm{CDCl}_{3}\right) \delta 5.80(\mathrm{~s}, 1 \mathrm{H}), 3.72(\mathrm{t}, J=6.4 \mathrm{~Hz}, 2 \mathrm{H}), 2.54(\mathrm{t}, J=6.4$ $\mathrm{Hz}, 2 \mathrm{H}), 2.22(\mathrm{~s}, 3 \mathrm{H}), 2.19(\mathrm{~s}, 3 \mathrm{H})$.

${ }^{13} \mathrm{C}$ NMR (100 MHz, $\left.\mathrm{CDCl}_{3}\right) \delta$ 149.9, 146.9, 115.7, 107.4, 62.9, 28.7, 13.7, 11.6.

HRMS (ESI') calcd for $\mathrm{C}_{8} \mathrm{H}_{12} \mathrm{O}_{2} \mathrm{Na}^{+}[\mathrm{M}+\mathrm{Na}]^{+}:$163.0730, found: 163.0738 .

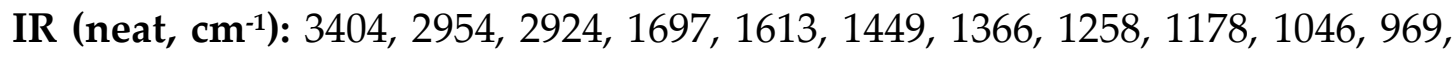
796.

\section{4,4,5,5-Tetramethyl-2-(2-(thiophen-2-yl)ethyl)-1,3,2-dioxaborolane (2aa)}

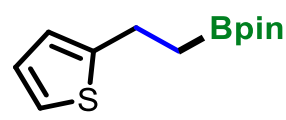

Compound 2aa was prepared following the GP1 using 1-(thiophen-2-yl)ethan1-one 1aa (37.8 mg, $0.3 \mathrm{mmol})$, [RhCl(cod)]2 (3.0 mg, $0.006 \mathrm{mmol}), \mathrm{P}(\mathrm{Oct}) 3$ (13.3 $\mathrm{mg}, 0.036 \mathrm{mmol}), B_{2}$ pin2 (137.0 mg, $\left.0.54 \mathrm{mmol}\right)$, HCOOK (12.6 mg, $0.15 \mathrm{mmol}$ ) ${ }^{i} \mathrm{PrOH}(22.9 \mathrm{mg}, 0.30 \mathrm{mmol})$ and IPE $(2.0 \mathrm{~mL})$. The product was obtained as a colourless oil in $65 \%$ yield $(46.0 \mathrm{mg}$, eluent : petroleum ether : EtOAc $=15: 1)$. $\mathbf{R}_{f}=0.71$ (petroleum ether : EtOAc $=10: 1$ ).

${ }^{1}$ H NMR (400 MHz, $\left.\mathrm{CDCl}_{3}\right) \delta 7.10-7.06(\mathrm{~m}, 1 \mathrm{H}), 6.91-6.87(\mathrm{~m}, 1 \mathrm{H}), 6.80(\mathrm{~d}, J$ $=4.6 \mathrm{~Hz}, 1 \mathrm{H}), 2.96(\mathrm{t}, J=8.1 \mathrm{~Hz}, 2 \mathrm{H}), 1.23-1.25(\mathrm{~m}, 14 \mathrm{H})$.

${ }^{13} \mathrm{C}$ NMR (100 MHz, $\left.\mathrm{CDCl}_{3}\right) \delta$ 148.0, 126.8, 123.6, 122.8, 83.5, 25.0, 24.6. The carbon signal attached to $B$ was not observed due to low intensity. Spectral data matched those previously reported. ${ }^{[10]}$ 


\section{4,4,5,5-Tetramethyl-2-octyl-1,3,2-dioxaborolane (2ab)}

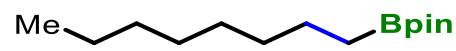

Compound 2ab was prepared following the GP1 using octan-2-one 1ab (38.4 $\mathrm{mg}$, $0.3 \mathrm{mmol})$, [RhCl(cod) $]_{2}(3.0 \mathrm{mg}, 0.006 \mathrm{mmol}), \mathrm{P}\left({ }^{n} \mathrm{Bu}\right) 3(7.3 \mathrm{mg}, 0.036 \mathrm{mmol})$, B2pin2 (137 mg, $0.54 \mathrm{mmol}$ ), ${ }^{2} \mathrm{PrOH}$ (22.9 mg, $\left.0.30 \mathrm{mmol}\right)$, HCOOK (12.6 mg, 0.15 $\mathrm{mmol})$ and IPE $(2.0 \mathrm{~mL})$. The product was obtained as a colourless oil in $38 \%$ yield $(27.1 \mathrm{mg}$, eluent : petroleum ether : EtOAc $=25: 1)$.

$\mathbf{R}_{f}=0.68$ (petroleum ether $:$ EtOAc $=20: 1$ ).

${ }^{1} \mathrm{H}$ NMR (400 MHz, $\left.\mathrm{CDCl}_{3}\right) \delta 1.53-1.50(\mathrm{~m}, 2 \mathrm{H}), 1.22-1.21(\mathrm{~m}, 22 \mathrm{H}), 0.85(\mathrm{t}, J$ $=7.1 \mathrm{~Hz}, 3 \mathrm{H}), 0.70(\mathrm{t}, J=8.0 \mathrm{~Hz}, 2 \mathrm{H})$.

${ }^{13} \mathrm{C}$ NMR (100 MHz, $\left.\mathrm{CDCl}_{3}\right) \delta$ 83.0, 32.7, 32.0, 29.7, 29.3, 25.0, 24.6, 22.8, 14.3. The carbon signal attached to B was not observed due to low intensity. Spectral data matched those previously reported..$^{[14]}$

\section{1-Phenylpropan-2-ol (2ac)}<smiles>CC(O)Cc1ccccc1</smiles>

Compound 2ac was prepared following the GP2 using propiophenone 1ac (40.2 mg, $0.3 \mathrm{mmol}),[\mathrm{RhCl}(\mathrm{cod})]_{2}(3.0 \mathrm{mg}, 0.006 \mathrm{mmol}), \mathrm{P}\left({ }^{t} \mathrm{Bu}\right){ }_{3} \cdot \mathrm{HBF}_{4}(10.4 \mathrm{mg}$, $0.036 \mathrm{mmol}), \mathrm{B}_{2}$ pin2 (137 mg, $\left.0.54 \mathrm{mmol}\right),{ }^{\mathrm{P}} \mathrm{PrOH}(22.9 \mathrm{mg}, 0.30 \mathrm{mmol})$, HCOOLi $(10.5 \mathrm{mg}, 0.15 \mathrm{mmol})$ and IPE $(2.0 \mathrm{~mL})$. The product was obtained as a colourless oil in $46 \%$ yield $(19.1 \mathrm{mg}$, eluent : petroleum ether : EtOAc $=5: 1)$.

$\mathbf{R}_{f}=0.49$ (petroleum ether : EtOAc $=2: 1$ ).

${ }^{1} \mathrm{H}$ NMR (400 MHz, $\left.\mathrm{CDCl}_{3}\right) 7.35(\mathrm{t}, J=7.2 \mathrm{~Hz}, 2 \mathrm{H}), 7.30-7.21(\mathrm{~m}, 3 \mathrm{H}), 4.15-$ $3.94(\mathrm{~m}, 1 \mathrm{H}), 2.90-2.69(\mathrm{~m}, 2 \mathrm{H}), 1.61(\mathrm{~s}, 1 \mathrm{H}), 1.28(\mathrm{~d}, J=6.2 \mathrm{~Hz}, 3 \mathrm{H})$. ${ }^{13} \mathrm{C}$ NMR (100 MHz, $\left.\mathrm{CDCl}_{3}\right) \delta$ 138.6, 129.5, 128.7, 126.6, 69.0, 45.9, 22.9. Spectral data matched those previously reported..$^{[16]}$ 


\section{General procedure for the synthesis of branched alkylboronates}

\section{General procedure 3 (GP3)}
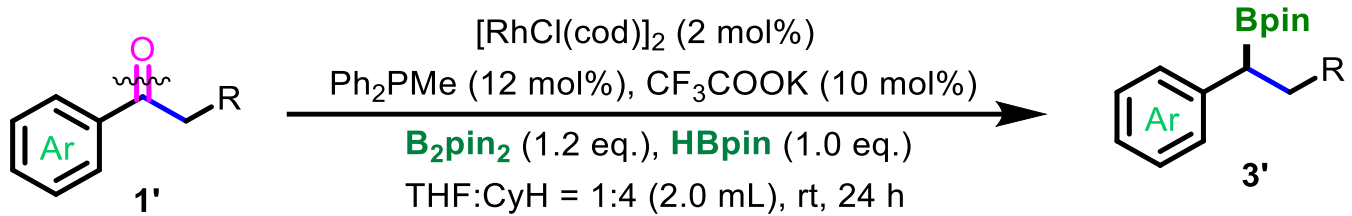

In glove box, $[\mathrm{RhCl}(\mathrm{cod})]_{2}(3.0 \mathrm{mg}, 0.006 \mathrm{mmol}), \mathrm{PPh}_{2} \mathrm{Me}(7.2 \mathrm{mg}, 0.036$ $\mathrm{mmol})$, and anhydrous THF $(0.4 \mathrm{~mL})$ were placed in a $4 \mathrm{~mL}$ glass tube and stirred for $30 \mathrm{~min}$. Then, $\mathrm{CF}_{3} \mathrm{COOK}$ (4.6 mg, $0.03 \mathrm{mmol}$ ), B2pinz (91.4 mg, 0.36 $\mathrm{mmol})$, HBpin ( $38.4 \mathrm{mg}, 0.30 \mathrm{mmol})$, and ketone $\mathbf{1}^{\prime}$ in $\mathrm{CyH}(1.6 \mathrm{~mL})$ were added. The reaction mixture was sealed, removed from the glovebox, and stirred at room tempreture for the duration of time indicated. After the reaction mixtrue was concentrated in vacuo, the residue was purified by silica gel chromatography using petroleum ether : EtOAc as the eluent to afford the desired products $3^{\prime}$.

\section{General procedure 4 (GP4)}

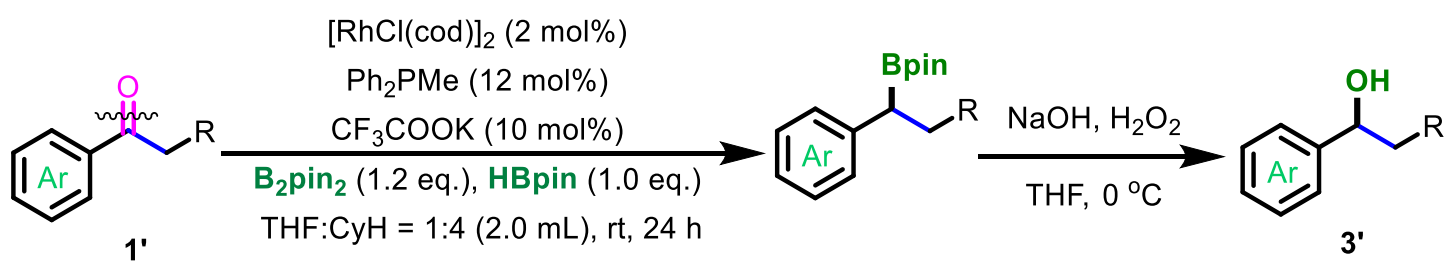

In glove box, $[\mathrm{RhCl}(\mathrm{cod})]_{2}(3.0 \mathrm{mg}, 0.006 \mathrm{mmol}), \mathrm{PPh}_{2} \mathrm{Me}(7.2 \mathrm{mg}, 0.036$ $\mathrm{mmol})$, and anhydrous THF $(0.4 \mathrm{~mL})$ were placed in a $4 \mathrm{~mL}$ glass tube and stirred for $30 \mathrm{~min}$. Then, $\mathrm{CF}_{3} \mathrm{COOK}$ (4.6 mg, $0.03 \mathrm{mmol}$ ), B2 pin $_{2}$ (91.4 mg, 0.36 mmol), HBpin (38.4 mg, $0.30 \mathrm{mmol})$, and ketone $\mathbf{1}^{\prime}$ in $\mathrm{CyH}(1.6 \mathrm{~mL})$ were added. The reaction mixture was sealed, removed from the glovebox, and stirred at room tempreture for the duration of time indicated. After the reaction mixture was concentrated in vacuo, to the stirred solution of the crude residue in THF (1.0 mL) was added $\mathrm{NaOH}(1.5$ equiv, $3.0 \mathrm{M})$ and $\mathrm{H}_{2} \mathrm{O}_{2}(1.5$ equiv, $30 \%)$ at $0{ }^{\circ} \mathrm{C}$. The reaction mixture was stirred for 0.5 hour, then extracted with EtOAc, dried with $\mathrm{Na}_{2} \mathrm{SO}_{4}$, filtered and concentrated. The residue was purified by silica gel chromatography using petroleum ether : EtOAc as the eluent to afford the 
desired products $\mathbf{3}^{\prime}$.

\section{General procedure 5 (GP5)}

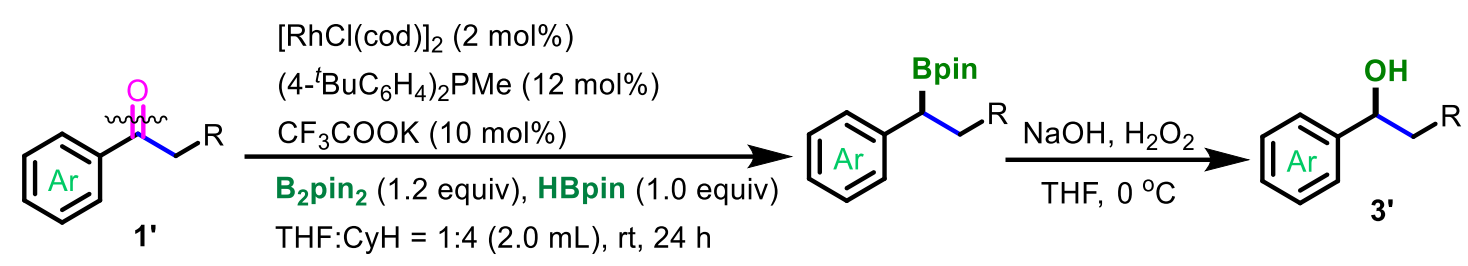

In glove box, $[\mathrm{RhCl}(\mathrm{cod})]_{2}(3.0 \mathrm{mg}, 0.006 \mathrm{mmol}),\left(4-{ }^{\mathrm{t}} \mathrm{BuC}_{6} \mathrm{H}_{4}\right)_{2} \mathrm{PMe}(11.2 \mathrm{mg}$, $0.036 \mathrm{mmol})$, and anhydrous THF $(0.4 \mathrm{~mL})$ were placed in a $4 \mathrm{~mL}$ glass tube and stirred for $30 \mathrm{~min}$. Then, $\mathrm{CF}_{3} \mathrm{COOK}$ (4.6 mg, $0.03 \mathrm{mmol}$ ), B2pin2 (91.4 mg, $0.36 \mathrm{mmol}), \mathrm{HBpin}(38.4 \mathrm{mg}, 0.30 \mathrm{mmol})$ and ketone $\mathbf{1}^{\prime}$ in $\mathrm{CyH}(1.6 \mathrm{~mL})$ were added. The reaction mixture was sealed, removed from the glovebox, and stirred at room tempreture for the duration of time indicated. After the reaction mixture was concentrated in vacuo, to the stirred solution of the crude residue in THF (1.0 mL) was added $\mathrm{NaOH}(1.5$ equiv, $3.0 \mathrm{M})$ and $\mathrm{H}_{2} \mathrm{O}_{2}$ (1.5 equiv, 30\%) at $0{ }^{\circ} \mathrm{C}$. The reaction mixture was stirred for 0.5 hour, then extracted with EtOAc, dried with $\mathrm{Na}_{2} \mathrm{SO}_{4}$, filtered and concentrated. The residue was purified by silica gel chromatography using petroleum ether : EtOAc as the eluent to afford the desired products $\mathbf{3}^{\prime}$.

\section{4,4,5,5-Tetramethyl-2-(1-phenylethyl)-1,3,2-dioxaborolane (3a').}<smiles>CC([B]c1ccccc1)c1ccccc1</smiles>

Compound 3a' was prepared following the GP3 using acetophenone 1a' (36.0 mg, $0.3 \mathrm{mmol})$, [RhCl(cod)]2 (3.0 mg, $0.006 \mathrm{mmol})$, PPh $2 \mathrm{Me}(7.2 \mathrm{mg}, 0.036 \mathrm{mmol})$, B2pinz (91.4 mg, $0.36 \mathrm{mmol}), \mathrm{HBpin}(38.4 \mathrm{mg}, 0.30 \mathrm{mmol}), \mathrm{CF}_{3} \mathrm{COOK}$ (4.6 mg, $0.03 \mathrm{mmol})$, THF $(0.4 \mathrm{~mL})$ and $\mathrm{CyH}(1.6 \mathrm{~mL})$. The product was obtained as a colourless oil in 78\% yield (54.3 mg, eluent : petroleum ether : EtOAc $=25: 1)$. $\mathbf{R}_{f}=0.50$ (petroleum ether : $\mathrm{EtOAc}=20: 1$ ).

${ }^{1} \mathrm{H}$ NMR (400 MHz, $\left.\mathrm{CDCl}_{3}\right) \delta 7.35-7.25(\mathrm{~m}, 4 \mathrm{H}), 7.22-7.15(\mathrm{~m}, 1 \mathrm{H}), 2.50(\mathrm{q}, J$ $=7.6 \mathrm{~Hz}, 1 \mathrm{H}), 1.39(\mathrm{~d}, J=7.2 \mathrm{~Hz}, 3 \mathrm{H}), 1.26(\mathrm{~d}, J=5.8 \mathrm{~Hz}, 12 \mathrm{H})$.

${ }^{13} \mathrm{C}$ NMR (100 MHz, $\left.\mathrm{CDCl}_{3}\right) \delta$ 145.0, 128.4, 127.9, 125.2, 83.4, 24.72, 24.68, 17.2. The carbon signal attached to $B$ was not observed due to low intensity. 
Spectral data matched those previously reported. ${ }^{[17]}$

4,4,5,5-Tetramethyl-2-(1-(p-tolyl)ethyl)-1,3,2-dioxaborolane (3b').<smiles>Cc1ccc(C(Br)Br)cc1</smiles>

Compound $\mathbf{3} \mathbf{b}^{\prime}$ was prepared following the GP3 using 1-(p-tolyl)ethan-1-one 1b' (40.2 mg, $0.3 \mathrm{mmol})$, [RhCl(cod)]2 (3.0 mg, $0.006 \mathrm{mmol}), \mathrm{PPh}_{2} \mathrm{Me}(7.2 \mathrm{mg}$, $0.036 \mathrm{mmol}), B_{2}$ pin $2(91.4 \mathrm{mg}, 0.36 \mathrm{mmol}), \mathrm{HBpin}(38.4 \mathrm{mg}, 0.30 \mathrm{mmol})$, $\mathrm{CF}_{3} \mathrm{COOK}(4.6 \mathrm{mg}, 0.03 \mathrm{mmol})$, THF $(0.4 \mathrm{~mL})$ and $\mathrm{CyH}(1.6 \mathrm{~mL})$. The product was obtained as a colourless oil in $72 \%$ yield $(53.2 \mathrm{mg}$, eluent : petroleum ether : $\mathrm{EtOAc}=30: 1)$.

$\mathbf{R}_{f}=0.63$ (petroleum ether : $\mathrm{EtOAc}=10: 1$ ).

${ }^{1} \mathrm{H}$ NMR (400 MHz, $\mathrm{CDCl}_{3}$ ) \& $7.17-7.05(\mathrm{~m}, 4 \mathrm{H}), 2.41$ (q, $\left.J=7.4 \mathrm{~Hz}, 1 \mathrm{H}\right), 2.32$ $(\mathrm{s}, 3 \mathrm{H}), 1.33(\mathrm{~d}, J=7.5 \mathrm{~Hz}, 3 \mathrm{H}), 1.23(\mathrm{~d}, J=5.1 \mathrm{~Hz}, 12 \mathrm{H})$.

${ }^{13} \mathrm{C}$ NMR (100 MHz, $\mathrm{CDCl}_{3}$ ) $\delta$ 142.0, 134.4, 129.1, 127.8, 83.3, 24.74, 24.71, 21.1, 17.4. The carbon signal attached to $B$ was not observed due to low intensity. Spectral data matched those previously reported. ${ }^{[18]}$

2-(1-(4-Methoxyphenyl)ethyl)-4,4,5,5-tetramethyl-1,3,2-dioxaborolane (3c').<smiles>COc1ccc(C(C)Cc2ccccc2)cc1</smiles>

Compound $3 \mathbf{c}^{\prime}$ was prepared following the GP3 using 1-(4methoxyphenyl)ethan-1-one $1 \mathbf{c}^{\prime}(45.0 \mathrm{mg}, 0.3 \mathrm{mmol})$, [ RhCl(cod)]2 $(3.0 \mathrm{mg}$, $0.006 \mathrm{mmol}), \mathrm{PPh}_{2} \mathrm{Me}$ (7.2 mg, $\left.0.036 \mathrm{mmol}\right), \mathrm{B}_{2} \mathrm{pin}_{2}$ (91.4 mg, $\left.0.36 \mathrm{mmol}\right), \mathrm{HBpin}$ (38.4 mg, $0.30 \mathrm{mmol}), \mathrm{CF}_{3} \mathrm{COOK}(4.6 \mathrm{mg}, 0.03 \mathrm{mmol})$, THF $(0.4 \mathrm{~mL})$ and $\mathrm{CyH}$ $(1.6 \mathrm{~mL})$. The product was obtained as a colourless oil in $76 \%$ yield $(60.2 \mathrm{mg}$, eluent : petroleum ether : $\mathrm{EtOAc}=25: 1)$.

$\mathbf{R}_{f}=0.45$ (petroleum ether : $\mathrm{EtOAc}=20: 1$ ).

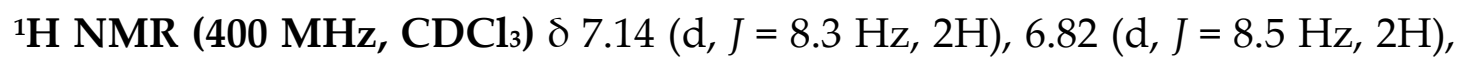
$3.78(\mathrm{~s}, 3 \mathrm{H}), 2.37(\mathrm{q}, J=7.5 \mathrm{~Hz}, 1 \mathrm{H}), 1.30(\mathrm{~d}, J=7.5 \mathrm{~Hz}, 3 \mathrm{H}), 1.21(\mathrm{~d}, J=5.1 \mathrm{~Hz}$, $12 \mathrm{H})$. 
${ }^{13} \mathrm{C}$ NMR (100 MHz, $\left.\mathrm{CDCl}_{3}\right) \delta 157.4,137.2,128.8,113.9,83.4,55.3,24.78,24.75$, 17.5. The carbon signal attached to $B$ was not observed due to low intensity. Spectral data matched those previously reported. ${ }^{[17]}$

4,4,5,5-Tetramethyl-2-(1-(4-(methylthio)phenyl)ethyl)-1,3,2-dioxaborolane $\left(3 d^{\prime}\right)$.<smiles>CSc1ccc(C(C)Cc2ccccc2)cc1</smiles>

Compound 3d' was prepared following the GP3 using 1-(4(methylthio)phenyl)ethan-1-one 1d' (49.8 mg, $0.3 \mathrm{mmol})$, [ $\mathrm{RhCl}(\mathrm{cod})]_{2}(3.0 \mathrm{mg}$, $0.006 \mathrm{mmol}), \mathrm{PPh}_{2} \mathrm{Me}$ (7.2 mg, $\left.0.036 \mathrm{mmol}\right), \mathrm{B}_{2}$ pin2 (91.4 mg, $\left.0.36 \mathrm{mmol}\right), \mathrm{HBpin}$ (38.4 mg, $0.30 \mathrm{mmol}), \mathrm{CF}_{3} \mathrm{COOK}(4.6 \mathrm{mg}, 0.03 \mathrm{mmol})$, THF $(0.4 \mathrm{~mL})$ and $\mathrm{CyH}$ $(1.6 \mathrm{~mL})$. The product was obtained as a colourless oil in $70 \%$ yield $(59.6 \mathrm{mg}$, eluent : petroleum ether : $\mathrm{EtOAc}=25: 1)$.

$\mathbf{R}_{f}=0.55$ (petroleum ether : $\mathrm{EtOAc}=10: 1$ ).

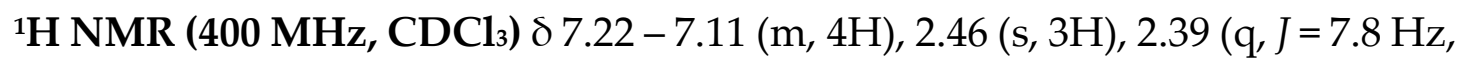
$1 \mathrm{H}), 1.31(\mathrm{~d}, J=7.7 \mathrm{~Hz}, 3 \mathrm{H}), 1.20(\mathrm{~d}, J=4.7 \mathrm{~Hz}, 12 \mathrm{H})$.

${ }^{13} \mathrm{C}$ NMR (100 MHz, $\left.\mathrm{CDCl}_{3}\right) \delta$ 142.4, 134.3, 128.5, 127.4, 83.5, 24.76, 24.72, 17.2, 16.5. The carbon signal attached to $B$ was not observed due to low intensity. Spectral data matched those previously reported. ${ }^{[18]}$

2-(1-(4-(Benzyloxy)phenyl)ethyl)-4,4,5,5-tetramethyl-1,3,2-dioxaborolane $\left(3 \mathrm{e}^{\prime}\right)$.<smiles>CC(Br)c1ccc(OCc2ccccc2)cc1</smiles>

Compound $3 \mathbf{e}^{\prime}$ was prepared following the GP3 using 1-(4(benzyloxy)phenyl)ethan-1-one 1e' (67.8 mg, $0.3 \mathrm{mmol})$, [ $\mathrm{RhCl}(\mathrm{cod})]_{2}(3.0 \mathrm{mg}$, $0.006 \mathrm{mmol}), \mathrm{PPh}_{2} \mathrm{Me}$ (7.2 mg, $\left.0.036 \mathrm{mmol}\right), \mathrm{B}_{2} \mathrm{Pin}_{2}(91.4 \mathrm{mg}, 0.36 \mathrm{mmol}), \mathrm{HBpin}$ (38.4 mg, $0.30 \mathrm{mmol}), \mathrm{CF}_{3} \mathrm{COOK}(4.6 \mathrm{mg}, 0.03 \mathrm{mmol})$, THF $(0.4 \mathrm{~mL})$ and $\mathrm{CyH}$ $(1.6 \mathrm{~mL})$. The product was obtained as a colourless oil in $81 \%$ yield $(81.8 \mathrm{mg}$, eluent : petroleum ether : $\mathrm{EtOAc}=15: 1$ ). 
$\mathbf{R}_{f}=0.65$ (petroleum ether $:$ EtOAc $=8: 1$ ).

${ }^{1} \mathrm{H}$ NMR (400 MHz, $\mathrm{CDCl}_{3}$ ) $\delta 7.47$ - 7.40 (m, 2H), 7.42 - $7.34(\mathrm{~m}, 2 \mathrm{H}), 7.36$ - 7.29 $(\mathrm{m}, 1 \mathrm{H}), 7.14(\mathrm{~d}, J=8.1 \mathrm{~Hz}, 2 \mathrm{H}), 6.90(\mathrm{~d}, J=8.1 \mathrm{~Hz}, 2 \mathrm{H}), 5.03(\mathrm{~s}, 2 \mathrm{H}), 2.38(\mathrm{q}, J=$ $7.5 \mathrm{~Hz}, 1 \mathrm{H}), 1.31(\mathrm{~d}, J=7.7 \mathrm{~Hz}, 3 \mathrm{H}), 1.21(\mathrm{~d}, J=5.0 \mathrm{~Hz}, 12 \mathrm{H})$.

${ }^{13} \mathrm{C}$ NMR (100 MHz, $\left.\mathrm{CDCl}_{3}\right) \delta$ 156.7, 137.5, 137.5, 128.8, 128.7, 128.0, 127.6, 114.9, $83.4,70.2,24.78,24.74,17.5$. The carbon signal attached to B was not observed due to low intensity.

Spectral data matched those previously reported. ${ }^{[17]}$

tert-Butyldimethyl(4-(1-(4,4,5,5-tetramethyl-1,3,2-dioxaborolan-2yl)ethyl)phenoxy)silane ( $\left.3 f^{\prime}\right)$.<smiles>CC(C)Cc1ccc(OC(C)C(C)c2ccccc2)cc1</smiles>

Compound $3 \mathbf{f}^{\prime}$ was prepared following the GP3 using 1-(4-((tert-

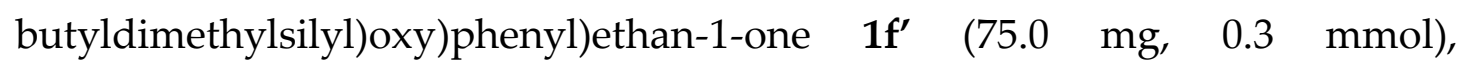
$[\mathrm{RhCl}(\mathrm{cod})]_{2}(3.0 \mathrm{mg}, 0.006 \mathrm{mmol}), \mathrm{PPh}_{2} \mathrm{Me}(7.2 \mathrm{mg}, 0.036 \mathrm{mmol}), \mathrm{B}_{2} \mathrm{pin}_{2}(114.0$ $\mathrm{mg}, 0.45 \mathrm{mmol}), \mathrm{HBpin}(38.4 \mathrm{mg}, 0.30 \mathrm{mmol}), \mathrm{CF}_{3} \mathrm{COOK}(4.6 \mathrm{mg}, 0.03 \mathrm{mmol}$ ), THF $(0.4 \mathrm{~mL})$ and $\mathrm{CyH}(1.6 \mathrm{~mL})$. The product was obtained as a colourless oil in $69 \%$ yield $(75.1 \mathrm{mg}$, eluent : petroleum ether : $\mathrm{EtOAc}=25: 1)$.

$\mathbf{R}_{f}=0.60$ (petroleum ether : $\mathrm{EtOAc}=20: 1$ ).

${ }^{1} \mathrm{H}$ NMR (400 MHz, $\left.\mathrm{CDCl}_{3}\right) \delta 7.06(\mathrm{~d}, J=8.9 \mathrm{~Hz}, 2 \mathrm{H}), 6.73(\mathrm{~d}, J=9.0 \mathrm{~Hz}, 2 \mathrm{H})$, $2.36(\mathrm{q}, J=7.6 \mathrm{~Hz}, 1 \mathrm{H}), 1.29(\mathrm{~d}, J=7.6 \mathrm{~Hz}, 3 \mathrm{H}), 1.19(\mathrm{~d}, J=5.6 \mathrm{~Hz}, 12 \mathrm{H}), 0.97$ (s, $9 \mathrm{H}), 0.17(\mathrm{~s}, 6 \mathrm{H})$.

${ }^{13} \mathrm{C}$ NMR (100 MHz, $\left.\mathrm{CDCl}_{3}\right) \delta$ 153.2, 137.6, 128.6, 119.9, 83.3, 25.9, 24.75, 24.70, $18.3,17.2,-4.3$. The carbon signal attached to $B$ was not observed due to low intensity.

Spectral data matched those previously reported.[17]

1-(4-(Dimethylamino)phenyl)ethan-1-ol (3g').<smiles>CC(O)c1ccc(N(C)C)cc1</smiles> 
Compound 3g' was prepared following the GP4 using 1-(4(dimethylamino)phenyl)ethan-1-one 1g' (48.9 mg, $0.3 \mathrm{mmol}),[\mathrm{RhCl}(\mathrm{cod})]_{2}(3.0$ $\mathrm{mg}, 0.006 \mathrm{mmol}), \mathrm{PPh}_{2} \mathrm{Me}(7.2 \mathrm{mg}, 0.036 \mathrm{mmol}), \mathrm{B}_{2}$ pin2 $(91.4 \mathrm{mg}, 0.36 \mathrm{mmol})$, HBpin (38.4 mg, $0.30 \mathrm{mmol}), \mathrm{CF}_{3} \mathrm{COOK}(4.6 \mathrm{mg}, 0.03 \mathrm{mmol}), \mathrm{THF}(0.4 \mathrm{~mL})$ and $\mathrm{CyH}(1.6 \mathrm{~mL})$. The product was obtained as a colourless oil in $85 \%$ yield (42.1 mg, eluent : petroleum ether : $\mathrm{EtOAc}=4: 1)$.

$\mathbf{R}_{f}=0.45$ (petroleum ether $: \mathrm{EtOAc}=2: 1$ ).

${ }^{1} \mathrm{H}$ NMR (400 MHz, $\left.\mathrm{CDCl}_{3}\right) \delta 7.40-7.32(\mathrm{~m}, 2 \mathrm{H}), 6.86-6.80(\mathrm{~m}, 2 \mathrm{H}), 4.91$ (q, J $=6.6 \mathrm{~Hz}, 1 \mathrm{H}), 3.03(\mathrm{~s}, 6 \mathrm{H}), 1.92(\mathrm{sbr}, 1 \mathrm{H}), 1.57(\mathrm{~d}, J=6.9 \mathrm{~Hz}, 3 \mathrm{H})$.

${ }^{13} \mathrm{C}$ NMR (100 MHz, $\left.\mathrm{CDCl}_{3}\right) \delta$ 150.4, 134.0, 126.7, 112.9, 70.3, 40.9, 24.9.

Spectral data matched those previously reported. ${ }^{[20]}$

\section{4,4,5,5-Tetramethyl-2-(4-(1-(4,4,5,5-tetramethyl-1,3,2-dioxaborolan-2-} yl)ethyl)phenyl)-1,3,2-dioxaborolane (3h').<smiles>CC(Br)c1ccc(Cc2ccccc2)cc1</smiles>

Compound $3 \mathbf{h}^{\prime}$ was prepared following the GP3 using 1-(4-(4,4,5,5tetramethyl-1,3,2-dioxaborolan-2-yl)phenyl)ethan-1-one $\mathbf{1 h}^{\prime}$ (73.8 $\mathbf{m g}, 0.3$ $\mathrm{mmol})$, [RhCl(cod)]2 (3.0 mg, $0.006 \mathrm{mmol}), \mathrm{PPh}_{2} \mathrm{Me}(7.2 \mathrm{mg}, 0.036 \mathrm{mmol}), \mathrm{B}_{2}$ pin 2 (91.4 mg, $0.36 \mathrm{mmol}$ ), HBpin (38.4 mg, $0.30 \mathrm{mmol}), \mathrm{CF}_{3} \mathrm{COOK}$ (4.6 mg, 0.03 $\mathrm{mmol})$, THF $(0.4 \mathrm{~mL})$ and $\mathrm{CyH}(1.6 \mathrm{~mL})$. The product was obtained as a white solid in $67 \%$ yield $(72.1 \mathrm{mg}$, eluent : petroleum ether : EtOAc $=15: 1)$.

$\mathbf{R}_{f}=0.60$ (petroleum ether $:$ EtOAc $=8: 1$ ).

${ }^{1} \mathrm{H}$ NMR (400 MHz, $\left.\mathrm{CDCl}_{3}\right) \delta 7.71(\mathrm{~d}, J=7.6 \mathrm{~Hz}, 2 \mathrm{H}), 7.23(\mathrm{~d}, J=7.6 \mathrm{~Hz}, 2 \mathrm{H})$, $2.45(\mathrm{q}, J=7.7 \mathrm{~Hz}, 1 \mathrm{H}), 1.33(\mathrm{~s}, 15 \mathrm{H}), 1.19(\mathrm{~d}, J=5.0 \mathrm{~Hz}, 12 \mathrm{H})$.

${ }^{13} \mathrm{C}$ NMR (100 MHz, $\left.\mathrm{CDCl}_{3}\right) \delta$ 148.7, 135.0, 127.4, 83.7, 83.5, 25.0, 24.75, 24.72, 16.9. The carbon signal attached to $B$ was not observed due to low intensity. Spectral data matched those previously reported. ${ }^{[19]}$

2-(1-([1,1'-Biphenyl]-4-yl)ethyl)-4,4,5,5-tetramethyl-1,3,2-dioxaborolane (3i'). 
<smiles>CC(Br)c1ccc(-c2ccccc2)cc1</smiles>

Compound 3i' was prepared following the GP3 using 1-([1,1'-biphenyl]-4yl)ethan-1-one 1i' (58.8 mg, $0.3 \mathrm{mmol}),[\mathrm{RhCl}(\mathrm{cod})]_{2}(3.0 \mathrm{mg}, 0.006 \mathrm{mmol})$, $\mathrm{PPh}_{2} \mathrm{Me}$ (7.2 mg, $0.036 \mathrm{mmol}$ ), B2pin2 (91.4 mg, $0.36 \mathrm{mmol}$ ), HBpin (38.4 mg, 0.30 $\mathrm{mmol}), \mathrm{CF}_{3} \mathrm{COOK}(4.6 \mathrm{mg}, 0.03 \mathrm{mmol})$, THF $(0.4 \mathrm{~mL})$ and $\mathrm{CyH}(1.6 \mathrm{~mL})$. The product was obtained as a white solid in $62 \%$ yield $(57.1 \mathrm{mg}$, eluent : petroleum ether : EtOAc $=30: 1)$.

$\mathbf{R}_{f}=0.62$ (petroleum ether : EtOAc $=10: 1$ ).

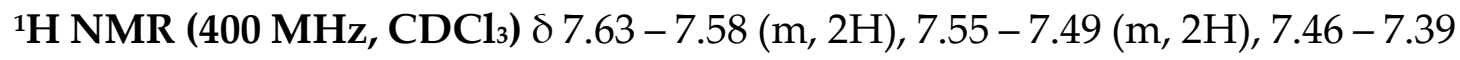
$(\mathrm{m}, 2 \mathrm{H}), 7.35-7.28(\mathrm{~m}, 3 \mathrm{H}), 2.50(\mathrm{q}, J=7.5 \mathrm{~Hz}, 1 \mathrm{H}), 1.39(\mathrm{~d}, J=7.5 \mathrm{~Hz}, 3 \mathrm{H}), 1.24$ $(\mathrm{d}, J=4.8 \mathrm{~Hz}, 12 \mathrm{H})$.

${ }^{13} \mathrm{C}$ NMR (100 MHz, CDCl ) $\delta$ 144.3, 141.4, 138.1, 128.8, 128.3, 127.2, 127.1, 126.9, $83.5,24.79,24.76,17.2$. The carbon signal attached to B was not observed due to low intensity.

Spectral data matched those previously reported. ${ }^{[17]}$

2-(1-(4-Fluorophenyl)ethyl)-4,4,5,5-tetramethyl-1,3,2-dioxaborolane (3j').<smiles>CC(Br)c1ccc(F)cc1</smiles>

Compound 3j' was prepared following the GP3 using 1-(4-fluorophenyl)ethan1-one 1j' (41.4. mg, $0.3 \mathrm{mmol})$, [RhCl(cod)]2 (3.0 mg, $0.006 \mathrm{mmol}), \mathrm{PPh}_{2} \mathrm{Me}$ (7.2 $\mathrm{mg}, 0.036 \mathrm{mmol}), \mathrm{B}_{2}$ pin2 $(91.4 \mathrm{mg}, 0.36 \mathrm{mmol}), \mathrm{HBpin}(38.4 \mathrm{mg}, 0.30 \mathrm{mmol})$, $\mathrm{CF}_{3} \mathrm{COOK}(4.6 \mathrm{mg}, 0.03 \mathrm{mmol})$, THF $(0.4 \mathrm{~mL})$ and $\mathrm{CyH}(1.6 \mathrm{~mL})$. The product was obtained as a colourless oil in $70 \%$ yield $(52.7 \mathrm{mg}$, eluent : petroleum ether : $\mathrm{EtOAc}=25: 1)$.

$\mathbf{R}_{f}=0.62$ (petroleum ether : $\mathrm{EtOAc}=20: 1$ ).

${ }^{1} \mathrm{H}$ NMR (400 MHz, $\left.\mathrm{CDCl}_{3}\right) \delta 7.19-7.12(\mathrm{~m}, 2 \mathrm{H}), 6.98-6.89(\mathrm{~m}, 2 \mathrm{H}), 2.41(\mathrm{q}, J$ $=7.5 \mathrm{~Hz}, 1 \mathrm{H}), 1.30(\mathrm{~d}, J=7.5 \mathrm{~Hz}, 3 \mathrm{H}), 1.20(\mathrm{~d}, J=4.7 \mathrm{~Hz}, 12 \mathrm{H})$. 
${ }^{13} \mathrm{C}$ NMR (100 MHz, $\left.\mathrm{CDCl}_{3}\right) \delta 161.0$ (d, JC-F $\left.=242.3 \mathrm{~Hz}\right), 140.7$ (d, JC-F $\left.=3.2 \mathrm{~Hz}\right)$, $129.1\left(\mathrm{~d}, J_{\mathrm{C}-\mathrm{F}}=7.6 \mathrm{~Hz}\right), 115.1\left(\mathrm{~d}, J_{\mathrm{C}-\mathrm{F}}=21.0 \mathrm{~Hz}\right), 83.5,24.76,24.72,17.4$. The carbon signal attached to B was not observed due to low intensity.

${ }^{19}$ F NMR (376 MHz, $\left.\mathrm{CDCl}_{3}\right)$ : $\delta$-119.0.

Spectral data matched those previously reported. ${ }^{[18]}$

2-(1-(4-Chlorophenyl)ethyl)-4,4,5,5-tetramethyl-1,3,2-dioxaborolane (3k').<smiles>CC(Br)c1ccc(Cl)cc1</smiles>

Compound $3 \mathbf{k}^{\prime}$ was prepared following the GP3 using 1-(4chlorophenyl)ethan-1-one 1k' (46.2. mg, $0.3 \mathrm{mmol}),[\mathrm{RhCl}(\mathrm{cod})]_{2}(3.0 \mathrm{mg}, 0.006$ mmol), PPh $2 \mathrm{Me}$ (7.2 mg, $0.036 \mathrm{mmol}$ ), B2pin2 (114.0 mg, $0.45 \mathrm{mmol}$ ), HBpin (38.4 $\mathrm{mg}, 0.30 \mathrm{mmol}), \mathrm{CF}_{3} \mathrm{COOK}(4.6 \mathrm{mg}, 0.03 \mathrm{mmol})$, THF (0.4 mL) and $\mathrm{CyH}(1.6$ $\mathrm{mL}$ ). The product was obtained as a colourless oil in $54 \%$ yield ( $42.9 \mathrm{mg}$, eluent : petroleum ether : $\mathrm{EtOAc}=20: 1)$.

$\mathbf{R}_{f}=0.70$ (petroleum ether : EtOAc $=10: 1$ ).

${ }^{1} \mathrm{H}$ NMR (400 MHz, $\left.\mathrm{CDCl}_{3}\right) \delta 7.25-7.19(\mathrm{~m}, 2 \mathrm{H}), 7.17-7.12(\mathrm{~m}, 2 \mathrm{H}), 2.40(\mathrm{q}, J$ $=7.6 \mathrm{~Hz}, 1 \mathrm{H}), 1.30(\mathrm{~d}, J=7.7 \mathrm{~Hz}, 3 \mathrm{H}), 1.20(\mathrm{~d}, J=4.2 \mathrm{~Hz}, 12 \mathrm{H})$.

${ }^{13} \mathrm{C}$ NMR (100 MHz, $\left.\mathrm{CDCl}_{3}\right) \delta$ 143.6, 130.9, 129.2, 128.5, 83.6, 24.76, 24.72, 17.1.

The carbon signal attached to B was not observed due to low intensity.

Spectral data matched those previously reported. ${ }^{[19]}$

4-(4-(1-(4,4,5,5-Tetramethyl-1,3,2-dioxaborolan-2-

yl)ethyl)phenyl)morpholine (31').<smiles>CC(Br)c1ccc(N2CCOCC2)cc1</smiles>

Compound 31' was prepared following the GP3 using 1-(4(dimethylamino)phenyl)ethan-1-one 11' (61.5 mg, $0.3 \mathrm{mmol}),[\mathrm{RhCl}(\mathrm{cod})]_{2}(3.0$ $\mathrm{mg}, 0.006 \mathrm{mmol}), \mathrm{PPh}_{2} \mathrm{Me}(7.2 \mathrm{mg}, 0.036 \mathrm{mmol}), \mathrm{B}_{2} \mathrm{pin} 2(91.4 \mathrm{mg}, 0.36 \mathrm{mmol})$, HBpin (38.4 mg, $0.30 \mathrm{mmol}), \mathrm{CF}_{3} \mathrm{COOK}(4.6 \mathrm{mg}, 0.03 \mathrm{mmol}), \mathrm{THF}(0.4 \mathrm{~mL})$ and 
CyH (1.6 mL). The product was obtained as a white solid in $66 \%$ yield $(62.7 \mathrm{mg}$, eluent : petroleum ether : $\mathrm{EtOAc}=4: 1)$.

$\mathbf{R}_{f}=0.82$ (petroleum ether : EtOAc $=2: 1$ ).

${ }^{1} \mathrm{H}$ NMR (400 MHz, $\left.\mathrm{CDCl}_{3}\right) \delta 7.13(\mathrm{~d}, J=8.4 \mathrm{~Hz}, 2 \mathrm{H}), 6.84(\mathrm{~d}, J=8.4 \mathrm{~Hz}, 2 \mathrm{H})$, $3.85(\mathrm{t}, J=7.6 \mathrm{~Hz}, 4 \mathrm{H}), 3.11(\mathrm{t}, J=7.6 \mathrm{~Hz}, 4 \mathrm{H}), 2.36(\mathrm{q}, J=7.5 \mathrm{~Hz}, 1 \mathrm{H}), 1.29(\mathrm{~d}, J=$ $7.4 \mathrm{~Hz}, 3 \mathrm{H}), 1.20(\mathrm{~d}, J=5.1 \mathrm{~Hz}, 12 \mathrm{H})$.

${ }^{13} \mathrm{C}$ NMR (100 MHz, $\left.\mathrm{CDCl}_{3}\right) \delta$ 149.0, 136.9, 128.6, 116.2, 83.4, 67.2, 50.0, 24.85, $24.82,17.5$. The carbon signal attached to $B$ was not observed due to low intensity.

HRMS (ESI') calcd for $\mathrm{C}_{18} \mathrm{H}_{29} \mathrm{BNO}_{3}{ }^{+}[\mathrm{M}+\mathrm{H}]^{+}:$318.2235, found: 318.2235.

IR (neat, cm-1): 2972, 1601, 1512, 1453, 1350, 1236, 1144, 1115, 982, 947, 826, 673.

\section{4,4,5,5-Tetramethyl-2-(1-(4-(methylsulfonyl)phenyl)ethyl)-1,3,2- dioxaborolane $\left(3 \mathrm{~m}^{\prime}\right)$.}<smiles>CC(O)c1ccc(S(C)(=O)=O)cc1</smiles>

Compound $3 \mathrm{~m}^{\prime}$ was prepared following the GP4 using 1-(4(methylsulfonyl)phenyl)ethan-1-one 1m' (59.4. $\mathrm{mg}, 0.3 \mathrm{mmol})$, [ $\mathrm{RhCl}(\mathrm{cod})]_{2}(3.0$ $\mathrm{mg}, 0.006 \mathrm{mmol}), \mathrm{PPh}_{2} \mathrm{Me}(7.2 \mathrm{mg}, 0.036 \mathrm{mmol}), \mathrm{B}_{2}$ pin2 $(114.0 \mathrm{mg}, 0.45 \mathrm{mmol})$, HBpin (38.4 mg, $0.30 \mathrm{mmol}), \mathrm{CF}_{3} \mathrm{COOK}(4.6 \mathrm{mg}, 0.03 \mathrm{mmol}), \mathrm{THF}(0.4 \mathrm{~mL})$ and $\mathrm{CyH}(1.6 \mathrm{~mL})$. The product was obtained as a colourless oil in $77 \%$ yield $(46.2$ $\mathrm{mg}$, eluent : petroleum ether : $\mathrm{EtOAc}=2: 1)$.

$\mathbf{R}_{f}=0.80$ (petroleum ether $:$ EtOAc $=1: 1$ ).

${ }^{1} \mathrm{H}$ NMR (400 MHz, $\left.\mathrm{CDCl}_{3}\right) \delta 7.83(\mathrm{~d}, J=7.9 \mathrm{~Hz}, 2 \mathrm{H}), 7.53(\mathrm{~d}, J=8.0 \mathrm{~Hz}, 2 \mathrm{H})$, $4.96(\mathrm{q}, J=6.6 \mathrm{~Hz}, 1 \mathrm{H}), 3.01(\mathrm{~s}, 3 \mathrm{H}), 2.47(\mathrm{~s}, 1 \mathrm{H}), 1.48(\mathrm{~d}, J=6.2 \mathrm{~Hz}, 3 \mathrm{H})$.

${ }^{13} \mathrm{C}$ NMR (100 MHz, $\left.\mathrm{CDCl}_{3}\right) \delta 1$ 152.6, 139.3, 127.7, 126.5, 69.7, 44.7, 25.6.

Spectral data matched those previously reported. ${ }^{[21]}$

4,4,5,5-Tetramethyl-2-(1-(4-(trifluoromethyl)phenyl)ethyl)-1,3,2-

dioxaborolane $\left(3 n^{\prime}\right)$. 
<smiles>CC(Cc1ccccc1)c1ccc(C(F)(F)F)cc1</smiles>

Compound 3n' was prepared following the GP3 using 1-(4(trifluoromethyl)phenyl)ethan-1-one 1n' (56.4. $\mathrm{mg}, 0.3 \mathrm{mmol}),[\mathrm{RhCl}(\mathrm{cod})]_{2}(3.0$ $\mathrm{mg}, 0.006 \mathrm{mmol}), \mathrm{PPh}_{2} \mathrm{Me}(7.2 \mathrm{mg}, 0.036 \mathrm{mmol}), \mathrm{B}_{2}$ pin2 $(91.3 \mathrm{mg}, 0.36 \mathrm{mmol}$ ), HBpin (38.4 mg, $0.30 \mathrm{mmol}), \mathrm{CF}_{3} \mathrm{COOK}(4.6 \mathrm{mg}, 0.03 \mathrm{mmol})$, THF (0.4 mL) and $\mathrm{CyH}(1.6 \mathrm{~mL})$. The product was obtained as a white solid in $53 \%$ yield $(48.1 \mathrm{mg}$, eluent : petroleum ether : $\mathrm{EtOAc}=25: 1)$.

$\mathbf{R}_{f}=0.65$ (petroleum ether : EtOAc $=20: 1$ ).

${ }^{1} \mathbf{H}$ NMR (400 MHz, $\left.\mathbf{C D C l}_{3}\right) \delta 7.51(\mathrm{~d}, J=7.9 \mathrm{~Hz}, 2 \mathrm{H}), 7.32(\mathrm{~d}, J=7.9 \mathrm{~Hz}, 2 \mathrm{H})$, $2.50(\mathrm{q}, J=7.5 \mathrm{~Hz}, 1 \mathrm{H}), 1.34(\mathrm{~d}, J=7.6 \mathrm{~Hz}, 3 \mathrm{H}), 1.21(\mathrm{~d}, J=4.1 \mathrm{~Hz}, 12 \mathrm{H})$.

$\left.{ }^{13} \mathrm{C} \mathrm{NMR} \mathrm{(100} \mathrm{MHz,} \mathrm{CDCl}_{3}\right) \delta$ 149.4, 128.1, 127.5 (JC-F $\left.=32.3\right), 125.3$ (JC-F $\left.=3.7 \mathrm{~Hz}\right)$, $124.7\left(q J_{C-F}=270.3\right), 83.7,24.76,24.72,16.9$. The carbon signal attached to $B$ was not observed due to low intensity.

${ }^{19}$ F NMR (376 MHz, $\left.\mathrm{CDCl}_{3}\right)$ : $\delta-62.2$.

Spectral data matched those previously reported. ${ }^{[18]}$

Methyl 4-(1-(4,4,5,5-tetramethyl-1,3,2-dioxaborolan-2-yl)ethyl)benzoate (3o').<smiles>CC(=O)c1ccc(C(C)Cc2ccccc2)cc1</smiles>

Compound 30' was prepared following the GP3 using methyl 4-acetylbenzoate 1o' (53.4. mg, $0.3 \mathrm{mmol})$, [ RhCl(cod)]2 (3.0 mg, $0.006 \mathrm{mmol}), \mathrm{PPh}_{2} \mathrm{Me}(7.2 \mathrm{mg}$, $0.036 \mathrm{mmol}), B_{2}$ pin 2 (114 mg, $\left.0.45 \mathrm{mmol}\right), \mathrm{HBpin}(38.4 \mathrm{mg}, 0.30 \mathrm{mmol}$ ), $\mathrm{CF}_{3} \mathrm{COOK}(4.6 \mathrm{mg}, 0.03 \mathrm{mmol})$, THF $(0.4 \mathrm{~mL})$ and $\mathrm{CyH}(1.6 \mathrm{~mL})$. The product was obtained as a colourless oil in $72 \%$ yield $(63.4 \mathrm{mg}$, eluent : petroleum ether : $\mathrm{EtOAc}=8: 1)$.

$\mathbf{R}_{f}=0.65$ (petroleum ether $: \mathrm{EtOAc}=5: 1$ ).

${ }^{1} \mathrm{H}$ NMR (400 MHz, $\left.\mathrm{CDCl}_{3}\right) \delta 7.93(\mathrm{~d}, J=8.9 \mathrm{~Hz}, 2 \mathrm{H}), 7.28(\mathrm{~d}, J=8.3 \mathrm{~Hz}, 2 \mathrm{H})$, $3.89(\mathrm{~s}, 3 \mathrm{H}), 2.51(\mathrm{q}, J=7.6 \mathrm{~Hz}, 1 \mathrm{H}), 1.35(\mathrm{~d}, J=7.4 \mathrm{~Hz}, 3 \mathrm{H}), 1.19(\mathrm{~d}, J=4.7 \mathrm{~Hz}$, $12 \mathrm{H})$.

${ }^{13} \mathrm{C}$ NMR (100 MHz, $\left.\mathrm{CDCl}_{3}\right) \delta$ 167.5, 150.9, 129.8, 127.8, 127.2, 83.6, 52.0, 24.73, 
$24.68,16.6$. The carbon signal attached to $B$ was not observed due to low intensity.

Spectral data matched those previously reported.[22]

\section{4-(1-Hydroxyethyl)phenol (3p').}<smiles>CC(O)c1ccc(O)cc1</smiles>

Compound 3p' was prepared following the GP4 using methyl 4-acetylbenzoate 1p' (40.8 mg, $0.3 \mathrm{mmol})$, [ $\mathrm{RhCl}(\mathrm{cod})]_{2}$ (3.0 mg, $\left.0.006 \mathrm{mmol}\right), \mathrm{PPh}_{2} \mathrm{Me}(7.2 \mathrm{mg}$, $0.036 \mathrm{mmol}), \mathrm{B}_{2}$ pin2 $(91 \mathrm{mg}, 0.36 \mathrm{mmol}), \mathrm{HB}$ in $(57.6 \mathrm{mg}, 0.45 \mathrm{mmol}), \mathrm{CF}_{3} \mathrm{COOK}$ $(4.6 \mathrm{mg}, 0.03 \mathrm{mmol}), \mathrm{THF}(0.4 \mathrm{~mL})$ and $\mathrm{CyH}(1.6 \mathrm{~mL})$. The product was obtained as a white solid in $67 \%$ yield $(27.7 \mathrm{mg}$, eluent : petroleum ether : EtOAc $=3: 1)$. $\mathbf{R}_{f}=0.45$ (petroleum ether : EtOAc $=1: 1$ ).

${ }^{1}$ H NMR (400 MHz, DMSO-d $) \delta 9.17(\mathrm{~s}, 1 \mathrm{H}), 7.11(\mathrm{~d}, J=8.5 \mathrm{~Hz}, 2 \mathrm{H}), 6.68(\mathrm{~d}, J$ $=8.4 \mathrm{~Hz}, 2 \mathrm{H}), 4.60(\mathrm{q}, J=6.4 \mathrm{~Hz}, 1 \mathrm{H}), 1.26(\mathrm{~d}, J=6.4 \mathrm{~Hz}, 3 \mathrm{H})$.

${ }^{13}$ C NMR (100 MHz, DMSO-d6) $\delta$ 155.9, 137.7, 126.4, 114.6, 67.7, 40.1, 25.9.

Spectral data matched those previously reported. ${ }^{[23]}$

\section{1-(3-Aminophenyl)ethan-1-ol (3q').}<smiles>CC(O)c1cccc(N)c1</smiles>

Compound 3q' was prepared following the GP4 using methyl 4-acetylbenzoate 1q' (40.5 mg, $0.3 \mathrm{mmol})$, [RhCl(cod)]2 (3.0 mg, $0.006 \mathrm{mmol}), \mathrm{PPh}_{2} \mathrm{Me}(7.2 \mathrm{mg}$, $0.036 \mathrm{mmol}), \mathrm{B}_{2}$ pin $2(228.6 \mathrm{mg}, 0.9 \mathrm{mmol}), \mathrm{CF}_{3} \mathrm{COOK}(4.6 \mathrm{mg}, 0.03 \mathrm{mmol}), \mathrm{THF}$ $(0.4 \mathrm{~mL})$ and $\mathrm{CyH}(1.6 \mathrm{~mL})$. The product was obtained as a white solid in $81 \%$ yield (33.3 $\mathrm{mg}$, eluent : DCM : EtOAc $=8: 1)$.

$\mathbf{R}_{f}=0.55(\mathrm{DCM}: \mathrm{EtOAC}=5: 1)$.

${ }^{1}$ H NMR (400 MHz, $\left.\mathrm{CDCl}_{3}\right) \delta 7.14-6.98(\mathrm{~m}, 1 \mathrm{H}), 6.79-6.68(\mathrm{~m}, 1 \mathrm{H}), 6.65(\mathrm{~m}$, $1 \mathrm{H}), 6.61-6.47(\mathrm{~m}, 1 \mathrm{H}), 4.73(\mathrm{q}, J=6.5 \mathrm{~Hz}, 1 \mathrm{H}), 1.42(\mathrm{~d}, J=6.5 \mathrm{~Hz}, 3 \mathrm{H})$.

${ }^{13} \mathrm{C}$ NMR (100 MHz, $\left.\mathrm{CDCl}_{3}\right)$ 8147.4, 146.4, 129.4, 115.9, 114.3, 112.3, 70.1, 25.0.

Spectral data matched those previously reported. ${ }^{[24]}$ 
2-(1-(3-Fluorophenyl)ethyl)-4,4,5,5-tetramethyl-1,3,2-dioxaborolane (3r').<smiles>CC(Br)c1cccc(F)c1</smiles>

Compound 3r' was prepared following the GP3 using 1-(3-fluorophenyl)ethan1-one 1r' (41.4. mg, $0.3 \mathrm{mmol})$, [ $\mathrm{RhCl}(\mathrm{cod})]_{2}$ (3.0 mg, $\left.0.006 \mathrm{mmol}\right), \mathrm{PPh}_{2} \mathrm{Me}(7.2$ $\mathrm{mg}, 0.036 \mathrm{mmol}), \mathrm{B}_{2}$ pin2 $(91.3 \mathrm{mg}, 0.36 \mathrm{mmol}), \mathrm{HBpin}(38.4 \mathrm{mg}, 0.30 \mathrm{mmol}$ ), $\mathrm{CF}_{3} \mathrm{COOK}(4.6 \mathrm{mg}, 0.03 \mathrm{mmol})$, THF $(0.4 \mathrm{~mL})$ and $\mathrm{CyH}(1.6 \mathrm{~mL})$. The product was obtained as a colourless oil in $67 \%$ yield ( $50.2 \mathrm{mg}$, eluent : petroleum ether : $\mathrm{EtOAc}=25: 1)$.

$\mathbf{R}_{f}=0.50$ (petroleum ether : $\mathrm{EtOAc}=20: 1$ ).

${ }^{1} \mathrm{H}$ NMR (400 MHz, $\left.\mathrm{CDCl}_{3}\right) \delta 7.24-7.17$ (m, 1H), 7.01 - $6.90(\mathrm{~m}, 2 \mathrm{H}), 6.87$ - 6.78 $(\mathrm{m}, 1 \mathrm{H}), 2.44(\mathrm{q}, J=7.5 \mathrm{~Hz}, 1 \mathrm{H}), 1.32(\mathrm{~d}, J=7.5 \mathrm{~Hz}, 3 \mathrm{H}), 1.21(\mathrm{~d}, J=4.3 \mathrm{~Hz}, 12 \mathrm{H})$.

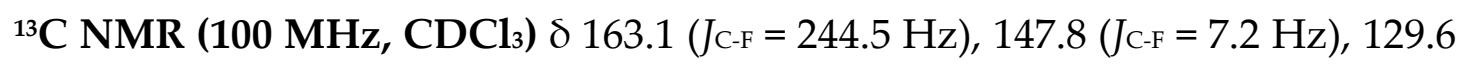
$\left(J_{\mathrm{C}-\mathrm{F}}=8.4 \mathrm{~Hz}\right), 123.6\left(J_{\mathrm{C}-\mathrm{F}}=2.7 \mathrm{~Hz}\right), 114.7\left(J_{\mathrm{C}-\mathrm{F}}=21.1 \mathrm{~Hz}\right), 112.0\left(J_{\mathrm{C}-\mathrm{F}}=21.1 \mathrm{~Hz}\right)$, $83.6,24.75,24.71,16.9$. The carbon signal attached to $B$ was not observed due to low intensity.

${ }^{19}$ F NMR (376 MHz, $\left.\mathrm{CDCl}_{3}\right)$ : $\delta-114.1$.

Spectral data matched those previously reported. ${ }^{[18]}$

2-(1-(3-Methoxyphenyl)ethyl)-4,4,5,5-tetramethyl-1,3,2-dioxaborolane (3s').<smiles>COc1cccc(C(C)Cc2ccccc2)c1</smiles>

Compound 3s' was prepared following the GP3 using 1-(3methoxyphenyl)ethan-1-one 1s' (45.0. mg, $0.3 \mathrm{mmol})$, [ $\mathrm{RhCl}(\mathrm{cod})]_{2}(3.0 \mathrm{mg}$, $0.006 \mathrm{mmol}), \mathrm{PPh}_{2} \mathrm{Me}(7.2 \mathrm{mg}, 0.036 \mathrm{mmol}), \mathrm{B}_{2} \mathrm{Pin}_{2}(91.3 \mathrm{mg}, 0.36 \mathrm{mmol}), \mathrm{HB}$ pin (38.4 mg, $0.30 \mathrm{mmol}), \mathrm{CF}_{3} \mathrm{COOK}(4.6 \mathrm{mg}, 0.03 \mathrm{mmol})$, THF $(0.4 \mathrm{~mL})$ and $\mathrm{CyH}$ $(1.6 \mathrm{~mL})$. The product was obtained as a colourless oil in $65 \%$ yield $(51.2 \mathrm{mg}$, eluent : petroleum ether : EtOAc $=25: 1)$.

$\mathbf{R}_{f}=0.48$ (petroleum ether : $\mathrm{EtOAc}=10: 1$ ).

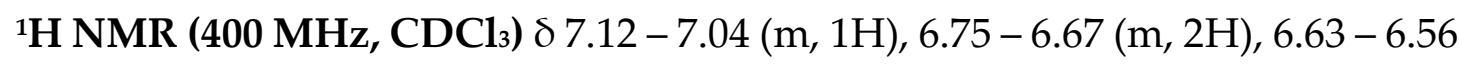


(m, 1H), $3.69(\mathrm{~s}, 3 \mathrm{H}), 2.31(\mathrm{q}, J=7.6 \mathrm{~Hz}, 1 \mathrm{H}), 1.22(\mathrm{~d}, J=7.5 \mathrm{~Hz}, 3 \mathrm{H}), 1.11(\mathrm{~d}, J=$ $5.1 \mathrm{~Hz}, 12 \mathrm{H})$.

${ }^{13} \mathrm{C}$ NMR (100 MHz, $\left.\mathrm{CDCl}_{3}\right) \delta$ 159.7, 146.8, 129.3, 120.4, 113.6, 110.7, 83.4, 55.2, $24.77,24.75,17.1$. The carbon signal attached to B was not observed due to low intensity.

Spectral data matched those previously reported. ${ }^{[22]}$

1-(2-Methoxyphenyl)ethan-1-ol (3t').<smiles>COc1ccccc1C(C)O</smiles>

Compound $3 \mathbf{t}^{\prime}$ was prepared following the GP4 using 1-(2methoxyphenyl)ethan-1-one $\mathbf{1 t}^{\prime}$ (45.0. $\left.\mathrm{mg}, 0.3 \mathrm{mmol}\right)$, [RhCl(cod)]2 (3.0 mg, $0.006 \mathrm{mmol}), \mathrm{PPh}_{2} \mathrm{Me}$ (7.2 mg, $\left.0.036 \mathrm{mmol}\right), \mathrm{B}_{2} \mathrm{Pin}_{2}(91.3 \mathrm{mg}, 0.36 \mathrm{mmol}), \mathrm{HB}$ in (38.4 mg, $0.30 \mathrm{mmol}), \mathrm{CF}_{3} \mathrm{COOK}(4.6 \mathrm{mg}, 0.03 \mathrm{mmol})$, THF $(0.4 \mathrm{~mL})$ and $\mathrm{CyH}$ $(1.6 \mathrm{~mL})$. The product was obtained as a colourless oil in $61 \%$ yield $(27.8 \mathrm{mg}$, eluent : petroleum ether : $\mathrm{EtOAc}=4: 1)$.

$\mathbf{R}_{f}=0.48$ (petroleum ether : $\mathrm{EtOAc}=2: 1$ ).

${ }^{1} \mathrm{H}$ NMR (400 MHz, $\left.\mathrm{CDCl}_{3}\right) \delta 7.37(\mathrm{~d}, J=7.5 \mathrm{~Hz}, 1 \mathrm{H}), 7.32-7.24(\mathrm{~m}, 1 \mathrm{H}), 7.03-$ $6.95(\mathrm{~m}, 1 \mathrm{H}), 6.91(\mathrm{~d}, J=8.2 \mathrm{~Hz}, 1 \mathrm{H}), 5.12(\mathrm{q}, J=6.6 \mathrm{~Hz}, 1 \mathrm{H}), 3.89(\mathrm{~s}, 3 \mathrm{H}), 2.66$ (s, $1 \mathrm{H}), 1.54(\mathrm{~d}, J=6.5 \mathrm{~Hz}, 3 \mathrm{H})$.

${ }^{13} \mathrm{C}$ NMR (100 MHz, $\left.\mathrm{CDCl}_{3}\right) \delta$ 156.7, 133.6, 128.4, 126.2, 121.0, 110.6, 66.7, 55.4, 23.0 .

Spectral data matched those previously reported. ${ }^{[20]}$

2-(1-(Benzo[d][1,3]dioxol-5-yl)ethyl)-4,4,5,5-tetramethyl-1,3,2-dioxaborolane $\left(3 \mathbf{u}^{\prime}\right)$.<smiles>CC(Br)c1ccc2c(c1)OCO2</smiles>

Compound 3u' was prepared following the GP3 using 1-(benzo[d][1,3]dioxol5-yl)ethan-1-ol 1u' (45.0. mg, $0.3 \mathrm{mmol})$, [RhCl(cod)]2 (3.0 mg, $0.006 \mathrm{mmol}$ ), $\mathrm{PPh}_{2} \mathrm{Me}$ (7.2 mg, $\left.0.036 \mathrm{mmol}\right), \mathrm{B}_{2}$ pin 2 (91.3 mg, $0.36 \mathrm{mmol}$ ), HBpin (38.4 mg, 0.30 
mmol), $\mathrm{CF}_{3} \mathrm{COOK}(4.6 \mathrm{mg}, 0.03 \mathrm{mmol})$, THF $(0.4 \mathrm{~mL})$ and $\mathrm{CyH}(1.6 \mathrm{~mL})$. The product was obtained as a colourless oil in $72 \%$ yield $(60.3 \mathrm{mg}$, eluent : petroleum ether : $\mathrm{EtOAc}=25: 1)$.

$\mathbf{R}_{f}=0.45$ (petroleum ether : EtOAc $=10: 1$ ).

${ }^{1} \mathrm{H}$ NMR (400 MHz, $\left.\mathrm{CDCl}_{3}\right) \delta 6.78-6.71(\mathrm{~m}, 2 \mathrm{H}), 6.70-6.66(\mathrm{~m}, 1 \mathrm{H}), 5.92(\mathrm{~s}$, $2 \mathrm{H}), 2.37(\mathrm{q}, J=7.5 \mathrm{~Hz}, 1 \mathrm{H}), 1.30(\mathrm{~d}, J=7.8 \mathrm{~Hz}, 3 \mathrm{H}), 1.24(\mathrm{~d}, J=4.6 \mathrm{~Hz}, 12 \mathrm{H})$.

${ }^{13} \mathrm{C}$ NMR (100 MHz, $\left.\mathrm{CDCl}_{3}\right) \delta$ 147.6, 145.2, 139.1, 120.5, 108.6, 108.3, 100.7, 83.4, $24.78,24.74,17.6$. The carbon signal attached to $B$ was not observed due to low intensity.

Spectral data matched those previously reported. ${ }^{[25]}$

4,4,5,5-Tetramethyl-2-(1-(3,4,5-trimethoxyphenyl)ethyl)-1,3,2-dioxaborolane $\left(3 v^{\prime}\right)$.<smiles>COc1cc(C([18OH])[Pb])cc(OC)c1OC</smiles>

Compound $3 \mathbf{v}^{\prime}$ was prepared following the GP3 using 1-(3,4,5trimethoxyphenyl)ethan-1-one $\mathbf{1 \mathbf { v } ^ { \prime }}$ (63.0. $\left.\mathrm{mg}, 0.3 \mathrm{mmol}\right),[\mathrm{RhCl}(\mathrm{cod})]_{2}(3.0 \mathrm{mg}$, $0.006 \mathrm{mmol}), \mathrm{PPh}_{2} \mathrm{Me}$ (7.2 mg, $\left.0.036 \mathrm{mmol}\right), \mathrm{B}_{2} \mathrm{Pin}_{2}(91.3 \mathrm{mg}, 0.36 \mathrm{mmol}), \mathrm{HB}$ pin (38.4 mg, $0.30 \mathrm{mmol}), \mathrm{CF}_{3} \mathrm{COOK}(4.6 \mathrm{mg}, 0.03 \mathrm{mmol})$, THF $(0.4 \mathrm{~mL})$ and $\mathrm{CyH}$ $(1.6 \mathrm{~mL})$. The product was obtained as a colourless oil in $63 \%$ yield $(61.3 \mathrm{mg}$, eluent : petroleum ether : $\mathrm{EtOAc}=8: 1)$.

$\mathbf{R}_{f}=0.45$ (petroleum ether $:$ EtOAc $=5: 1$ ).

${ }^{1} \mathrm{H}$ NMR (400 MHz, $\left.\mathrm{CDCl}_{3}\right) \delta 6.44(\mathrm{~s}, 2 \mathrm{H}), 3.84(\mathrm{~s}, 6 \mathrm{H}), 3.82(\mathrm{~s}, 3 \mathrm{H}), 2.36(\mathrm{q}, J=$ $6.8 \mathrm{~Hz}, 1 \mathrm{H}), 1.31(\mathrm{~d}, J=6.8 \mathrm{~Hz}, 3 \mathrm{H}), 1.22(\mathrm{~d}, J=3.9 \mathrm{~Hz}, 12 \mathrm{H})$.

${ }^{13} \mathrm{C}$ NMR (100 MHz, $\left.\mathrm{CDCl}_{3}\right) \delta$ 153.1, 140.8, 135.7, 104.9, 83.5, 61.0, 56.1, 24.81, 24.77, 17.4. The carbon signal attached to $B$ was not observed due to low intensity.

HRMS (ESI') calcd for $\mathrm{C}_{17} \mathrm{H}_{27} \mathrm{BO}_{5} \mathrm{Na}^{+}[\mathrm{M}+\mathrm{Na}]^{+}$: 345.1844 , found: 345.1843 .

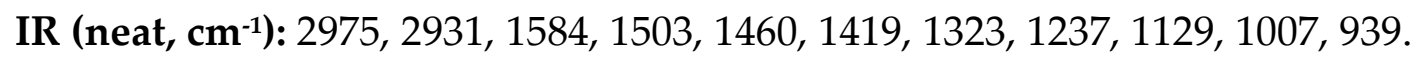

2-(1-(6-Methoxynaphthalen-2-yl)ethyl)-4,4,5,5-tetramethyl-1,3,2dioxaborolane $\left(3 w^{\prime}\right)$. 


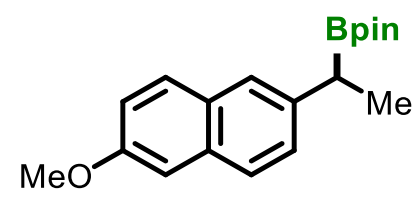

Compound $3 \mathbf{w}^{\prime}$ was prepared following the GP3 using 1-(6methoxynaphthalen-2-yl)ethan-1-one $\mathbf{1} \mathbf{w}^{\prime}$ (60.0. $\left.\mathrm{mg}, 0.3 \mathrm{mmol}\right)$, [ $\left.\mathrm{RhCl}(\mathrm{cod})\right]_{2}$ ( $3.0 \mathrm{mg}, 0.006 \mathrm{mmol}$ ), PPh2Me (7.2 mg, $0.036 \mathrm{mmol}$ ), B2 pin $_{2}(91.3 \mathrm{mg}, 0.36 \mathrm{mmol}$ ), HBpin (38.4 mg, $0.30 \mathrm{mmol}), \mathrm{CF}_{3} \mathrm{COOK}(4.6 \mathrm{mg}, 0.03 \mathrm{mmol}), \mathrm{THF}(0.4 \mathrm{~mL})$ and $\mathrm{CyH}(1.6 \mathrm{~mL})$. The product was obtained as a white solid in $63 \%$ yield $(59.1 \mathrm{mg}$, eluent : petroleum ether : $\mathrm{EtOAc}=8: 1)$.

$\mathbf{R}_{f}=0.45$ (petroleum ether $:$ EtOAc $=5: 1$ ).

${ }^{1} \mathrm{H}$ NMR (400 MHz, CDCl $)$ d $7.70-7.63(\mathrm{~m}, 2 \mathrm{H}), 7.58(\mathrm{~s}, 1 \mathrm{H}), 7.36(\mathrm{~d}, J=8.5 \mathrm{~Hz}$, $1 \mathrm{H}), 7.14-7.08(\mathrm{~m}, 2 \mathrm{H}), 3.91(\mathrm{~s}, 3 \mathrm{H}), 2.58(\mathrm{q}, J=7.6 \mathrm{~Hz}, 1 \mathrm{H}), 1.42(\mathrm{~d}, J=7.6 \mathrm{~Hz}$, $3 \mathrm{H}), 1.21(\mathrm{~d}, J=6.4 \mathrm{~Hz}, 12 \mathrm{H})$.

${ }^{13} \mathrm{C}$ NMR (100 MHz, CDCl $)$ ) $157.1,140.3,132.8,129.5,129.1,127.8,126.7,125.3$, $118.5,105.8,83.5,55.4,24.77,24.73,17.0$. The carbon signal attached to B was not observed due to low intensity.

Spectral data matched those previously reported. ${ }^{[26]}$

1-Methyl-3-(1-(4,4,5,5-tetramethyl-1,3,2-dioxaborolan-2-yl)ethyl)-1H-indole $\left(3 x^{\prime}\right)$.

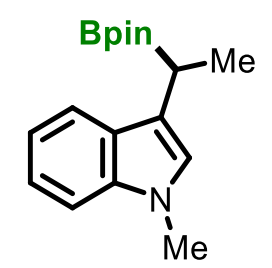

Compound $3 \mathbf{x}^{\prime}$ was prepared following the GP3 using 1-(1-methyl-1H-indol-3yl)ethan-1-one $1 \mathbf{x}^{\prime}$ (51.9. mg, $\left.0.3 \mathrm{mmol}\right)$, [ $\left.\mathrm{RhCl}(\mathrm{cod})\right]_{2}(3.0 \mathrm{mg}, 0.006 \mathrm{mmol})$, $\mathrm{PPh}_{2} \mathrm{Me}$ (7.2 mg, $0.036 \mathrm{mmol}$ ), B2pinz (91.3 mg, $0.36 \mathrm{mmol}$ ), HBpin (38.4 mg, 0.30 $\mathrm{mmol}), \mathrm{CF}_{3} \mathrm{COOK}(4.6 \mathrm{mg}, 0.03 \mathrm{mmol})$, THF (0.4 mL) and CyH (1.6 mL). The product was obtained as a colourless oil in $70 \%$ yield $(60.1 \mathrm{mg}$, eluent : petroleum ether : $\mathrm{EtOAc}=10: 1)$.

$\mathbf{R}_{f}=0.65$ (petroleum ether $:$ EtOAc $=5: 1$ ).

${ }^{1} \mathrm{H}$ NMR $\left(400 \mathrm{MHz}, \mathrm{CDCl}_{3}\right) \delta 7.72(\mathrm{~d}, J=7.9 \mathrm{~Hz}, 1 \mathrm{H}), 7.32(\mathrm{~d}, J=8.3 \mathrm{~Hz}, 1 \mathrm{H})$, $7.28-7.23(\mathrm{~m}, 1 \mathrm{H}), 7.16-7.09(\mathrm{~m}, 1 \mathrm{H}), 6.94(\mathrm{~s}, 1 \mathrm{H}), 3.78(\mathrm{~s}, 3 \mathrm{H}), 2.72(\mathrm{q}, J=7.5$ 
$\mathrm{Hz}, 1 \mathrm{H}), 1.46(\mathrm{~d}, J=7.5 \mathrm{~Hz}, 3 \mathrm{H}), 1.29(\mathrm{~d}, J=4.3 \mathrm{~Hz}, 12 \mathrm{H})$.

${ }^{13} \mathrm{C}$ NMR (100 MHz, $\mathrm{CDCl}_{3}$ ) $\delta$ 137.3, 127.9, 125.4, 121.3, 119.7, 118.2, 117.7, 109.0, 83.4, 32.7, 24.87, 24.81, 16.8. The carbon signal attached to B was not observed due to low intensity.

HRMS (ESI ${ }^{+}$) calcd for $\mathrm{C}_{17} \mathrm{H}_{25} \mathrm{BNO}_{2}{ }^{+}[\mathrm{M}+\mathrm{H}]^{+}:$: 286.1973, found: 286.1973 .

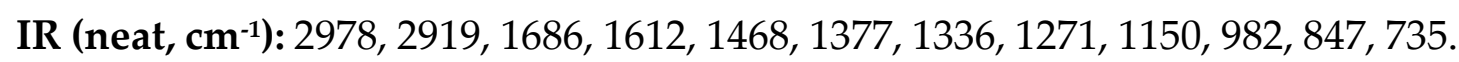

\section{3-(1-(4,4,5,5-Tetramethyl-1,3,2-dioxaborolan-2-yl)ethyl)-9-tosyl-9H-carbazole} $\left(3 y^{\prime}\right)$.<smiles>[Y9]n1c2ccccc2c2cc(C(Br)Br)ccc21</smiles>

Compound $3 \mathbf{y}^{\prime}$ was prepared following the GP3 using 1-(6-

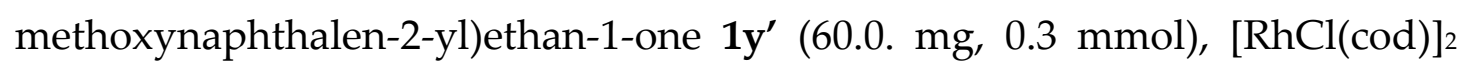
(3.0 mg, $0.006 \mathrm{mmol}), \mathrm{PPh}_{2} \mathrm{Me}$ (7.2 mg, $\left.0.036 \mathrm{mmol}\right), \mathrm{B}_{2}$ pin 2 (114.0 mg, 0.45 $\mathrm{mmol})$, HBpin ( $38.4 \mathrm{mg}, 0.30 \mathrm{mmol}), \mathrm{CF}_{3} \mathrm{COOK}(4.6 \mathrm{mg}, 0.03 \mathrm{mmol})$, THF (0.4 $\mathrm{mL})$ and $\mathrm{CyH}(1.6 \mathrm{~mL})$. The product was obtained as a white solid in $48 \%$ yield $(65.3 \mathrm{mg}$, eluent : petroleum ether : $\mathrm{EtOAc}=8: 1)$.

$\mathbf{R}_{f}=0.60$ (petroleum ether : EtOAc $=5: 1$ ).

${ }^{1} \mathrm{H}$ NMR (400 MHz, CDCl $\left.{ }_{3}\right) \delta 8.30(\mathrm{~d}, J=8.4 \mathrm{~Hz}, 1 \mathrm{H}), 8.20(\mathrm{~d}, J=8.6 \mathrm{~Hz}, 1 \mathrm{H})$, $7.87(\mathrm{~d}, J=7.8 \mathrm{~Hz}, 1 \mathrm{H}), 7.74(\mathrm{~s}, 1 \mathrm{H}), 7.68(\mathrm{~d}, J=8.0 \mathrm{~Hz}, 2 \mathrm{H}), 7.49-7.42(\mathrm{~m}, 1 \mathrm{H})$, $7.40-7.29(\mathrm{~m}, 2 \mathrm{H}), 7.07(\mathrm{~d}, J=8.0 \mathrm{~Hz}, 2 \mathrm{H}), 2.58(\mathrm{q}, J=7.5 \mathrm{~Hz}, 1 \mathrm{H}), 2.24(\mathrm{~s}, 3 \mathrm{H})$, $1.40(\mathrm{~d}, J=7.5 \mathrm{~Hz}, 3 \mathrm{H}), 1.21(\mathrm{~d}, J=6.8 \mathrm{~Hz}, 12 \mathrm{H})$.

${ }^{13} \mathrm{C}$ NMR (100 MHz, CDCl $)$ ) $\delta$ 144.8, 140.9, 138.7, 136.5, 135.2, 129.7, 127.7, 127.1, 126.8, 126.7, 126.6, 123.8, 120.0, 118.8, 115.3, 115.1, 83.5, 24.74, 24.71, 21.6, 17.3. The carbon signal attached to $B$ was not observed due to low intensity. HRMS (ESI+) calcd for $\mathrm{C}_{27} \mathrm{H}_{30} \mathrm{BNO}_{4} \mathrm{SNa}^{+}[\mathrm{M}+\mathrm{Na}]^{+}: 498.1881$, found: 498.1879 .

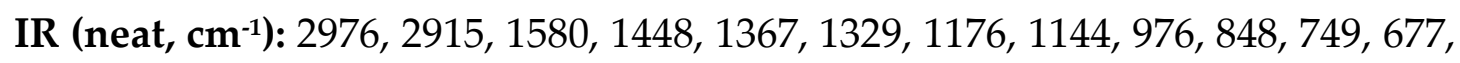
581.

1-(2,5-Dimethylfuran-3-yl)ethan-1-ol (3z'). 
<smiles>Cc1cc(C(C)O)c(C)o1</smiles>

Compound 3z' was prepared following the GP4 using 1-(2,5-dimethylfuran-3yl)ethan-1-one 1z' (41.4 mg, $0.3 \mathrm{mmol})$, [RhCl(cod)]2 (3.0 mg, $0.006 \mathrm{mmol})$, $\mathrm{PPh}_{2} \mathrm{Me}$ (7.2 mg, $0.036 \mathrm{mmol}$ ), B2pin2 (91.0 mg, $0.36 \mathrm{mmol}$ ), HBpin (38.4 mg, 0.30 $\mathrm{mmol}), \mathrm{CF}_{3} \mathrm{COOK}(4.6 \mathrm{mg}, 0.03 \mathrm{mmol}), \mathrm{THF}(0.4 \mathrm{~mL})$ and $\mathrm{CyH}(1.6 \mathrm{~mL})$. The product was obtained as a colourless oil in $42 \%$ yield $(17.6 \mathrm{mg}$, eluent : petroleum ether : $\mathrm{EtOAc}=10: 1)$.

$\mathbf{R}_{f}=0.45$ (petroleum ether $:$ EtOAc $=4: 1$ ).

${ }^{1} \mathrm{H}$ NMR (400 MHz, $\mathrm{CDCl}_{3}$ ) $\delta 5.94(\mathrm{~s}, 1 \mathrm{H}), 4.77$ (q, J = 6.4 Hz, 1H), 2.23 (s, 3H), $2.22(\mathrm{~s}, 3 \mathrm{H}), 1.41(\mathrm{~d}, J=6.5 \mathrm{~Hz}, 3 \mathrm{H})$.

${ }^{13} \mathrm{C}$ NMR (100 MHz, $\mathrm{CDCl}_{3}$ ) $\delta 150.1,145.8,124.3,104.3,63.0,24.1,13.7,11.9$.

Spectral data matched those previously reported. ${ }^{[29]}$

\section{1-(Thiophen-3-yl)ethan-1-ol (3aa').}<smiles>[C]C(O)c1ccsc1</smiles>

Compound 3aa' was prepared following the GP4 using 1-(thiophen-3-yl)ethan1-one 1aa' (37.8 mg, $0.3 \mathrm{mmol}),[\mathrm{RhCl}(\mathrm{cod})]_{2}$ (3.0 mg, $\left.0.006 \mathrm{mmol}\right), \mathrm{PPh}_{2} \mathrm{Me}(7.2$ $\mathrm{mg}, 0.036 \mathrm{mmol}), B_{2}$ pin2 $(91.0 \mathrm{mg}, 0.36 \mathrm{mmol}), \mathrm{HBpin}(38.4 \mathrm{mg}, 0.30 \mathrm{mmol})$, $\mathrm{CF}_{3} \mathrm{COOK}(4.6 \mathrm{mg}, 0.03 \mathrm{mmol})$, THF $(0.4 \mathrm{~mL})$ and $\mathrm{CyH}(1.6 \mathrm{~mL})$. The product was obtained as a colourless oil in $58 \%$ yield $(22.5 \mathrm{mg}$, eluent : petroleum ether : $\mathrm{EtOAc}=10: 1)$.

$\mathbf{R}_{f}=0.35$ (petroleum ether $:$ EtOAc $=5: 1$ ).

${ }^{1} \mathrm{H}$ NMR (400 MHz, $\mathrm{CDCl}_{3}$ ) $\delta 7.32$ - 7.28 (m, 1H), 7.20 - $7.16(\mathrm{~m}, 1 \mathrm{H}), 7.11$ - 7.07 $(\mathrm{m}, 1 \mathrm{H}), 4.95(\mathrm{q}, J=6.5 \mathrm{~Hz}, 1 \mathrm{H}), 2.08(\mathrm{~s}, 1 \mathrm{H}), 1.51(\mathrm{~d}, J=6.4 \mathrm{~Hz}, 3 \mathrm{H})$.

${ }^{13} \mathrm{C}$ NMR (100 MHz, $\left.\mathrm{CDCl}_{3}\right)$ 8147.5, 126.3, 125.9, 120.4, 66.7, 24.6.

Spectral data matched those previously reported. ${ }^{[21]}$

1-(4-((3,7-Dimethyloct-6-en-1-yl)oxy)phenyl)ethan-1-ol (3ab'). 
<smiles>CC(C)=CCCC(C)CCOc1ccc(C(C)O)cc1</smiles>

Compound 3ab' was prepared following the GP4 using 1-(4-((3,7-dimethyloct6-en-1-yl)oxy)phenyl)ethan-1-one 1ab' (82.2 mg, $0.3 \mathrm{mmol}),[\mathrm{RhCl}(\mathrm{cod})]_{2}(3.0$ $\mathrm{mg}, 0.006 \mathrm{mmol}), \mathrm{PPh}_{2} \mathrm{Me}(7.2 \mathrm{mg}, 0.036 \mathrm{mmol}), \mathrm{B}_{2} \mathrm{pin}_{2}(114.0 \mathrm{mg}, 0.45 \mathrm{mmol})$, HBpin ( $38.4 \mathrm{mg}, 0.30 \mathrm{mmol}), \mathrm{CF}_{3} \mathrm{COOK}(4.6 \mathrm{mg}, 0.03 \mathrm{mmol})$, THF $(0.4 \mathrm{~mL})$ and $\mathrm{CyH}(1.6 \mathrm{~mL})$. The product was obtained as a colourless oil in $56 \%$ yield (46.3 $\mathrm{mg}$, eluent : petroleum ether : $\mathrm{EtOAc}=5: 1)$.

$\mathbf{R}_{f}=0.75$ (petroleum ether $:$ EtOAc $=2: 1$ ).

${ }^{1} \mathrm{H}$ NMR (400 MHz, $\left.\mathrm{CDCl}_{3}\right)$ \& $7.35-7.25(\mathrm{~m}, 2 \mathrm{H}), 6.95-6.84(\mathrm{~m}, 2 \mathrm{H}), 5.13(\mathrm{t}, J=$ $7.3 \mathrm{~Hz}, 1 \mathrm{H}), 4.87(\mathrm{q}, J=6.5 \mathrm{~Hz}, 1 \mathrm{H}), 4.11-3.96(\mathrm{~m}, 2 \mathrm{H}), 2.13-1.96(\mathrm{~m}, 2 \mathrm{H}), 1.88$ $-1.77(\mathrm{~m}, 2 \mathrm{H}), 1.71(\mathrm{~s}, 3 \mathrm{H}), 1.64(\mathrm{~s}, 4 \mathrm{H}), 1.51(\mathrm{~d}, J=6.5 \mathrm{~Hz}, 3 \mathrm{H}), 1.48-1.37(\mathrm{~m}$, 1H), $1.30-1.23(\mathrm{~m}, 1 \mathrm{H}), 0.98(\mathrm{~d}, J=6.4 \mathrm{~Hz}, 3 \mathrm{H})$.

${ }^{13} \mathrm{C}$ NMR (100 MHz, $\left.\mathrm{CDCl}_{3}\right) \delta 158.7,137.9,131.4,126.8,124.8,114.6,70.2,66.5$, 37.3, 36.3, 29.7, 25.9, 25.6, 25.1, 19.7, 17.8.

HRMS (ESI+) calcd for $\mathrm{C}_{18} \mathrm{H}_{29} \mathrm{O}_{2}{ }^{+}[\mathrm{M}+\mathrm{H}]^{+}: 277.2162$, found: 277.2163 .

IR (neat, cm-1): 2958, 2916, 1609, 1512, 1450, 1380, 1241, 1174, 1079, 1018, 832.

1-(3-(((2S,5R)-2-Isopropyl-5-methylcyclohexyl)oxy)phenyl)ethan-1-ol (3ac').<smiles>CC1CC[C@@H](C(C)C)C(Oc2ccc(C(C)O)cc2)C1</smiles>

Compound 3ac' was prepared following the GP4 using 1-(4-(( $2 S, 5 R)-2-$ isopropyl-5-methylcyclohexyl)oxy)phenyl)ethan-1-one $\mathbf{1 a c}^{\prime}$ (82.2 $\mathrm{mg}, \quad 0.3$ $\mathrm{mmol}$ ), [ $\mathrm{RhCl}(\mathrm{cod})]_{2}$ (3.0 mg, $\left.0.006 \mathrm{mmol}\right), \mathrm{PPh}_{2} \mathrm{Me}$ (7.2 mg, $\left.0.036 \mathrm{mmol}\right), \mathrm{B}_{2}$ pin 2 (91.3 mg, $0.36 \mathrm{mmol}$ ), HBpin (38.4 mg, $0.30 \mathrm{mmol}$ ), CF 3 COOK (4.6 mg, 0.03 $\mathrm{mmol})$, THF $(0.4 \mathrm{~mL})$ and $\mathrm{CyH}(1.6 \mathrm{~mL})$. The product was obtained as a colourless oil in $73 \%$ yield $(60.4 \mathrm{mg}$, eluent : petroleum ether : EtOAc $=5: 1)$. $\mathbf{R}_{f}=0.75$ (petroleum ether : EtOAc $=2: 1$ ).

${ }^{1} \mathrm{H}$ NMR $\left(400 \mathrm{MHz}, \mathrm{CDCl}_{3}\right)$ \& $7.30(\mathrm{~d}, J=8.4 \mathrm{~Hz}, 2 \mathrm{H}), 6.90(\mathrm{~d}, J=8.1 \mathrm{~Hz}, 2 \mathrm{H})$, 
$4.87(\mathrm{q}, J=6.6 \mathrm{~Hz}, 1 \mathrm{H}), 4.65(\mathrm{~s}, 1 \mathrm{H}), 2.17-2.05(\mathrm{~m}, 1 \mathrm{H}), 1.85-1.60(\mathrm{~m}, 5 \mathrm{H}), 1.51$ $(\mathrm{d}, J=6.5 \mathrm{~Hz}, 3 \mathrm{H}), 1.12-0.93(\mathrm{~m}, 6 \mathrm{H}), 0.89(\mathrm{~d}, J=6.7 \mathrm{~Hz}, 3 \mathrm{H}), 0.84(\mathrm{~d}, J=6.6 \mathrm{~Hz}$, $3 \mathrm{H})$.

${ }^{13} \mathrm{C}$ NMR (100 MHz, $\left.\mathrm{CDCl}_{3}\right) \delta$ 157.8, 137.4, 126.9, 115.7, 73.4, 70.2, 48.0, 37.80, $37.78,35.2,29.4,26.3,25.0,22.4,21.2,21.0$.

HRMS (ESI ${ }^{+}$) calcd for $\mathrm{C}_{18} \mathrm{H}_{29} \mathrm{O}_{2}{ }^{+}[\mathrm{M}+\mathrm{H}]^{+}:$277.2162, found: 277.2162.

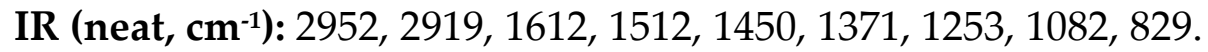

2-(1-(4-(17-Methoxy-13-methyl-7,8,9,11,12,13,14,15,16,17-decahydro-6Hcyclopenta[a]phenanthren-2-yl)phenyl)ethyl)-4,4,5,5-tetramethyl-1,3,2dioxaborolane (3ad').

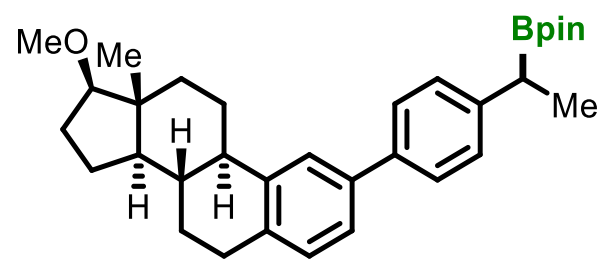

Compound 3ad' was prepared following the GP3 using 1-(4-(17-methoxy-13methyl-7,8,9,11,12,13,14,15,16,17-decahydro-6H-cyclopenta[a]phenanthren-2yl)phenyl)ethan-1-one 1ad' (116.4. mg, $0.3 \mathrm{mmol})$, [ RhCl(cod)]2 (3.0 mg, 0.006 $\mathrm{mmol})$, PPh $2 \mathrm{Me}$ (7.2 mg, $0.036 \mathrm{mmol}), \mathrm{B}_{2}$ pin2 $(91.3 \mathrm{mg}, 0.36 \mathrm{mmol})$, HBpin (38.4 $\mathrm{mg}, 0.30 \mathrm{mmol}), \mathrm{CF}_{3} \mathrm{COOK}(4.6 \mathrm{mg}, 0.03 \mathrm{mmol})$, THF $(0.4 \mathrm{~mL})$ and $\mathrm{CyH}(1.6$ $\mathrm{mL})$. The product was obtained as a white solid in $72 \%$ yield $(107.8 \mathrm{mg}$, eluent : petroleum ether : $\mathrm{EtOAc}=10: 1)$.

$\mathbf{R}_{f}=0.65$ (petroleum ether $:$ EtOAc $=5: 1$ ).

${ }^{1} \mathrm{H}$ NMR (400 MHz, $\left.\mathrm{CDCl}_{3}\right) \delta 7.55$ - $7.48(\mathrm{~m}, 2 \mathrm{H}), 7.42$ - $7.36(\mathrm{~m}, 2 \mathrm{H}), 7.35$ - 7.27 $(\mathrm{m}, 3 \mathrm{H}), 3.42(\mathrm{~s}, 3 \mathrm{H}), 3.36(\mathrm{t}, J=8.3 \mathrm{~Hz}, 1 \mathrm{H}), 3.00-2.91(\mathrm{~m}, 2 \mathrm{H}), 2.51(\mathrm{q}, J=7.6$ $\mathrm{Hz}, 1 \mathrm{H}), 2.43-2.27(\mathrm{~m}, 2 \mathrm{H}), 2.17-2.05(\mathrm{~m}, 2 \mathrm{H}), 2.01-1.91(\mathrm{~m}, 1 \mathrm{H}), 1.80-1.70$ $(\mathrm{m}, 1 \mathrm{H}), 1.65-1.33(\mathrm{~m}, 10 \mathrm{H}), 1.26(\mathrm{~d}, J=4.8 \mathrm{~Hz}, 12 \mathrm{H}), 0.85(\mathrm{~s}, 3 \mathrm{H})$.

${ }^{13} \mathrm{C}$ NMR (100 MHz, $\left.\mathrm{CDCl}_{3}\right) \delta$ 143.9, 139.2, 138.7, 138.0, 137.0, 128.2, 127.6, 127.0, $125.8,124.3,90.9,83.5,58.0,50.6,44.5,43.4,38.6,38.3,29.8,27.9,27.4,26.4,24.78$, $24.74,23.2,17.2,11.7$. The carbon signal attached to B was not observed due to low intensity.

HRMS (ESI ${ }^{+}$) calcd for $\mathrm{C}_{33} \mathrm{H}_{45} \mathrm{BO}_{3} \mathrm{Na}^{+}[\mathrm{M}+\mathrm{Na}]^{+}: 523.3354$, found: 523.3353 .

IR (neat, cm-1): 2972, 2925, 2869, 1489, 1456, 1373, 1350, 1321, 1266, 1138, 1100, $848,813$. 


\section{1-Phenylpropan-1-ol (3ae')}<smiles>CCC(O)c1ccccc1</smiles>

Compound 3ae' was prepared following the GP5 using propiophenone 1ae' (40.2 mg, $0.3 \mathrm{mmol})$, [ $\mathrm{RhCl}(\mathrm{cod})]_{2}(3.0 \mathrm{mg}, 0.006 \mathrm{mmol}), \mathrm{P}\left(4-{ }^{-} \mathrm{BuC}_{6} \mathrm{H}_{4}\right)_{2} \mathrm{Me}(11.2$ mg, $0.036 \mathrm{mmol}), B_{2}$ pin2 (91.3 mg, $\left.0.36 \mathrm{mmol}\right), \mathrm{HBpin}(38.4 \mathrm{mg}, 0.30 \mathrm{mmol})$, $\mathrm{CF}_{3} \mathrm{COOK}(4.6 \mathrm{mg}, 0.03 \mathrm{mmol})$, THF $(0.4 \mathrm{~mL})$ and $\mathrm{CyH}(1.6 \mathrm{~mL})$. The product was obtained as a colourless oil in $54 \%$ yield ( $22.3 \mathrm{mg}$, eluent : petroleum ether : EtOAc $=10: 1)$.

$\mathbf{R}_{f}=0.55$ (petroleum ether $:$ EtOAc $=5: 1$ ).

${ }^{1} \mathrm{H}$ NMR (400 MHz, $\left.\mathrm{CDCl}_{3}\right) \delta 7.41-7.34(\mathrm{~m}, 4 \mathrm{H}), 7.33-7.27(\mathrm{~m}, 1 \mathrm{H}), 4.60(\mathrm{t}, J=$ $6.8 \mathrm{~Hz}, 1 \mathrm{H}), 1.89-1.72(\mathrm{~m}, 2 \mathrm{H}), 0.94(\mathrm{t}, J=7.4 \mathrm{~Hz}, 3 \mathrm{H})$.

${ }^{13} \mathrm{C}$ NMR (100 MHz, $\left.\mathrm{CDCl}_{3}\right) \delta$ 144.7, 128.5, 127.6, 126.1, 76.1, 32.0, 10.3.

Spectral data matched those previously reported. ${ }^{[20]}$

\section{1-(4-Methoxyphenyl)propan-1-ol (3af')}<smiles>CCC(O)c1ccc(OC)cc1</smiles>

Compound 3af' was prepared following the GP5 using 1-(4methoxyphenyl)propan-1-one 1af' (49.2 mg, $0.3 \mathrm{mmol})$, [RhCl(cod)]2 (3.0 mg, $0.006 \mathrm{mmol}), \mathrm{P}\left(4{ }^{-}{ }^{-} \mathrm{BuC}_{6} \mathrm{H}_{4}\right)_{2} \mathrm{Me}(11.2 \mathrm{mg}, 0.036 \mathrm{mmol}), \mathrm{B}_{2}$ pin2 (91.3 mg, 0.36 $\mathrm{mmol}), \mathrm{HB}$ in $(38.4 \mathrm{mg}, 0.30 \mathrm{mmol}), \mathrm{CF}_{3} \mathrm{COOK}(4.6 \mathrm{mg}, 0.03 \mathrm{mmol}), \mathrm{THF}$ (0.4 $\mathrm{mL})$ and $\mathrm{CyH}(1.6 \mathrm{~mL})$. The product was obtained as a colourless oil in $62 \%$ yield (27.2 $\mathrm{mg}$, eluent : petroleum ether : EtOAc $=8: 1)$.

$\mathbf{R}_{f}=0.55$ (petroleum ether : EtOAc $=4: 1$ ).

${ }^{1} \mathrm{H}$ NMR (400 MHz, $\left.\mathrm{CDCl}_{3}\right) \delta 7.37-7.23(\mathrm{~m}, 2 \mathrm{H}), 6.96-6.83(\mathrm{~m}, 2 \mathrm{H}), 4.55(\mathrm{t}, J=$ $6.8 \mathrm{~Hz}, 1 \mathrm{H}), 3.83(\mathrm{~s}, 3 \mathrm{H}), 1.96(\mathrm{~s}, 1 \mathrm{H}), 1.85-1.68(\mathrm{~m}, 2 \mathrm{H}), 0.92(\mathrm{t}, J=7.3 \mathrm{~Hz}, 3 \mathrm{H})$. ${ }^{13} \mathrm{C}$ NMR (100 MHz, $\left.\mathrm{CDCl}_{3}\right) \delta$ 159.1, 136.9, 127.3, 113.9, 75.8, 55.4, 31.9, 10.3.

Spectral data matched those previously reported.[27]

\section{1-Phenylbutan-1-ol (3ag').}


<smiles>CCCC(O)c1ccccc1</smiles>

Compound 3ag' was prepared following the GP5 using 1-phenylbutan-1-one

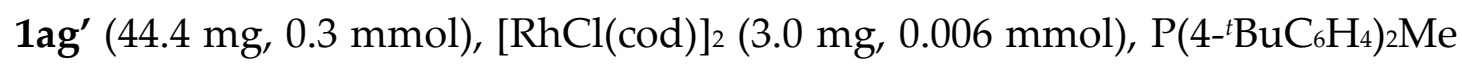
(11.2 mg, $0.036 \mathrm{mmol}), B_{2}$ pin $2(91.3 \mathrm{mg}, 0.36 \mathrm{mmol}), \mathrm{HBpin}(38.4 \mathrm{mg}, 0.30 \mathrm{mmol})$, $\mathrm{CF}_{3} \mathrm{COOK}$ (4.6 mg, $\left.0.03 \mathrm{mmol}\right)$, THF $(0.4 \mathrm{~mL})$ and $\mathrm{CyH}(1.6 \mathrm{~mL})$. The product was obtained as a colourless oil in $66 \%$ yield $(29.7 \mathrm{mg}$, eluent : petroleum ether : $\mathrm{EtOAc}=8: 1)$.

$\mathbf{R}_{f}=0.50$ (petroleum ether $: \mathrm{EtOAc}=4: 1$ ).

${ }^{1} \mathrm{H}$ NMR (400 MHz, $\left.\mathrm{CDCl}_{3}\right) \delta 7.43-7.33(\mathrm{~m}, 4 \mathrm{H}), 7.34-7.26(\mathrm{~m}, 1 \mathrm{H}), 4.70(\mathrm{t}, J=$ $6.8 \mathrm{~Hz}, 1 \mathrm{H}), 1.95-1.77(\mathrm{~m}, 2 \mathrm{H}), 1.77-1.66(\mathrm{~m}, 1 \mathrm{H}), 1.52-1.43(\mathrm{~m}, 1 \mathrm{H}), 1.37$ $1.30(\mathrm{~m}, 1 \mathrm{H}), 0.96(\mathrm{t}, J=7.4 \mathrm{~Hz}, 3 \mathrm{H})$.

${ }^{13} \mathrm{C}$ NMR (100 MHz, $\left.\mathrm{CDCl}_{3}\right) \delta 145.1,128.5,127.6,126.0,74.6,41.4,19.2,14.1$.

Spectral data matched those previously reported. ${ }^{[20]}$

6-((tert-Butyldimethylsilyl)oxy)-1-phenylhexan-1-ol (3ah').<smiles>OC(CCCCC[Se-][Sb])c1ccccc1</smiles>

Compound 3ah' was prepared following the GP5 using 6-((tertbutyldimethylsilyl)oxy)-1-phenylhexan-1-one 1ah' (91.8 mg, $0.3 \mathrm{mmol}$ ), $[\mathrm{RhCl}(\mathrm{cod})]_{2}(3.0 \mathrm{mg}, 0.006 \mathrm{mmol}), \mathrm{P}\left(4{ }^{-} \mathrm{BuC}_{6} \mathrm{H}_{4}\right)_{2} \mathrm{Me}(11.2 \mathrm{mg}, 0.036 \mathrm{mmol})$, B2pinz (91.3 mg, $0.36 \mathrm{mmol}), \mathrm{HBpin}(38.4 \mathrm{mg}, 0.30 \mathrm{mmol}), \mathrm{CF}_{3} \mathrm{COOK}$ (4.6 mg, $0.03 \mathrm{mmol})$, THF $(0.4 \mathrm{~mL})$ and $\mathrm{CyH}(1.6 \mathrm{~mL})$. The product was obtained as a colourless oil in $65 \%$ yield $(60.1 \mathrm{mg}$, eluent : petroleum ether : EtOAc $=8: 1)$.

$\mathbf{R}_{f}=0.52($ petroleum ether $:$ EtOAc $=4: 1)$.

${ }^{1} \mathrm{H}$ NMR (400 MHz, $\left.\mathrm{CDCl}_{3}\right) \delta 7.42-7.34(\mathrm{~m}, 4 \mathrm{H}), 7.33-7.26(\mathrm{~m}, 1 \mathrm{H}), 4.69(\mathrm{t}, J=$ $6.8 \mathrm{~Hz}, 1 \mathrm{H}), 3.61(\mathrm{t}, J=6.6 \mathrm{~Hz}, 2 \mathrm{H}), 1.88-1.71(\mathrm{~m}, 3 \mathrm{H}), 1.56-1.30(\mathrm{~m}, 6 \mathrm{H}), 0.91$ $(\mathrm{s}, 9 \mathrm{H}), 0.06(\mathrm{~s}, 6 \mathrm{H})$.

${ }^{13} \mathrm{C}$ NMR (100 MHz, $\left.\mathrm{CDCl}_{3}\right) \delta 145.0,128.6,127.6,126.0,74.8,63.3,39.2,32.9,26.1$, $25.9,25.8,18.5,-5.1$.

Spectral data matched those previously reported. ${ }^{[28]}$ 
General procedure for the synthesis of 1,1,2-trisboronates (GP6).
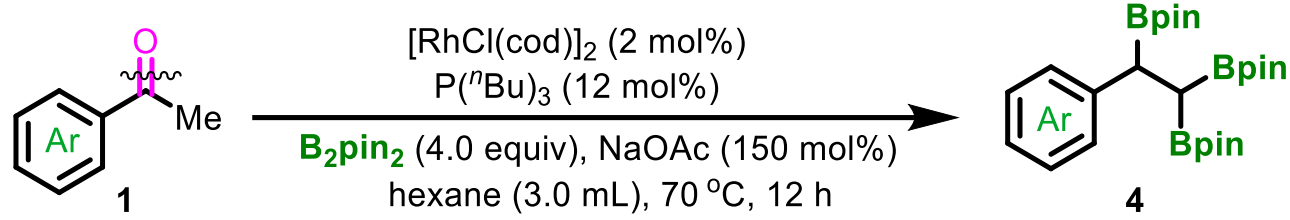

In glove box, $[\mathrm{RhCl}(\mathrm{cod})]_{2}(3.0 \mathrm{mg}, 0.006 \mathrm{mmol}), \mathrm{P}\left({ }^{n} \mathrm{Bu}\right)_{3}(7.3 \mathrm{mg}, 0.036$ $\mathrm{mmol})$, and hexane $(1.0 \mathrm{~mL})$ were placed in a $4 \mathrm{~mL}$ glass tube and stirred for 30 min. Then, NaOAc (36.9 mg, $0.45 \mathrm{mmol}), \mathrm{B}_{2}$ pin 2 (304.8 mg, $1.2 \mathrm{mmol}$ ), and ketone 1 in hexane $(2.0 \mathrm{~mL})$ were added. The reaction mixture was sealed, removed from the glovebox, and stirred at $70{ }^{\circ} \mathrm{C}$ for the duration of time indicated. After cooled to room temperature, the mixture was concentrated in vacuo and purified by silica gel chromatography using petroleum ether : EtOAc as the eluent to afford the desired products 4 .

\section{2,2',2'-(2-Phenylethane-1,1,2-triyl)tris(4,4,5,5-tetramethyl-1,3,2-}

dioxaborolane) (4a)<smiles>BrC(Br)C(Br)C(Br)C(Br)c1ccccc1</smiles>

Compound 4a was prepared following the GP6 using acetophenone 1a (36.0 $\mathrm{mg}, 0.3 \mathrm{mmol})$, [ $\mathrm{RhCl}(\mathrm{cod})]_{2}(3.0 \mathrm{mg}, 0.006 \mathrm{mmol}), \mathrm{P}\left({ }^{n} \mathrm{Bu}\right)_{3}(7.3 \mathrm{mg}, 0.036 \mathrm{mmol})$, $B_{2} \operatorname{pin}_{2}(304.8 \mathrm{mg}, 1.20 \mathrm{mmol}), \mathrm{NaOAc}(36.9 \mathrm{mg}, 0.45 \mathrm{mmol})$ and hexane $(3.0 \mathrm{~mL})$. The product was obtained as a white solid in $66 \%$ yield $(95.8 \mathrm{mg}$, eluent : petroleum ether : $\mathrm{EtOAc}=10: 1)$.

$\mathbf{R}_{f}=0.45$ (petroleum ether $:$ EtOAc $=5: 1$ ).

${ }^{1} \mathrm{H}$ NMR (400 MHz, $\left.\mathrm{CDCl}_{3}\right)$ d $7.21-7.12(\mathrm{~m}, 4 \mathrm{H}), 7.02(\mathrm{t}, J=7.2 \mathrm{~Hz}, 1 \mathrm{H}), 2.65(\mathrm{~d}$, $J=12.7 \mathrm{~Hz}, 1 \mathrm{H}), 1.43(\mathrm{~d}, J=12.8 \mathrm{~Hz}, 1 \mathrm{H}), 1.23(\mathrm{~d}, J=5.1 \mathrm{~Hz}, 12 \mathrm{H}), 1.13(\mathrm{~d}, J=8.4$ $\mathrm{Hz}, 12 \mathrm{H}), 0.93(\mathrm{~d}, J=7.3 \mathrm{~Hz}, 12 \mathrm{H})$.

${ }^{13} \mathrm{C}$ NMR (100 MHz, $\mathrm{CDCl}_{3}$ ) $\delta$ 145.4, 128.6, 127.9, 124.7, 83.12, 83.08, 82.7, 24.9, $24.7,24.5,24.4,24.3$. The carbon signal attached to B was not observed due to low intensity.

Spectral data matched those previously reported. ${ }^{[30]}$ 
<smiles>COc1ccc(C(C(Cc2ccccc2)Cc2ccccc2)C(Br)c2ccccc2)cc1</smiles>

Compound $4 \mathbf{b}$ was prepared following the GP6 using 1-(4methoxyphenyl)ethan-1-one $1 \mathbf{c}^{\prime}(45.0 \mathrm{mg}, 0.3 \mathrm{mmol})$, [ $\left.\mathrm{RhCl}(\mathrm{cod})\right]_{2}$ (3.0 mg, $0.006 \mathrm{mmol}), \mathrm{P}\left({ }^{n} \mathrm{Bu}\right) 3(7.3 \mathrm{mg}, 0.036 \mathrm{mmol}), \mathrm{B}_{2}$ pin2 $(304.8 \mathrm{mg}, 1.20 \mathrm{mmol}$ ), $\mathrm{NaOAc}(36.9 \mathrm{mg}, 0.45 \mathrm{mmol})$ and hexane $(3.0 \mathrm{~mL})$. The product was obtained as a white solid in $61 \%$ yield $(94.1 \mathrm{mg}$, eluent : petroleum ether : EtOAc $=8: 1)$. $\mathbf{R}_{f}=0.42$ (petroleum ether $:$ EtOAc $=5: 1$ ).

${ }^{1} \mathrm{H}$ NMR (400 MHz, $\mathrm{CDCl}_{3}$ ) $\delta 7.14-7.09$ (m, 2H), $6.75-6.70$ (m, 2H), 3.73 (s, $3 \mathrm{H}), 2.60(\mathrm{~d}, J=12.7 \mathrm{~Hz}, 1 \mathrm{H}), 1.37(\mathrm{~d}, J=12.7 \mathrm{~Hz}, 1 \mathrm{H}), 1.22(\mathrm{~d}, J=4.9 \mathrm{~Hz}, 12 \mathrm{H})$, $1.14(\mathrm{~d}, J=7.2 \mathrm{~Hz}, 12 \mathrm{H}), 0.95(\mathrm{~d}, J=3.7 \mathrm{~Hz}, 12 \mathrm{H})$.

${ }^{13} \mathrm{C}$ NMR (100 MHz, $\left.\mathrm{CDCl}_{3}\right) \delta$ 157.1, 137.6, 129.4, 113.4, 83.11, 83.10, 82.7, 55.3, $24.99,24.97,24.8,24.6,24.5,24.4$. The carbon signal attached to B was not observed due to low intensity.

Spectral data matched those previously reported. ${ }^{[31]}$

2,2',2'-(2-(4-Fluorophenyl)ethane-1,1,2-triyl)tris(4,4,5,5-tetramethyl-1,3,2dioxaborolane) (4c)<smiles>Fc1ccc(C(C(Br)c2ccccc2)C(Br)c2ccccc2)cc1</smiles>

Compound 4c was prepared following the GP6 using 1-(4-fluorophenyl)ethan1-one $1 \mathrm{j}$ (41.4 mg, $0.3 \mathrm{mmol})$, [ RhCl(cod)]2 (3.0 mg, $0.006 \mathrm{mmol}), \mathrm{P}\left({ }^{n} \mathrm{Bu}\right) 3(7.3 \mathrm{mg}$, $0.036 \mathrm{mmol}), \mathrm{B}_{2}$ pin $2(304.8 \mathrm{mg}, 1.20 \mathrm{mmol}), \mathrm{NaOAc}(36.9 \mathrm{mg}, 0.45 \mathrm{mmol})$ and hexane $(3.0 \mathrm{~mL})$. The product was obtained as a white solid in $68 \%$ yield $(102.4$ $\mathrm{mg}$, eluent : petroleum ether : $\mathrm{EtOAc}=8: 1)$.

$\mathbf{R}_{f}=0.45$ (petroleum ether : $\mathrm{EtOAc}=5: 1$ ).

${ }^{1}$ H NMR (400 MHz, $\left.\mathrm{CDCl}_{3}\right) \delta 7.17-7.12(\mathrm{~m}, 2 \mathrm{H}), 6.89-6.82(\mathrm{~m}, 2 \mathrm{H}), 2.64(\mathrm{~d}, J$ 
$=12.7 \mathrm{~Hz}, 1 \mathrm{H}), 1.38(\mathrm{~d}, J=12.8 \mathrm{~Hz}, 1 \mathrm{H}), 1.22(\mathrm{~d}, J=4.7 \mathrm{~Hz}, 12 \mathrm{H}), 1.13(\mathrm{~d}, J=7.1$ $\mathrm{Hz}, 12 \mathrm{H}), 0.94(\mathrm{~d}, J=9.7 \mathrm{~Hz}, 12 \mathrm{H})$.

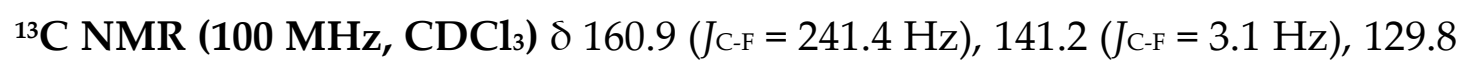
$\left(J_{C-F}=7.7 \mathrm{~Hz}\right), 114.6\left(J_{C-F}=20.9 \mathrm{~Hz}\right), 83.3,83.2,82.9,25.0,24.9,24.8,24.6,24.5$, 24.4. The carbon signal attached to $B$ was not observed due to low intensity. ${ }^{19}$ F NMR (376 MHz, $\mathrm{CDCl}_{3}$ ): $\delta$-119.6.

Spectral data matched those previously reported. ${ }^{[30]}$

\section{2,2',2"-(2-(4-(Trifluoromethyl)phenyl)ethane-1,1,2-triyl)tris(4,4,5,5-} tetramethyl-1,3,2-dioxaborolane) (4d)<smiles>FC(F)(F)c1ccc(C(Br)C(Br)c2ccccc2)cc1</smiles>

Compound 4d was prepared following the GP6 using 1-(4(trifluoromethyl)phenyl)ethan-1-one 1n' (56.4 mg, $0.3 \mathrm{mmol})$, [ RhCl(cod) $]_{2}(3.0$ $\mathrm{mg}, 0.006 \mathrm{mmol}), \mathrm{P}\left({ }^{n} \mathrm{Bu}\right)_{3}(7.3 \mathrm{mg}, 0.036 \mathrm{mmol}), \mathrm{B}_{2} \operatorname{pin}_{2}(304.8 \mathrm{mg}, 1.20 \mathrm{mmol})$, $\mathrm{NaOAc}(36.9 \mathrm{mg}, 0.45 \mathrm{mmol})$ and hexane $(3.0 \mathrm{~mL})$. The product was obtained as a white solid in $65 \%$ yield $(107.6 \mathrm{mg}$, eluent : petroleum ether : $\mathrm{EtOAc}=8$ : 1).

$\mathbf{R}_{f}=0.50$ (petroleum ether : EtOAc $=5: 1$ ).

${ }^{1} \mathrm{H}$ NMR (400 MHz, CDCl $) \delta 7.43(\mathrm{~d}, J=8.1 \mathrm{~Hz}, 2 \mathrm{H}), 7.31(\mathrm{~d}, J=8.0 \mathrm{~Hz}, 2 \mathrm{H})$, $2.74(\mathrm{~d}, J=12.6 \mathrm{~Hz}, 1 \mathrm{H}), 1.44(\mathrm{~d}, J=12.6 \mathrm{~Hz}, 1 \mathrm{H}), 1.23(\mathrm{~d}, J=4.4 \mathrm{~Hz}, 12 \mathrm{H}), 1.14$ $(\mathrm{d}, J=6.9 \mathrm{~Hz}, 12 \mathrm{H}), 0.92(\mathrm{~d}, J=16.5 \mathrm{~Hz}, 12 \mathrm{H})$.

${ }^{13} \mathrm{C}$ NMR (100 MHz, $\left.\mathrm{CDCl}_{3}\right) \delta$ 150.1, 128.8, 127.1 (JC-F $\left.=32.0 \mathrm{~Hz}\right), 124.9$ (JC-F $=3.7$ $\mathrm{Hz}), 124.7(\mathrm{Jc}-\mathrm{F}=271.4 \mathrm{~Hz}), 83.5,83.3,83.0,25.0,24.9,24.8$, 24.5, 24.39, 24.37. The carbon signal attached to $B$ was not observed due to low intensity.

${ }^{19} \mathrm{~F}$ NMR (376 MHz, $\mathrm{CDCl}_{3}$ ): $\delta$-62.2.

Spectral data matched those previously reported. ${ }^{[32]}$

2,2',2"-(2-(4-(4,4,5,5-Tetramethyl-1,3,2-dioxaborolan-2-yl)phenyl)ethane1,1,2-triyl)tris (4,4,5,5-tetramethyl-1,3,2-dioxaborolane) (4e) 
<smiles>BrC(c1ccccc1)c1ccccc1</smiles>

Compound 4e was prepared following the GP6 using 1-(4-(4,4,5,5-tetramethyl1,3,2-dioxaborolan-2-yl)phenyl)ethan-1-one $\mathbf{1 h} \quad(73.8 \mathrm{mg}, \quad 0.3 \mathrm{mmol})$, $[\mathrm{RhCl}(\mathrm{cod})]_{2}(3.0 \mathrm{mg}, 0.006 \mathrm{mmol}), \mathrm{P}\left({ }^{n} \mathrm{Bu}\right)_{3}(7.3 \mathrm{mg}, 0.036 \mathrm{mmol}), \mathrm{B}_{2}$ pin2 (304.8 $\mathrm{mg}, 1.20 \mathrm{mmol}), \mathrm{NaOAc}(36.9 \mathrm{mg}, 0.45 \mathrm{mmol})$ and hexane $(3.0 \mathrm{~mL})$. The product was obtained as a white solid in $62 \%$ yield $(113.5 \mathrm{mg}$, eluent : petroleum ether : $\mathrm{EtOAc}=8: 1)$.

$\mathbf{R}_{f}=0.40$ (petroleum ether : EtOAc $=5: 1$ ).

${ }^{1} \mathrm{H}$ NMR (400 MHz, $\left.\mathrm{CDCl}_{3}\right) \delta 7.63-7.59(\mathrm{~m}, 2 \mathrm{H}), 7.24-7.19(\mathrm{~m}, 2 \mathrm{H}), 2.69$ (d, J $=12.6 \mathrm{~Hz}, 1 \mathrm{H}), 1.43(\mathrm{~d}, J=12.5 \mathrm{~Hz}, 1 \mathrm{H}), 1.32(\mathrm{~d}, J=1.8 \mathrm{~Hz}, 12 \mathrm{H}), 1.22(\mathrm{~d}, J=3.8$ $\mathrm{Hz}, 12 \mathrm{H}), 1.12(\mathrm{~d}, J=6.3 \mathrm{~Hz}, 12 \mathrm{H}), 0.94(\mathrm{~d}, J=12.1 \mathrm{~Hz}, 12 \mathrm{H})$.

${ }^{13} \mathrm{C}$ NMR (100 MHz, $\left.\mathrm{CDCl}_{3}\right) \delta$ 149.1, 134.6, 128.0, 83.5, 83.21, 83.18, 82.8, 25.1, $25.02,24.99,24.9,24.7,24.6,24.4,24.3$. The carbon signal attached to B was not observed due to low intensity.

HRMS (ESI') calcd for $\mathrm{C}_{32} \mathrm{H}_{54} \mathrm{~B}_{4} \mathrm{O}_{8} \mathrm{Na}^{+}[\mathrm{M}+\mathrm{Na}]^{+}$: 633.4083, found: 633.4082.

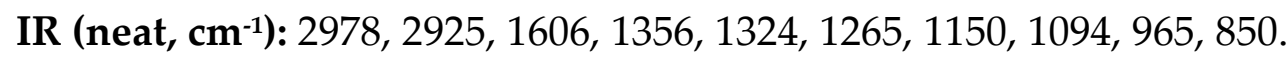

\section{Methyl}

yl)ethyl)benzoate (4f)

\section{4-(1,2,2-tris(4,4,5,5-tetramethyl-1,3,2-dioxaborolan-2-}<smiles>CC(=O)c1ccc(C(Cc2ccccc2)C(Cc2ccccc2)Cc2ccccc2)cc1</smiles>

Compound $4 \mathbf{f}$ was prepared following the GP6 using methyl 4-acetylbenzoate 1o' (53.4 mg, $0.3 \mathrm{mmol})$, [RhCl(cod)]2 (3.0 mg, $0.006 \mathrm{mmol}), \mathrm{P}\left({ }^{n} \mathrm{Bu}\right)_{3}(7.3 \mathrm{mg}$, $0.036 \mathrm{mmol}), B_{2}$ pin2 (304.8 mg, $\left.1.20 \mathrm{mmol}\right), \mathrm{NaOAc}(36.9 \mathrm{mg}, 0.45 \mathrm{mmol})$ and hexane $(3.0 \mathrm{~mL})$. The product was obtained as a white solid in $54 \%$ yield $(87.8$ $\mathrm{mg}$, eluent : petroleum ether : $\mathrm{EtOAc}=8: 1)$.

$\mathbf{R}_{f}=0.35$ (petroleum ether : EtOAc $=5: 1$ ).

${ }^{1} \mathrm{H}$ NMR (400 MHz, $\left.\mathrm{CDCl}_{3}\right) \delta 7.88(\mathrm{~d}, J=7.9 \mathrm{~Hz}, 2 \mathrm{H}), 7.30(\mathrm{~d}, J=8.3 \mathrm{~Hz}, 2 \mathrm{H})$, $3.88(\mathrm{~s}, 3 \mathrm{H}), 2.78(\mathrm{~d}, J=12.6 \mathrm{~Hz}, 1 \mathrm{H}), 1.48(\mathrm{~d}, J=12.6 \mathrm{~Hz}, 1 \mathrm{H}), 1.25(\mathrm{~d}, J=4.1 \mathrm{~Hz}$, $12 \mathrm{H}), 1.14(\mathrm{~d}, J=7.9 \mathrm{~Hz}, 12 \mathrm{H}), 0.95(\mathrm{~d}, J=20.2 \mathrm{~Hz}, 12 \mathrm{H})$. 
${ }^{13} \mathrm{C}$ NMR (100 MHz, $\left.\mathrm{CDCl}_{3}\right) \delta$ 167.7, 151.7, 129.4, 128.5, 126.7, 83.4, 83.3, 82.9, $51.9,25.0,24.9,24.8,24.6,24.42,24.38$. The carbon signal attached to B was not observed due to low intensity.

HRMS (ESI ${ }^{+}$) calcd for $\mathrm{C}_{28} \mathrm{H}_{45} \mathrm{~B}_{3} \mathrm{O}_{8} \mathrm{Na}^{+}[\mathrm{M}+\mathrm{Na}]^{+}$: 565.3286, found: 565.3284 .

IR (neat, cm-1): 2978, 2925, 1718, 1605, 1368, 1315, 1277, 1141, 1112, 966, 856.

\section{4-(1,2,2-Tris(4,4,5,5-tetramethyl-1,3,2-dioxaborolan-2-yl)ethyl)phenol (4g)}<smiles>Oc1ccc(C(Br)C(Br)Cc2ccccc2)cc1</smiles>

Compound $4 \mathrm{~g}$ was prepared following the GP6 using methyl 4-acetylbenzoate 1 p (40.8 mg, $0.3 \mathrm{mmol})$, [RhCl(cod) $]_{2}(3.0 \mathrm{mg}, 0.006 \mathrm{mmol}), \mathrm{P}\left({ }^{n} \mathrm{Bu}\right)_{3}(7.3 \mathrm{mg}, 0.036$ $\mathrm{mmol}), \mathrm{B}_{2}$ pin2 $(304.8 \mathrm{mg}, 1.20 \mathrm{mmol}), \mathrm{NaOAc}(36.9 \mathrm{mg}, 0.45 \mathrm{mmol})$ and hexane $(3.0 \mathrm{~mL})$. The product was obtained as a white solid in $70 \%$ yield $(105.0 \mathrm{mg}$, eluent : DCM : $\mathrm{EtOAC}=8: 1)$.

$\mathbf{R}_{f}=0.65(\mathrm{DCM}: \mathrm{EtOAc}=5: 1)$.

${ }^{1} \mathrm{H}$ NMR (400 MHz, $\left.\mathrm{CDCl}_{3}\right) \delta 7.16-6.89(\mathrm{~m}, 2 \mathrm{H}), 6.79-6.40(\mathrm{~m}, 2 \mathrm{H}), 4.84(\mathrm{~s}$, $1 \mathrm{H}), 2.60(\mathrm{~d}, J=12.8 \mathrm{~Hz}, 1 \mathrm{H}), 1.38(\mathrm{~d}, J=12.7 \mathrm{~Hz}, 1 \mathrm{H}), 1.23(\mathrm{~d}, J=5.0 \mathrm{~Hz}, 12 \mathrm{H})$, $1.14(\mathrm{~d}, J=7.2 \mathrm{~Hz}, 12 \mathrm{H}), 0.96(\mathrm{~d}, J=5.3 \mathrm{~Hz}, 12 \mathrm{H})$.

${ }^{13} \mathrm{C}$ NMR (100 MHz, $\left.\mathrm{CDCl}_{3}\right) \delta$ 153.1, 137.5, 129.6, 114.9, 83.22, 83.19, 82.9, 25.0, $24.8,24.6,24.5,24.4$. The carbon signal attached to B was not observed due to low intensity.

HRMS (ESI ${ }^{+}$) calcd for $\mathrm{C}_{26} \mathrm{H}_{43} \mathrm{~B}_{3} \mathrm{O}_{7} \mathrm{Na}^{+}[\mathrm{M}+\mathrm{Na}]^{+}$: 523.3180, found: 523.3191 .

IR (neat, cm-1): 3357, 2981, 1604, 1512, 1440, 1348, 1330, 1139, 978, 846, 673.

3-(1,2,2-Tris (4,4,5,5-tetramethyl-1,3,2-dioxaborolan-2-yl)ethyl)aniline (4h)<smiles>Nc1cccc(C(Br)C(Br)c2ccccc2)c1</smiles>

Compound 4h was prepared following the GP6 using methyl 4-acetylbenzoate 1q (40.5 mg, $0.3 \mathrm{mmol}),[\mathrm{RhCl}(\mathrm{cod})]_{2}(3.0 \mathrm{mg}, 0.006 \mathrm{mmol}), \mathrm{P}\left({ }^{n} \mathrm{Bu}\right)_{3}(7.3 \mathrm{mg}, 0.036$ $\mathrm{mmol}), \mathrm{B}_{2}$ pin2 $(304.8 \mathrm{mg}, 1.20 \mathrm{mmol}), \mathrm{NaOAc}(36.9 \mathrm{mg}, 0.45 \mathrm{mmol})$ and hexane $(3.0 \mathrm{~mL})$. The product was obtained as a white solid in $86 \%$ yield (128.8 $\mathrm{mg}$, 
eluent : petroleum ether : $\mathrm{EtOAc}=2: 1)$.

$\mathbf{R}_{f}=0.55$ (petroleum ether : EtOAc $=1: 1$ ).

${ }^{1} \mathrm{H}$ NMR (400 MHz, $\left.\mathrm{CDCl}_{3}\right) \delta 7.09-6.77$ (m, 1H), $6.69-6.57$ (m, 1H), $6.57-6.53$ (m, 1H), $6.43-6.33(\mathrm{~m}, 1 \mathrm{H}), 3.31(\mathrm{~s}, 2 \mathrm{H}), 2.57(\mathrm{~d}, J=12.8 \mathrm{~Hz}, 1 \mathrm{H}), 1.39(\mathrm{~d}, J=12.8$ $\mathrm{Hz}, 1 \mathrm{H}), 1.22(\mathrm{~d}, J=4.7 \mathrm{~Hz}, 12 \mathrm{H}), 1.14(\mathrm{~d}, J=7.0 \mathrm{~Hz}, 12 \mathrm{H}), 0.97(\mathrm{~d}, J=8.0 \mathrm{~Hz}$, $12 \mathrm{H})$.

${ }^{13} \mathrm{C}$ NMR (100 MHz, $\left.\mathrm{CDCl}_{3}\right) \delta$ 146.5, 145.9, 128.8, 119.3, 115.9, 112.0, 83.13, 83.09, $82.7,25.0,24.8,24.6,24.5,24.4$. The carbon signal attached to $B$ was not observed due to low intensity.

HRMS (ESI') calcd for $\mathrm{C}_{26} \mathrm{H}_{44} \mathrm{~B}_{3} \mathrm{NO}_{6} \mathrm{Na}^{+}[\mathrm{M}+\mathrm{Na}]^{+}: 522.3340$, found: 522.3355 .

IR (neat, cm $\left.{ }^{-1}\right):$ 2978, 1470, 1315, 1264, 1139, 966, 843, 667.

\section{2,2',2"-(2-(m-Tolyl)ethane-1,1,2-triyl)tris(4,4,5,5-tetramethyl-1,3,2-}

dioxaborolane) (4i)<smiles>Cc1cccc(C(Cc2ccccc2)C(Br)c2ccccc2)c1</smiles>

Compound 4i was prepared following the GP6 using 1-(m-tolyl)ethan-1-one $1 \mathrm{~m}$ (40.2 mg, $0.3 \mathrm{mmol}),[\mathrm{RhCl}(\mathrm{cod})]_{2}(3.0 \mathrm{mg}, 0.006 \mathrm{mmol}), \mathrm{P}\left({ }^{n} \mathrm{Bu}\right)_{3}(7.3 \mathrm{mg}$, $0.036 \mathrm{mmol}), B_{2}$ pin2 $(304.8 \mathrm{mg}, 1.20 \mathrm{mmol}), \mathrm{NaOAc}(36.9 \mathrm{mg}, 0.45 \mathrm{mmol})$ and hexane $(3.0 \mathrm{~mL})$. The product was obtained as a white solid in $78 \%$ yield $(118.0$ $\mathrm{mg}$, eluent : petroleum ether $: \mathrm{EtOAc}=5: 1)$.

$\mathbf{R}_{f}=0.50$ (petroleum ether $:$ EtOAc $=5: 1$ ).

${ }^{1} \mathrm{H}$ NMR (400 MHz, $\left.\mathrm{CDCl}_{3}\right) \delta 7.07-6.97(\mathrm{~m}, 3 \mathrm{H}), 6.86-6.81(\mathrm{~m}, 1 \mathrm{H}), 2.61(\mathrm{~d}, J$ $=12.7 \mathrm{~Hz}, 1 \mathrm{H}), 2.24(\mathrm{~s}, 3 \mathrm{H}), 1.40(\mathrm{~d}, J=12.7 \mathrm{~Hz}, 1 \mathrm{H}), 1.23(\mathrm{~d}, J=4.9 \mathrm{~Hz}, 12 \mathrm{H})$, $1.15(\mathrm{~d}, J=7.9 \mathrm{~Hz}, 12 \mathrm{H}), 0.94(\mathrm{~d}, J=3.5 \mathrm{~Hz}, 12 \mathrm{H})$.

${ }^{13} \mathrm{C}$ NMR (100 MHz, $\left.\mathrm{CDCl}_{3}\right) \delta$ 145.3, 137.1, 129.7, 127.9, 125.6, 125.5, 83.14, 83.09, $82.7,24.99,24.98,24.8,24.54,24.48,24.3,21.5$. The carbon signal attached to B was not observed due to low intensity.

HRMS (ESI ${ }^{+}$) calcd for $\mathrm{C}_{27} \mathrm{H}_{45} \mathrm{~B}_{3} \mathrm{O}_{6} \mathrm{Na}^{+}[\mathrm{M}+\mathrm{Na}]^{+}$: 521.3388, found: 521.3387 .

IR (neat, cm$^{-1}$ ): 2978, 2931, 1465, 1365, 1318, 1271, 1147, 971, 850. 


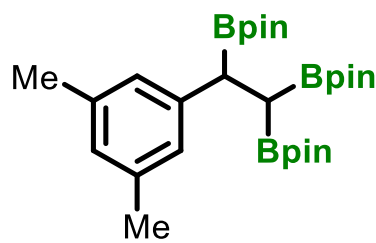

Compound $\mathbf{4} \mathbf{j}$ was prepared following the GP6 using 1-(3,5dimethylphenyl)ethan-1-one 1s (44.4 mg, $0.3 \mathrm{mmol})$, [ RhCl(cod) $]_{2}(3.0 \mathrm{mg}, 0.006$ mmol), $\mathrm{P}\left({ }^{n} \mathrm{Bu}\right.$ ) 3 (7.3 mg, $0.036 \mathrm{mmol}$ ), B ${ }_{2} \mathrm{pin}_{2}$ (304.8 mg, $1.20 \mathrm{mmol}$ ), NaOAc (36.9 $\mathrm{mg}, 0.45 \mathrm{mmol})$ and hexane $(3.0 \mathrm{~mL})$. The product was obtained as a white solid in $80 \%$ yield $(123.0 \mathrm{mg}$, eluent : petroleum ether : EtOAc $=10: 1)$.

$\mathbf{R}_{f}=0.55$ (petroleum ether : EtOAc $=5: 1$ ).

${ }^{1} \mathrm{H}$ NMR (400 MHz, CDCl $) \delta 6.81(\mathrm{~s}, 2 \mathrm{H}), 6.65(\mathrm{~s}, 1 \mathrm{H}), 2.57(\mathrm{~d}, J=12.6 \mathrm{~Hz}, 1 \mathrm{H})$, $2.20(\mathrm{~s}, 6 \mathrm{H}), 1.36(\mathrm{~d}, J=12.6 \mathrm{~Hz}, 1 \mathrm{H}), 1.22(\mathrm{~d}, J=4.5 \mathrm{~Hz}, 12 \mathrm{H}), 1.16(\mathrm{~d}, J=7.6 \mathrm{~Hz}$, $12 \mathrm{H}), 0.96(\mathrm{~s}, 12 \mathrm{H})$.

${ }^{13} \mathrm{C}$ NMR (100 MHz, $\mathrm{CDCl}_{3}$ ) $\delta$ 145.1, 137.0, 126.7, 126.4, 83.12, 83.07, 82.7, 25.02, $25.99,24.8,24.54,24.52,24.3,21.4$. The carbon signal attached to B was not observed due to low intensity.

HRMS (ESI') calcd for $\mathrm{C}_{28} \mathrm{H}_{48} \mathrm{~B}_{3} \mathrm{O}_{6} \mathrm{Na}^{+}[\mathrm{M}+\mathrm{Na}]^{+}: 535.3544$, found: 535.3553 .

IR (neat, cm$\left.^{-1}\right):$ 2978, 2926, 1367, 1315, 1266, 1144, 966, 845.

2,2',2"-(2-(3,4,5-Trimethoxyphenyl)ethane-1,1,2-triyl)tris(4,4,5,5-tetramethyl1,3,2-dioxaborolane) (4k)<smiles>COc1cc(C(Br)C(Br)c2ccccc2)cc(OC)c1OC</smiles>

Compound 4k was prepared following the GP6 using 1-(3,4,5trimethoxyphenyl)ethan-1-one $1 \mathbf{v}(63.0 \mathrm{mg}, 0.3 \mathrm{mmol})$, [ $\mathrm{RhCl}(\operatorname{cod})]_{2}(3.0 \mathrm{mg}$, $0.006 \mathrm{mmol}), \mathrm{P}\left({ }^{n} \mathrm{Bu}\right)_{3}(7.3 \mathrm{mg}, 0.036 \mathrm{mmol}), B_{2}$ pin $2(304.8 \mathrm{mg}, 1.20 \mathrm{mmol})$, $\mathrm{NaOAc}(36.9 \mathrm{mg}, 0.45 \mathrm{mmol})$ and hexane $(3.0 \mathrm{~mL})$. The product was obtained as a white solid in $82 \%$ yield $(141.2 \mathrm{mg}$, eluent : petroleum ether : EtOAc $=4$ : $1)$. 
$\mathbf{R}_{f}=0.55$ (petroleum ether : $\mathrm{EtOAc}=2: 1$ ).

${ }^{1} \mathrm{H}$ NMR (400 MHz, $\left.\mathrm{CDCl}_{3}\right) \delta 6.44(\mathrm{~s}, 2 \mathrm{H}), 3.79(\mathrm{~s}, 6 \mathrm{H}), 3.74(\mathrm{~s}, 3 \mathrm{H}), 2.57(\mathrm{~d}, J=$ $12.8 \mathrm{~Hz}, 1 \mathrm{H}), 1.39(\mathrm{~d}, J=12.6 \mathrm{~Hz}, 1 \mathrm{H}), 1.22(\mathrm{~d}, J=1.6 \mathrm{~Hz}, 12 \mathrm{H}), 1.16(\mathrm{~d}, J=6.0$ $\mathrm{Hz}, 12 \mathrm{H}), 0.96$ (s, 12H).

${ }^{13} \mathrm{C}$ NMR (100 MHz, $\left.\mathrm{CDCl}_{3}\right) \delta$ 152.6, 141.2, 135.3, 105.4, 83.3, 83.2, 82.8, 60.9, 55.9, $25.1,25.0,24.8,24.59,24.55,24.3$. The carbon signal attached to B was not observed due to low intensity.

HRMS (ESI') calcd for $\mathrm{C}_{29} \mathrm{H}_{49} \mathrm{~B}_{3} \mathrm{O}_{9} \mathrm{Na}^{+}[\mathrm{M}+\mathrm{Na}]^{+}$: 597.3548, found: 597.3547.

IR (neat, cm-1): 2981, 2934, 1592, 1500, 1456, 1377, 1321, 1271, 1135, 971, 844.

\section{2,2',2"-(2-(Naphthalen-2-yl)ethane-1,1,2-triyl)tris(4,4,5,5-tetramethyl-1,3,2-} dioxaborolane) (41)<smiles>BrC(C(Cc1ccccc1)c1ccccc1)C(Cc1ccccc1)c1ccc2ccccc2c1</smiles>

Compound 41 was prepared following the GP6 using 1-(naphthalen-2yl)ethan-1-one 1ai (51.0 mg, $0.3 \mathrm{mmol})$, [RhCl(cod)]2 (3.0 mg, $0.006 \mathrm{mmol})$, $\mathrm{P}\left({ }^{n} \mathrm{Bu}\right)_{3}(7.3 \mathrm{mg}, 0.036 \mathrm{mmol}), \mathrm{B}_{2}$ pin $2(304.8 \mathrm{mg}, 1.20 \mathrm{mmol}), \mathrm{NaOAc}$ (36.9 mg, $0.45 \mathrm{mmol})$ and hexane $(3.0 \mathrm{~mL})$. The product was obtained as a white solid in $52 \%$ yield ( $83.3 \mathrm{mg}$, eluent : petroleum ether : $\mathrm{EtOAc}=8: 1$ ).

$\mathbf{R}_{f}=0.55$ (petroleum ether : EtOAc $=5: 1$ ).

${ }^{1} \mathrm{H}$ NMR (400 MHz, $\left.\mathrm{CDCl}_{3}\right) \delta 7.76-7.70(\mathrm{~m}, 2 \mathrm{H}), 7.69-7.63(\mathrm{~m}, 2 \mathrm{H}), 7.41-7.30$ $(\mathrm{m}, 3 \mathrm{H}), 2.87(\mathrm{~d}, J=12.6 \mathrm{~Hz}, 1 \mathrm{H}), 1.56(\mathrm{~d}, J=12.6 \mathrm{~Hz}, 1 \mathrm{H}), 1.26(\mathrm{~d}, J=4.9 \mathrm{~Hz}$, $12 \mathrm{H}), 1.14(\mathrm{~d}, J=11.1 \mathrm{~Hz}, 12 \mathrm{H}), 0.86(\mathrm{~d}, J=11.9 \mathrm{~Hz}, 12 \mathrm{H})$.

${ }^{13} \mathrm{C}$ NMR (100 MHz, $\left.\mathrm{CDCl}_{3}\right) \delta$ 143.2, 133.8, 131.7, 128.0, 127.6, 127.5, 127.3, 126.3, $125.3,124.4,83.3,83.2,82.8,25.01,24.98,24.8,24.6,24.4$. The carbon signal attached to $\mathrm{B}$ was not observed due to low intensity.

HRMS (ESI') calcd for $\mathrm{C}_{30} \mathrm{H}_{45} \mathrm{~B}_{3} \mathrm{O}_{6} \mathrm{Na}^{+}[\mathrm{M}+\mathrm{Na}]^{+}$: 557.3388, found: 557.3386.

IR (neat, cm$^{-1}$ ): 2976, 2928, 1462, 1369, 1316, 1268, 1146, 965, 852.

1-Methyl-5-(1,2,2-tris(4,4,5,5-tetramethyl-1,3,2-dioxaborolan-2-yl)ethyl)-1Hindole $(4 \mathrm{~m})$ 


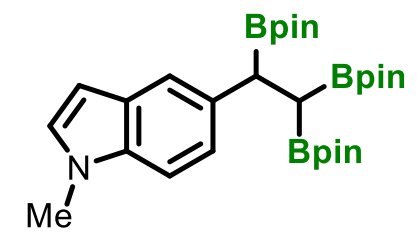

Compound 4m was prepared following the GP6 using 1-(1-methyl-1H-indol5-yl)ethan-1-one 1y (51.9 mg, $0.3 \mathrm{mmol})$, [ RhCl(cod)]2 (3.0 mg, $0.006 \mathrm{mmol})$, $\mathrm{P}\left({ }^{n} \mathrm{Bu}\right)_{3}(7.3 \mathrm{mg}, 0.036 \mathrm{mmol}), \mathrm{B}_{2}$ pin 2 (304.8 mg, $\left.1.20 \mathrm{mmol}\right), \mathrm{NaOAc}(36.9 \mathrm{mg}$, $0.45 \mathrm{mmol})$ and hexane $(3.0 \mathrm{~mL})$. The product was obtained as a white solid in $49 \%$ yield $(78.9 \mathrm{mg}$, eluent : petroleum ether : EtOAc $=10: 1)$.

$\mathbf{R}_{f}=0.45$ (petroleum ether : EtOAc $=5: 1$ ).

${ }^{1} \mathrm{H}$ NMR (400 MHz, $\left.\mathrm{CDCl}_{3}\right) \delta 7.44(\mathrm{~s}, 1 \mathrm{H}), 7.16-7.08(\mathrm{~m}, 2 \mathrm{H}), 6.92(\mathrm{~d}, J=3.1 \mathrm{~Hz}$, $1 \mathrm{H}), 6.33(\mathrm{~d}, J=3.0 \mathrm{~Hz}, 1 \mathrm{H}), 3.71(\mathrm{~s}, 3 \mathrm{H}), 2.75(\mathrm{~d}, J=12.6 \mathrm{~Hz}, 1 \mathrm{H}), 1.47(\mathrm{~d}, J=12.7$ $\mathrm{Hz}, 1 \mathrm{H}), 1.24(\mathrm{~d}, J=5.1 \mathrm{~Hz}, 12 \mathrm{H}), 1.14(\mathrm{~d}, J=11.3 \mathrm{~Hz}, 12 \mathrm{H}), 0.89$ (s, 12H).

${ }^{13} \mathrm{C}$ NMR (100 MHz, $\left.\mathrm{CDCl}_{3}\right) \delta$ 136.0, 135.2, 128.6, 128.1, 123.1, 120.4, 108.6, 100.6, $83.1,83.0,82.7,32.9,25.1,25.0,24.8,24.6,24.5,24.4$. The carbon signal attached to $\mathrm{B}$ was not observed due to low intensity.

HRMS (ESI ${ }^{+}$) calcd for $\mathrm{C}_{29} \mathrm{H}_{46} \mathrm{~B}_{3} \mathrm{NO}_{6} \mathrm{Na}^{+}[\mathrm{M}+\mathrm{Na}]^{+}$: 560.3497, found: 560.3495 .

IR (neat, cm$^{-1}$ ): 2978, 2916, 1477, 1371, 1327, 1262, 1147, 971, 847.

\section{Compound $4 n$}

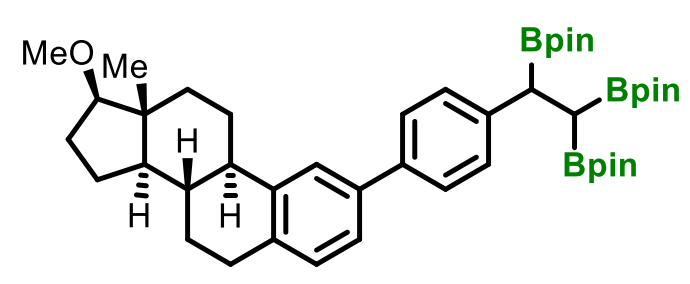

Compound 4n was prepared following the GP6 using the ketone 1ad' (116.4 $\mathrm{mg}, 0.3 \mathrm{mmol}),[\mathrm{RhCl}(\mathrm{cod})]_{2}(3.0 \mathrm{mg}, 0.006 \mathrm{mmol}), \mathrm{P}\left({ }^{n} \mathrm{Bu}\right)_{3}(7.3 \mathrm{mg}, 0.036 \mathrm{mmol})$, $\mathrm{B}_{2}$ pinz $(304.8 \mathrm{mg}, 1.20 \mathrm{mmol}), \mathrm{NaOAc}(36.9 \mathrm{mg}, 0.45 \mathrm{mmol})$ and hexane $(3.0 \mathrm{~mL})$. The product was obtained as a white solid in $57 \%$ yield $(128.6 \mathrm{mg}$, eluent : petroleum ether : $\mathrm{EtOAc}=10: 1)$.

$\mathbf{R}_{f}=0.45$ (petroleum ether : $\mathrm{EtOAc}=3: 1$ ).

${ }^{1} \mathrm{H}$ NMR (400 MHz, $\left.\mathrm{CDCl}_{3}\right) \delta 7.46(\mathrm{~d}, J=7.8 \mathrm{~Hz}, 2 \mathrm{H}), 7.42-7.36(\mathrm{~m}, 2 \mathrm{H}), 7.34$ (s, 1H), $7.33-7.28(\mathrm{~m}, 2 \mathrm{H}), 3.43(\mathrm{~s}, 3 \mathrm{H}), 3.38(\mathrm{t}, J=8.2 \mathrm{~Hz}, 1 \mathrm{H}), 3.01-2.93(\mathrm{~m}$, $2 \mathrm{H}), 2.75(\mathrm{~d}, \mathrm{~J}=12.7 \mathrm{~Hz}, 1 \mathrm{H}), 2.44-2.27(\mathrm{~m}, 2 \mathrm{H}), 2.18-2.06(\mathrm{~m}, 3 \mathrm{H}), 2.01-1.92$ 
$(\mathrm{m}, 1 \mathrm{H}), 1.80-1.71(\mathrm{~m}, 1 \mathrm{H}), 1.61-1.39(\mathrm{~m}, 7 \mathrm{H}), 1.30(\mathrm{~d}, J=5.3 \mathrm{~Hz}, 12 \mathrm{H}), 1.21(\mathrm{~d}$, $J=8.6 \mathrm{~Hz}, 12 \mathrm{H}), 0.99(\mathrm{~d}, J=4.0 \mathrm{~Hz}, 12 \mathrm{H}), 0.85(\mathrm{~s}, 3 \mathrm{H})$.

${ }^{13} \mathrm{C}$ NMR (100 MHz, $\left.\mathrm{CDCl}_{3}\right) \delta$ 144.4, 139.0, 138.9, 137.4, 137.0, 128.9, 127.4, 126.5, $125.8,124.2$, 90.9, 83.24, 83.15, 82.8, 58.0, 50.5, 44.4, 43.3, 38.6, 38.2, 29.8, 27.9, 27.4, $26.4,25.0,24.9,24.8,24.52,24.46,24.4,23.2,11.6$. The carbon signal attached to B was not observed due to low intensity.

HRMS (ESI') calcd for $\mathrm{C}_{45} \mathrm{H}_{67} \mathrm{~B}_{3} \mathrm{O}_{7} \mathrm{Na}^{+}[\mathrm{M}+\mathrm{Na}]^{+}$: 775.5058, found: 775.5056.

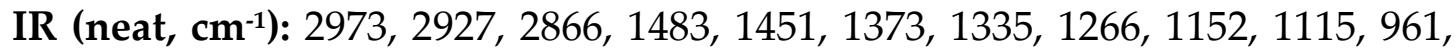
845.

\section{Synthetic Applications}

\section{Gram scale Synthesis}

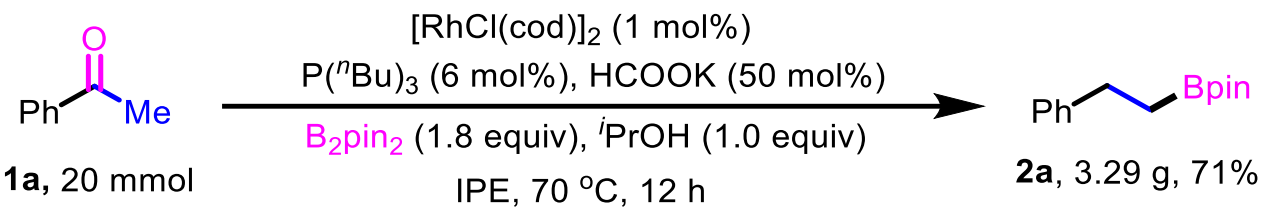

In a glove box, $[\mathrm{RhCl}(\mathrm{cod})]_{2}(98.6 \mathrm{mg}, 0.2 \mathrm{mmol}), \mathrm{P}\left({ }^{n} \mathrm{Bu}\right)_{3}(242.8 \mathrm{mg}, 1.2$ $\mathrm{mmol})$, and anhydrous IPE $(20.0 \mathrm{~mL})$ were placed in a $100 \mathrm{~mL}$ round bottom flask and stirred for $30 \mathrm{~min}$. Then, HCOOK (841.1 mg, $10.0 \mathrm{mmol}), \mathrm{B}_{2}$ pin2 (9.14 $\mathrm{g}$, $36.0 \mathrm{mmol}),{ }^{i} \mathrm{PrOH}(600.0 \mathrm{mg}, 10.0 \mathrm{mmol})$, and ketone $1 \mathrm{a}(2.4 \mathrm{~g}, 20 \mathrm{mmol})$ in IPE $(20.0 \mathrm{~mL})$ were added. The reaction mixture was sealed, removed from the glovebox, and stirred at $70{ }^{\circ} \mathrm{C}$ for 12 hours. After cooled to room temperature, the mixture was quenched with saturated $\mathrm{NH}_{4} \mathrm{Cl}(20 \mathrm{~mL})$, extracted with EtOAc $\left(3^{*} 10 \mathrm{~mL}\right)$, dried with $\mathrm{Na}_{2} \mathrm{SO}_{4}$, filtered and concentrated. The residue was purified by silica gel chromatography using petroleum ether : EtOAc $=50: 1$ as the eluent to afford the desired product $2 \mathrm{a}$ ( $3.29 \mathrm{~g}, 71 \%$ yield).

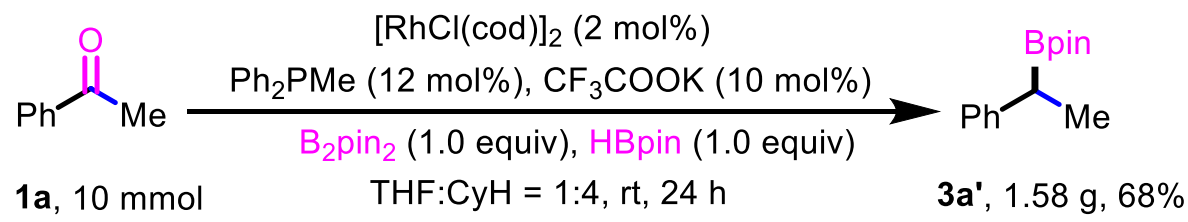

In a glove box, $[\mathrm{RhCl}(\mathrm{cod})]_{2}(98.6 \mathrm{mg}, 0.2 \mathrm{mmol}), \mathrm{PPh}_{2} \mathrm{Me}(240.0 \mathrm{mg}, 1.2$ 
$\mathrm{mmol})$, and anhydrous THF $(6.0 \mathrm{~mL})$ were placed in a $100 \mathrm{~mL}$ round bottom flask and stirred for $30 \mathrm{~min}$. Then, $\mathrm{CF}_{3} \mathrm{COOK}(153.3 \mathrm{mg}, 1.0 \mathrm{mmol}), \mathrm{B}_{2}$ pin 2 (2.54g, $10.0 \mathrm{mmol})$, HBpin $(1.28 \mathrm{~g}, 10.0 \mathrm{mmol})$, and ketone $1 \mathrm{a}^{\prime}(1.2 \mathrm{~g}, 10 \mathrm{mmol})$ in CyH $(24 \mathrm{~mL})$ were added. The reaction mixture was sealed, removed from the glovebox, and stirred at room temperature for 24 hours. The mixture was quenched with saturated $\mathrm{NH}_{4} \mathrm{Cl}(20 \mathrm{~mL})$, extracted with EtOAc $\left(3^{*} 10 \mathrm{~mL}\right)$, dried with $\mathrm{Na}_{2} \mathrm{SO}_{4}$, filtered and concentrated. The residue was purified by silica gel chromatography using petroleum ether : EtOAc $=25: 1$ as the eluent to afford the desired product 3a' (1.58 g, 68\% yield).

\section{Synthetic transformations}

\section{Synthesis of $5 a$}

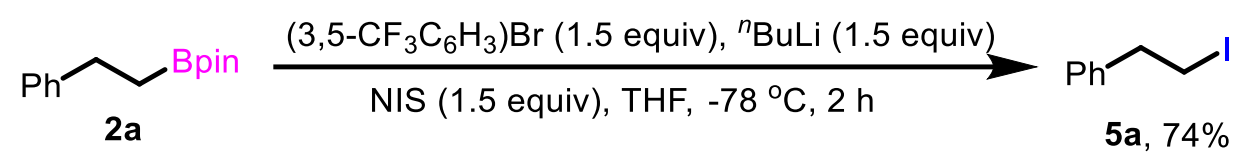

According to a literature procedure ${ }^{[33]}$, to a solution of 3,5bis(trifluoromethyl)-1-bromobenzene (436.5 mg, $1.5 \mathrm{mmol}$ ) in THF (5.0 mL) was added ${ }^{n} \mathrm{BuLi}(2.5 \mathrm{M}$ in hexane, $1.5 \mathrm{mmol})$ at $-78^{\circ} \mathrm{C}$. The mixture was stirred at $78{ }^{\circ} \mathrm{C}$ for 1 hour, and a solution of compound $2 \mathrm{a}(232.0 \mathrm{mg}, 1.0 \mathrm{mmol})$ in THF $(2.0 \mathrm{~mL})$ was then added. The reaction mixture was stirred at $-78{ }^{\circ} \mathrm{C}$ for $30 \mathrm{~min}$ and at room temperature for $30 \mathrm{~min}$, followed by the addition of a solution of NIS (337.5 mg, $1.5 \mathrm{mmol}$ ) in THF (2.0 mL) dropwise. Upon completion of the reaction, the reaction mixture was quenched with $\mathrm{Na}_{2} \mathrm{~S}_{2} \mathrm{O}_{3}$ (aq.), extracted with EtOAc, dried over $\mathrm{NaSO}_{4}$, filtered and concentrated. The resulting residue was purified by silica gel flash column chromatography (petroleum ether) to provide the title compound $5 \mathbf{a}$ (170.9 $\mathrm{mg}, 74 \%$ yield).

${ }^{1} \mathrm{H}$ NMR (400 MHz, $\left.\mathrm{CDCl}_{3}\right) \delta 7.38-7.28(\mathrm{~m}, 3 \mathrm{H}), 7.26-7.19(\mathrm{~m}, 2 \mathrm{H}), 3.38(\mathrm{t}, J=$ $7.8 \mathrm{~Hz}, 2 \mathrm{H}), 3.21(\mathrm{t}, J=7.9 \mathrm{~Hz}, 2 \mathrm{H})$.

${ }^{13} \mathrm{C}$ NMR (100 MHz, $\left.\mathrm{CDCl}_{3}\right) \delta 140.8,128.8,128.5,127.0,40.5,5.7$.

Spectral data matched those previously reported. ${ }^{[34]}$

\section{Synthesis of $\mathbf{5 b}$}




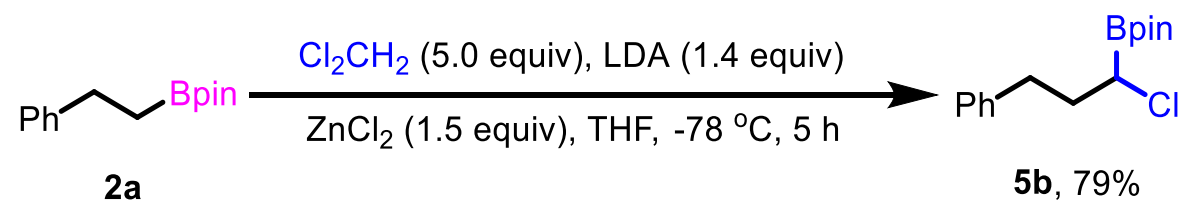

According to a literature procedure ${ }^{[35]}$, a flame-dried round bottom flask with a stirred bar was charged with $2 \mathbf{a}(232 \mathrm{mg}, 1.0 \mathrm{mmol}), \mathrm{CH}_{2} \mathrm{Cl}_{2}(0.3 \mathrm{~mL}, 5.0$ $\mathrm{mmol})$, and THF $(8 \mathrm{~mL})$ and cooled to $-78^{\circ} \mathrm{C}$. The LDA was then added over 5 minutes. $\mathrm{ZnCl}_{2}(205 \mathrm{mg}, 1.5 \mathrm{mmol})$ was dissolved in THF $(6 \mathrm{~mL})$ and added dropwise over 10 minutes. The resulting mixture was stirred for an additional 5 hours at $-78^{\circ} \mathrm{C}$. After warmed to room temperature, the reaction mixture was quenched with $\mathrm{NH}_{4} \mathrm{Cl}$ (aq.), extracted with EtOAc, dried over $\mathrm{NaSO}_{4}$, filtered and concentrated. The resulting residue was purified by silica gel flash column chromatography (petroleum ether/EtOAc $=20: 1$ ) to provide the title compound $5 \mathbf{b}$ (221 mg, 79\%).

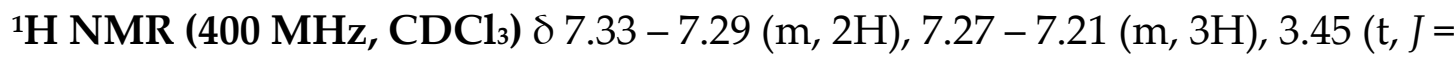
$7.4 \mathrm{~Hz}, 1 \mathrm{H}), 2.94-2.74(\mathrm{~m}, 2 \mathrm{H}), 2.15(\mathrm{q}, J=7.6 \mathrm{~Hz}, 2 \mathrm{H}), 1.32(\mathrm{~s}, 12 \mathrm{H})$.

${ }^{13} \mathrm{C}$ NMR (100 MHz, $\left.\mathrm{CDCl}_{3}\right) \delta$ 141.2, 128.7, 128.5, 126.1, 84.6, 35.8, 33.4, 24.74, 24.72. The carbon signal attached to B was not observed due to low intensity. Spectral data matched those previously reported. ${ }^{[36]}$

\section{Synthesis of $5 c$}
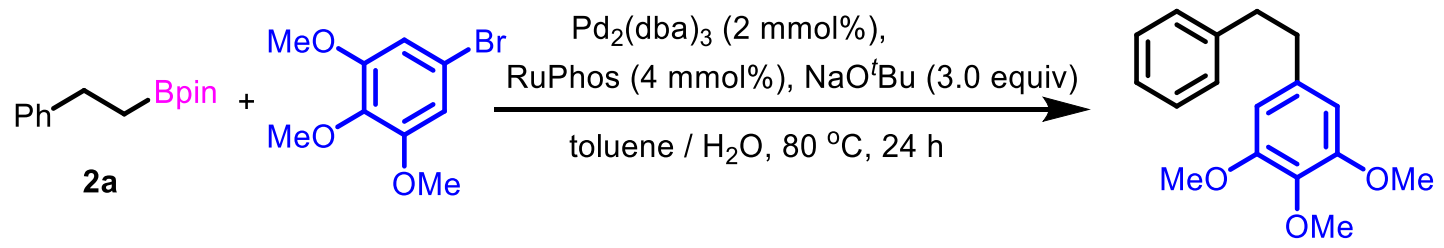

$5 c, 88 \%$

According to a literature procedure ${ }^{[37]}$, in a glove box, to a Schlenk tube equipped with a magnetic stir bar was added $\mathrm{Pd}_{2}(\mathrm{dba})_{3}(9.3 \mathrm{mg}, 0.01 \mathrm{mmol})$, Ruphos (9.3 mg, $0.02 \mathrm{mmol}), \mathrm{NaO}{ }^{t} \mathrm{Bu}(144.0 \mathrm{mg}, 1.5 \mathrm{mmol})$ and $2 \mathrm{a}(116.0 \mathrm{mg}$, $0.5 \mathrm{mmol})$. Toluene (10.0 mL), $\mathrm{H}_{2} \mathrm{O}(1.0 \mathrm{~mL})$, and 5-bromo-1,2,3trimethoxybenzene $(123.0 \mathrm{mg}, 0.5 \mathrm{mmol})$ were then added by syringe. The resulting reaction mixture was stirred vigorously at $80{ }^{\circ} \mathrm{C}$ for 24 hours. After the reaction was cooled to room temperature, the reaction mixture was diluted 
with water, extracted with EtOAc, washed with brine, dried over $\mathrm{NaSO}_{4}$, filtered and concentrated. The resulting residue was purified by silica gel flash column chromatography (petroleum ether $/ \mathrm{EtOAc}=10: 1$ ) to afford the desired products $5 \mathrm{c}$ (119.7 $\mathrm{mg}, 88 \%$ yield).

${ }^{1} \mathrm{H}$ NMR (400 MHz, $\left.\mathrm{CDCl}_{3}\right) \delta 7.36-7.28(\mathrm{~m}, 2 \mathrm{H}), 7.27-7.17(\mathrm{~m}, 3 \mathrm{H}), 6.39$ (s, $2 \mathrm{H}), 3.86(\mathrm{~s}, 3 \mathrm{H}), 3.85(\mathrm{~s}, 6 \mathrm{H}), 2.99-2.84(\mathrm{~m}, 4 \mathrm{H})$.

${ }^{13} \mathrm{C}$ NMR (100 MHz, $\left.\mathrm{CDCl}_{3}\right) \delta$ 153.1, 141.7, 137.6, 136.3, 128.6, 128.5, 126.1, 105.5, 61.0, 56.2, 38.4, 38.1 .

Spectral data matched those previously reported. ${ }^{[38]}$

\section{Synthesis of $5 \mathrm{~d}$}

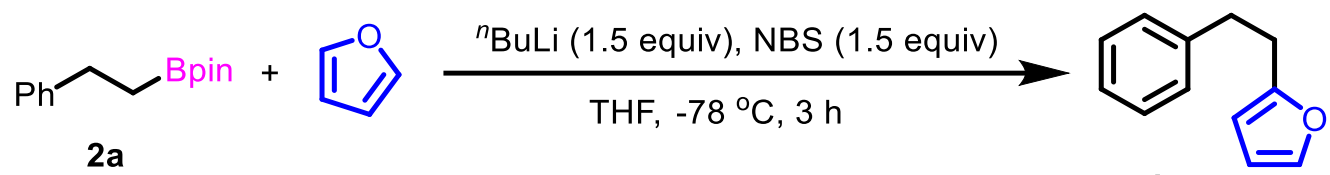

$5 d, 84 \%$

According to a literature procedure ${ }^{[39]}$, to a solution of furan $(68.0 \mathrm{mg}, 1.0$ $\mathrm{mmol})$ in THF $(5.0 \mathrm{~mL})$ was added ${ }^{n} \mathrm{BuLi}(2.5 \mathrm{M}$ in hexane, $0.75 \mathrm{mmol})$ at $-78^{\circ} \mathrm{C}$. The resultant mixture was allowed to warm to room temperature and stirred for 1 hour. Subsequently, the reaction mixture was cooled back to $-78^{\circ} \mathrm{C}$, and a solution of $2 \mathrm{a}(116.0 \mathrm{mg}, 0.5 \mathrm{mmol})$ in THF $(2.0 \mathrm{~mL})$ was added dropwise. The resulting mixture was stirred at the same temperature for 1 hour. A solution of NBS (138.5 mg, $0.75 \mathrm{mmol})$ in THF $(5.0 \mathrm{~mL})$ was then added dropwise. After stirred at $-78{ }^{\circ} \mathrm{C}$ for 1 hour, saturated aqueous $\mathrm{Na}_{2} \mathrm{~S}_{2} \mathrm{O}_{3}$ solution $(4.0 \mathrm{~mL})$ was added and the reaction mixture was allowed to warm to room temperature. The reaction mixture was diluted with water, extracted with EtOAc, dried over $\mathrm{NaSO}_{4}$, filtered and concentrated under reduced pressure. The crude material was purified by flash column chromatography (petroleum ether/EtOAc $=200: 1$ ) to provide the title compound $5 \mathrm{~d}$ (72.2 $\mathrm{mg}, 84 \%$ yield).

${ }^{1} \mathrm{H}$ NMR (400 MHz, $\mathbf{C D C l}_{3}$ ) $\delta 7.42-7.34(\mathrm{~m}, 3 \mathrm{H}), 7.31-7.23(\mathrm{~m}, 3 \mathrm{H}), 6.36$ (s, $1 \mathrm{H}), 6.05(\mathrm{~s}, 1 \mathrm{H}), 3.03(\mathrm{~s}, 4 \mathrm{H})$.

${ }^{13} \mathrm{C}$ NMR (100 MHz, $\left.\mathrm{CDCl}_{3}\right) \delta$ 155.5, 141.3, 141.0, 128.5, 128.5, 126.2, 110.2, 105.3, $34.5,30.0$.

Spectral data matched those previously reported. ${ }^{[40]}$ 


\section{Synthesis of $5 e$}

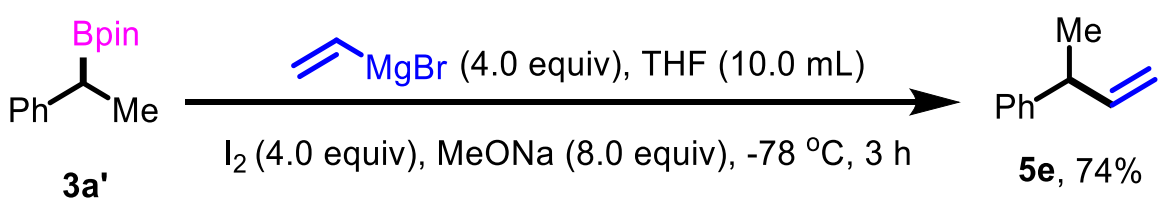

According to a literature procedure ${ }^{[41]}$, to a solution of $3 \mathbf{a}^{\prime}(1.0 \mathrm{mmol})$ in anhydrous THF $(10 \mathrm{~mL}, 0.1 \mathrm{M})$ at room temperature was added a vinylmagnesium bromide $(1.0 \mathrm{M}$ in THF, $4.0 \mathrm{mmol})$ dropwise. The resulting mixture was stirred at room temperature for $30 \mathrm{~min}$ and cooled down to $-78^{\circ} \mathrm{C}$. A solution of iodine $(4.0 \mathrm{mmol})$ in $\mathrm{MeOH}(4 \mathrm{~mL})$ was added dropwise. After stirred for $30 \mathrm{~min}$, a solution of $\mathrm{MeONa}(8.0 \mathrm{mmol})$ in $\mathrm{MeOH}(8 \mathrm{~mL})$ was added. The reaction mixture was then allowed to warm to room temperature and stirred for an additional 1 hour, then quenched with a $20 \%$ aq. $\mathrm{NaS}_{2} \mathrm{O}_{3}$ solution $(5 \mathrm{~mL})$ and water $(10 \mathrm{~mL})$, extracted with EtOAc $(2 \times 10 \mathrm{~mL})$, washed with brine, dried with $\mathrm{NaSO}_{4}$, filtered and concentrated in vacuo. The crude material was purified by flash column chromatography (petroleum ether/EtOAc $=50: 1$ ) to provide the title compound $5 \mathbf{e}$ (97.7 $\mathrm{mg}, 74 \%$ yield).

${ }^{1}$ H NMR (400 MHz, $\mathrm{CDCl}_{3}$ ) $\delta 7.43$ - 7.35 (m, 2H), $7.34-7.25$ (m, 3H), 6.19 - 6.00 (m, 1H), $5.20-5.04(\mathrm{~m}, 2 \mathrm{H}), 3.62-3.49(\mathrm{~m}, 1 \mathrm{H}), 1.45(\mathrm{~d}, J=7.1 \mathrm{~Hz}, 3 \mathrm{H})$.

${ }^{13} \mathrm{C}$ NMR (100 MHz, $\left.\mathrm{CDCl}_{3}\right) \delta$ 145.7, 143.4, 128.5, 127.4, 126.3, 113.2, 43.3, 20.9. Spectral data matched those previously reported. ${ }^{[42]}$

\section{Synthesis of $5 \mathrm{f}$}

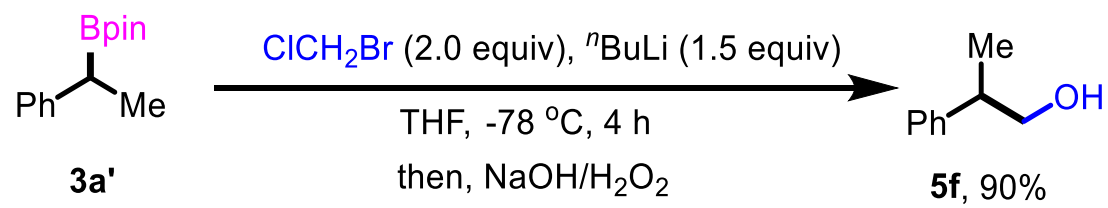

According to a literature procedure ${ }^{[33]}$, to a solution of $3 \mathbf{a}^{\prime}(232.0 \mathrm{mg}, 1.0$ $\mathrm{mmol})$ and bromochloromethane $(254.0 \mathrm{mg}, 2.0 \mathrm{mmol})$ in THF $(10.0 \mathrm{~mL})$ was added ${ }^{n} \mathrm{BuLi}(2.5 \mathrm{M}$ in hexane, $1.5 \mathrm{mmol})$ at $-78^{\circ} \mathrm{C}$. After stirring for $10 \mathrm{~min}$, the reaction mixture was warmed to room temperature and additionally stirred for 2 hours. The reaction mixture was then quenched with saturated $\mathrm{NH}_{4} \mathrm{Cl}$, extracted with EtOAc, dried with $\mathrm{Na}_{2} \mathrm{SO}_{4}$, filtered and concentrated. The crude 
residue was dissolved in THF $(10 \mathrm{~mL})$ and cooled to $0{ }^{\circ} \mathrm{C}$, then $3 \mathrm{M} \mathrm{NaOH}(2.5$ $\mathrm{mL})$ and $30 \% \mathrm{H}_{2} \mathrm{O}_{2}(2.5 \mathrm{~mL})$ were added and stirred for 0.5 hour. After the reaction was quenched by saturated aqueous $\mathrm{Na}_{2} \mathrm{~S}_{2} \mathrm{O}_{3}$ solution $(4.0 \mathrm{~mL})$, the mixture was extracted with EtOAc, washed with brine, dried with $\mathrm{Na}_{2} \mathrm{SO}_{4}$, filtered and concentrated in vacuo. The crude material was purified by flash column chromatography (petroleum ether/EtOAc $=10: 1$ ) to provide the title compound 5 f (122.4 $\mathrm{mg}$, $90 \%$ yield).

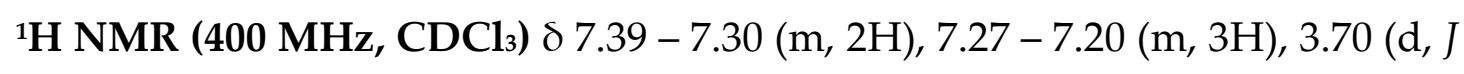
$=6.6 \mathrm{~Hz}, 2 \mathrm{H}), 3.07-2.87(\mathrm{~m}, 1 \mathrm{H}), 1.28(\mathrm{~d}, J=7.2 \mathrm{~Hz}, 3 \mathrm{H})$.

${ }^{13} \mathrm{C}$ NMR (100 MHz, $\left.\mathrm{CDCl}_{3}\right) \delta$ 143.8, 128.8, 127.6, 126.8, 68.8, 42.6, 17.7.

Spectral data matched those previously reported. ${ }^{[43]}$

\section{Synthesis of $5 \mathrm{~g}$}

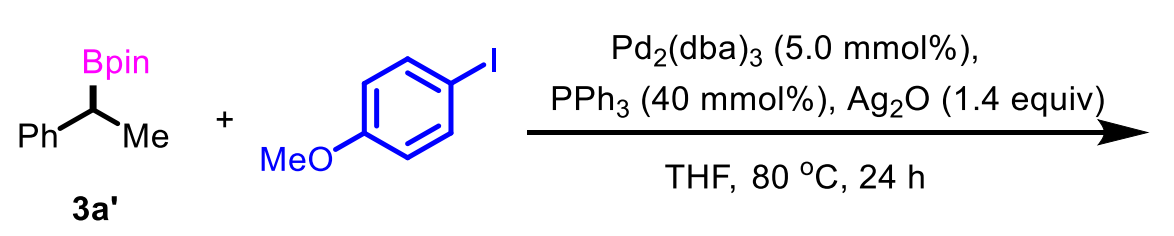

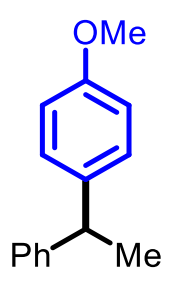

5g, $74 \%$

According to a literature procedure ${ }^{[44]}$, in a glove box, to a Schlenk tube equipped with a magnetic stir bar was added $\mathrm{Ag}_{2} \mathrm{O}(259.0 \mathrm{mg}, 1.4 \mathrm{mmol})$, $\mathrm{Pd}_{2}(\mathrm{dba})_{3}$ (45.5 mg, $0.05 \mathrm{mmol}$ ), $\mathrm{PPh}_{3}$ (104.8 mg, $0.4 \mathrm{mmol}$ ), 3a' (324.8 mg, 1.4 $\mathrm{mmol})$, and 1-iodo-3- methoxybenzene (233.9 mg, $1.0 \mathrm{mmol}$ ). THF (10.0 mL, $0.1 \mathrm{M}$ ) was then added and the tube was sealed. The mixture was stirred at 80 ${ }^{\circ} \mathrm{C}$ for 24 hours. After cooled to room temperature, water was added and the mixture was extracted with EtOAc. The combined organic layer was washed with brine, dried with $\mathrm{Na}_{2} \mathrm{SO}_{4}$, filtered and concentrated. The crude material was purified by flash column chromatography (petroleum ether/EtOAc $=50: 1$ ) to provide the title compound $5 \mathrm{~g}$ (156.9 $\mathrm{mg}$, 74\% yield).

${ }^{1} \mathbf{H}$ NMR (400 MHz, $\left.\mathrm{CDCl}_{3}\right) \delta 7.32(\mathrm{t}, J=7.5 \mathrm{~Hz}, 2 \mathrm{H}), 7.29-7.15(\mathrm{~m}, 5 \mathrm{H}), 6.95$ $6.81(\mathrm{~m}, 2 \mathrm{H}), 4.16(\mathrm{q}, J=7.3 \mathrm{~Hz}, 1 \mathrm{H}), 3.82(\mathrm{~s}, 3 \mathrm{H}), 1.67(\mathrm{~d}, J=7.1 \mathrm{~Hz}, 3 \mathrm{H})$.

${ }^{13} \mathrm{C}$ NMR (100 MHz, $\left.\mathrm{CDCl}_{3}\right) \delta$ 158.0, 146.9, 138.7, 128.6, 128.5, 127.7, 126.1, 113.9, $55.4,44.1,22.2$. 
Spectral data matched those previously reported. ${ }^{[4]}$

\section{Synthesis of $5 \mathrm{~h}$}

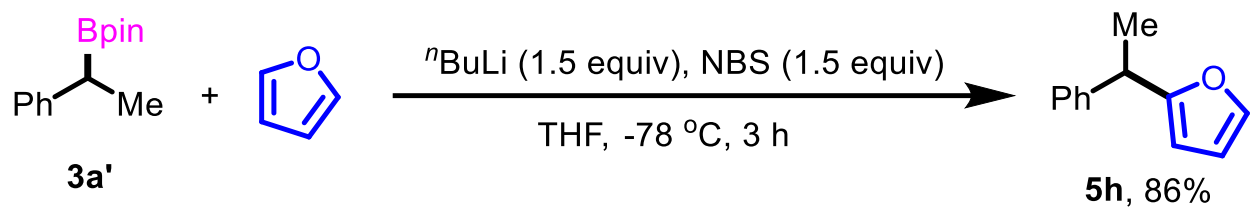

According to a literature procedure ${ }^{[39]}$, to a solution of furan $(136.0 \mathrm{mg}, 2.0$ $\mathrm{mmol})$ in THF $(5.0 \mathrm{~mL})$ was added ${ }^{n} \mathrm{BuLi}(2.5 \mathrm{M}$ in hexane, $1.5 \mathrm{mmol})$ at $-78^{\circ} \mathrm{C}$. The resultant mixture was allowed to warm to room temperature and stirred for 1 hour. Subsequently, the reaction mixture was cooled back to $-78^{\circ} \mathrm{C}$. A solution of 3a' $(232.0 \mathrm{mg}, 1.0 \mathrm{mmol})$ in THF $(2.0 \mathrm{~mL})$ was then added dropwise and stirred for 1 hour, followed by the addition of a solution of NBS $(267.1 \mathrm{mg}$, $1.5 \mathrm{mmol})$ in THF $(5.0 \mathrm{~mL})$. After stirred at $-78{ }^{\circ} \mathrm{C}$ for 1 hour, saturated aqueous $\mathrm{Na}_{2} \mathrm{~S}_{2} \mathrm{O}_{3}$ solution $(4.0 \mathrm{~mL})$ was added. The reaction mixture was allowed to warm to room temperature, diluted with water, extracted with EtOAc, dried with $\mathrm{NaSO}_{4}$, filtered and concentrated under reduced pressure. The crude material was purified by flash column chromatography (petroleum ether $/ \mathrm{EtOAc}=200: 1)$ to provide the title compound $5 \mathrm{~h}(147.9 \mathrm{mg}, 86 \%$ yield $)$. ${ }^{1} \mathrm{H}$ NMR (400 MHz, CDCl$)$ \& $7.32-7.26(\mathrm{~m}, 3 \mathrm{H}), 7.24-7.17(\mathrm{~m}, 3 \mathrm{H}), 6.28(\mathrm{~d}, J$ $=2.6 \mathrm{~Hz}, 1 \mathrm{H}), 6.11-5.99(\mathrm{~m}, 1 \mathrm{H}), 4.11(\mathrm{q}, J=7.3 \mathrm{~Hz}, 1 \mathrm{H}), 1.59(\mathrm{~d}, J=7.5 \mathrm{~Hz}, 3 \mathrm{H})$. ${ }^{13} \mathrm{C}$ NMR (100 MHz, CDCl $)$ ) $\delta$ 159.1, 144.3, 141.4, 128.6, 127.4, 126.6, 110.1, 105.0, 39.4, 20.7.

Spectral data matched those previously reported. ${ }^{[39]}$

\section{Control Experiments}

\section{Synthesis of $d-1$ a}

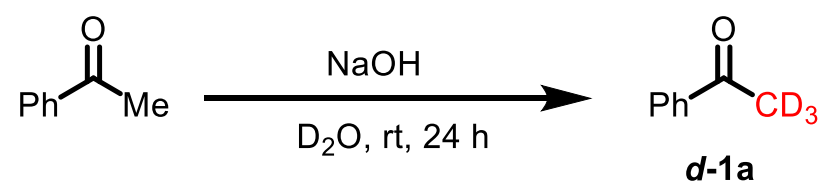

According to a literature procedure ${ }^{[4]}$, a mixture of acetophenone $(1.2 \mathrm{~g}, 10$ 
$\mathrm{mmol}), \mathrm{NaOH}(80 \mathrm{mg}, 2.0 \mathrm{mmol})$ and $\mathrm{D}_{2} \mathrm{O}(99.9 \% \mathrm{D}, \mathrm{CIL})(5 \mathrm{~mL})$ was stirred at room temperature for 24 hours under nitrogen atmosphere. Upon completion of the reaction, the reaction mixture was extracted with $\mathrm{Et}_{2} \mathrm{O}(5 \mathrm{~mL} \times 3)$, dried over $\mathrm{Na}_{2} \mathrm{SO}_{4}$, filtered and concentrated. The residue was purified by flash chromatography over silica gel using petroleum ether/EtOAc $=30: 1$ as an eluent to afford the desired product $d-1$ a $(84 \%, 1.03 \mathrm{~g}, 97 \% \mathrm{D})$ as a colorless oil.

\section{Deuterium-labelling Experiments}

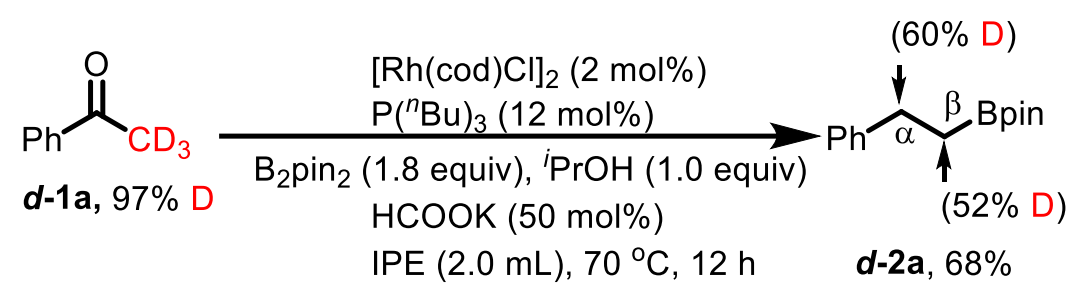

In a glove box, $[\mathrm{RhCl}(\mathrm{cod})]_{2}(4.9 \mathrm{mg}, 0.01 \mathrm{mmol}), \mathrm{P}\left({ }^{n} \mathrm{Bu}\right) 3(12.1 \mathrm{mg}, 0.06$ $\mathrm{mmol})$, and IPE $(1.0 \mathrm{~mL})$ were placed in a $4 \mathrm{~mL}$ glass tube and stirred for 30 min. Then, HCOOK (21.0 mg, $0.25 \mathrm{mmol}), \mathrm{B}_{2}$ pin2 $(228.6 \mathrm{mg}, 0.90 \mathrm{mmol}),{ }^{\mathrm{i}} \mathrm{PrOH}$ (38.1 mg, $0.50 \mathrm{mmol}$ ) was added, and stirred for another $30 \mathrm{~min}$. After ketone $\boldsymbol{d}$-1a $(61.5 \mathrm{mg}, 0.5 \mathrm{mmol}, 97 \% \mathrm{D})$ in IPE $(1.0 \mathrm{~mL})$ was added, the reaction mixture was sealed, removed from the glovebox and stirred at $70{ }^{\circ} \mathrm{C}$ for 12 hours. After cooled to room temperature, the mixture was concentrated in vacuo and purified by silica gel chromatography using petroleum ether:EtOAc $=50: 1$ as the eluent to afford the desired products $d-2 \mathrm{a}$ as a colorless oil $(79 \mathrm{mg}, 68 \%$ yield).

${ }^{1} \mathrm{H}$ NMR (400 MHz, $\left.\mathrm{CDCl}_{3}\right) \delta 7.33-7.26(\mathrm{~m}, 4 \mathrm{H}), 7.23-7.17(\mathrm{~m}, 1 \mathrm{H}), 2.78(\mathrm{t}, J=$ $7.6 \mathrm{~Hz}, 0.81 \mathrm{H}), 1.27(\mathrm{~s}, 12 \mathrm{H}), 1.17(\mathrm{t}, J=8.3 \mathrm{~Hz}, 0.96 \mathrm{H})$. 


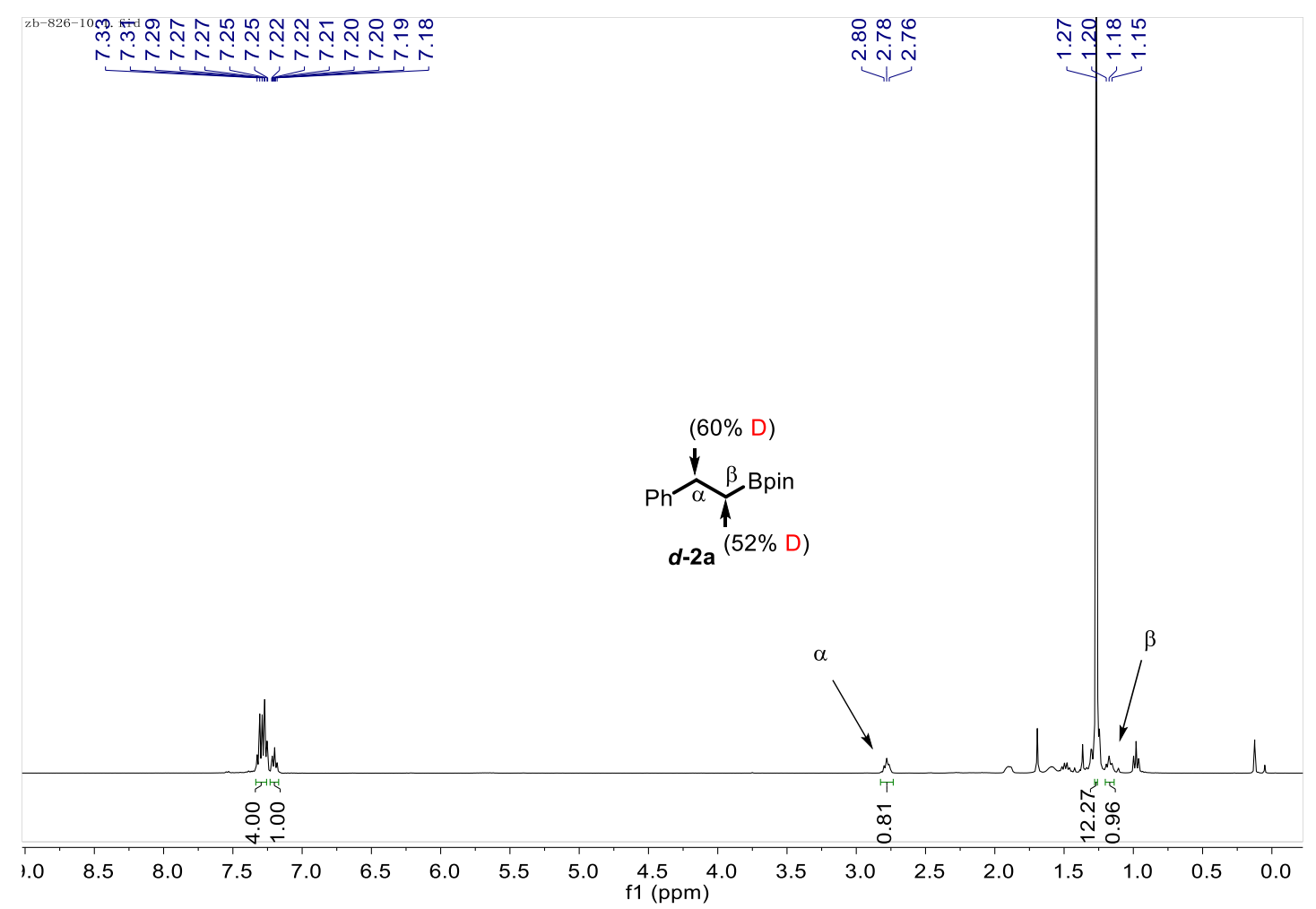

${ }^{1} \mathrm{H}$ NMR spectrum of $\boldsymbol{d}$-2a

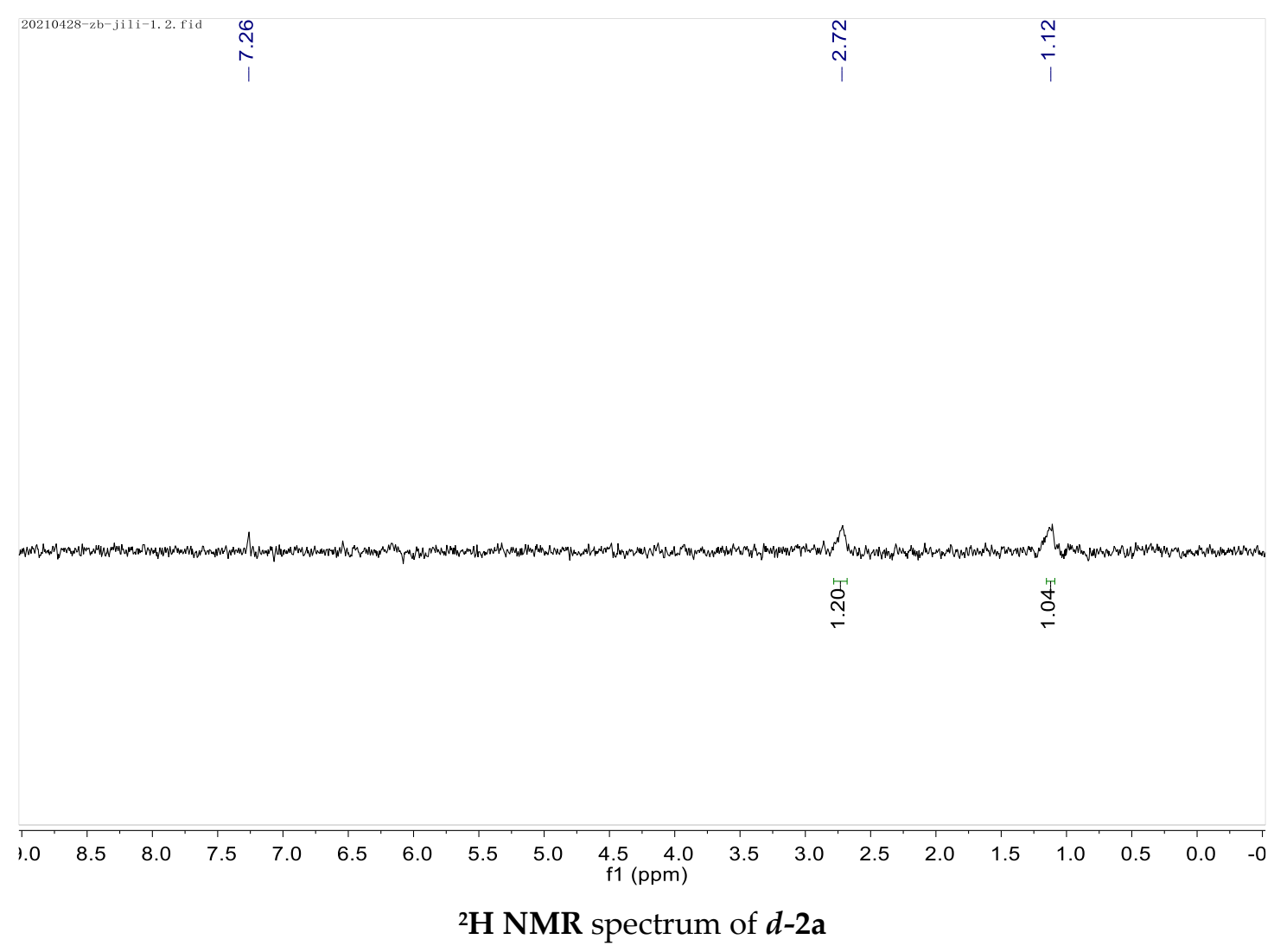




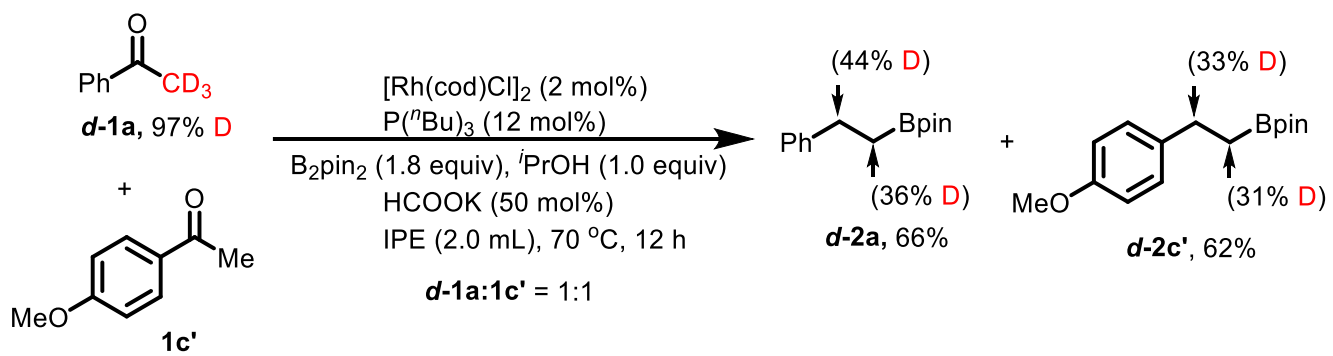

In a glove box, $[\mathrm{RhCl}(\mathrm{cod})]_{2}(4.9 \mathrm{mg}, 0.01 \mathrm{mmol}), \mathrm{P}\left({ }^{n} \mathrm{Bu}\right)_{3}(12.1 \mathrm{mg}, 0.06$ $\mathrm{mmol})$, and IPE $(1.0 \mathrm{~mL})$ were placed in a $4 \mathrm{~mL}$ glass tube and stirred for 30 min. Then, HCOOK (21.0 mg, $0.25 \mathrm{mmol}), \mathrm{B}_{2} \mathrm{pin}_{2}(228.6 \mathrm{mg}, 0.90 \mathrm{mmol})$, and ${ }^{i} \mathrm{PrOH}(38.1 \mathrm{mg}, 0.50 \mathrm{mmol})$ were added, and stirred for another $30 \mathrm{~min}$. Ketone $\boldsymbol{d}-\mathbf{1 a}(30.8 \mathrm{mg}, 0.25 \mathrm{~mol})$ and $\mathbf{1} \mathbf{c}^{\prime}(45 \mathrm{mg}, 0.25 \mathrm{mmol})$ in IPE $(1.0 \mathrm{~mL})$ were added. The reaction mixture was sealed, removed from the glovebox and stirred at 70 ${ }^{\circ} \mathrm{C}$ for 12 hours. After cooled to ambient temperature, the mixture was concentrated in vacuo and purified by silica gel chromatography using petroleum ether:EtOAc $=50: 1-30: 1$ as the eluent to afford the H/D scrambled products $\boldsymbol{d}$-2a ( $40 \mathrm{mg}, 66 \%$ yield) and $\boldsymbol{d}-\mathbf{2} \mathbf{c}^{\prime}$ ( $40.3 \mathrm{mg}, 62 \%$ yield) as colorless oil. ${ }^{1} \mathrm{H}$ NMR (d-2a, $\left.400 \mathrm{MHz}, \mathrm{CDCl}_{3}\right) \delta 8.34-7.25(\mathrm{~m}, 4 \mathrm{H}), 7.23-7.17$ (m, 1H), 2.79 $(\mathrm{t}, J=8.0 \mathrm{~Hz}, 1.13 \mathrm{H}), 1.27(\mathrm{~s}, 12 \mathrm{H}), 1.19(\mathrm{t}, J=8.2 \mathrm{~Hz}, 1.26 \mathrm{H})$.

${ }^{1} \mathrm{H}$ NMR (d-2c', $\left.400 \mathrm{MHz}, \mathrm{CDCl}_{3}\right) \delta 7.15(\mathrm{~d}, J=8.2 \mathrm{~Hz}, 2 \mathrm{H}), 6.86(\mathrm{~d}, J=8.1 \mathrm{~Hz}$, $2 \mathrm{H}), 3.79(\mathrm{~m}, 4.38 \mathrm{H}), 2.80(\mathrm{t}, J=6.5 \mathrm{~Hz}, 1.33 \mathrm{H})$. 


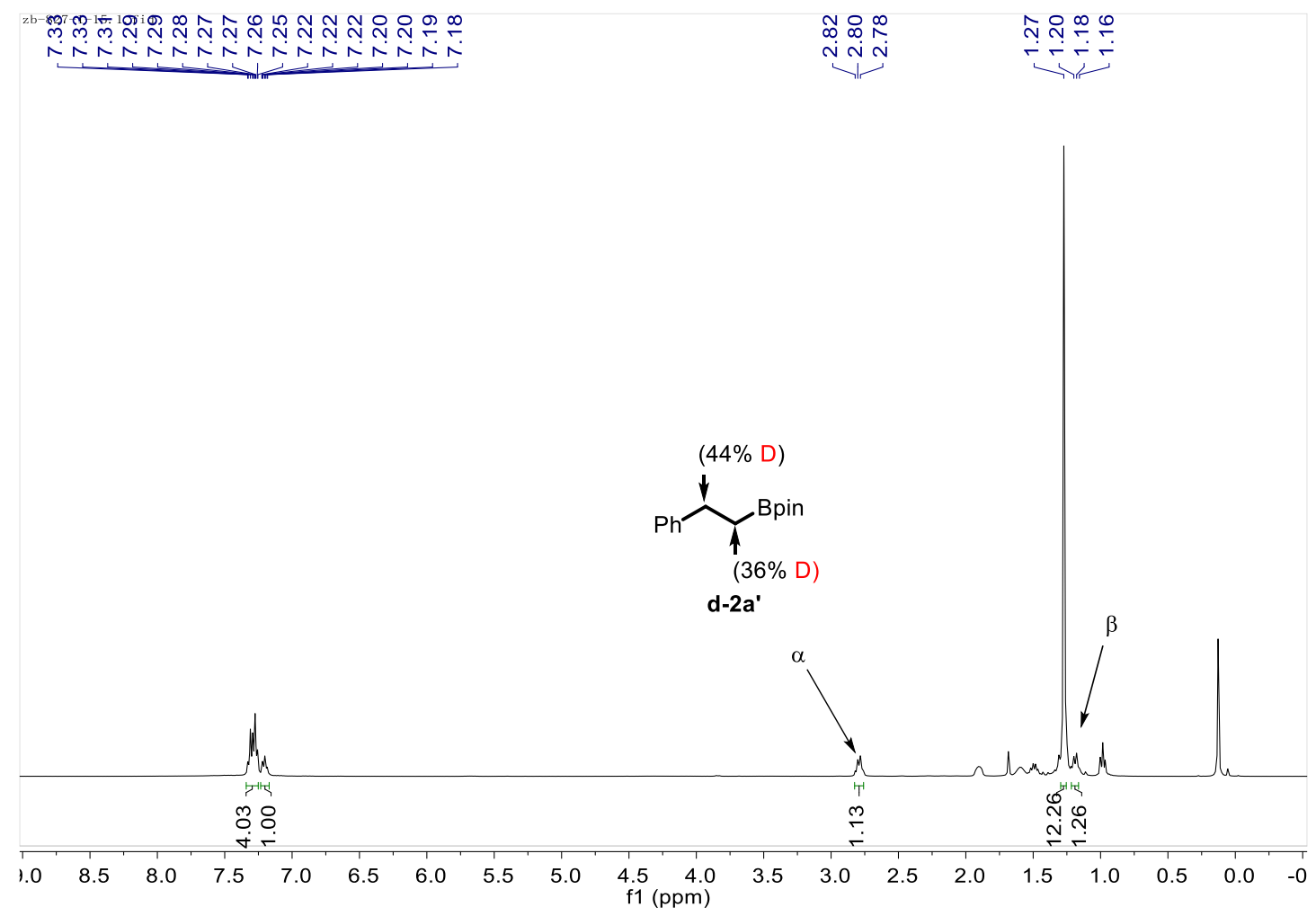

${ }^{1} \mathrm{H}$ NMR spectrum of $\boldsymbol{d}$-2a

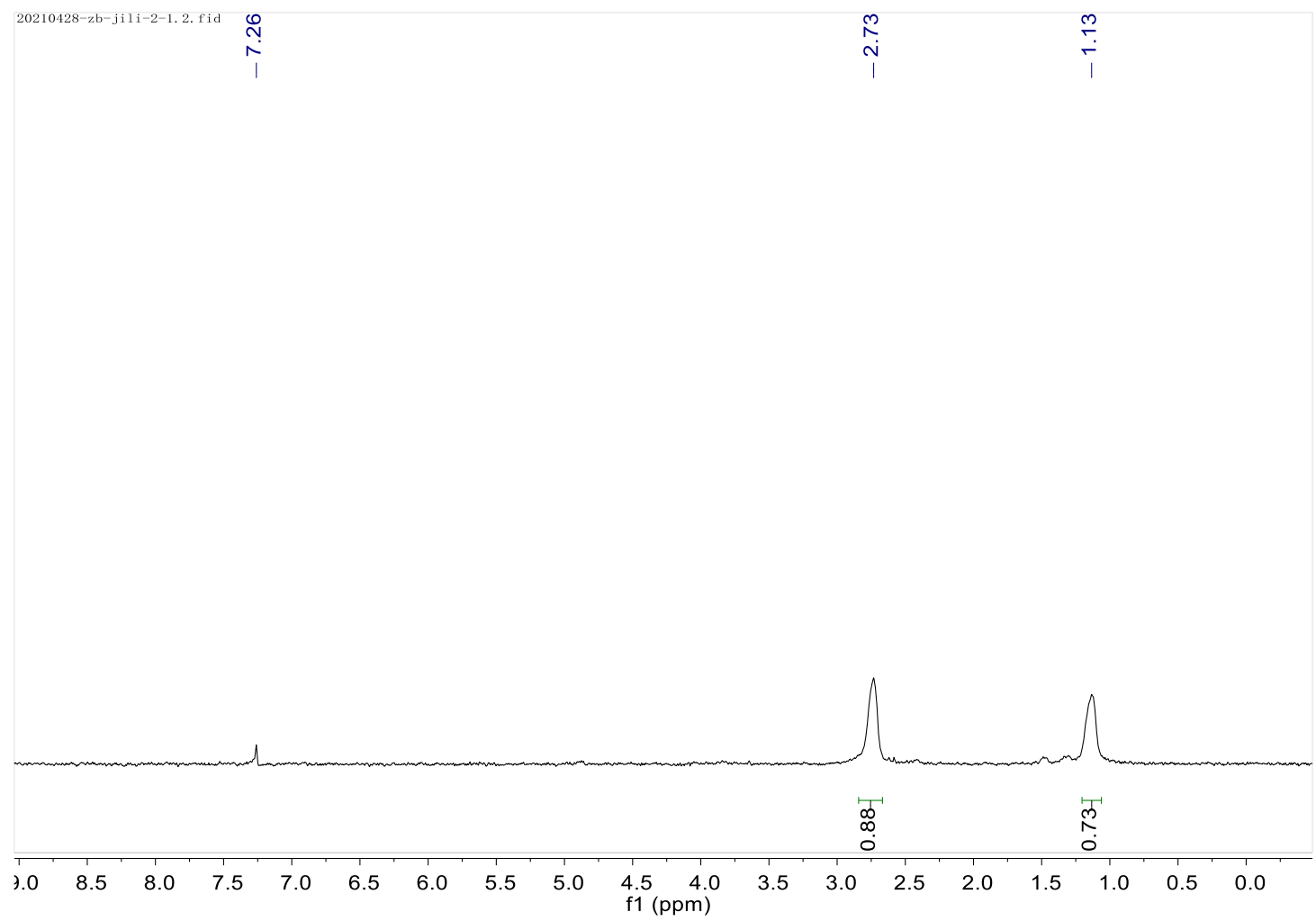

${ }^{2} \mathrm{H}$ NMR spectrum of $\boldsymbol{d}-\mathbf{2 a}$ 


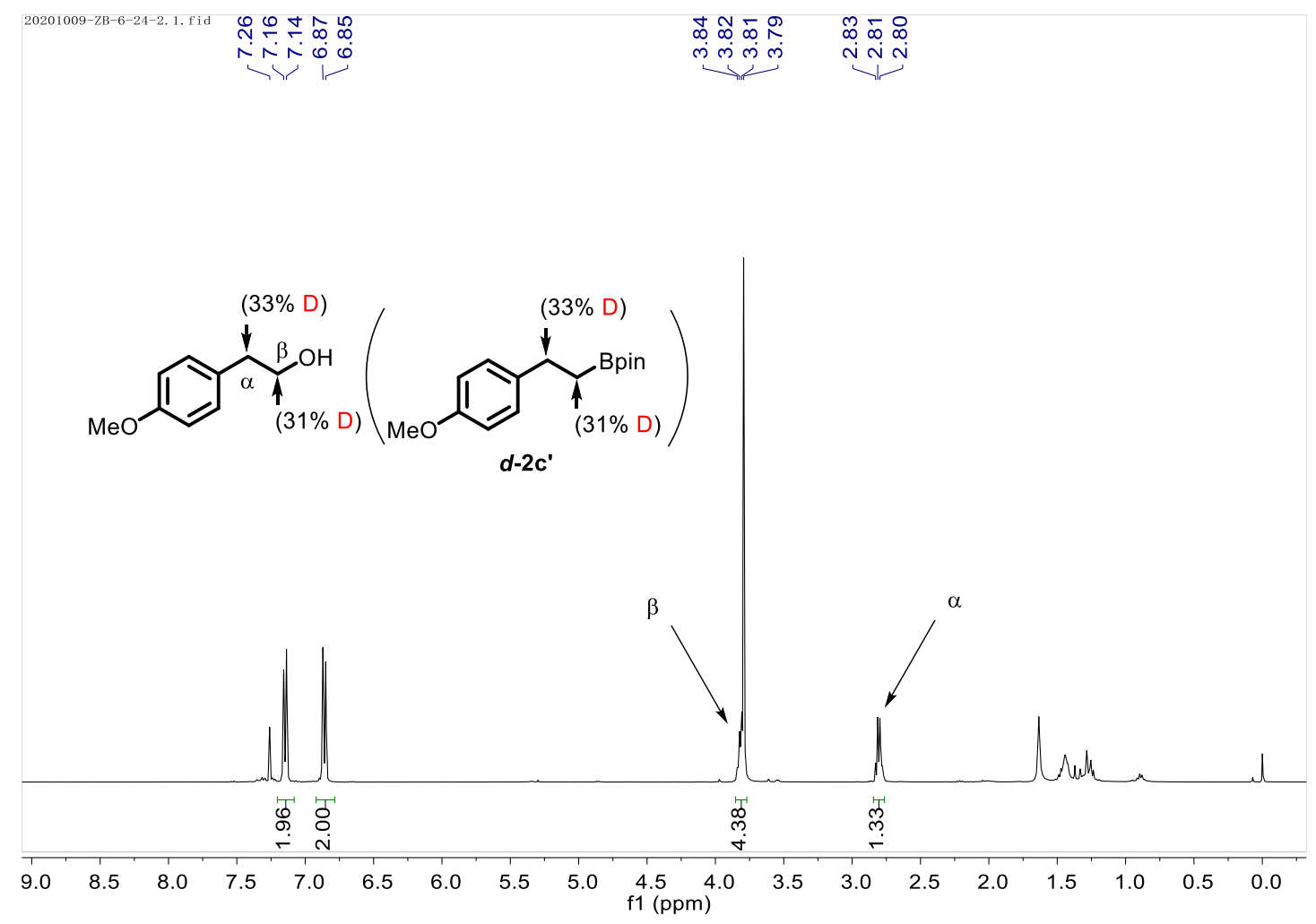

${ }^{1} \mathrm{H}$ NMR spectrum of $\boldsymbol{d}-\mathbf{2} \mathrm{c}^{\prime}$

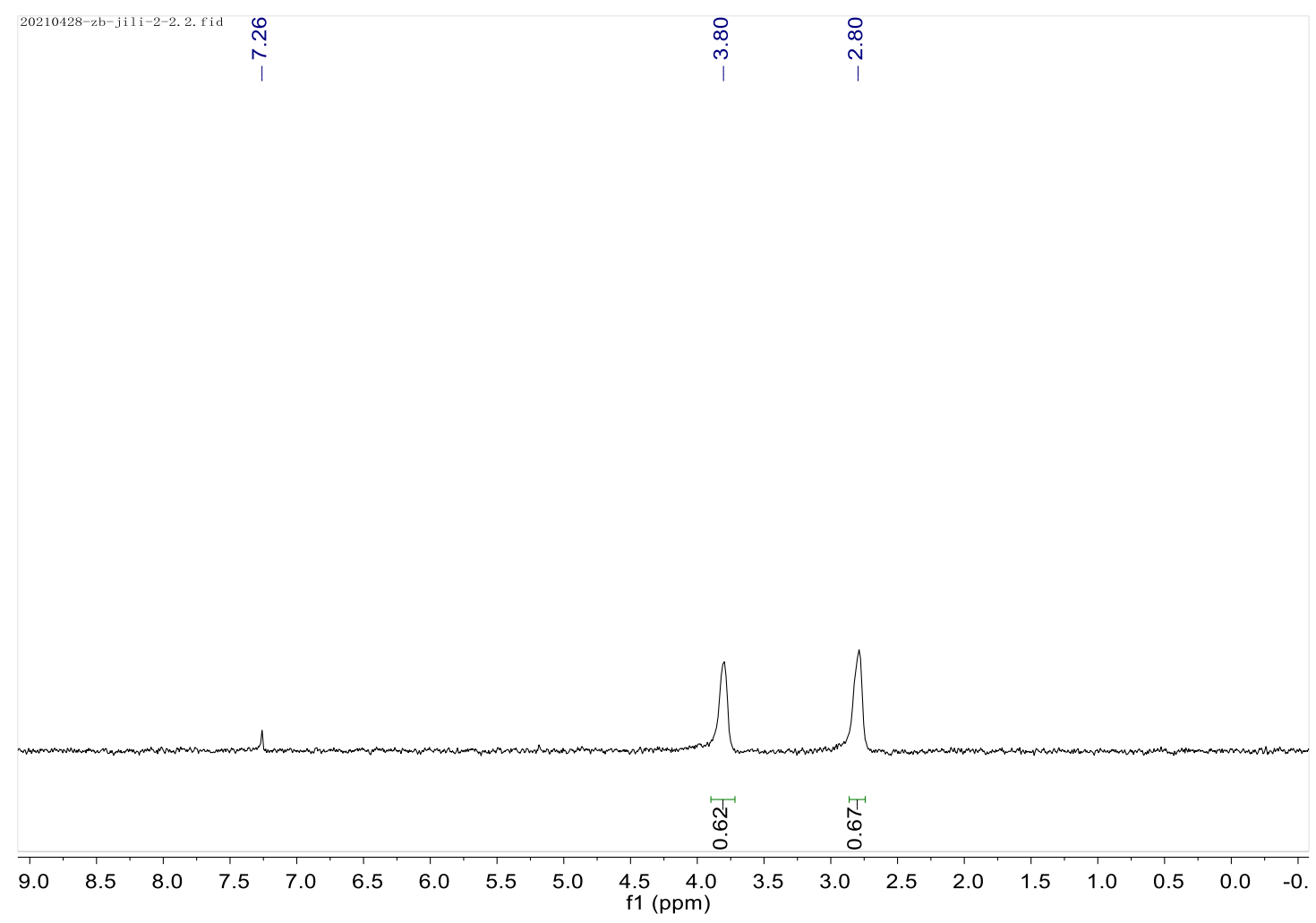

${ }^{2} \mathrm{H}$ NMR spectrum of $\boldsymbol{d}-\mathbf{2} \mathrm{c}^{\prime}$ 


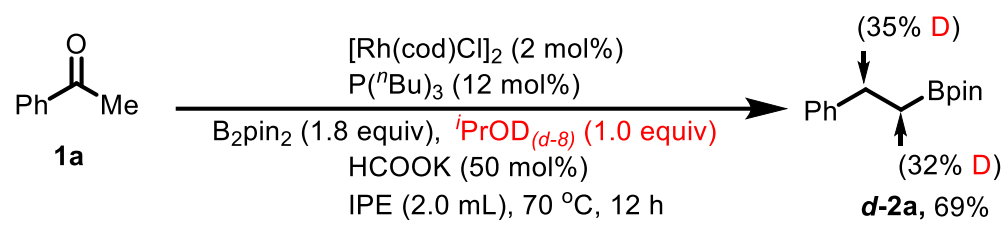

In a glove box, $[\mathrm{RhCl}(\mathrm{cod})]_{2}(3.0 \mathrm{mg}, 0.006 \mathrm{mmol}), \mathrm{P}\left({ }^{n} \mathrm{Bu}\right) 3(7.3 \mathrm{mg}, 0.036$ $\mathrm{mmol})$, and IPE $(1.0 \mathrm{~mL})$ were placed in a $4 \mathrm{~mL}$ glass tube and stirred for 30 min. Then, HCOOK (12.6 mg, $0.15 \mathrm{mmol}), \mathrm{B}_{2}$ pinz $(137 \mathrm{mg}, 0.54 \mathrm{mmol})$, and ${ }^{i} \operatorname{PrOD}(d-8)(22.9 \mathrm{mg}, 0.30 \mathrm{mmol})$ were added, and stirred for another $30 \mathrm{~min} .1 \mathrm{a}$ (36 mg, $0.3 \mathrm{~mol})$ in IPE $(1.0 \mathrm{~mL})$ was added. The reaction mixture was sealed, removed from the glovebox and stirred at $70{ }^{\circ} \mathrm{C}$ for 12 hours. After cooled to ambient temperature, the mixture was concentrated in vacuo and purified by silica gel chromatography using petroleum ether : $\mathrm{EtOAc}=50: 1$ as the eluent to afford the product $\boldsymbol{d}-\mathbf{2 a}$ ( $48 \mathrm{mg}, 69 \%$ yield) as colorless oil.

${ }^{1} \mathrm{H}$ NMR (400 MHz, $\left.\mathrm{CDCl}_{3}\right) \delta$ 8 7.36 - $7.28(\mathrm{~m}, 4 \mathrm{H}), 7.27$ - $7.19(\mathrm{~m}, 1 \mathrm{H}), 2.85$ - 2.78 $(\mathrm{m}, 1.31 \mathrm{H}), 1.29(\mathrm{~s}, 12 \mathrm{H}), 1.25-1.19(\mathrm{~m}, 1.36 \mathrm{H})$.

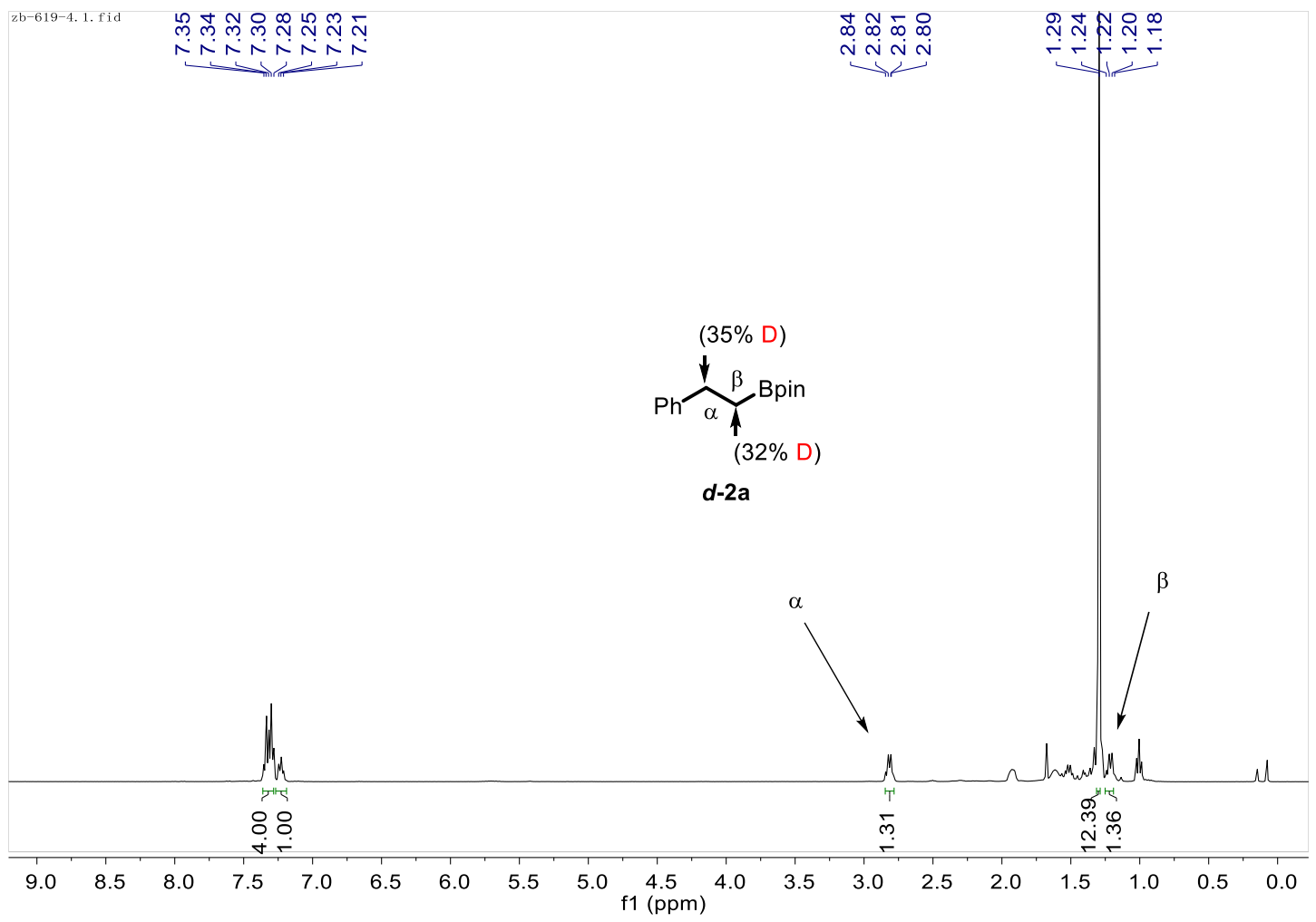

${ }^{1} \mathrm{H}$ NMR spectrum of $\boldsymbol{d}-\mathbf{2 a}$ 


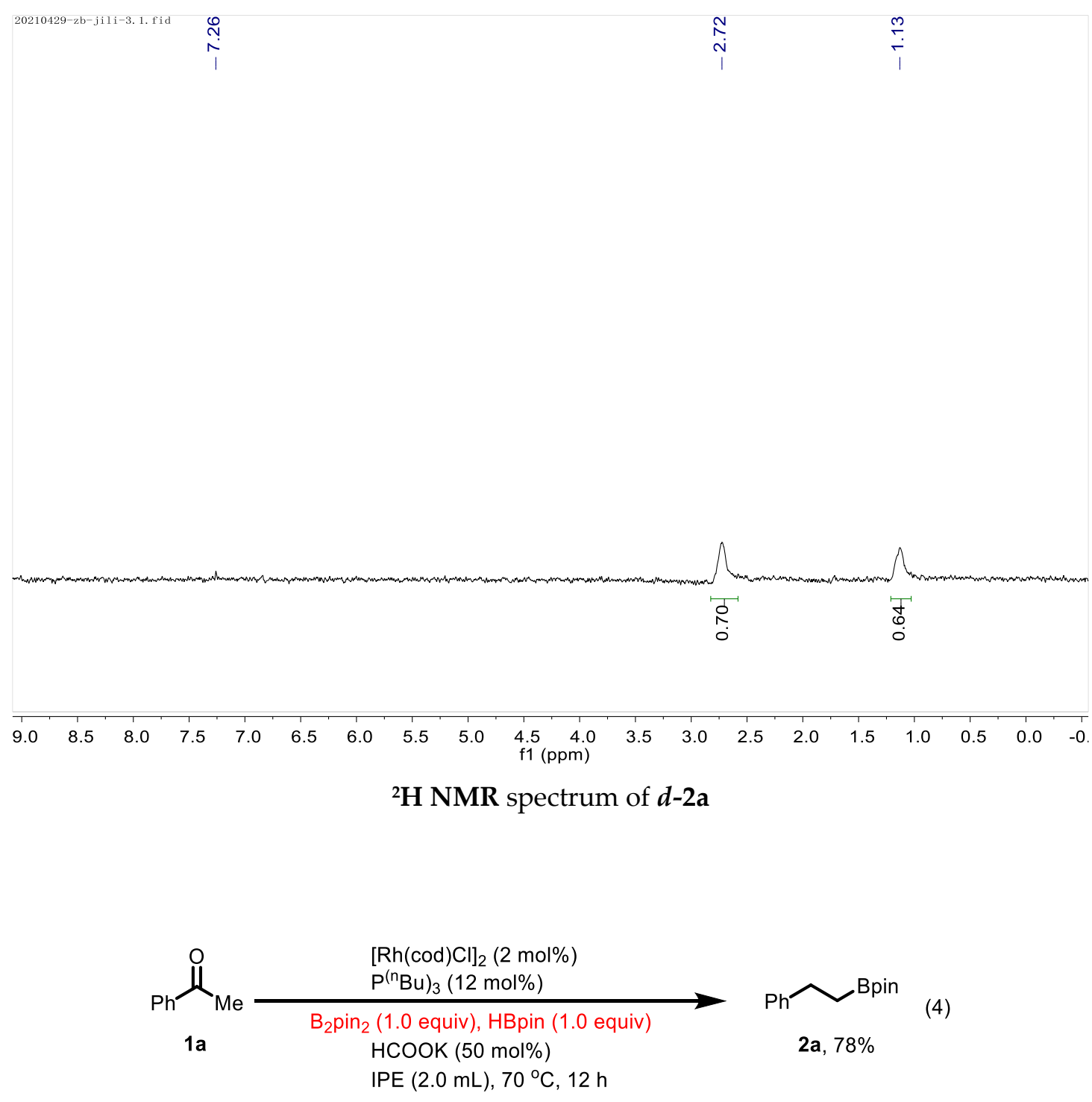

In a glove box, $[\mathrm{RhCl}(\mathrm{cod})]_{2}(3.0 \mathrm{mg}, 0.006 \mathrm{mmol}), \mathrm{P}\left({ }^{\mathrm{n} B u}\right)_{3}(7.3 \mathrm{mg}, 0.036$ $\mathrm{mmol})$, and IPE $(1.0 \mathrm{~mL})$ were placed in a $4 \mathrm{~mL}$ glass tube and stirred for 30 min. Then, HCOOK (12.6 mg, $0.15 \mathrm{mmol}), \mathrm{B}_{2}$ pin 2 (76 mg, $\left.0.3 \mathrm{mmol}\right)$, and HBpin (38.4 mg, $0.30 \mathrm{mmol}$ ) were added, and stirred for another $30 \mathrm{~min}$. 1a (36 mg, 0.3 $\mathrm{mmol})$ in IPE $(1.0 \mathrm{~mL})$ was added. The reaction mixture was sealed, removed from the glovebox and stirred at $70{ }^{\circ} \mathrm{C}$ for $12 \mathrm{~h}$. After cooled to ambient temperature, the mixture was concentrated in vacuo and purified by silica gel chromatography using petroleum ether : EtOAc $=50: 1$ as the eluent to afford the products $2 \mathrm{a}(54.2 \mathrm{mg}, 78 \%$ yield) as colorless oil. 


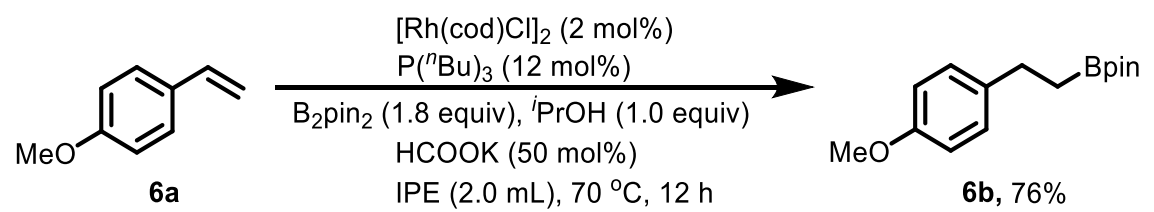

$6 b, 76 \%$

In a glove box, $[\mathrm{RhCl}(\mathrm{cod})]_{2}(3.0 \mathrm{mg}, 0.006 \mathrm{mmol}), \mathrm{P}\left({ }^{n} \mathrm{Bu}\right)_{3}(7.3 \mathrm{mg}, 0.036$ $\mathrm{mmol})$, and IPE $(1.0 \mathrm{~mL})$ were placed in a $4 \mathrm{~mL}$ glass tube and stirred for 30 min. Then, HCOOK (12.6 mg, $0.15 \mathrm{mmol}), \mathrm{B}_{2}$ pin2 (137 mg, $\left.0.54 \mathrm{mmol}\right)$, and ${ }^{i} \mathrm{PrOH}(22.9 \mathrm{mg}, 0.30 \mathrm{mmol})$ were added, and stirred for another $30 \mathrm{~min}$. 6a (40.2 $\mathrm{mg}, 0.3 \mathrm{~mol})$ in IPE $(1.0 \mathrm{~mL})$ was added. The reaction mixture was sealed, removed from the glovebox and stirred at $70{ }^{\circ} \mathrm{C}$ for 12 hours. After cooled to ambient temperature, the mixture was filtered through a silica gel pad with EtOAc and concentrated under vacuo. The residue was dissolved in $\mathrm{CDCl}_{3}(0.6$ $\mathrm{mL}$ ), and the yield was determined by ${ }^{1} \mathrm{H}-\mathrm{NMR}$ analysis with $\mathrm{CH}_{2} \mathrm{Br}_{2}(0.3 \mathrm{mmol})$ as the internal standard. The ${ }^{1} \mathrm{H}-\mathrm{NMR}$ spectra showed that the $6 \mathrm{~b}$ was produced with $76 \%$ yield.

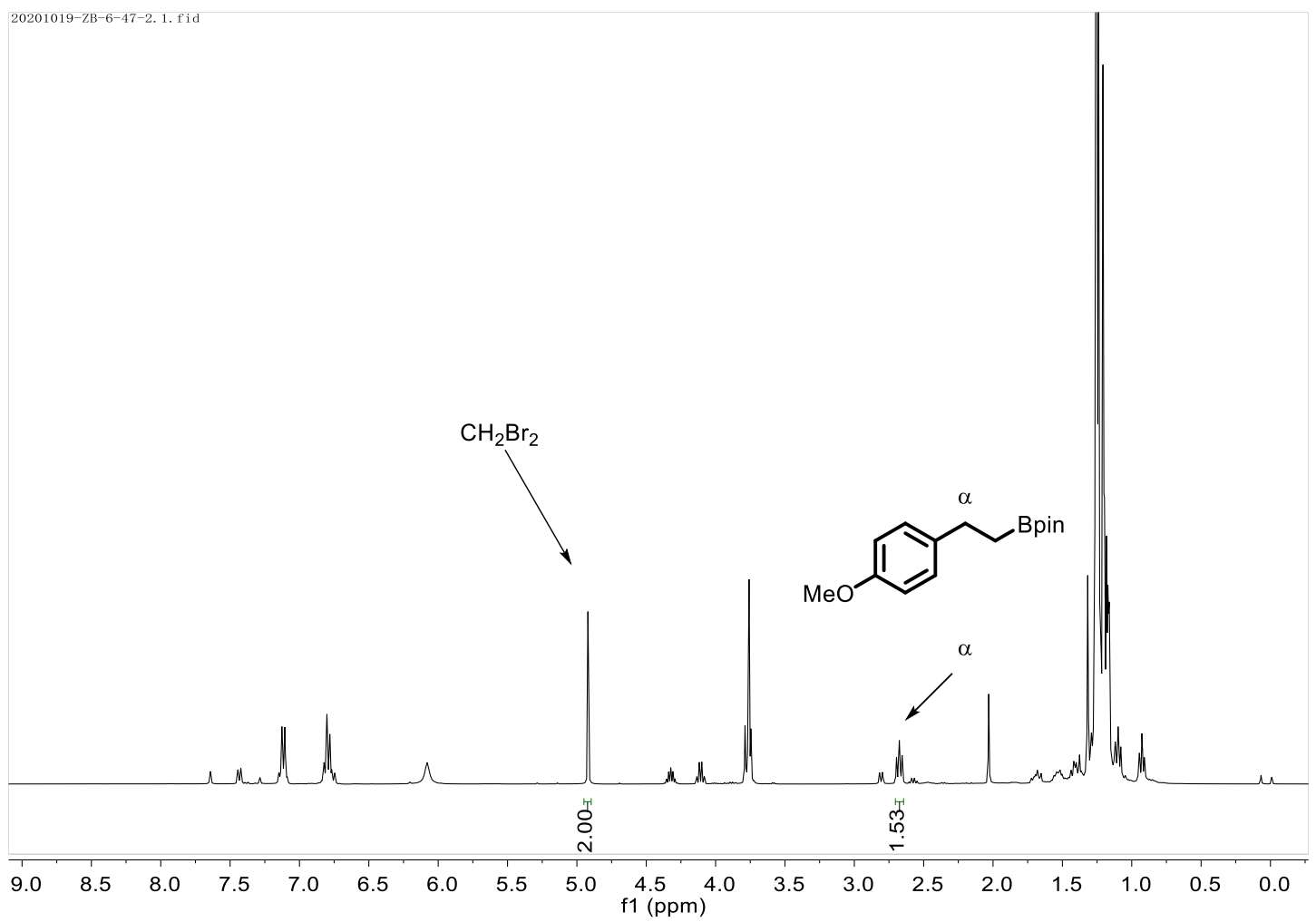




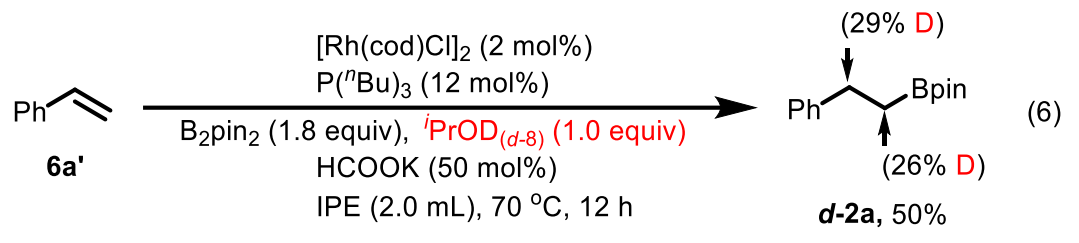

In a glove box, $[\mathrm{RhCl}(\mathrm{cod})]_{2}(3.0 \mathrm{mg}, 0.006 \mathrm{mmol}), \mathrm{P}\left({ }^{n} \mathrm{Bu}\right) 3(7.3 \mathrm{mg}, 0.036$ $\mathrm{mmol})$, and IPE $(1.0 \mathrm{~mL})$ were placed in a $4 \mathrm{~mL}$ glass tube and stirred for 30 min. Then, HCOOK (12.6 mg, $0.15 \mathrm{mmol}$ ), B2pinz (137 mg, $0.54 \mathrm{mmol}$ ), and ${ }^{i} \operatorname{PrOD}_{(d-8)}(23.0 \mathrm{mg}, 0.30 \mathrm{mmol})$ were added, and stirred for another $30 \mathrm{~min} . \mathbf{6 a}^{\prime}$ $(40.2 \mathrm{mg}, 0.3 \mathrm{~mol})$ in IPE $(1.0 \mathrm{~mL})$ was added. The reaction mixture was sealed, removed from the glovebox and stirred at $70{ }^{\circ} \mathrm{C}$ for 12 hours. After cooled to ambient temperature, the mixture was concentrated in vacuo and purified by silica gel chromatography using petroleum ether : $\mathrm{EtOAc}=50: 1$ as the eluent to afford the product $\boldsymbol{d}-\mathbf{2 a}$ ( $34.8 \mathrm{mg}, 50 \%$ yield) as colorless oil.

${ }^{1} \mathrm{H}$ NMR (400 MHz, CDCl$)$ ) $7.40-7.34(\mathrm{~m}, 2 \mathrm{H}), 7.31-7.26(\mathrm{~m}, 3 \mathrm{H}), 3.91(\mathrm{t}, J=$ $6.5 \mathrm{~Hz}, 1.48 \mathrm{H}), 2.92(\mathrm{t}, J=6.5 \mathrm{~Hz}, 1.42 \mathrm{H})$.

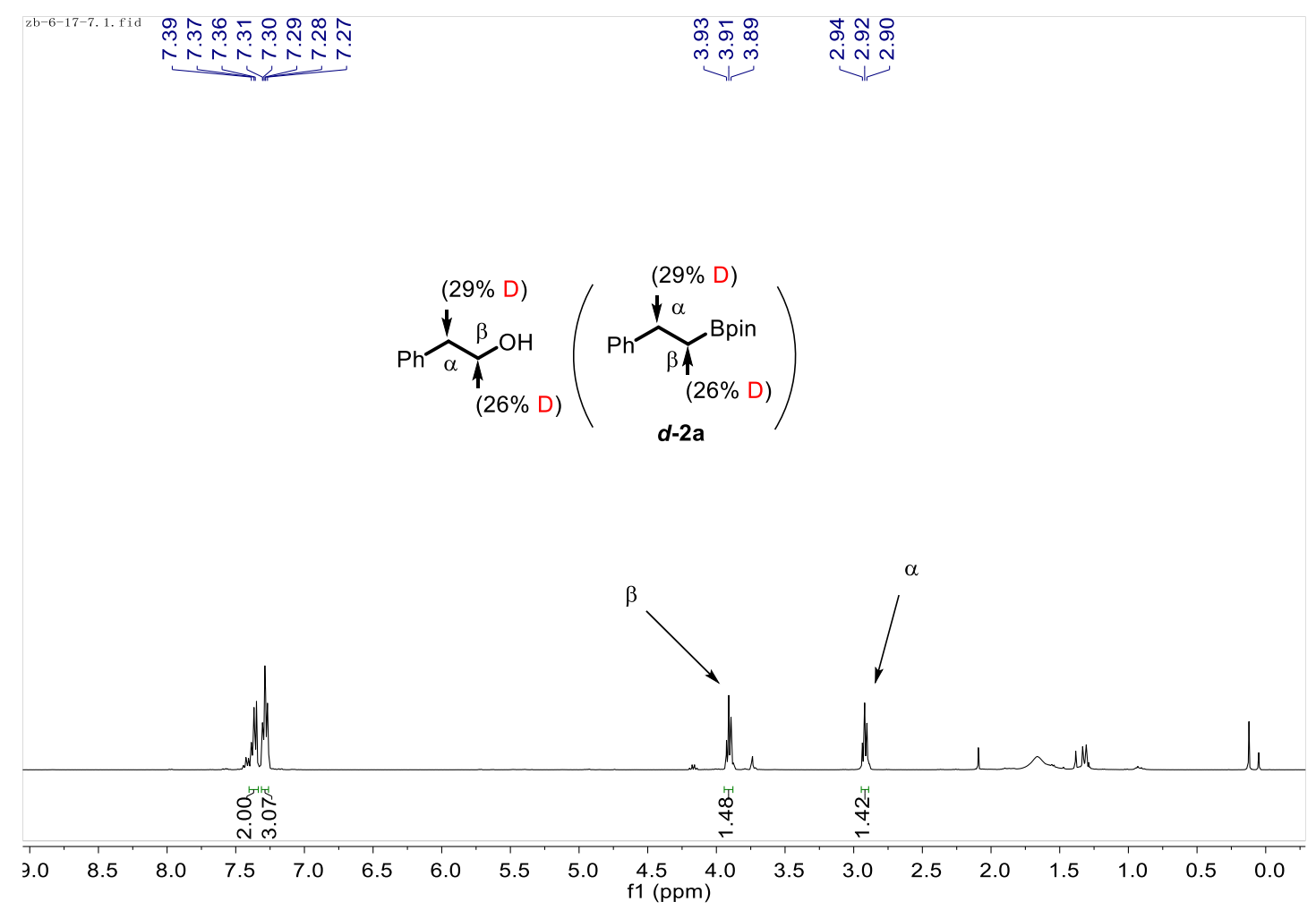

${ }^{1} \mathrm{H}$ NMR spectrum of $\boldsymbol{d}$-2a 


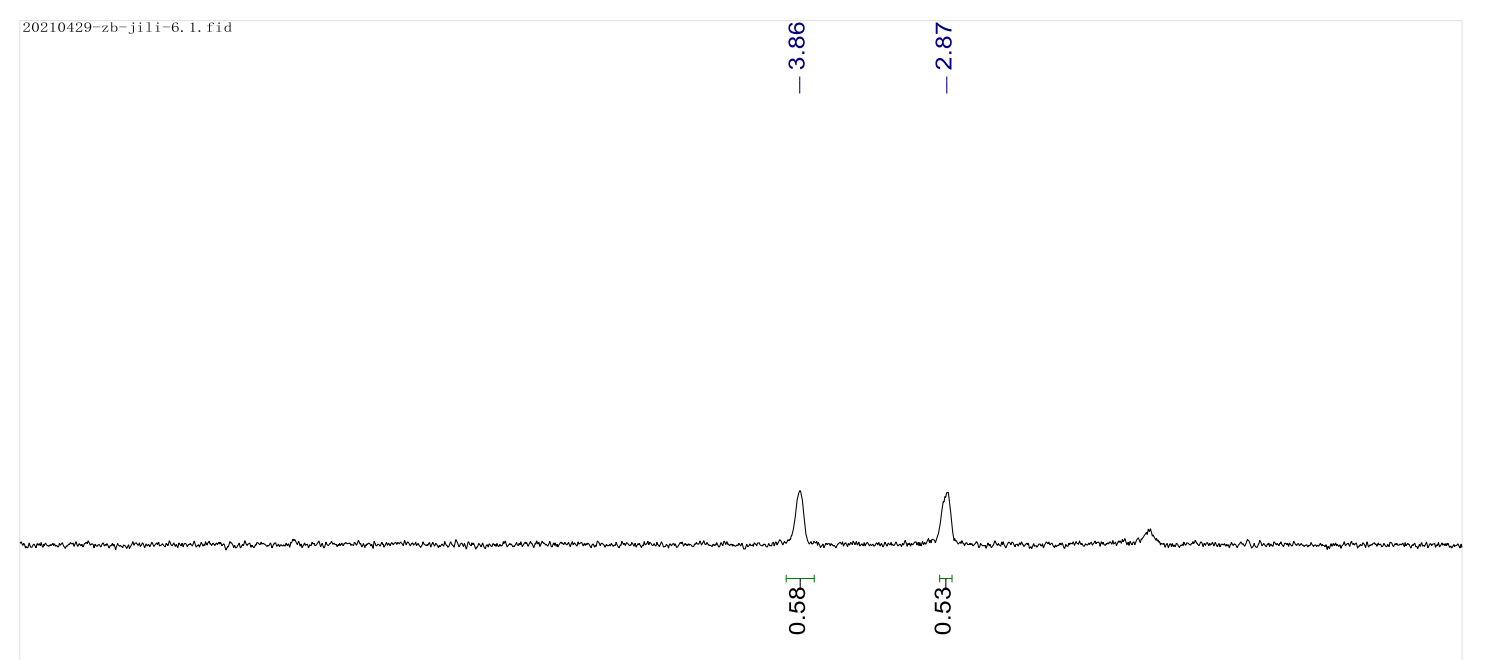

\begin{tabular}{|c|c|c|c|c|c|c|c|c|c|c|c|c|c|c|c|c|c|c|c|}
\hline 9.0 & 8.5 & 8.0 & 7.5 & 7.0 & 6.5 & 6.0 & 5.5 & 5.0 & $\begin{array}{l}4.5 \\
\mathrm{f} 1(\mathrm{p}\end{array}$ & $\begin{array}{c}4.0 \\
\mathrm{pm})\end{array}$ & 3.5 & 3.0 & 2.5 & 2.0 & 1.5 & 1.0 & 0.5 & 0.0 & -0 \\
\hline
\end{tabular}

${ }^{2} \mathrm{H}$ NMR spectrum of $\boldsymbol{d}-\mathbf{2 a}$

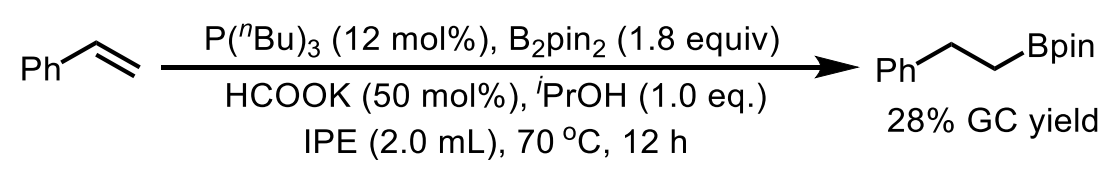

In a glove box, $\mathrm{P}\left({ }^{n} \mathrm{Bu}\right)_{3}(7.3 \mathrm{mg}, 0.036 \mathrm{mmol})$, and IPE $(1.0 \mathrm{~mL})$ were placed in a $4 \mathrm{~mL}$ glass tube and stirred for $30 \mathrm{~min}$. Then, HCOOK (12.6 mg, $0.15 \mathrm{mmol})$, B $_{2}$ pin 2 (137 mg, $\left.0.54 \mathrm{mmol}\right)$, and ${ }^{\mathrm{i}} \mathrm{PrOH}(22.9 \mathrm{mg}, 0.30 \mathrm{mmol})$ were added, and stirred for another $30 \mathrm{~min}$. styrene $(31.2 \mathrm{mg}, 0.3 \mathrm{~mol})$ in IPE $(1.0 \mathrm{~mL})$ was added. The reaction mixture was sealed, removed from the glovebox and stirred at 70 ${ }^{\circ} \mathrm{C}$ for 12 hours. After cooled to ambient temperature, the mixture was filtered through a silica gel pad with EtOAc and concentrated under vacuo. The yield was determined by GC-MS analysis with dodecane as the internal standard. The GC-MS showed that the $2 \mathbf{a}$ was produced in $28 \%$ yield.

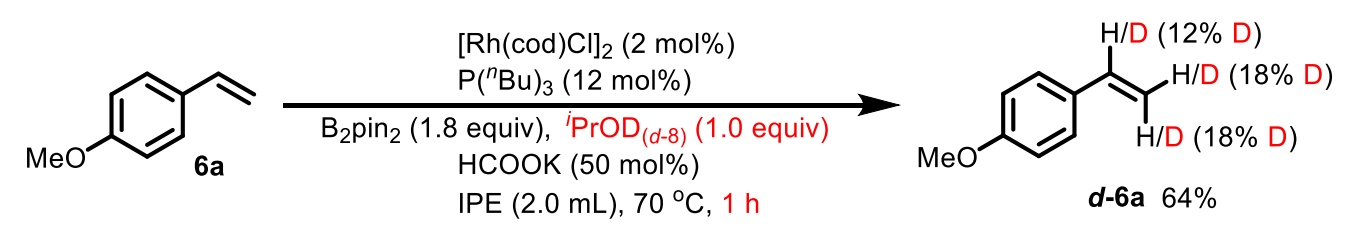


In a glove box, $[\mathrm{RhCl}(\mathrm{cod})]_{2}(3.0 \mathrm{mg}, 0.006 \mathrm{mmol}), \mathrm{P}\left({ }^{n} \mathrm{Bu}\right)_{3}(7.3 \mathrm{mg}, 0.036$ $\mathrm{mmol})$, and IPE $(1.0 \mathrm{~mL})$ were placed in a $4 \mathrm{~mL}$ glass tube and stirred for 30 min. Then, HCOOK (12.6 mg, $0.15 \mathrm{mmol}$ ), B2pinz (137 mg, $0.54 \mathrm{mmol})$, and ${ }^{i} \operatorname{PrOD}(d-8)(23.0 \mathrm{mg}, 0.30 \mathrm{mmol})$ were added, and stirred for another $30 \mathrm{~min}$. 6a $(40.2 \mathrm{mg}, 0.3 \mathrm{~mol})$ in IPE $(1.0 \mathrm{~mL})$ was added. The reaction mixture was sealed, removed from the glovebox and stirred at $70{ }^{\circ} \mathrm{C}$ for 1 hour. The mixture was then quenched with saturated $\mathrm{NH}_{4} \mathrm{Cl}$, extracted with EtOAc, dried with $\mathrm{Na}_{2} \mathrm{SO}_{4}$. The reaction mixture was concentrated in vacuo and purified by silica gel chromatography using petroleum ether : EtOAc $=20: 1$ as the eluent to afford $\boldsymbol{d}$ 6a (25.3 mg, 64\% yield) as colorless oil.

${ }^{1} \mathrm{H}$ NMR (400 MHz, $\left.\mathrm{CDCl}_{3}\right)$ \& 7.41 - $7.35(\mathrm{~m}, 2 \mathrm{H}), 6.92-6.85(\mathrm{~m}, 2 \mathrm{H}), 6.75-6.65$ $(\mathrm{m}, 0.88 \mathrm{H}), 5.64(\mathrm{~d}, J=17.5 \mathrm{~Hz}, 0.82 \mathrm{H}), 5.16(\mathrm{~d}, J=10.8 \mathrm{~Hz}, 0.82 \mathrm{H}), 3.84(\mathrm{~s}, 3 \mathrm{H})$.

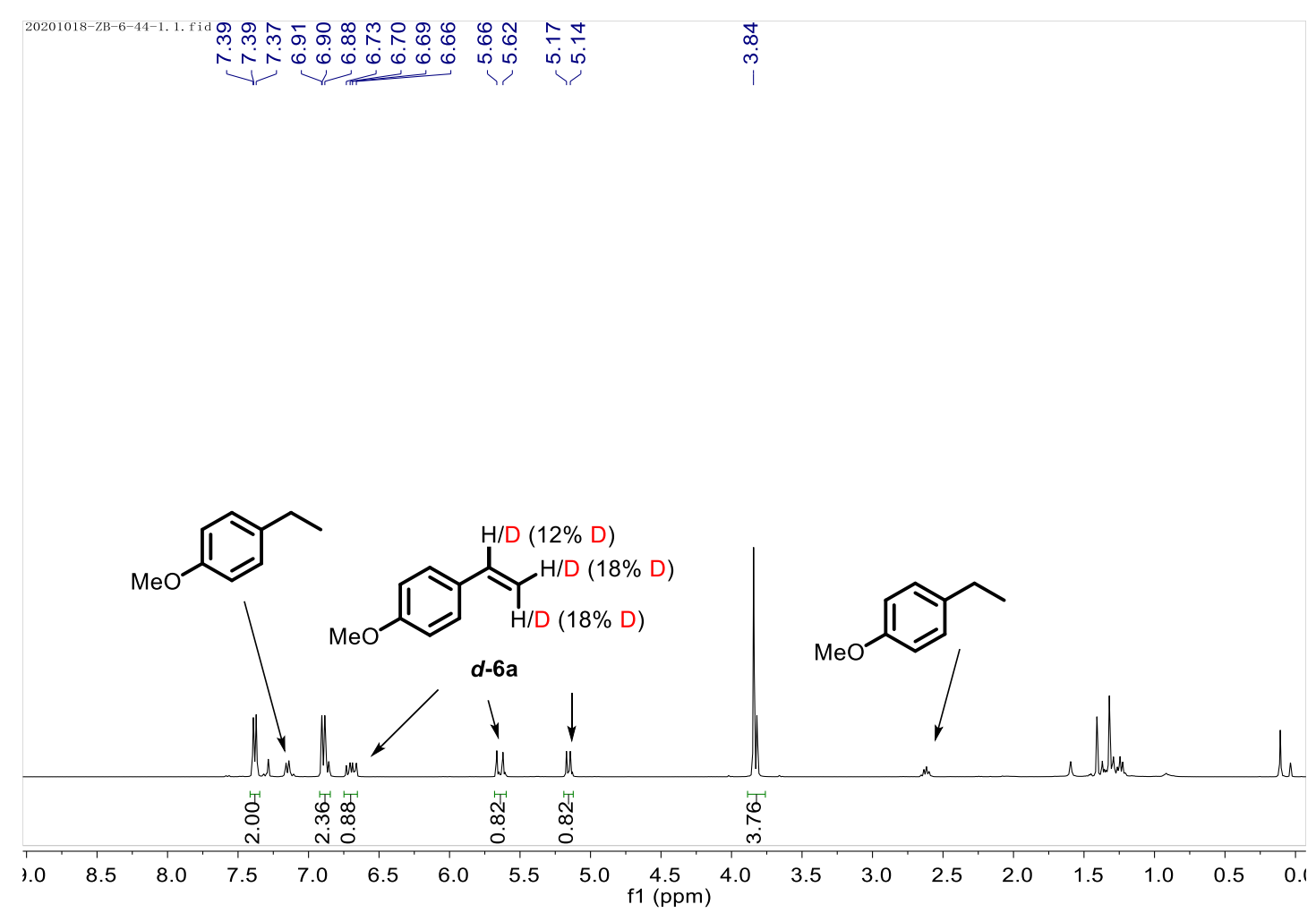

${ }^{1} \mathrm{H}$ NMR spectrum of $\boldsymbol{d}$-6a 


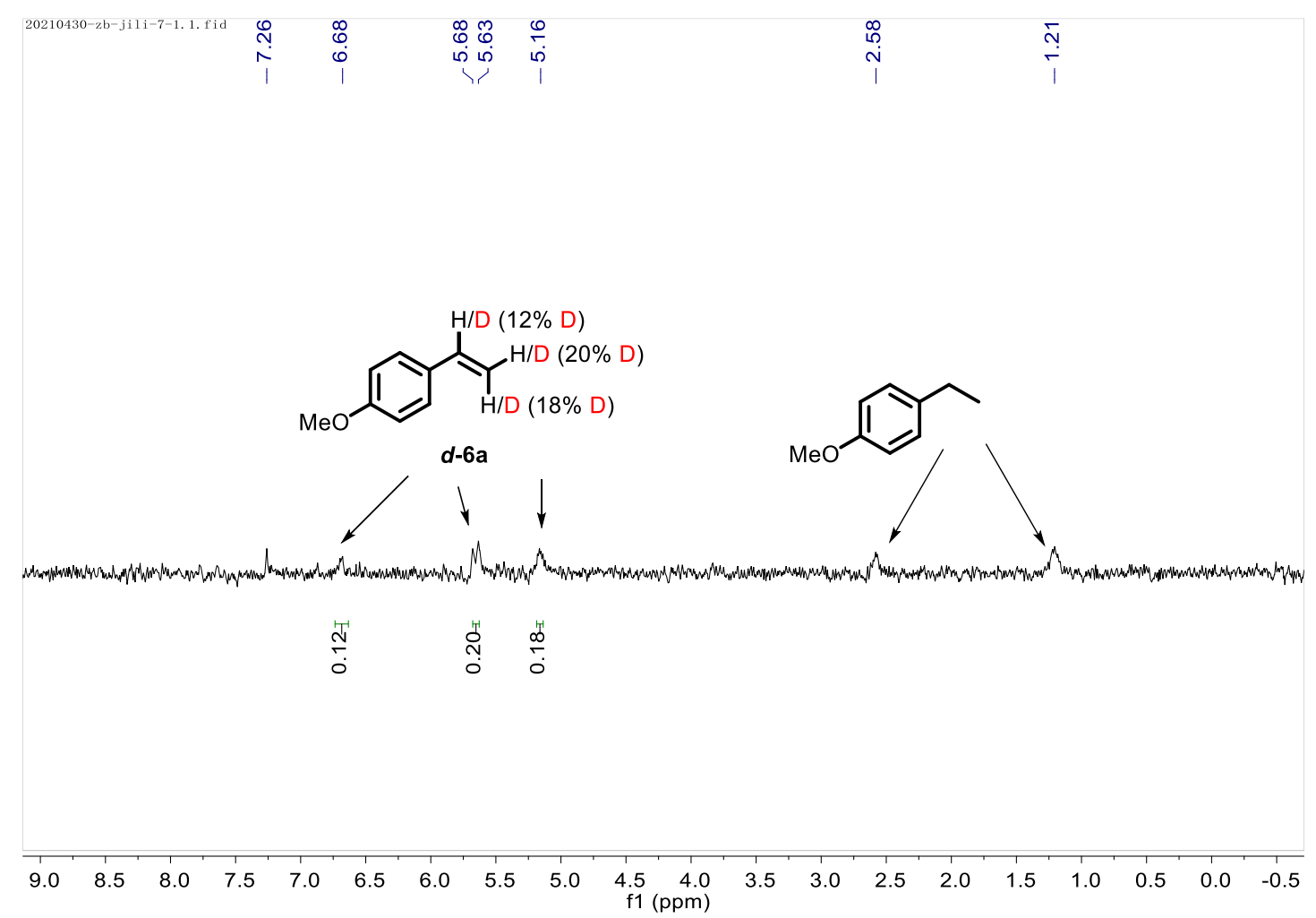

${ }^{2} \mathrm{H}$ NMR spectrum of $\boldsymbol{d}-\mathbf{2 a}$

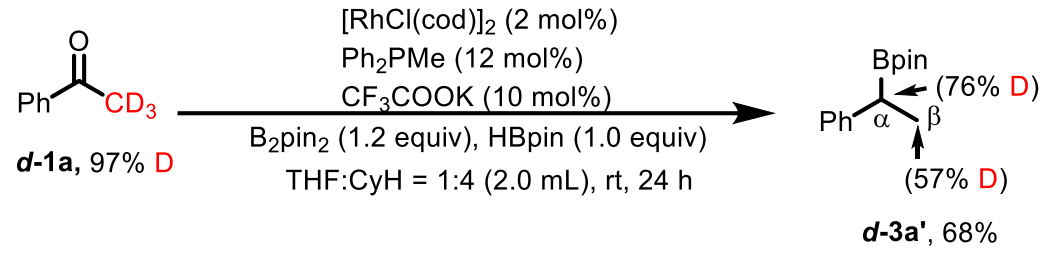

In a glove box, $[\mathrm{RhCl}(\mathrm{cod})]_{2}(3.0 \mathrm{mg}, 0.006 \mathrm{mmol}), \mathrm{PPh}_{2} \mathrm{Me}(7.2 \mathrm{mg}, 0.036$ $\mathrm{mmol})$, and anhydrous THF $(0.4 \mathrm{~mL})$ were placed in a $4 \mathrm{~mL}$ glass tube and stirred for $30 \mathrm{~min}$. Then, $\mathrm{CF}_{3} \mathrm{COOK}$ (4.6 mg, $\left.0.03 \mathrm{mmol}\right), \mathrm{B}_{2}$ pin2 $(91.4 \mathrm{mg}, 0.36$ $\mathrm{mmol}), \mathrm{HBpin}(38.4 \mathrm{mg}, 0.30 \mathrm{mmol})$ and ketone $\boldsymbol{d}$-1a in $\mathrm{CyH}(1.6 \mathrm{~mL})$ were added. The reaction mixture was sealed, removed from the glovebox and stirred at room temperature for $24 \mathrm{~h}$. The mixture was concentrated in vacuo and purified by silica gel chromatography using petroleum ether : EtOAc $=20: 1$ as the eluent to afford the product $d-3 \mathbf{a}^{\prime}$ (47.3 $\mathrm{mg}, 68 \%$ yield) as colorless oil. ${ }^{1} \mathbf{H}$ NMR (400 MHz, $\left.\mathrm{CDCl}_{3}\right) \delta 7.43$ - 7.35 (m, 4H), 7.34 - 7.29 (m, 1H), 4.49-4.41 $(\mathrm{m}, 0.24 \mathrm{H}) 1.54-1.47(\mathrm{~m}, 1.30 \mathrm{H})$. 


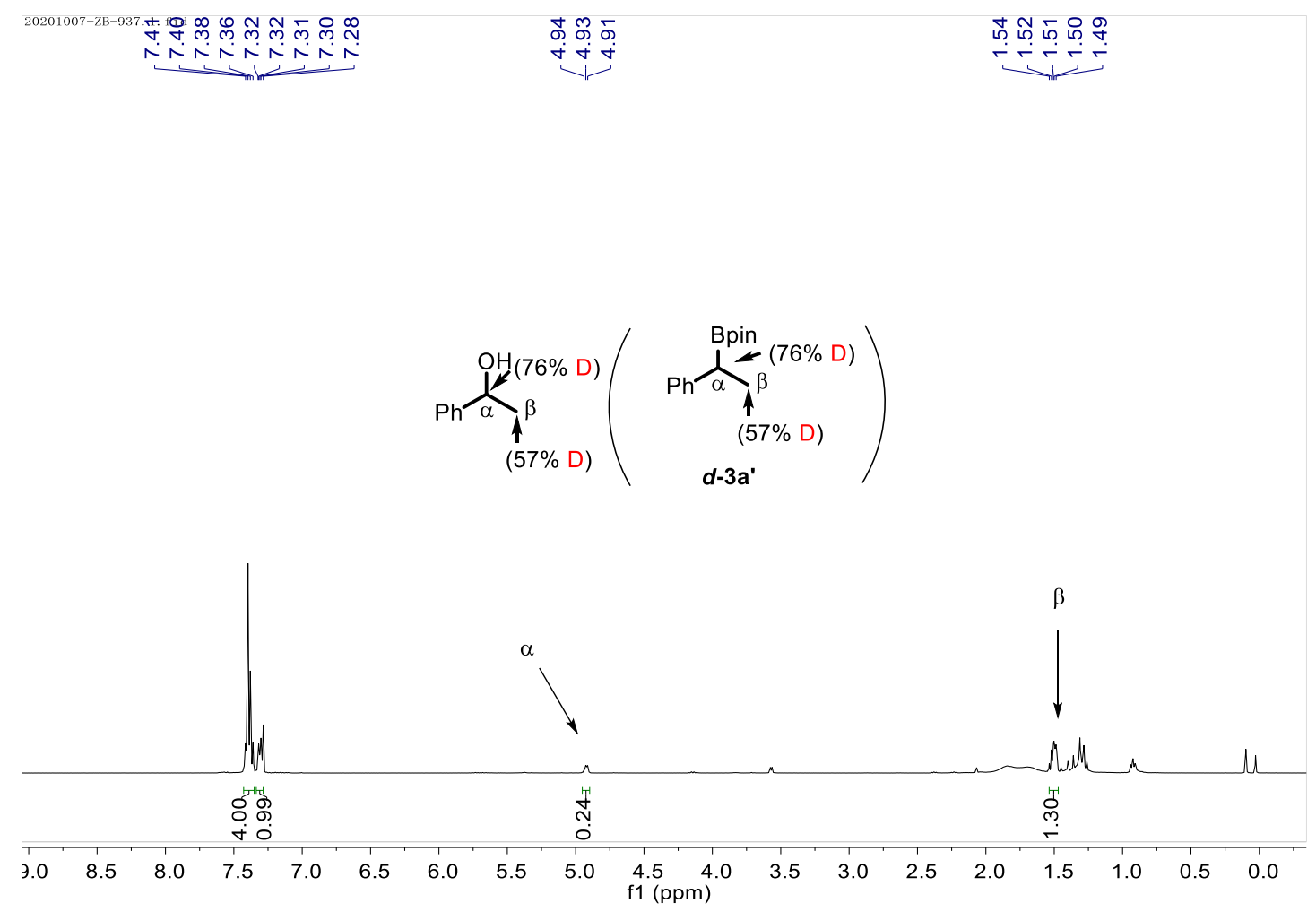

${ }^{1} \mathrm{H}$ NMR spectrum of $\boldsymbol{d}-\mathbf{3} \mathrm{a}^{\prime}$

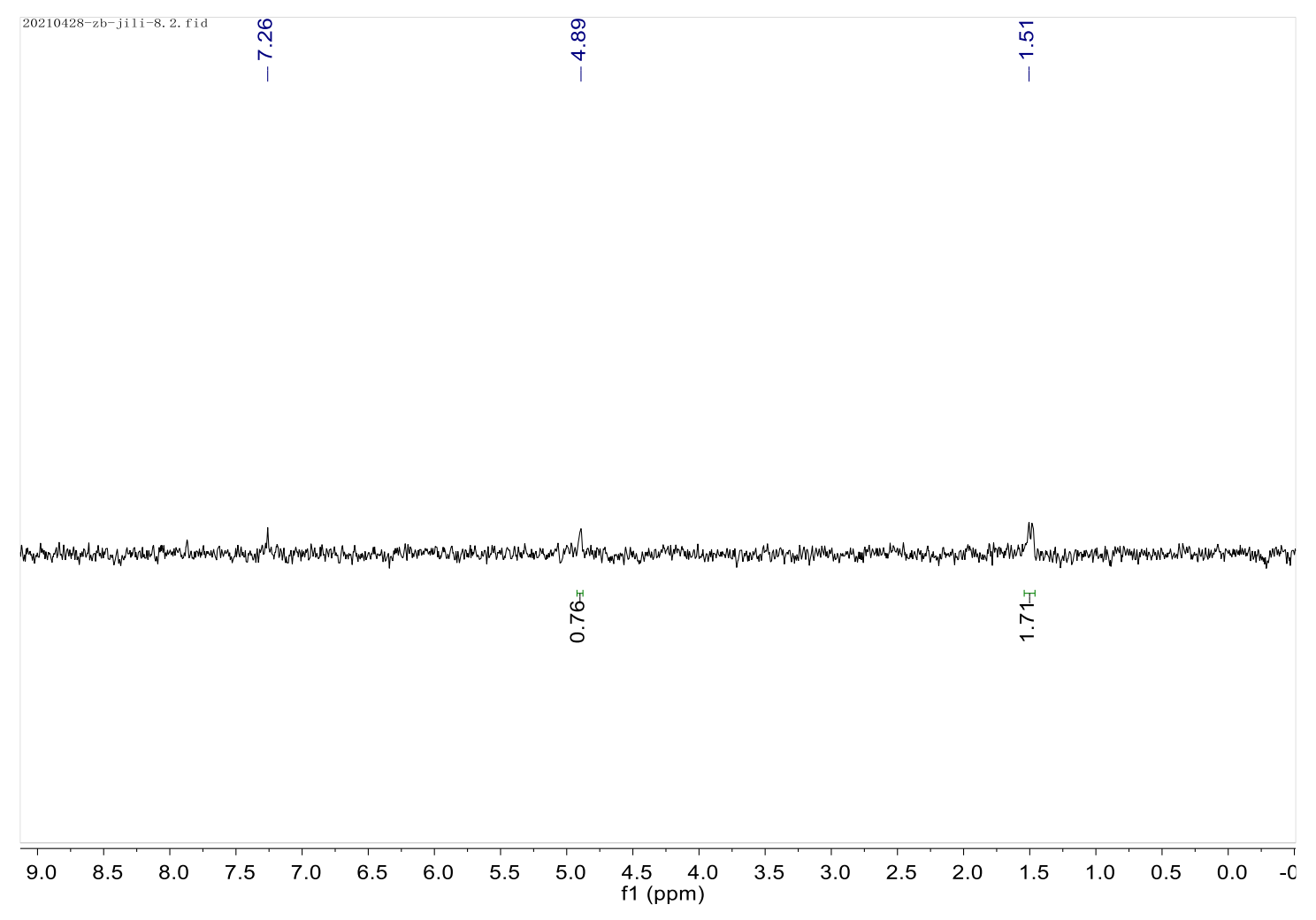

${ }^{2} \mathrm{H}$ NMR spectrum of $\boldsymbol{d}-\mathbf{3 a}{ }^{\prime}$ 


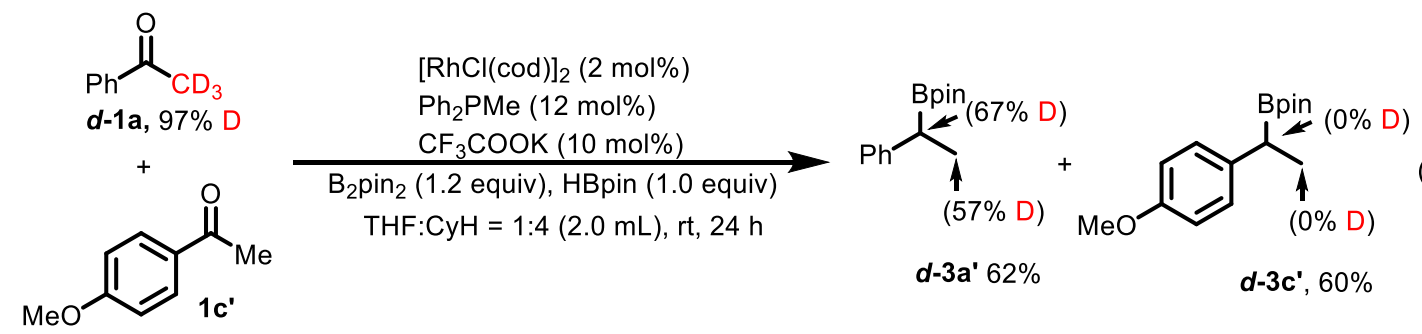

In a glove box, $[\mathrm{RhCl}(\mathrm{cod})]_{2}(6.0 \mathrm{mg}, 0.012 \mathrm{mmol}), \mathrm{PPh}_{2} \mathrm{Me}(14.2 \mathrm{mg}, 0.072$ $\mathrm{mmol})$, and anhydrous THF $(0.6 \mathrm{~mL})$ were placed in a $4 \mathrm{~mL}$ glass tube and stirred for $30 \mathrm{~min}$. Then, $\mathrm{CF}_{3} \mathrm{COOK}(9.2 \mathrm{mg}, 0.06 \mathrm{mmol}), \mathrm{B}_{2}$ pin2 (182 mg, 0.72 $\mathrm{mmol})$, HBpin (76.8 mg, $0.60 \mathrm{mmol})$ and ketone $\boldsymbol{d}-1 \mathrm{a}(36.9 \mathrm{mg}, 0.3 \mathrm{mmol})$ and $1 \mathbf{c}^{\prime}$ (45 mg, $\left.0.3 \mathrm{mmol}\right)$ in $\mathrm{CyH}(2.4 \mathrm{~mL})$ were added and stirred at room temperature for 24 hours. The mixture was concentrated in vacuo and purified by silica gel chromatography using petroleum ether : EtOAc $=50: 1-30: 1$ as the eluent to afford the product $d-3 \mathbf{a}^{\prime}$ (43.9 $\mathrm{mg}, 62 \%$ yield) and $d-3 c^{\prime}(47.1 \mathrm{mg}, 60 \%$ yield) as colorless oil.

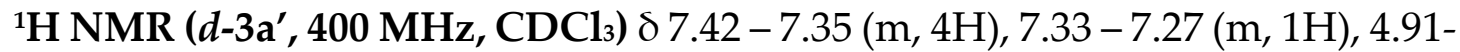
$4.90(\mathrm{~m}, 0.33 \mathrm{H}), 1.54-1.46(\mathrm{~m}, 1.29 \mathrm{H})$.

${ }^{1} \mathrm{H}$ NMR $\left(d-3 \mathrm{c}^{\prime}, 400 \mathrm{MHz}, \mathrm{CDCl}_{3}\right) \delta 7.32(\mathrm{~d}, J=8.5 \mathrm{~Hz}, 2 \mathrm{H}), 6.90(\mathrm{~d}, J=8.5 \mathrm{~Hz}$, $2 \mathrm{H}), 4.86(\mathrm{q}, J=6.4 \mathrm{~Hz}, 1 \mathrm{H}), 3.82(\mathrm{~s}, 3 \mathrm{H}), 1.49(\mathrm{~d}, J=6.5 \mathrm{~Hz}, 3 \mathrm{H})$.

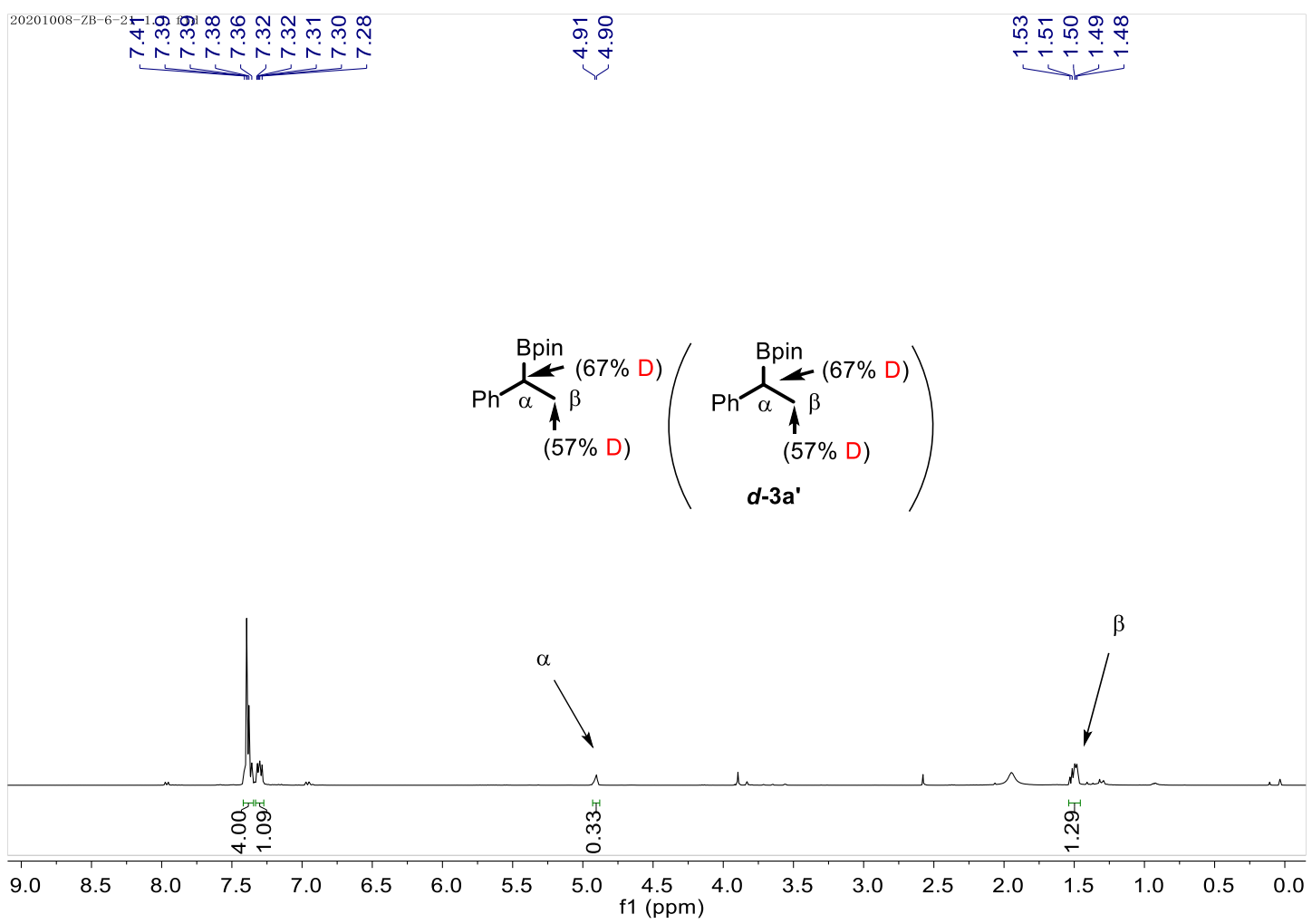

${ }^{1} \mathrm{H}$ NMR spectrum of $d-3 \mathrm{a}^{\prime}$ 


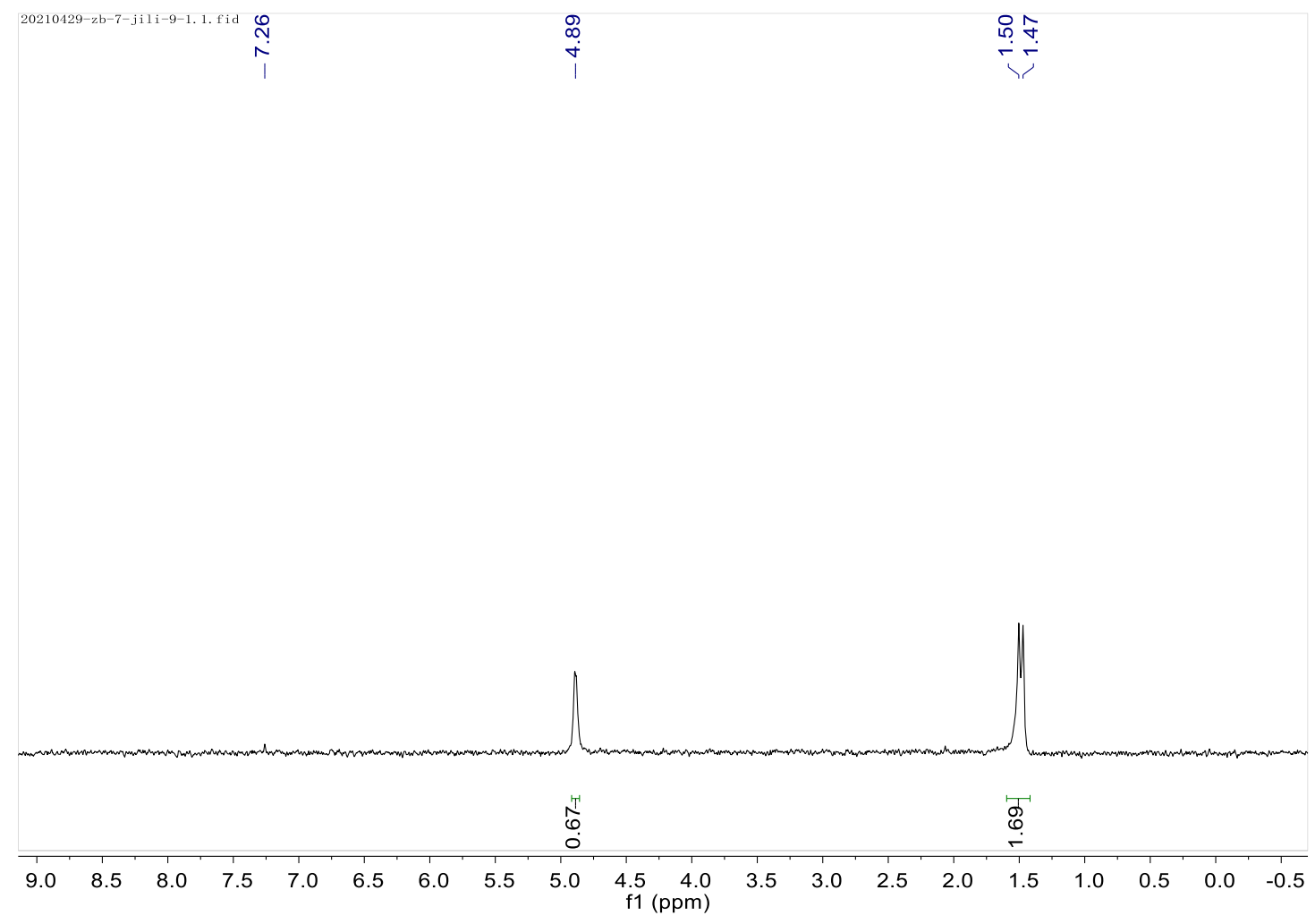

${ }^{2} \mathrm{H}$ NMR spectrum of $\boldsymbol{d}-\mathbf{3 a} \mathbf{a}^{\prime}$

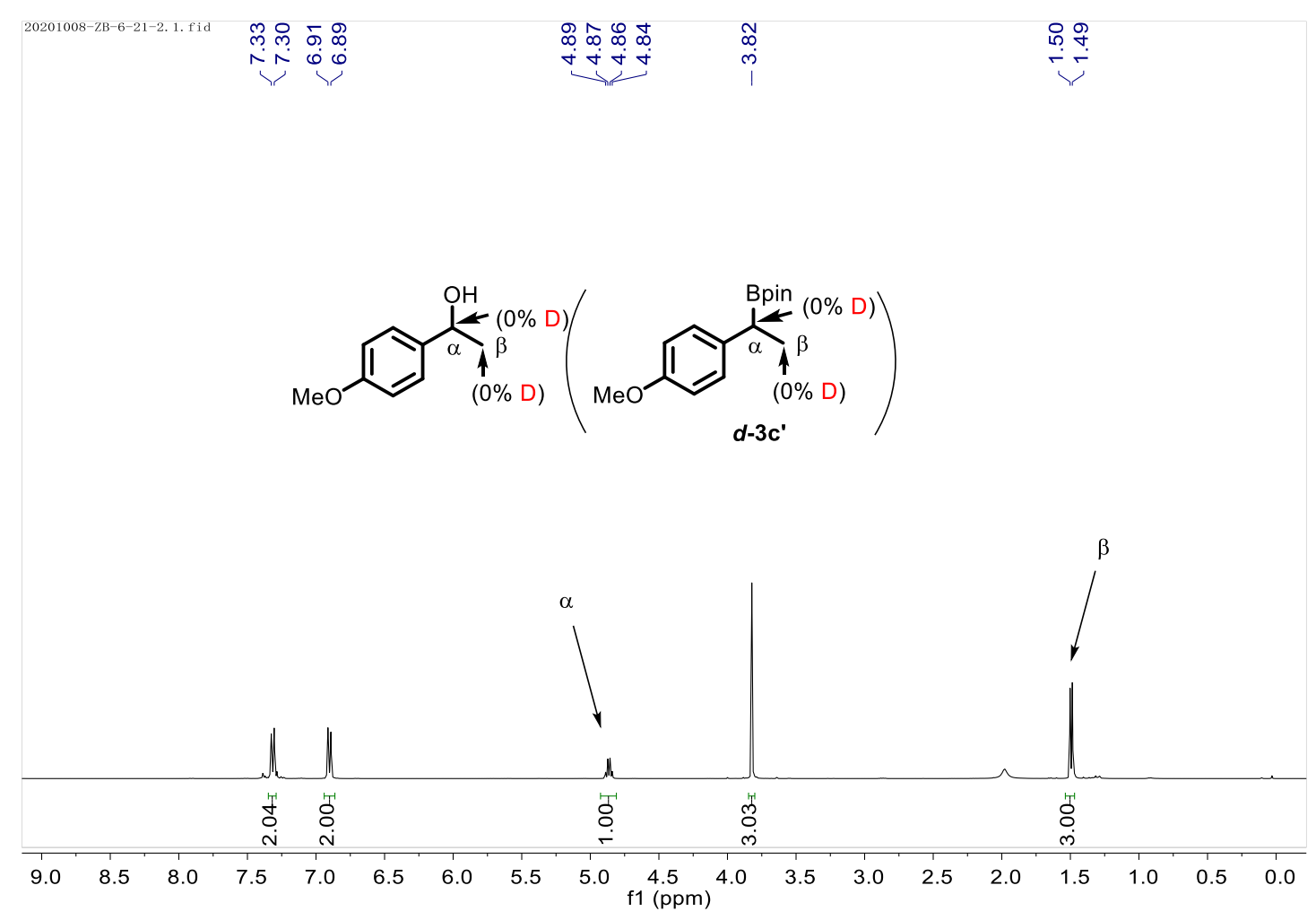

${ }^{1} \mathrm{H}$ NMR spectrum of $\boldsymbol{d}-\mathbf{3 c} \mathrm{c}^{\prime}$ 
${ }^{2} \mathrm{H}$ NMR spectrum of $\boldsymbol{d}-3 \mathrm{c}^{\prime}$
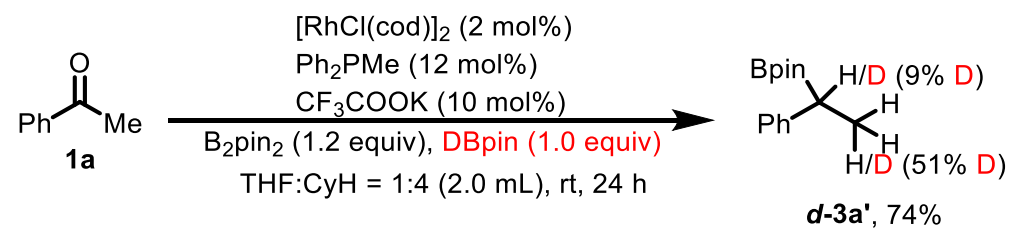

In a glove box, $[\mathrm{RhCl}(\mathrm{cod})]_{2}(3.0 \mathrm{mg}, 0.006 \mathrm{mmol}), \mathrm{PPh}_{2} \mathrm{Me}(7.2 \mathrm{mg}, 0.036$ $\mathrm{mmol})$, and anhydrous THF $(0.4 \mathrm{~mL})$ were placed in a $4 \mathrm{~mL}$ glass tube and stirred for $30 \mathrm{~min}$. Then, $\mathrm{CF}_{3} \mathrm{COOK}$ (4.6 mg, $\left.0.03 \mathrm{mmol}\right)$, B2pin2 (91.4 mg, 0.36 $\mathrm{mmol})$, DBpin (38.4 mg, $0.30 \mathrm{mmol})$ and ketone $1 \mathrm{a}(36 \mathrm{mg}, 0.3 \mathrm{mmol})$ in $\mathrm{CyH}$ $(1.6 \mathrm{~mL})$ were added and stirred at room temperature for 24 hours. The mixture was concentrated in vacuo and purified by silica gel chromatography using petroleum ether : EtOAc $=20: 1$ as the eluent to afford the product $\boldsymbol{d}-\mathbf{3} \mathbf{a}^{\prime}$ (51.2 $\mathrm{mg}, 74 \%$ yield) as colorless oil.

${ }^{1} \mathrm{H}$ NMR (400 MHz, $\left.\mathrm{CDCl}_{3}\right) \delta 7.43-7.35(\mathrm{~m}, 4 \mathrm{H}), 7.33-7.28(\mathrm{~m}, 1 \mathrm{H}), 4.93(\mathrm{q}, J$ $=6.2 \mathrm{~Hz}, 0.92 \mathrm{H}), 1.55-1.49(\mathrm{~m}, 2.49 \mathrm{H})$. 


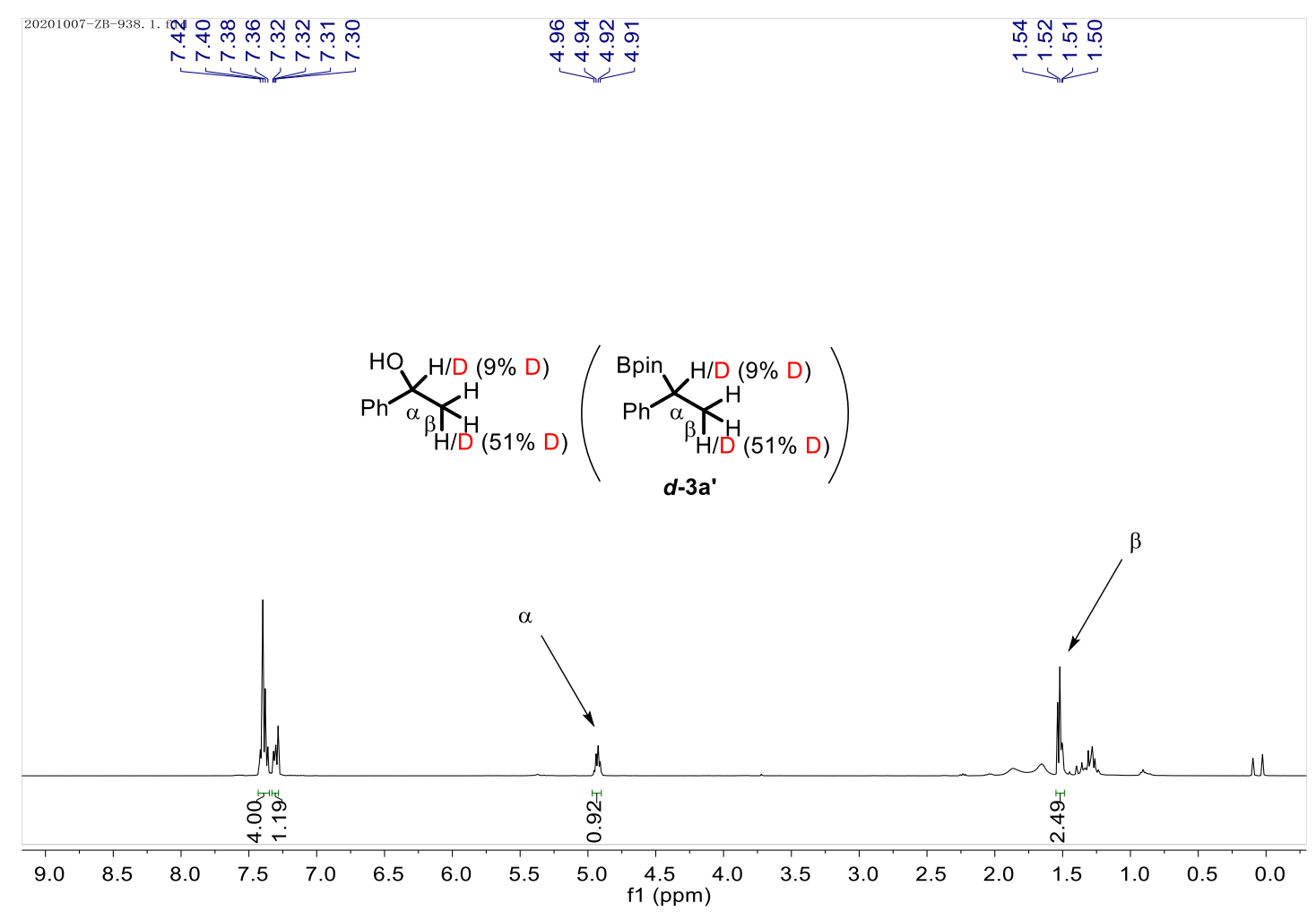

${ }^{1} \mathrm{H}$ NMR spectrum of $\boldsymbol{d}-\mathbf{3 a} \mathbf{a}^{\prime}$

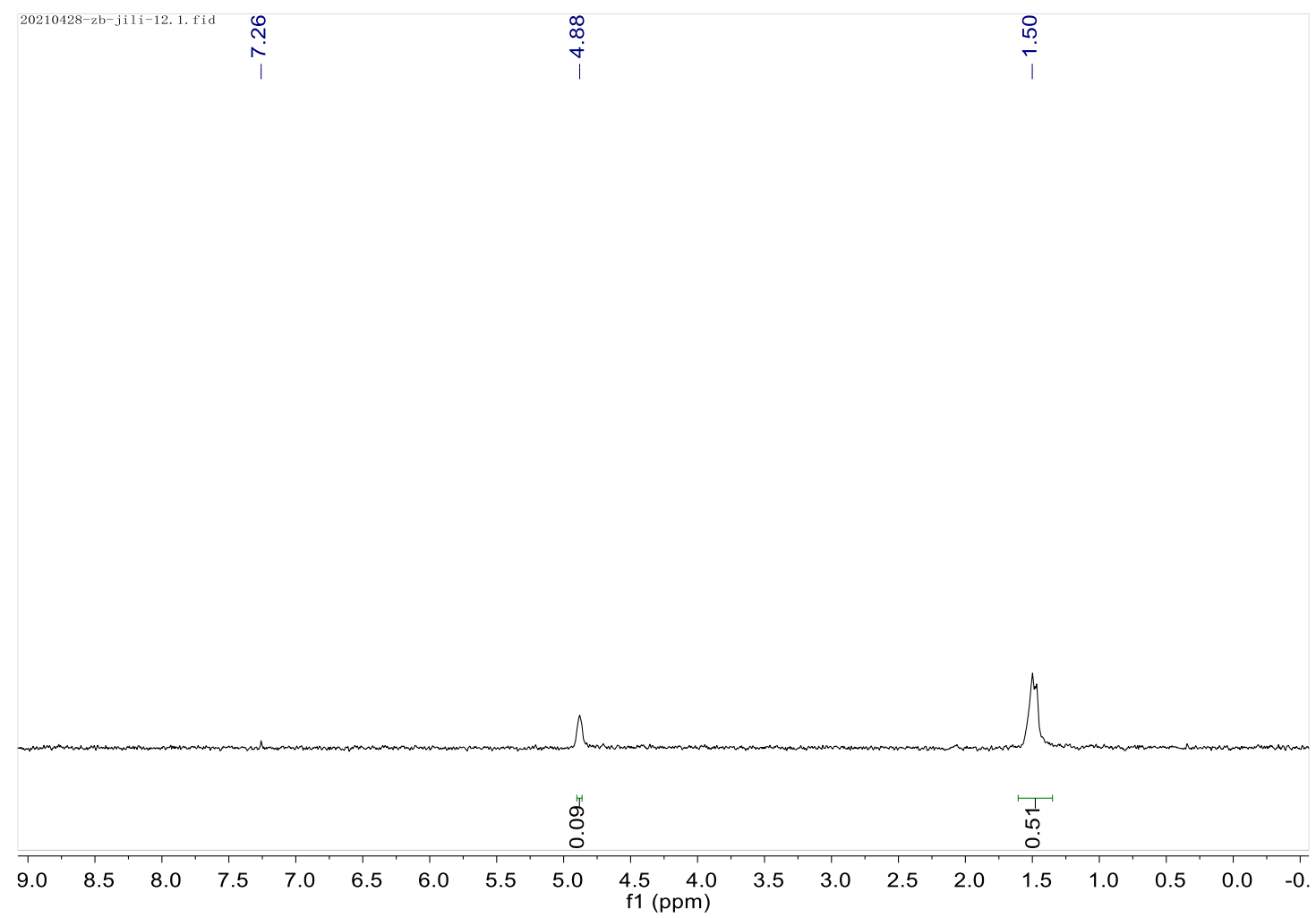

${ }^{2} \mathrm{H}$ NMR spectrum of $\boldsymbol{d}-\mathbf{3 a}{ }^{\prime}$ 


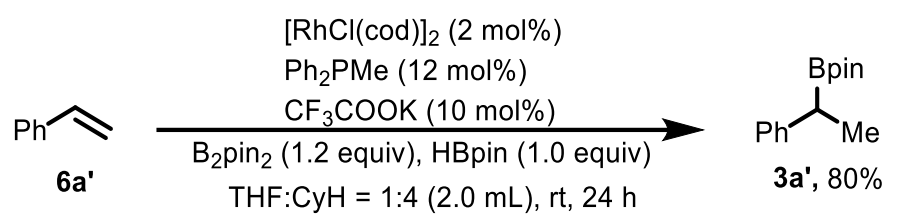

In a glove box, $[\mathrm{RhCl}(\mathrm{cod})]_{2}(3.0 \mathrm{mg}, 0.006 \mathrm{mmol}), \mathrm{PPh}_{2} \mathrm{Me}(7.2 \mathrm{mg}, 0.036$ $\mathrm{mmol})$, and anhydrous THF $(0.4 \mathrm{~mL})$ were placed in a $4 \mathrm{~mL}$ glass tube and stirred for $30 \mathrm{~min}$. Then, $\mathrm{CF}_{3} \mathrm{COOK}$ (4.6 mg, $0.03 \mathrm{mmol}$ ), B2pinz (91.4 mg, 0.36 mmol), HBpin (38.4 mg, $0.30 \mathrm{mmol})$ and 6a' $(31.2 \mathrm{mg}, 0.3 \mathrm{mmol})$ in СyH (1.6 $\mathrm{mL}$ ) were added and stirred at room temperature for 24 hours. The resulting mixture was filtered through a silica gel pad with EtOAc and concentrated under vacuo. The yield was determined by GC-MS analysis with dodecane as the internal standard. The GC-MS showed that the $3 a^{\prime}$ was produced with $80 \%$ yield.

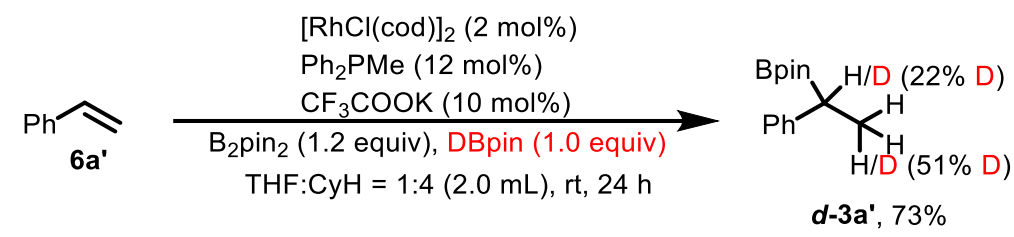

In a glove box, $[\mathrm{RhCl}(\mathrm{cod})]_{2}(3.0 \mathrm{mg}, 0.006 \mathrm{mmol}), \mathrm{PPh}_{2} \mathrm{Me}(7.2 \mathrm{mg}, 0.036$ $\mathrm{mmol})$, and anhydrous THF $(0.4 \mathrm{~mL})$ were placed in a $4 \mathrm{~mL}$ glass tube and stirred for $30 \mathrm{~min}$. Then, $\mathrm{CF}_{3} \mathrm{COOK}$ ( $4.6 \mathrm{mg}$, $0.03 \mathrm{mmol}$ ), B ${ }_{2}$ pinz $(91.4 \mathrm{mg}$, 0.36 mmol), DBpin (38.4 mg, $0.30 \mathrm{mmol})$ and 6a' $(31.2 \mathrm{mg}, 0.3 \mathrm{mmol})$ in СyH (1.6 $\mathrm{mL}$ ) were added and stirred at room temperature for $24 \mathrm{~h}$. The mixture was concentrated in vacuo and purified by silica gel chromatography using petroleum ether : EtOAc $=20: 1$ as the eluent to afford the product $\boldsymbol{d}-\mathbf{3} \mathbf{a}^{\prime}$ (50.8 $\mathrm{mg}, 73 \%$ yield) as colorless oil.

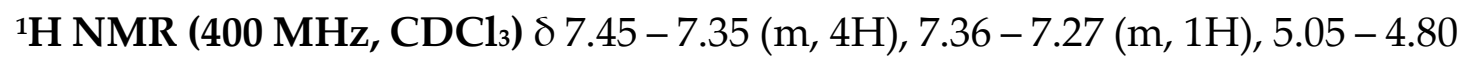
$(\mathrm{m}, 0.78 \mathrm{H}), 1.54-1.51(\mathrm{~m}, 2.49 \mathrm{H})$. 


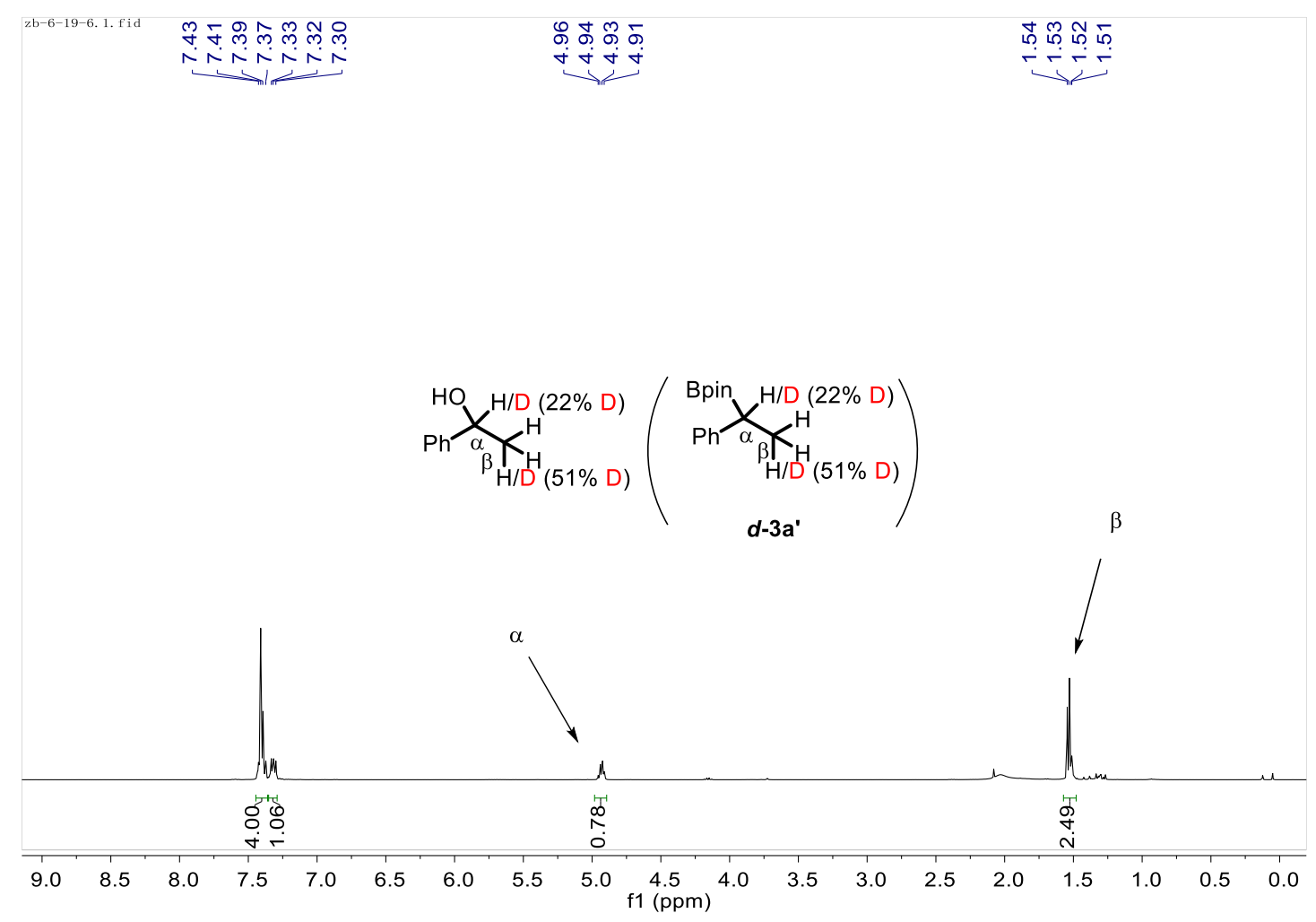

${ }^{1} \mathrm{H}$ NMR spectrum of $\boldsymbol{d}-\mathbf{3} \mathrm{a}^{\prime}$

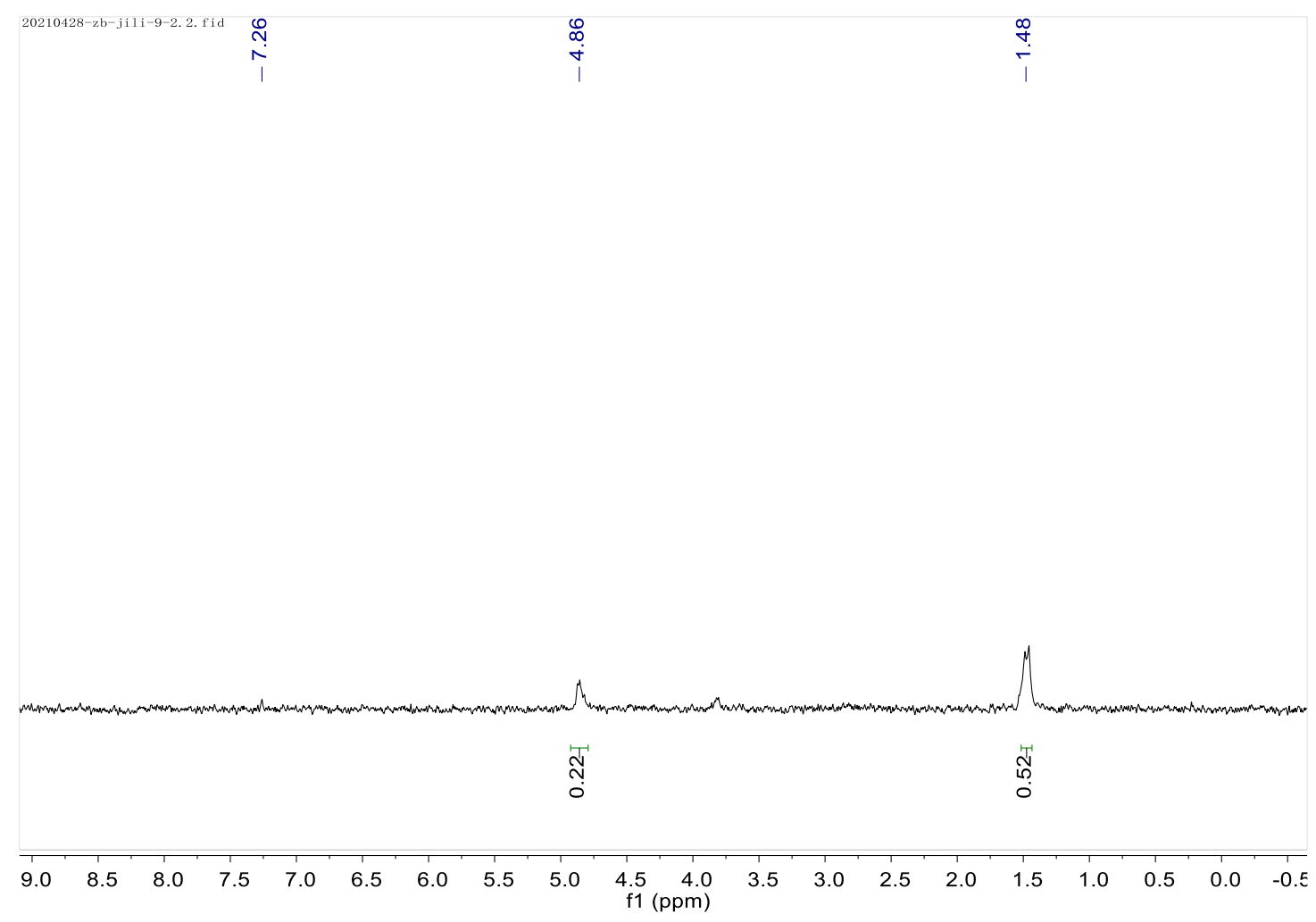

${ }^{2} \mathrm{H}$ NMR spectrum of $d-3 \mathbf{a}^{\prime}$ 


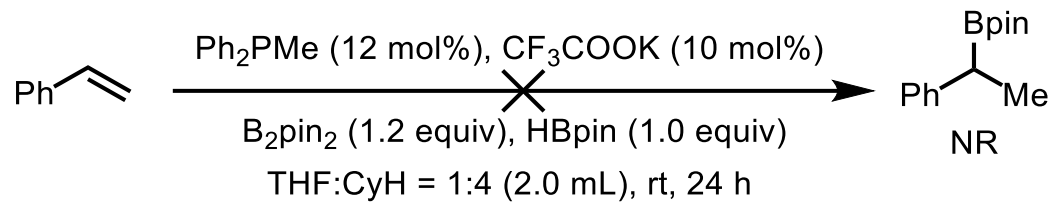

In a glove box, $\mathrm{PPh} 2 \mathrm{Me}(7.2 \mathrm{mg}, 0.036 \mathrm{mmol})$, and anhydrous THF $(0.4 \mathrm{~mL})$ were placed in a $4 \mathrm{~mL}$ glass tube and stirred for $30 \mathrm{~min}$. Then, $\mathrm{CF}_{3} \mathrm{COOK}$ (4.6 $\mathrm{mg}, 0.03 \mathrm{mmol}$ ), B2pinz (91.4 mg, $0.36 \mathrm{mmol}), \mathrm{HB}$ pin $(38.4 \mathrm{mg}, 0.30 \mathrm{mmol})$ and styrene $(31.2 \mathrm{mg}, 0.3 \mathrm{~mol})$ in $\mathrm{CyH}(1.6 \mathrm{~mL})$ were added and stirred at room temperature for 24 hours. The resulting mixture was filtered through a silica gel pad with EtOAc and concentrated under vacuo. The GC-MS and TLC analysis showed that no reaction was observed.

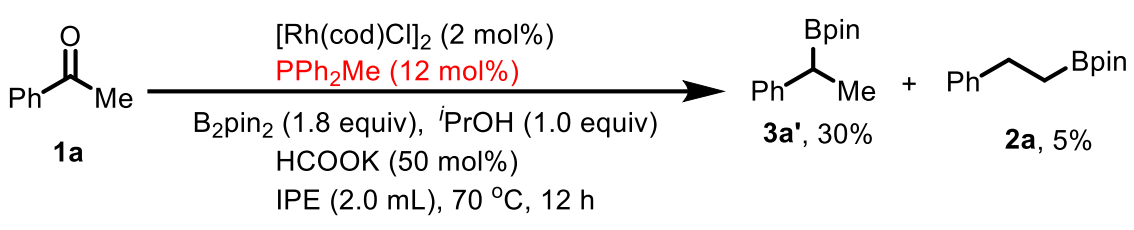

In a glove box, $[\mathrm{RhCl}(\mathrm{cod})]_{2}(3.0 \mathrm{mg}, 0.006 \mathrm{mmol}), \mathrm{PPh}_{2} \mathrm{Me}(7.2 \mathrm{mg}, 0.036$ $\mathrm{mmol})$, and IPE $(1.0 \mathrm{~mL})$ were placed in a $4 \mathrm{~mL}$ glass tube and stirred for 30 min. Then, HCOOK (12.6 mg, $0.15 \mathrm{mmol})$, B2pinz (137 mg, $0.54 \mathrm{mmol}$ ), and ${ }^{i} \mathrm{PrOH}(22.9 \mathrm{mg}, 0.30 \mathrm{mmol})$ were added, and stirred for another $30 \mathrm{~min}$. 1a (36 $\mathrm{mg}, 0.3 \mathrm{mmol})$ in IPE $(1.0 \mathrm{~mL})$ was added. The reaction mixture was sealed, removed from the glovebox and stirred at $70{ }^{\circ} \mathrm{C}$ for 12 hours. The resulting mixture was cooled to ambient temperature, filtered through a silica gel pad with EtOAc. The yields were determined by GC-MS analysis with dodecane as the internal standard. The GC-MS showed that the reaction afforded a mixture of product $\mathbf{2 a}$ and $3 \mathbf{a}^{\prime}$ in $5 \%$ and $30 \%$ yields, respectively.

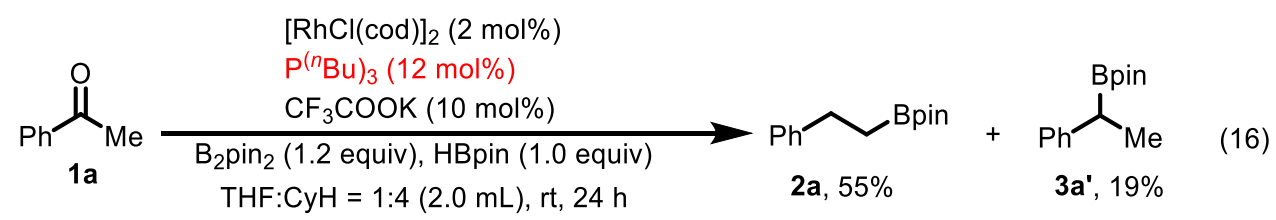

In a glove box, $[\mathrm{RhCl}(\mathrm{cod})]_{2}(3.0 \mathrm{mg}, 0.006 \mathrm{mmol}), \mathrm{P}\left({ }^{n} \mathrm{Bu}\right)_{3}(7.3 \mathrm{mg}, 0.036$ $\mathrm{mmol})$, and anhydrous THF $(0.4 \mathrm{~mL})$ were placed in a $4 \mathrm{~mL}$ glass tube and 
stirred for $30 \mathrm{~min}$. Then, $\mathrm{CF}_{3} \mathrm{COOK}$ (4.6 mg, $0.03 \mathrm{mmol}$ ), B2pin2 (91.4 mg, 0.36 $\mathrm{mmol})$, HBpin (38.4 mg, $0.30 \mathrm{mmol})$ and $1 \mathrm{a}(36 \mathrm{mg}, 0.3 \mathrm{mmol})$ in $\mathrm{CyH}(1.6 \mathrm{~mL})$ were added and stirred at room temperature for 24 hours. The resulting mixture was filtered through a silica gel pad with EtOAc. The yields were determined by GC-MS analysis with dodecane as the internal standard. The GC-MS showed that the reaction afforded a mixture of product $\mathbf{2} \mathbf{a}$ and $\mathbf{3} \mathbf{a}^{\prime}$ in $55 \%$ and $19 \%$ yields, respectively.
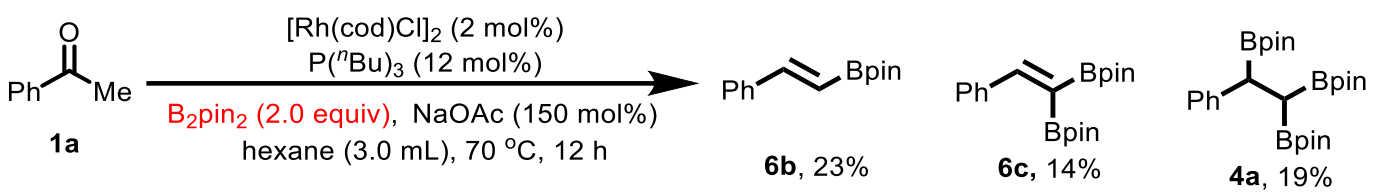

In a glove box, $[\mathrm{RhCl}(\mathrm{cod})]_{2}(3.0 \mathrm{mg}, 0.006 \mathrm{mmol}), \mathrm{P}\left({ }^{n} \mathrm{Bu}\right)_{3}(7.3 \mathrm{mg}, 0.036$ $\mathrm{mmol})$, and hexane $(1.0 \mathrm{~mL})$ were placed in a $4 \mathrm{~mL}$ glass tube and stirred for 30 min. Then, NaOAc (36.9 mg, $0.45 \mathrm{mmol}), \mathrm{B}_{2}$ pin 2 (152.0 mg, $\left.0.6 \mathrm{mmol}\right)$, and 1a $(31.2 \mathrm{mg}, 0.3 \mathrm{mmol})$ in hexane $(2.0 \mathrm{~mL})$ were added. The reaction mixture was sealed, removed from the glovebox and stirred at $70{ }^{\circ} \mathrm{C}$ for 12 hours. The resulting mixture was cooled to ambient temperature and filtered through a silica gel pad with EtOAc. The yield was determined by ${ }^{1} \mathrm{H}$ NMR analysis with $\mathrm{CH}_{2} \mathrm{Br}_{2}$ as the internal standard. The ${ }^{1} \mathrm{H}$ NMR showed that the $\mathbf{6} \mathbf{b}, \mathbf{6} \mathbf{c}, \mathbf{4 a}$ were produced in $23 \%, 14 \%$ and $19 \%$ yields.
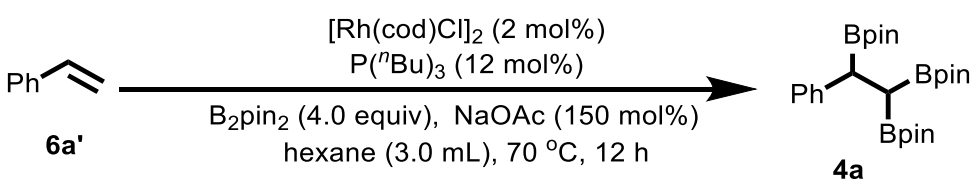

In a glove box, $[\mathrm{RhCl}(\mathrm{cod})]_{2}(3.0 \mathrm{mg}, 0.006 \mathrm{mmol}), \mathrm{P}\left({ }^{n} \mathrm{Bu}\right)_{3}(7.3 \mathrm{mg}, 0.036$ $\mathrm{mmol})$, and hexane $(1.0 \mathrm{~mL})$ were placed in a $4 \mathrm{~mL}$ glass tube and stirred for 30 min. Then, NaOAc (36.9 mg, $0.45 \mathrm{mmol}), \mathrm{B}_{2}$ pin2 (304.8 mg, $\left.1.2 \mathrm{mmol}\right)$, and 6a' $(31.2 \mathrm{mg}, 0.3 \mathrm{mmol})$ in hexane $(2.0 \mathrm{~mL})$ were added. The reaction mixture was sealed, removed from the glovebox and stirred at $70{ }^{\circ} \mathrm{C}$ for 12 hours. The resulting mixture was cooled to ambient temperature and filtered through a silica gel pad with EtOAc. The yield was determined by GC-MS analysis with dodecane as the internal standard. The GC-MS showed that the $4 \mathbf{a}$ was 
produced in $77 \%$ yield.

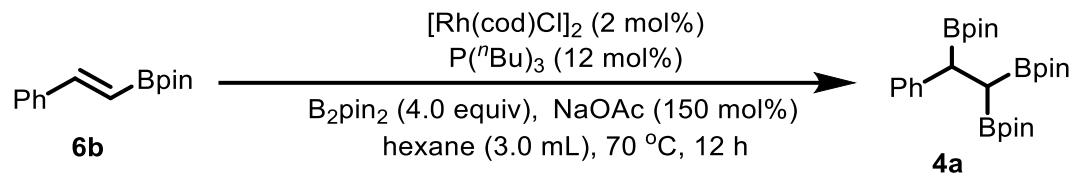

In a glove box, $[\mathrm{RhCl}(\mathrm{cod})]_{2}(3.0 \mathrm{mg}, 0.006 \mathrm{mmol}), \mathrm{P}\left({ }^{n} \mathrm{Bu}\right)_{3}(7.3 \mathrm{mg}, 0.036$ $\mathrm{mmol})$, and hexane $(1.0 \mathrm{~mL})$ were placed in a $4 \mathrm{~mL}$ glass tube and stirred for 30 min. Then, NaOAc (36.9 mg, $0.45 \mathrm{mmol}$ ), B2pin 2 (304.8 mg, $1.2 \mathrm{mmol}$ ), and $6 \mathbf{b}$ $(69 \mathrm{mg}, 0.3 \mathrm{mmol})$ in hexane $(2.0 \mathrm{~mL})$ were added. The reaction mixture was sealed, removed from the glovebox and stirred at $70{ }^{\circ} \mathrm{C}$ for 12 hours. The resulting mixture was cooled to ambient temperature and filtered through a silica gel pad with EtOAc. The yield was determined by GC-MS analysis with dodecane as the internal standard. The GC-MS showed that the 4a was produced in $62 \%$ yield.

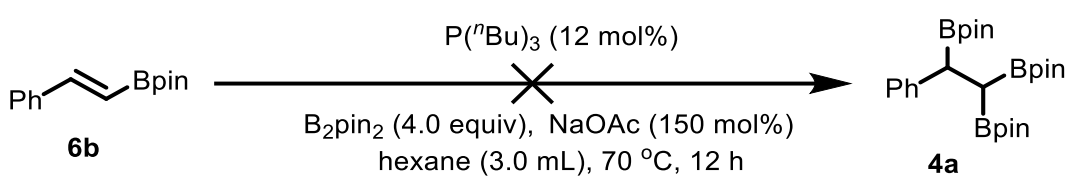

In a glove box, $\mathrm{P}\left({ }^{n} \mathrm{Bu}\right)_{3}(7.3 \mathrm{mg}, 0.036 \mathrm{mmol})$, and hexane $(1.0 \mathrm{~mL})$ were placed in a $4 \mathrm{~mL}$ glass tube and stirred for $30 \mathrm{~min}$. Then, $\mathrm{NaOAc}(36.9 \mathrm{mg}, 0.45$ mmol), B2pin $(304.8 \mathrm{mg}, 1.2 \mathrm{mmol})$, and $6 \mathbf{b}(69 \mathrm{mg}, 0.3 \mathrm{mmol})$ in hexane (2.0 $\mathrm{mL}$ ) were added. The reaction mixture was sealed, removed from the glovebox and stirred at $70{ }^{\circ} \mathrm{C}$ for 12 hours. The resulting mixture was cooled to ambient temperature and filtered through a silica gel pad with EtOAc. The GC-MS and TLC analysis showed that no reaction was observed.

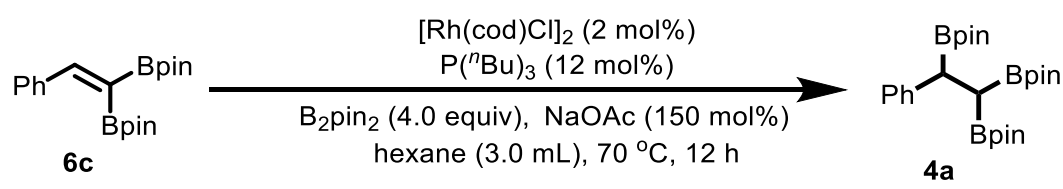

In a glove box, $[\mathrm{RhCl}(\mathrm{cod})]_{2}(3.0 \mathrm{mg}, 0.006 \mathrm{mmol}), \mathrm{P}\left({ }^{n} \mathrm{Bu}\right)_{3}(7.3 \mathrm{mg}, 0.036$ $\mathrm{mmol})$, and hexane $(1.0 \mathrm{~mL})$ were placed in a $4 \mathrm{~mL}$ glass tube and stirred for 30 min. Then, NaOAc (36.9 mg, $0.45 \mathrm{mmol}), \mathrm{B}_{2}$ pin 2 (304.8 mg, $\left.1.2 \mathrm{mmol}\right)$, and $6 \mathrm{c}$ 
$(106.8 \mathrm{mg}, 0.3 \mathrm{mmol})$ in hexane $(2.0 \mathrm{~mL})$ were added. The reaction mixture was sealed, removed from the glovebox and stirred at $70{ }^{\circ} \mathrm{C}$ for $12 \mathrm{~h}$. The resulting mixture was cooled to ambient temperature and filtered through a silica gel pad with EtOAc. The yield was determined by GC-MS analysis with dodecane as the internal standard. The GC-MS showed that the $4 a$ was produced in $62 \%$ yield.

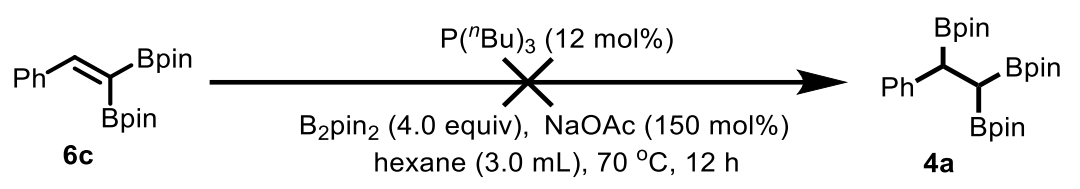

In a glove box, $\mathrm{P}\left({ }^{n} \mathrm{Bu}\right)_{3}(7.3 \mathrm{mg}, 0.036 \mathrm{mmol})$, and hexane $(1.0 \mathrm{~mL})$ were placed in a $4 \mathrm{~mL}$ glass tube and stirred for $30 \mathrm{~min}$. Then, $\mathrm{NaOAc}(36.9 \mathrm{mg}, 0.45$ $\mathrm{mmol}), \mathrm{B}_{2}$ pin $2(304.8 \mathrm{mg}, 1.2 \mathrm{mmol})$, and 6c $(106.8 \mathrm{mg}, 0.3 \mathrm{mmol})$ in hexane $(2.0$ $\mathrm{mL}$ ) were added. The reaction mixture was sealed, removed from the glovebox and stirred at $70{ }^{\circ} \mathrm{C}$ for $12 \mathrm{~h}$. The resulting mixture was cooled to ambient temperature and filtered through a silica gel pad with EtOAc. The GC-MS and TLC analysis showed that no reaction was observed.

(23) $P h$

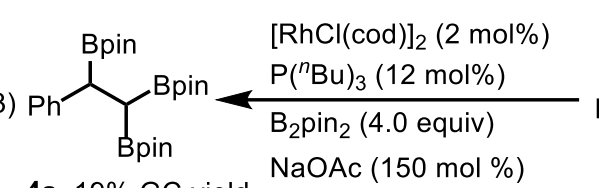

4a, 19\% GC yield

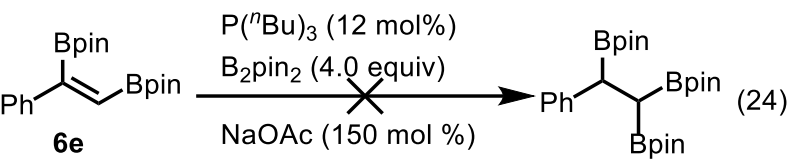

hexane $(3.0 \mathrm{~mL}), 70^{\circ} \mathrm{C}, 12 \mathrm{~h} \quad \mathrm{NR}$

In a glove box, $[\mathrm{RhCl}(\mathrm{cod})]_{2}(3.0 \mathrm{mg}, 0.006 \mathrm{mmol}), \mathrm{P}\left({ }^{n} \mathrm{Bu}\right)_{3}(7.3 \mathrm{mg}, 0.036$ $\mathrm{mmol})$, and hexane $(1.0 \mathrm{~mL})$ were placed in a $4 \mathrm{~mL}$ glass tube and stirred for 30 min. Then, NaOAc (36.9 mg, $0.45 \mathrm{mmol}$ ), B2pin2 (304.8 mg, $1.2 \mathrm{mmol}$ ), and 1,2diborated styrene $\mathbf{6 e}(106.8 \mathrm{mg}, 0.3 \mathrm{mmol})$ in hexane $(2.0 \mathrm{~mL})$ were added. The reaction mixture was sealed, removed from the glovebox and stirred at $70{ }^{\circ} \mathrm{C}$ for $12 \mathrm{~h}$. The resulting mixture was cooled to ambient temperature and filtered through a silica gel pad with EtOAc. The yield was determined by GC-MS analysis with dodecane as the internal standard. The GC-MS showed that the 4a was produced in 19\% yield (eq. 23).

In a glove box, $\mathrm{P}\left({ }^{n} \mathrm{Bu}\right)_{3}(7.3 \mathrm{mg}, 0.036 \mathrm{mmol})$, and hexane $(1.0 \mathrm{~mL})$ were placed in a $4 \mathrm{~mL}$ glass tube and stirred for $30 \mathrm{~min}$. Then, $\mathrm{NaOAc}(36.9 \mathrm{mg}, 0.45$ mmol), B2pin2 (304.8 mg, $1.2 \mathrm{mmol})$, and 1,2-diborated styrene 6e (106.8 mg, 
$0.3 \mathrm{mmol})$ in hexane $(2.0 \mathrm{~mL})$ were added. The reaction mixture was sealed, removed from the glovebox and stirred at $70^{\circ} \mathrm{C}$ for $12 \mathrm{~h}$. The resulting mixture was cooled to ambient temperature and filtered through a silica gel pad with EtOAc. The GC-MS and TLC analysis showed that no reaction was observed (eq. 24).

\section{DFT Computational Details}

All structures were optimized using the long-range corrected (LC) hybrid density functional $\omega B$ 97X-D of density functional theory. ${ }^{[46]}$ The basis set 6$31 \mathrm{G}(\mathrm{d}, \mathrm{p})$ was used to describe $\mathrm{C}, \mathrm{H}, \mathrm{B}, \mathrm{O}$ atoms, while the Lanl2DZ basis set ${ }^{[47]}$ with polarization functions was used for $\mathrm{P}\left(\xi_{\mathrm{d}}=0.387\right)^{[48]}$ and $\mathrm{Rh}\left(\xi_{\mathrm{F}}=1.350\right) \cdot{ }^{[49]}$ Frequency calculations were performed at the same level to ensure the optimized structures are local minima or transition states. Intrinsic reaction coordinate (IRC) ${ }^{[50]}$ calculations were carried out to make sure every transition state links two relevant intermediates. All of calculations were performed with Gaussian 09 package. ${ }^{[51]}$ 


\section{References}

[1]. Giordano, G.; iCrabtree, R. H.; Heintz, R. M.; Forster, D.; Morris, D. E. Inorg. Synth. 1990, 28, 88-90.

[2]. Tao, L.; Guo, X.; Li, J.; Li, R.; Lin, Z.; Zhao, W. Rhodium-Catalyzed Deoxygenation and Borylation of Ketones: A Combined Experimental and Theoretical Investigation. J. Am. Chem. Soc. 2020, 142, 18118-18127.

[3]. Duczynski, J.; Sobolev, A. N.; Moggach, S. A.; Dorta, R.; Stewart, S. G. The Synthesis and Catalytic Activity of New Mixed NHC-Phosphite Nickel(0) Complexes. Organometallics 2020, 39, 105-115.

[4]. Wang, X.; Li, C.; Wang, X.; Wang, Q.; Dong, X.-Q.; Duan, A.; Zhao, W. Metal-Free Etherification of Aryl Methyl Ether Derivatives by C-OMe Bond Cleavage. Org. Lett. 2018, 20, 4267-4272.

[5]. St. Jean, D. J.; Poon, S. F.; Schwarzbach, J. L. A Tandem CrossCoupling/SNAr Approach to Functionalized Carbazoles. Org. Lett. 2007, 9, 4893-4896.

[6]. Schirmer, M.-L.; Jopp, S.; Holz, J.; Spannenberg, A.; Werner, T. Organocatalyzed Reduction of Tertiary Phosphine Oxides. Adv. Synth. Catal. 2016, 358, 26-29.

[7]. Thomas, J. C.; Peters, J. C. Bis(phosphino)borates: A New Family of Monoanionic Chelating Phosphine Ligands. Inorg. Chem. 2003, 42, 50555073.

[8].Li, J.-F.; Wei, Z.-Z.; Wang, Y.-Q.; Ye, M. Base-free Nickel-Catalyzed Hydroboration of Simple Alkenes with Bis(pinacolato)diboron in An Alcoholic Solvent. Green Chem. 2017, 19, 4498-4502.

[9]. Qiao, L.; Zhang, L.; Liu, G.; Huang, Z. A Highly Efficient Cobalt-Catalyzed Deuterogenolysis of Diboron: Synthesis of Deuterated Pinacolborane and Vinylboronates. Tetrahedron 2019, 75, 4138-4142.

[10]. Liu, Y.; Zhou, Y.; Wang, H.; Qu, J. FeCl2-Catalyzed Hydroboration of Aryl Alkenes with Bis(pinacolato)diboron. RSC Adv. 2015, 5, 73705-73713.

[11]. Hong, S.; Liu, M.; Zhang, W.; Zeng, Q.; Deng, W. Copper-Catalyzed Hydroboration of Arylalkenes at Room Temperature. Tetrahedron Lett. 2015, $56,2297-2302$.

[12]. Jaladi, A. K.; Shin, W. K.; An, D. K. Alkene Hydroboration with Pinacolborane Catalysed by Lithium Diisobutyl-tert-butoxyaluminum 
Hydride. RSC Adv. 2019, 9, 26483-26486.

[13]. Berthold, D.; Breit, B. Asymmetric Total Syntheses of (-)-Angustureine and (-)-Cuspareine via Rhodium-Catalyzed Hydroamination. Org. Lett. 2020, 22, 565-568.

[14]. Jones, M. R.; Fast, C. D.; Schley, N. D. Iridium-Catalyzed $\mathrm{sp}^{3} \mathrm{C}-\mathrm{H}$ Borylation in Hydrocarbon Solvent Enabled by 2,2'-Dipyridylarylmethane Ligands. J. Am. Chem. Soc. 2020, 142, 6488-6492.

[15]. Vogels, C. M.; Decken, A.; Westcott, S. A. Catalyzed Hydroboration of Nitrostyrenes and 4-Vinylaniline: A Mild and Selective Route to Aniline Derivatives Containing Boronate Esters. Tetrahedron Lett. 2006, 47, 24192422.

[16]. Liu, X.; Longwitz, L.; Spiegelberg, B.; Tönjes, J.; Beweries, T.; Werner, T. Erbium-Catalyzed Regioselective Isomerization-Cobalt-Catalyzed Transfer Hydrogenation Sequence for the Synthesis of anti-Markovnikov Alcohols from Epoxides under Mild Conditions. ACS Catal. 2020, 10, 13659-13667.

[17]. Hashimoto, T.; Shiota, K.; Yamaguchi, Y. Selective Synthesis of Secondary Alkylboronates: Markovnikov-Selective Hydroboration of Vinylarenes with Bis(pinacolato)diboron Catalyzed by a Nickel Pincer Complex. Org. Lett. 2020, 22, 4033-4037.

[18]. Chen, X.; Cheng, Z.; Lu, Z. Iron-Catalyzed, Markovnikov-Selective Hydroboration of Styrenes. Org. Lett. 2017, 19, 969-971.

[19]. DiBenedetto, T. A.; Parsons, A. M.; Jones, W. D. Markovnikov-Selective Hydroboration of Olefins Catalyzed by a Copper N-Heterocyclic Carbene Complex. Organometallics 2019, 38, 3322-3326.

[20]. Guo, J.; Chen, J.; Lu, Z. Cobalt-Catalyzed Asymmetric Hydroboration of Aryl Ketones with Pinacolborane. Chem. Commun. 2015, 51, 5725-5727.

[21]. Xuan, Q.; Zhao, C.; Song, Q. Umpolung of Protons from $\mathrm{H}_{2} \mathrm{O}$ : A MetalFree Chemoselective Reduction of Carbonyl Compounds via $\mathrm{B}_{2} \mathrm{pin}_{2} / \mathrm{H}_{2} \mathrm{O}$ Systems. Org. Biomol. Chem. 2017, 15, 5140-5144.

[22]. Yang, C.; Gao, Y.; Bai, S.; Jiang, C.; Qi, X. Chemoselective Cross-Coupling of gem-Borazirconocene Alkanes with Aryl Halides. J. Am. Chem. Soc. 2020, 142, 11506-11513.

[23].Ye, T.-N.; Lu, Y.; Li, J.; Nakao, T.; Yang, H.; Tada, T.; Kitano, M.; Hosono, H. 
Copper-Based Intermetallic Electride Catalyst for Chemoselective Hydrogenation Reactions. J. Am. Chem. Soc. 2017, 139, 17089-17097.

[24].Krishnan, S.; Patel, P. N.; Balasubramanian, K. K.; Chadha, A. Yeast supported gold nanoparticles: an efficient catalyst for the synthesis of commercially important aryl amines. New J. Chem. 2021, 45, 1915-1923.

[25]. Huang, J.; Yan, W.; Tan, C.; Wu, W.; Jiang, H. Palladium-Catalyzed Regioselective Hydroboration of Aryl Alkenes with B2pinz. Chem. Commun. 2018, 54, 1770-1773.

[26]. Vijaykumar, G.; Bhunia, M.; Mandal, S. K. A Phenalenyl-Based Nickel Catalyst for the Hydroboration of Olefins under Ambient Conditions. Dalton Trans. 2019, 48, 5779-5784.

[27]. Roesner, S.; Blair, D. J.; Aggarwal, V. K. Enantioselective Installation of Adjacent Tertiary Benzylic Stereocentres using Lithiation-BorylationProtodeboronation Methodology. Application to the Synthesis of Bifluranol and Fluorohexestrol. Chem. Sci. 2015, 6, 3718-3723.

[28]. Ooi, T.; Sakai, D.; Takada, M.; Komatsu, N.; Maruoka, K. o-(oIodophenyl)phenylselenenyl Ethers/t-BuLi System as an Alkyllithium Equivalent with Structural Variations. Synlett 2001, 2001, 0791-0792.

[29]. Krabbe, S. W.; Hatcher, M. A.; Bowman, R. K.; Mitchell, M. B.; McClure, M. S.; Johnson, J. S. Copper-Catalyzed Asymmetric Hydrogenation of Aryl and Heteroaryl Ketones. Org. Lett. 2013, 15, 4560-4563.

[30]. Gao, G.; Yan, J.; Yang, K.; Chen, F.; Song, Q. Base-controlled Highly Selective Synthesis of Alkyl 1,2-Bis(boronates) or 1,1,2-Tris(boronates) from Terminal Alkynes. Green Chem. 2017, 19, 3997-4001.

[31]. Yukimori, D.; Nagashima, Y.; Wang, C.; Muranaka, A.; Uchiyama, M. Quadruple Borylation of Terminal Alkynes. J. Am. Chem. Soc. 2019, 141, 9819-9822.

[32]. Gao, G.; Kuang, Z.; Song, Q. Functionalized Geminal-Diborylalkanes from Various Electron-Deficient Alkynes and B2pin.. Org. Chem. Front. 2018, 5, 2249-2253.

[33]. Larouche-Gauthier, R.; Elford, T. G.; Aggarwal, V. K. Ate Complexes of Secondary Boronic Esters as Chiral Organometallic-Type Nucleophiles for Asymmetric Synthesis. J. Am. Chem. Soc. 2011, 133, 16794-16797.

[34]. Ai, H.-J.; Wang, H.; Li, C.-L.; Wu, X.-F. Rhodium-Catalyzed Carbonylative 
Coupling of Alkyl Halides with Phenols under Low CO Pressure. ACS Catal. 2020, 10, 5147-5152.

[35]. Sadhu, K. M.; Matteson, D. S. (Chloromethyl)lithium: Efficient Generation and Capture by Boronic Esters and a Simple Preparation of Diisopropyl (chloromethyl)boronate. Organometallics 1985, 4, 1687-1689.

[36]. Atack, T. C.; Cook, S. P. Manganese-Catalyzed Borylation of Unactivated Alkyl Chlorides. J. Am. Chem. Soc. 2016, 138, 6139-6142.

[37]. Yang, C.-T.; Zhang, Z.-Q.; Tajuddin, H.; Wu, C.-C.; Liang, J.; Liu, J.-H.; Fu, Y.; Czyzewska, M.; Steel, P. G.; Marder, T. B.; Liu, L. Alkylboronic Esters from Copper-Catalyzed Borylation of Primary and Secondary Alkyl Halides and Pseudohalides. Angew. Chem. Int. Ed. 2012, 51, 528-532.

[38]. Manvar, A.; Fleming, P.; O'Shea, D. F. General Ambient Temperature Benzylic Metalations Using Mixed-Metal Li/K-TMP Amide. J. Org. Chem. 2015, 80, 8727-8738.

[39]. Bonet, A.; Odachowski, M.; Leonori, D.; Essafi, S.; Aggarwal, V. K. Enantiospecific sp ${ }^{2}-\mathrm{sp}^{3}$ Coupling of Secondary and Tertiary Boronic Esters. Nat. Chem. 2014, 6, 584-589.

[40]. Lauer, M. G.; Henderson, W. H.; Awad, A.; Stambuli, J. P. PalladiumCatalyzed Reactions of Enol Ethers: Access to Enals, Furans, and Dihydrofurans. Org. Lett. 2012, 14, 6000-6003.

[41]. Sonawane, R. P.; Jheengut, V.; Rabalakos, C.; Larouche-Gauthier, R.; Scott, H. K.; Aggarwal, V. K. Enantioselective Construction of Quaternary Stereogenic Centers from Tertiary Boronic Esters: Methodology and Applications. Angew. Chem. Int. Ed. 2011, 50, 3760-3763.

[42]. Zhang, S.; Bedi, D.; Cheng, L.; Unruh, D. K.; Li, G.; Findlater, M. Cobalt(II)Catalyzed Stereoselective Olefin Isomerization: Facile Access to Acyclic Trisubstituted Alkenes. J. Am. Chem. Soc. 2020, 142, 8910-8917.

[43]. Oikawa, K.; Itoh, S.; Yano, H.; Kawasaki, H.; Obora, Y. Preparation and Use of DMF-Stabilized Iridium Nanoclusters as Methylation Catalysts Using Methanol as the $\mathrm{C}_{1}$ Source. Chem. Commun. 2017, 53, 1080-1083.

[44]. Imao, D.; Glasspoole, B. W.; Laberge, V. S.; Crudden, C. M. Cross Coupling Reactions of Chiral Secondary Organoboronic Esters With Retention of Configuration. J. Am. Chem. Soc. 2009, 131, 5024-5025.

[45]. Chang, Y.; Yesilcimen, A.; Cao, M.; Zhang, Y.; Zhang, B.; Chan, J. Z.; Wasa, 
M. Catalytic Deuterium Incorporation within Metabolically Stable $\beta$ Amino C-H Bonds of Drug Molecules. J. Am. Chem. Soc. 2019, 141, 1457014575.

[46]. Chai, J. Da; Head-Gordon, M. Long-Range Corrected Hybrid Density Functionals with Damped Atom-Atom Dispersion Corrections. Phys. Chem. Chem. Phys. 2008, 10, 6615-6620.

[47]. Wadt, W. R.; Hay, P. J. Ab Initio Effective Core Potentials for Molecular Calculations. Potentials for Main Group Elements Na to Bi. J. Chem. Phys. 1985, 82, 284-298.

[48]. Höllwarth, A.; Böhme, M.; Dapprich, S.; Ehlers, A. W.; Gobbi, A.; Jonas, V.; Köhler, K. F.; Stegmann, R.; Veldkamp, A.; Frenking, G. A Set of DPolarization Functions for Pseudo-Potential Basis Sets of the Main Group Elements AlBi and f-Type Polarization Functions for $\mathrm{Zn}, \mathrm{Cd}, \mathrm{Hg}$. Chem. Phys. Lett. 1993, 208, 237-240.

[49]. Ehlers, A. W.; Böhme, M.; Dapprich, S.; Gobbi, A.; Höllwarth, A.; Jonas, V.; Köhler, K. F.; Stegmann, R.; Veldkamp, A.; Frenking, G. A Set of FPolarization Functions for Pseudo-Potential Basis Sets of the Transition Metals Sc-Cu, Y-Ag and La-Au. Chem. Phys. Lett. 1993, 208, 237-240.

[50]. Fukui, K. The Path of Chemical Reactions - The IRC Approach. Acc. Chem. Res. 1981, 14, 363-368.

[51]. Frisch, M. J.; Trucks, G. W.; Schlegel, H. B.; Scuseria, G. E.; Robb, M. A.; Cheeseman, J. R.;Scalmani, G.; Barone, V.; Mennucci, B.; Petersson, G. A.; Nakatsuji, H.; Caricato, M.; Li, X.;Hratchian, H. P.; Izmaylov, A. F.; Bloino, J.; Zheng, G.; Sonnenberg, J. L.; Hada, M.; Ehara, M.;Toyota, K.; Fukuda, R.; Hasegawa, J.; Ishida, M.; Nakajima, T.; Honda, Y.; Kitao, O.; Nakai, H.; Vreven, T.; Montgomery, Jr., J. A.; Peralta, J. E.; Ogliaro, F.; Bearpark, M.; Heyd, J. J.; Brothers, E.;Kudin, K. N.; Staroverov, V. N.; Kobayashi, R.; Normand, J.; Raghavachari, K.; Rendell, A.; Burant,J. C.; Iyengar, S. S.; Tomasi, J.; Cossi, M.; Rega, N.; Millam, J. M.; Klene, M.; Knox, J. E.; Cross, J.B.; Bakken, V.; Adamo, C.; Jaramillo, J.; Gomperts, R.; Stratmann, R. E.; Yazyev, O.; Austin, A. J.;Cammi, R.; Pomelli, C.; Ochterski, J. W.; Martin, R. L.; Morokuma, K.; Zakrzewski, V. G.; Voth, G.A.; Salvador, P.; Dannenberg, J. J.; Dapprich, S.; Daniels, A. D.; Farkas, O. .; Foresman, J. B.; Ortiz,J. V.; Cioslowski, J.; Fox, D. J. Gaussian 09, Revision D.01; Gaussian, Inc.: 
Wallingford, CT, 2009. 


\section{NMR Spectra}

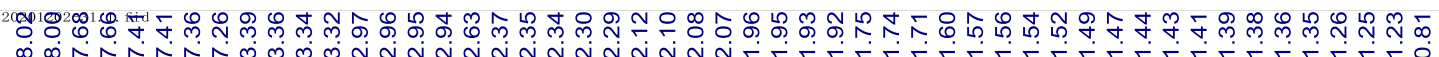

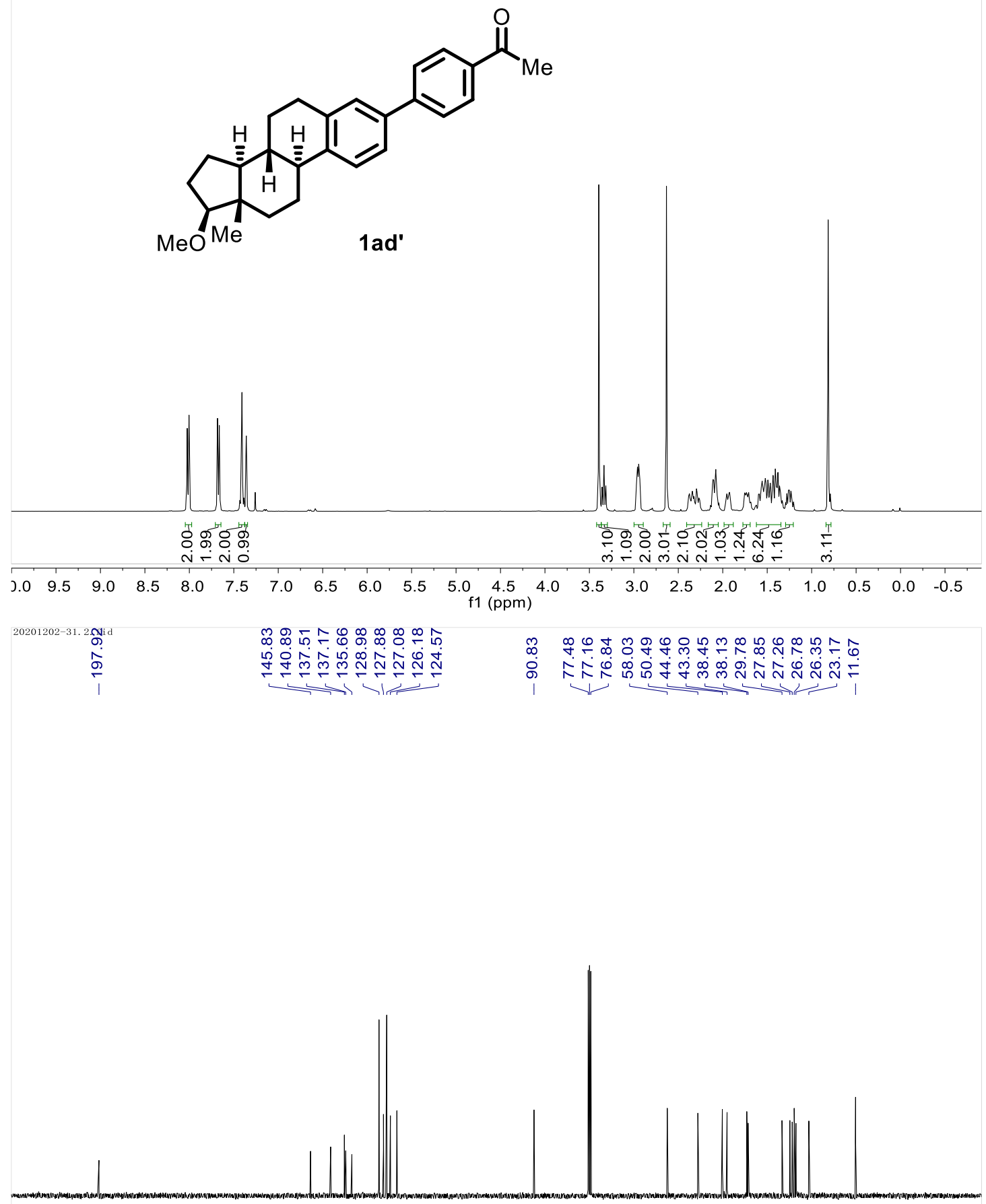

$\begin{array}{llllllllllllllllllllll}210 & 200 & 190 & 180 & 170 & 160 & 150 & 140 & 130 & 120 & \begin{array}{c}110 \\ \mathrm{f} 1\end{array}(\mathrm{ppm}) \\ (\mathrm{ppo}) & 90 & 70 & 60 & 50 & 40 & 30 & 20 & 10 & 0 & -10\end{array}$ 


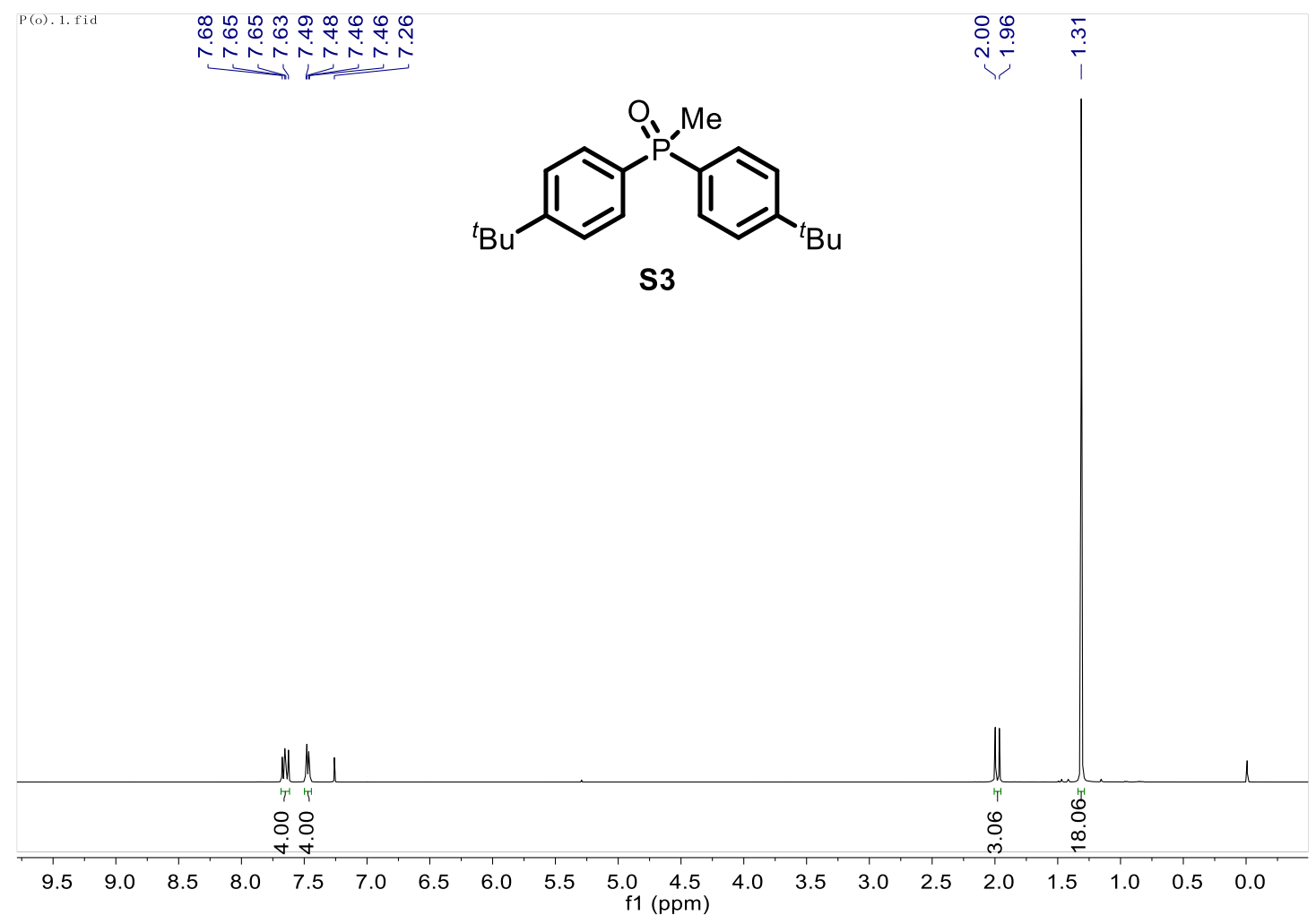

20210106-1-12.2.fid

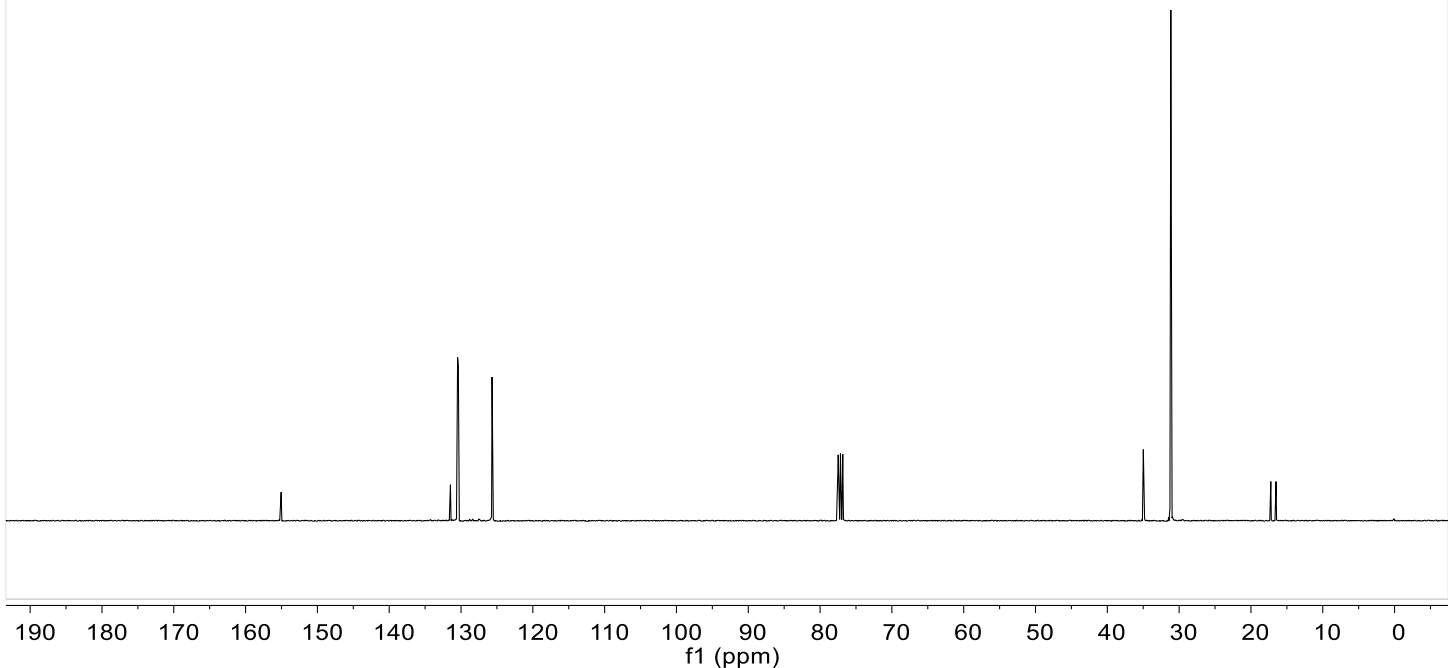




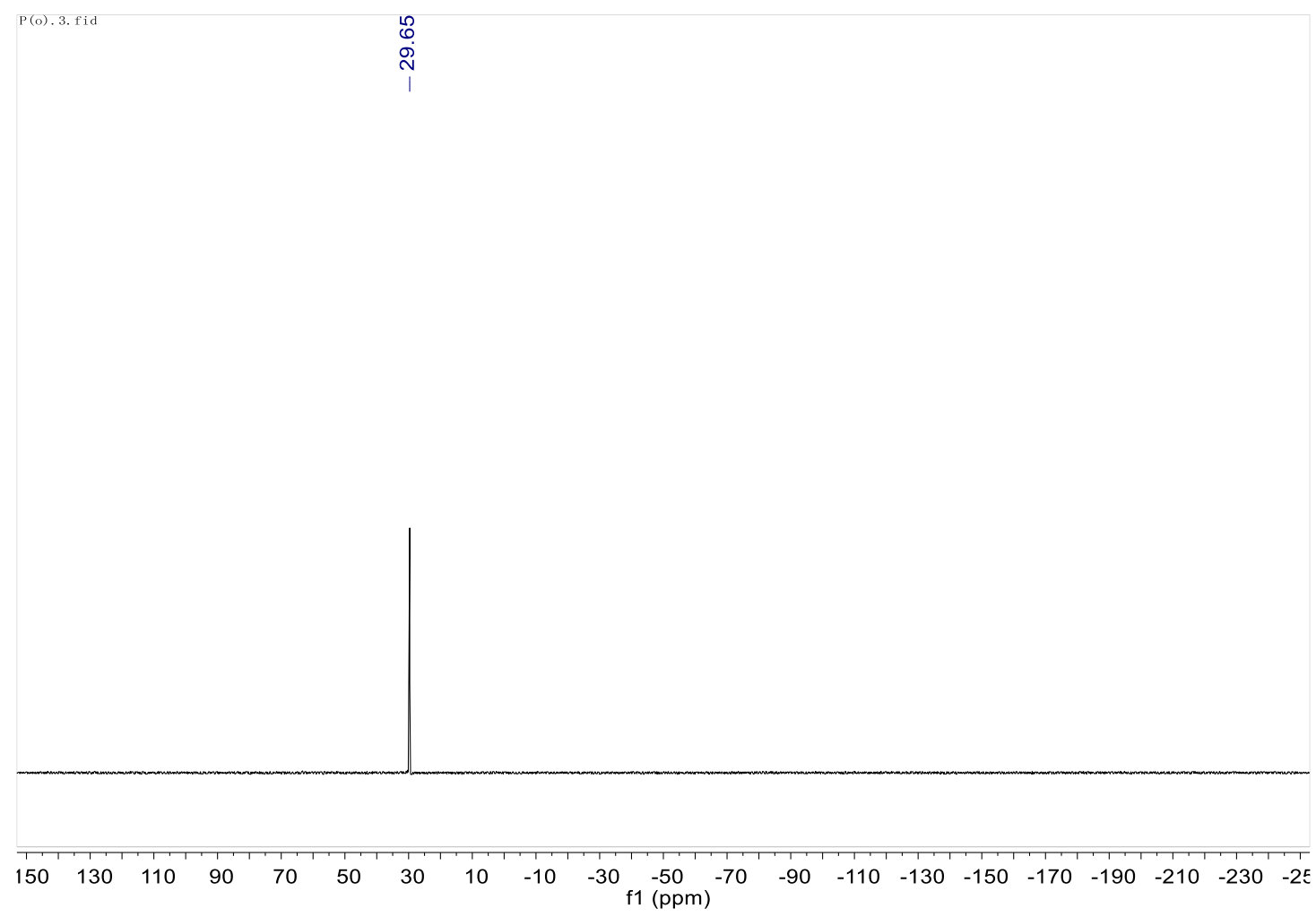



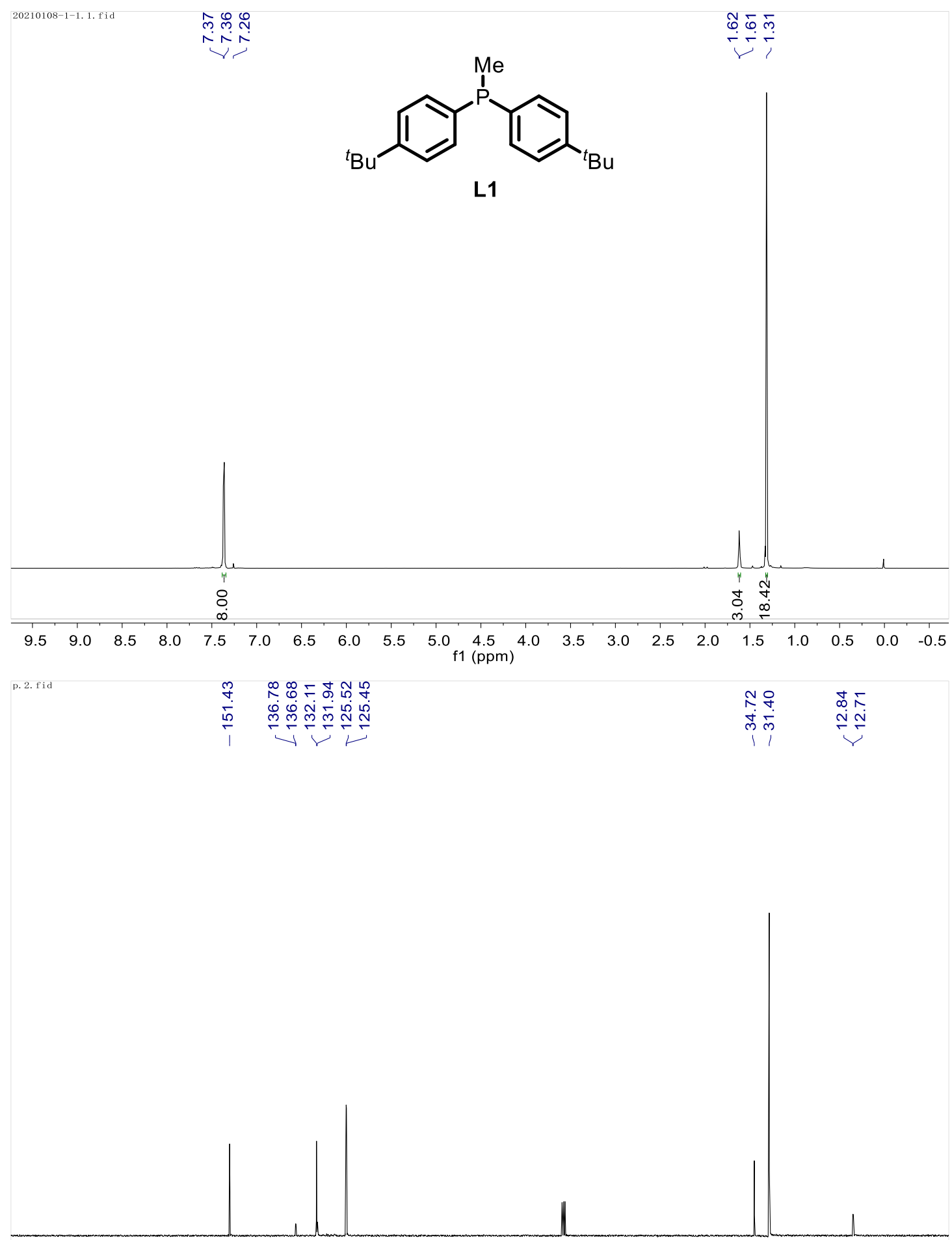

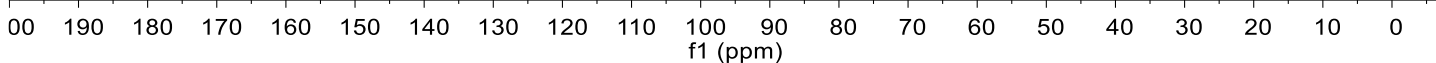




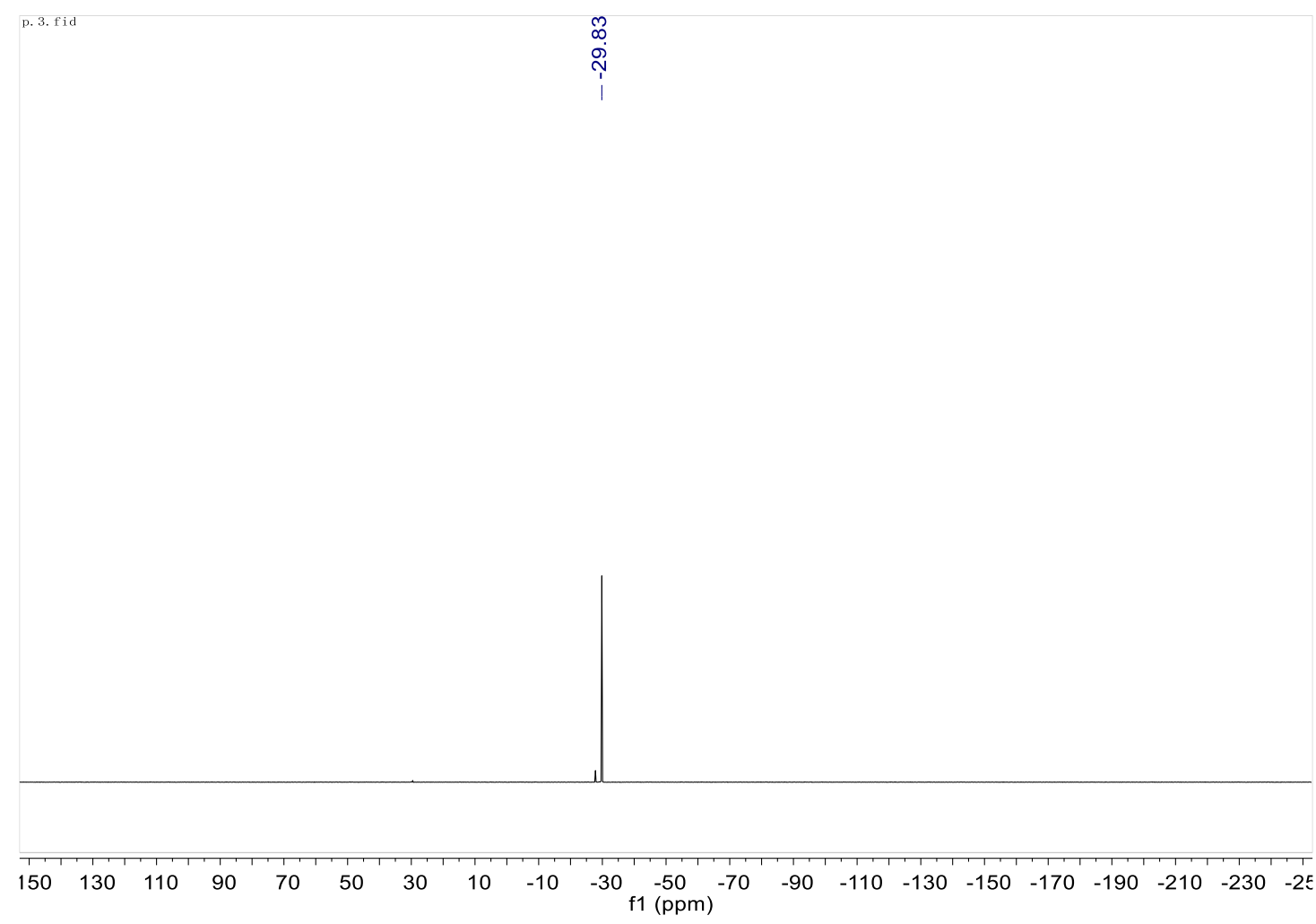




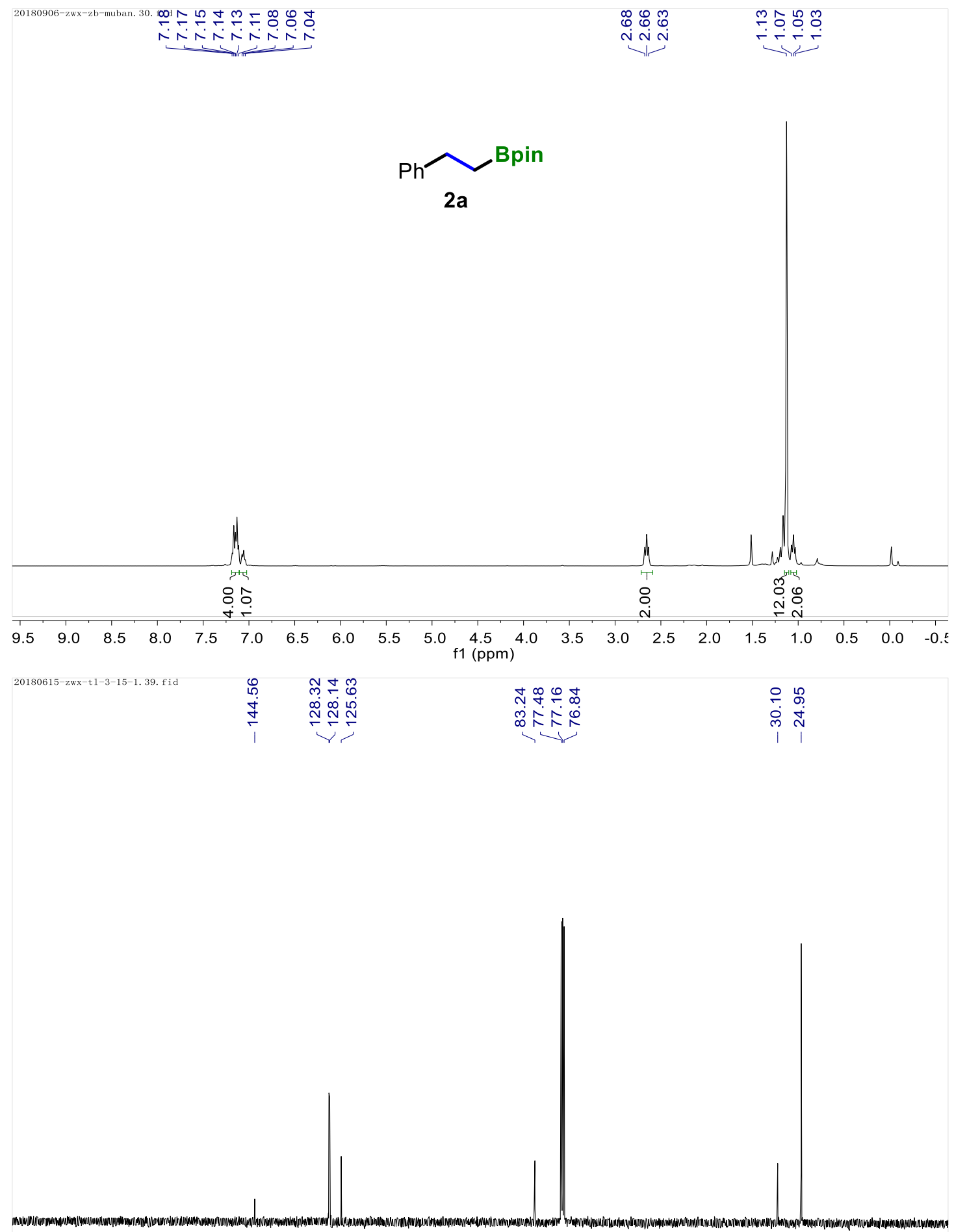

$\begin{array}{llllllllllllllllllll}190 & 180 & 170 & 160 & 150 & 140 & 130 & 120 & 110 & 100 & 90 & 80 & 70 & 60 & 50 & 40 & 30 & 20 & 10 & 0\end{array}$ f1 (ppm) 


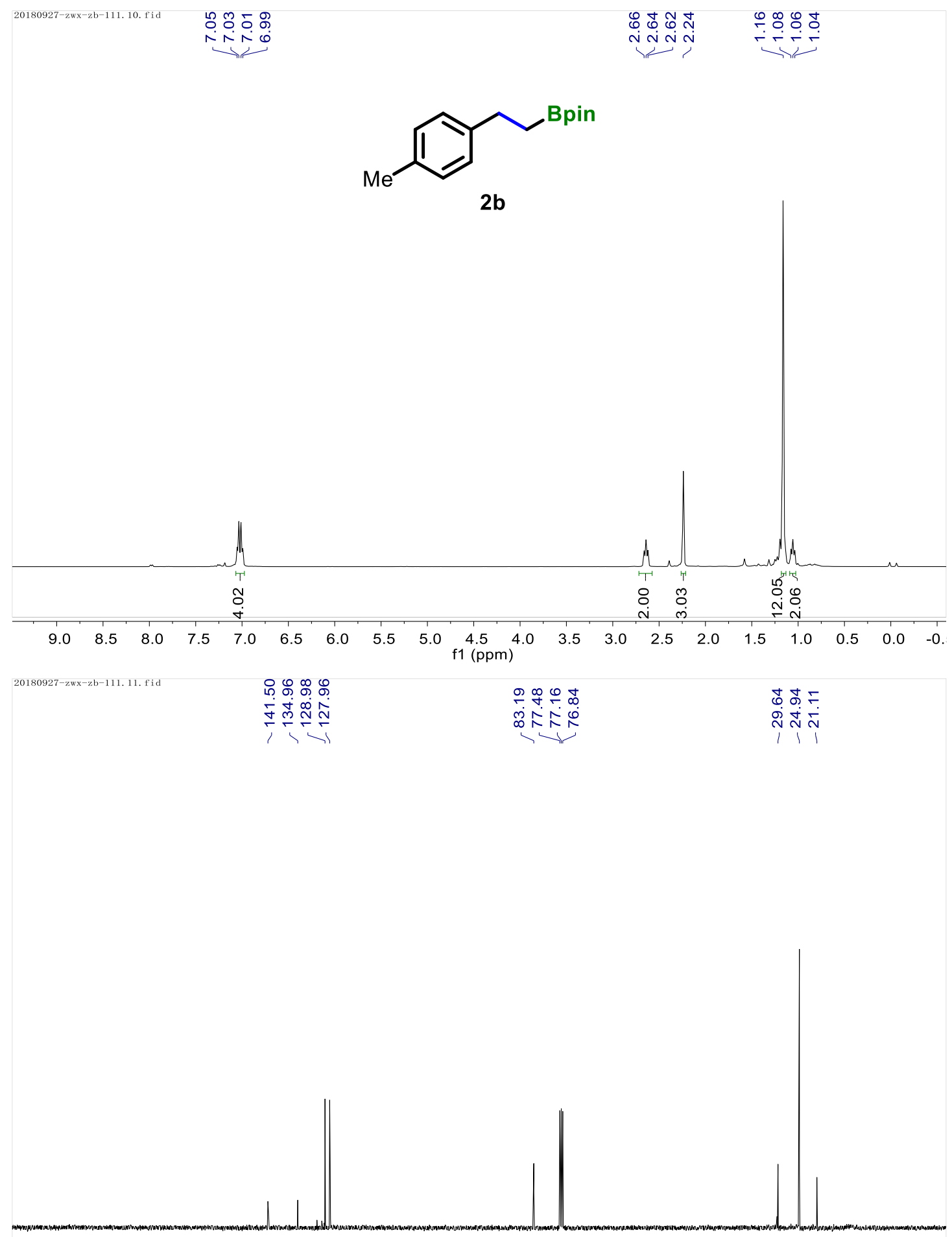

$\begin{array}{llllllllllllllllllll}190 & 180 & 170 & 160 & 150 & 140 & 130 & 120 & 110 & 100 & 90 & 80 & 70 & 60 & 50 & 40 & 30 & 20 & 10 & 0\end{array}$ f1 (ppm) 


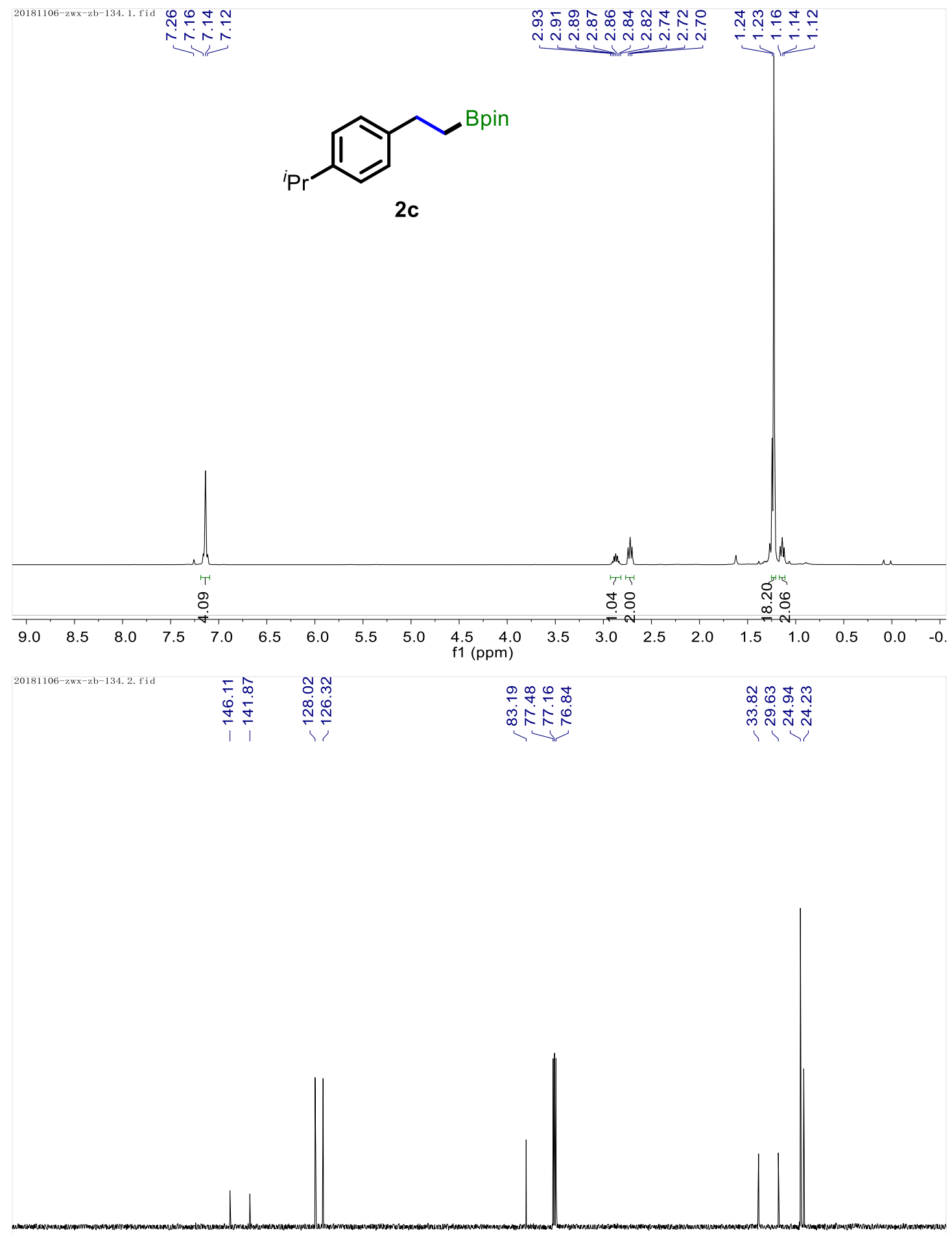

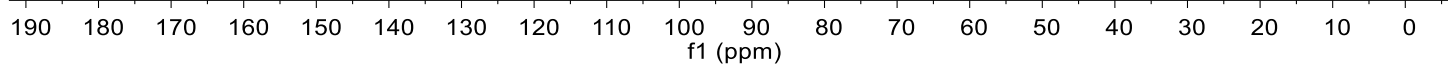




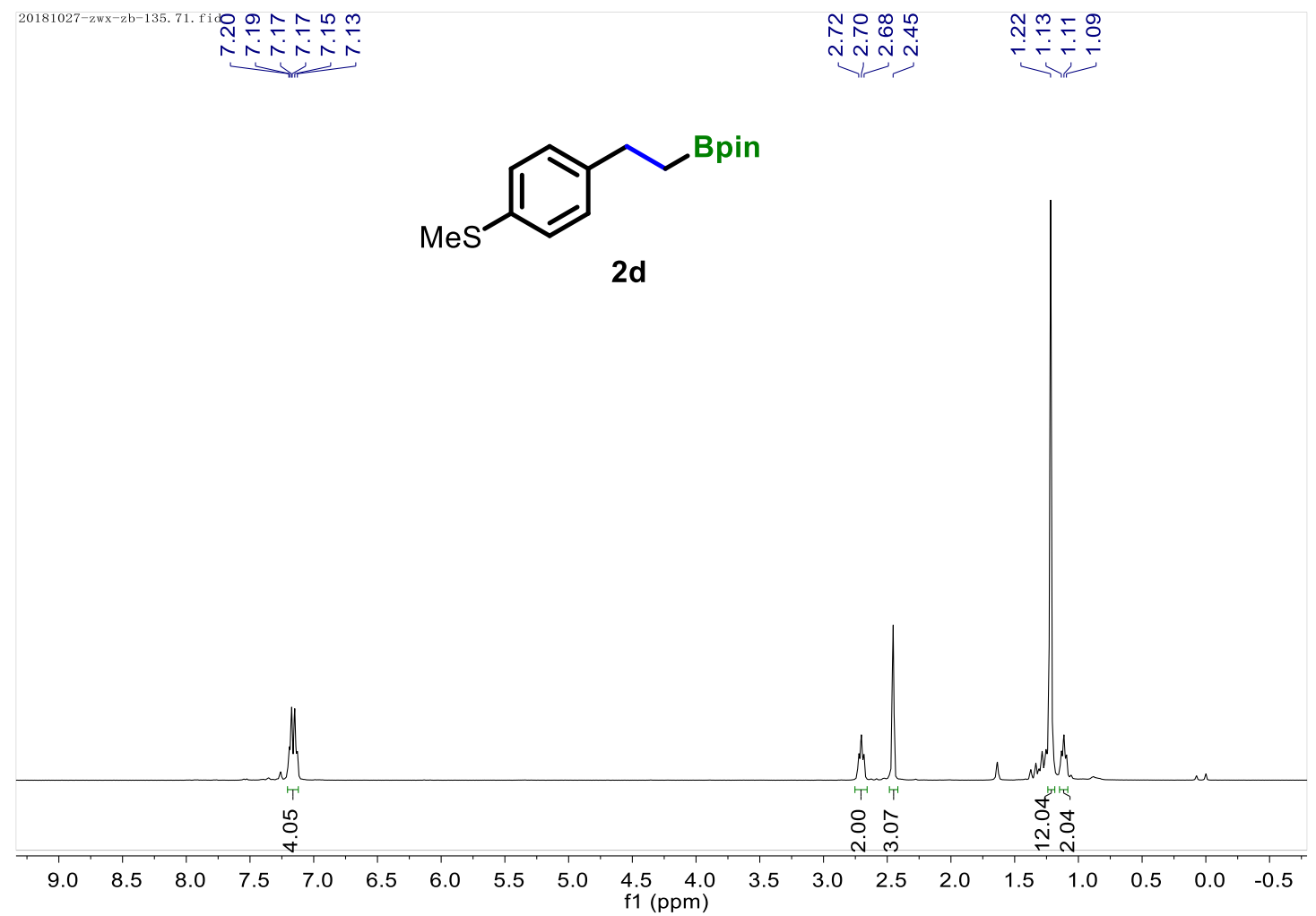

20181101-zwx-zb-135. 1. fid $\quad$ j

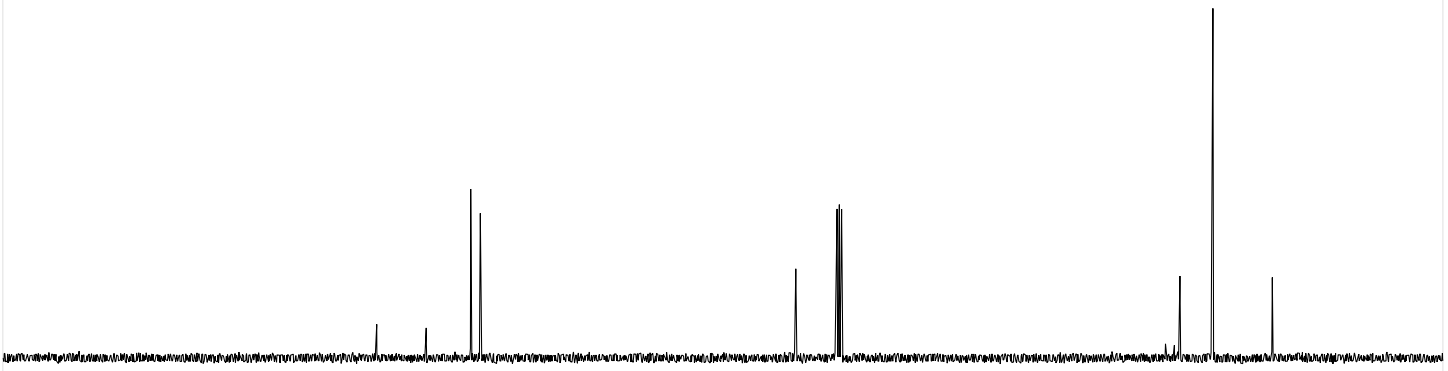

$\begin{array}{llllllllllllllllllll}190 & 180 & 170 & 160 & 150 & 140 & 130 & 120 & 110 & \begin{array}{c}100 \\ \mathrm{f} 1\end{array}(\mathrm{ppm}) & 90 & 70 & 60 & 50 & 40 & 30 & 20 & 10 & 0\end{array}$ 


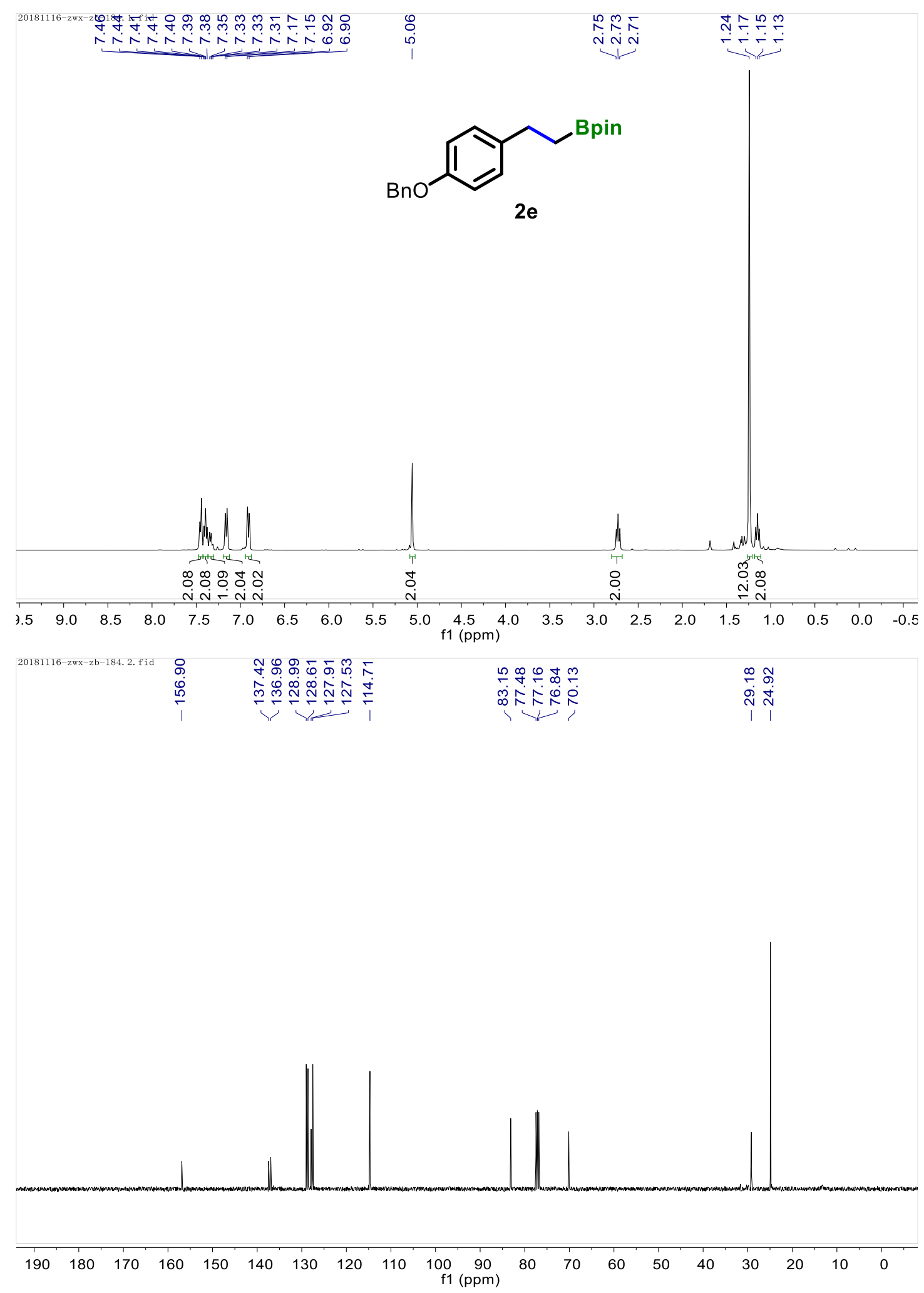



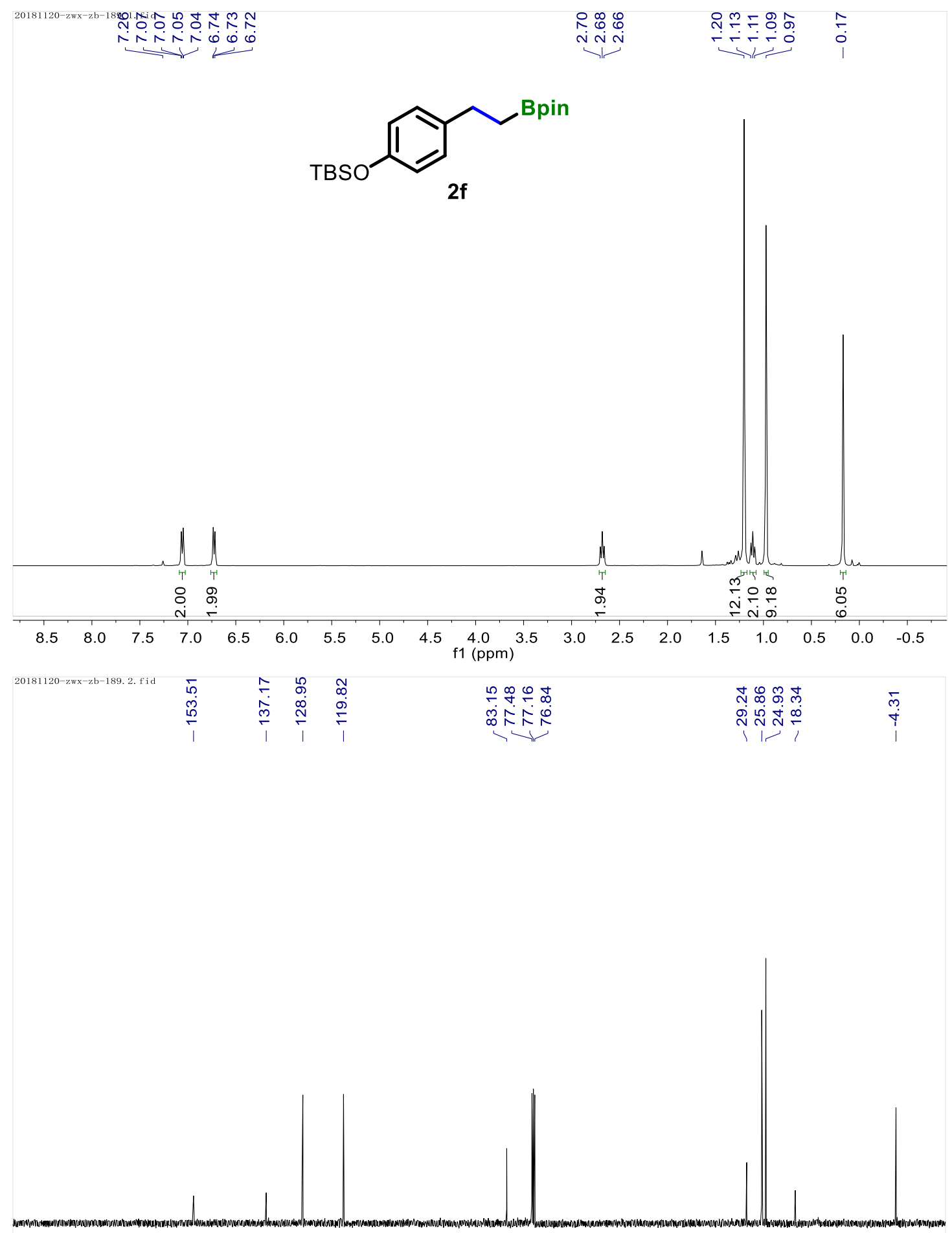

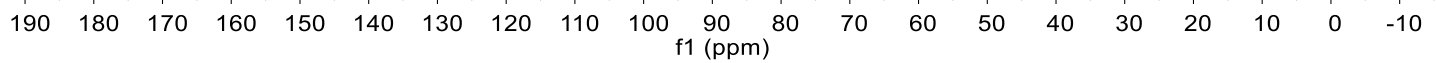




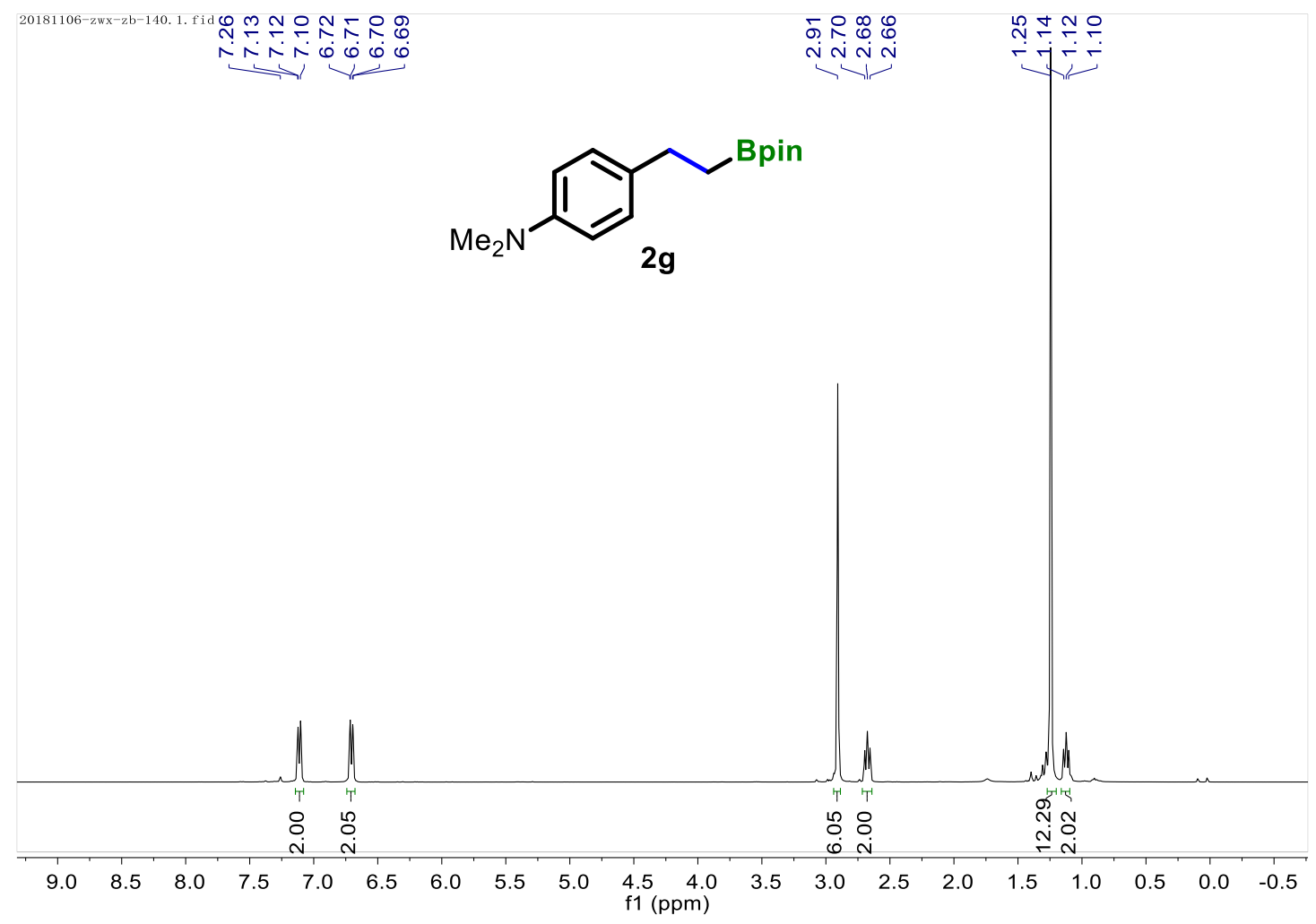

\begin{tabular}{|c|c|c|c|c|c|c|}
\hline $20181106-z w x-z b-140.2 . f i d ~$ & $\begin{array}{l}\stackrel{0}{0} \\
\dot{0} \\
\stackrel{+}{\leftarrow} \\
\text { I }\end{array}$ & 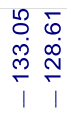 & $\begin{array}{l}\bar{N} \\
\stackrel{m}{\sigma}\end{array}$ & 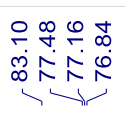 & $\frac{\stackrel{60}{\leftarrow}}{\dot{\tau}}$ & 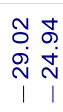 \\
\hline
\end{tabular}

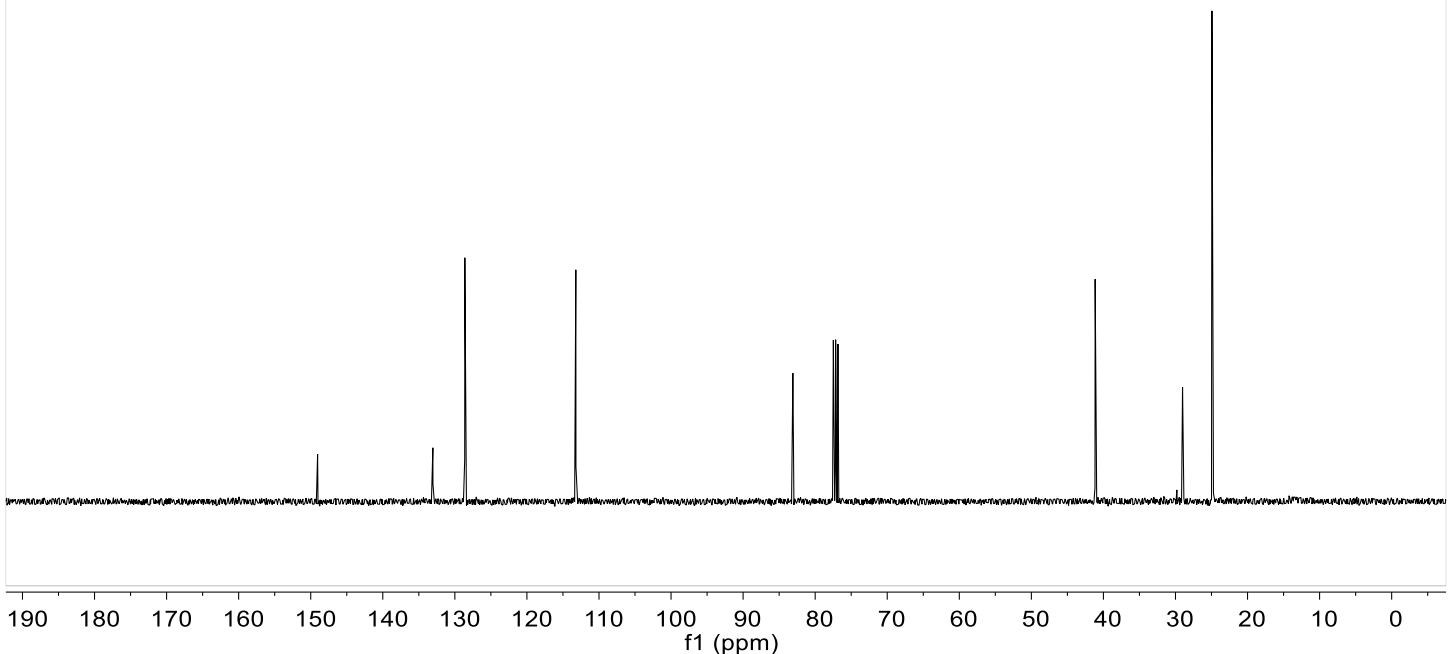




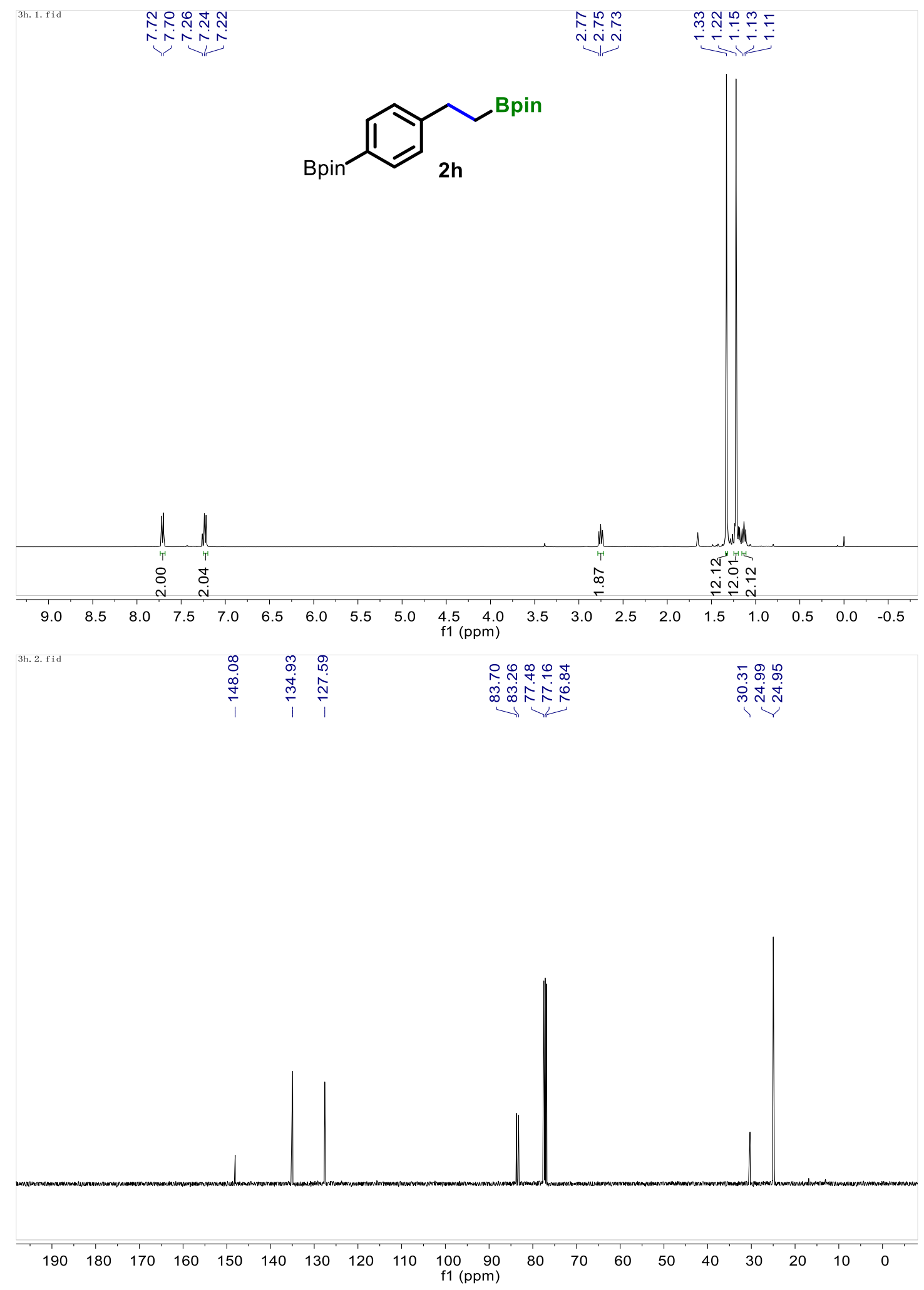




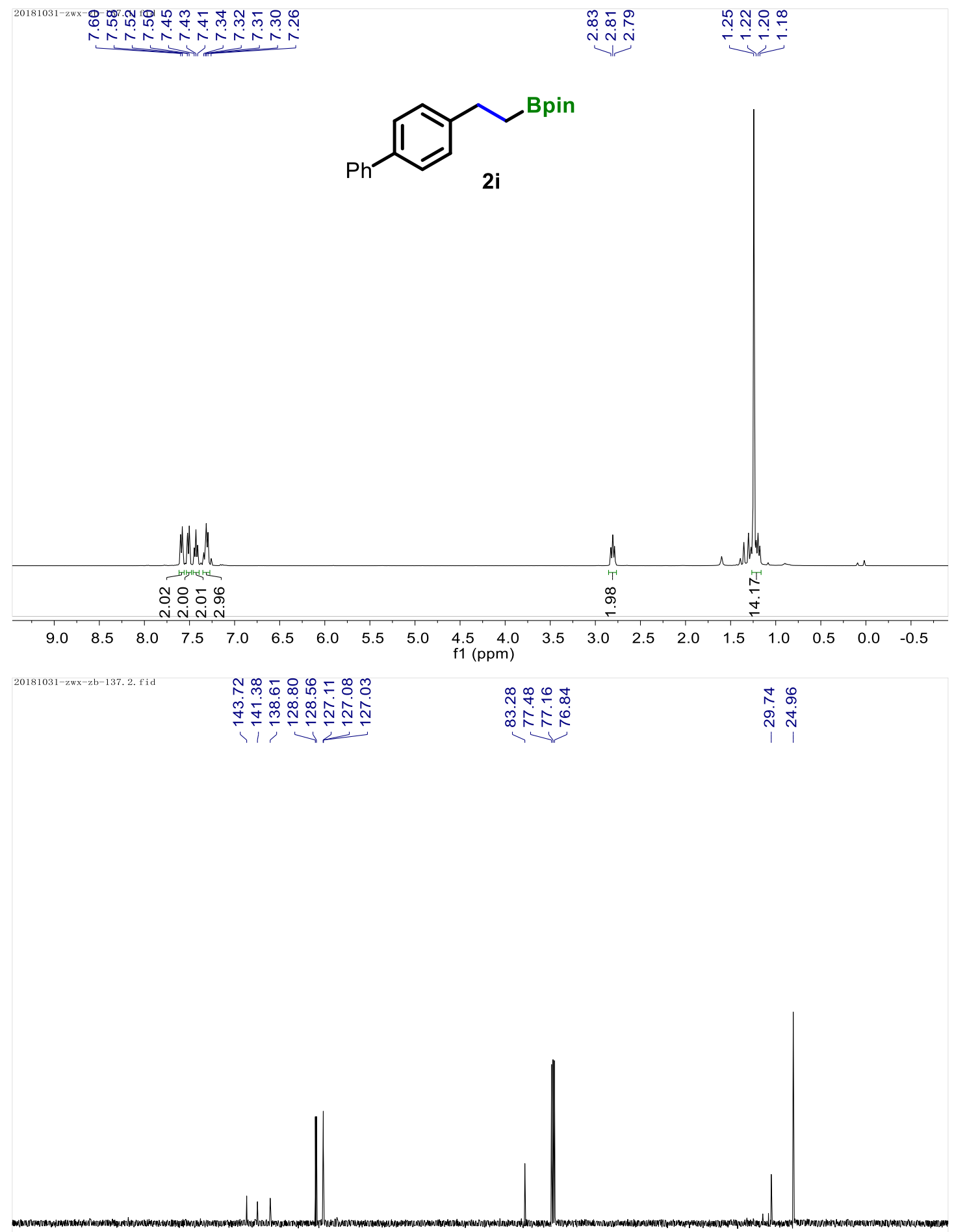

$\begin{array}{lllllllllllllllllll}190 & 180 & 170 & 160 & 150 & 140 & 130 & 120 & 110 & \begin{array}{c}100 \\ \mathrm{f} 1(\mathrm{ppm})\end{array} & 80 & 70 & 60 & 50 & 40 & 30 & 20 & 10 & 0\end{array}$ 


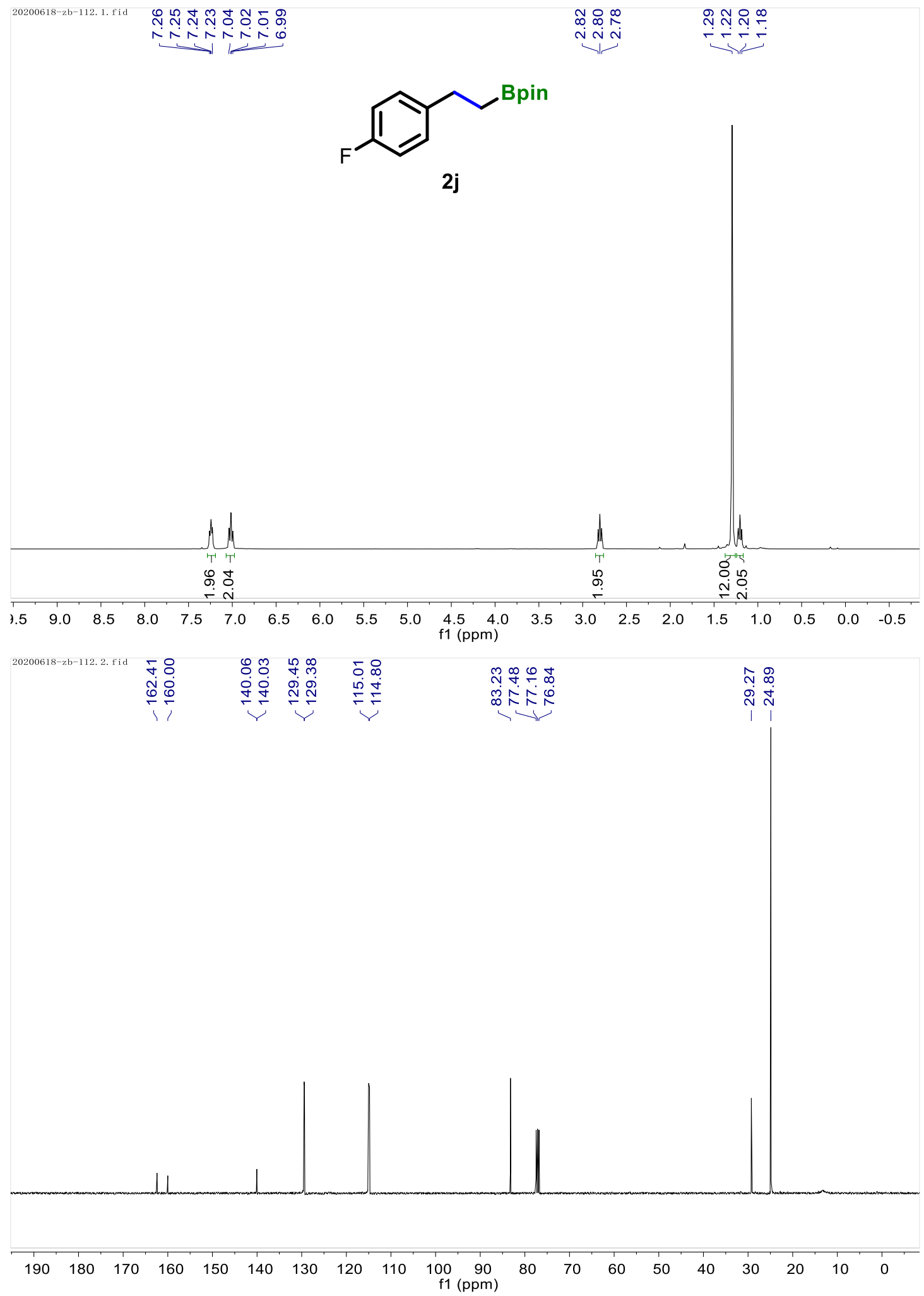




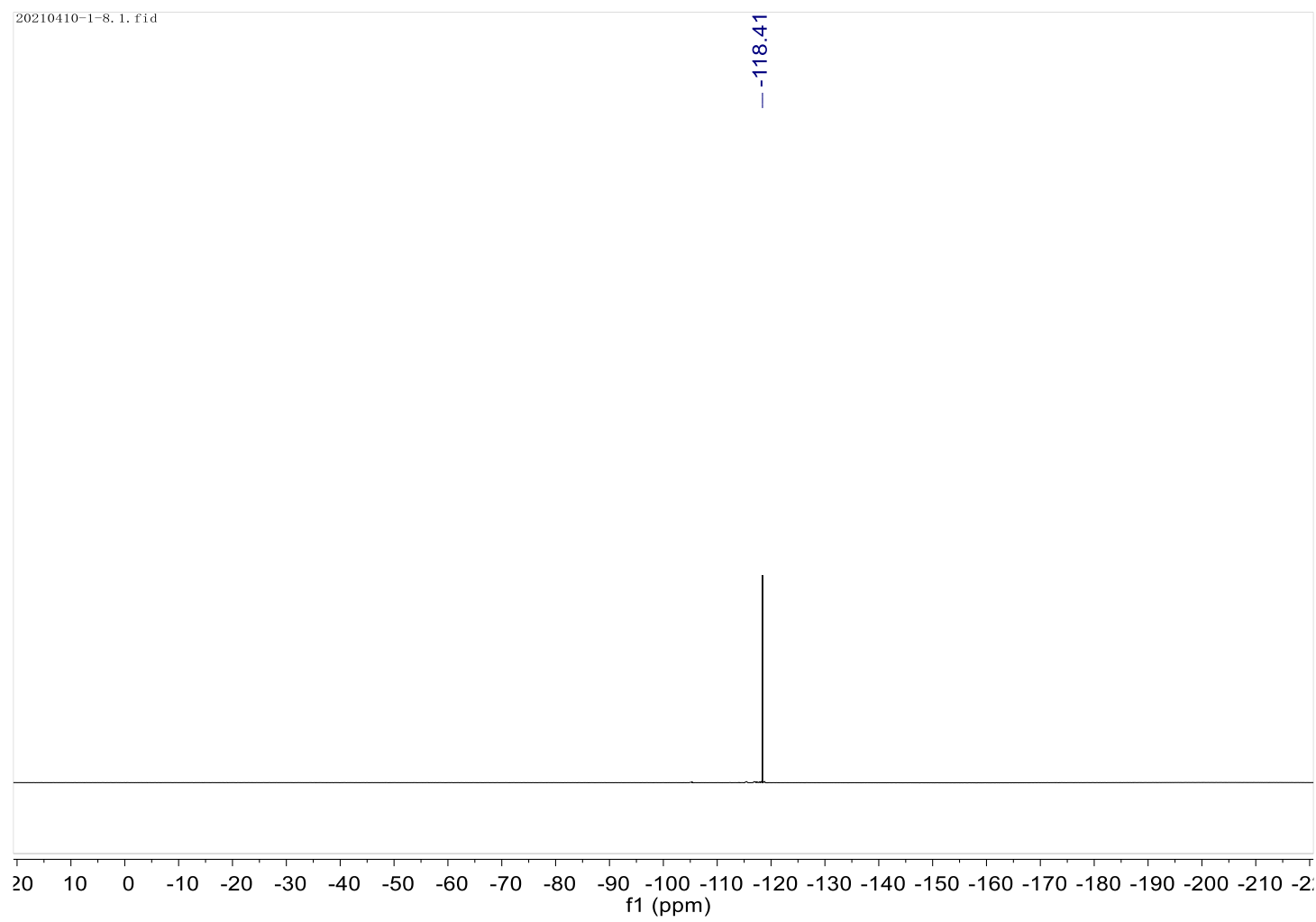




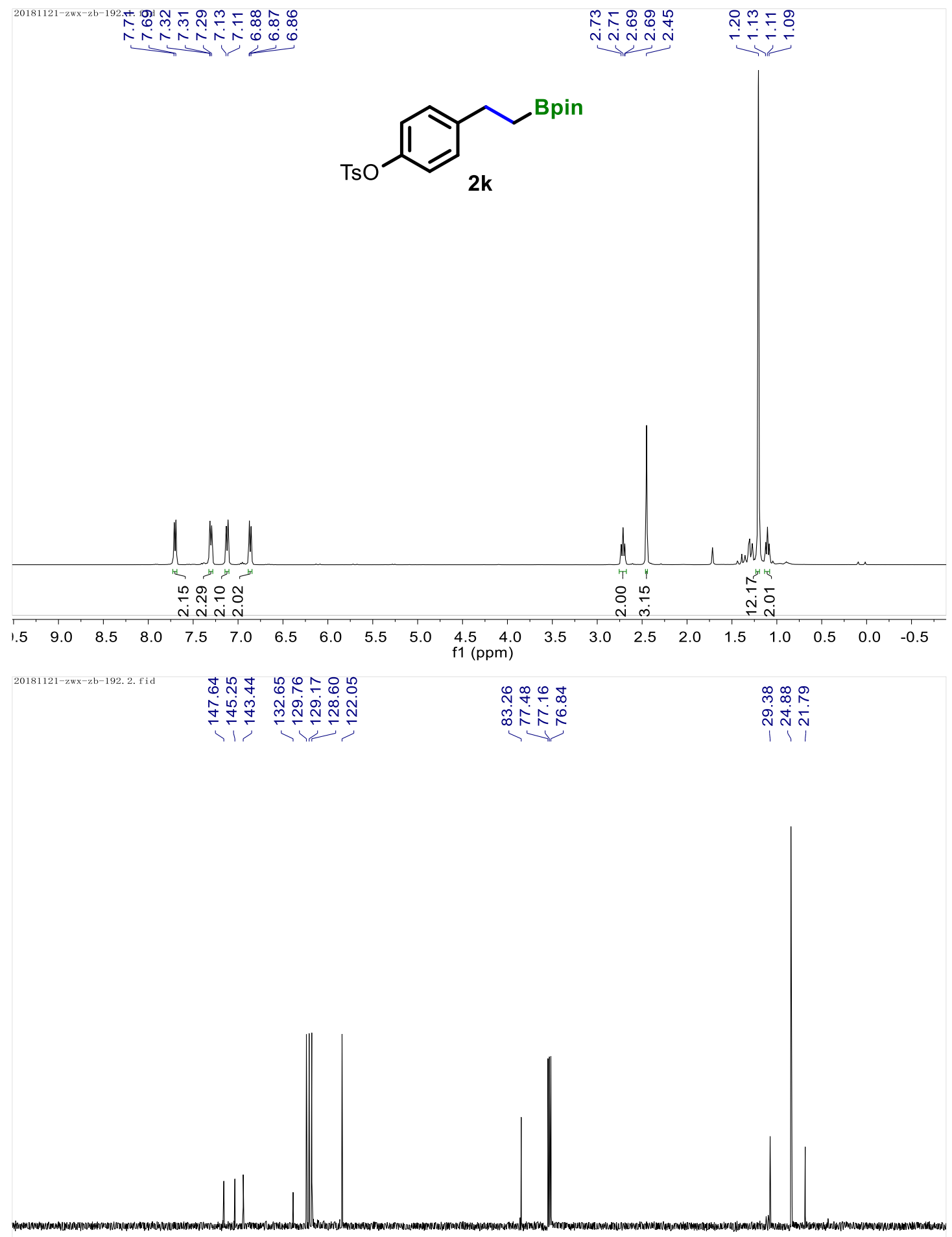

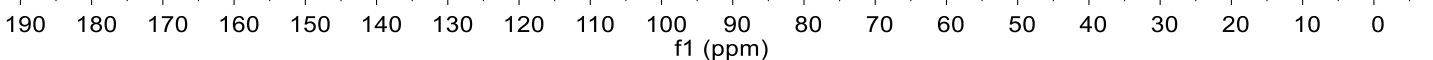




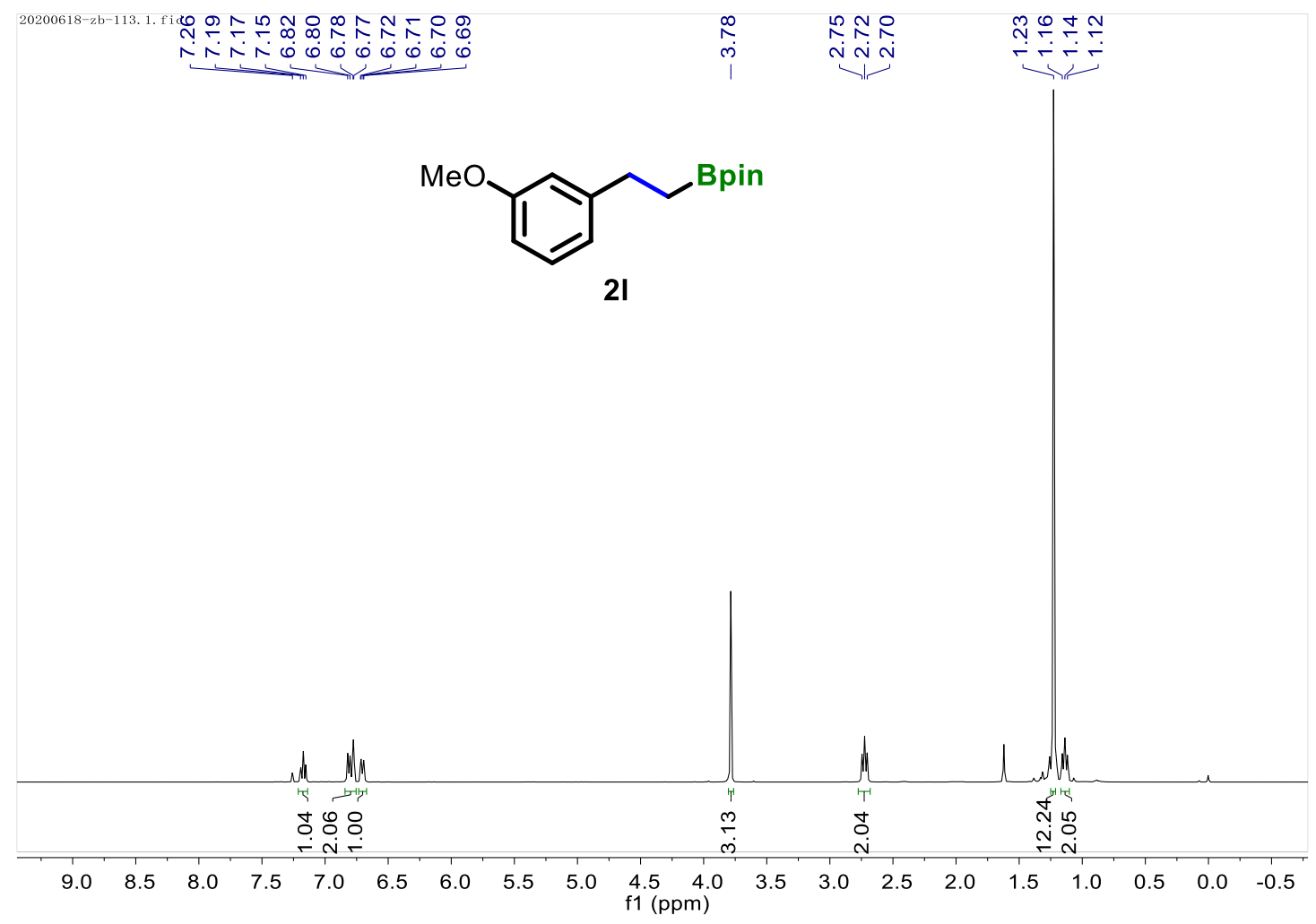

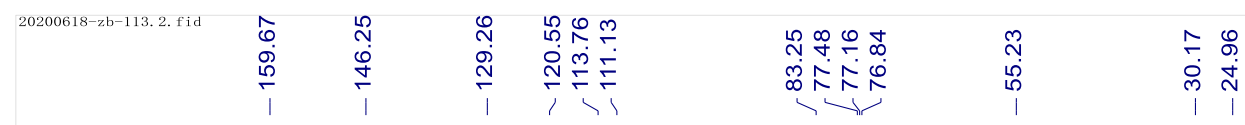

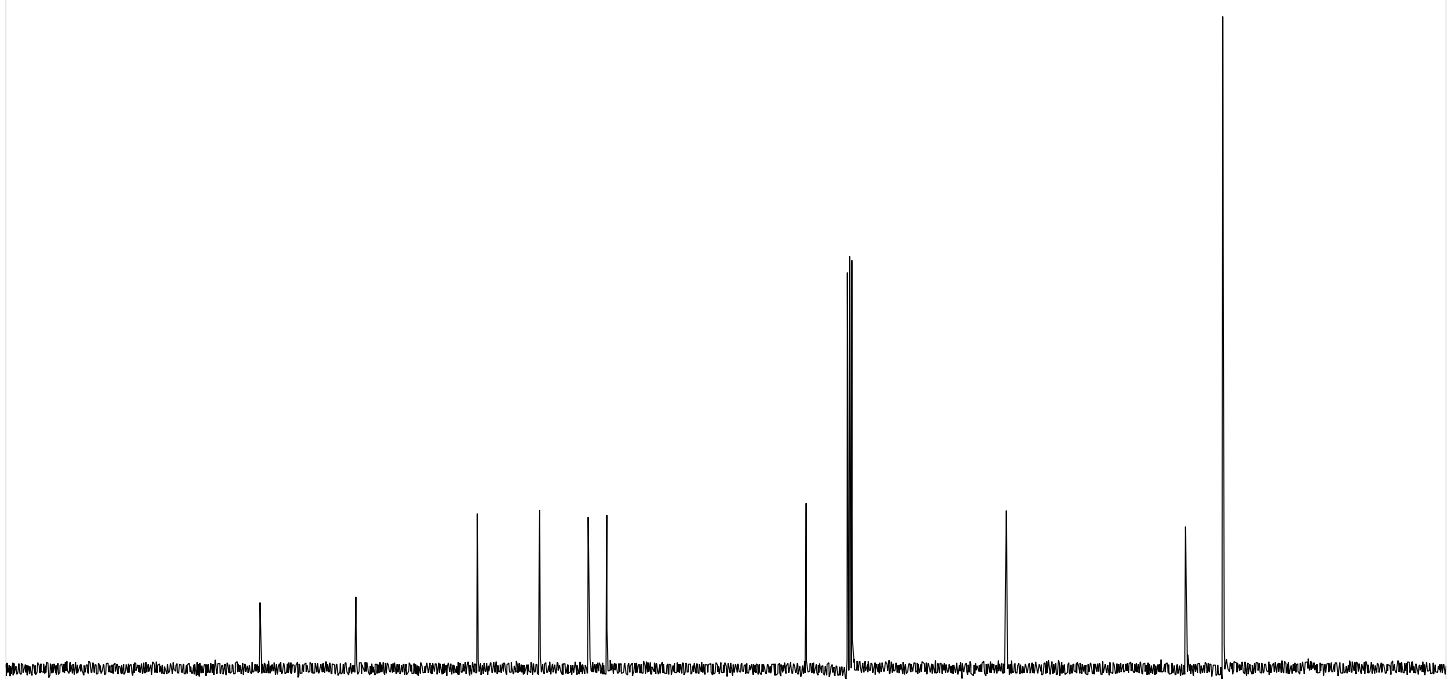

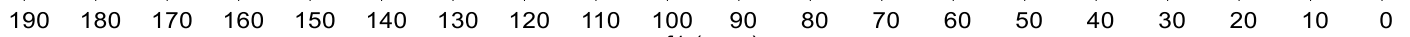
f1 (ppm) 

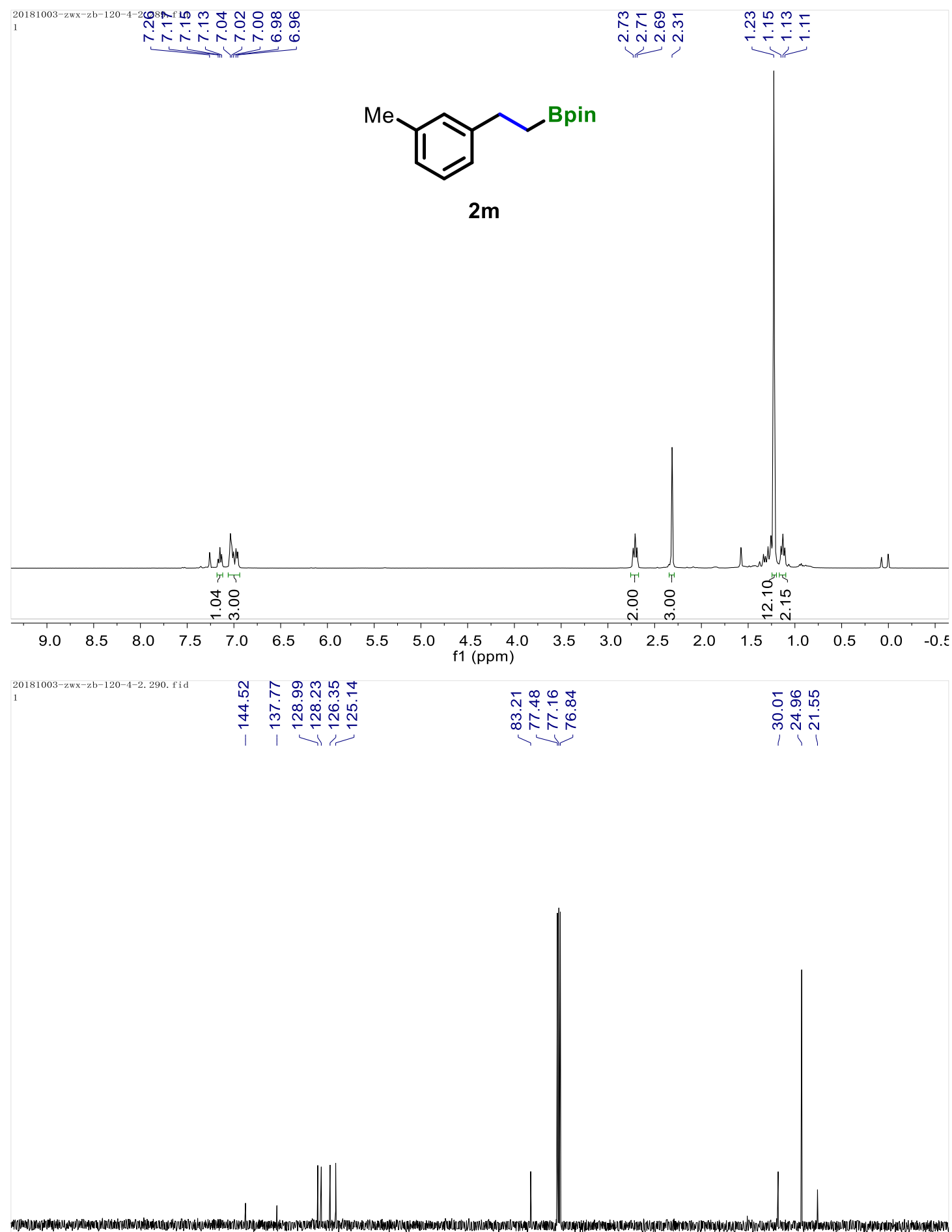

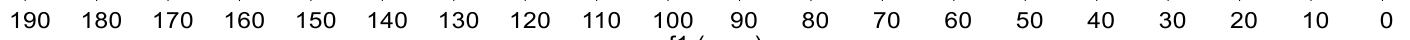
f1 (ppm) 

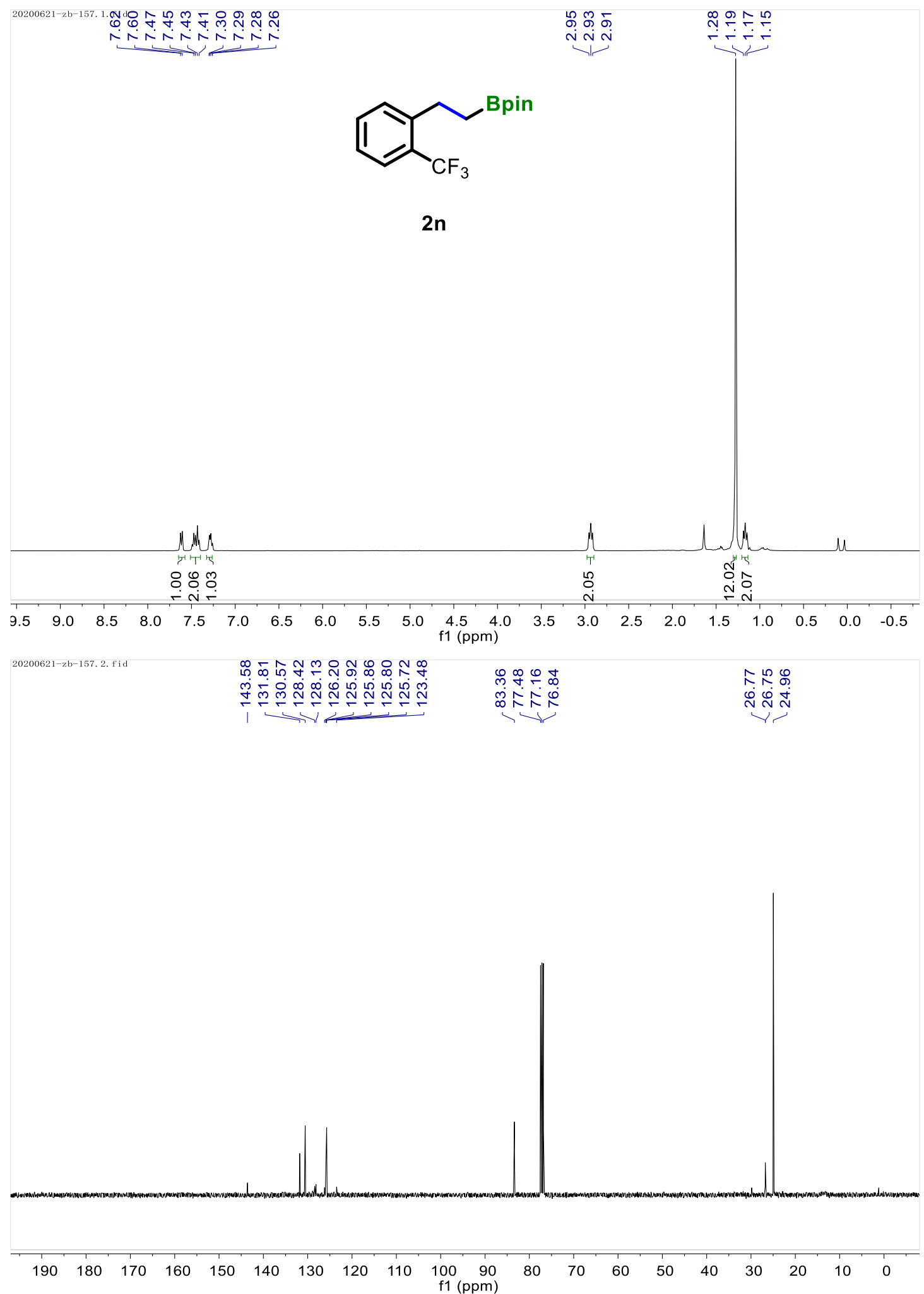


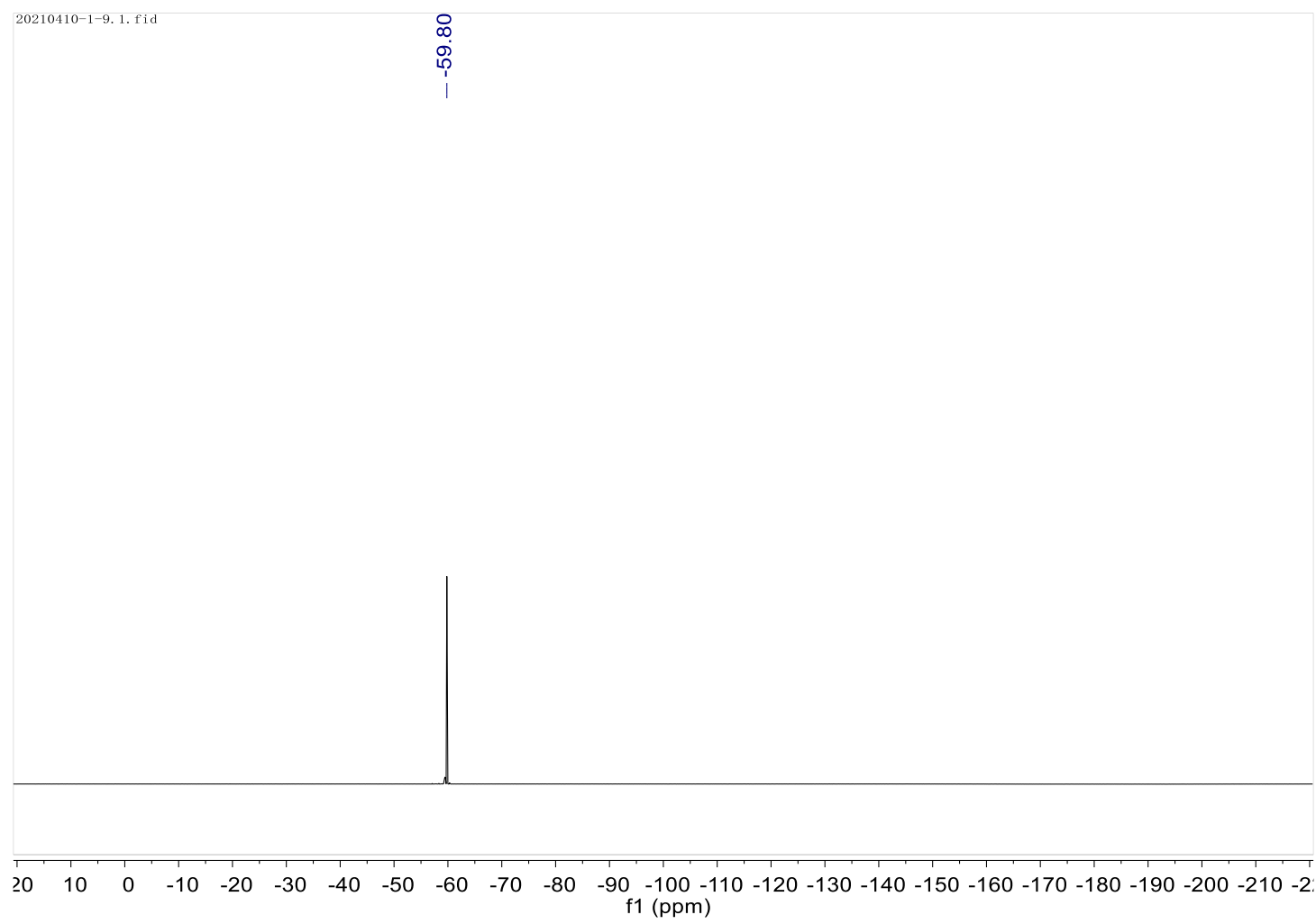



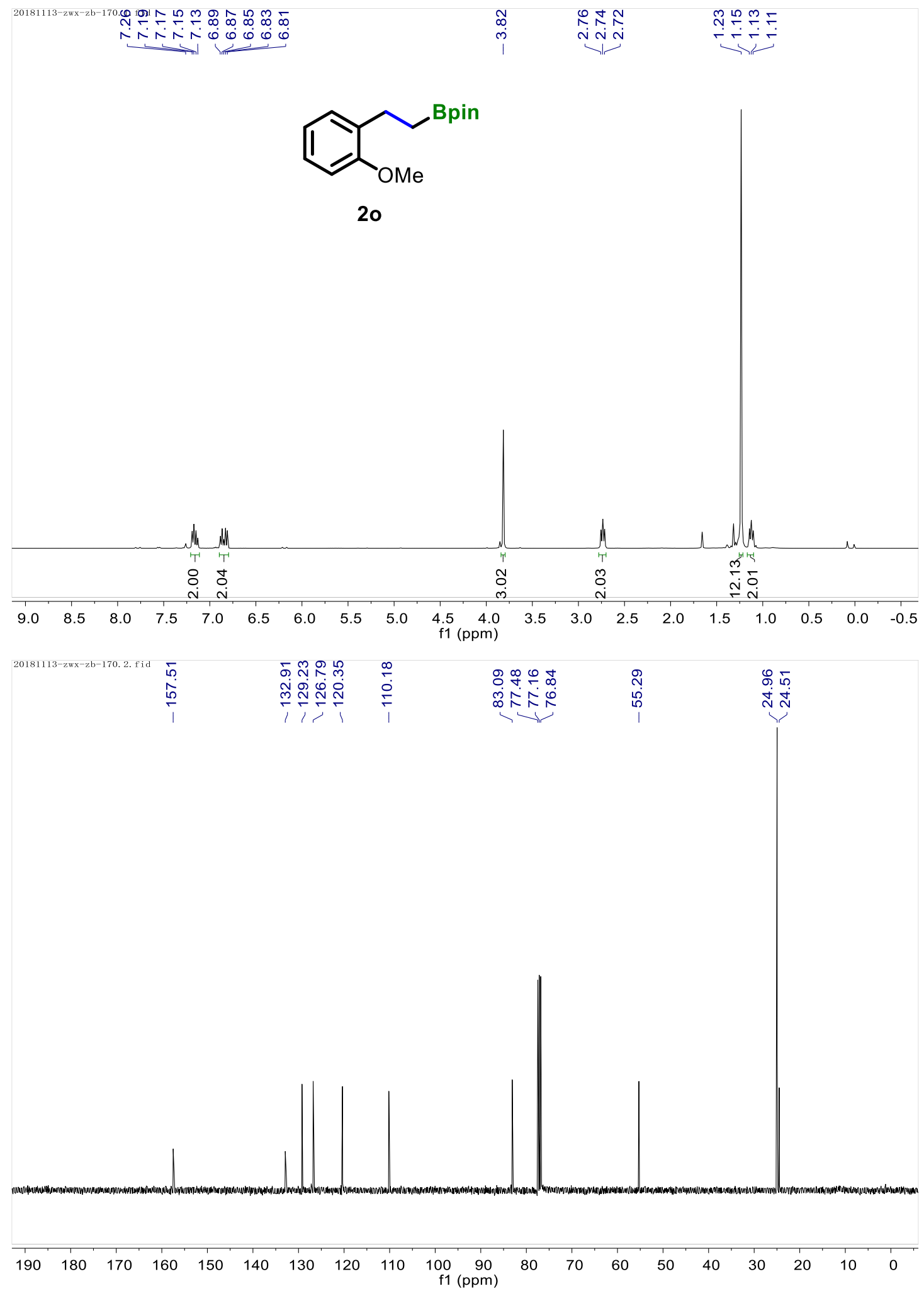


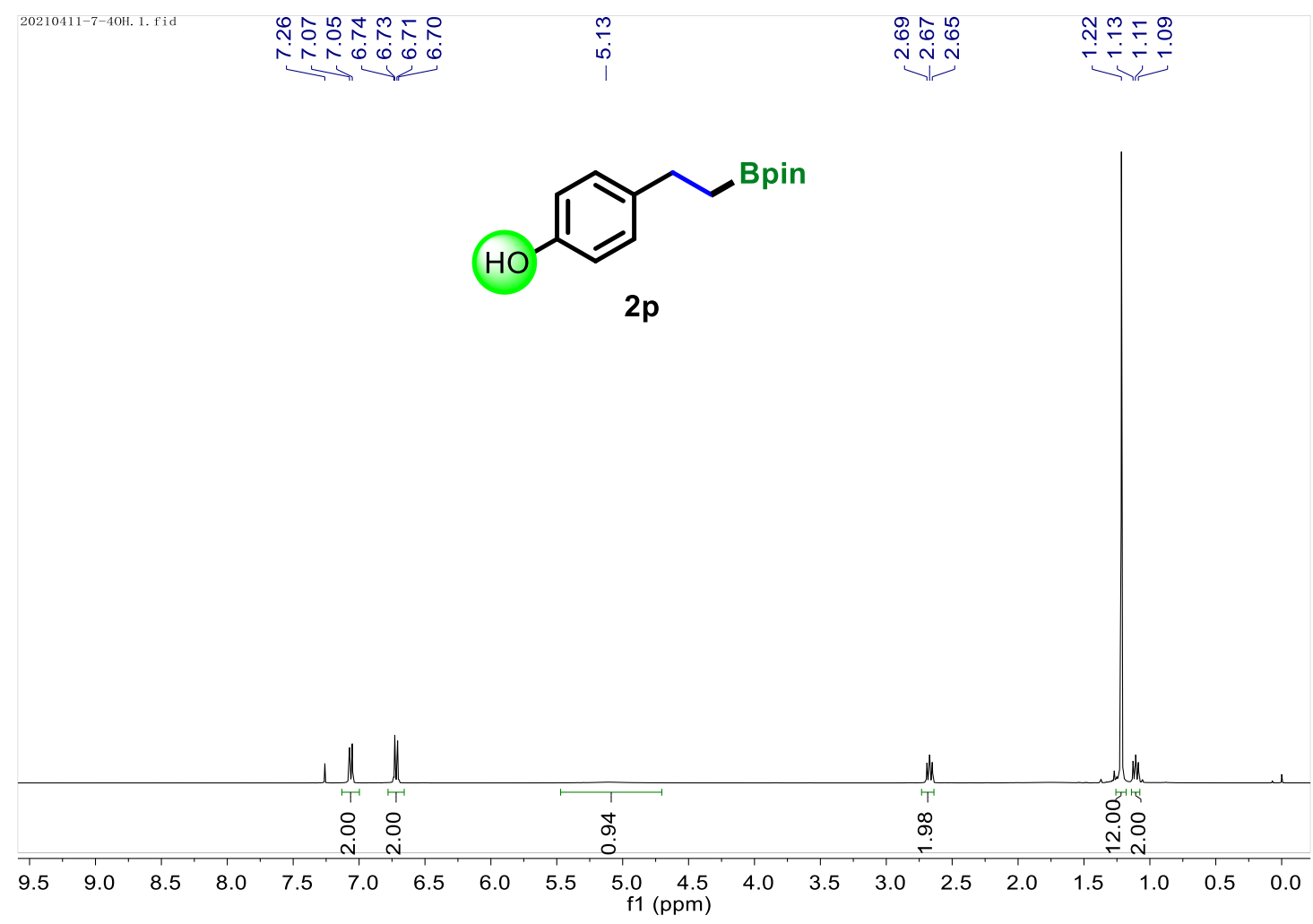

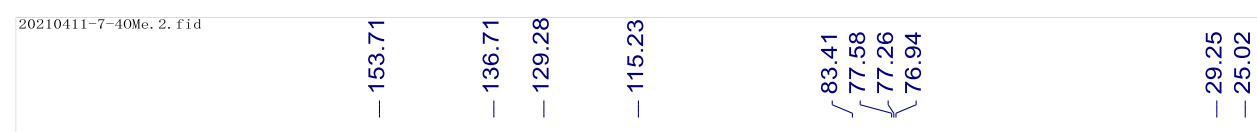

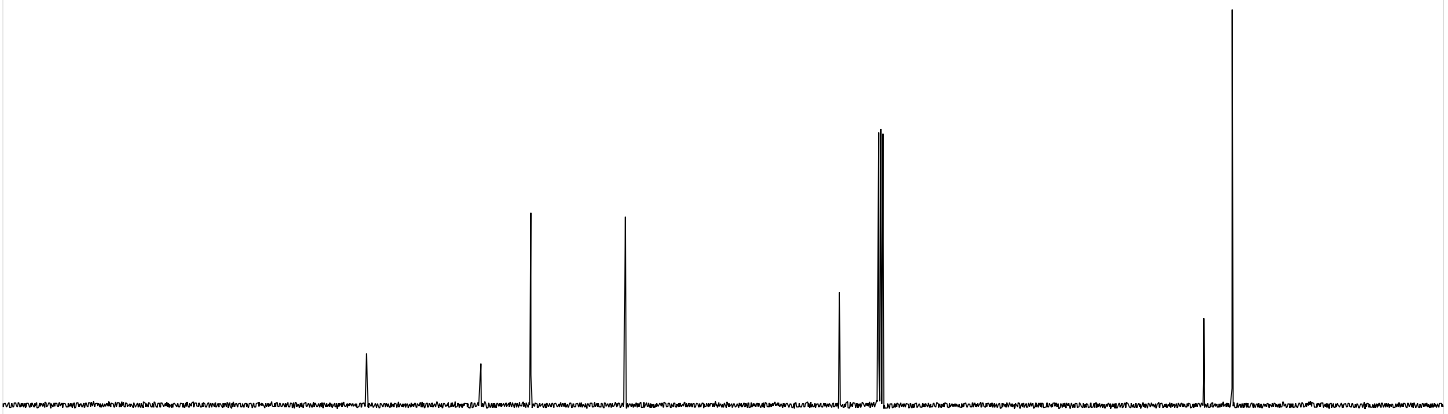

$\begin{array}{llllllllllllllllllllllllll}200 & 190 & 180 & 170 & 160 & 150 & 140 & 130 & 120 & 110 & 100 & 90 & 80 & 70 & 60 & 50 & 40 & 30 & 20 & 10 & 0\end{array}$ 


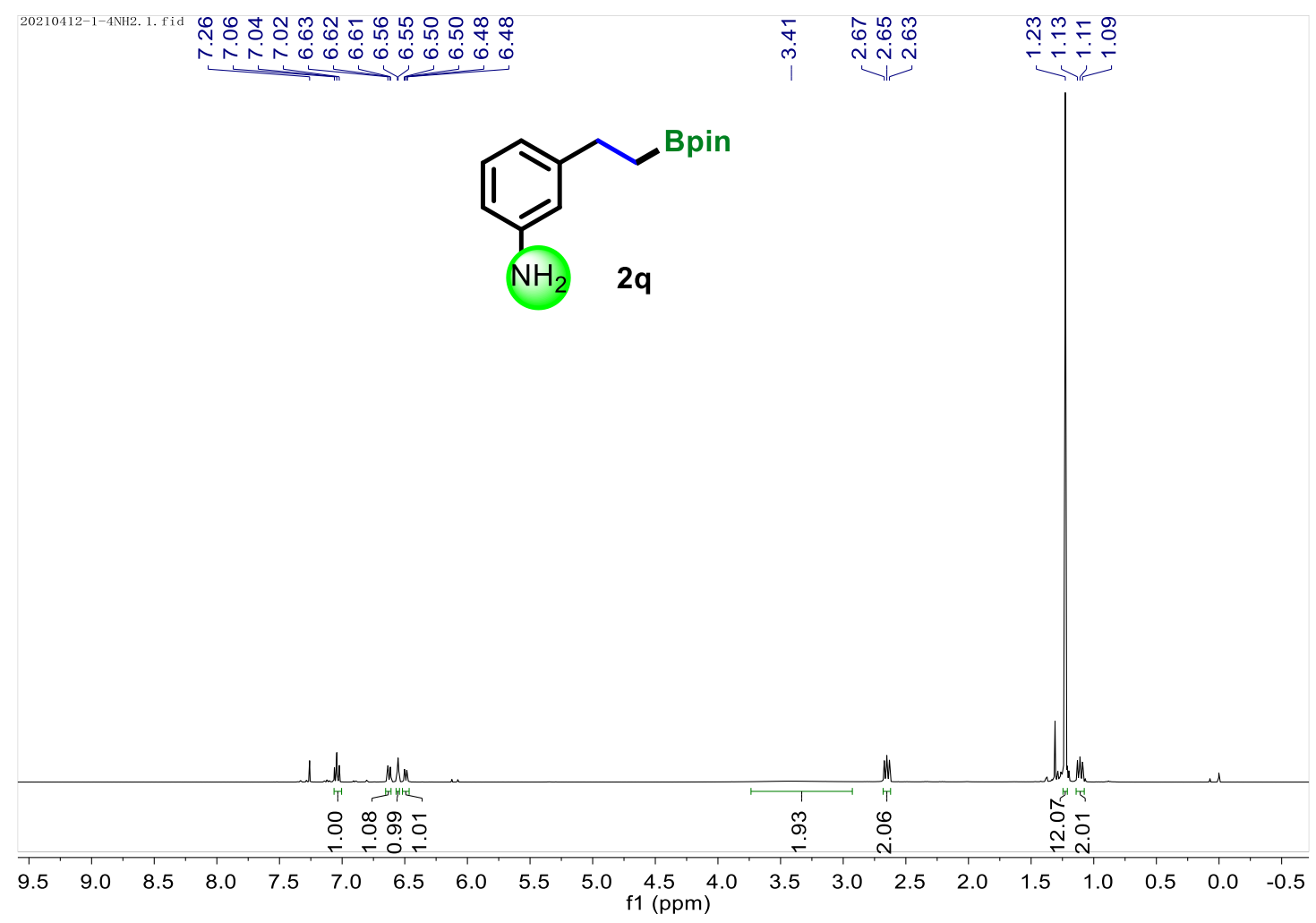

\begin{tabular}{|c|c|c|c|c|}
\hline 20210412-1-18.2.fid & 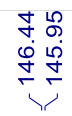 & 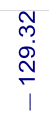 & 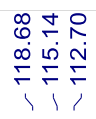 & 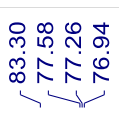 \\
\hline
\end{tabular}

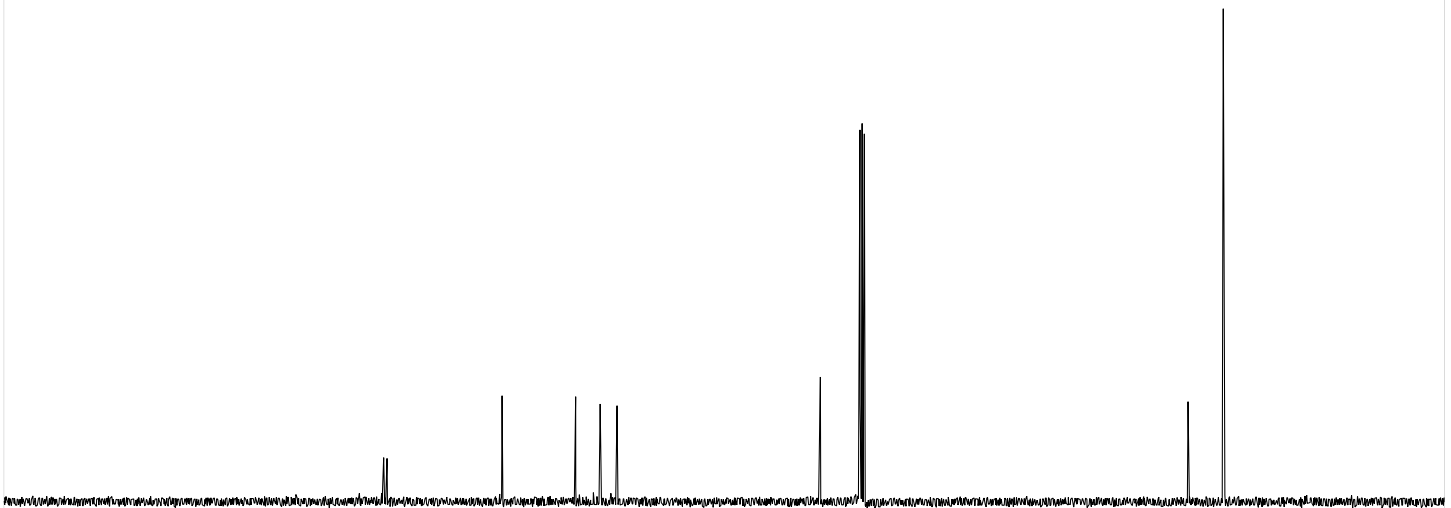

$\begin{array}{llllllllllllllllllllll}200 & 190 & 180 & 170 & 160 & 150 & 140 & 130 & 120 & 110 & 100 & 90 & 80 & 70 & 60 & 50 & 40 & 30 & 20 & 10 & 0\end{array}$ 

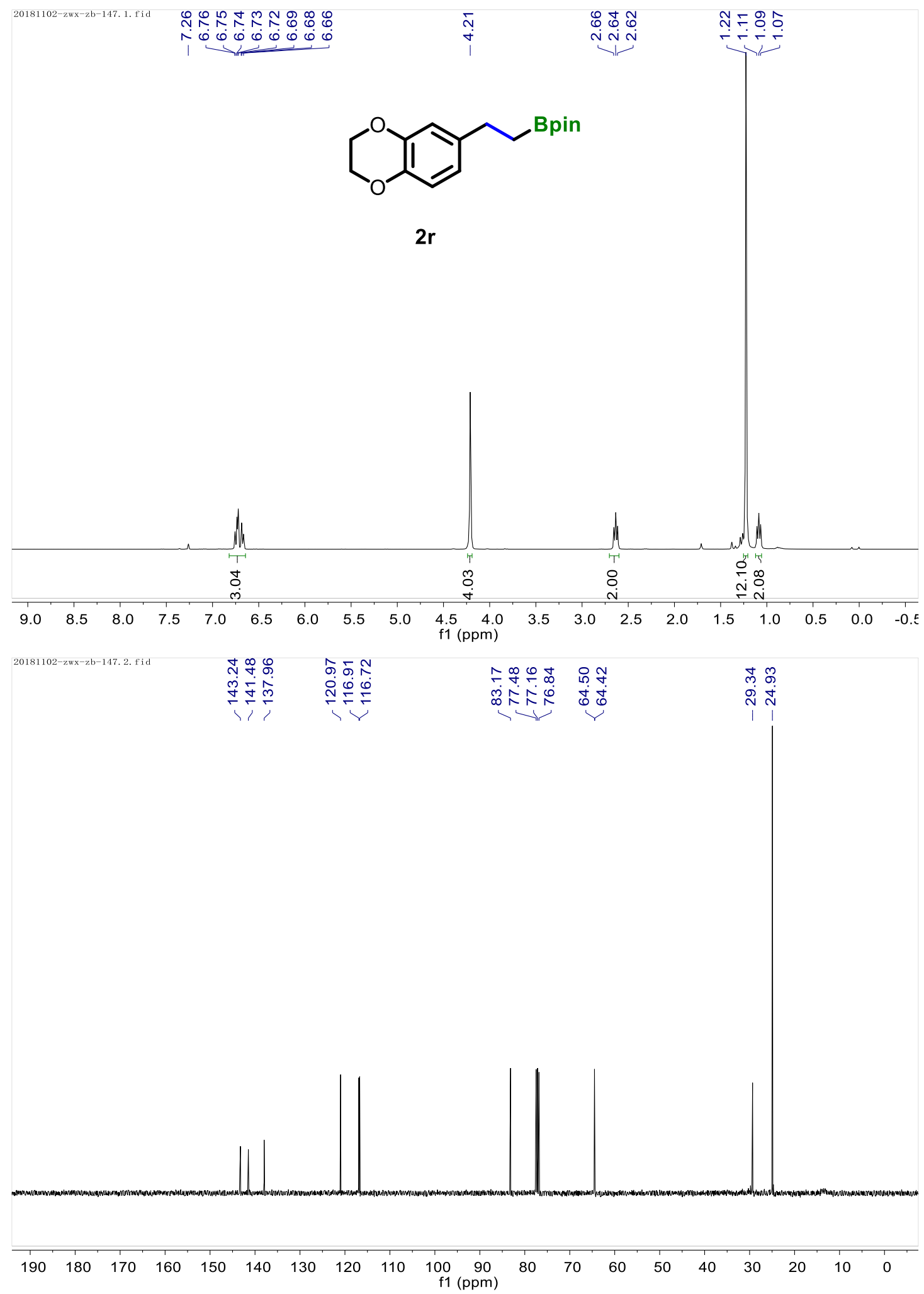


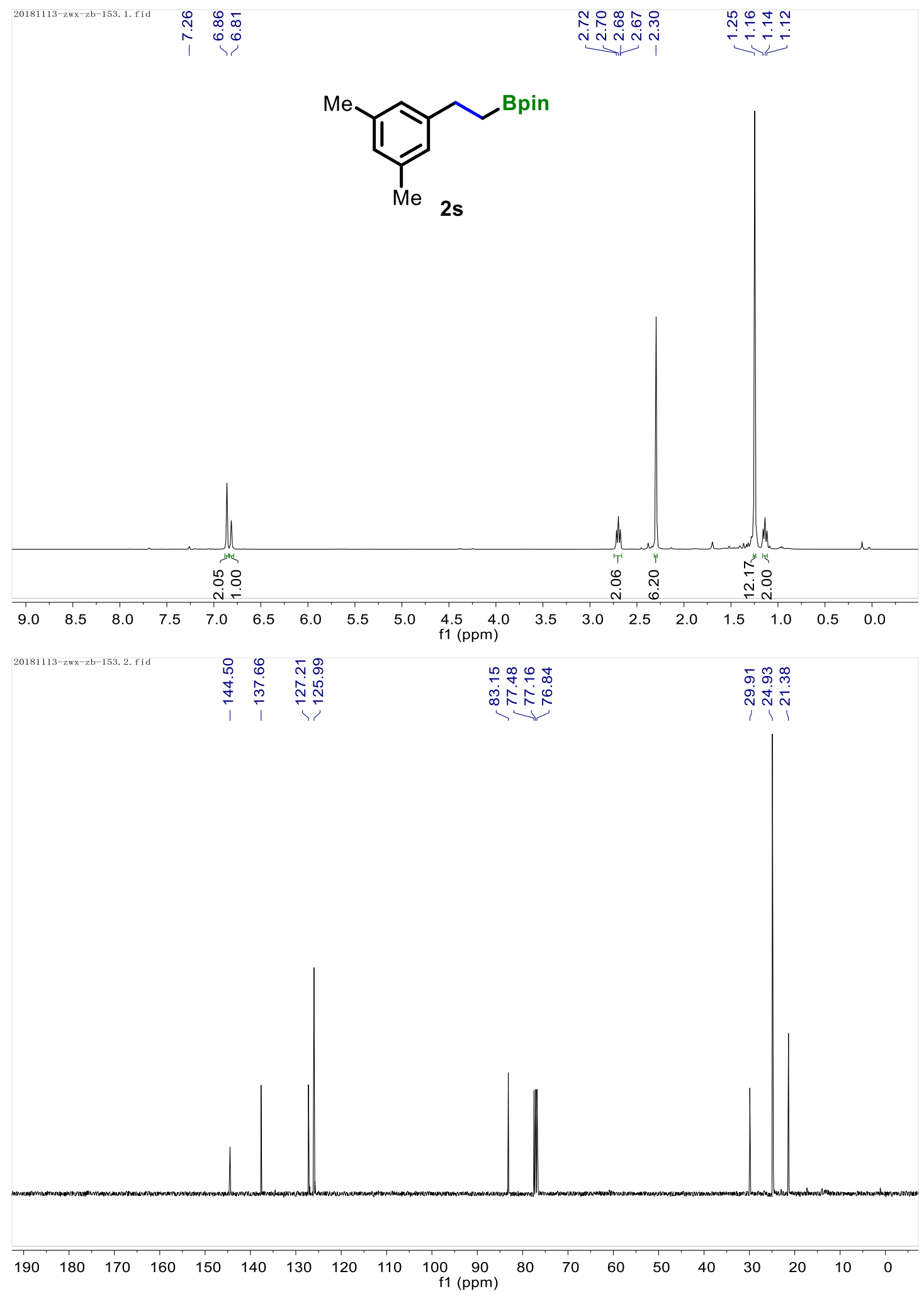




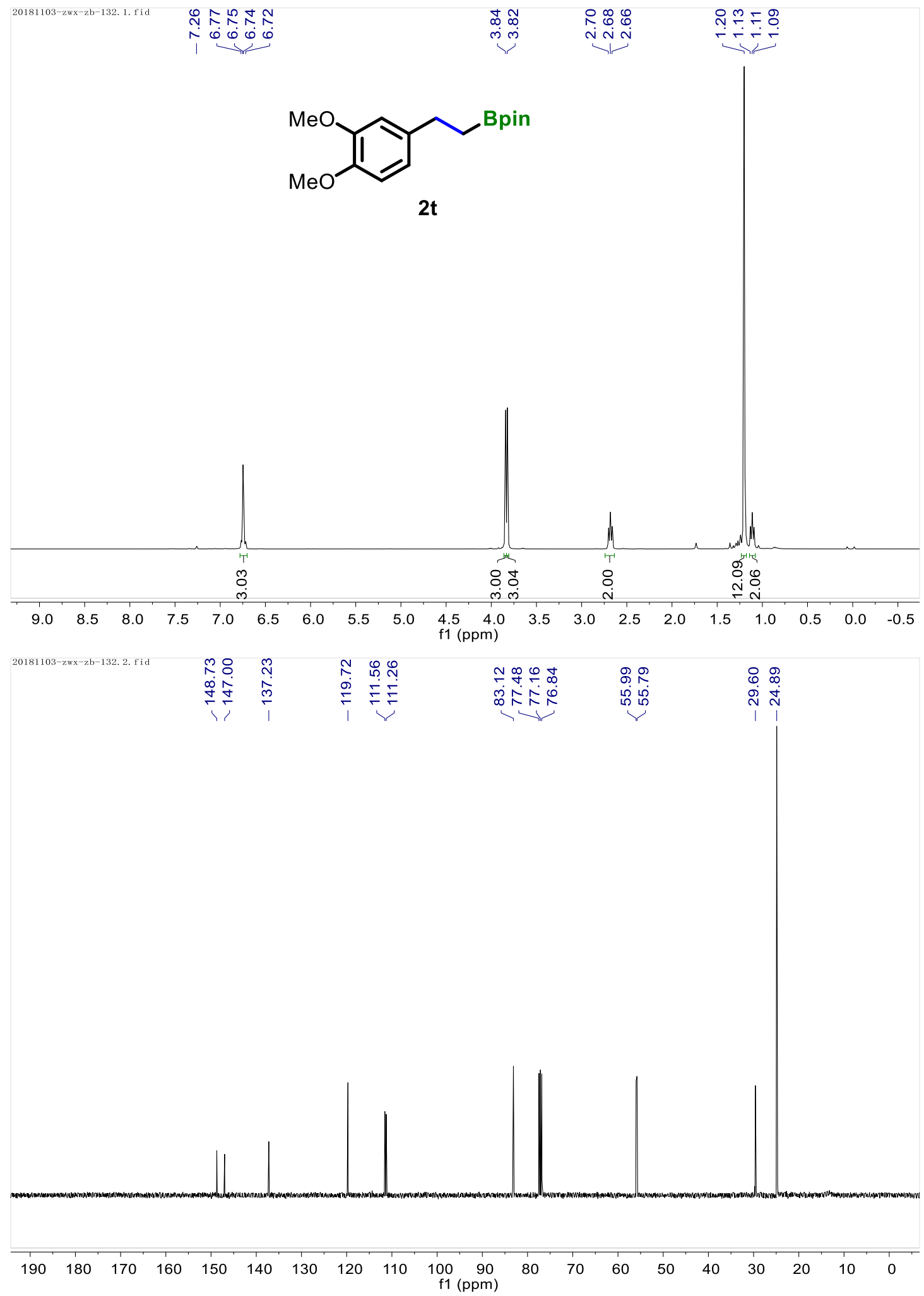




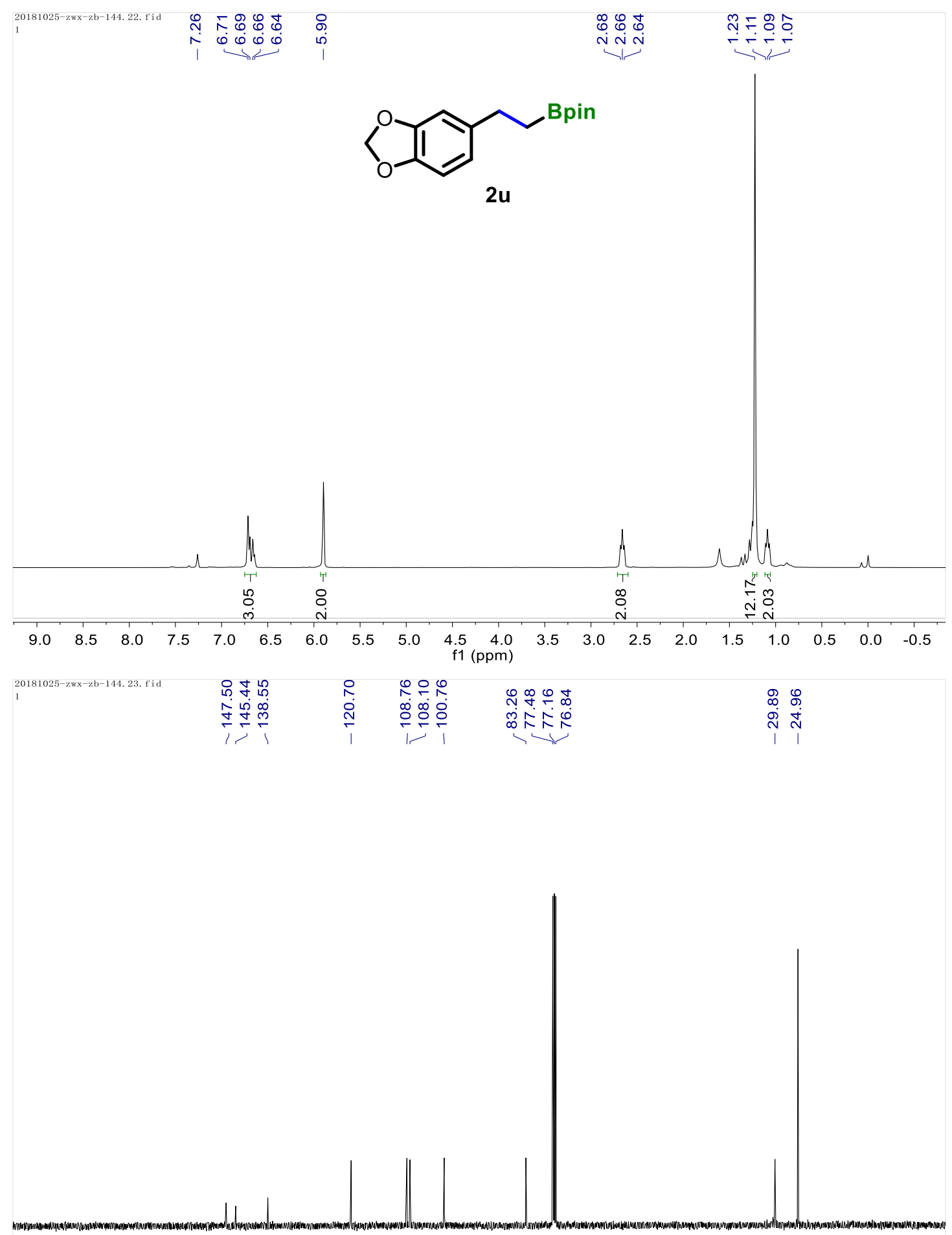

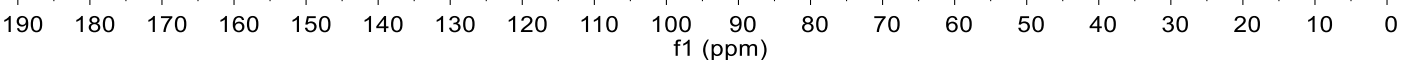




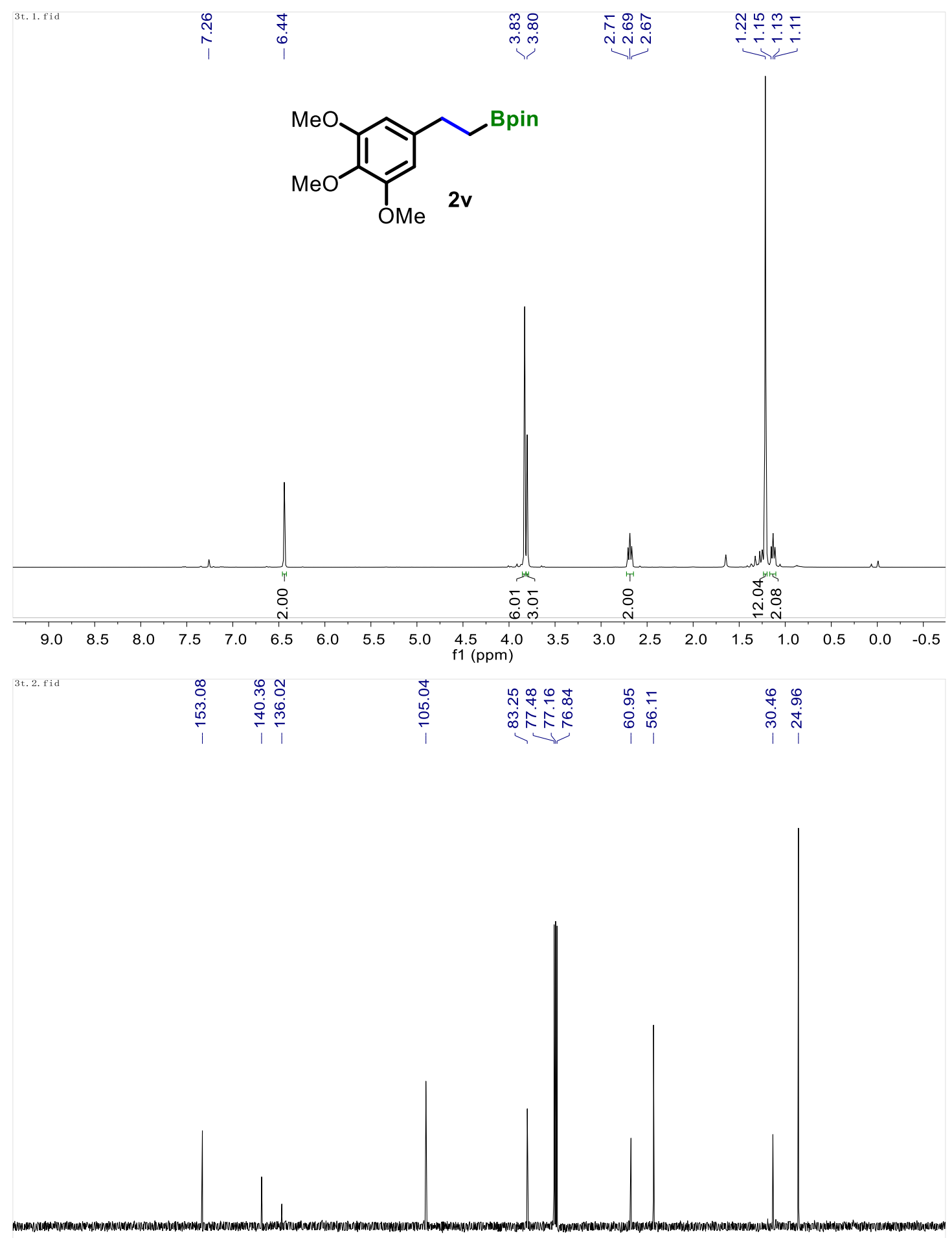

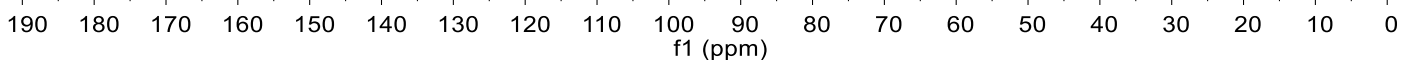


<smiles>CC(C)=CCCC(C)CCOc1ccc(CCc2ccccc2)cc1</smiles>
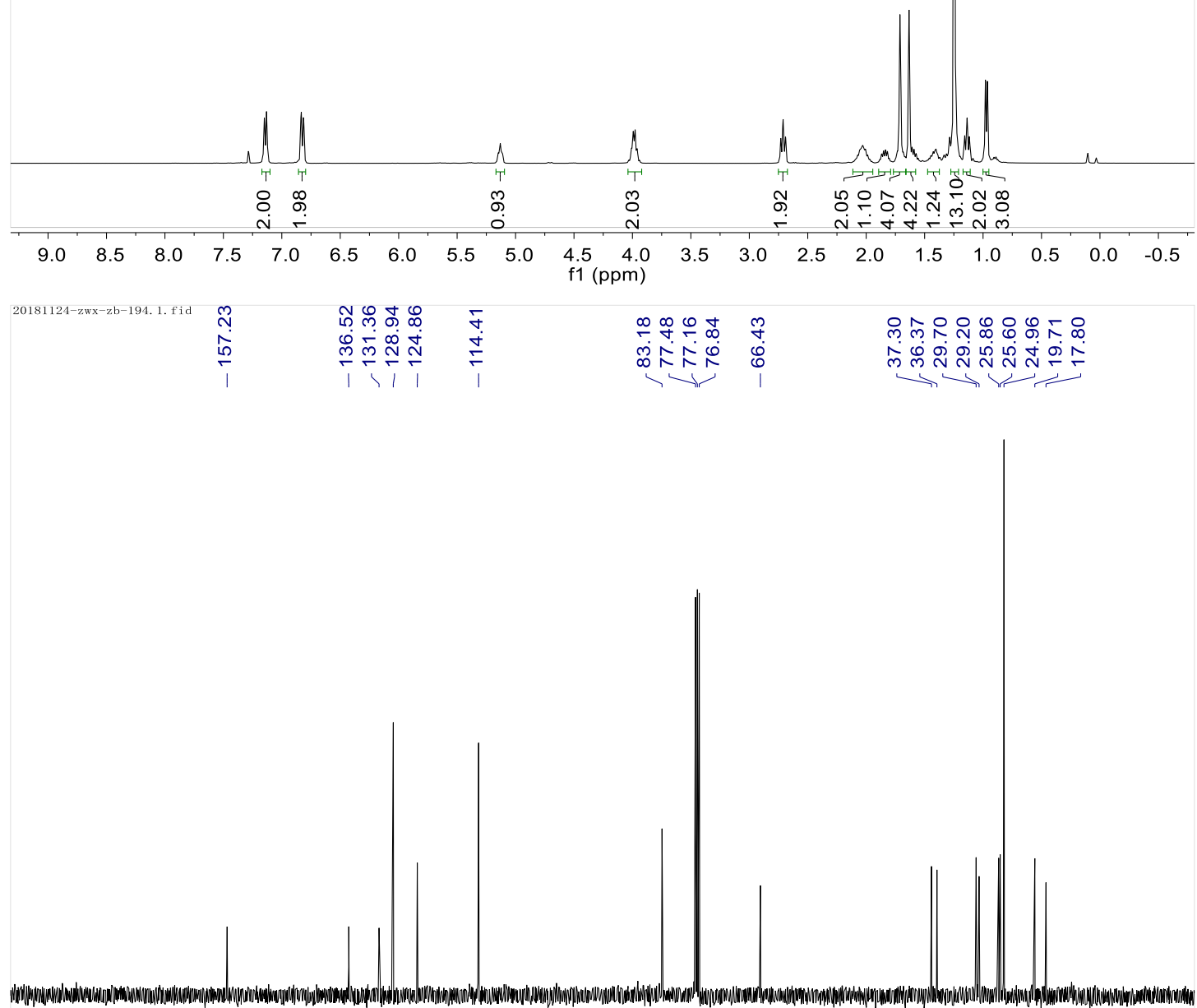

$\begin{array}{lllllllllllllllllll}190 & 180 & 170 & 160 & 150 & 140 & 130 & 120 & 110 & \begin{array}{c}100 \\ \mathrm{f} 1(\mathrm{ppm})\end{array} & 80 & 70 & 60 & 50 & 40 & 30 & 20 & 10 & 0\end{array}$ 

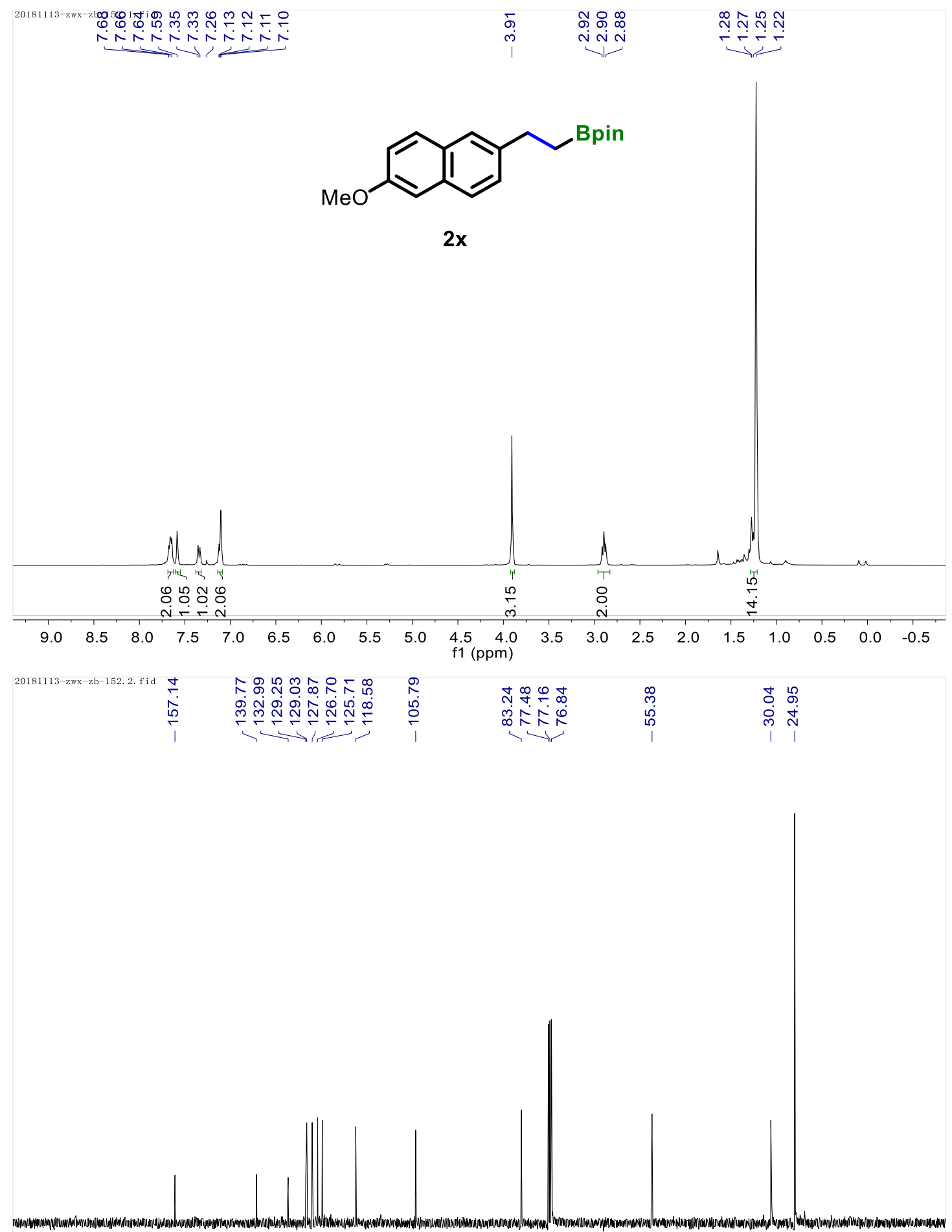

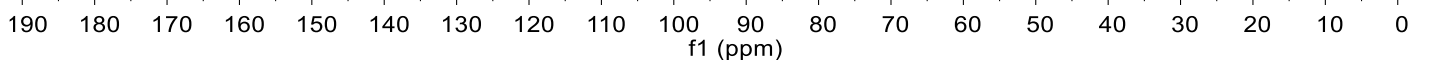




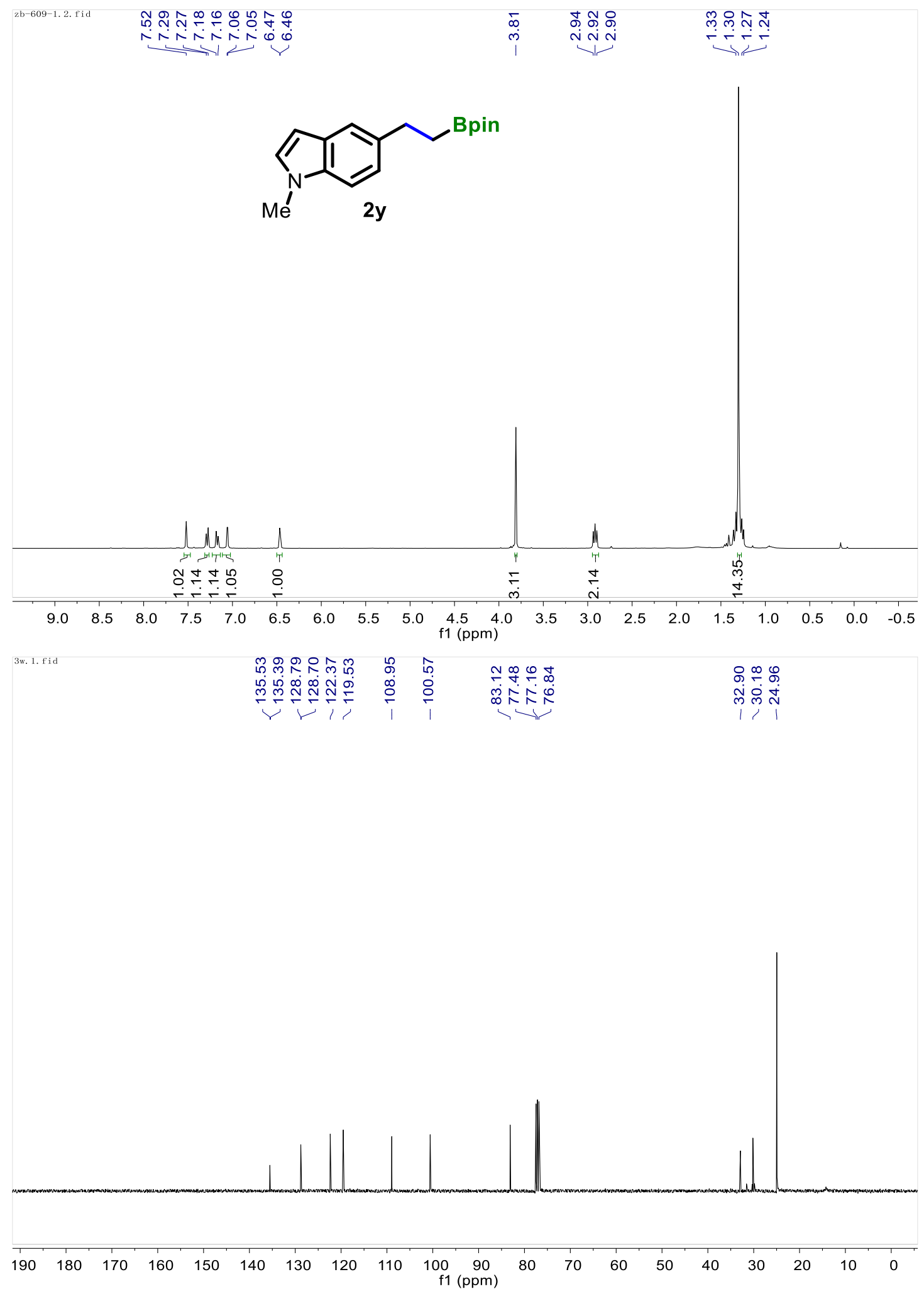




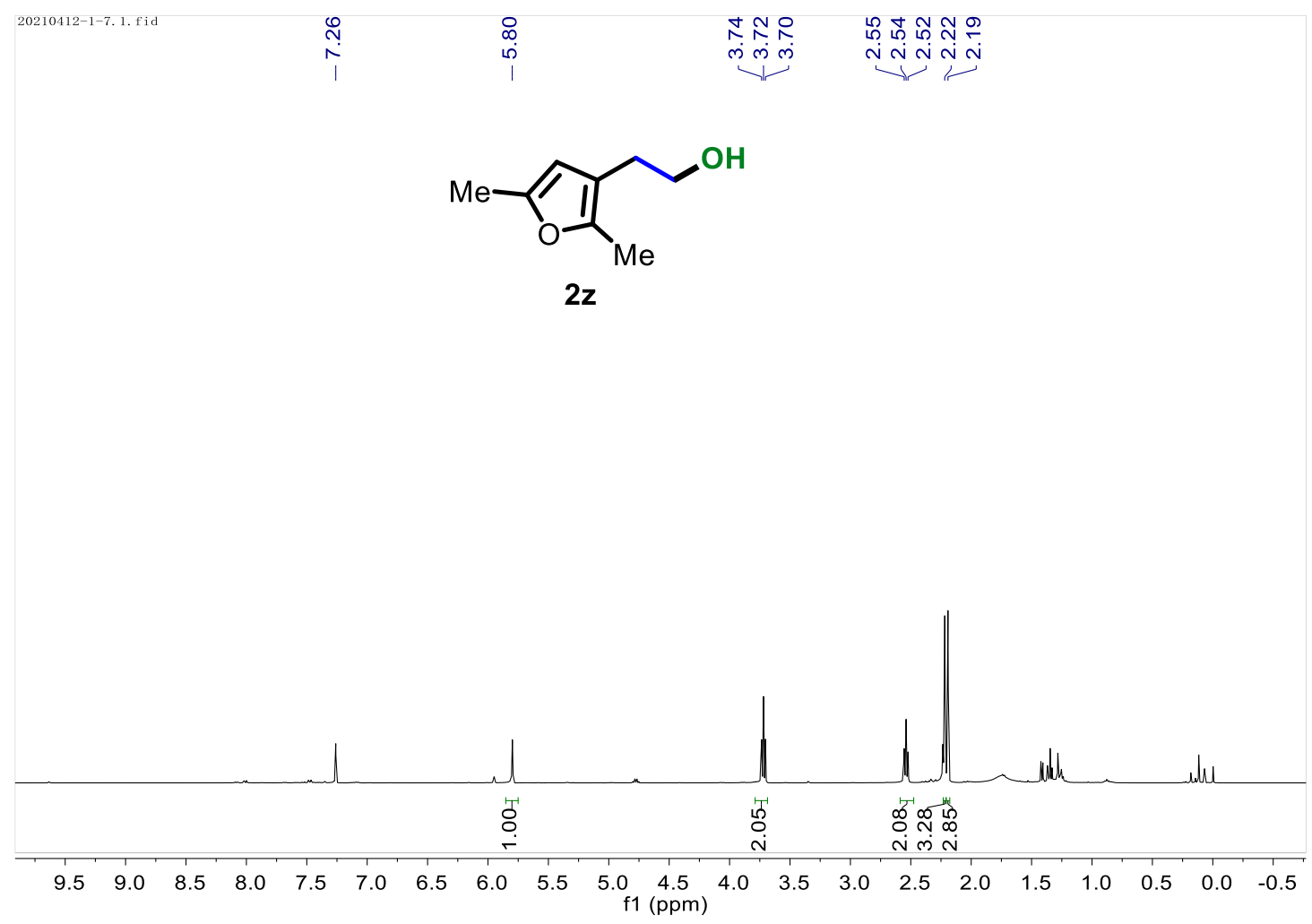

\begin{tabular}{|c|c|c|c|c|c|c|c|}
\hline $20210412-1-7.2 . \mathrm{fid}$ & 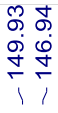 & $\begin{array}{l}\frac{0}{r} \\
\frac{10}{5} \\
i\end{array}$ & $\begin{array}{l}\stackrel{3}{+} \\
\stackrel{5}{\circ} \\
\frac{1}{1}\end{array}$ & 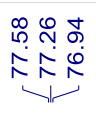 & $\begin{array}{l}\text { ठ } \\
\text { ก̃ } \\
\text { । }\end{array}$ & $\begin{array}{l}\hat{0} \\
\infty \\
\stackrel{\infty}{N} \\
1\end{array}$ & 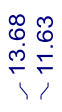 \\
\hline
\end{tabular}

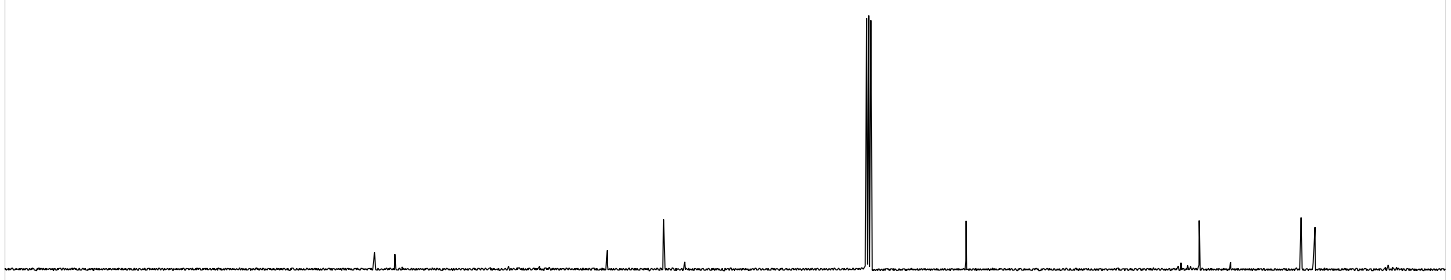

$\begin{array}{lllllllllllllllllll}200 & 190 & 180 & 170 & 160 & 150 & 140 & 130 & 120 & 110 \begin{array}{c}100 \\ \mathrm{f} 1(\mathrm{ppm})\end{array} & 80 & 70 & 60 & 50 & 40 & 30 & 20 & 10 & 0\end{array}$ 


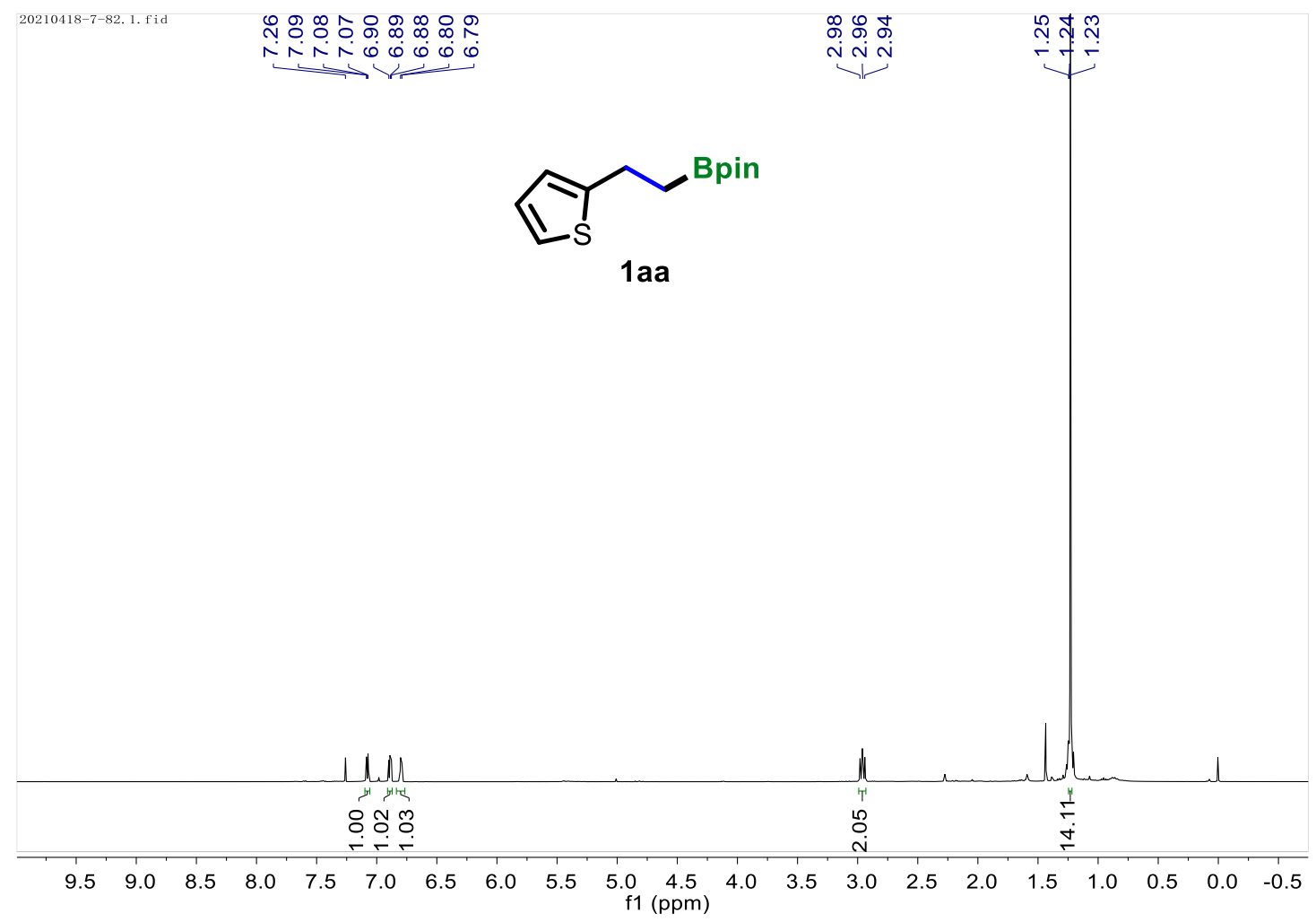

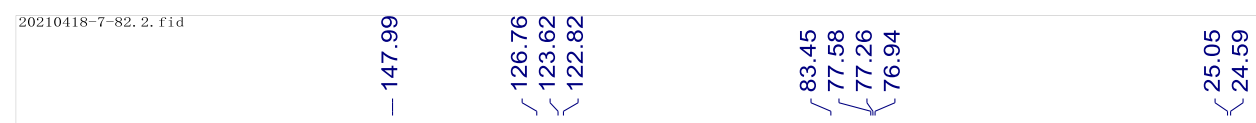

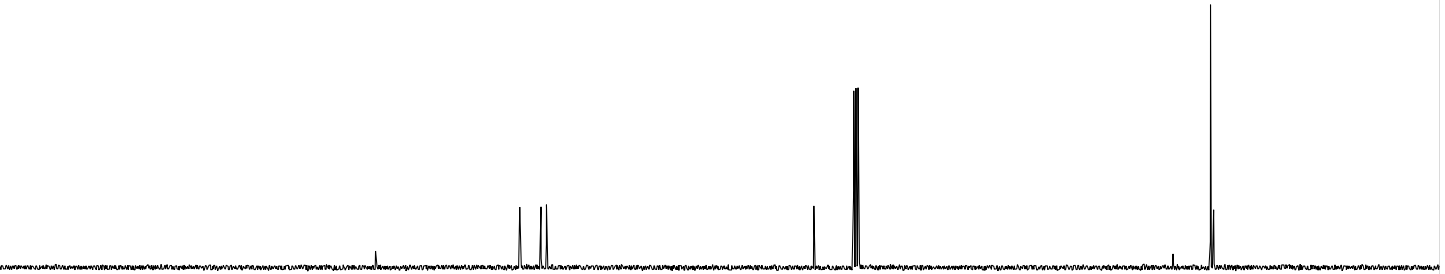

$\begin{array}{lllllllllllllllllllll}200 & 190 & 180 & 170 & 160 & 150 & 140 & 130 & 120 & 110 & 100 & 90 & 80 & 70 & 60 & 50 & 40 & 30 & 20 & 10 & 0\end{array}$ f1 (ppm) 


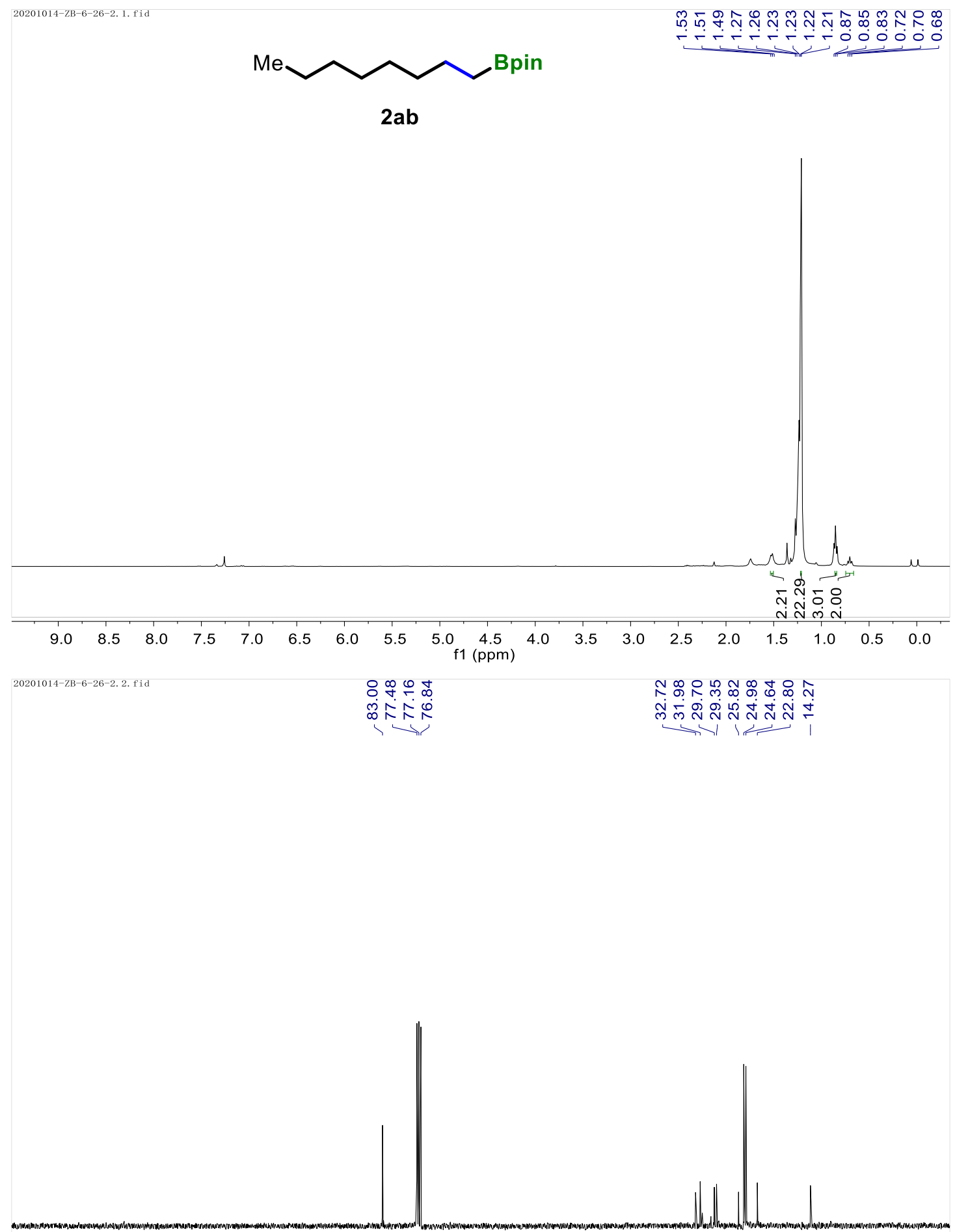

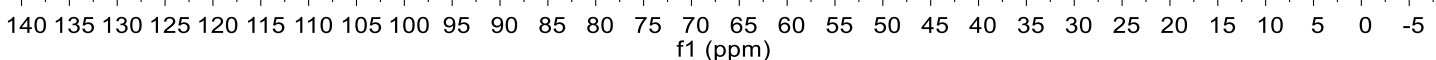




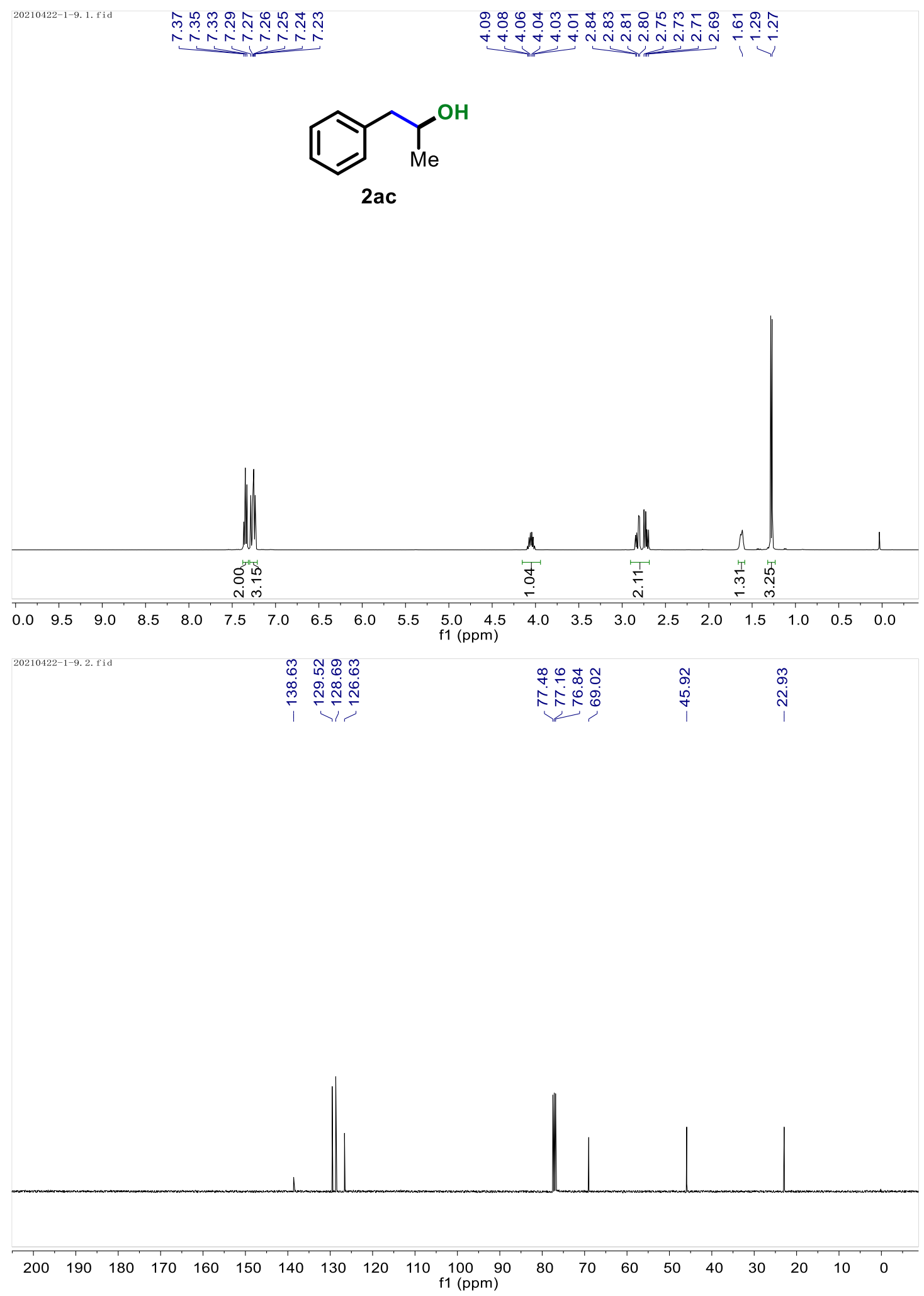




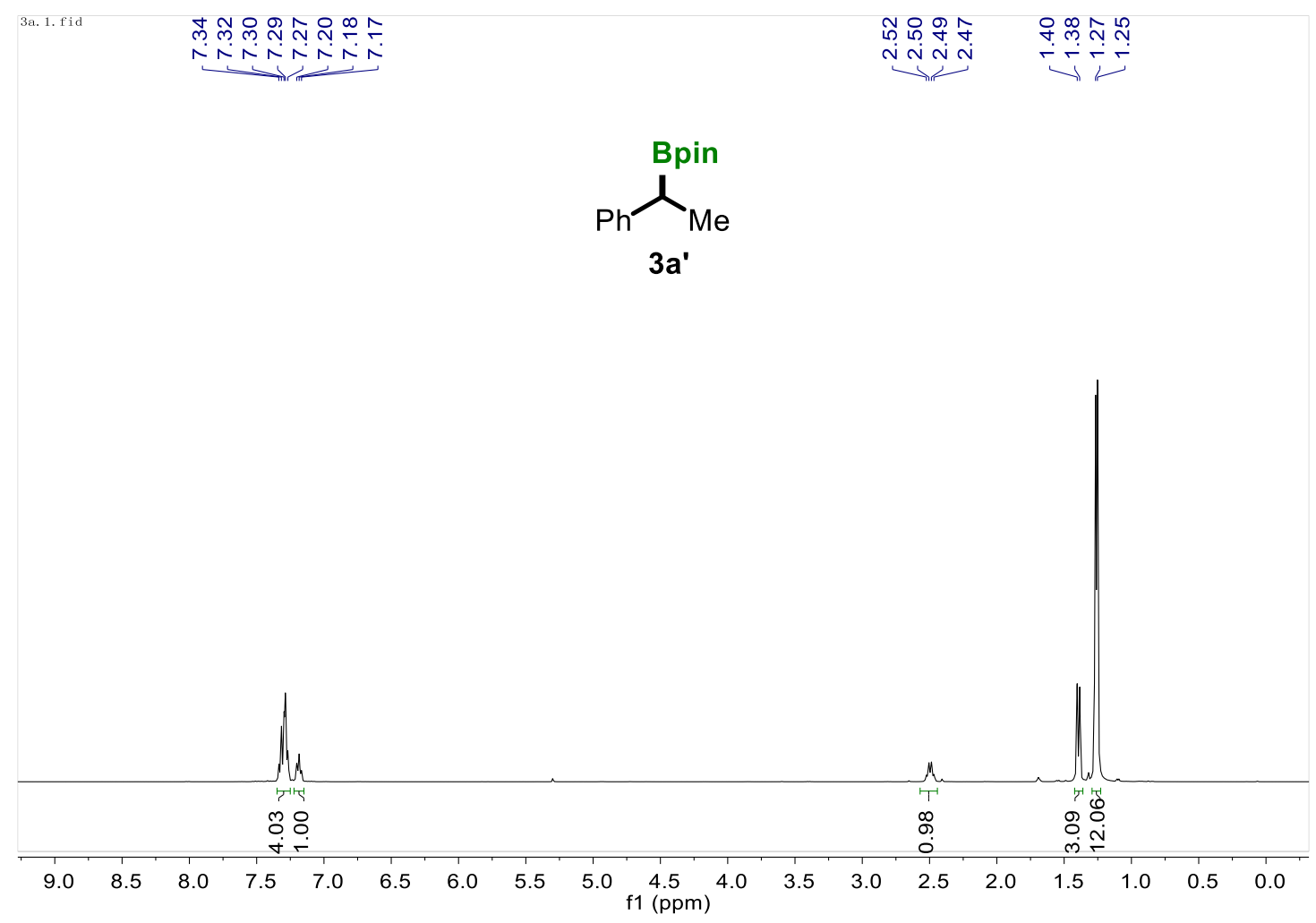

\begin{tabular}{|c|c|c|c|c|}
\hline 3a. 2. fid & \begin{tabular}{c}
$m$ \\
0 \\
in \\
\multirow{2}{*}{} \\
1
\end{tabular} & 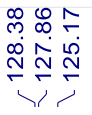 & 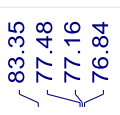 & 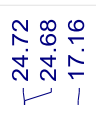 \\
\hline
\end{tabular}

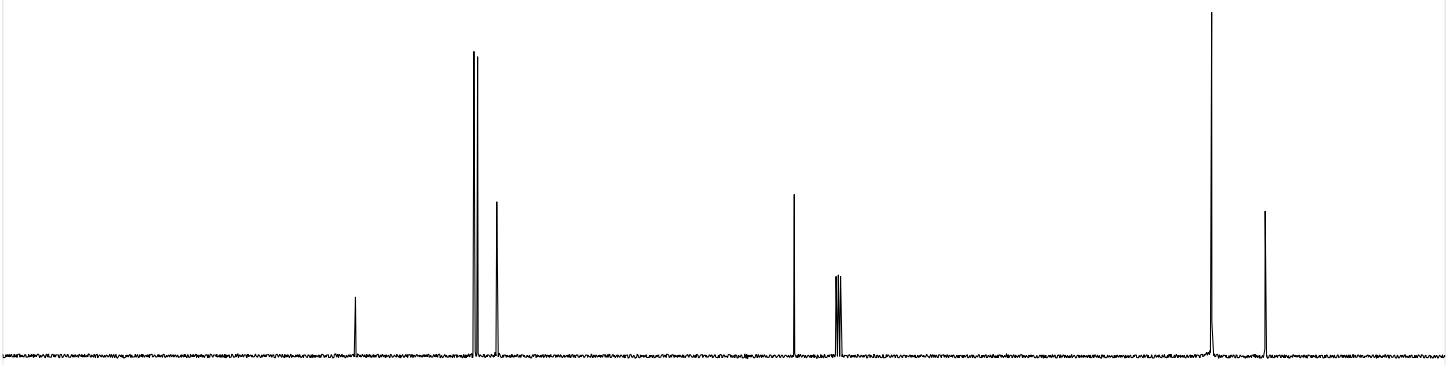

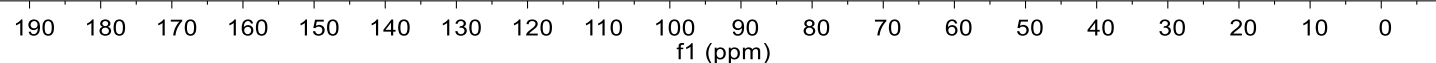




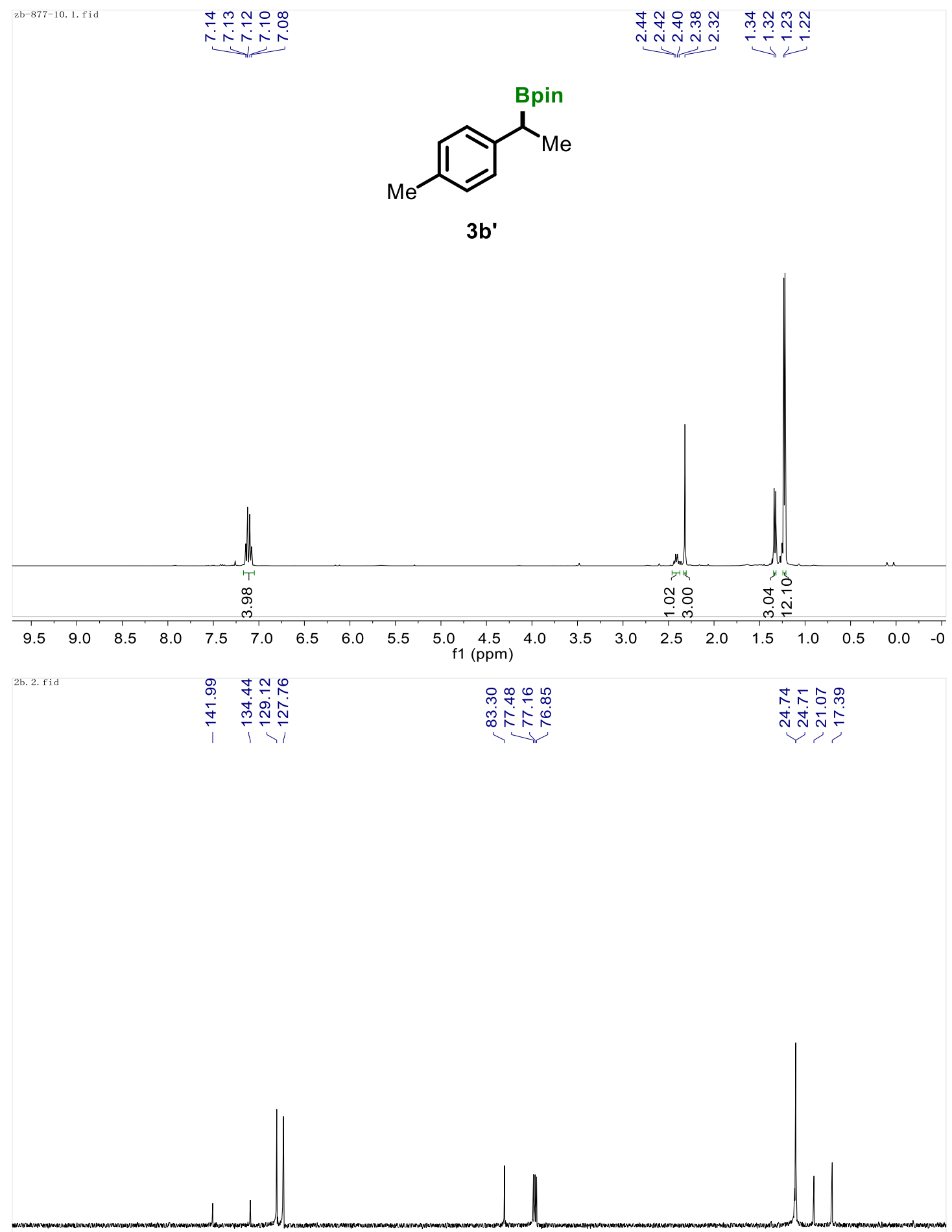

$\begin{array}{llllllllllllllllllll}180 & 170 & 160 & 150 & 140 & 130 & 120 & 110 & 100 & \begin{array}{c}90 \\ \mathrm{f} 1(\mathrm{ppm})\end{array} & 80 & 70 & 60 & 50 & 40 & 30 & 20 & 10 & 0\end{array}$ 


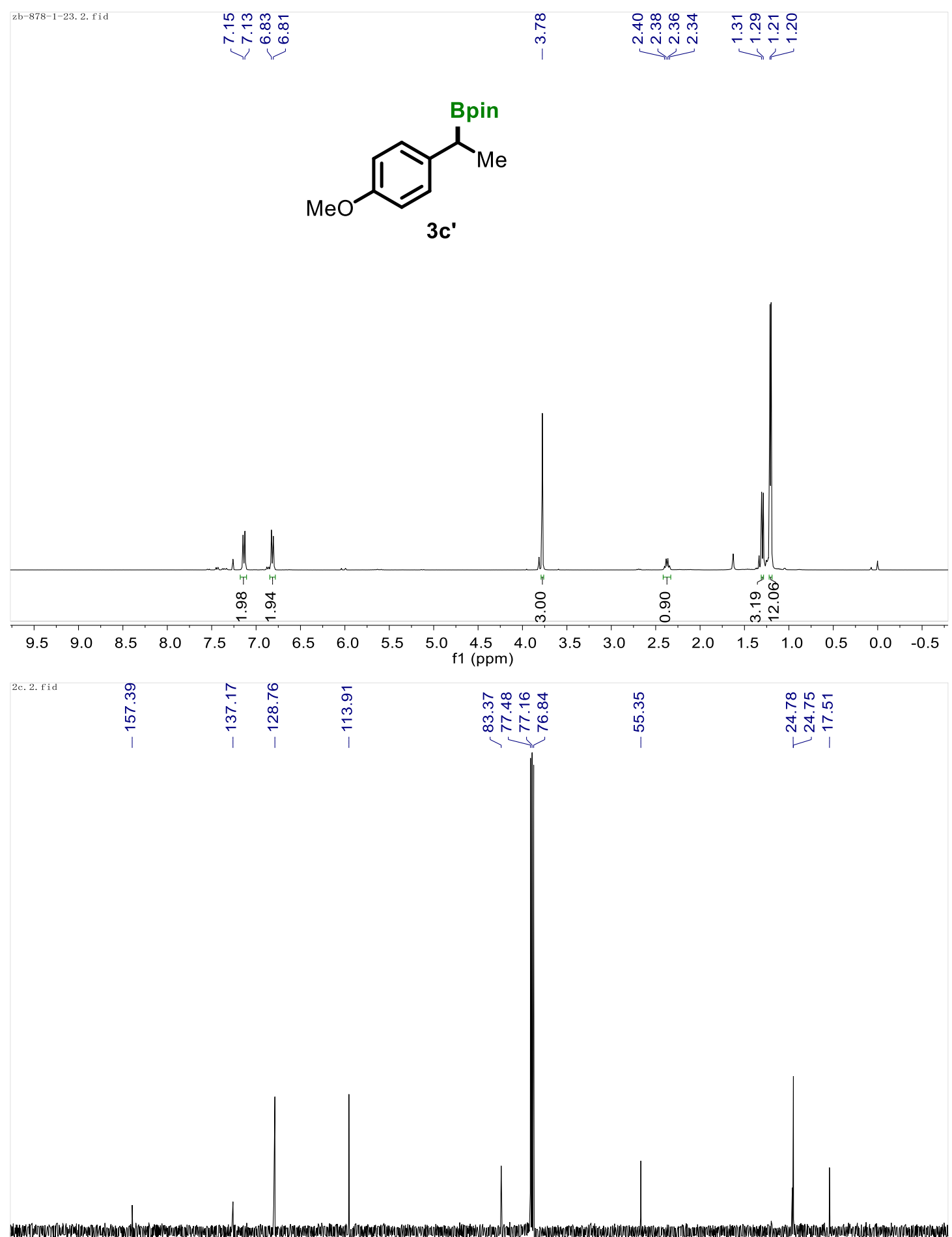

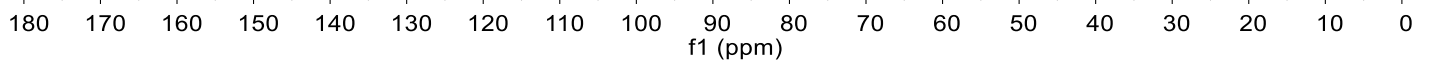




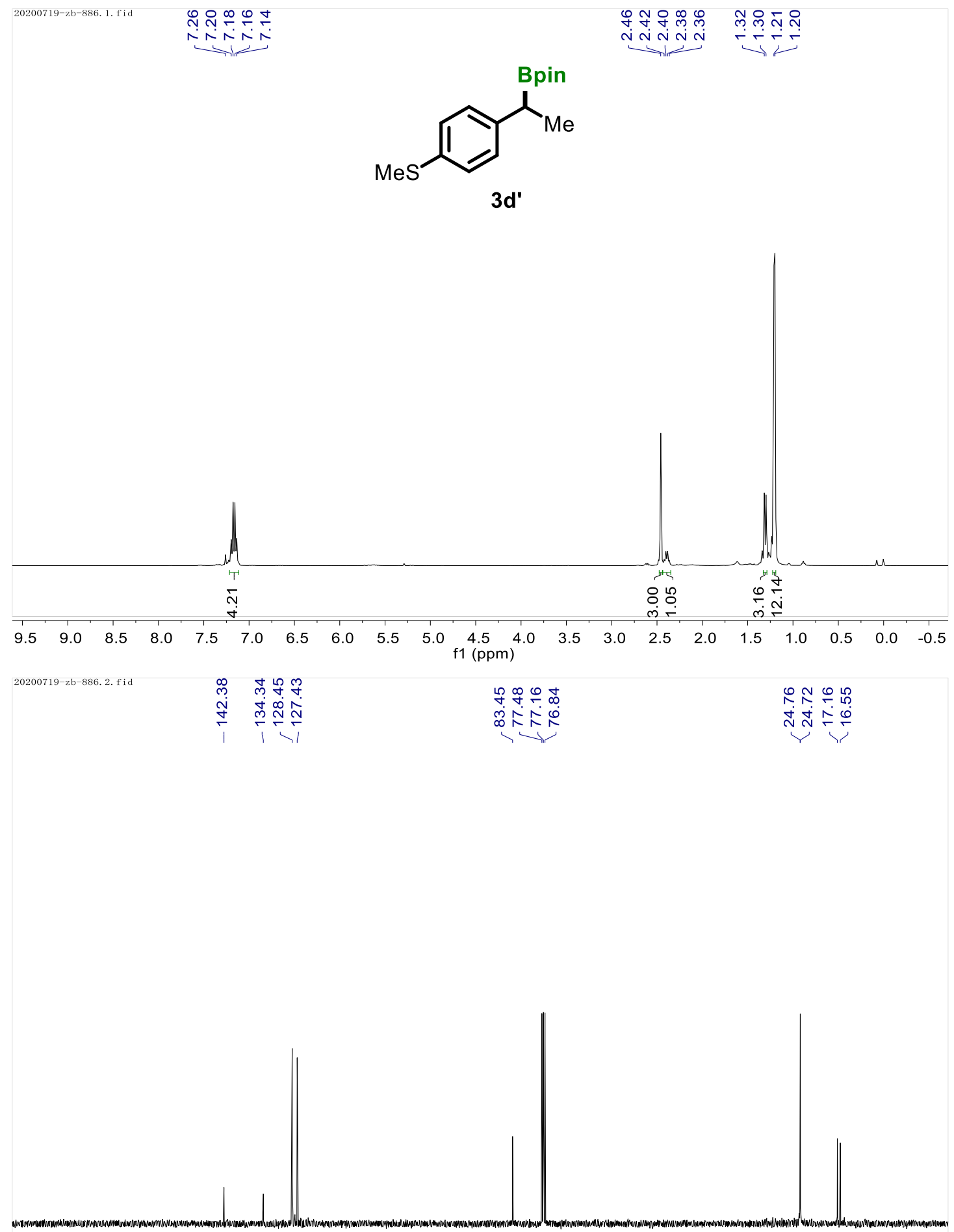

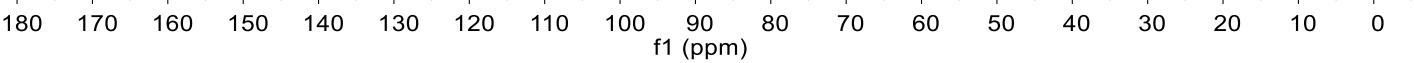



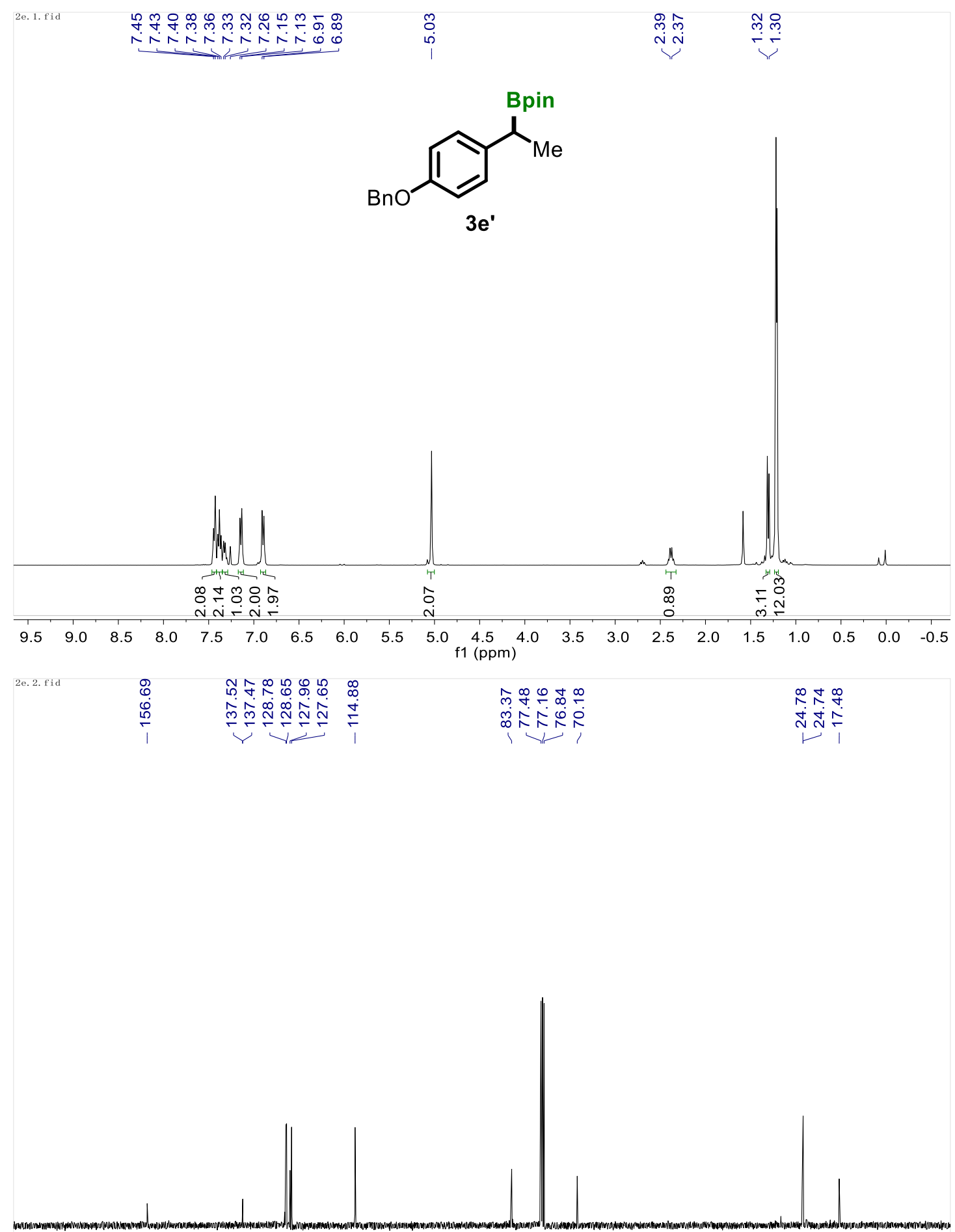

$\begin{array}{lllllllllllllllllll}180 & 170 & 160 & 150 & 140 & 130 & 120 & 110 & 100 & 90 & 80 & 70 & 60 & 50 & 40 & 30 & 20 & 10 & 0\end{array}$ 


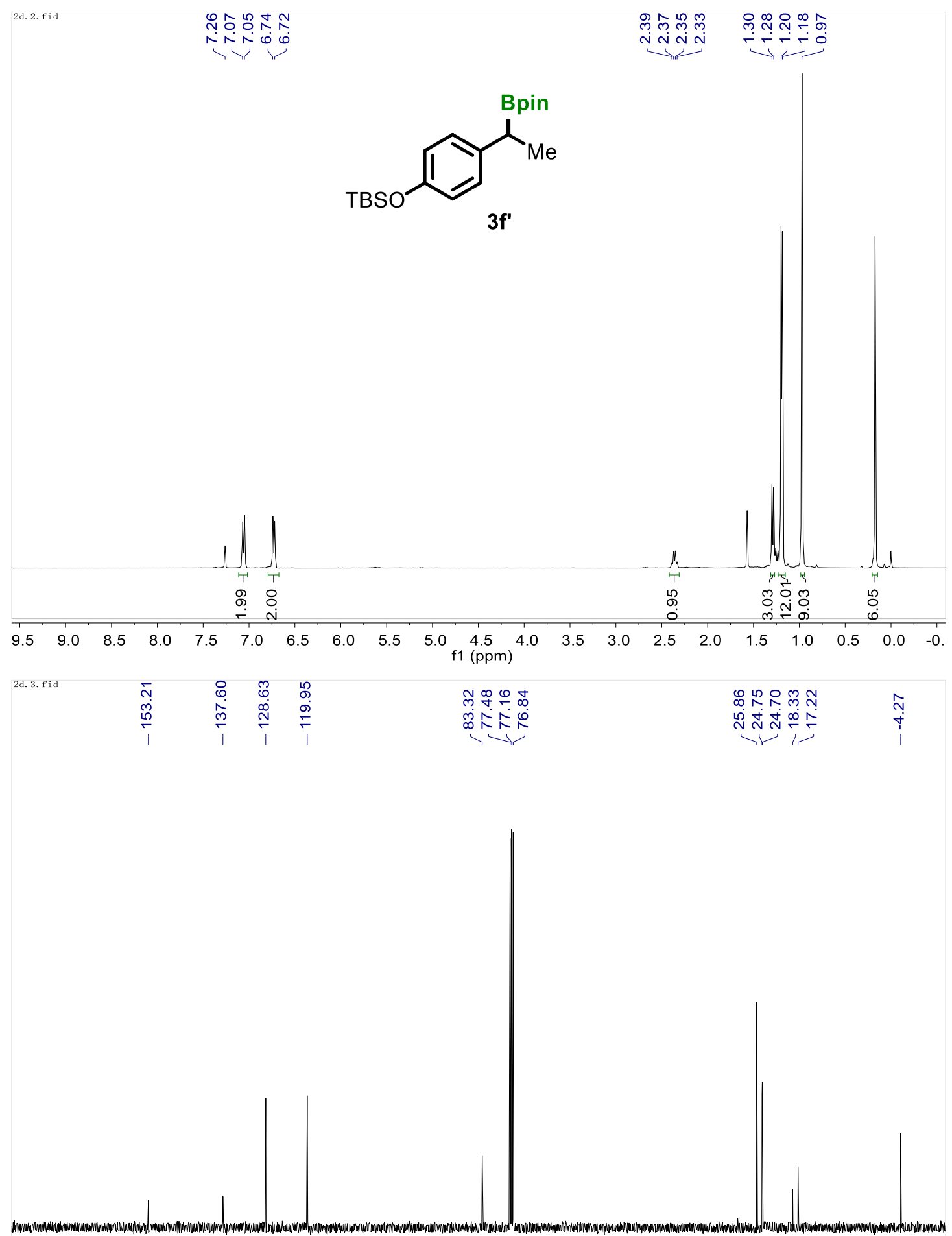

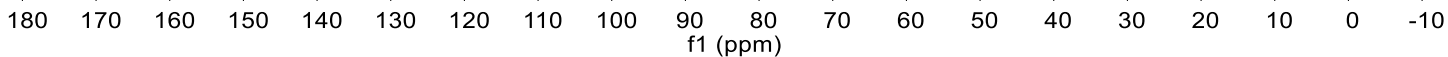




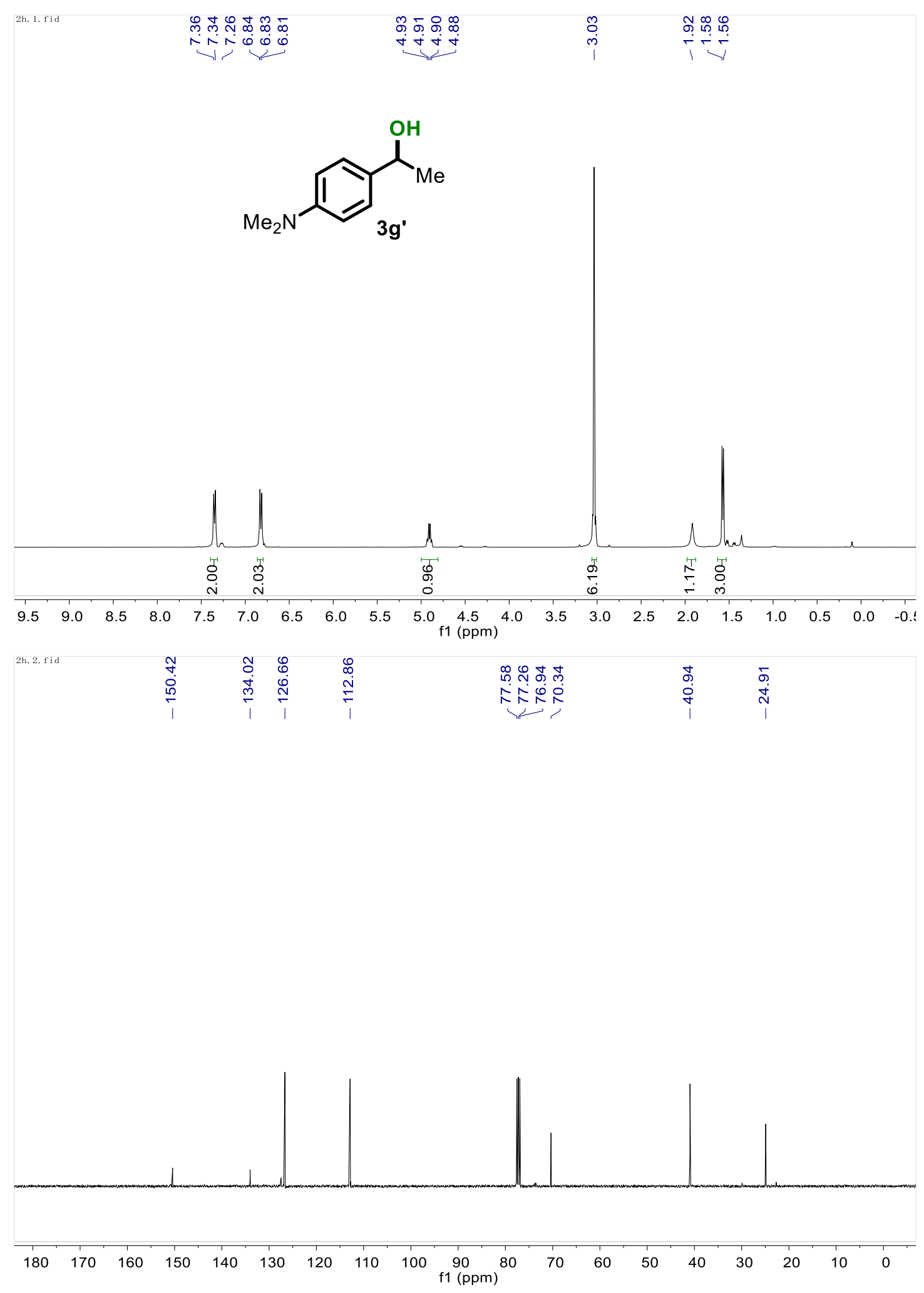



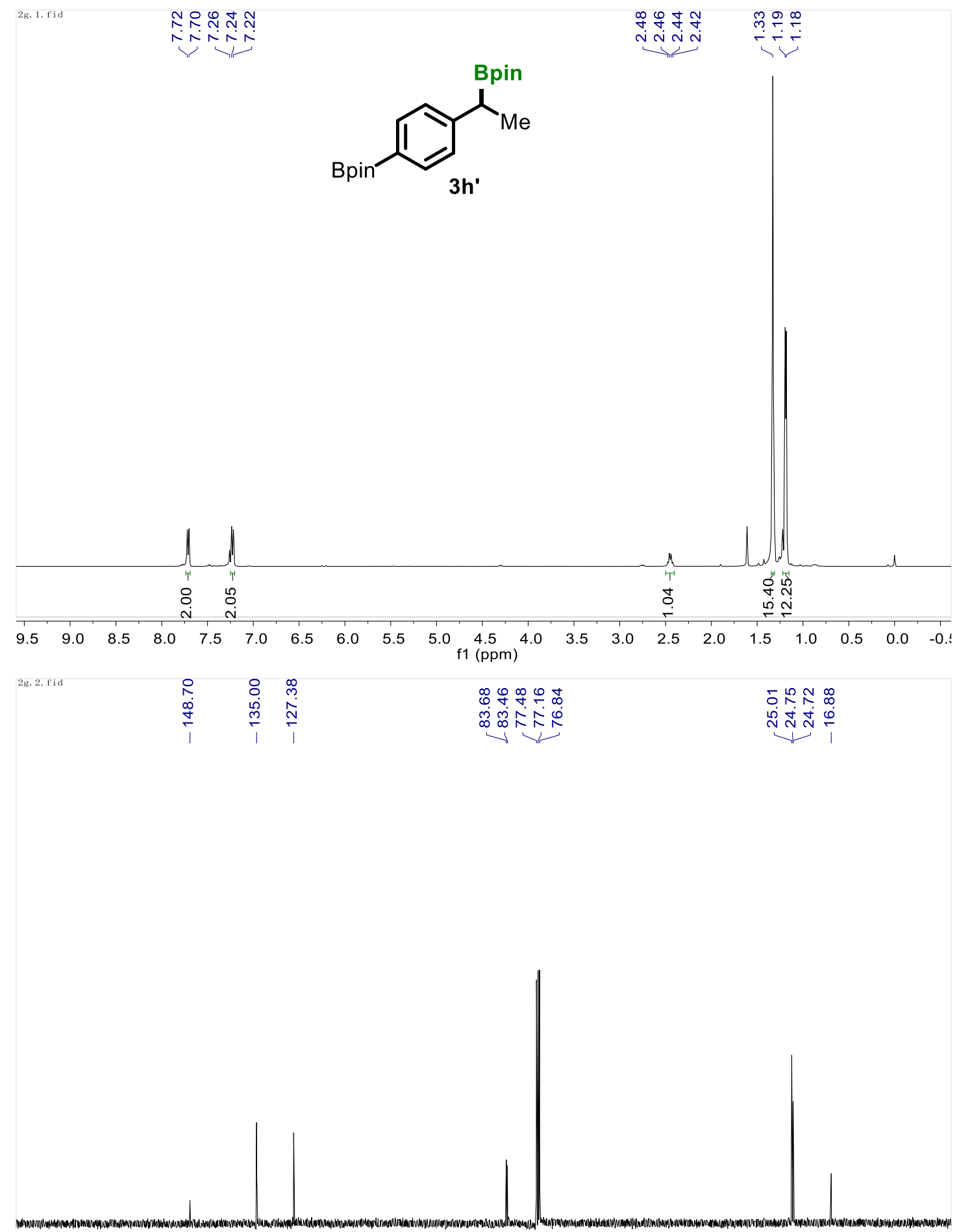

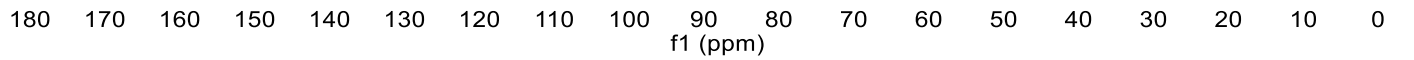



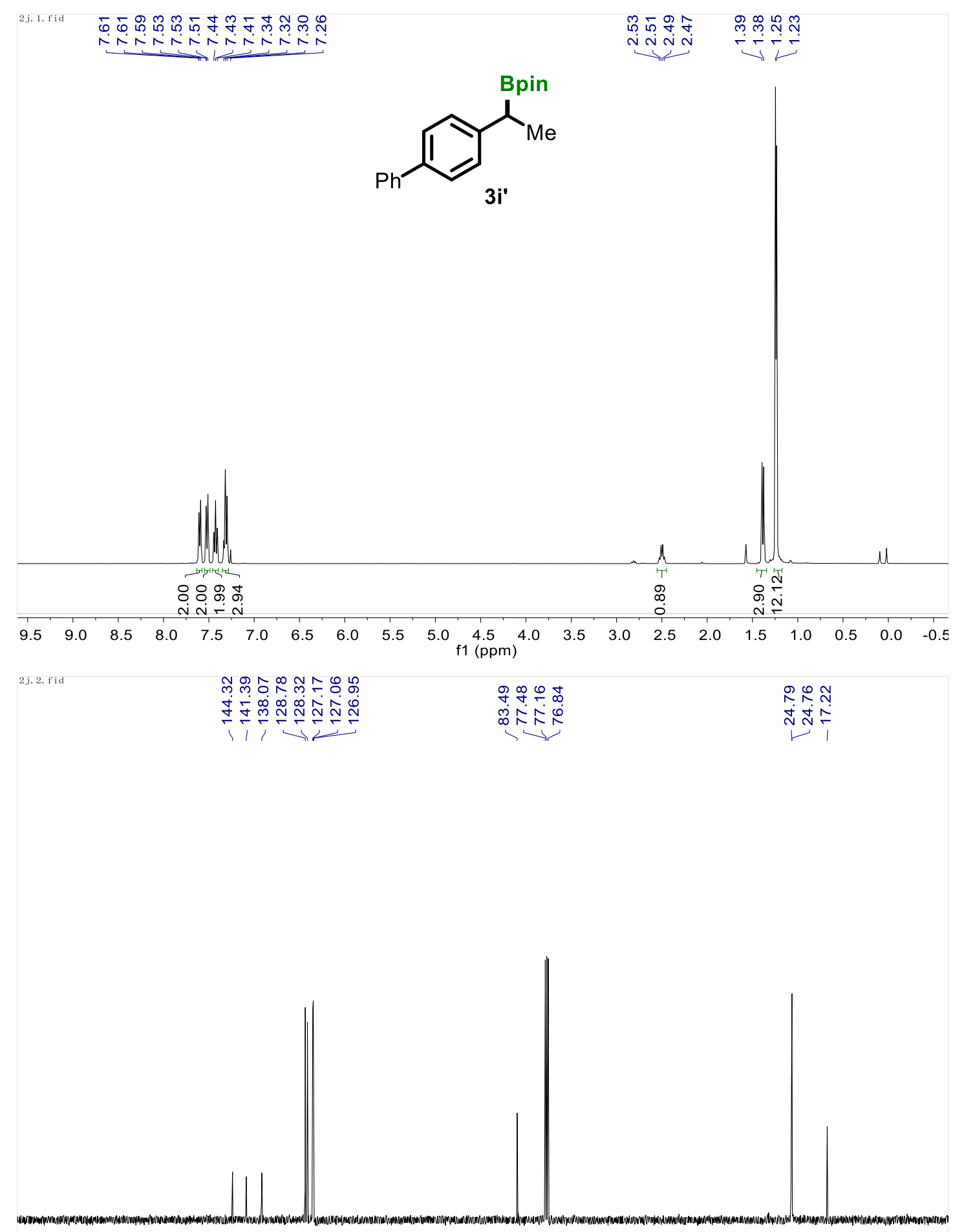

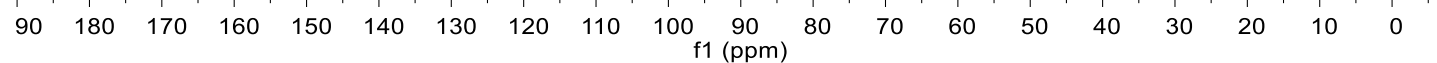




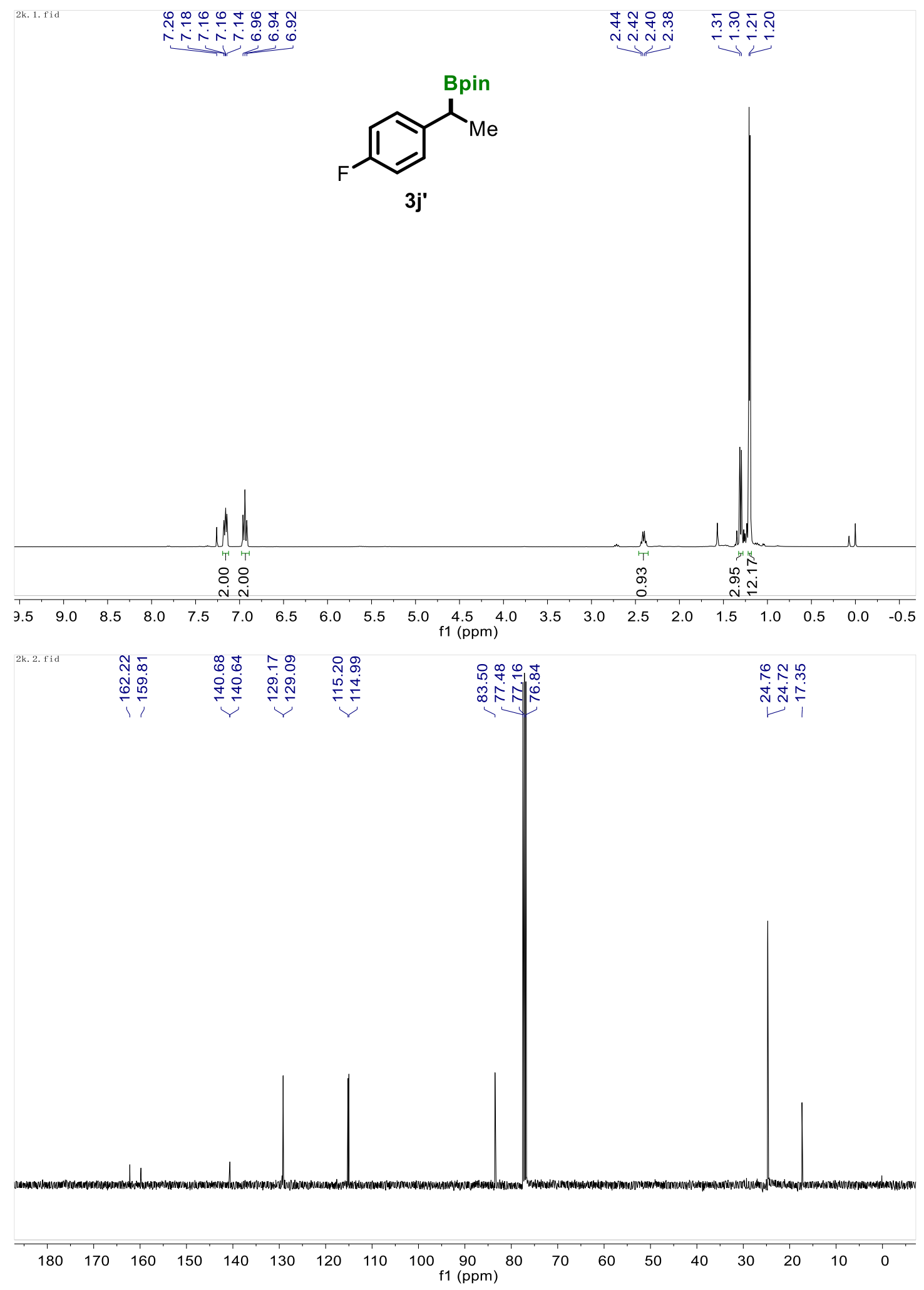




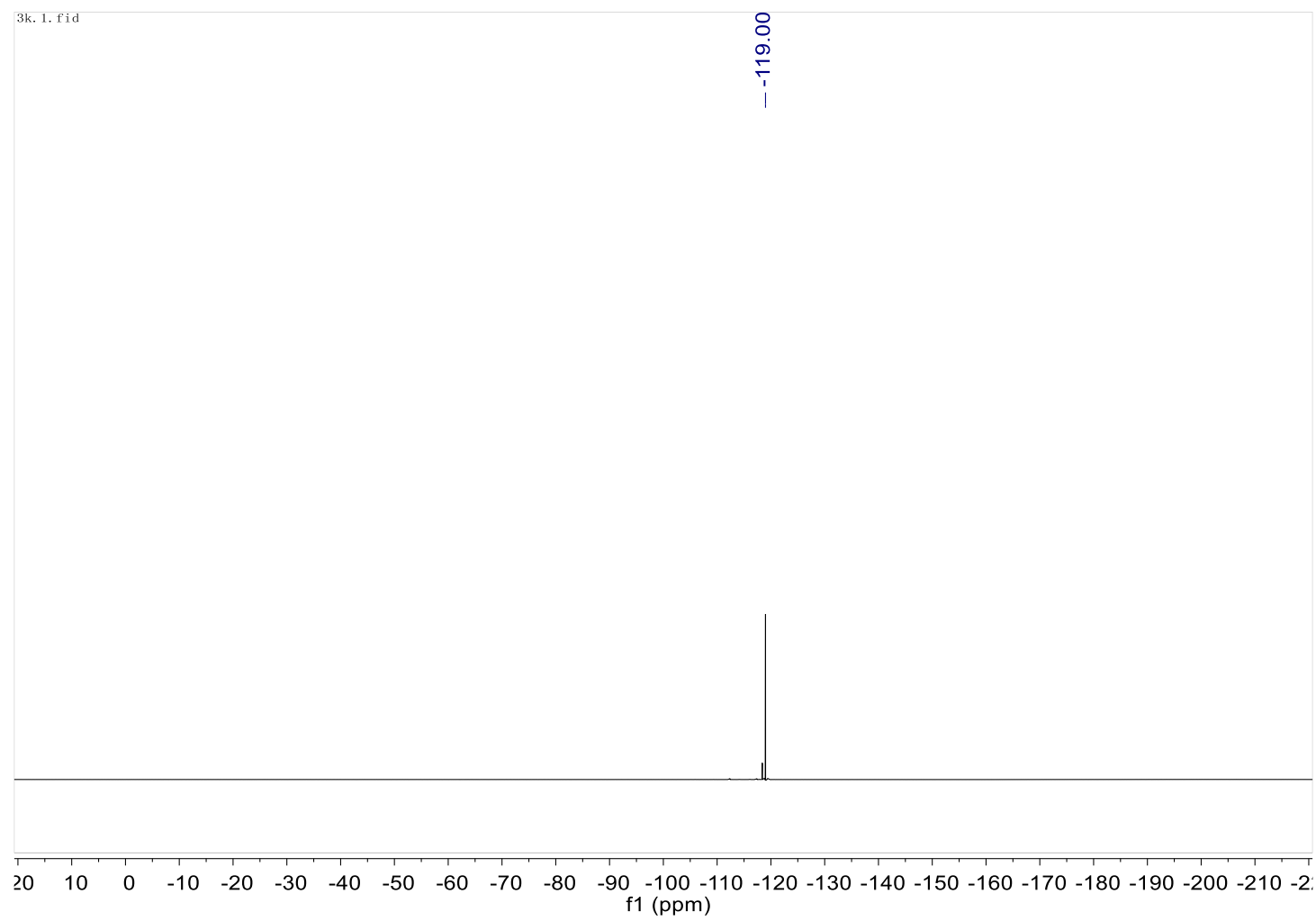



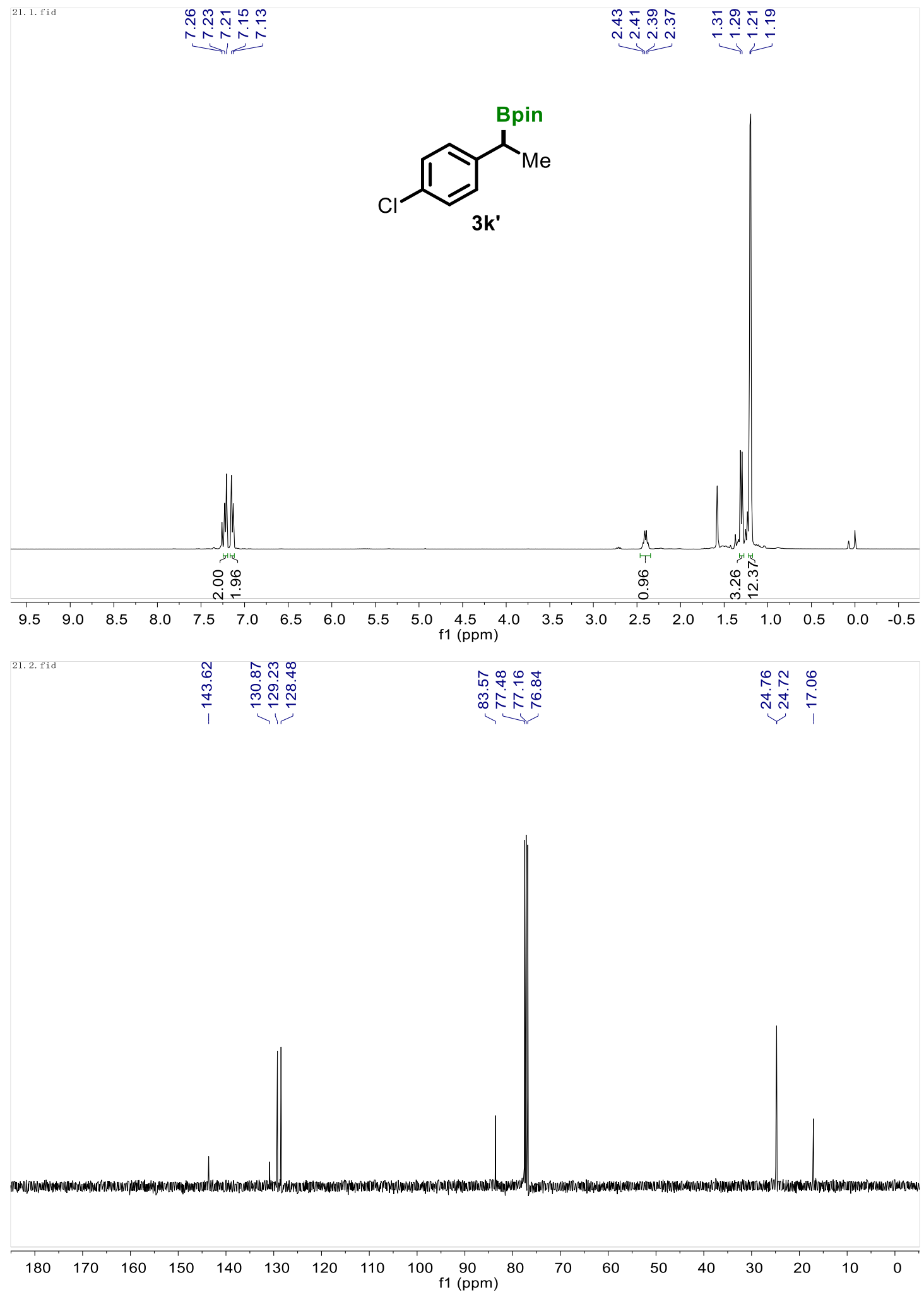

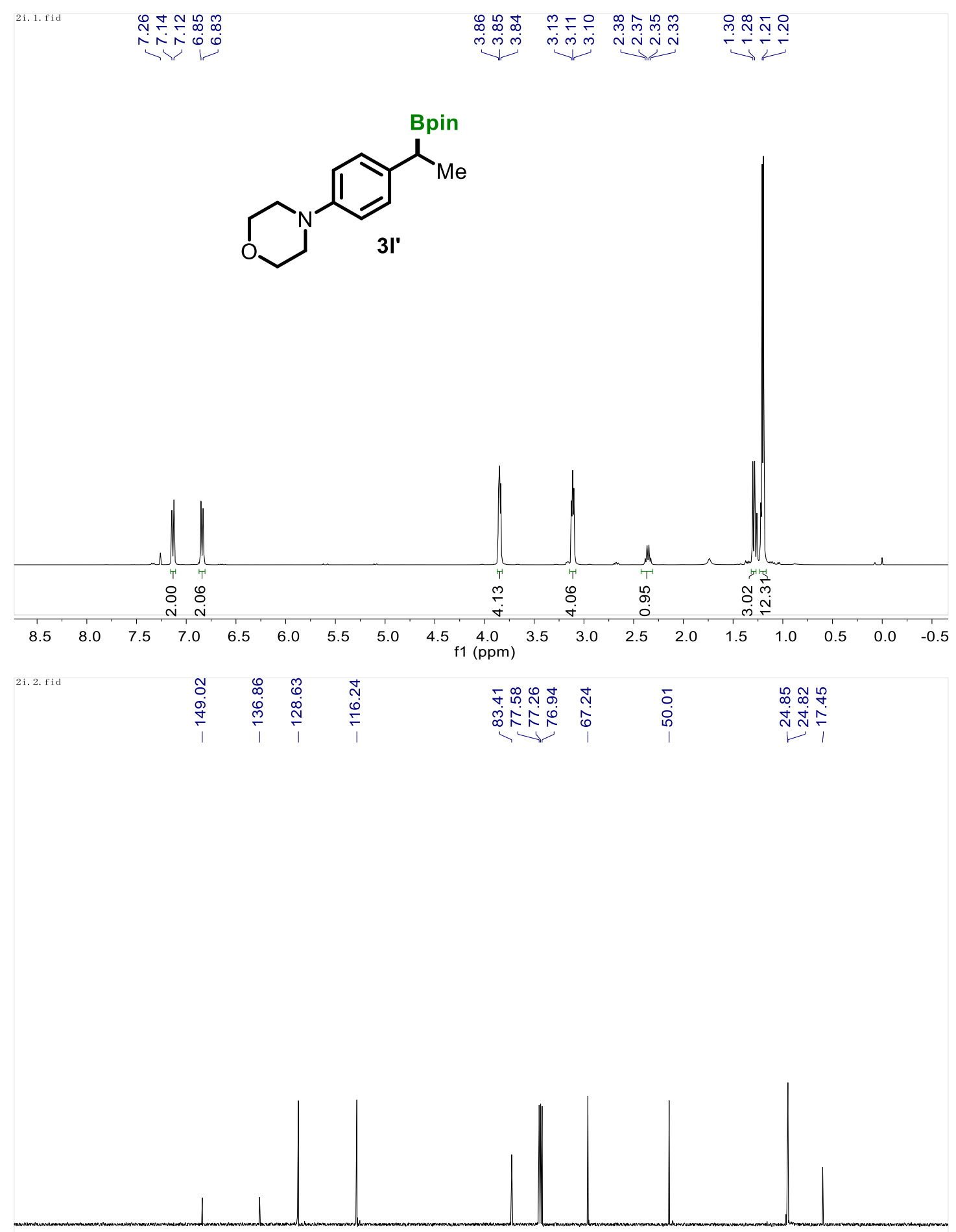

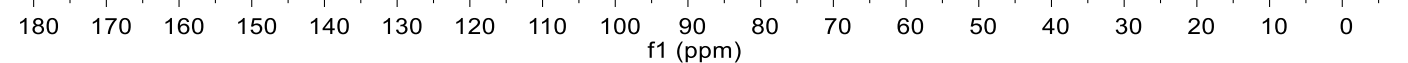




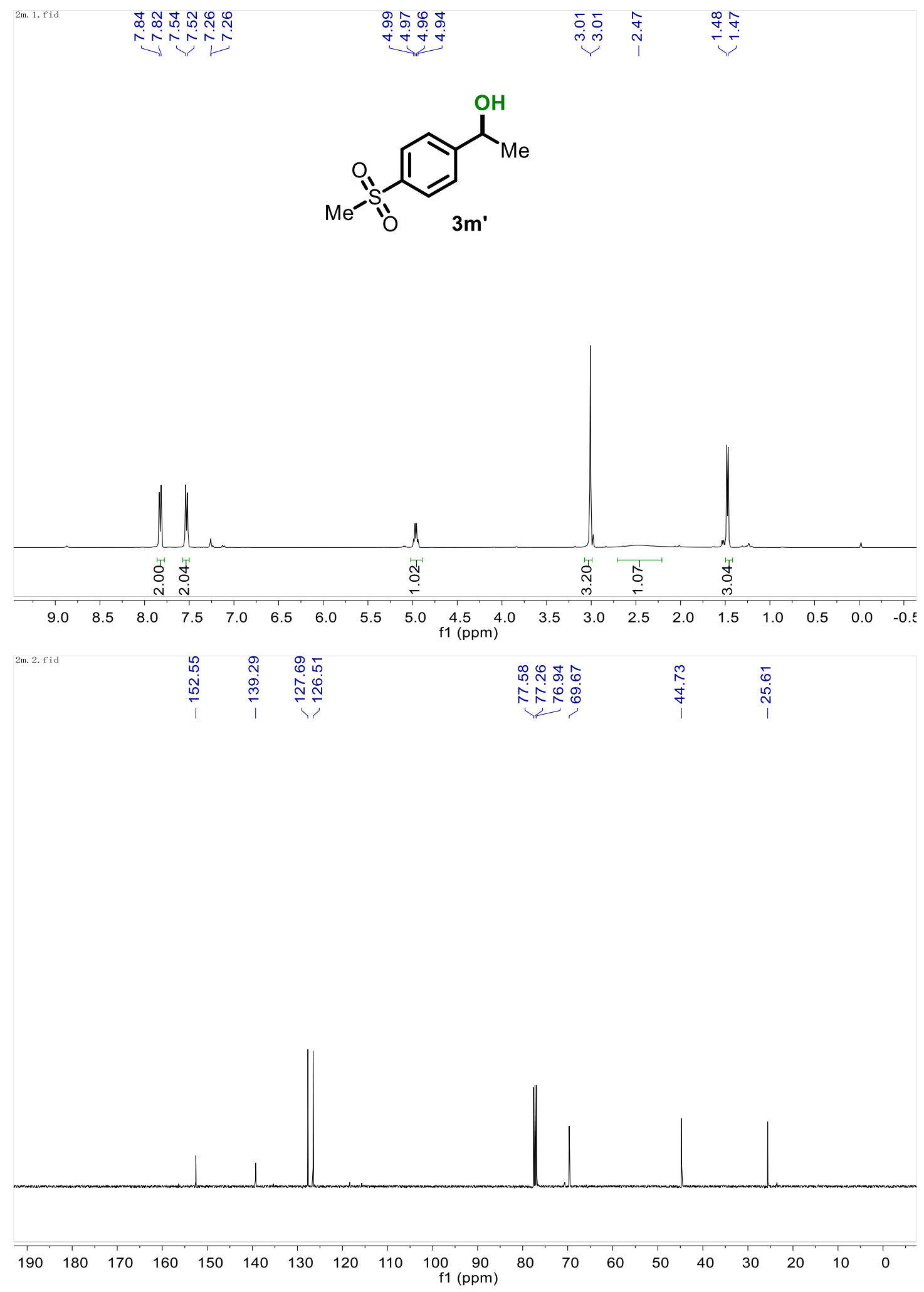



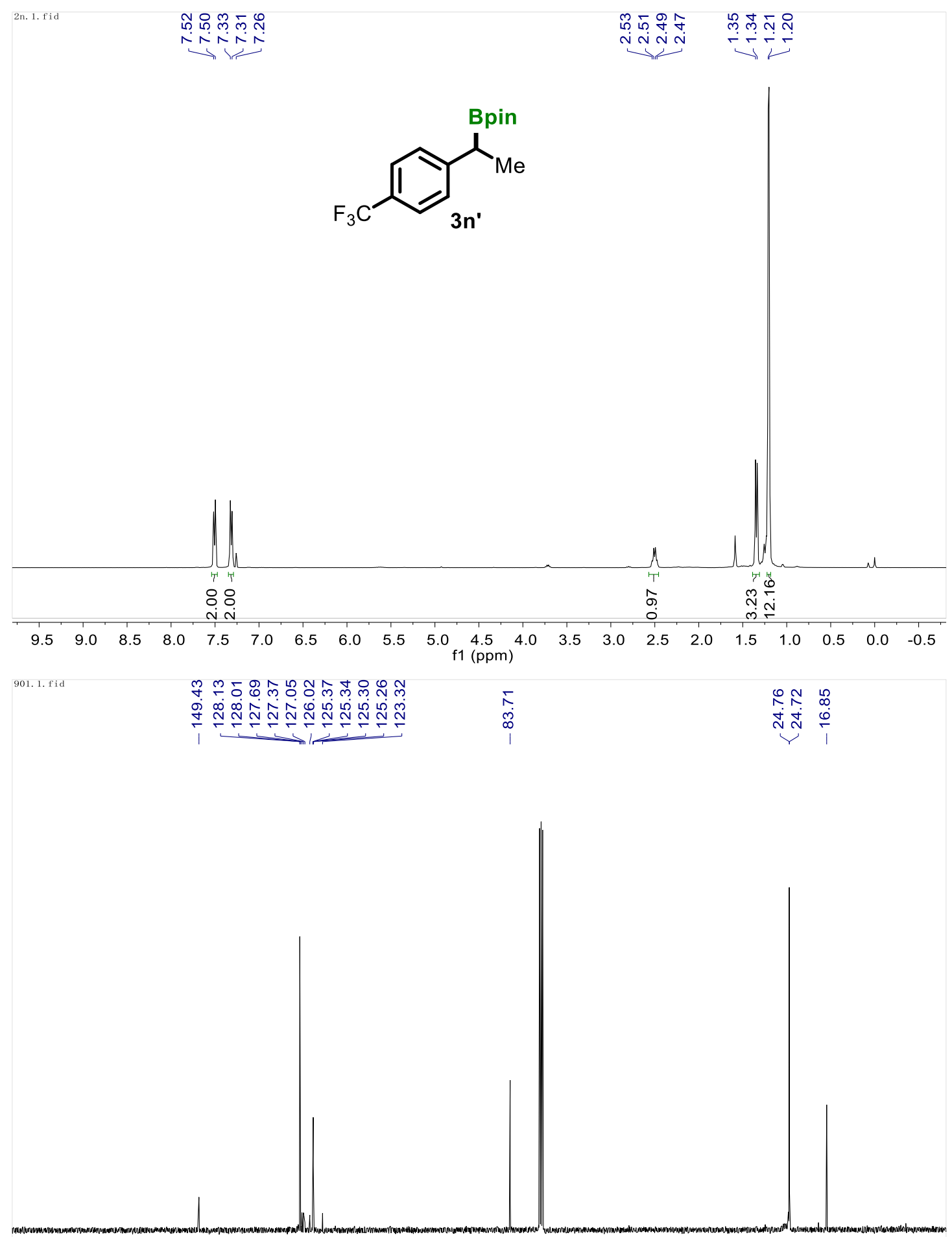

$\begin{array}{llllllllllllllllll}180 & 170 & 160 & 150 & 140 & 130 & 120 & 110 & 100 \underset{\mathrm{f} 1}{\stackrel{90}{(\mathrm{ppm})}} \mathbf{8 0} & 70 & 60 & 50 & 40 & 30 & 20 & 10 & 0\end{array}$ 


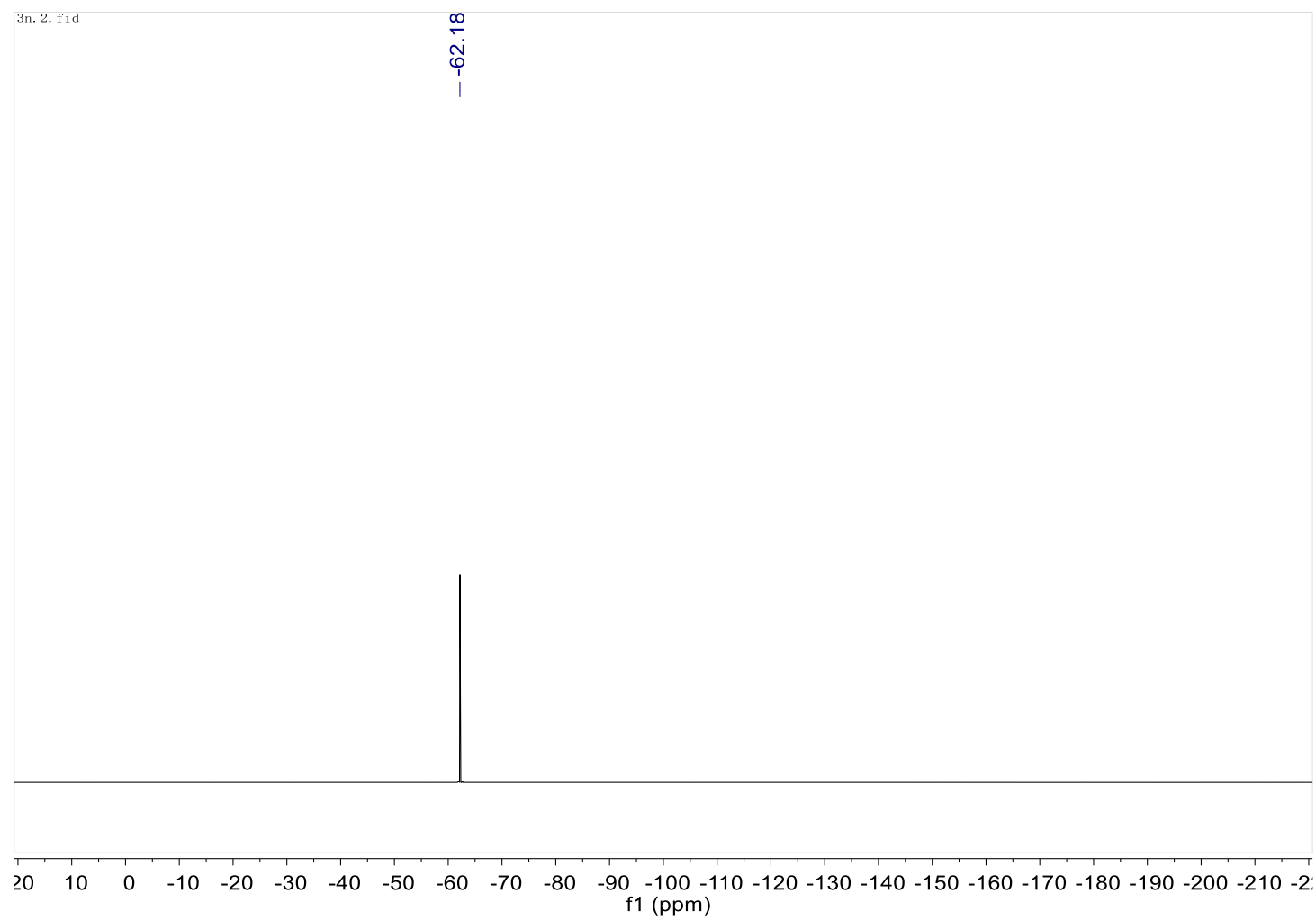



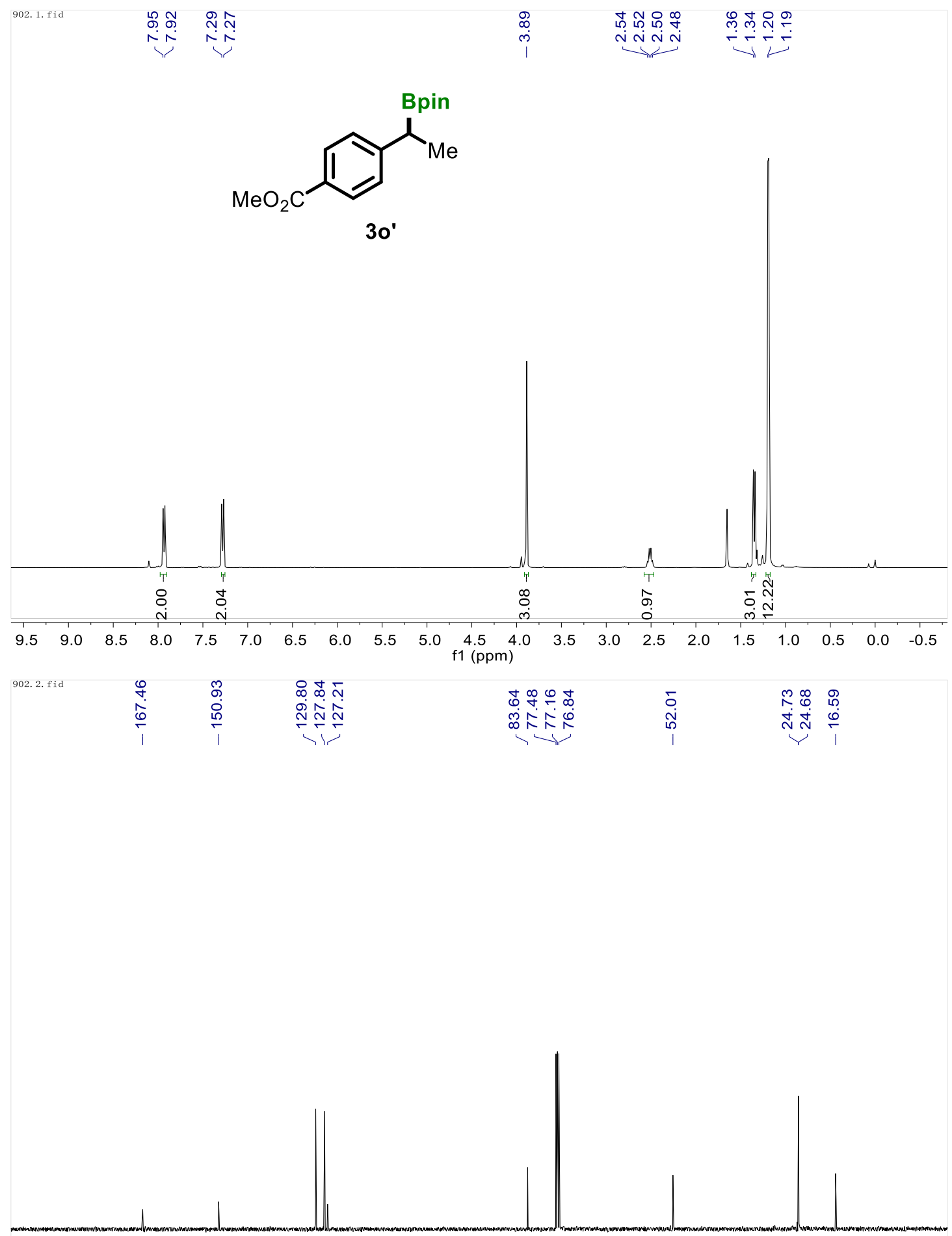

$\begin{array}{lllllllllllllllllllllll}190 & 180 & 170 & 160 & 150 & 140 & 130 & 120 & 110 & 100 & 90 & 80 & 70 & 60 & 50 & 40 & 30 & 20 & 10 & 0\end{array}$ 

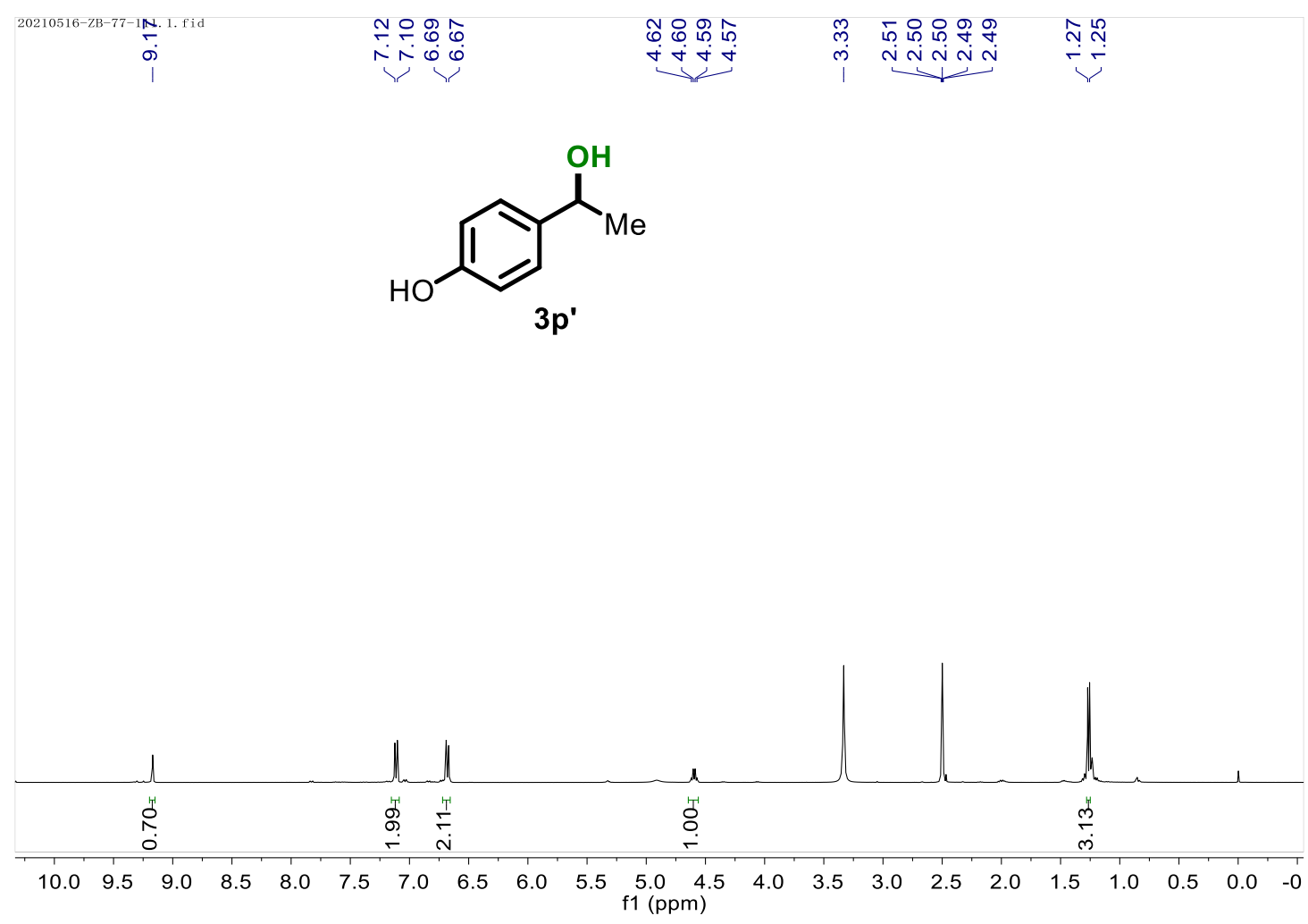

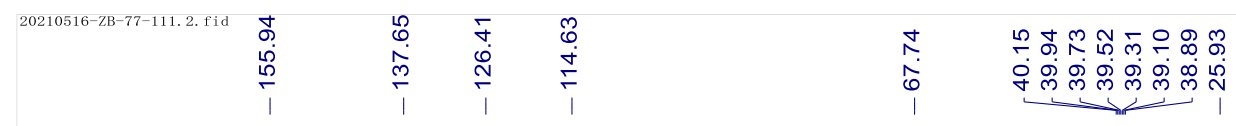

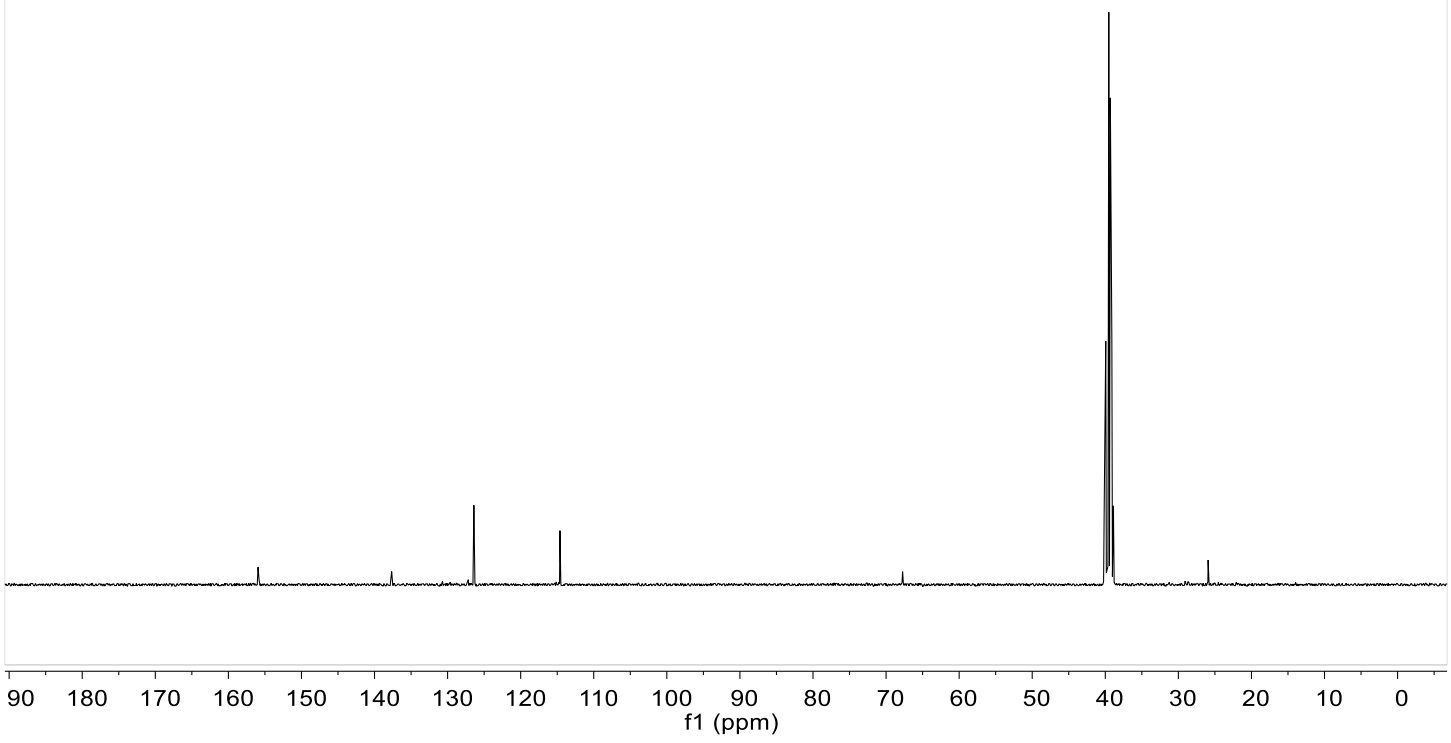



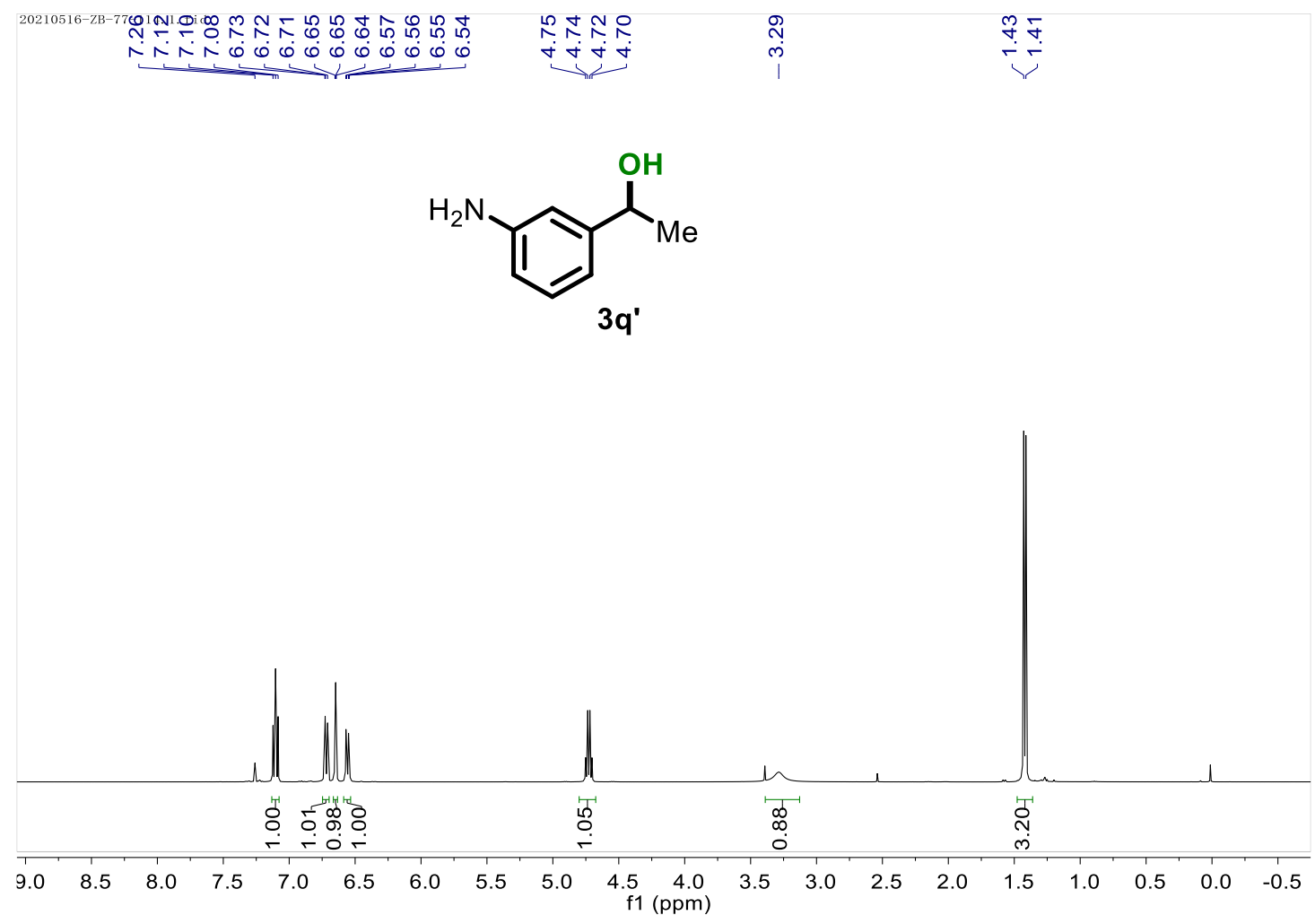

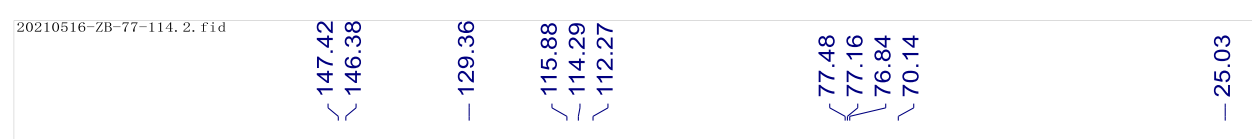

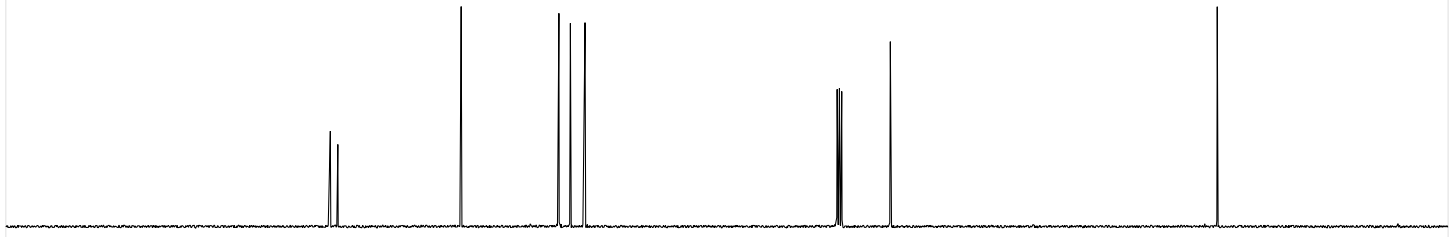

$\begin{array}{llllllllllllllllllll}190 & 180 & 170 & 160 & 150 & 140 & 130 & 120 & 110 & \underset{\mathrm{f} 1}{100}(\mathrm{ppm}) & 90 & 70 & 60 & 50 & 40 & 30 & 20 & 10 & 0\end{array}$ 

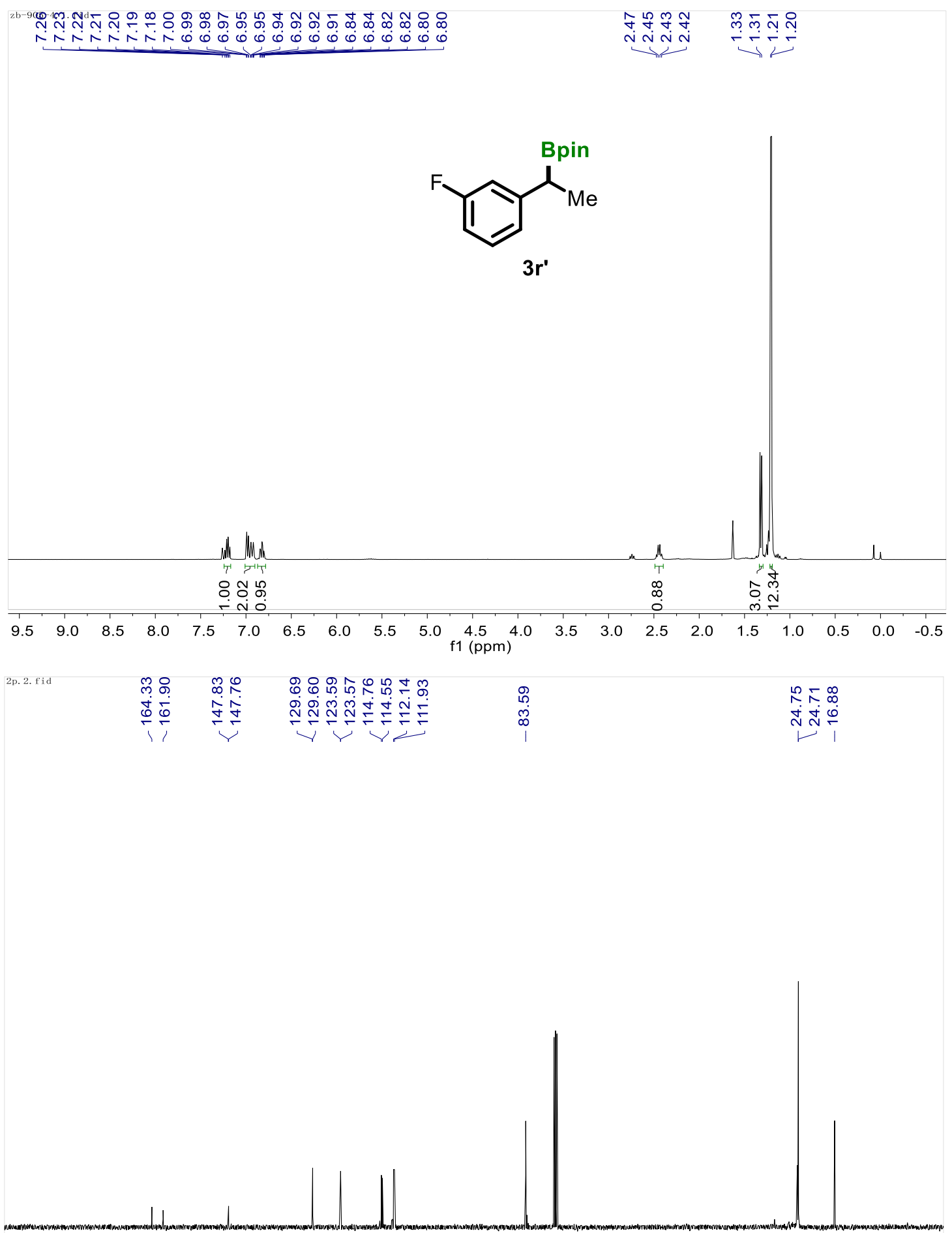

$\begin{array}{lllllllllllllllllllllll}190 & 180 & 170 & 160 & 150 & 140 & 130 & 120 & 110 & 100 & 90 & 80 & 70 & 60 & 50 & 40 & 30 & 20 & 10 & 0\end{array}$ f1 (ppm) 


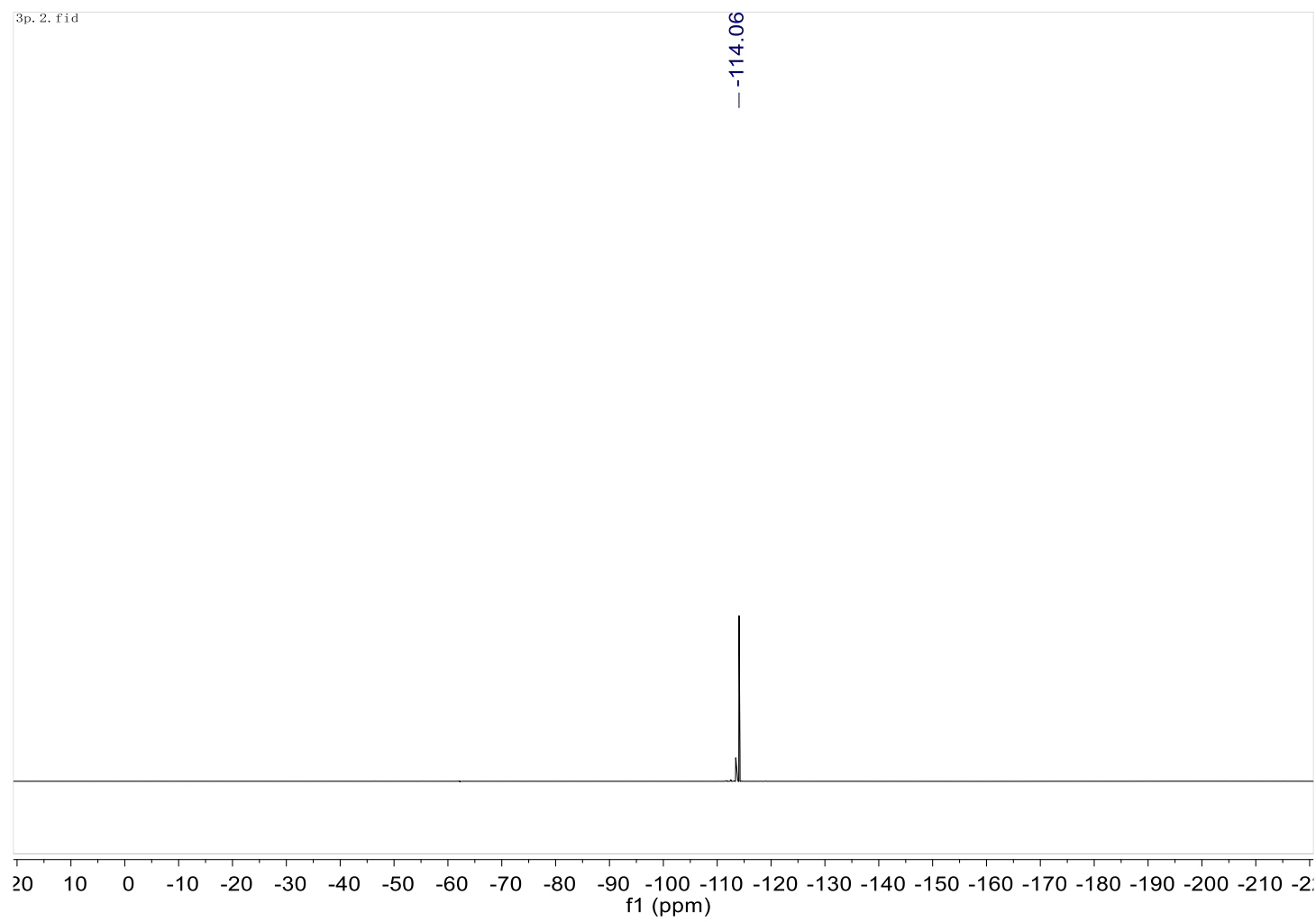




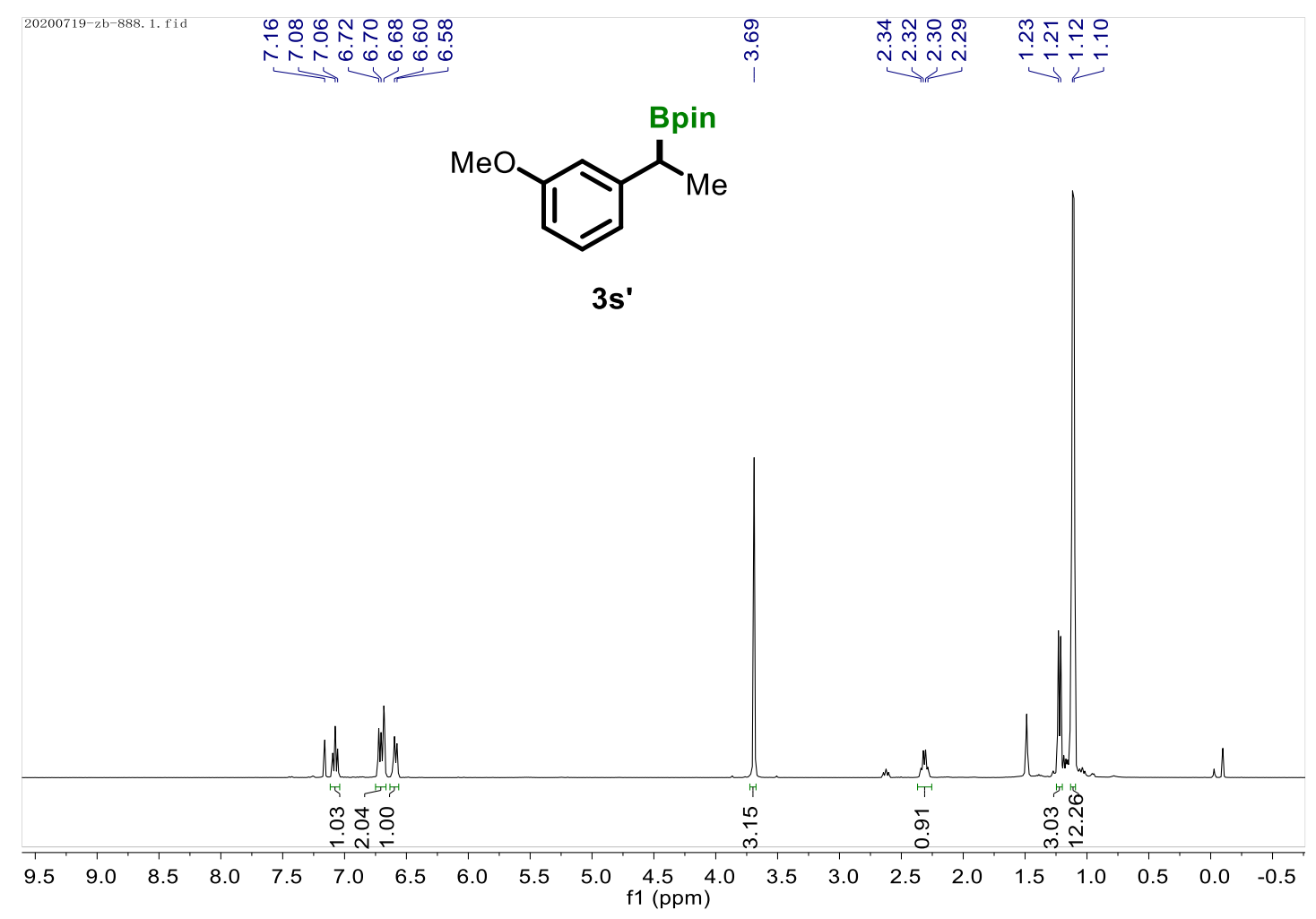

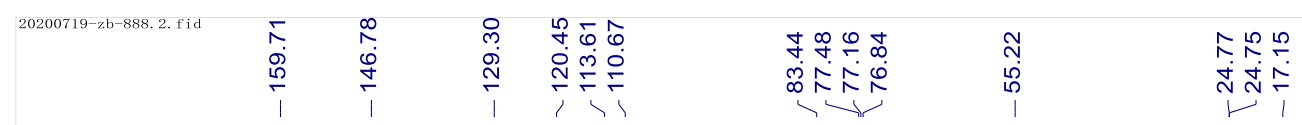

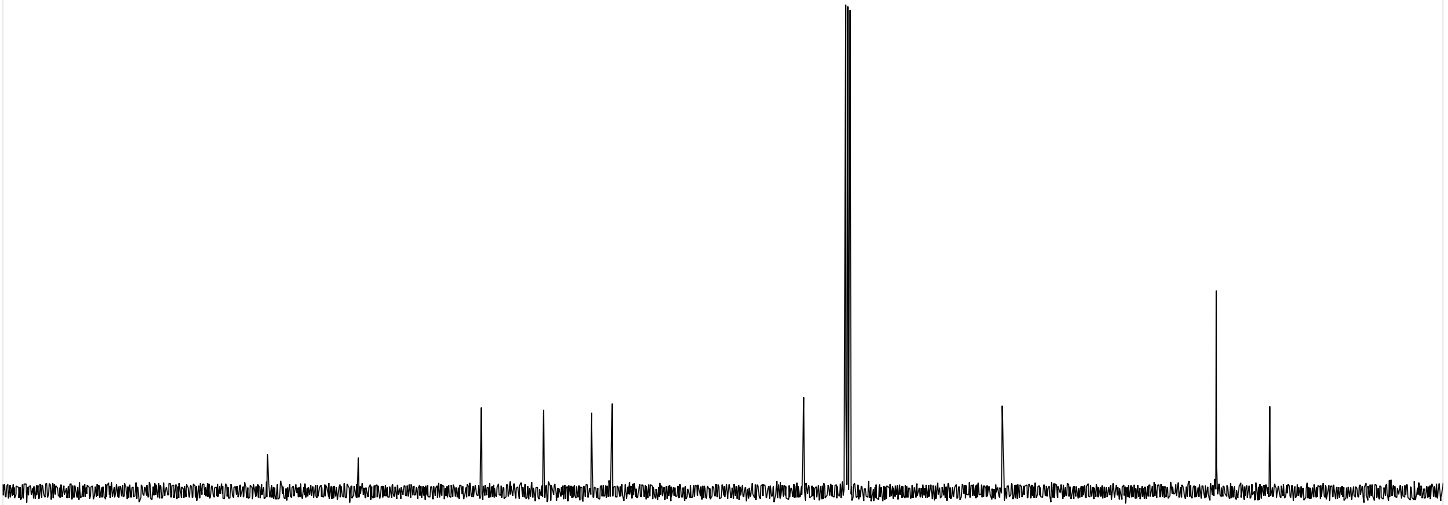

$\begin{array}{lllllllllllllllllllllll}190 & 180 & 170 & 160 & 150 & 140 & 130 & 120 & 110 & 100 & 90 & 80 & 70 & 60 & 50 & 40 & 30 & 20 & 10 & 0\end{array}$ 


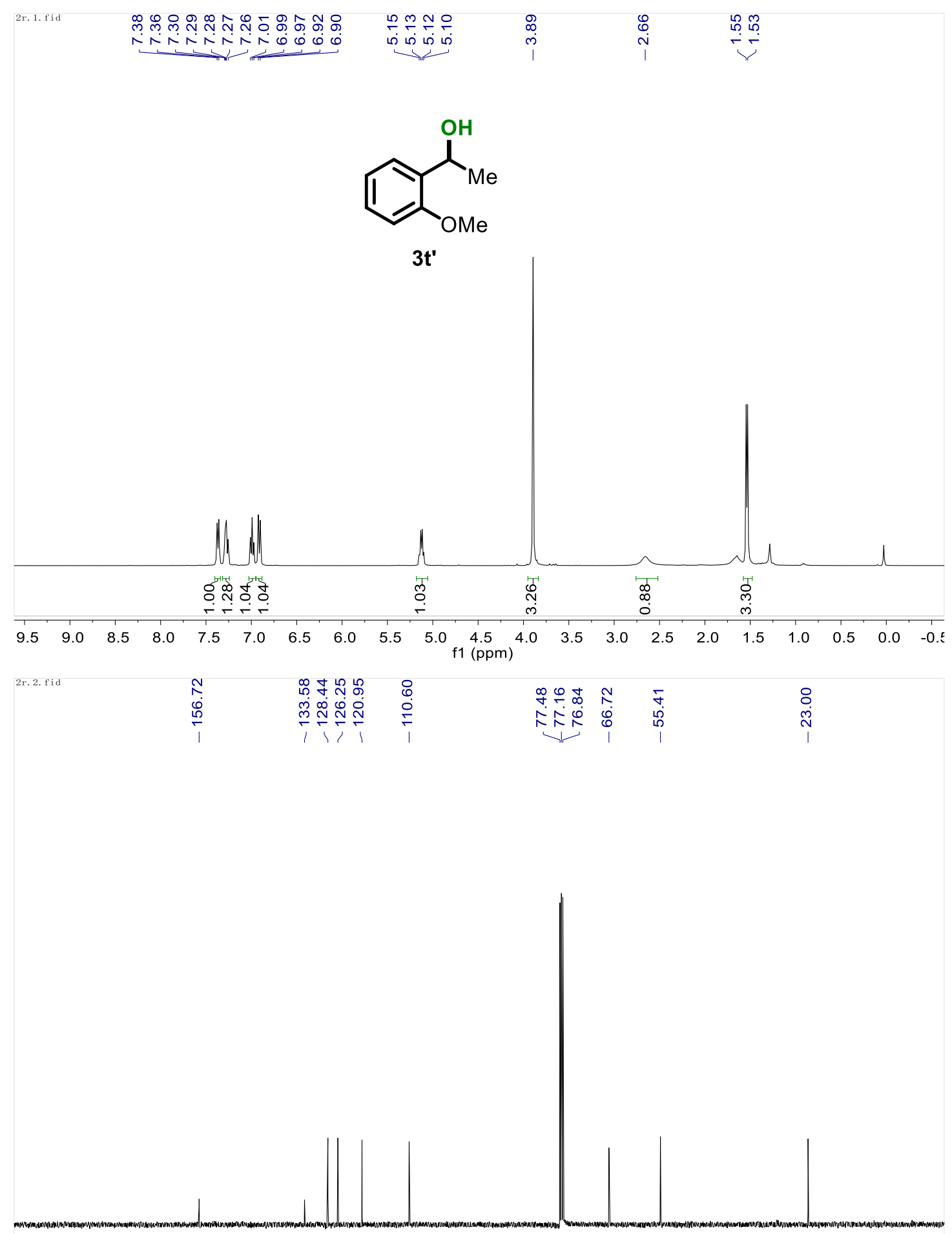

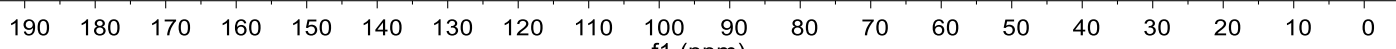



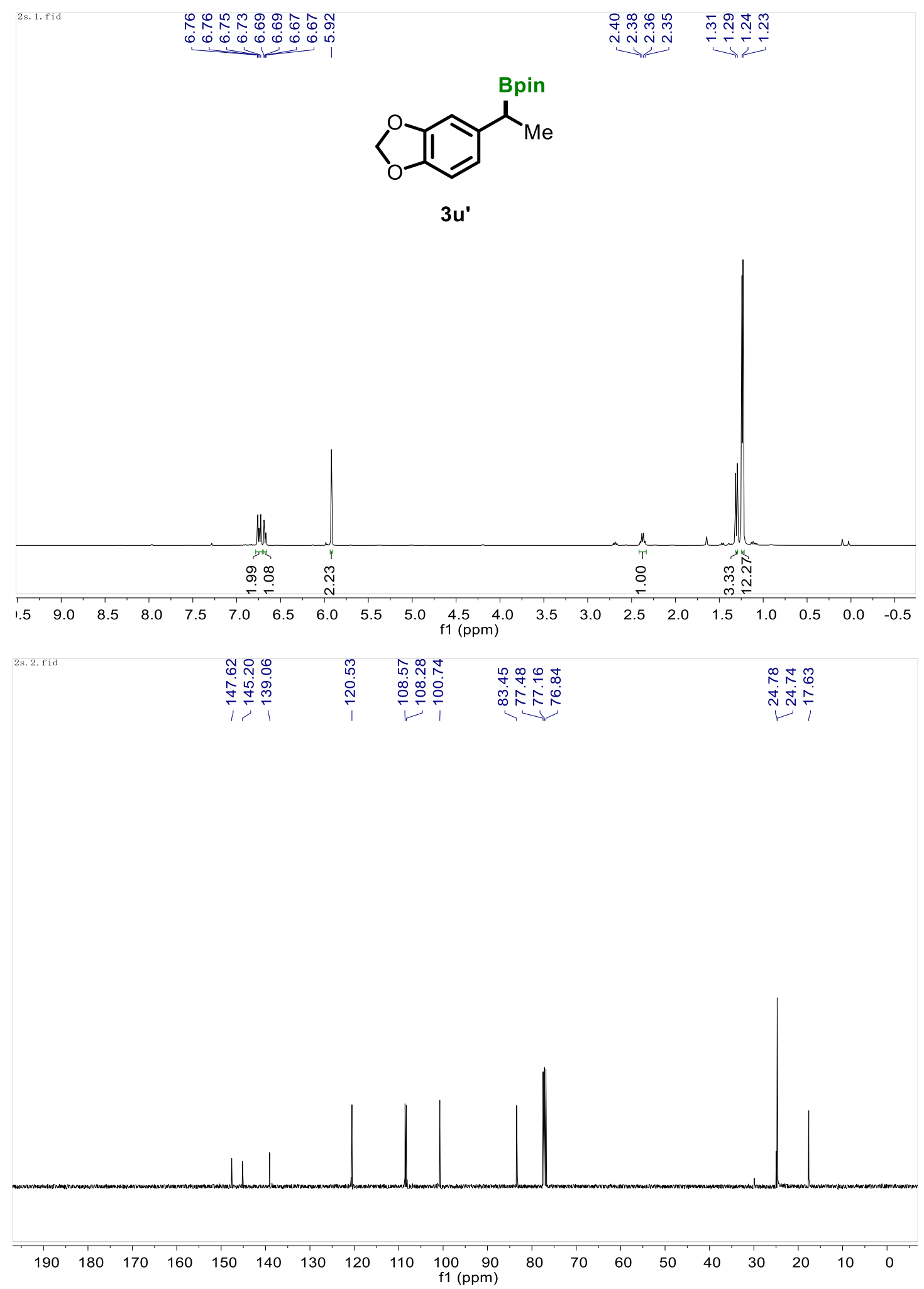


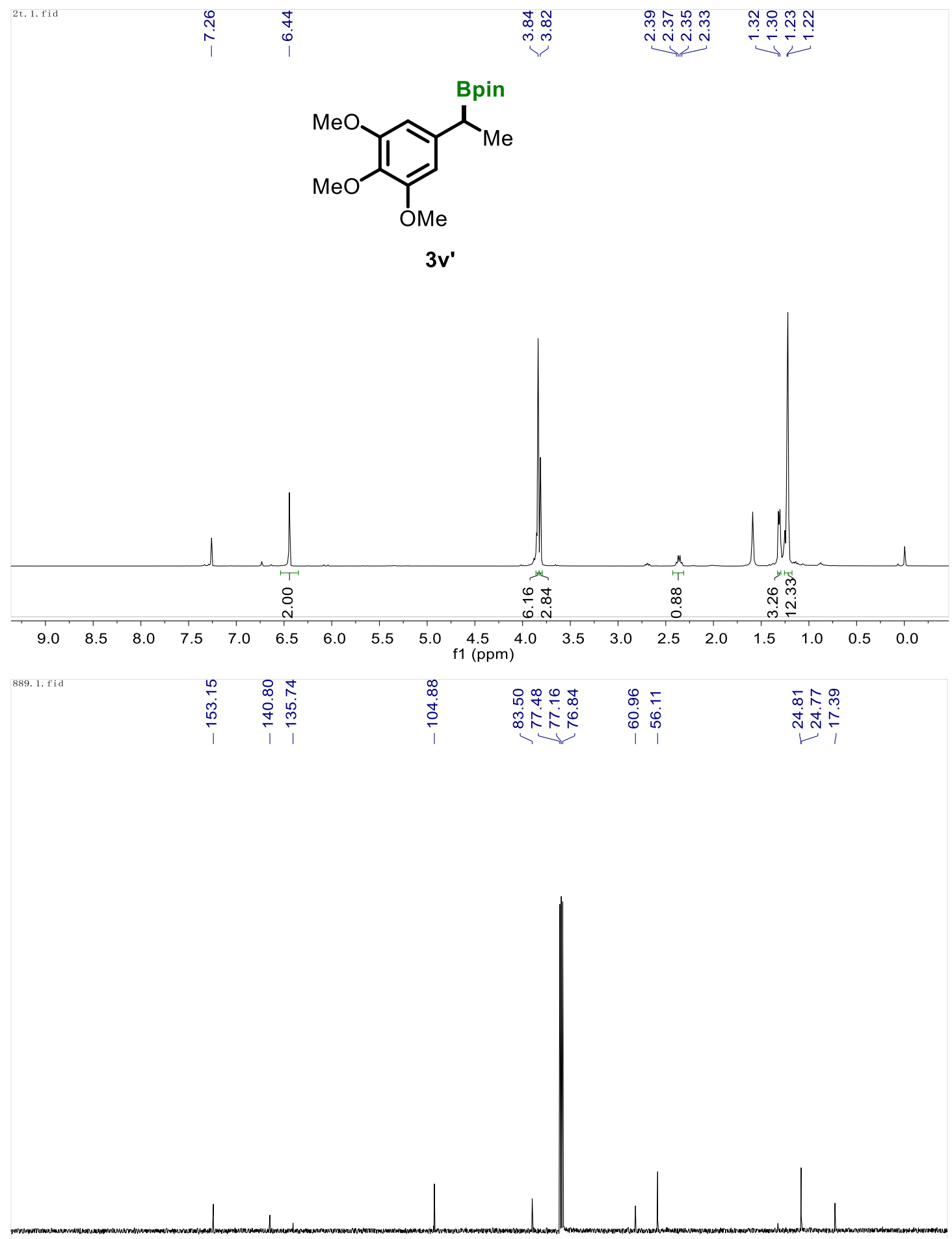

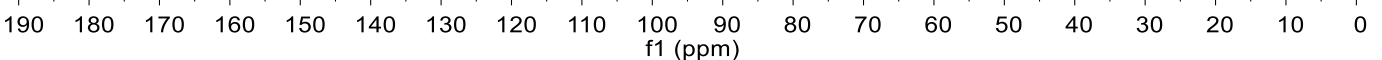



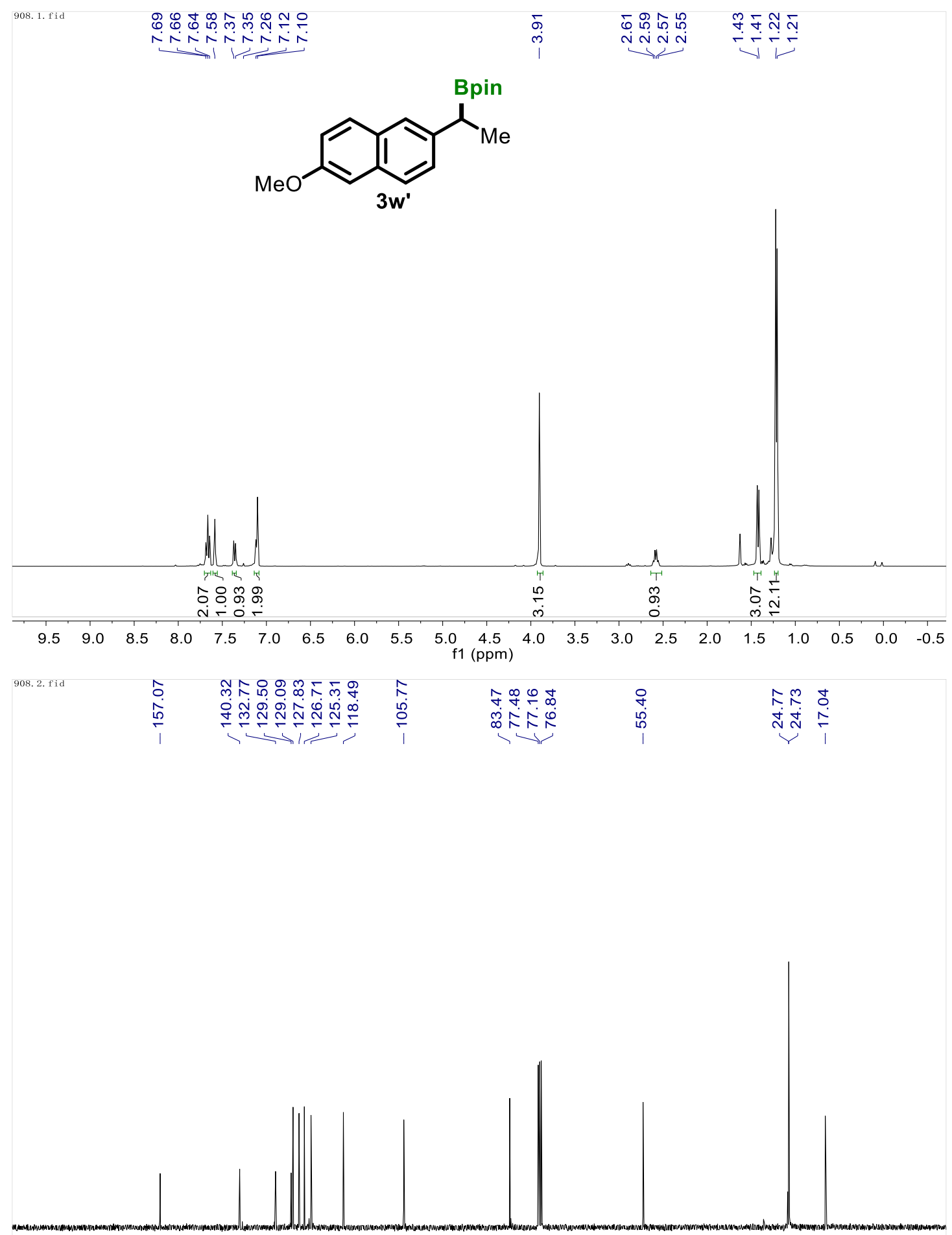

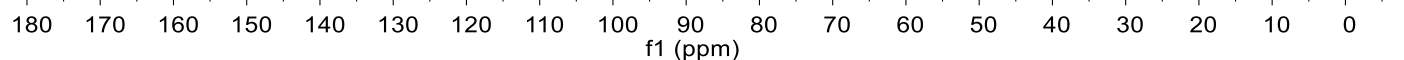




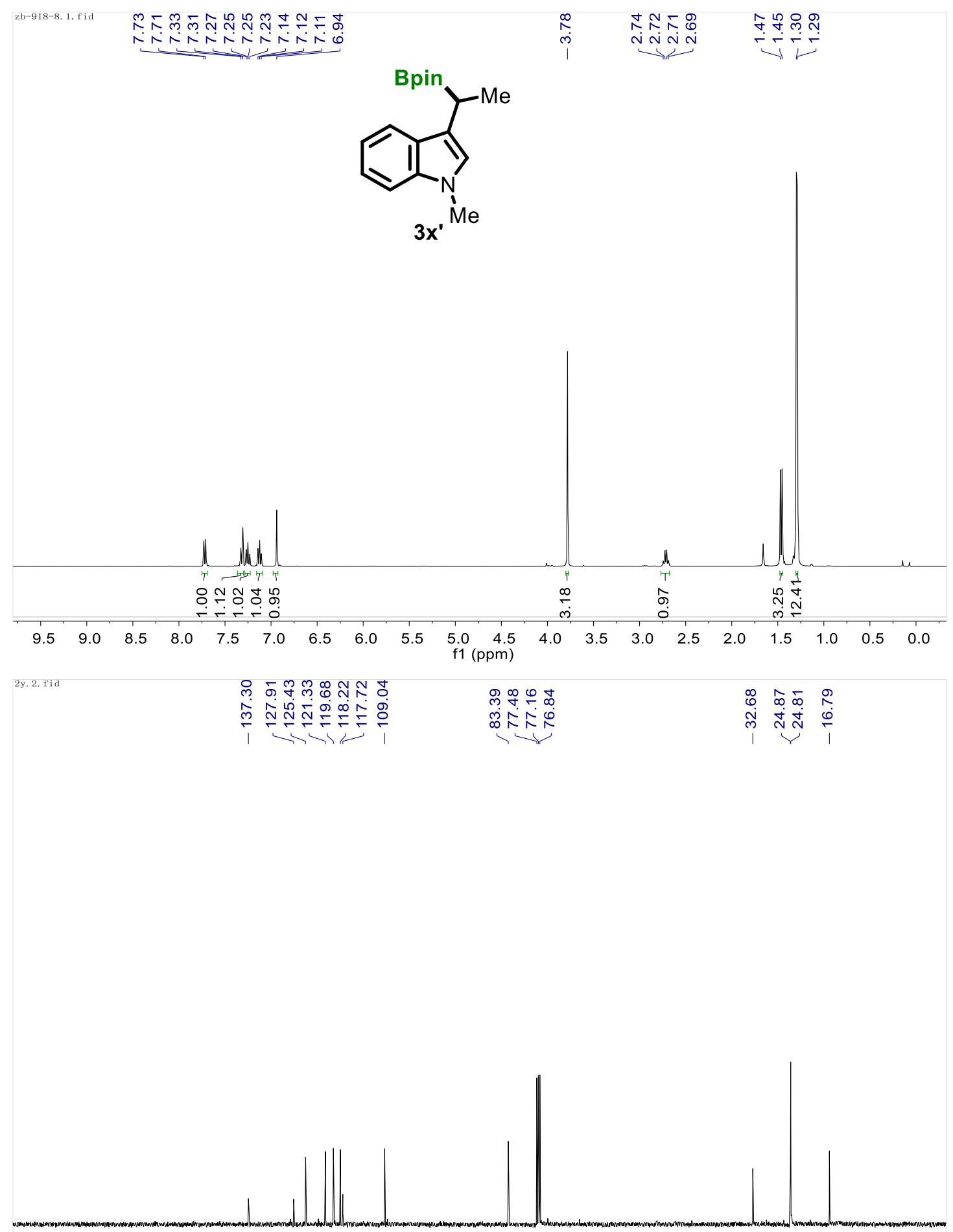

$\begin{array}{llllllllllllllllll}180 & 170 & 160 & 150 & 140 & 130 & 120 & 110 & 100 \underset{\mathrm{f} 1}{90}(\mathrm{ppm}) & 80 & 70 & 60 & 50 & 40 & 30 & 20 & 10 & 0\end{array}$ 


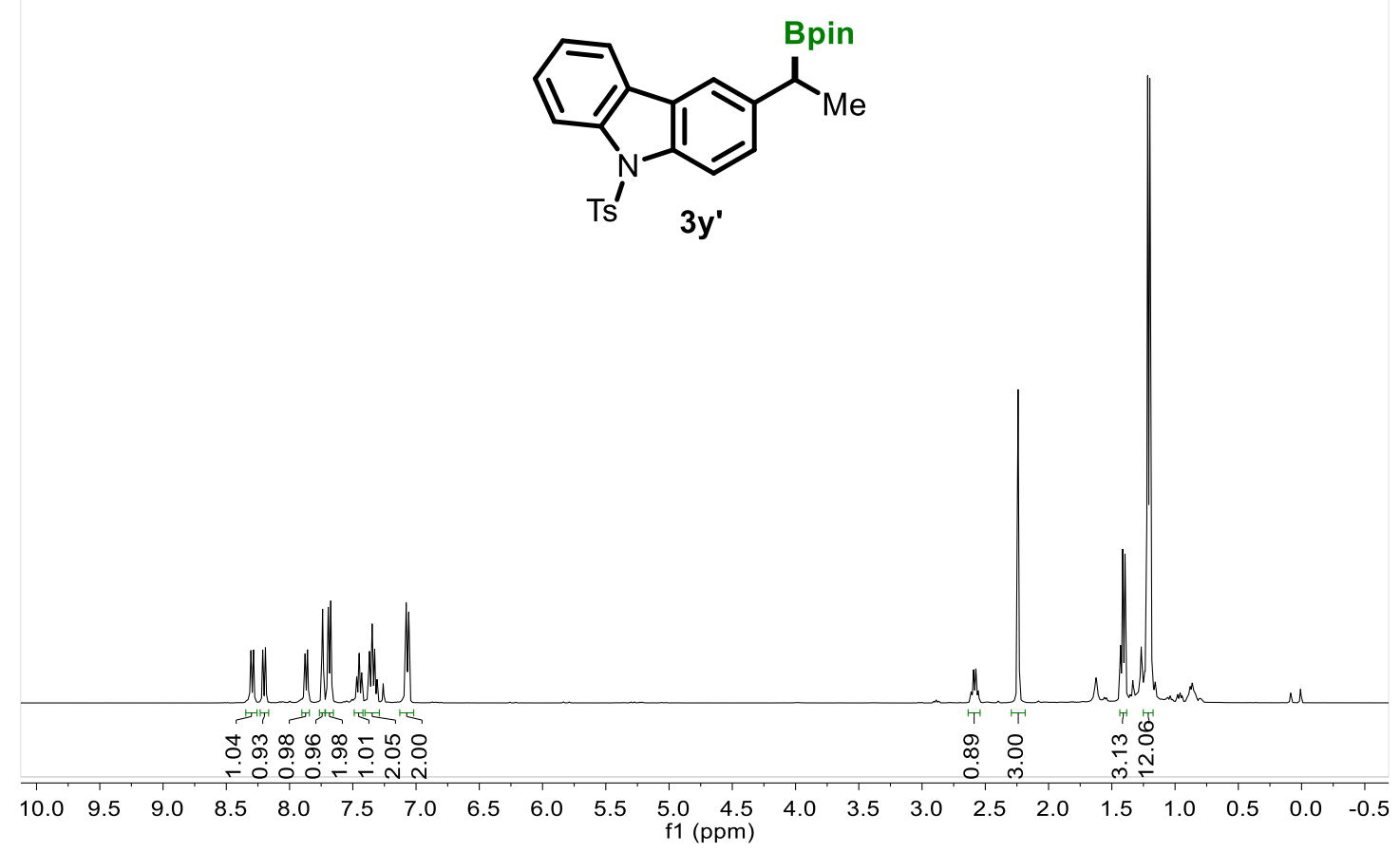
$2 x^{2}, \mathrm{fid}$

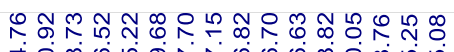

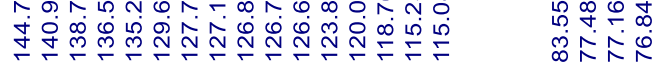

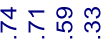

ปั่

化

$\checkmark<$

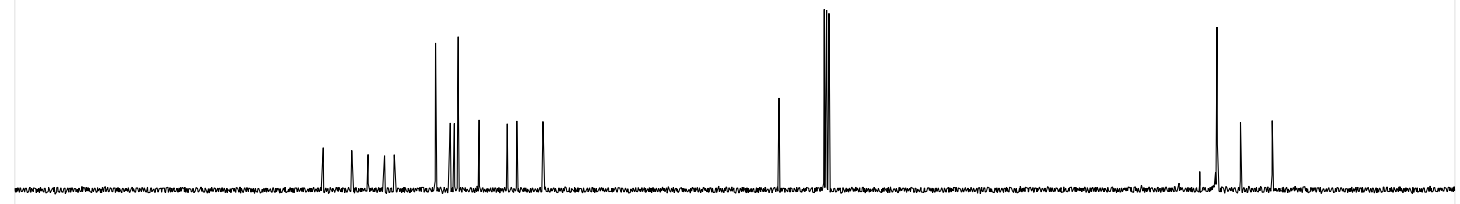

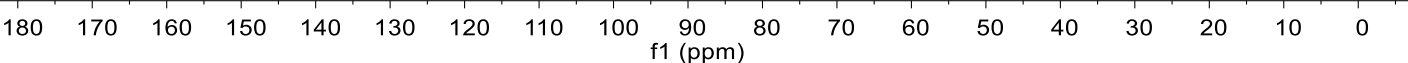




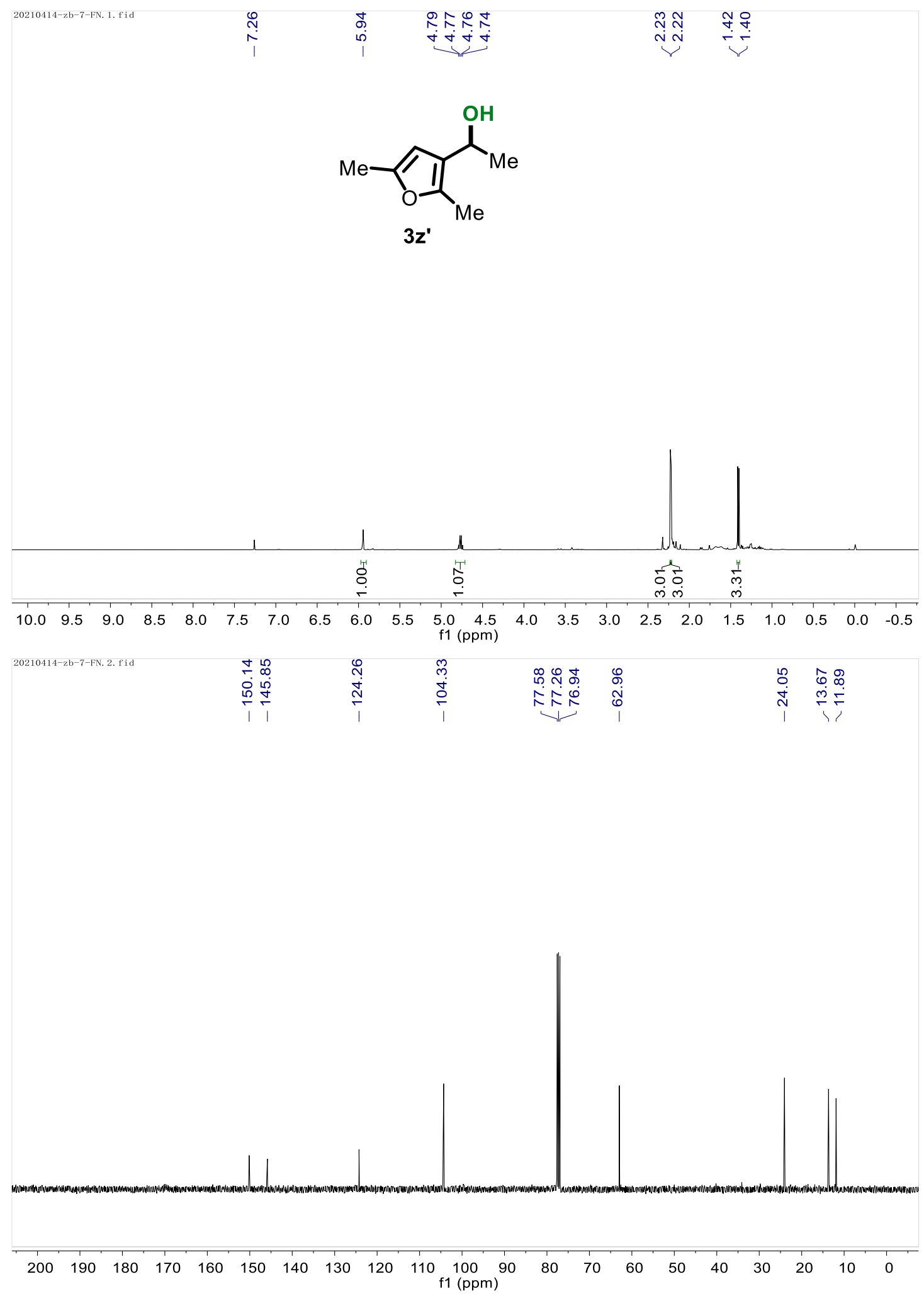




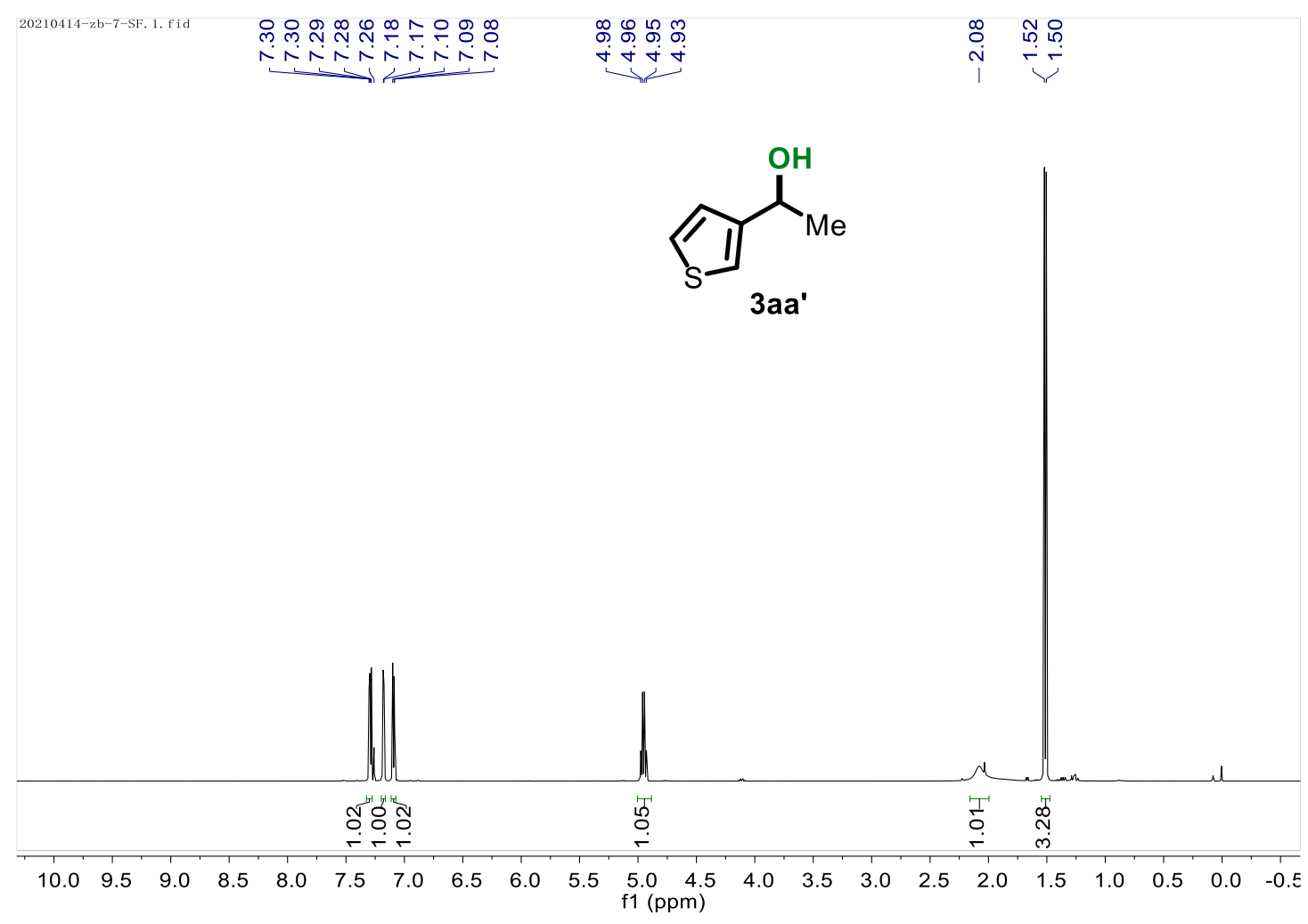

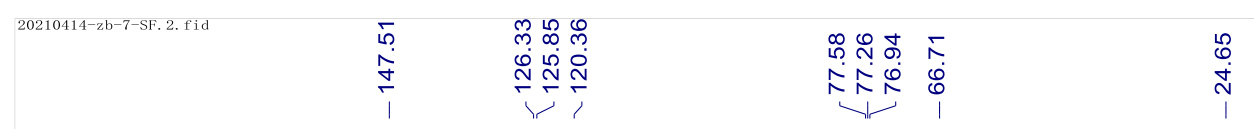

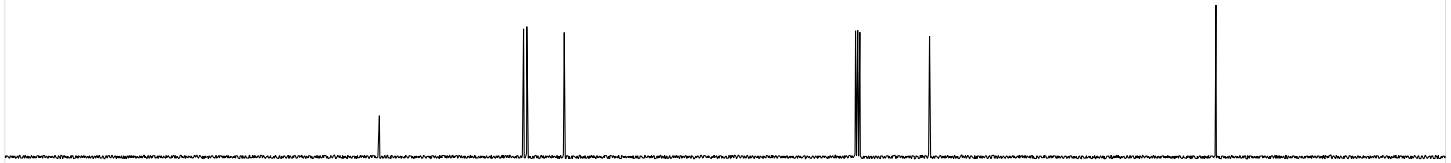
$\begin{array}{llllllllllllllllllll}200 & 190 & 180 & 170 & 160 & 150 & 140 & 130 & 120 & 110 & \begin{array}{c}100 \\ \mathrm{f} 1(\mathrm{ppm})\end{array} & 80 & 70 & 60 & 50 & 40 & 30 & 20 & 10 & 0\end{array}$ 
<smiles>CC(C)=CCCC(C)CCOc1ccc(C(C)O)cc1</smiles>

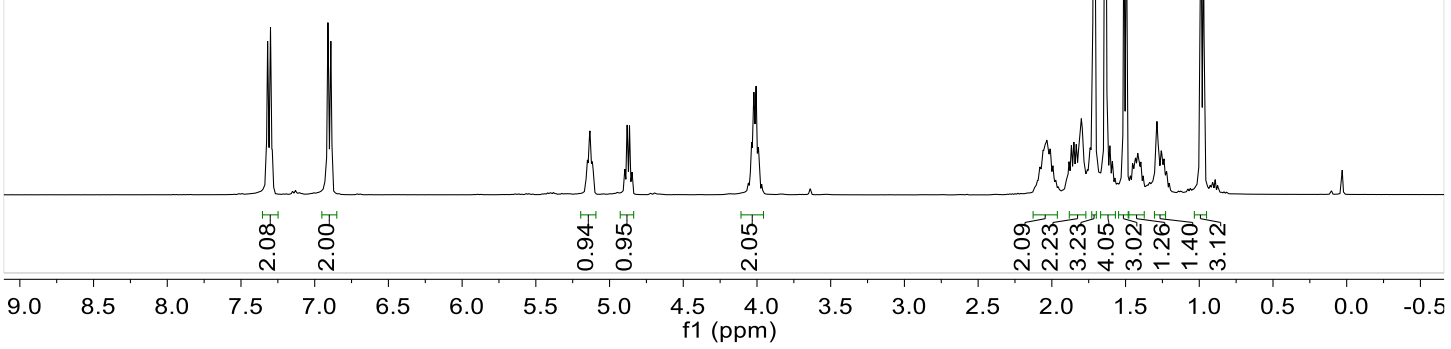

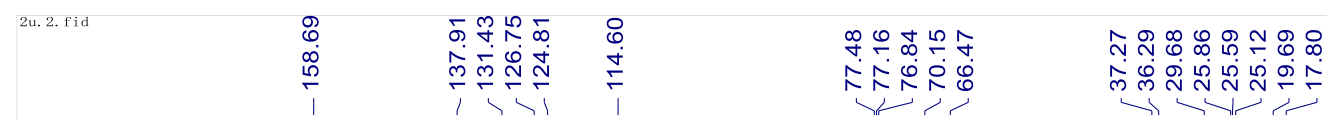

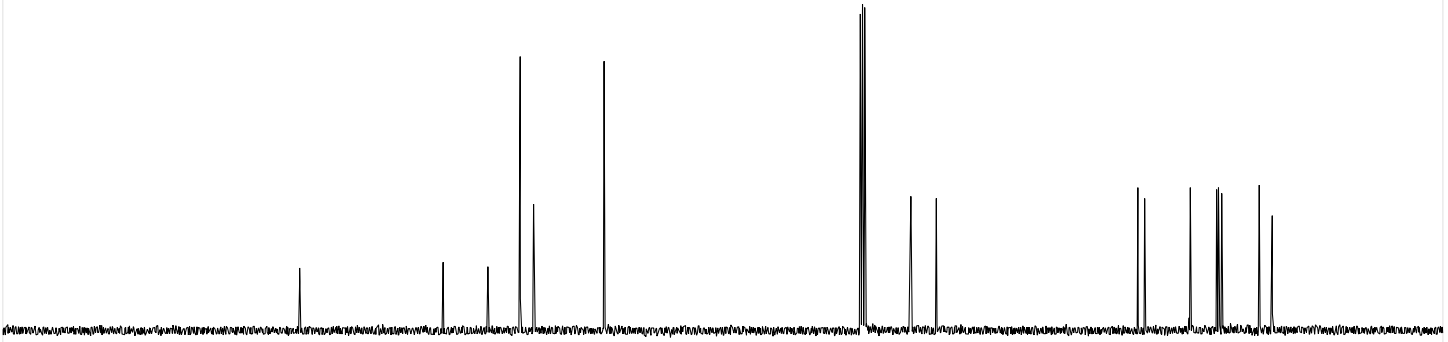

$\begin{array}{llllllllllllllllllllllll}200 & 190 & 180 & 170 & 160 & 150 & 140 & 130 & 120 & 110 & 100 & 90 & 80 & 70 & 60 & 50 & 40 & 30 & 20 & 10 & 0\end{array}$ 


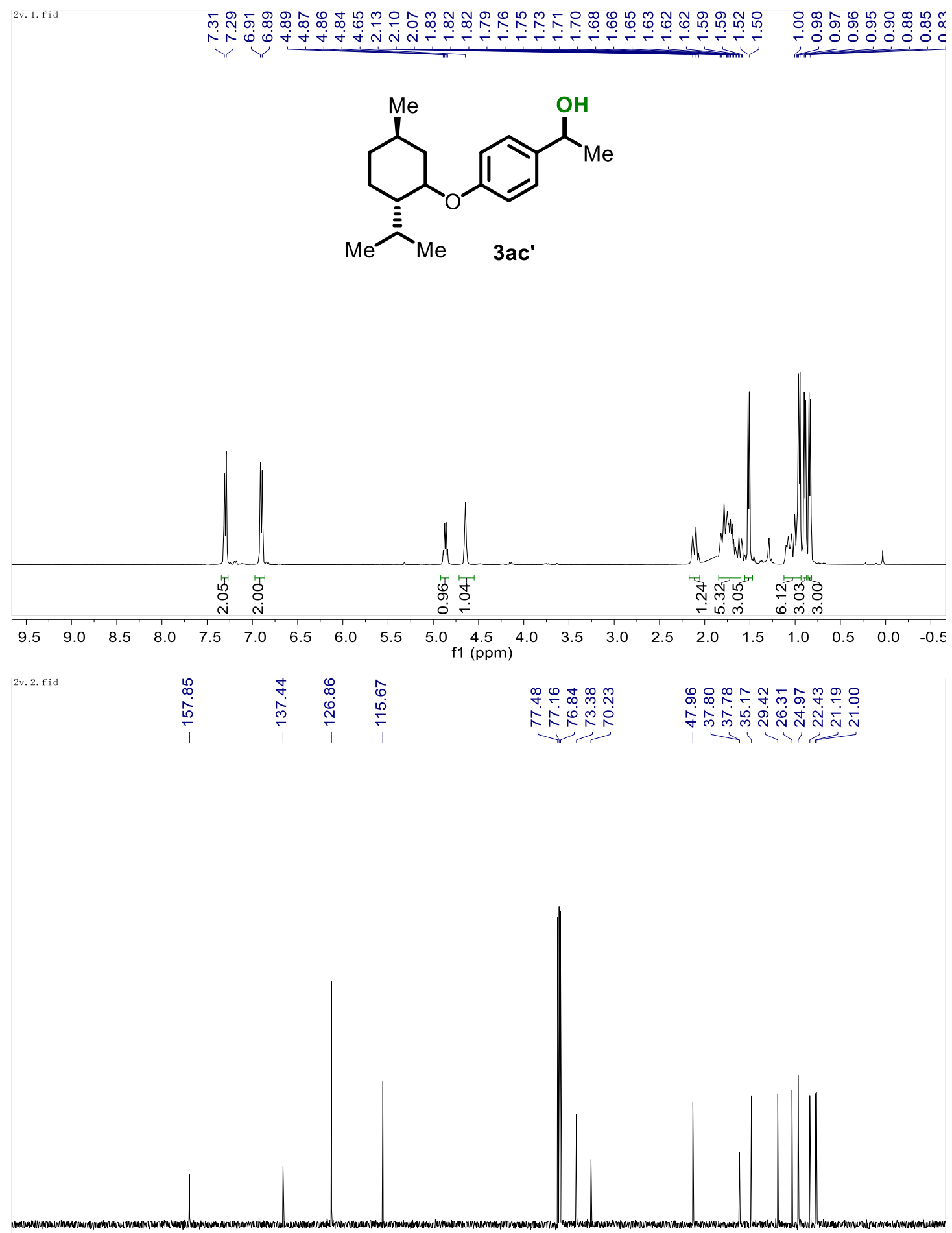

$\begin{array}{llllllllllllllllllll}190 & 180 & 170 & 160 & 150 & 140 & 130 & 120 & 110 & 100 & 90 & 80 & 70 & 60 & 50 & 40 & 30 & 20 & 10 & 0\end{array}$ 


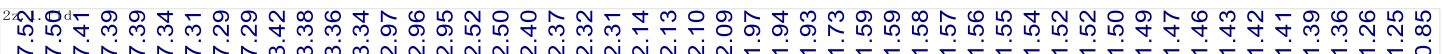

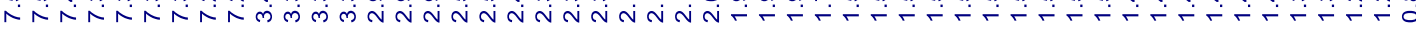<smiles>COC1CC[C@H]2[C@H]3CCc4ccc(-c5ccc(C(C)Cc6ccccc6)cc5)cc4[C@H]3CC[C@]12C</smiles>
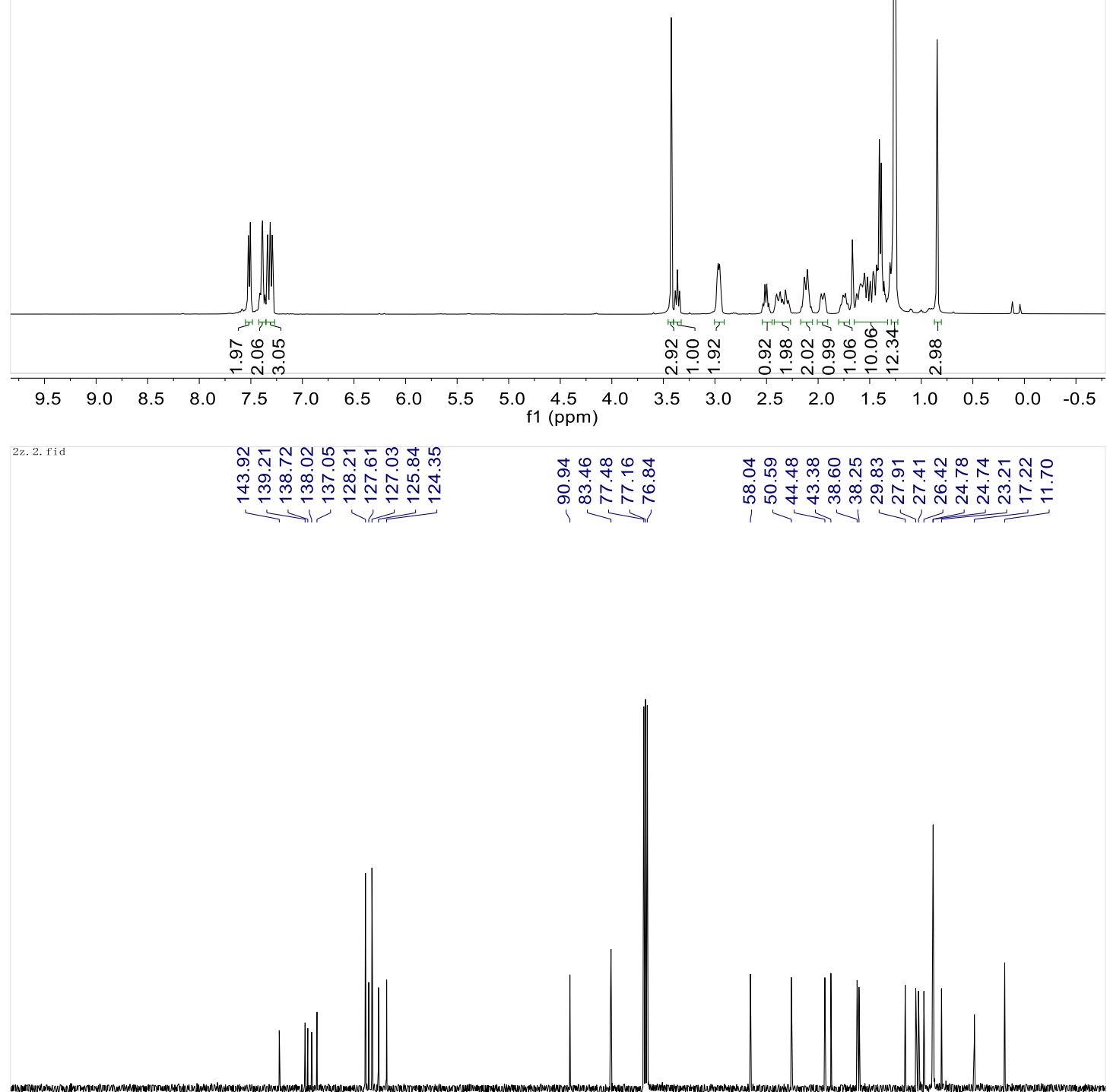

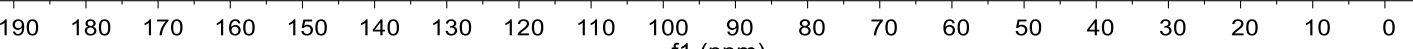




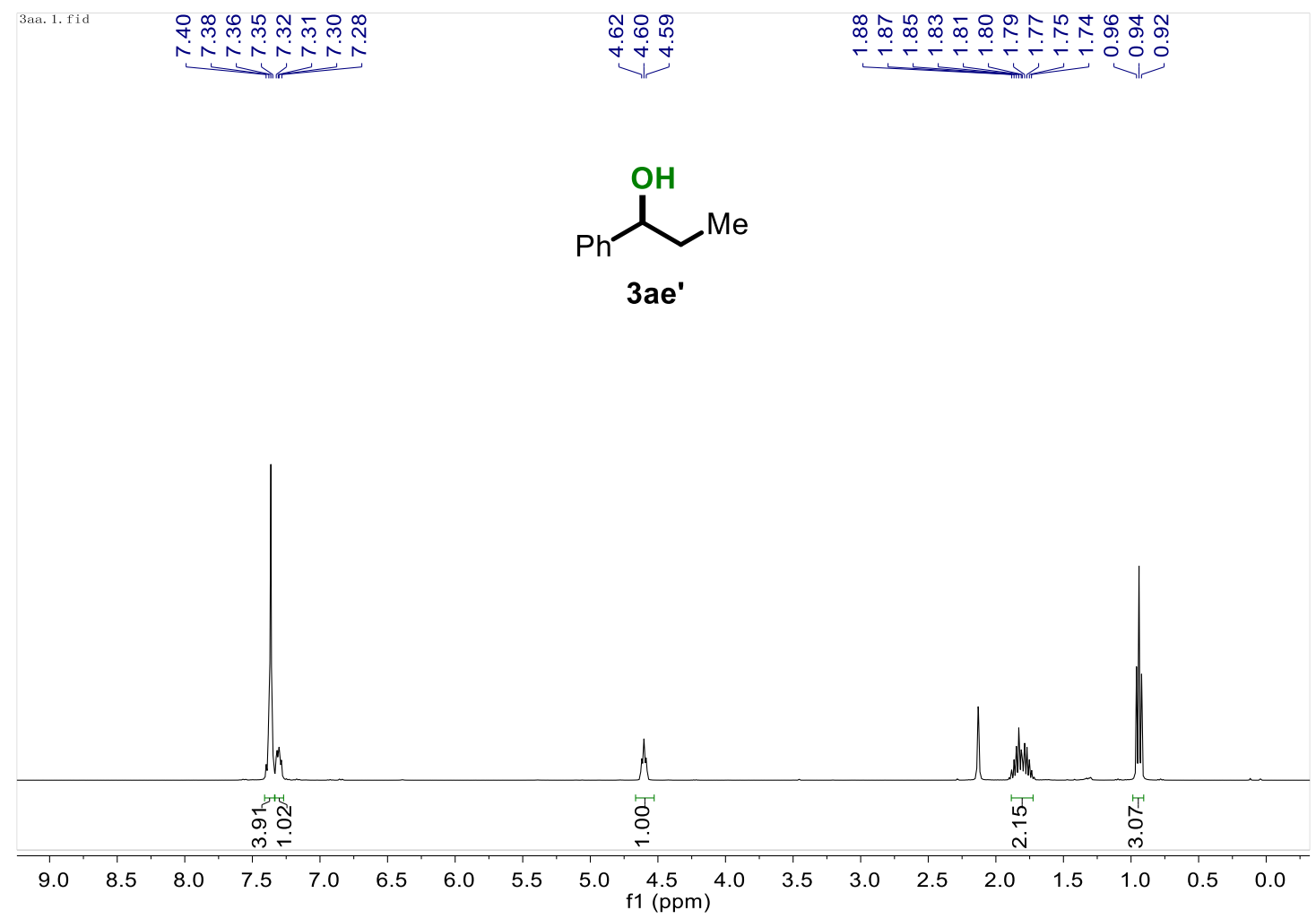

\begin{tabular}{|c|c|c|c|c|c|}
\hline 3aa. 2. fid & $\begin{array}{l}\infty \\
0 \\
\dot{j} \\
\dot{+} \\
i\end{array}$ & 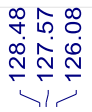 & 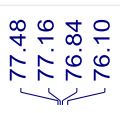 & $\frac{\hat{\sigma}}{\dot{m}}$ & $\begin{array}{l}\stackrel{0}{N} \\
\stackrel{\circ}{\leftarrow} \\
i\end{array}$ \\
\hline
\end{tabular}

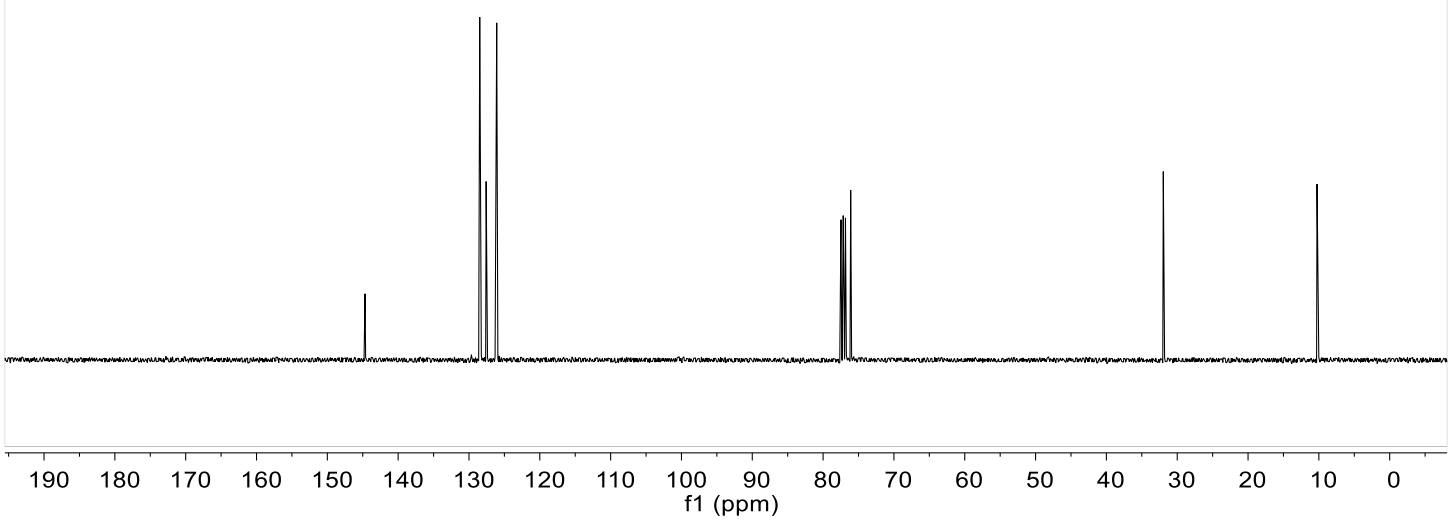




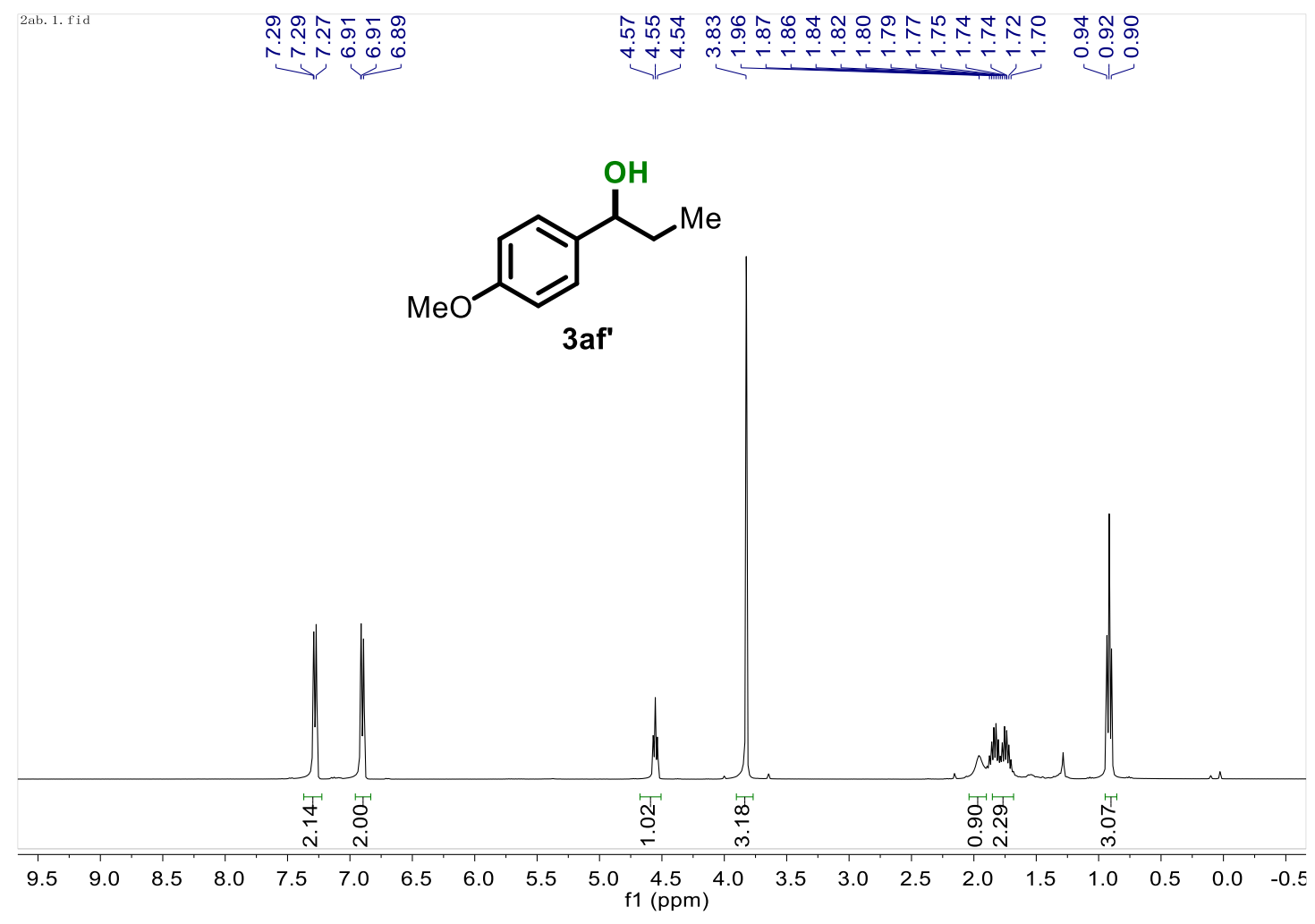

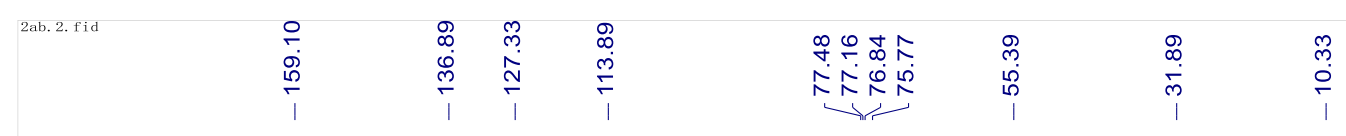

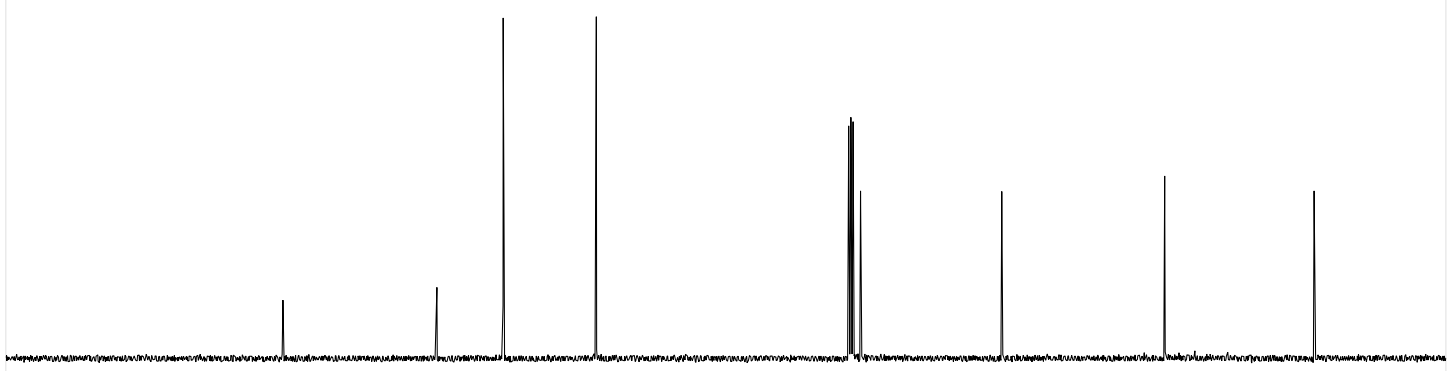

$\begin{array}{llllllllllllllllllll}190 & 180 & 170 & 160 & 150 & 140 & 130 & 120 & 110 & 100 & 90 & 80 & 70 & 60 & 50 & 40 & 30 & 20 & 10 & 0\end{array}$ 
<smiles>CCCC(O)c1ccccc1</smiles>

3 ag'

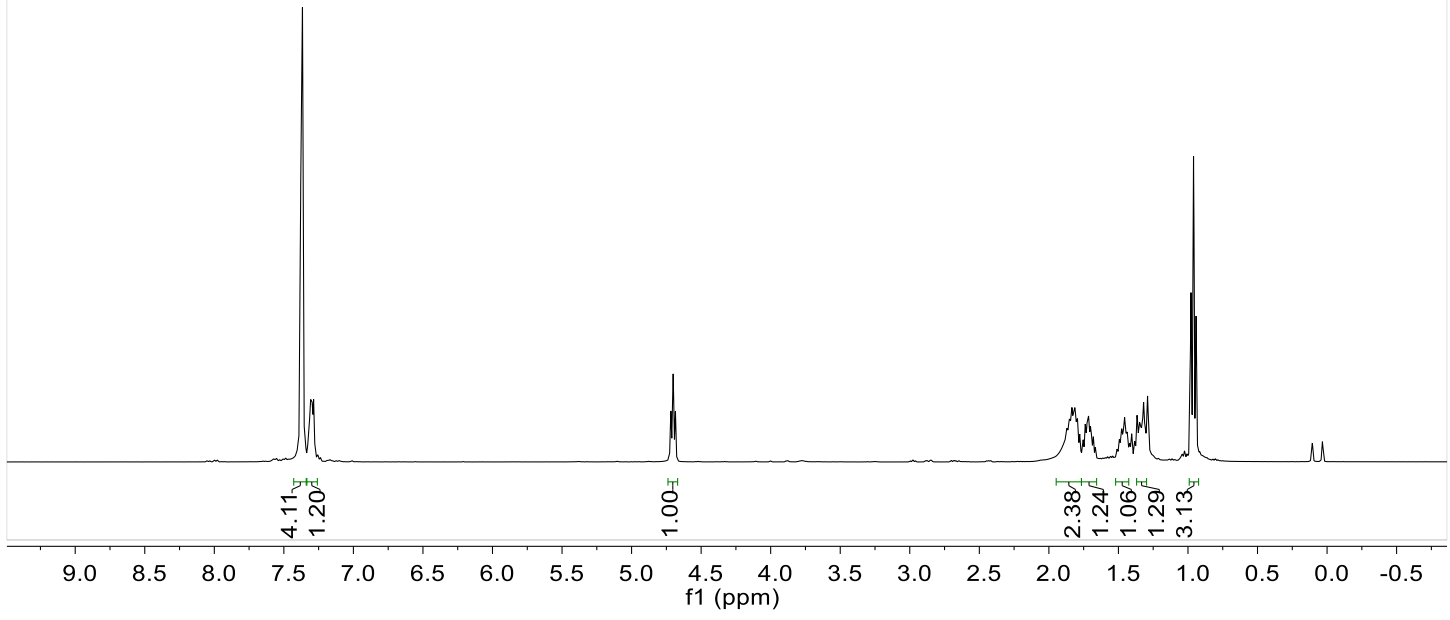

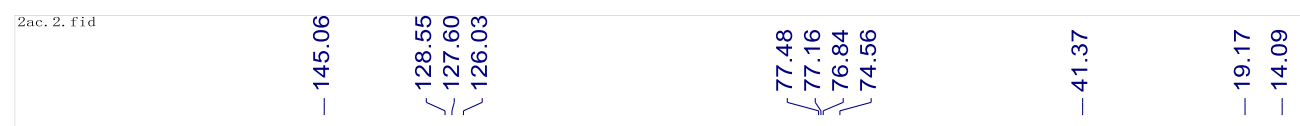

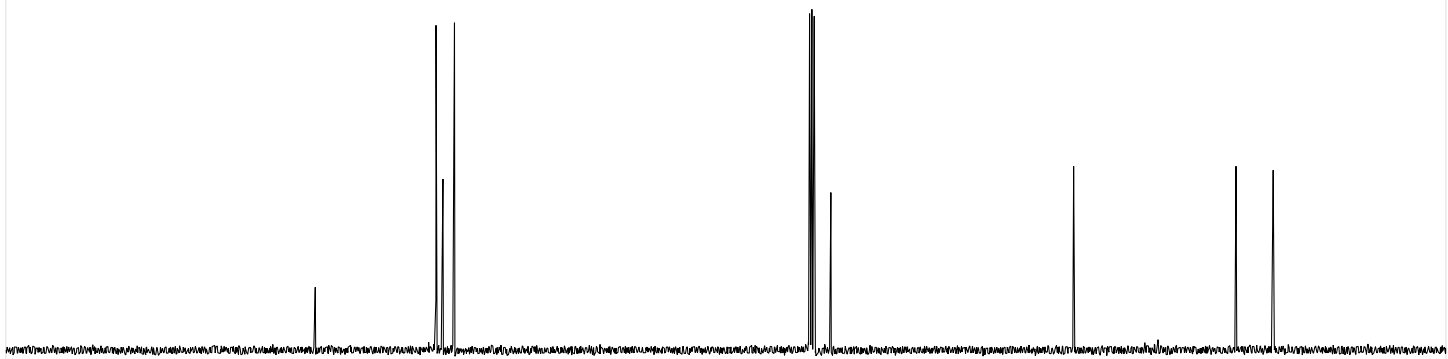

$\begin{array}{lllllllllllllllllllll}180 & 170 & 160 & 150 & 140 & 130 & 120 & 110 & 100 & 90 & 80 & 70 & 60 & 50 & 40 & 30 & 20 & 10 & 0\end{array}$ 

2ad.1.fid

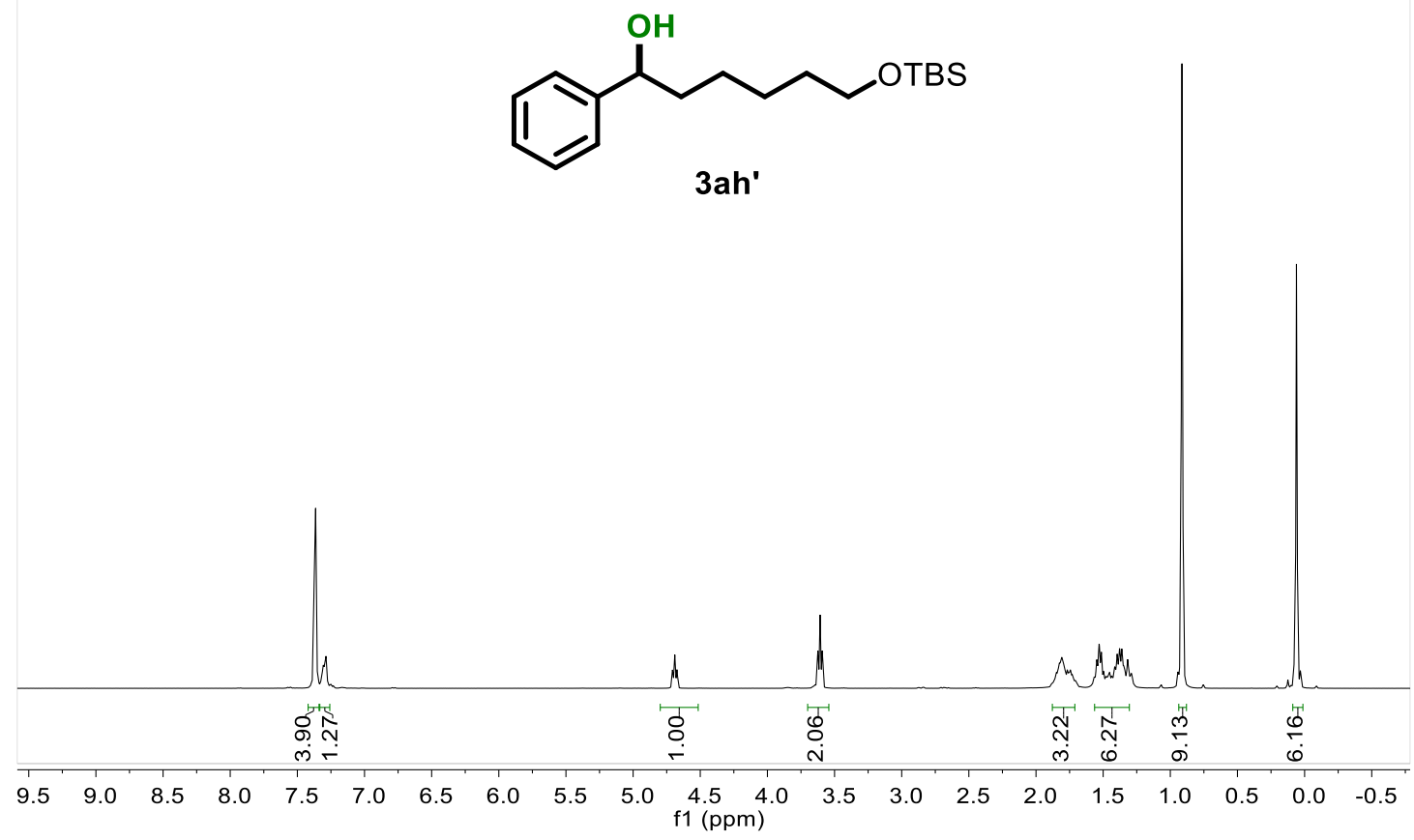

\begin{tabular}{|c|c|c|c|c|c|}
\hline 2ad. 2. fid & 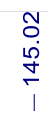 & 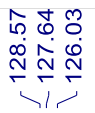 & 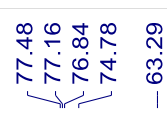 & 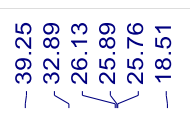 & $i$ \\
\hline
\end{tabular}

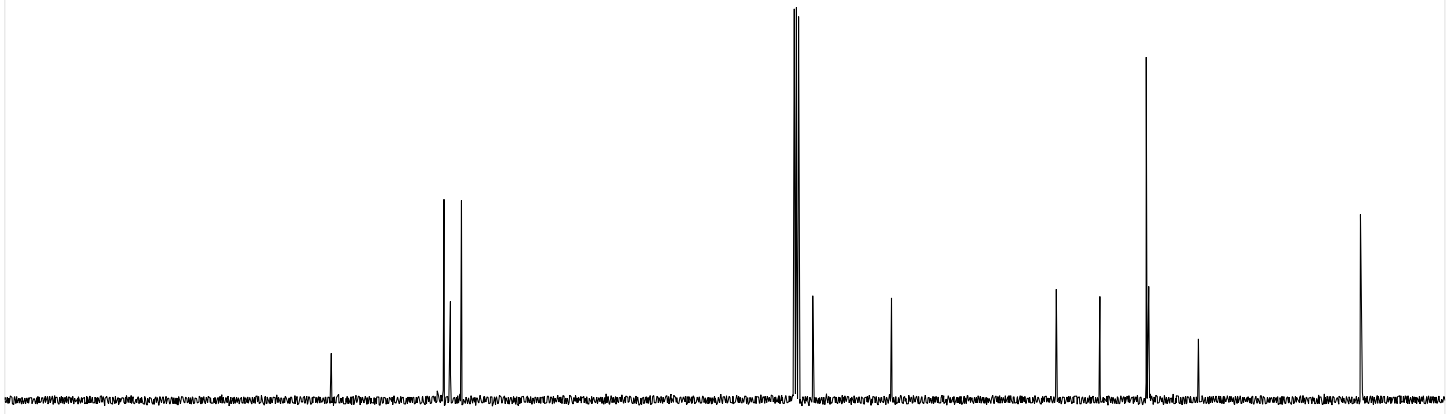

$\begin{array}{lllllllllllllllllllll}190 & 180 & 170 & 160 & 150 & 140 & 130 & 120 & 110 & 100 & \begin{array}{c}90 \\ \mathrm{f} 1(\mathrm{ppm})\end{array} & 70 & 60 & 50 & 40 & 30 & 20 & 10 & 0 & -10\end{array}$ 

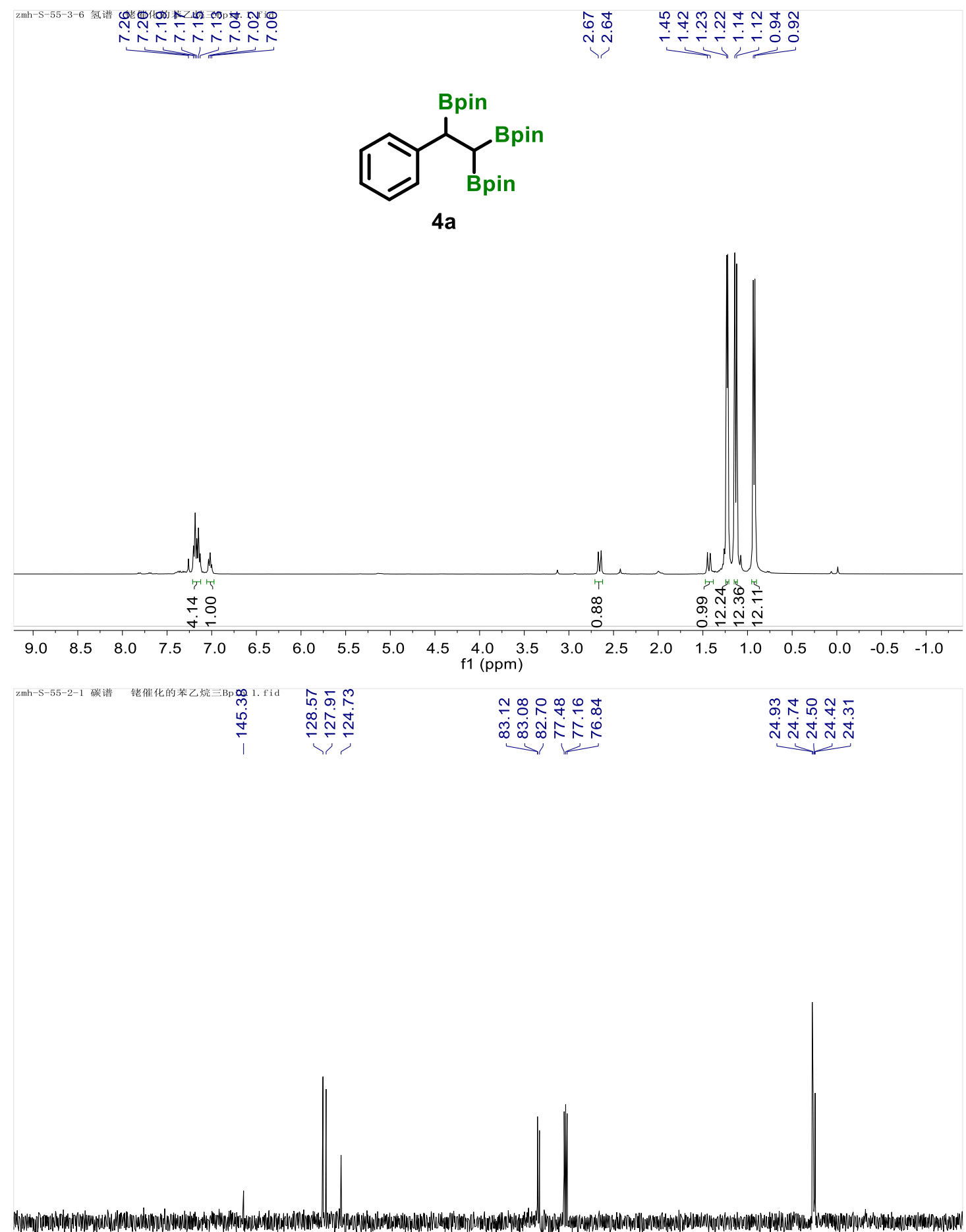

$\begin{array}{lllllllllllllllllll}190 & 180 & 170 & 160 & 150 & 140 & 130 & 120 & 110 & \begin{array}{r}100 \\ \mathrm{f} 1(\mathrm{ppm})\end{array} & 80 & 70 & 60 & 50 & 40 & 30 & 20 & 10 & 0\end{array}$ 


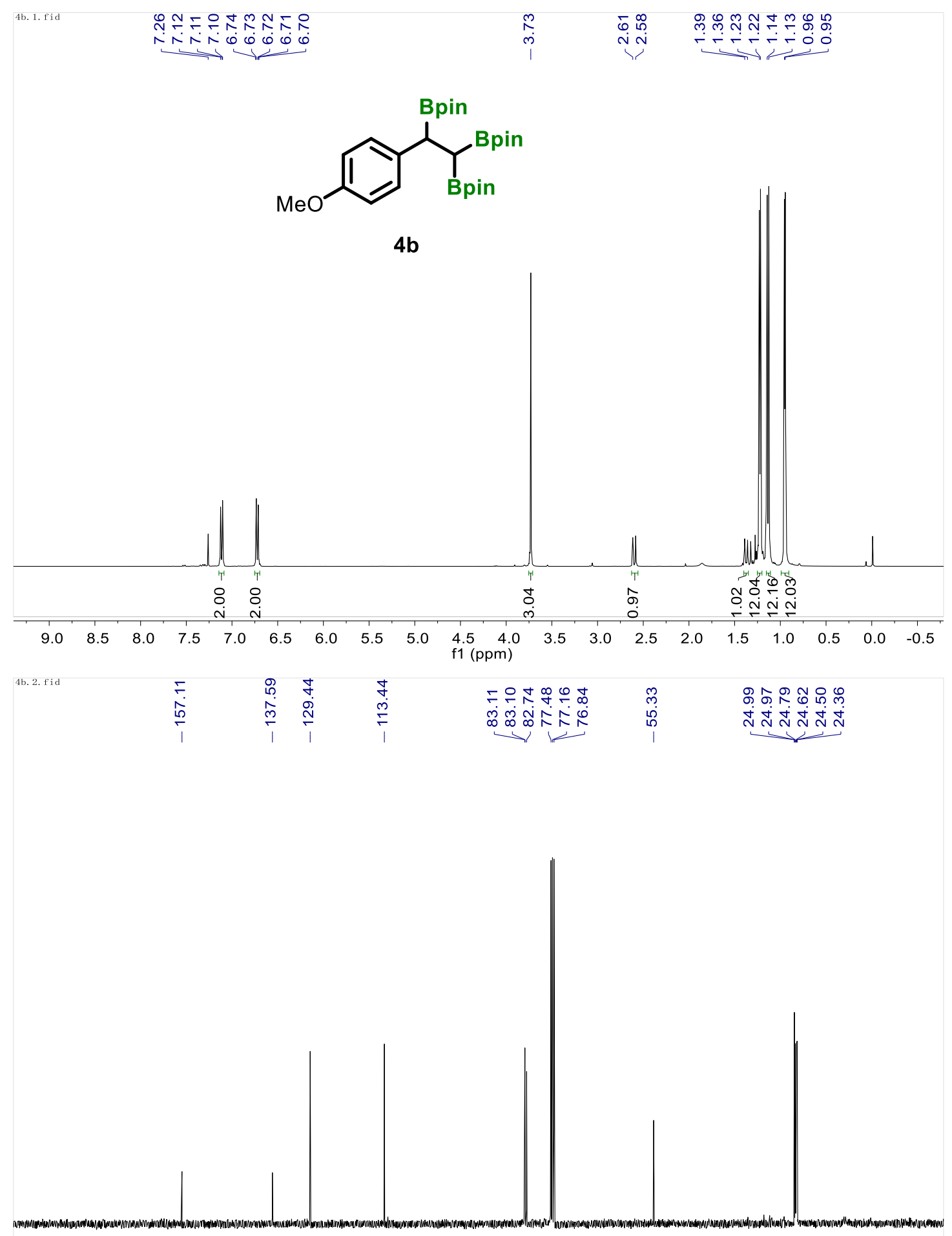

$\begin{array}{lllllllllllllllllll}190 & 180 & 170 & 160 & 150 & 140 & 130 & 120 & 110 & \begin{array}{c}100 \\ \mathrm{f} 1(\mathrm{ppm})\end{array} & 80 & 70 & 60 & 50 & 40 & 30 & 20 & 10 & 0\end{array}$ 


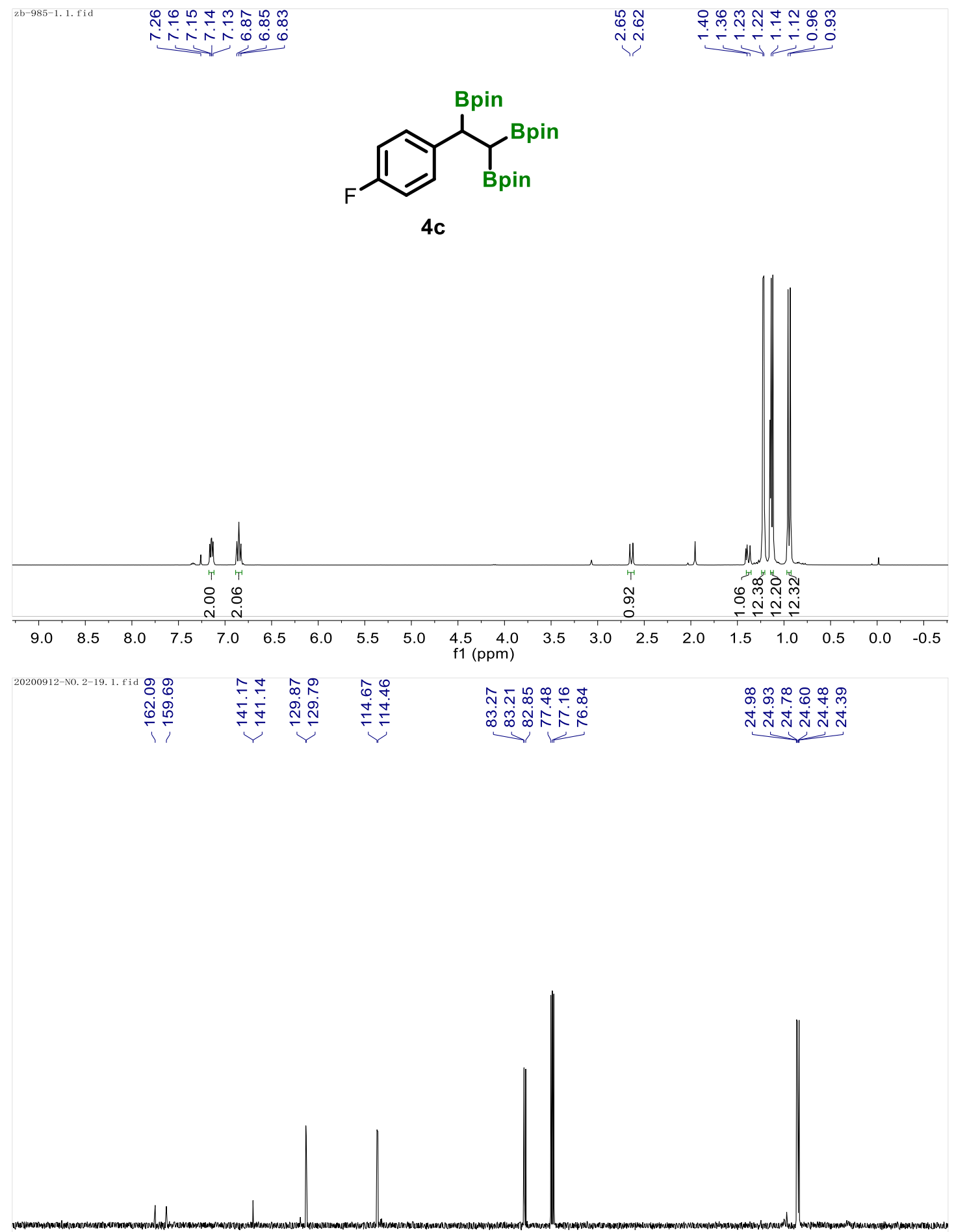

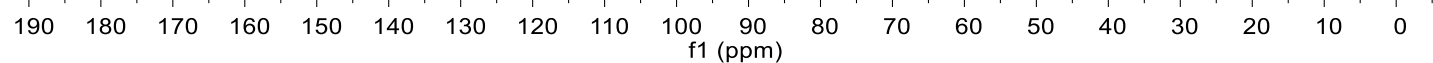




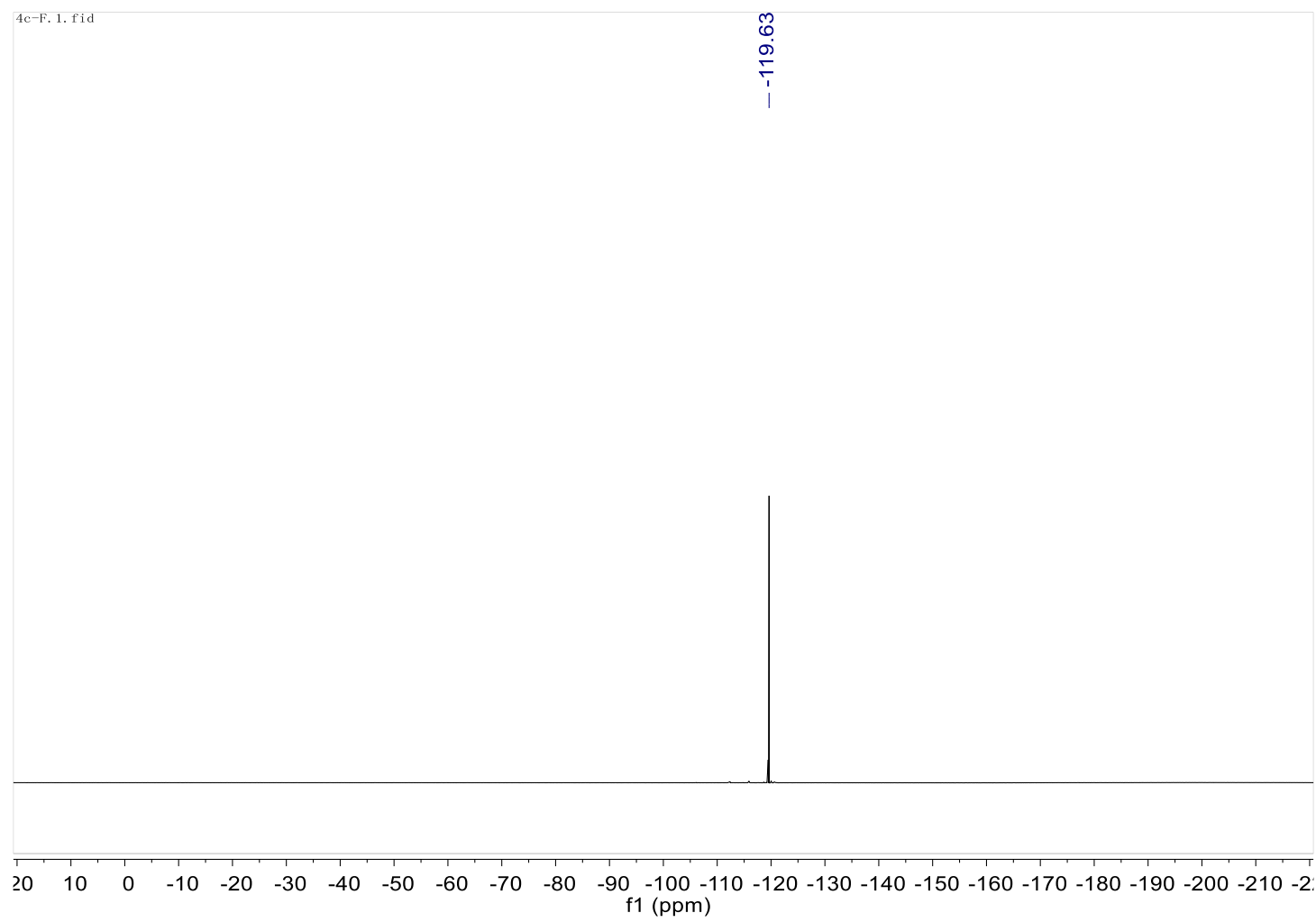




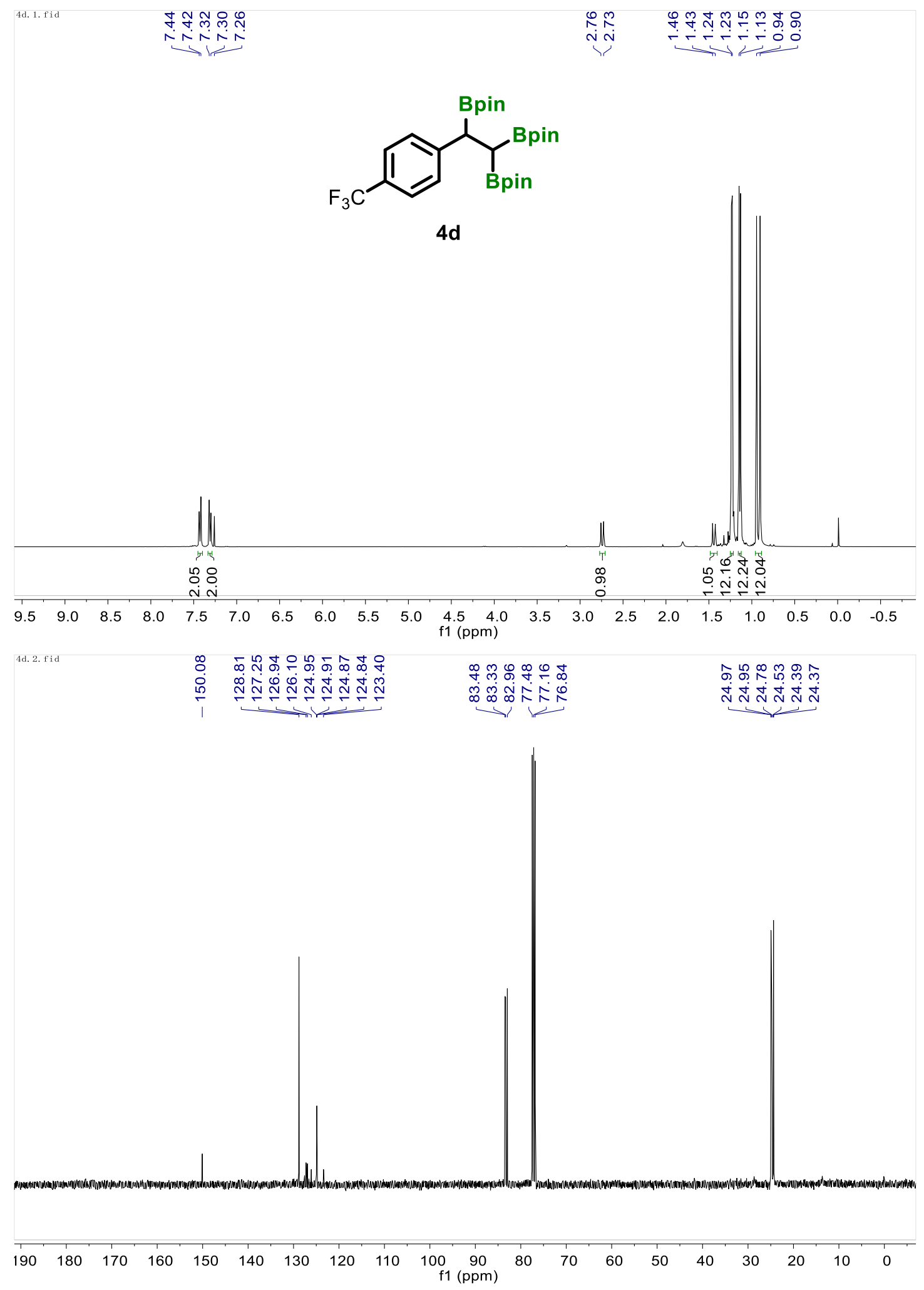




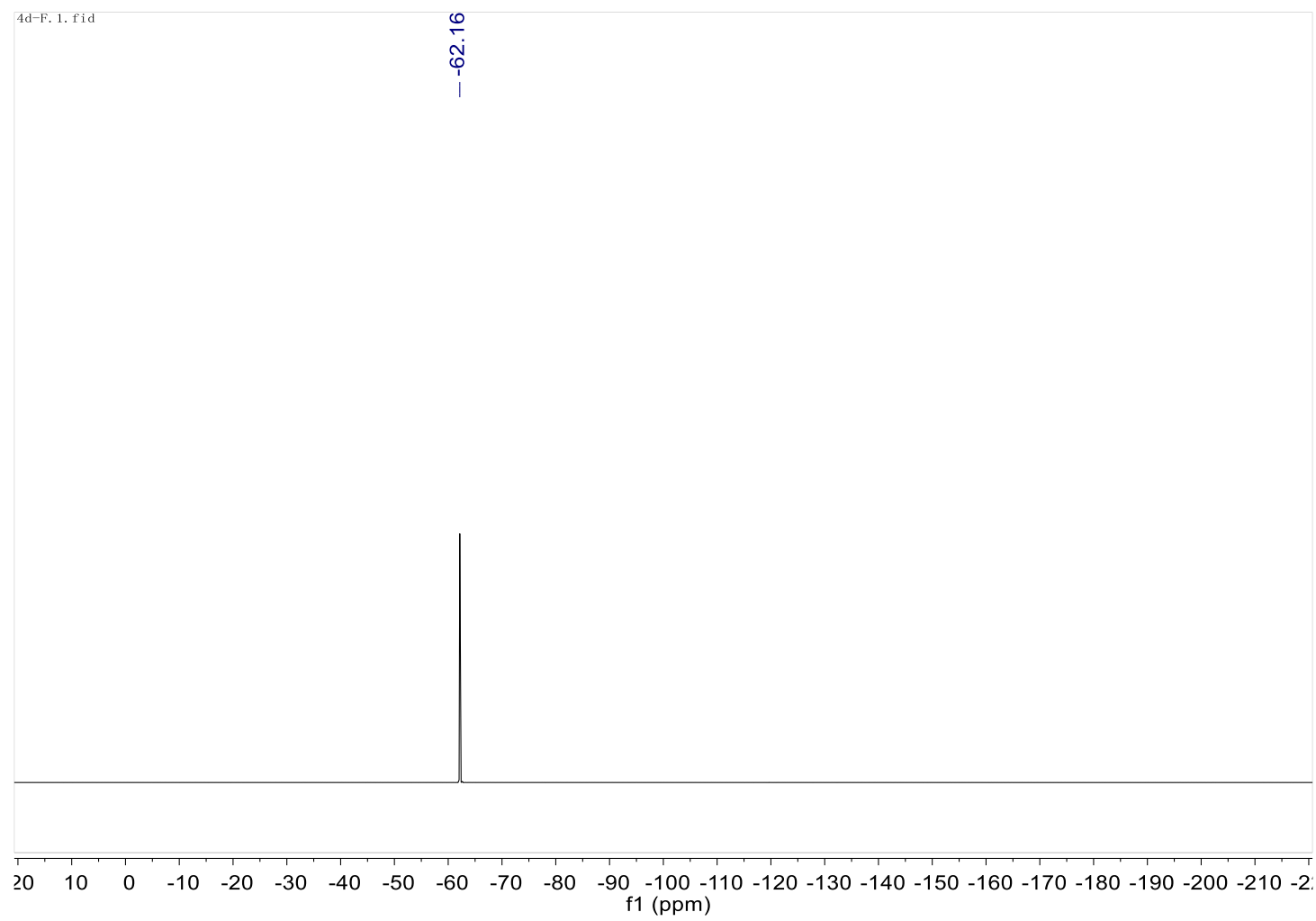




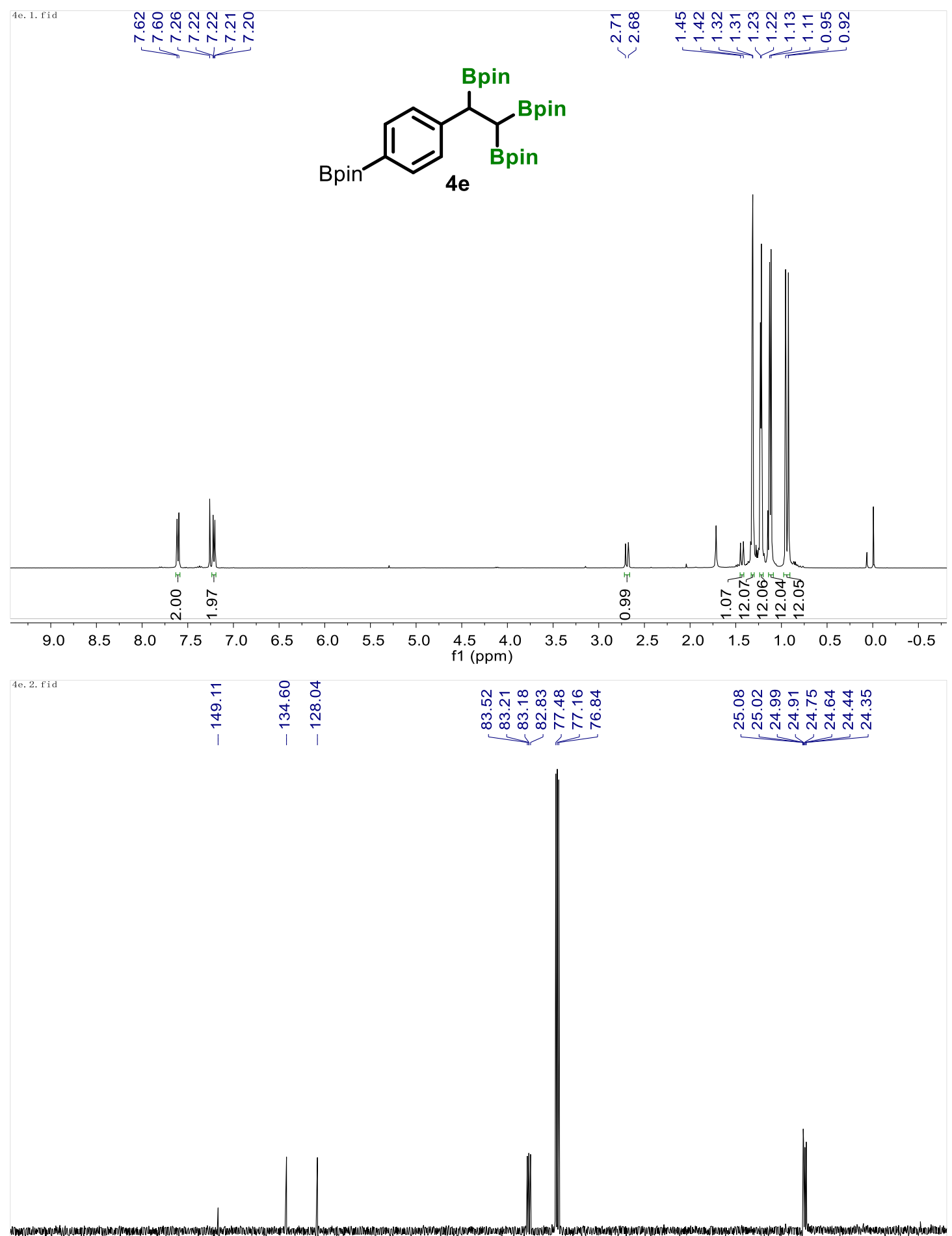

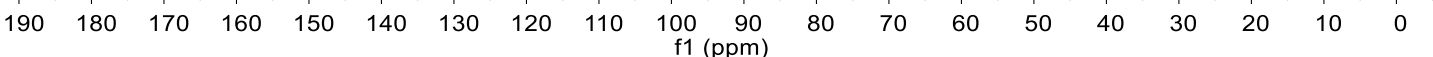




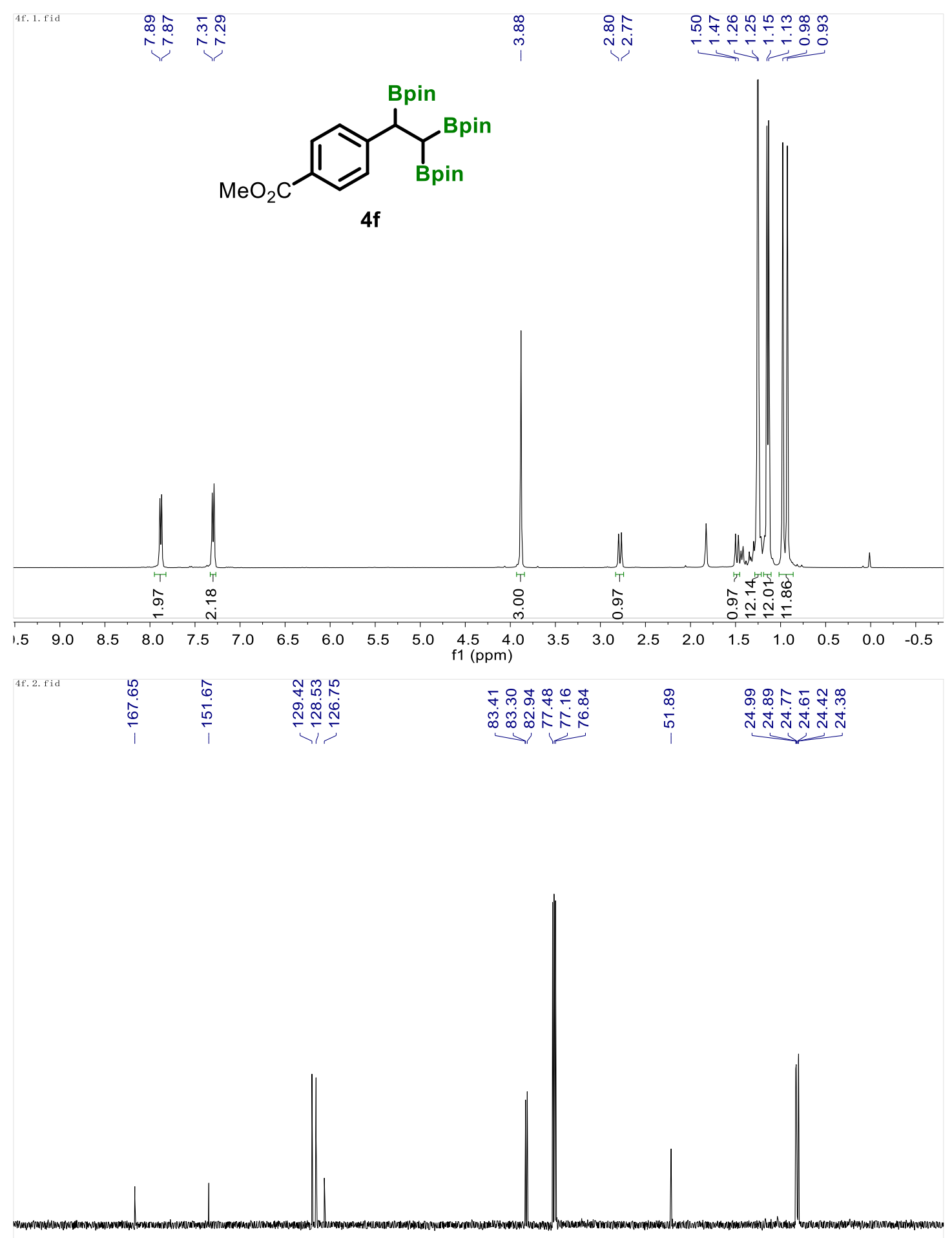

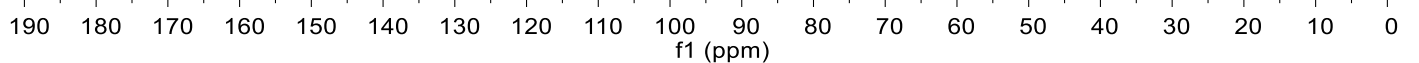




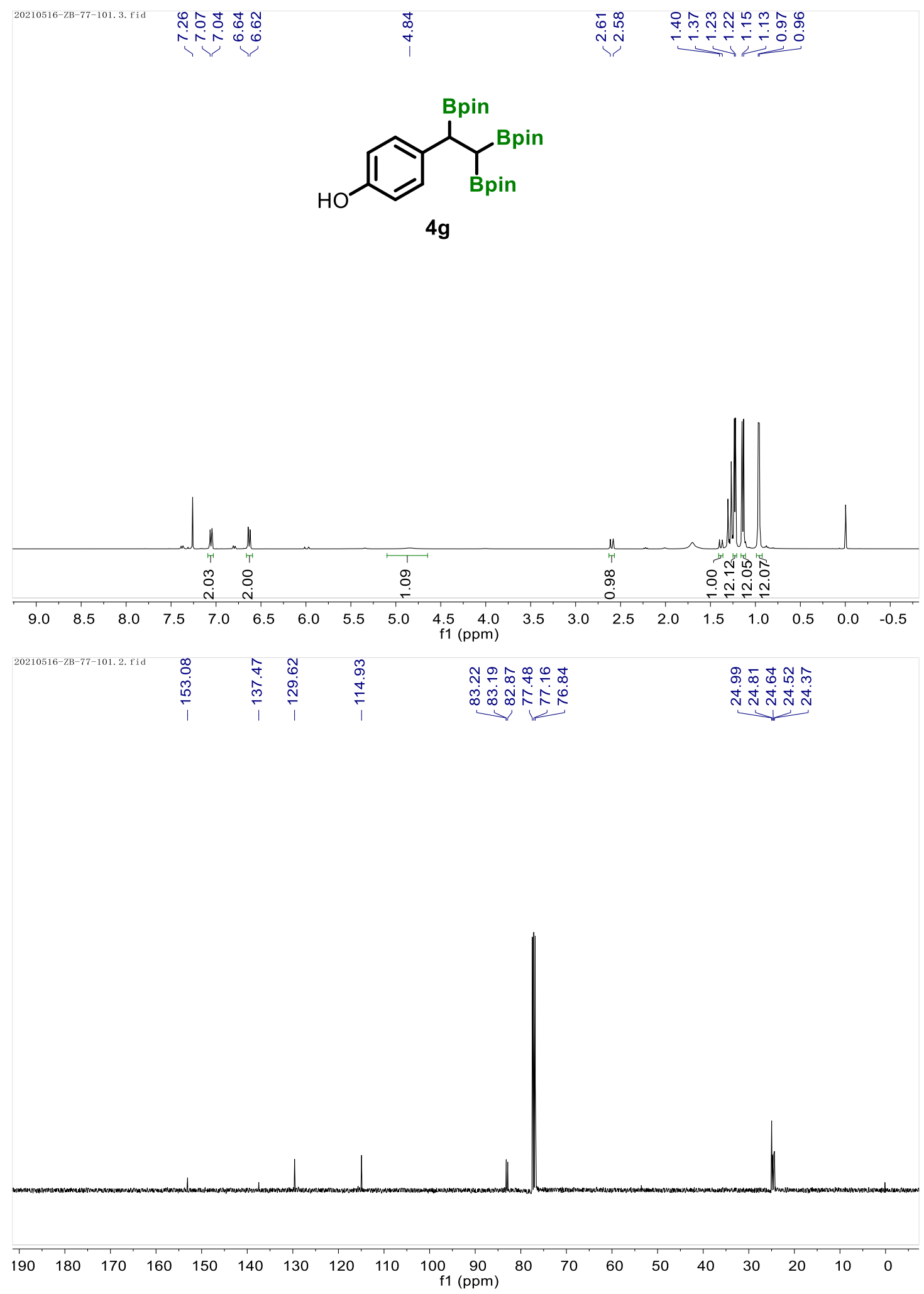




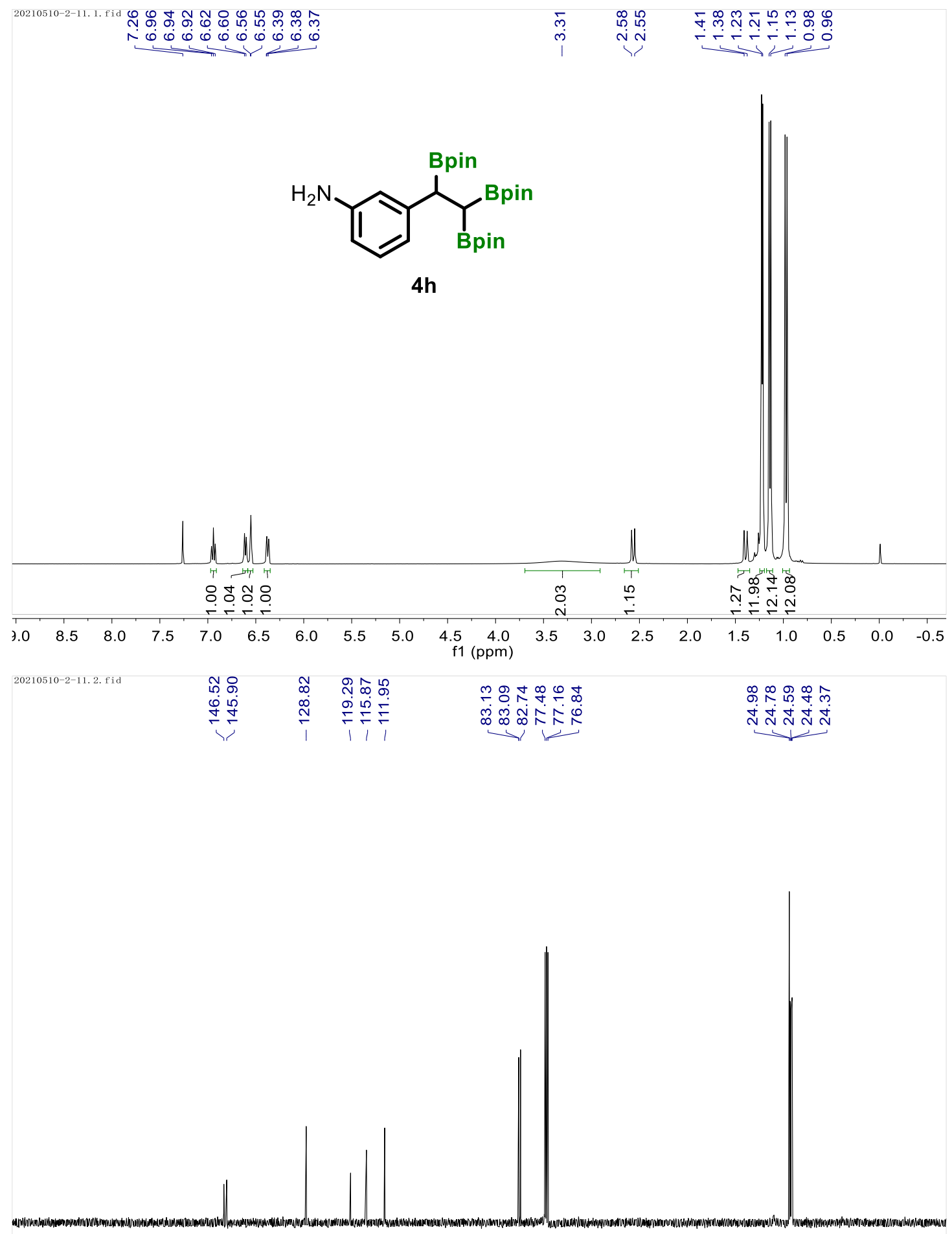

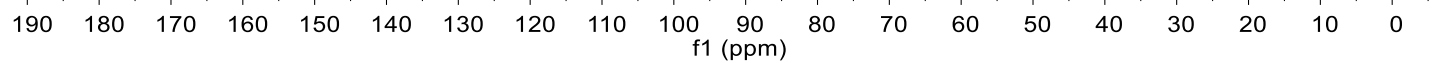




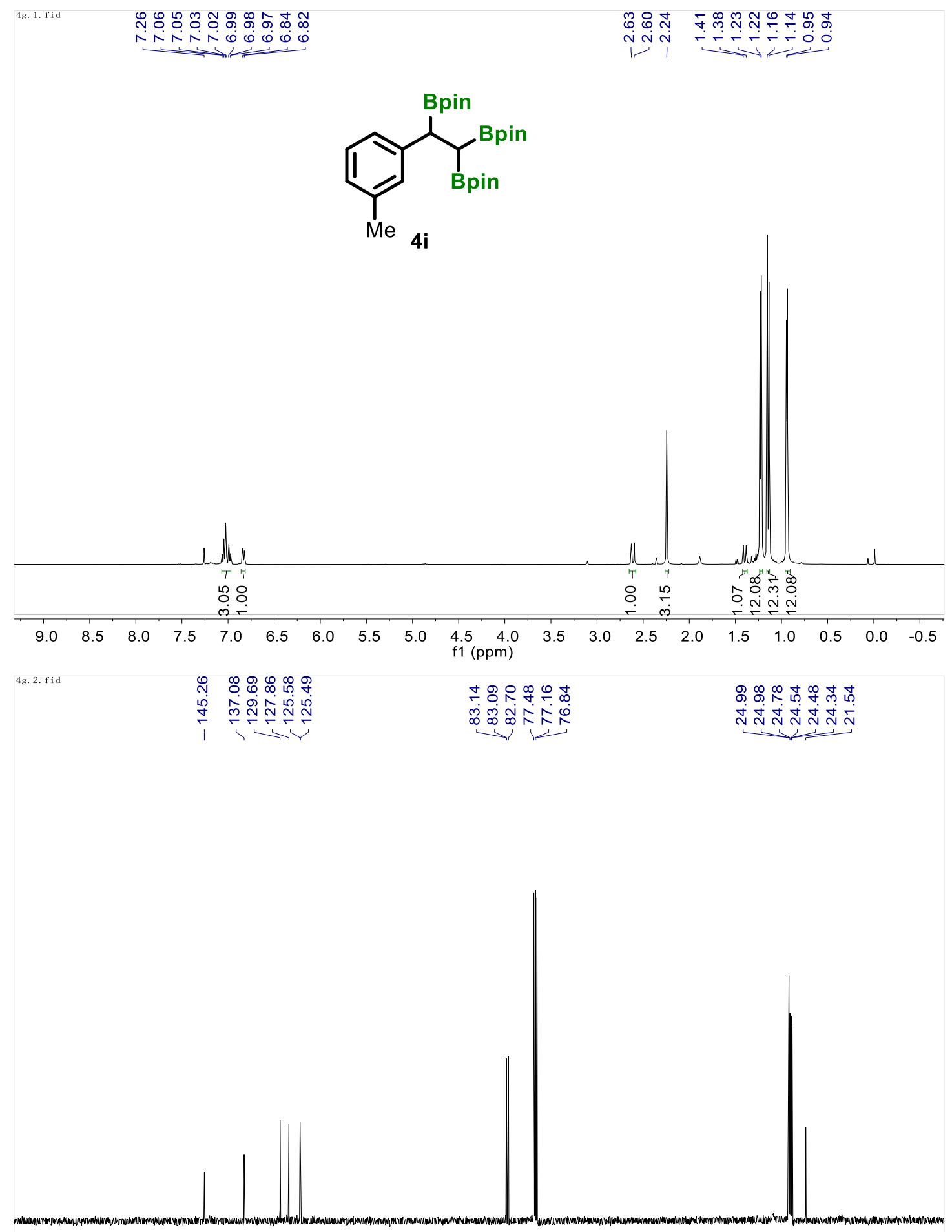

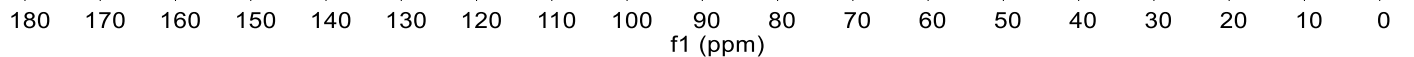




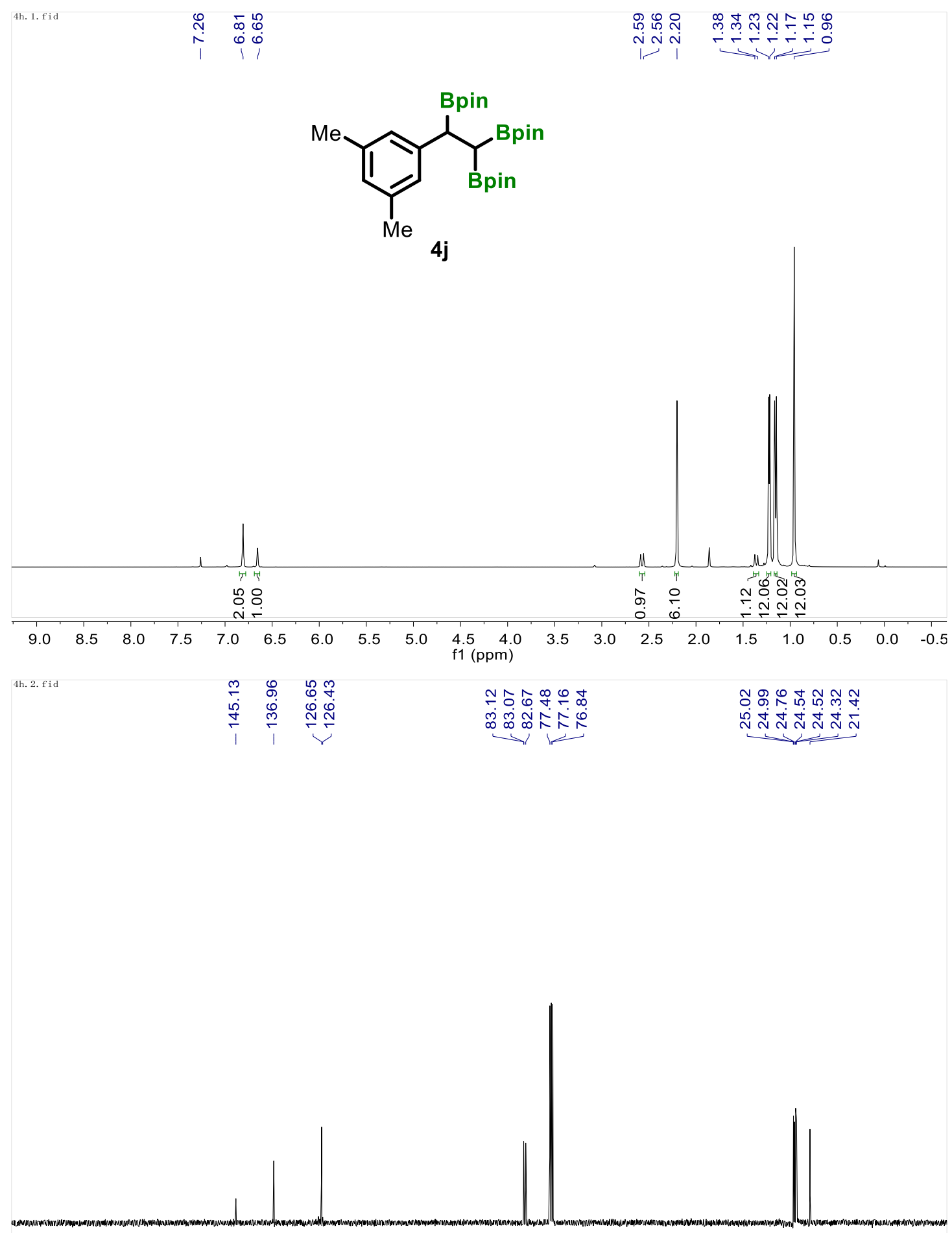

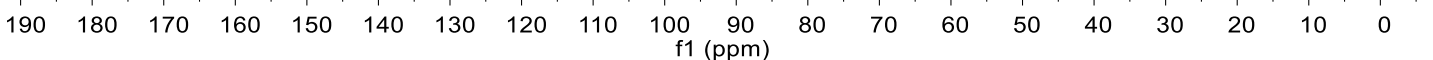



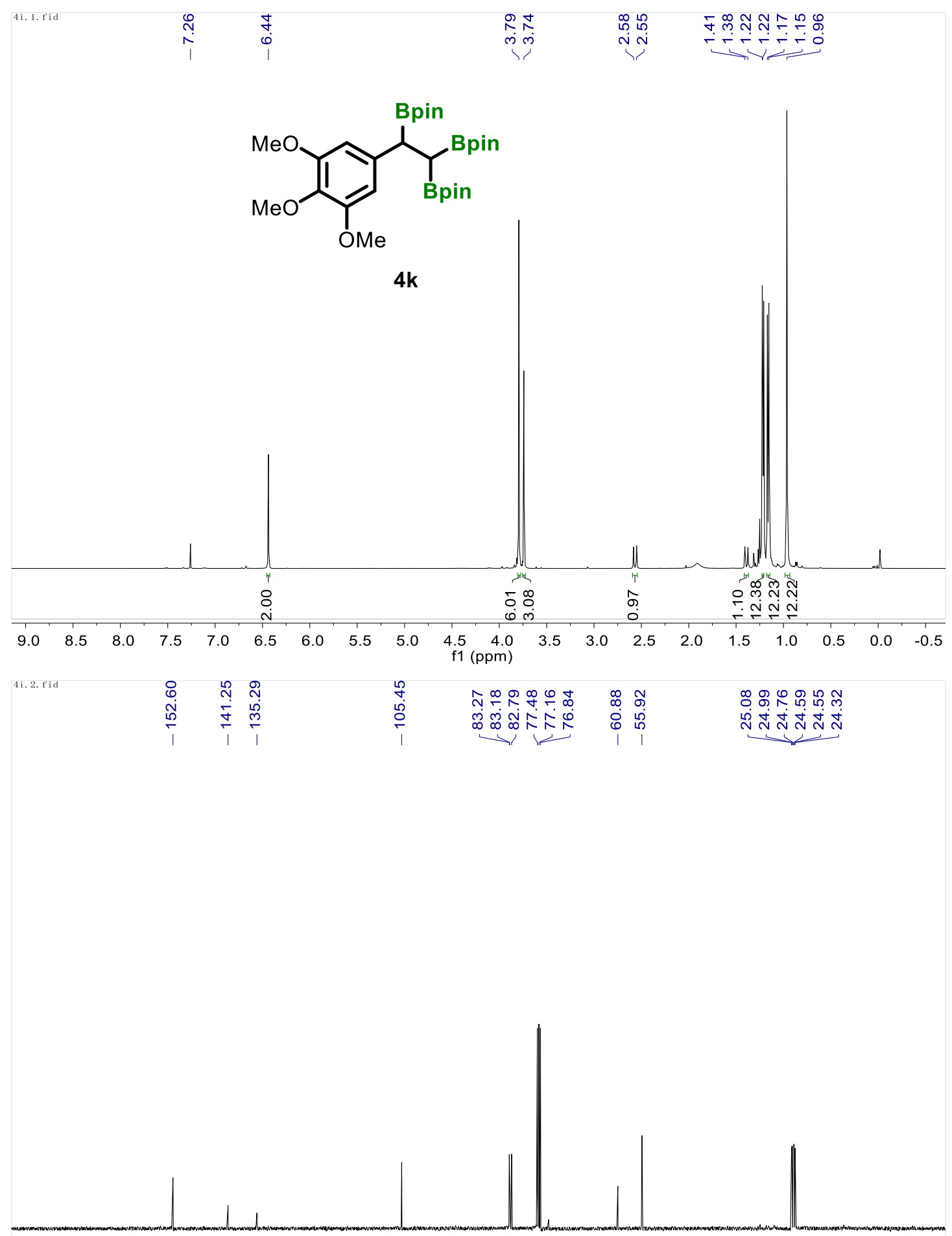

$\begin{array}{lllllllllllllllll}180 & 170 & 160 & 150 & 140 & 130 & 120 & 110 & 100 \underset{\mathrm{f} 1}{9(\mathrm{ppm})} \mathbf{8 0} & 70 & 60 & 50 & 40 & 30 & 20 & 10 & 0\end{array}$ 


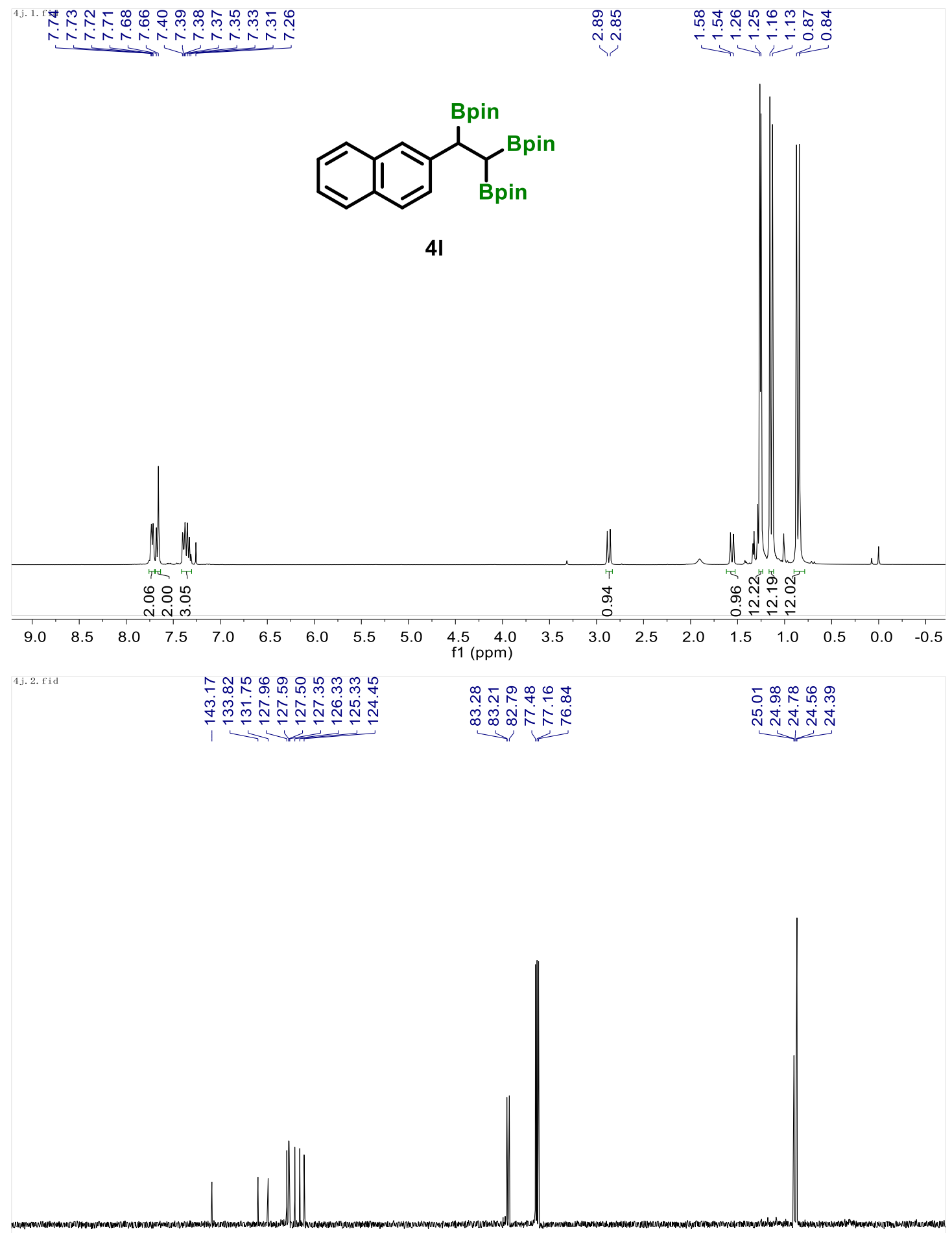

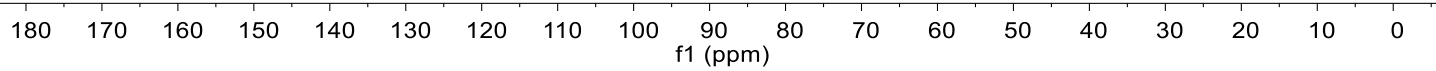




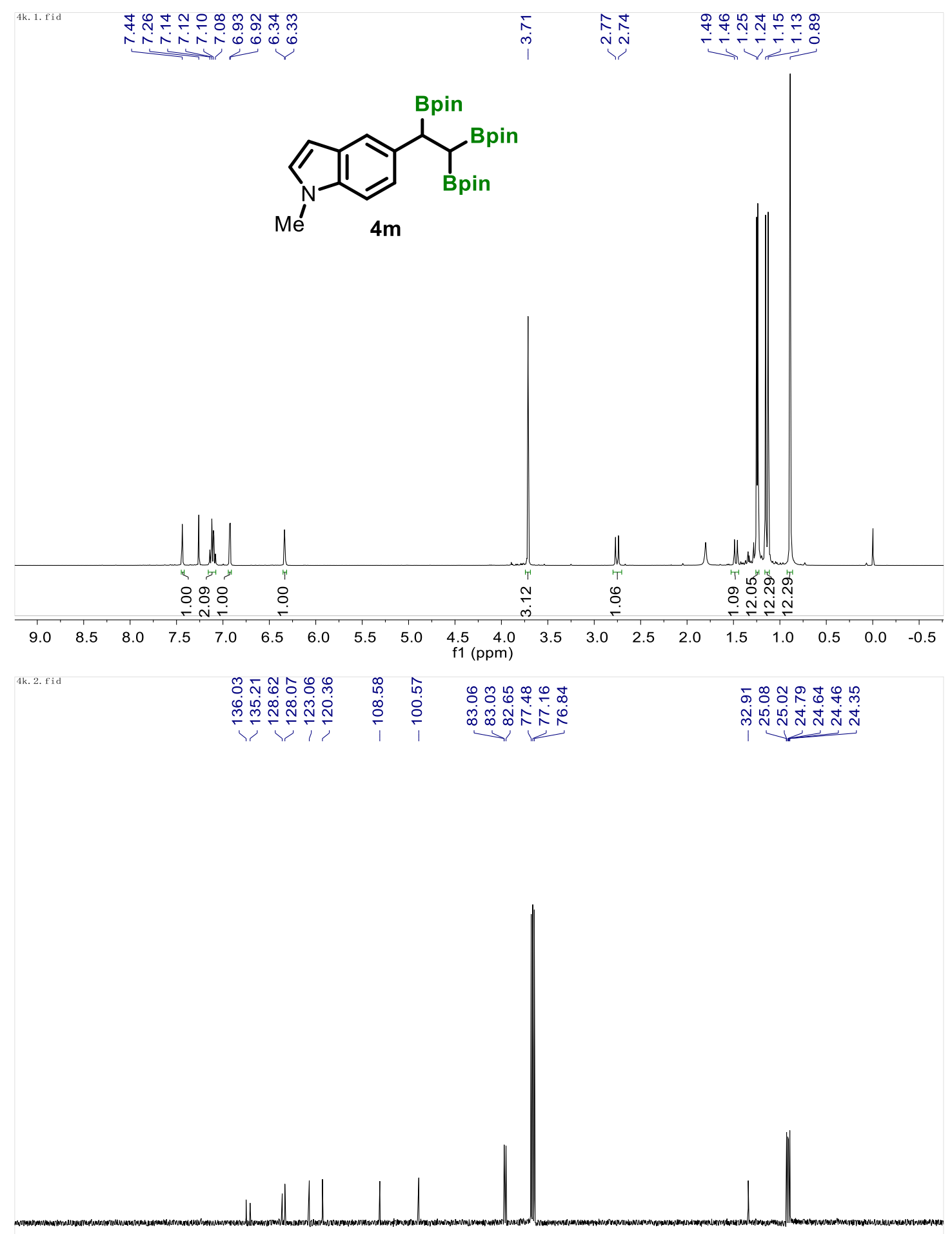

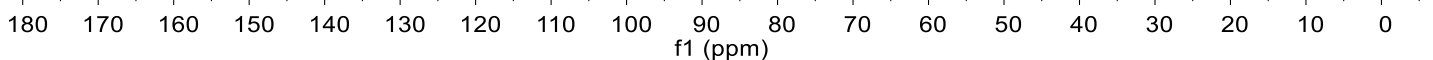



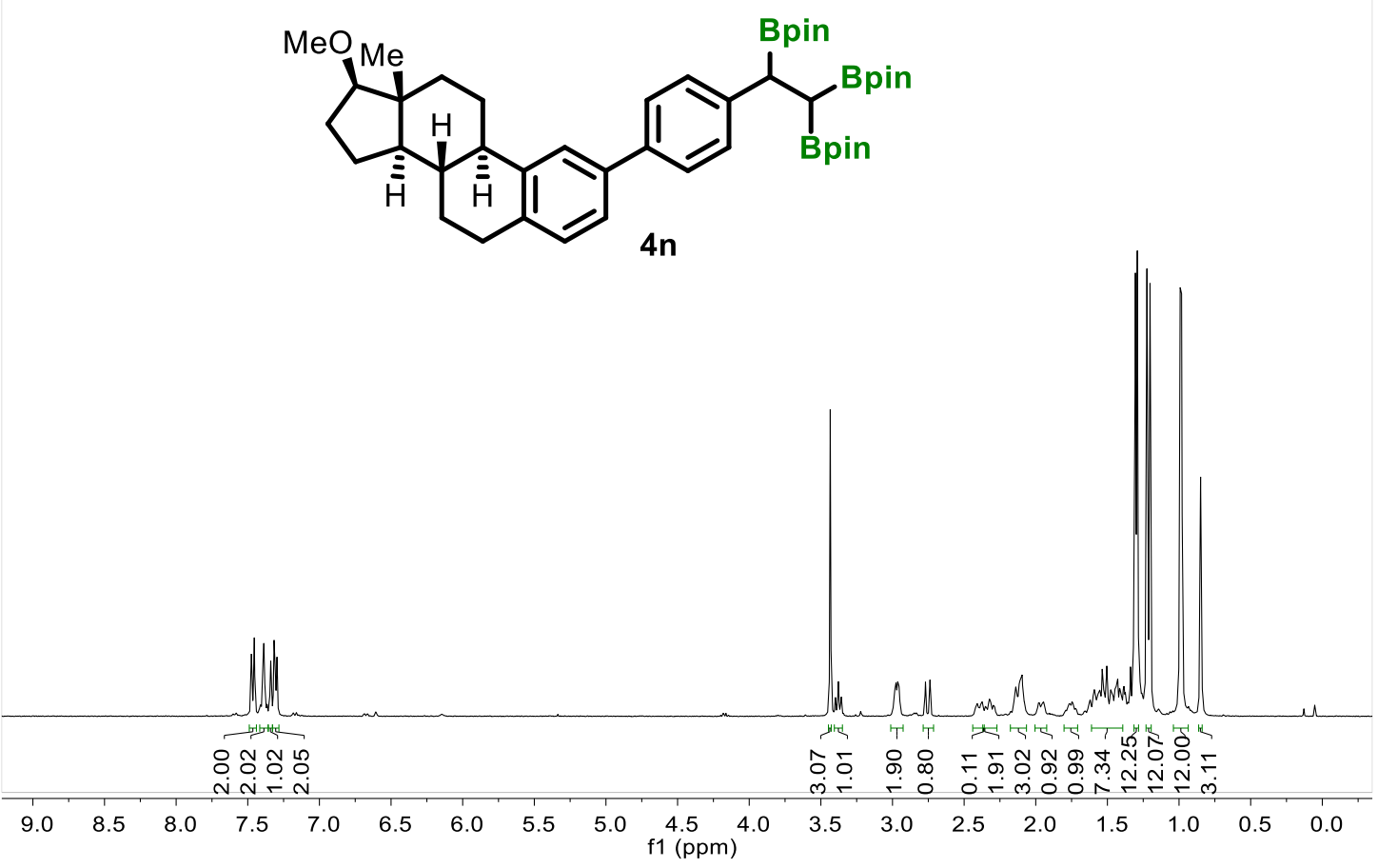

\begin{tabular}{|c|c|c|c|}
\hline 41.2. fid & 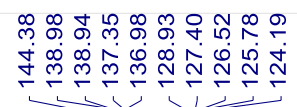 & 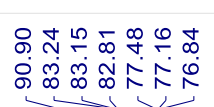 & 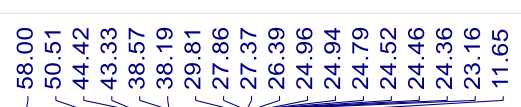 \\
\hline
\end{tabular}

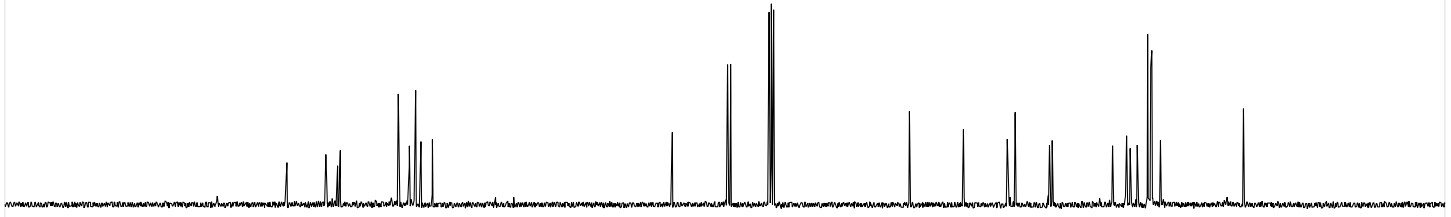

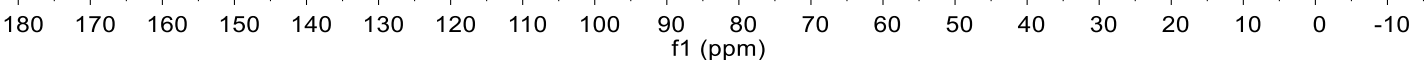




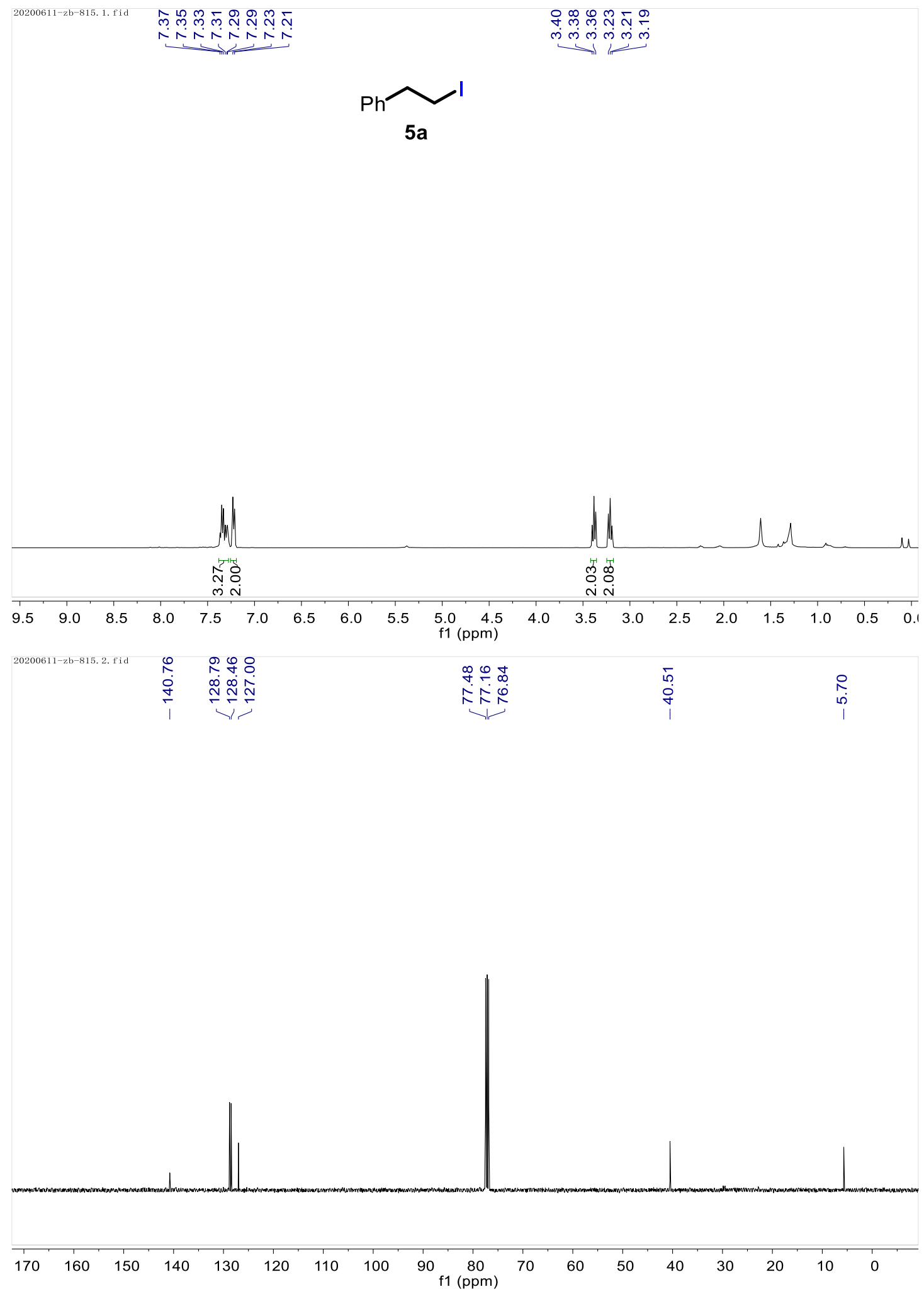




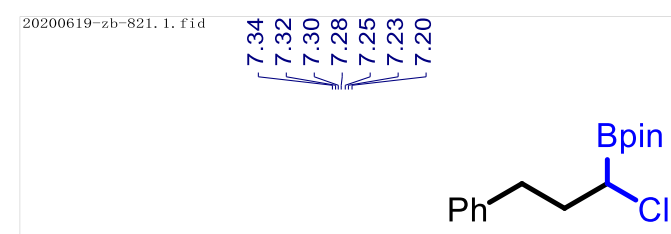

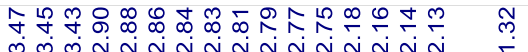
vimiñ

$5 b$

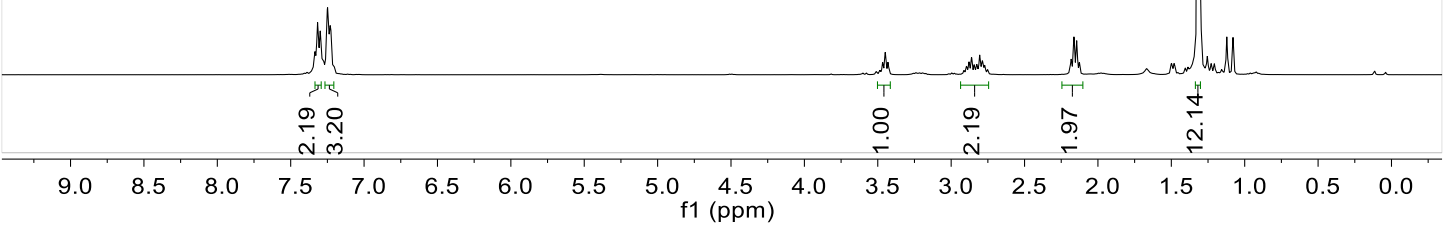

\begin{tabular}{|c|c|c|c|c|}
\hline $20200619-\mathrm{zb}-821.2 . \mathrm{fid}$ & 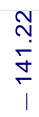 & 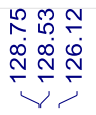 & 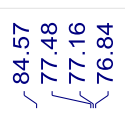 & $\begin{array}{ll}N & m \\
\infty & ? \\
\omega & m \\
m & m \\
1 & 1\end{array}$ \\
\hline
\end{tabular}

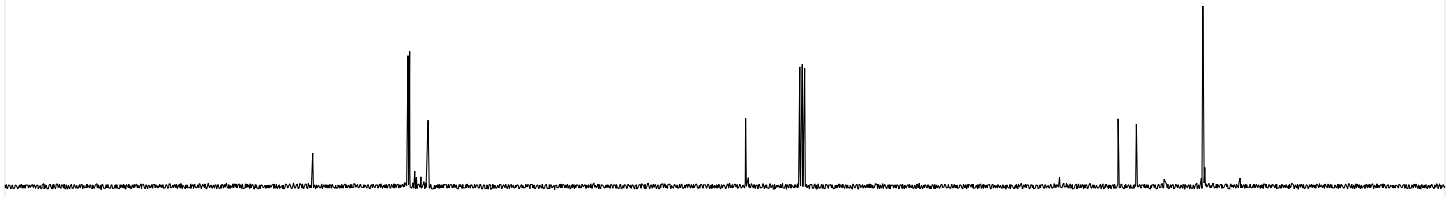

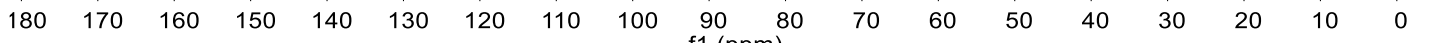




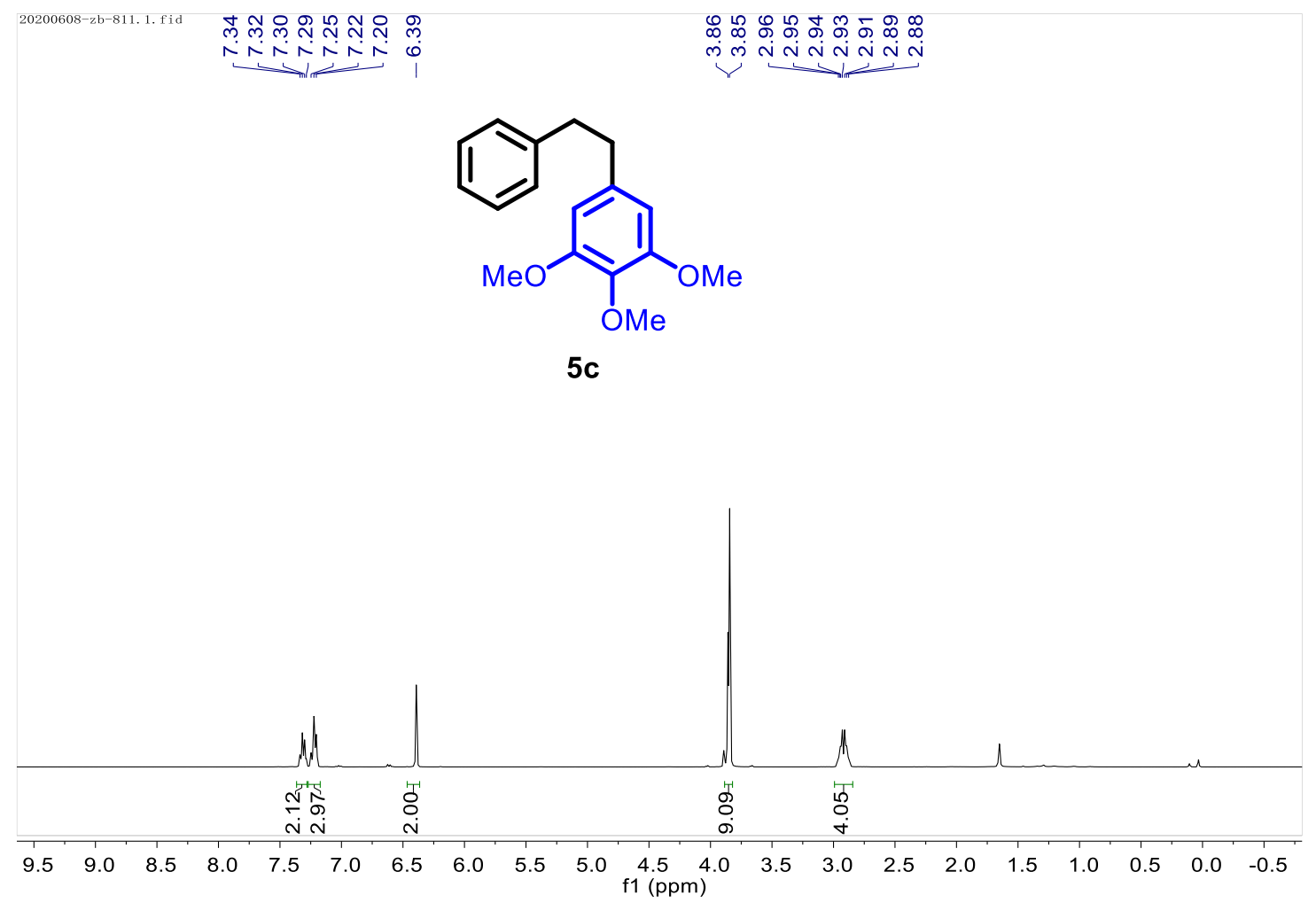

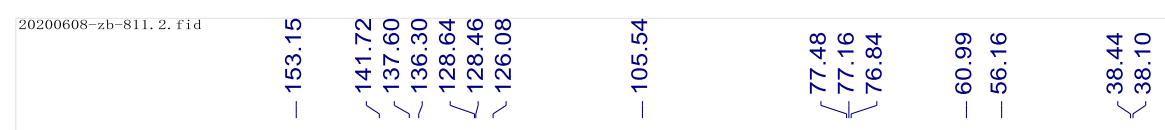

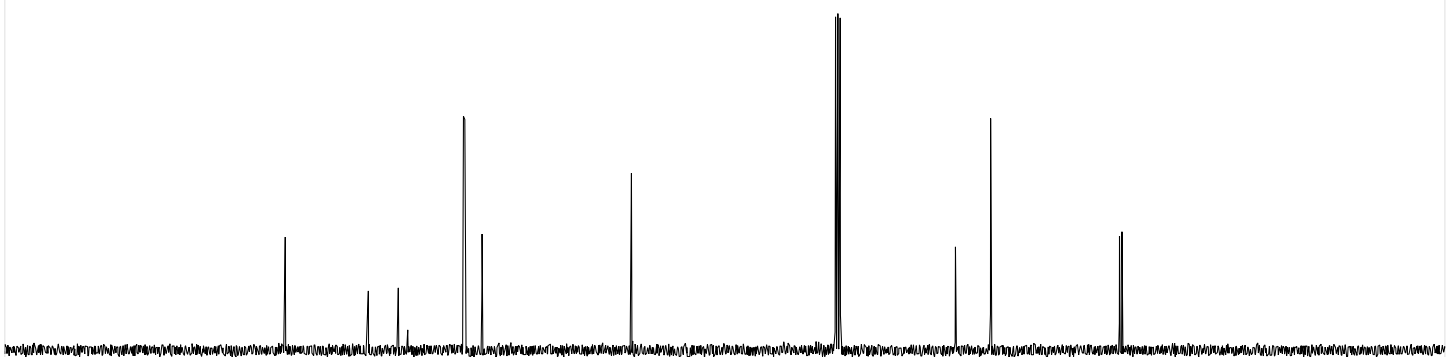

$\begin{array}{llllllllllllllllllll}190 & 180 & 170 & 160 & 150 & 140 & 130 & 120 & 110 & \begin{array}{c}100 \\ \mathrm{f} 1(\mathrm{ppm})\end{array} & 80 & 70 & 60 & 50 & 40 & 30 & 20 & 10 & 0\end{array}$ 
<smiles>c1ccc(CCc2ccco2)cc1</smiles>

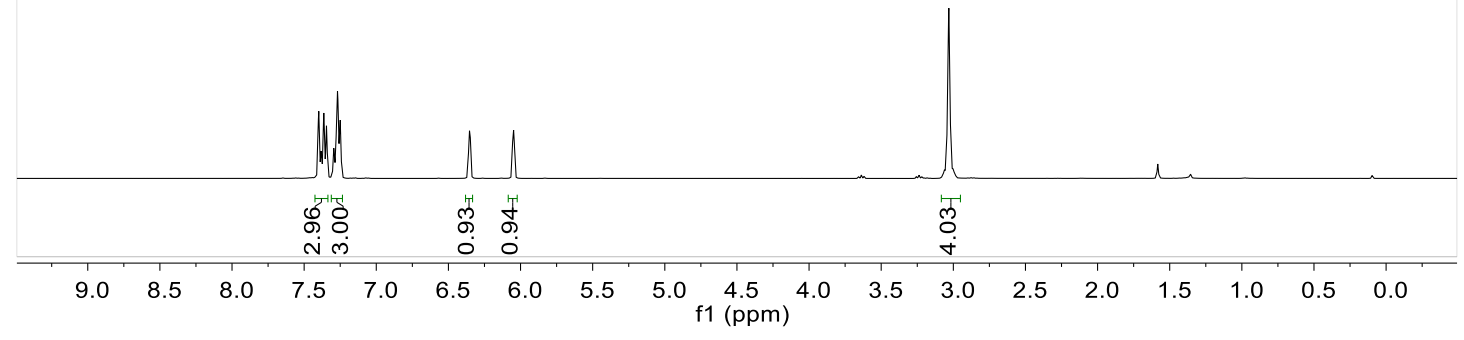

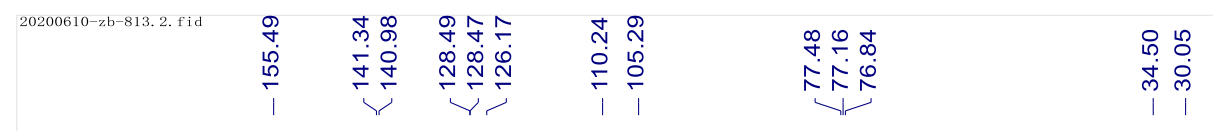

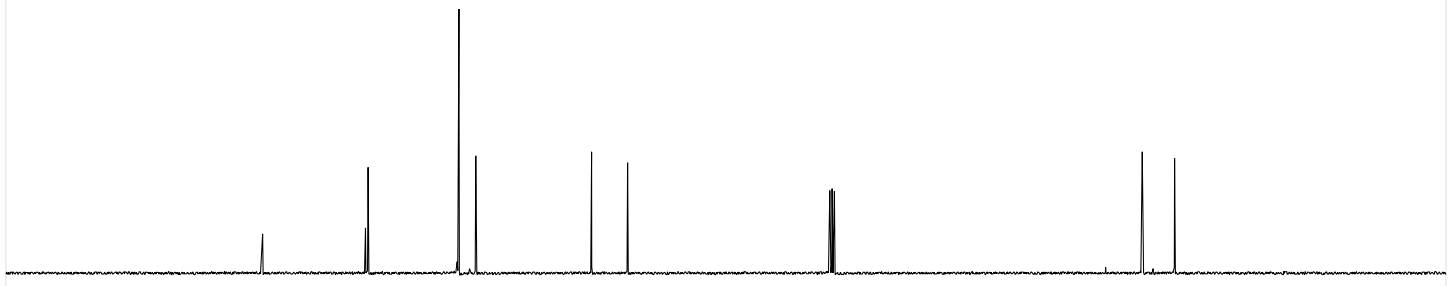

$\begin{array}{llllllllllllllllllll}90 & 180 & 170 & 160 & 150 & 140 & 130 & 120 & 110 & 100 & 90 & 80 & 70 & 60 & 50 & 40 & 30 & 20 & 10 & 0\end{array}$ 


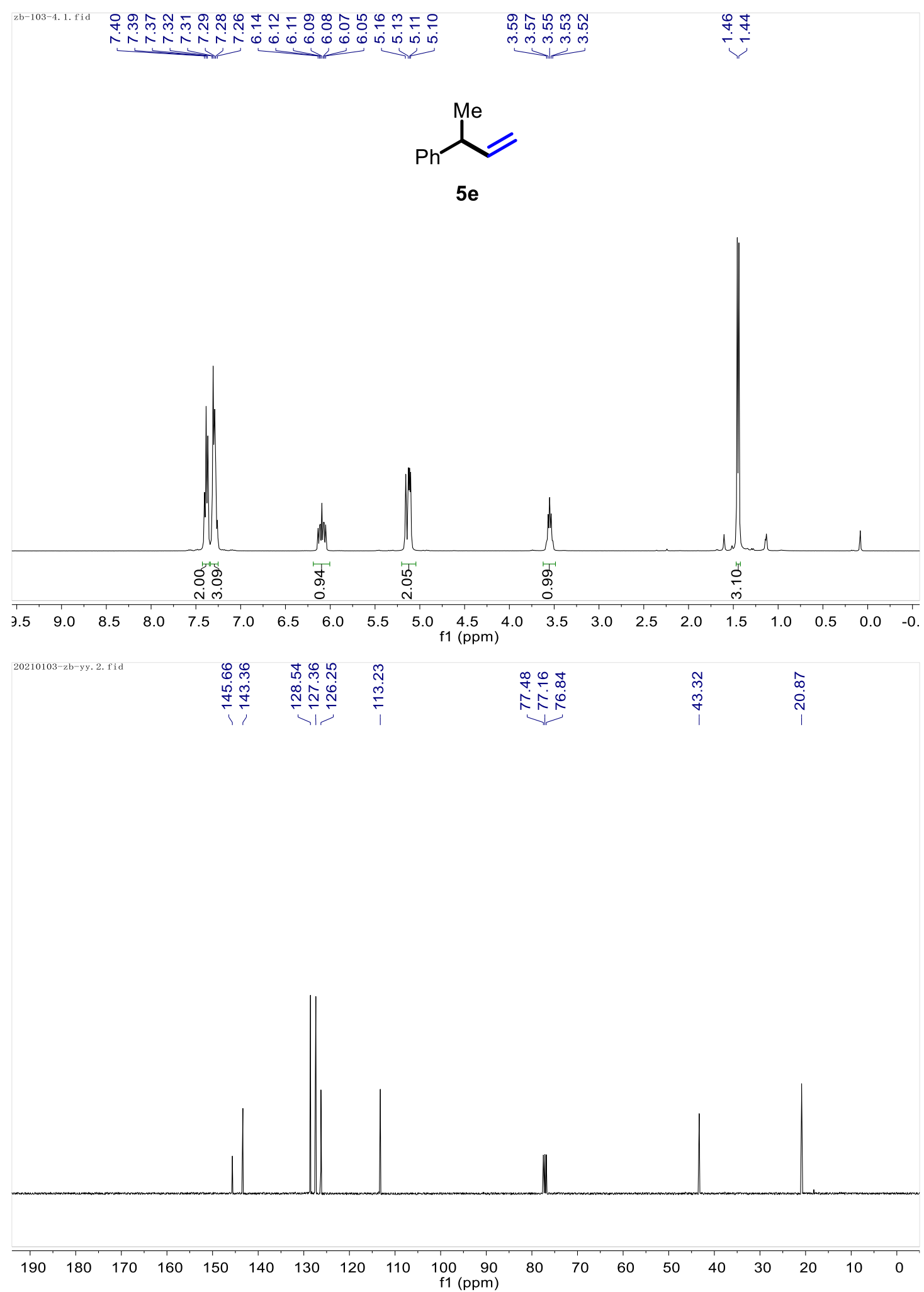



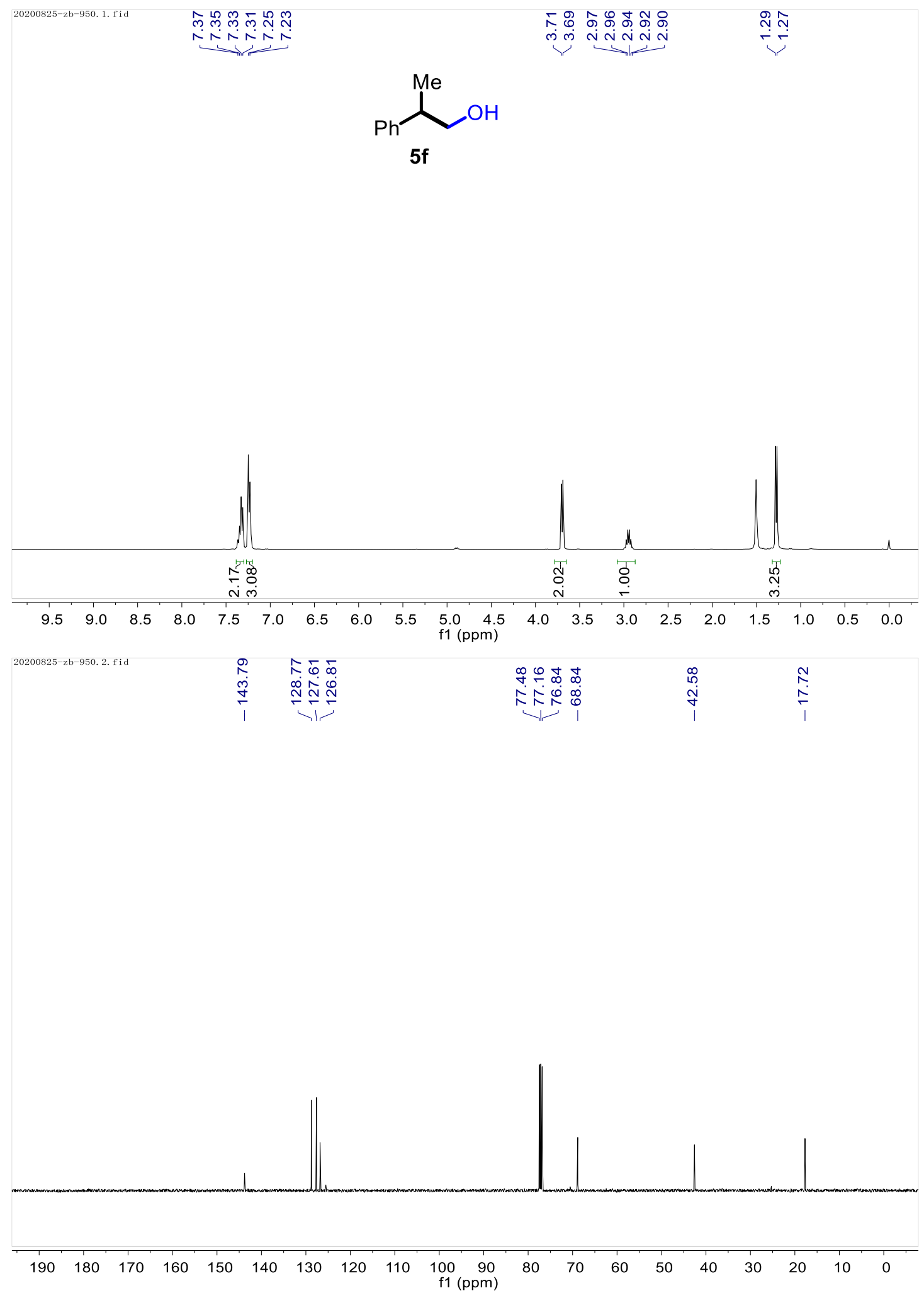


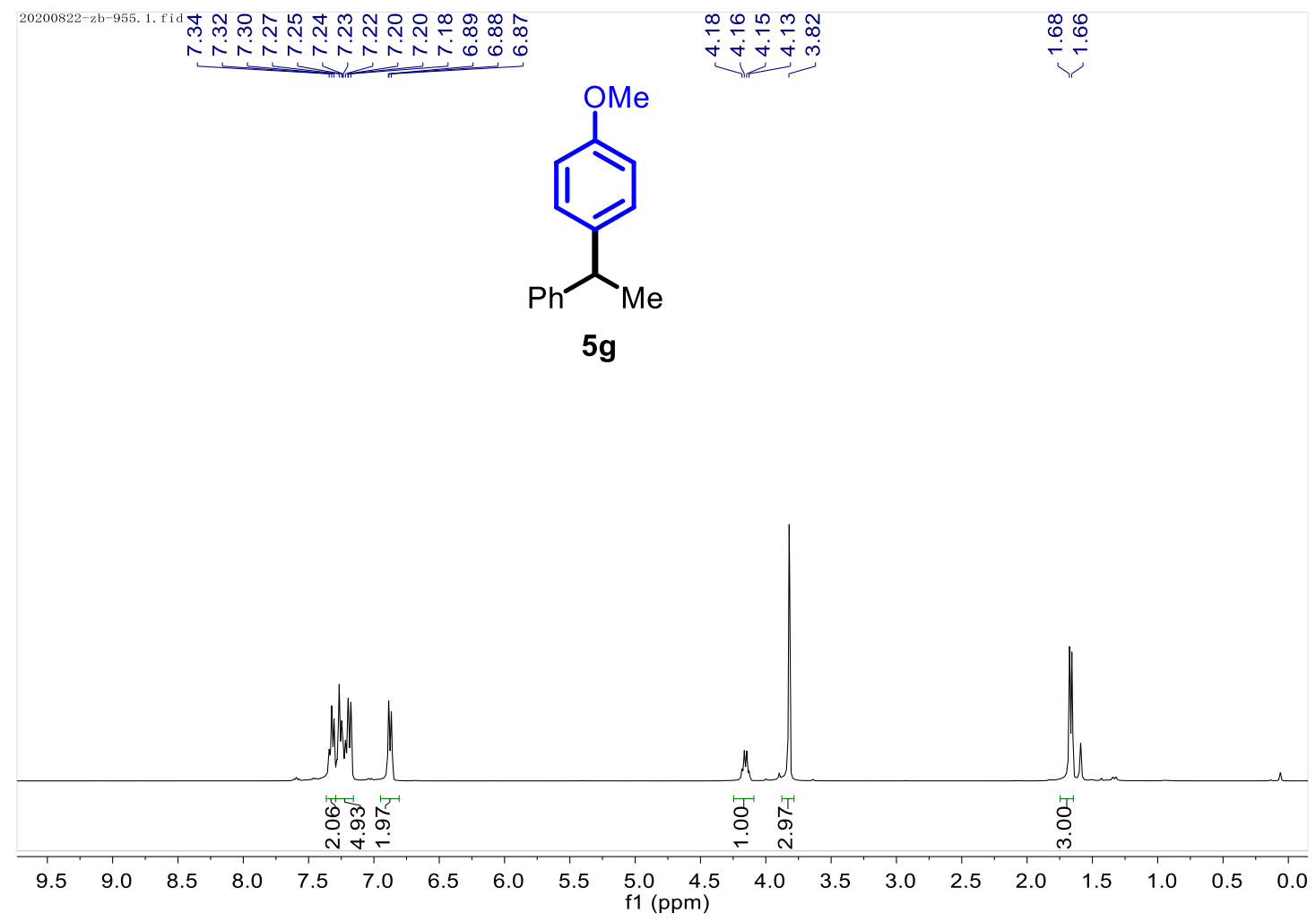

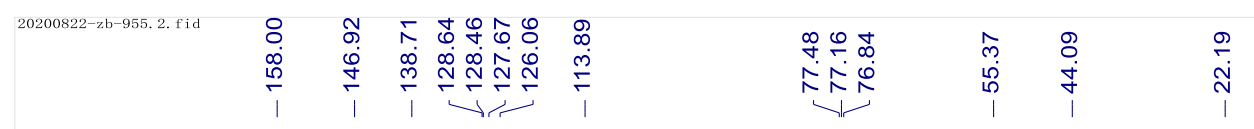

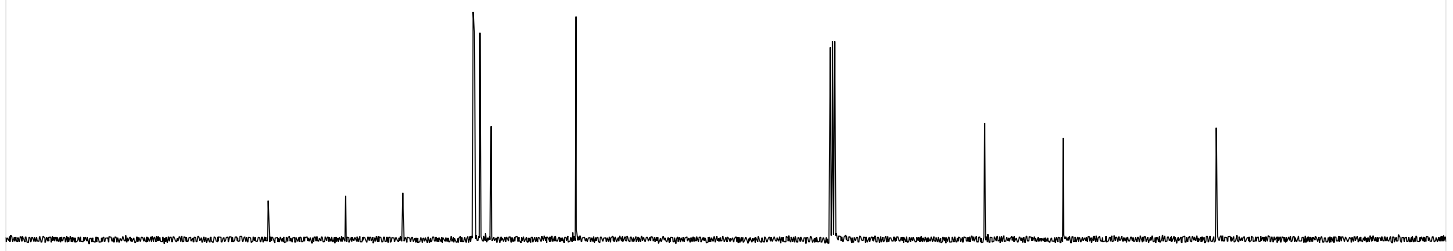
$\begin{array}{llllllllllllllllllll}190 & 180 & 170 & 160 & 150 & 140 & 130 & 120 & 110 & \begin{array}{c}100 \\ \mathrm{f} 1(\mathrm{ppm})\end{array} & 80 & 70 & 60 & 50 & 40 & 30 & 20 & 10 & 0 & -1\end{array}$ 


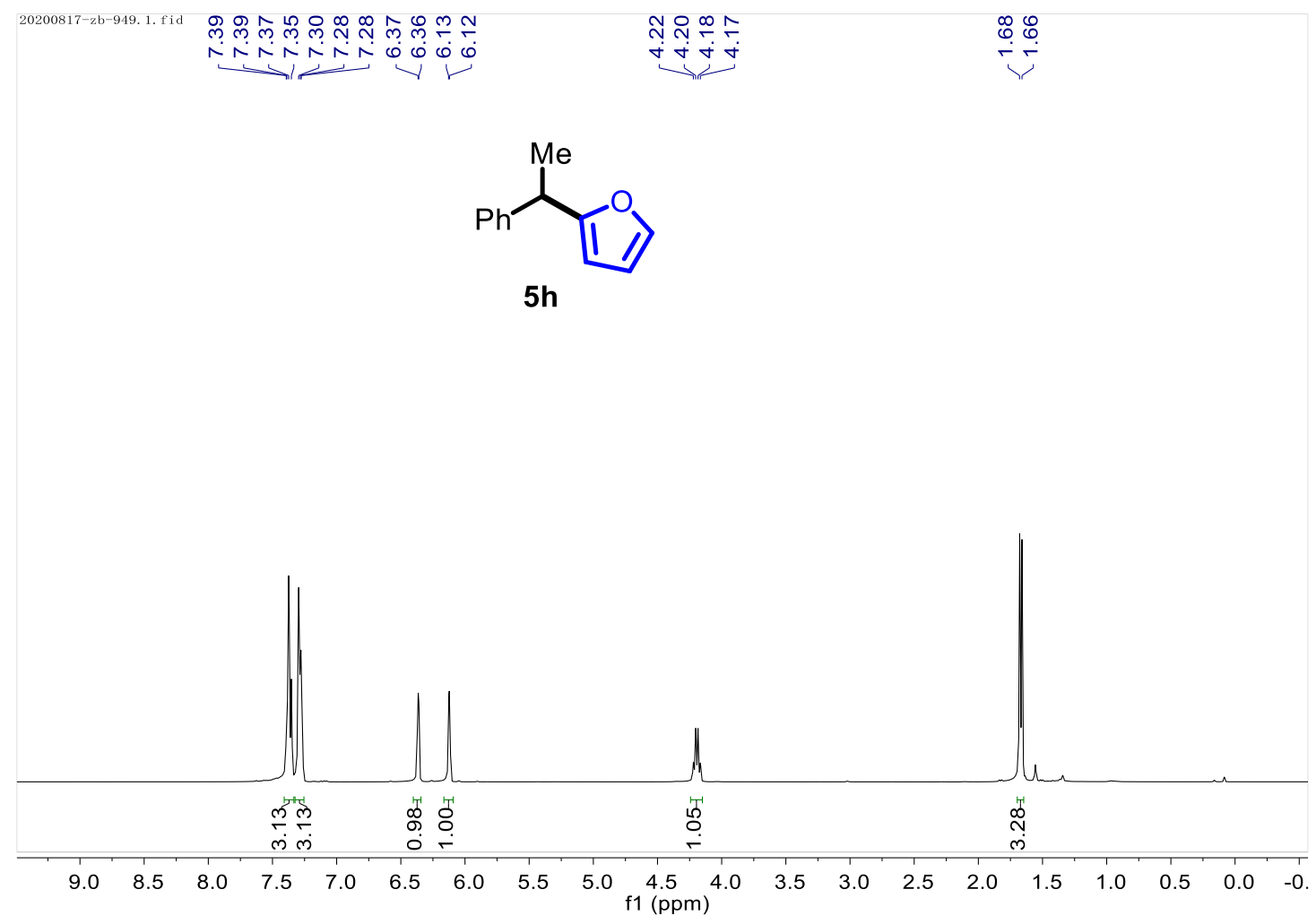

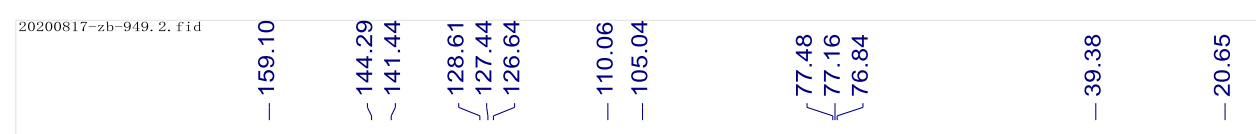

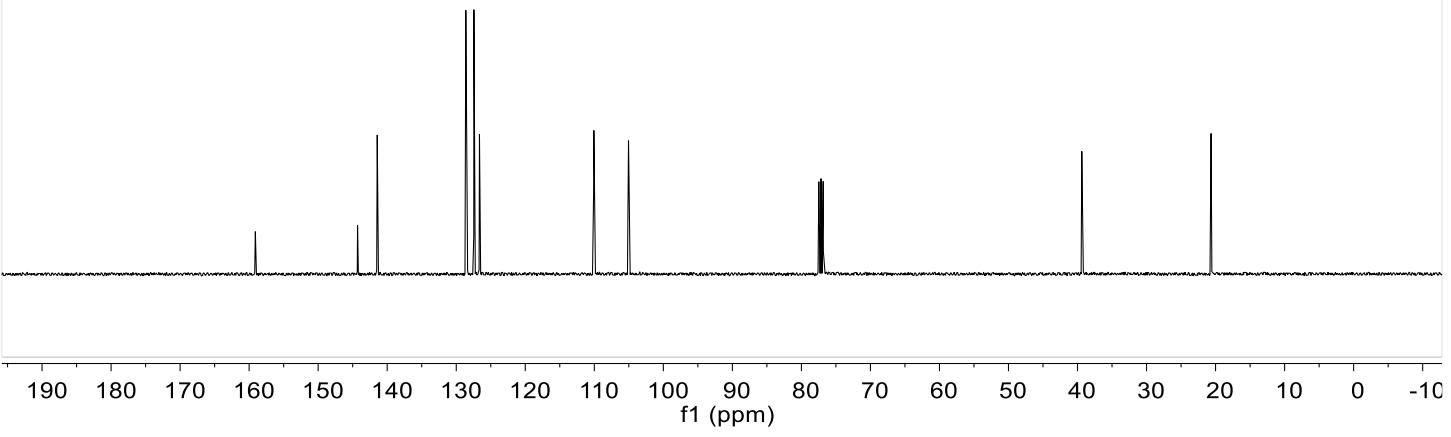




\section{Cartesian Coordinates}<smiles>CCP(CC)[PH](CC)CC</smiles>

\section{B1}

$\mathrm{E}=-842.682203$

C

$\mathrm{H}$

H

C

$\mathrm{H}$

$\mathrm{H}$

C

$\mathrm{H}$

$\mathrm{H}$

P

C

$$
G=-842.129398
$$

$$
-3.29844700 \quad-0.07587200 \quad-1.63159500
$$$$
-4.36755800 \quad-0.27734500 \quad-1.49763300
$$$$
\begin{array}{lll}
-3.18265500 & 1.01222300 & -1.67047000
\end{array}
$$$$
\begin{array}{lll}
3.57057300 & -0.07452500 & 0.31514900
\end{array}
$$$$
3.28748500 \quad 0.44665200 \quad 1.23714500
$$$$
3.60296500 \quad 0.69213100 \quad-0.46623800
$$$$
\begin{array}{lll}
-0.18929500 & 2.43193900 & 1.96508300
\end{array}
$$$$
\begin{array}{lll}
-1.23595700 & 2.22516700 \quad 2.21570800
\end{array}
$$$$
\begin{array}{lll}
-0.04354300 & 3.51500000 & 2.05008200
\end{array}
$$$$
-2.34413100 \quad-0.65239900 \quad-0.14346900
$$$$
2.12065700 \quad-1.17573400 \quad-0.10825000
$$$$
\begin{array}{lll}
0.05696200 & 1.87412900 & 0.20444200
\end{array}
$$$$
\begin{array}{lll}
1.60601600 & 2.81321600 & -0.22457900
\end{array}
$$$$
\begin{array}{lll}
2.40401200 & 2.41454800 & 0.40755500
\end{array}
$$$$
\begin{array}{lll}
1.48622900 & 3.86787400 & 0.04713100
\end{array}
$$$$
-1.22094800 \quad 2.90245500 \quad-0.69171300
$$$$
\begin{array}{lll}
-1.20072900 & 2.56392400 & -1.73384100
\end{array}
$$$$
\begin{array}{lll}
-2.18822600 & 2.57802000 & -0.29332800
\end{array}
$$$$
\begin{array}{lll}
-3.29339900 & 0.18009600 & 1.22521400
\end{array}
$$$$
\begin{array}{lll}
-3.13891700 \quad 1.25826800 & 1.10972400
\end{array}
$$$$
-4.36776100 \quad 0.00273100
$$$$
-2.90626400 \quad-2.41900200
$$$$
1.10186600
$$$$
0.03220600
$$$$
-2.47392900 \quad-2.93861900 \quad-0.82853400
$$$$
-2.36537500 \quad-2.80855000
$$$$
2.32006000 \quad-2.55384800
$$$$
1.62739300 \quad-3.33329300
$$$$
3.33356000 \quad-2.96731900
$$$$
2.70531500 \quad-2.01337900 \quad-1.65801500
$$$$
3.69192100 \quad-2.46375400 \quad-1.49925700
$$$$
\begin{array}{lll}
1.99384500 & -2.82829000 & -1.82378300
\end{array}
$$$$
\begin{array}{lll}
-0.05527300 & -0.43490900 & -0.17913300
\end{array}
$$$$
\begin{array}{lll}
-0.14846600 & -2.01607000 & -0.50441600
\end{array}
$$$$
\begin{array}{lll}
-4.40370400 & -2.69474700 & 0.15026500
\end{array}
$$$$
\begin{array}{lll}
-4.83551500 & -2.22907900 & 1.04163200
\end{array}
$$$$
\begin{array}{lll}
-4.95615700 & -2.32768800 & -0.72029200
\end{array}
$$
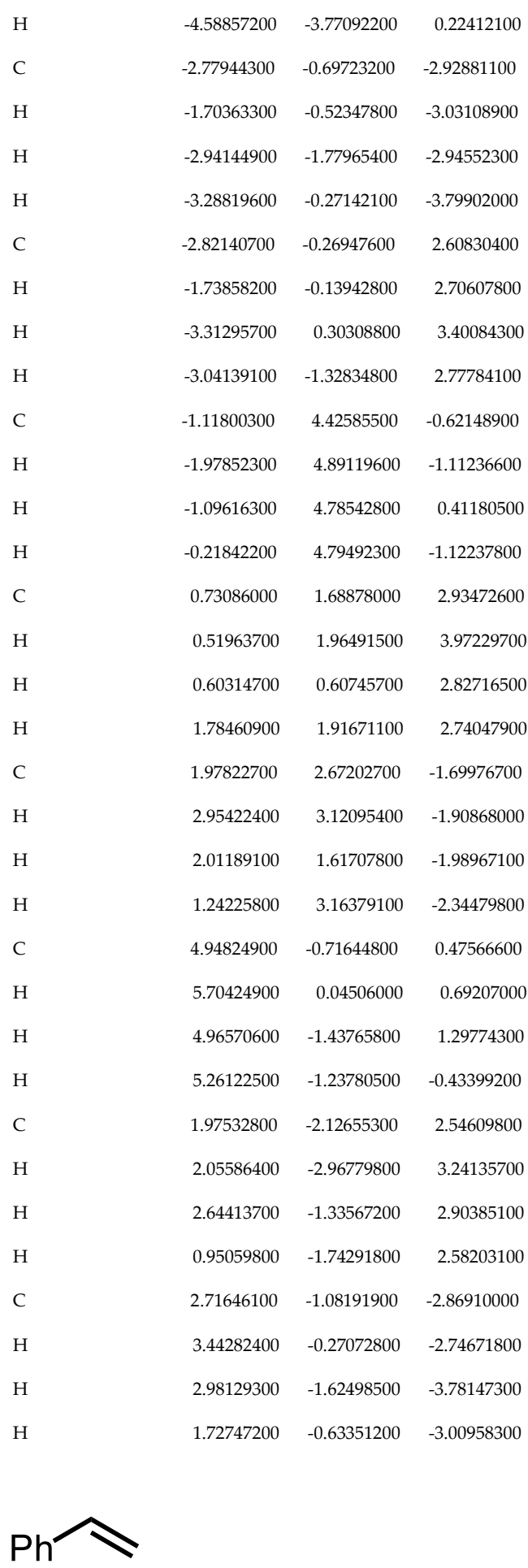

$E=-309.543827$

$G=-309.439932$

$\begin{array}{rrr}-2.25566700 & 0.26163400 & 0.03776200 \\ -1.77361600 & -1.04337400 & 0.04713400 \\ -0.40361300 & -1.27961000 & 0.00240300 \\ 0.51172100 & -0.22175200 & -0.04584100 \\ 0.01056200 & 1.08683300 & -0.06548400 \\ -1.35679600 & 1.32531400 & -0.02102000 \\ -3.32405600 & 0.45031400 & 0.07082800\end{array}$




\begin{tabular}{|c|c|c|c|c|c|c|c|}
\hline $\mathrm{H}$ & -2.46527200 & -1.87918100 & 0.08716500 & $\mathrm{H}$ & -1.39692300 & 3.84432500 & -1.63483200 \\
\hline $\mathrm{H}$ & -0.03240000 & -2.30128900 & 0.01064100 & $\mathrm{H}$ & 0.06163700 & 3.16011100 & -0.91640500 \\
\hline $\mathrm{H}$ & 0.69611100 & 1.92626800 & -0.12835400 & C & -1.11275200 & -2.78470000 & 1.45383500 \\
\hline $\mathrm{H}$ & -1.72548400 & 2.34640500 & -0.03920600 & $\mathrm{H}$ & -1.32994300 & -1.98091700 & 2.16537500 \\
\hline C & 1.95494500 & -0.52520000 & -0.07727500 & $\mathrm{H}$ & -0.02847700 & -2.92922100 & 1.49498800 \\
\hline $\mathrm{H}$ & 2.19757000 & -1.57200200 & -0.25556100 & C & 0.23333900 & 2.05710400 & 2.57411700 \\
\hline C & 2.95744300 & 0.33540600 & 0.10359800 & $\mathrm{H}$ & -0.00885000 & 2.93088900 & 1.95954000 \\
\hline $\mathrm{H}$ & 3.98942500 & 0.00467600 & 0.05800300 & $\mathrm{H}$ & 0.91109500 & 2.40487100 & 3.36006500 \\
\hline \multirow[t]{2}{*}{$\mathrm{H}$} & 2.79422900 & 1.38930000 & 0.30882200 & $\mathrm{P}$ & -1.79731100 & 1.67881300 & -0.50674700 \\
\hline & & & & $\mathrm{P}$ & -1.47575500 & -2.05600200 & -0.22786900 \\
\hline HBpin & & & & $\mathrm{P}$ & 1.12800500 & 0.85155800 & 1.46398600 \\
\hline$E=-411.761622$ & \multicolumn{2}{|c|}{$G=-411.601291$} & & C & 1.48092300 & -0.45699400 & 2.74868500 \\
\hline $\mathrm{O}$ & -1.07023700 & 1.18916500 & -0.40191300 & $\mathrm{H}$ & 0.51863600 & -0.92568300 & 2.97287900 \\
\hline $\mathrm{O}$ & 1.07027100 & 1.18929300 & 0.40171000 & $\mathrm{H}$ & 1.81556500 & 0.04024200 & 3.66745900 \\
\hline C & -0.78322300 & -0.18385200 & -0.05336700 & C & 2.78540400 & 1.69694200 & 1.32144700 \\
\hline C & 0.78324000 & -0.18380600 & 0.05345000 & $\mathrm{H}$ & 3.41188500 & 1.04021700 & 0.71686700 \\
\hline C & -1.35231800 & -1.09513200 & -1.13143200 & $\mathrm{H}$ & 2.61631900 & 2.58813900 & 0.70561600 \\
\hline $\mathrm{H}$ & -1.09489700 & -2.13931900 & -0.92714800 & C & -2.67410800 & 2.91502300 & 0.60886400 \\
\hline $\mathrm{H}$ & -2.44177500 & -1.00871100 & -1.14597900 & $\mathrm{H}$ & -1.93583400 & 3.28771900 & 1.32404400 \\
\hline $\mathrm{H}$ & -0.97868100 & -0.82899300 & -2.12136100 & $\mathrm{H}$ & -2.94994500 & 3.77005100 & -0.01863400 \\
\hline C & -1.47743400 & -0.45334100 & 1.28291600 & C & -3.19226000 & 1.26392100 & -1.67759200 \\
\hline $\mathrm{H}$ & -2.54489800 & -0.24680100 & 1.17411500 & $\mathrm{H}$ & -2.74635500 & 0.62391900 & -2.44683700 \\
\hline $\mathrm{H}$ & -1.35446000 & -1.49440300 & 1.59418400 & $\mathrm{H}$ & -3.87884100 & 0.62199200 & -1.11721600 \\
\hline $\mathrm{H}$ & -1.08440400 & 0.19605000 & 2.07004400 & C & -3.34441200 & -1.97997900 & -0.16499500 \\
\hline C & 1.35250400 & -1.09490300 & 1.13157200 & $\mathrm{H}$ & -3.66840900 & -1.55780900 & -1.12172300 \\
\hline $\mathrm{H}$ & 1.09493600 & -2.13911000 & 0.92757600 & $\mathrm{H}$ & -3.75358700 & -2.99520200 & -0.11827000 \\
\hline $\mathrm{H}$ & 2.44197500 & -1.00857200 & 1.14583900 & C & -1.35955900 & -3.59512900 & -1.30151700 \\
\hline $\mathrm{H}$ & 0.97914300 & -0.82851900 & 2.12153900 & $\mathrm{H}$ & -2.16862100 & -4.26158200 & -0.98119400 \\
\hline C & 1.47725300 & -0.45351800 & -1.28287000 & $\mathrm{H}$ & -1.60249300 & -3.29559300 & -2.32724500 \\
\hline $\mathrm{H}$ & 2.54470900 & -0.24685000 & -1.17426600 & $\mathrm{Rh}$ & -0.17558300 & -0.08544000 & -0.25389500 \\
\hline $\mathrm{H}$ & 1.35431300 & -1.49465800 & -1.59390600 & $\mathrm{H}$ & 0.88057600 & -0.41950300 & -3.17367500 \\
\hline $\mathrm{H}$ & 1.08405800 & 0.19567100 & -2.07008500 & C & -3.96624600 & 2.41924300 & -2.31292400 \\
\hline B & -0.00007900 & 1.93578900 & -0.00001500 & $\mathrm{H}$ & -4.49263100 & 3.01626600 & -1.56228500 \\
\hline $\mathrm{H}$ & -0.00002900 & 3.12491200 & -0.00046200 & $\mathrm{H}$ & -3.31117500 & 3.09278200 & -2.87302600 \\
\hline & & & & $\mathrm{H}$ & -4.71744500 & 2.03791800 & -3.01172100 \\
\hline & & & & $\mathrm{C}$ & -0.39358800 & 2.38340800 & -2.88161000 \\
\hline & & & & $\mathrm{H}$ & 0.05419800 & 1.39186100 & -2.78220500 \\
\hline & & & & $\mathrm{H}$ & -1.24390400 & 2.29316800 & -3.56439400 \\
\hline & & & & $\mathrm{H}$ & 0.33978400 & 3.04664200 & -3.35001900 \\
\hline & & & & C & -3.91438500 & 2.42531800 & 1.35684100 \\
\hline & & & & $\mathrm{H}$ & -3.67422800 & 1.65771500 & 2.09490800 \\
\hline INT1 & & & & $\mathrm{H}$ & -4.38156000 & 3.25845300 & 1.89128900 \\
\hline$E=-1152.236448$ & $\mathrm{G}=-11$ & 51.543321 & & $\mathrm{H}$ & -4.66428600 & 2.01119800 & 0.67544700 \\
\hline C & 273500 & 2.91806100 & -1.51538500 & C & 3.53614400 & 2.06208100 & 2.60264400 \\
\hline
\end{tabular}




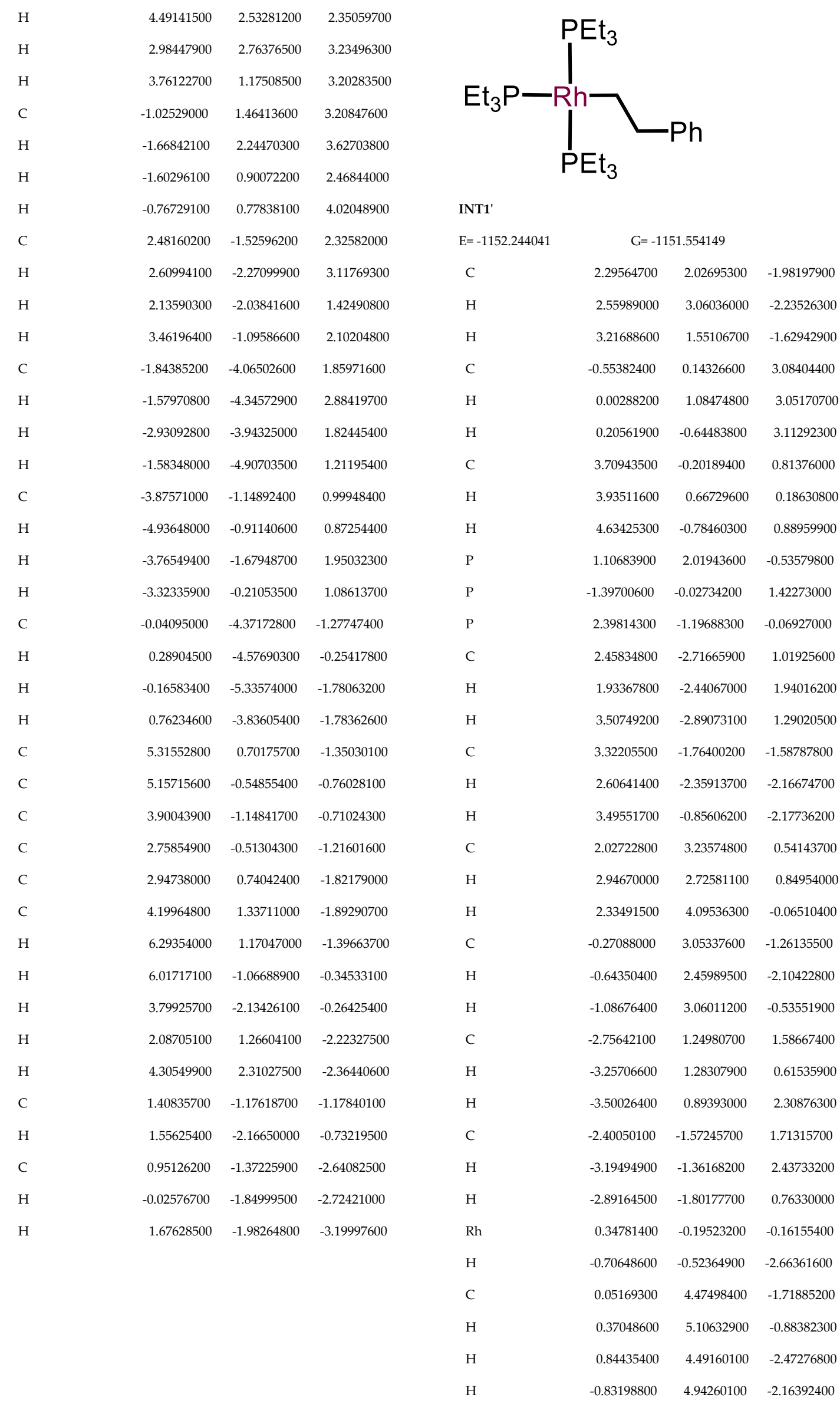




\begin{tabular}{|c|c|c|c|c|c|c|c|}
\hline C & 1.77599100 & 1.29182500 & -3.21614100 & $\mathrm{H}$ & -1.18078200 & -2.16245100 & -3.05532500 \\
\hline $\mathrm{H}$ & 1.46599100 & 0.27376400 & -2.95864500 & $\mathrm{C}$ & -0.42440900 & -1.95201100 & -1.02805800 \\
\hline $\mathrm{H}$ & 0.91373800 & 1.80271500 & -3.65640400 & $\mathrm{H}$ & 0.35360800 & -2.62251700 & -1.41120000 \\
\hline $\mathrm{H}$ & 2.54942200 & 1.23116400 & -3.98807800 & $\mathrm{H}$ & -1.09310300 & -2.58552700 & -0.43451900 \\
\hline C & 1.25708100 & 3.70303600 & 1.77279800 & & & & \\
\hline $\mathrm{H}$ & 0.91245900 & 2.85391000 & 2.36981900 & & & & \\
\hline $\mathrm{H}$ & 1.88507200 & 4.33136200 & 2.41142200 & & & & \\
\hline $\mathrm{H}$ & 0.37606300 & 4.29034200 & 1.49498400 & & & & \\
\hline C & 4.63155800 & -2.53309600 & -1.40647100 & & & & \\
\hline $\mathrm{H}$ & 5.06513400 & -2.78816500 & -2.37858600 & & & & \\
\hline $\mathrm{H}$ & 5.37608300 & -1.94451600 & -0.86255900 & & & & \\
\hline $\mathrm{H}$ & 4.48185800 & -3.46821100 & -0.85905300 & INT2 & & & \\
\hline C & 3.26410200 & 0.25749000 & 2.20354600 & $E=-1564.035193$ & $G=-15$ & 63.160337 & \\
\hline $\mathrm{H}$ & 3.98316900 & 0.96244200 & 2.63318700 & $\mathrm{H}$ & 0.99674700 & 2.38799900 & 2.07848500 \\
\hline $\mathrm{H}$ & 2.28495100 & 0.74344700 & 2.15298300 & $\mathrm{C}$ & -2.84083900 & 4.99254200 & 0.56574500 \\
\hline $\mathrm{H}$ & 3.17869100 & -0.58763200 & 2.89341300 & $\mathrm{C}$ & -3.33659200 & 4.10976400 & 1.51951200 \\
\hline C & 1.85137600 & -4.00048700 & 0.45344300 & C & -2.65840300 & 2.92306900 & 1.78793100 \\
\hline $\mathrm{H}$ & 2.01628400 & -4.83111300 & 1.14694100 & $\mathrm{C}$ & -1.48791200 & 2.56453500 & 1.10350900 \\
\hline $\mathrm{H}$ & 0.77674800 & -3.90004100 & 0.29791100 & C & -0.99188400 & 3.48162400 & 0.16778900 \\
\hline $\mathrm{H}$ & 2.30075100 & -4.27592800 & -0.50609800 & $\mathrm{C}$ & -1.65797100 & 4.67381700 & -0.09802800 \\
\hline C & -1.43230400 & 0.08885300 & 4.33517500 & $\mathrm{H}$ & -3.36258200 & 5.91989300 & 0.34859400 \\
\hline $\mathrm{H}$ & -0.82500100 & 0.21508000 & 5.23725400 & $\mathrm{H}$ & -4.25127900 & 4.34300600 & 2.05784200 \\
\hline $\mathrm{H}$ & -2.19111000 & 0.87658200 & 4.33788700 & $\mathrm{H}$ & -3.05383800 & 2.24100000 & 2.53864900 \\
\hline $\mathrm{H}$ & -1.95472100 & -0.86806400 & 4.42360500 & $\mathrm{H}$ & -0.07075600 & 3.24373400 & -0.35206500 \\
\hline C & -2.27599200 & 2.64335400 & 1.98760400 & $\mathrm{H}$ & -1.25242100 & 5.35910300 & -0.83792900 \\
\hline $\mathrm{H}$ & -3.07877300 & 3.38015900 & 1.88606600 & $\mathrm{C}$ & -0.77999900 & 1.27650600 & 1.47388900 \\
\hline $\mathrm{H}$ & -1.93081900 & 2.67240600 & 3.02551000 & $\mathrm{H}$ & -1.52997000 & 0.68804300 & 2.01609200 \\
\hline $\mathrm{H}$ & -1.43971800 & 2.97188700 & 1.36816700 & $\mathrm{C}$ & 0.30671000 & 1.65440700 & 2.50359800 \\
\hline C & -1.55400100 & -2.75644500 & 2.18767200 & $\mathrm{H}$ & 0.90400000 & 0.79364900 & 2.82702000 \\
\hline $\mathrm{H}$ & -1.31085200 & -2.67221300 & 3.25138200 & $\mathrm{H}$ & -0.13722200 & 2.11213400 & 3.40067100 \\
\hline $\mathrm{H}$ & -2.08363500 & -3.70292600 & 2.04333200 & $\mathrm{O}$ & 2.88263600 & 0.24996800 & 0.18187400 \\
\hline $\mathrm{H}$ & -0.60991200 & -2.80766800 & 1.63867200 & $\mathrm{O}$ & 1.83484200 & 2.20853400 & -0.23103900 \\
\hline C & -5.34988600 & -0.49879000 & -1.42941300 & $\mathrm{C}$ & 3.89222300 & 1.25124900 & 0.36427000 \\
\hline C & -4.43544400 & 0.53036700 & -1.63052400 & $\mathrm{C}$ & 3.22663700 & 2.53020600 & -0.26058800 \\
\hline C & -3.09859400 & 0.23879300 & -1.89416000 & $\mathrm{C}$ & 5.16546700 & 0.78614400 & -0.33328500 \\
\hline C & -2.64870900 & -1.07983300 & -1.97305200 & $\mathrm{H}$ & 5.94839700 & 1.54822300 & -0.26478200 \\
\hline C & -3.58295300 & -2.10412000 & -1.78187100 & $\mathrm{H}$ & 5.53458500 & -0.12489400 & 0.14601200 \\
\hline C & -4.91714600 & -1.82112300 & -1.50767100 & $\mathrm{H}$ & 4.98689900 & 0.56521600 & -1.38769800 \\
\hline $\mathrm{H}$ & -6.39155100 & -0.27508800 & -1.22126500 & C & 4.14261500 & 1.36373200 & 1.87075800 \\
\hline $\mathrm{H}$ & -4.76063500 & 1.56572000 & -1.57596200 & $\mathrm{H}$ & 4.47479000 & 0.39224200 & 2.24866800 \\
\hline $\mathrm{H}$ & -2.38407900 & 1.04634900 & -2.02679400 & $\mathrm{H}$ & 4.91506400 & 2.10288800 & 2.10123800 \\
\hline $\mathrm{H}$ & -3.25132700 & -3.13785700 & -1.84623700 & $\mathrm{H}$ & 3.22647900 & 1.63480800 & 2.40151200 \\
\hline $\mathrm{H}$ & -5.62345700 & -2.63297600 & -1.36063400 & C & 3.44533400 & 3.81386700 & 0.53503900 \\
\hline C & -1.20400600 & -1.41296600 & -2.25006900 & $\mathrm{H}$ & 4.51051800 & 4.06102300 & 0.59371800 \\
\hline
\end{tabular}




\begin{tabular}{|c|c|c|c|c|c|c|c|}
\hline $\mathrm{H}$ & 2.92872100 & 4.64114500 & 0.04137800 & $\mathrm{H}$ & -2.86834900 & -3.18912900 & 0.41638100 \\
\hline $\mathrm{H}$ & 3.04761800 & 3.72959400 & 1.54770700 & $\mathrm{H}$ & -4.22002000 & -3.88280500 & -0.48887600 \\
\hline $\mathrm{C}$ & 3.60262700 & 2.77868300 & -1.72384000 & $\mathrm{H}$ & -4.46219100 & -2.44879600 & 0.51674100 \\
\hline $\mathrm{H}$ & 2.97145100 & 3.58141500 & -2.11345400 & C & -2.59936300 & 1.90343400 & -2.53899200 \\
\hline $\mathrm{H}$ & 4.64995300 & 3.07638800 & -1.83058300 & $\mathrm{H}$ & -2.61972500 & 2.30468500 & -3.55718100 \\
\hline $\mathrm{H}$ & 3.42777600 & 1.89389300 & -2.33962100 & $\mathrm{H}$ & -1.67005600 & 2.22055700 & -2.06220800 \\
\hline B & 1.63041400 & 0.84722100 & -0.02100500 & $\mathrm{H}$ & -3.42103800 & 2.36485200 & -1.98280400 \\
\hline $\mathrm{H}$ & -0.26315400 & 1.07044500 & -1.05914800 & $\mathrm{C}$ & -0.07150000 & -2.56833700 & -2.91364700 \\
\hline $\mathrm{Rh}$ & -0.16617400 & -0.12533900 & -0.08442200 & $\mathrm{H}$ & -0.95204300 & -2.02694800 & -3.27483400 \\
\hline $\mathrm{P}$ & 0.18391500 & -1.79992100 & 1.73248600 & $\mathrm{H}$ & 0.53146000 & -2.80191900 & -3.79847500 \\
\hline $\mathrm{P}$ & -2.56752500 & -0.45541900 & -0.90281500 & $\mathrm{C}$ & -0.49241600 & -3.85927400 & -2.21138800 \\
\hline $\mathrm{P}$ & 0.88394400 & -1.39574300 & -1.82029700 & $\mathrm{H}$ & 0.37428400 & -4.48557100 & -1.97781900 \\
\hline $\mathrm{C}$ & -0.84258500 & -1.55608800 & 3.26983900 & $\mathrm{H}$ & -1.16233100 & -4.44934600 & -2.84434700 \\
\hline $\mathrm{H}$ & -0.46409900 & -2.19525600 & 4.07206600 & $\mathrm{H}$ & -1.01215800 & -3.65379200 & -1.27178000 \\
\hline $\mathrm{H}$ & -0.69863100 & -0.52387500 & 3.59625000 & $\mathrm{C}$ & 1.74136600 & -0.35985000 & -3.10715400 \\
\hline $\mathrm{C}$ & -2.32723000 & -1.82865900 & 3.03723600 & $\mathrm{H}$ & 2.62077600 & 0.04946700 & -2.59850400 \\
\hline $\mathrm{H}$ & -2.91984100 & -1.51508500 & 3.90162100 & $\mathrm{H}$ & 2.11204000 & -1.02253600 & -3.89669300 \\
\hline $\mathrm{H}$ & -2.52107500 & -2.89313900 & 2.87058500 & $\mathrm{C}$ & 0.91018200 & 0.76746300 & -3.71239600 \\
\hline $\mathrm{H}$ & -2.69025000 & -1.28649900 & 2.16067000 & $\mathrm{H}$ & 0.03305300 & 0.38040800 & -4.24148100 \\
\hline $\mathrm{C}$ & 1.94199600 & -1.83200500 & 2.38289200 & $\mathrm{H}$ & 1.50616500 & 1.33529900 & -4.43378100 \\
\hline $\mathrm{H}$ & 2.42666400 & -2.69427900 & 1.90847900 & $\mathrm{H}$ & 0.56256000 & 1.45359500 & -2.93655600 \\
\hline $\mathrm{H}$ & 2.42441800 & -0.95456100 & 1.95199900 & $\mathrm{C}$ & 2.31067600 & -2.46880500 & -1.28731100 \\
\hline $\mathrm{C}$ & 2.16729200 & -1.86506700 & 3.89452800 & $\mathrm{H}$ & 2.94476600 & -1.80828900 & -0.69006300 \\
\hline $\mathrm{H}$ & 1.72067600 & -0.99346400 & 4.38271200 & $\mathrm{H}$ & 1.90292300 & -3.20919200 & -0.59407500 \\
\hline $\mathrm{H}$ & 3.24049400 & -1.84533800 & 4.11024900 & $\mathrm{C}$ & 3.12134700 & -3.17070800 & -2.37727600 \\
\hline $\mathrm{H}$ & 1.75204200 & -2.76066600 & 4.36601400 & $\mathrm{H}$ & 2.49692200 & -3.80007500 & -3.02001000 \\
\hline $\mathrm{C}$ & -0.11566300 & -3.61993500 & 1.43524100 & $\mathrm{H}$ & 3.88054000 & -3.81707800 & -1.92596500 \\
\hline $\mathrm{H}$ & -1.14217900 & -3.71616400 & 1.07144400 & $\mathrm{H}$ & 3.64448700 & -2.45503000 & -3.01729400 \\
\hline $\mathrm{H}$ & 0.51809100 & -3.90546000 & 0.59107800 & $\mathrm{C}$ & -2.71506200 & 0.38126800 & -2.56717200 \\
\hline $\mathrm{C}$ & 0.11985200 & -4.57816700 & 2.60222400 & $\mathrm{H}$ & -3.66097100 & 0.08312400 & -3.03442800 \\
\hline $\mathrm{H}$ & -0.05876400 & -5.61237500 & 2.29172300 & $\mathrm{H}$ & -1.91230300 & -0.03347600 & -3.18505100 \\
\hline $\mathrm{H}$ & -0.54965100 & -4.36810200 & 3.44149700 & & & & \\
\hline $\mathrm{H}$ & 1.14808600 & -4.51767700 & 2.97062100 & & & & \\
\hline $\mathrm{C}$ & -3.93006300 & 0.38800600 & 0.04161500 & & & & \\
\hline $\mathrm{H}$ & -3.94471700 & -0.04032500 & 1.04950700 & & & & \\
\hline $\mathrm{H}$ & -3.59371000 & 1.41742100 & 0.17499500 & & & & \\
\hline $\mathrm{C}$ & -5.33458800 & 0.37162900 & -0.55968700 & & & & \\
\hline $\mathrm{H}$ & -6.00973200 & 0.97569600 & 0.05423400 & & & & \\
\hline $\mathrm{H}$ & -5.75500900 & -0.63722600 & -0.61462600 & & & & \\
\hline $\mathrm{H}$ & -5.34664800 & 0.79527500 & -1.56862000 & INT2' & & & \\
\hline $\mathrm{C}$ & -3.41873400 & -2.05785700 & -1.35621800 & $E=-1564.041852$ & $\mathrm{G}=-1$ & 63.167827 & \\
\hline $\mathrm{H}$ & -4.33076800 & -1.81737600 & -1.91370900 & $\mathrm{H}$ & -2.51986400 & 0.61160200 & -1.40814600 \\
\hline $\mathrm{H}$ & -2.76741800 & -2.59438500 & -2.05041700 & $\mathrm{C}$ & -6.73739300 & -0.60758000 & 0.53213600 \\
\hline C & -3.76145600 & -2.94447900 & -0.16277600 & C & -6.17863100 & -1.67705800 & -0.16060700 \\
\hline
\end{tabular}




$\begin{array}{rrr}-4.87462000 & -1.59157800 & -0.64407600 \\ -4.10579500 & -0.44414900 & -0.44372200 \\ -4.67907000 & 0.62252200 & 0.25847800 \\ -5.98107100 & 0.54519800 & 0.74005600 \\ -7.75365600 & -0.67096200 & 0.90944200 \\ -6.75861500 & -2.58030300 & -0.32765100 \\ -4.44470900 & -2.42960900 & -1.18893500 \\ -4.07859800 & 1.51347600 & 0.43042900 \\ -6.41022300 & 1.38594600 & 1.27792600 \\ -2.67511400 & -0.35186700 & -0.91478000 \\ -2.48943600 & -1.11887000 & -1.67528200 \\ -1.68190900 & -0.48153500 & 0.24814800 \\ -1.84170100 & -1.45384500 & 0.73160000 \\ -1.96332500 & 0.27469100 & 0.98743000\end{array}$

$\begin{array}{lll}-1.96332500 & 0.27469100 & 0.98743000\end{array}$

$\begin{array}{lll}-1.29951200 & 2.31824100 & 0.07635200\end{array}$

$\begin{array}{lll}0.61501700 & 2.70912700 & -1.06636100\end{array}$

$-1.45050500 \quad 3.62080600 \quad-0.49671400$

$\begin{array}{lll}0.02466300 & 3.99610200 & -0.85280200\end{array}$

$\begin{array}{lll}-2.11791300 \quad 4.53306100 & 0.52416900\end{array}$

$-2.17514400 \quad 5.56033100 \quad 0.14916900$

$\begin{array}{lll}-3.13658100 & 4.18459400 & 0.71580400\end{array}$

$-1.57781300 \quad 4.53450900 \quad 1.47306000$

$-2.34608600 \quad 3.47393200 \quad-1.72955100$

$\begin{array}{lll}-3.29611200 & 3.02517400 & -1.42653700\end{array}$

$\begin{array}{lll}-2.55187100 & 4.43995000 & -2.20010400\end{array}$

$\begin{array}{lll}-1.88539700 & 2.81242900 & -2.46903000\end{array}$

$0.19019100 \quad 4.82823000 \quad-2.11841800$

$\begin{array}{lll}-0.31807900 & 5.79297900 & -2.01825100\end{array}$

$\begin{array}{lll}1.25242200 & 5.02072600 & -2.29493000\end{array}$

$-0.20957000 \quad 4.31020900 \quad-2.99152700$

$\begin{array}{lll}0.77346000 & 4.66391700 & 0.30390900\end{array}$

$\begin{array}{lll}1.83468900 & 4.73481400 & 0.04768900\end{array}$

$0.40051800 \quad 5.67403700 \quad 0.49685000$

$\begin{array}{lll}0.68389600 & 4.07685500 & 1.22185400\end{array}$

$-0.13291500 \quad 1.72106000 \quad-0.41023000$

$\begin{array}{lll}-0.10016100 & 0.08641600 & -1.67916000\end{array}$

$\begin{array}{lll}0.38020700 & -0.25339000 & -0.20990500\end{array}$

$\begin{array}{lll}0.70369700 & -0.14747200 & 2.20736500\end{array}$

$\begin{array}{lll}0.44823600 & -2.66503000 & -0.89941600\end{array}$

$\begin{array}{lll}2.61174500 & 0.32634600 & -0.92457100\end{array}$

$\begin{array}{lll}-0.55987700 & -0.99196400 & 3.27883100\end{array}$

$\begin{array}{lll}-0.43837800 & -0.65507300 \quad 4.31252300\end{array}$

$\begin{array}{lll}-1.53688400 & -0.63688300 & 2.93880800\end{array}$

$\begin{array}{lll}-0.50641200 & -2.51531300 & 3.21797900\end{array}$

\begin{tabular}{|c|c|c|}
\hline & & \\
\hline 0.41130300 & -2.90812600 & 3.66788900 \\
\hline-0.54484400 & -2.86379800 & 2.18414600 \\
\hline 0.54682500 & 1.62435400 & 2.78266100 \\
\hline 1.51320200 & 2.09324000 & 2.55758100 \\
\hline-0.17642700 & 2.06446300 & 2.09150000 \\
\hline 0.10384200 & 1.94084500 & 4.21323100 \\
\hline-0.92449500 & 1.61216500 & 4.38679300 \\
\hline 0.12780600 & 3.02365200 & 4.37443500 \\
\hline 0.73407000 & 1.48033800 & 4.97662200 \\
\hline 2.29212900 & -0.73332100 & 2.99334000 \\
\hline 2.34508100 & -1.80364000 & 2.76909100 \\
\hline 3.10640500 & -0.28283700 & 2.42024300 \\
\hline 2.52890100 & -0.50606500 & 4.48589300 \\
\hline 3.43864900 & -1.02308700 & 4.80751600 \\
\hline 1.70370600 & -0.88333900 & 5.09733700 \\
\hline 2.66063800 & 0.55522500 & 4.71122000 \\
\hline-1.10507600 & -3.65210600 & -0.58846500 \\
\hline-1.24358700 & -3.70614300 & 0.49549400 \\
\hline-1.93053700 & -3.03305600 & -0.95012400 \\
\hline-1.18992600 & -5.05236500 & -1.19362300 \\
\hline-2.16242900 & -5.50256400 & -0.97201600 \\
\hline-0.42204100 & -5.72059300 & -0.79176900 \\
\hline-1.07902600 & -5.03343600 & -2.28190800 \\
\hline 1.75851400 & -3.91660900 & -0.44290700 \\
\hline 1.62875900 & -4.81186000 & -1.06131100 \\
\hline 2.72125000 & -3.48245000 & -0.72819200 \\
\hline 1.77508200 & -4.29860900 & 1.03368400 \\
\hline 1.87677000 & -3.41302800 & 1.66578100 \\
\hline 2.60761600 & -4.97239800 & 1.25699900 \\
\hline 0.84929800 & -4.80373300 & 1.32758700 \\
\hline-0.57140800 & -2.25186500 & -3.54248600 \\
\hline-0.36616400 & -2.28970000 & -4.61667500 \\
\hline-0.76491300 & -1.21216800 & -3.26477000 \\
\hline-1.48940700 & -2.82051400 & -3.36092900 \\
\hline 3.96067600 & -0.95084000 & -1.09390500 \\
\hline 3.57084700 & -1.72532700 & -1.76415900 \\
\hline 4.81219200 & -0.49608300 & -1.61354900 \\
\hline 4.42107300 & -1.57067500 & 0.22255600 \\
\hline 4.89077200 & -0.82247800 & 0.86976400 \\
\hline 5.15536900 & -2.36334400 & 0.04852200 \\
\hline 3.58172400 & -2.00302400 & 0.77250500 \\
\hline 2.74534300 & 1.11862800 & -2.60297500 \\
\hline 00 & 2.05175100 & -2.523757 \\
\hline
\end{tabular}




$\begin{array}{llllllll}\mathrm{H} & 3.79773700 & 1.36398200 & -2.78454300 & \mathrm{C} & -3.21605200 & -0.92425100 & -1.05193100 \\ \mathrm{C} & 2.20517100 & 0.27803100 & -3.75605700 & \mathrm{C} & -2.76755500 & 0.38589500 & -0.91908500 \\ \mathrm{H} & 2.76982200 & -0.65194900 & -3.88463800 & \mathrm{C} & -2.17898800 & 0.82863200 & 0.26917300 \\ \mathrm{H} & 2.27514300 & 0.83153200 & -4.69754700 & \mathrm{C} & -2.04914400 & -0.08027700 & 1.32241700 \\ \mathrm{H} & 1.15579200 & 0.02381500 & -3.58849900 & \mathrm{C} & -2.50123000 & -1.39169200 & 1.19689600 \\ \mathrm{C} & 3.42940900 & 1.62448100 & 0.13212700 & \mathrm{H} & -3.43549100 & -2.84150500 & -0.09458300 \\ \mathrm{H} & 2.70179000 & 2.44034000 & 0.14990400 & \mathrm{H} & -3.66937900 & -1.24812100 & -1.98382700 \\ \mathrm{H} & 3.46665900 & 1.22345000 & 1.14935200 & \mathrm{H} & -2.87156200 & 1.07771400 & -1.75126900 \\ \mathrm{C} & 4.81143000 & 2.13300300 & -0.27566300 & \mathrm{H} & -1.57068500 & 0.24222400 & 2.24253200 \\ \mathrm{H} & 5.54909700 & 1.32531400 & -0.32238200 & \mathrm{H} & -2.39210500 & -2.08223200 & 2.02804300 \\ \mathrm{H} & 5.17568300 & 2.86591300 & 0.45128100 & \mathrm{C} & -1.63928400 & 2.23806700 & 0.38444200 \\ \mathrm{H} & 4.79080000 & 2.62501200 & -1.25159700 & \mathrm{H} & -2.43102300 & 2.95877300 & 0.14827500 \\ \mathrm{C} & 0.60418800 & -2.81972400 & -2.75114700 & \mathrm{C} & -0.42292400 & 2.47458700 & -0.54050200 \\ \mathrm{H} & & & & & & \\ \mathrm{H} & 1.52215800 & -2.28699200 & -3.01695000 & \mathrm{H} & -0.01132500 & 3.45750200 & -0.32145400 \\ & & & & -0.74226300 & 2.49743200 & -1.58783100\end{array}$

\section{$\mathrm{Ph}^{\text {Bpin }}$}

2a

$E=-721.369245$

$G=-721.079226$

O

$\mathrm{O}$

C

C

$\mathrm{H}$

$\mathrm{H}$

H

C

H

H

$\mathrm{H}$

C

$\mathrm{H}$

$\mathrm{H}$

H

C

$\mathrm{H}$

$\mathrm{H}$

$\mathrm{H}$

B

$\mathrm{H}$

C

\section{$\overbrace{\mathrm{Me}}^{\text {Bpin }}$}

$3 a$

$E=-721.365606$

$\mathrm{H}$

C

C

C

C

C

C

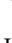

$\mathrm{H}$

$\mathrm{H}$

$\mathrm{H}$

$\mathrm{H}$

$\mathrm{H}$

C

$\mathrm{H}$

C

c

$\mathrm{H}$

$\mathrm{H}$

$\mathrm{O}$

$\mathrm{O}$

C

C

C

$$
G=-721.076639
$$

$0.81818500 \quad 3.00063100 \quad 0.48370200$

$3.89187700 \quad-1.30856700 \quad 0.63413900$

$3.14695900 \quad-1.65708600 \quad-0.49073300$

$2.21157600 \quad-0.77116800 \quad-1.01076300$

$1.99941800 \quad 0.48451000 \quad-0.42628000$

$2.74745600 \quad 0.81860700 \quad 0.70492800$

$3.68662200 \quad-0.06845500 \quad 1.22813000$

$4.62477300 \quad-1.99815200 \quad 1.04130300$

$3.29612100 \quad-2.62227200 \quad-0.96559400$

$1.62414500 \quad-1.05330400 \quad-1.88040100$

$2.60302800 \quad 1.78011700 \quad 1.18648000$

$\begin{array}{lll}4.25882500 & 0.21524200 & 2.10658600\end{array}$

$0.92629900 \quad 1.40228800 \quad-1.00249400$

$\begin{array}{lll}0.99670100 & 1.33339600 & -2.09485100\end{array}$

$1.03809300 \quad 2.86947500 \quad-0.57984400$

$0.31815700 \quad 3.47925000 \quad-1.13322600$

$2.03867200 \quad 3.26948600 \quad-0.77478900$

$\begin{array}{lll}-1.07962200 & 1.04478400 \quad 0.60515000\end{array}$

$-1.01919400 \quad-0.30125000 \quad-1.24248400$

$\begin{array}{lll}-2.28999400 & 0.26008100 & 0.64423400\end{array}$

$-1.94911400 \quad-0.92146400 \quad-0.32921500$

$\begin{array}{lll}-3.41859500 & 1.15400700 & 0.12872500\end{array}$ 


\begin{tabular}{|c|c|c|c|c|c|c|c|}
\hline $\mathrm{H}$ & -4.38917900 & 0.65516400 & 0.20056000 & $\mathrm{H}$ & 0.36527600 & 2.58578300 & 2.44954400 \\
\hline $\mathrm{H}$ & -3.45088500 & 2.06543400 & 0.73082600 & $\mathrm{H}$ & -0.25059800 & 4.10929800 & 1.84101400 \\
\hline $\mathrm{H}$ & -3.24884800 & 1.43965000 & -0.91317400 & $\mathrm{C}$ & -1.68768100 & 3.24775900 & -0.72616600 \\
\hline $\mathrm{C}$ & -2.56026900 & -0.15512400 & 2.08323000 & $\mathrm{H}$ & -1.56743900 & 3.02404200 & -1.79222600 \\
\hline $\mathrm{H}$ & -2.79599100 & 0.72744600 & 2.68359200 & $\mathrm{H}$ & -2.59740000 & 2.72143300 & -0.42041100 \\
\hline $\mathrm{H}$ & -3.41339300 & -0.83949700 & 2.13144900 & $\mathrm{C}$ & -3.78425100 & -0.01292400 & -0.95803100 \\
\hline $\mathrm{H}$ & -1.69132600 & -0.64444700 & 2.52586300 & $\mathrm{H}$ & -3.52103200 & 0.81034700 & -1.63193200 \\
\hline $\mathrm{C}$ & -1.19389200 & -2.06165000 & 0.35724600 & $\mathrm{H}$ & -4.61334600 & -0.55718000 & -1.42616800 \\
\hline $\mathrm{H}$ & -1.84366800 & -2.62698400 & 1.03111300 & $\mathrm{C}$ & -2.38174500 & -1.81353800 & -2.59742600 \\
\hline $\mathrm{H}$ & -0.81208600 & -2.74193700 & -0.40818200 & $\mathrm{H}$ & -3.42494200 & -2.06675200 & -2.81992000 \\
\hline $\mathrm{H}$ & -0.33814100 & -1.68340600 & 0.92398000 & $\mathrm{H}$ & -2.10414700 & -1.01074400 & -3.29022800 \\
\hline $\mathrm{C}$ & -3.13234000 & -1.46607700 & -1.11515200 & $\mathrm{Rh}$ & -0.17193900 & 0.06121300 & -0.44967400 \\
\hline $\mathrm{H}$ & -2.79993400 & -2.27960200 & -1.76512000 & $\mathrm{H}$ & -0.45162000 & 0.66862900 & -1.93080100 \\
\hline $\mathrm{H}$ & -3.89376600 & -1.86137800 & -0.43516200 & C & -1.84047400 & 4.75305700 & -0.50618100 \\
\hline $\mathrm{H}$ & -3.58651300 & -0.69679500 & -1.74173700 & $\mathrm{H}$ & -2.01956500 & 4.99424700 & 0.54603500 \\
\hline \multirow[t]{8}{*}{ B } & -0.42978800 & 0.73148400 & -0.55829000 & $\mathrm{H}$ & -0.94923200 & 5.30403600 & -0.82171000 \\
\hline & & & & $\mathrm{H}$ & -2.68782900 & 5.14286700 & -1.07914000 \\
\hline & & & & C & 1.29596500 & 3.63694800 & -1.91054600 \\
\hline & & & & $\mathrm{H}$ & 1.23150500 & 2.67871100 & -2.43138300 \\
\hline & & & & $\mathrm{H}$ & 0.50417300 & 4.27591700 & -2.31299900 \\
\hline & & & & $\mathrm{H}$ & 2.25494000 & 4.09665700 & -2.16784000 \\
\hline & & & & C & -1.79685800 & 2.77316500 & 2.52355300 \\
\hline & & & & $\mathrm{H}$ & -2.05653900 & 1.71398900 & 2.51046300 \\
\hline TS1 & & & & $\mathrm{H}$ & -1.78954600 & 3.10375100 & 3.56710100 \\
\hline$E=-1152.219319$ & $G=-11$ & 51.534098 & & $\mathrm{H}$ & -2.60019900 & 3.31238800 & 2.01190100 \\
\hline $\mathrm{C}$ & 1.15478800 & 3.46593600 & -0.39775200 & C & 2.92702200 & -1.28372100 & 3.16520300 \\
\hline $\mathrm{H}$ & 1.05310900 & 4.45145300 & 0.07265100 & $\mathrm{H}$ & 3.92346300 & -0.88268700 & 3.37362600 \\
\hline $\mathrm{H}$ & 2.05619300 & 3.00743600 & 0.02260900 & $\mathrm{H}$ & 2.36711900 & -1.27887200 & 4.10608800 \\
\hline C & -2.86903700 & -2.53230100 & 0.13307900 & $\mathrm{H}$ & 3.05543800 & -2.32554700 & 2.85611700 \\
\hline $\mathrm{H}$ & -2.91167800 & -2.18658000 & 1.17037100 & $\mathrm{C}$ & -1.82676600 & -0.74089700 & 3.28798000 \\
\hline $\mathrm{H}$ & -2.06386200 & -3.27541900 & 0.10219100 & $\mathrm{H}$ & -2.29691800 & -0.26900500 & 4.15621600 \\
\hline C & -0.34734500 & -0.37175300 & 3.20050700 & $\mathrm{H}$ & -2.36345700 & -0.41514300 & 2.39264600 \\
\hline $\mathrm{H}$ & -0.22106500 & 0.70816800 & 3.30758700 & $\mathrm{H}$ & -1.96639600 & -1.82151000 & 3.38832000 \\
\hline $\mathrm{H}$ & 0.19826800 & -0.83333300 & 4.03119000 & $\mathrm{C}$ & 1.02204100 & -3.53002300 & 0.72002200 \\
\hline $\mathrm{P}$ & -0.27239700 & 2.36245800 & 0.12011300 & $\mathrm{H}$ & 0.94056100 & -4.59926600 & 0.93914900 \\
\hline $\mathrm{P}$ & -2.25375000 & -1.08392700 & -0.88025000 & $\mathrm{H}$ & 0.53044000 & -3.33896600 & -0.23775300 \\
\hline $\mathrm{P}$ & 0.49242600 & -0.85295200 & 1.60573000 & $\mathrm{H}$ & 2.08239800 & -3.28715000 & 0.60644200 \\
\hline C & 0.37729200 & -2.70003700 & 1.82265200 & C & -4.19784000 & -3.17776100 & -0.25837900 \\
\hline $\mathrm{H}$ & -0.69141900 & -2.92781200 & 1.87440700 & $\mathrm{H}$ & -4.44340100 & -3.99956000 & 0.42197200 \\
\hline $\mathrm{H}$ & 0.80006900 & -2.95822000 & 2.80060400 & $\mathrm{H}$ & -5.02271100 & -2.46013900 & -0.21582200 \\
\hline $\mathrm{C}$ & 2.25348900 & -0.46027500 & 2.06847000 & $\mathrm{H}$ & -4.16607400 & -3.58959200 & -1.27162400 \\
\hline $\mathrm{H}$ & 2.84008500 & -0.52164500 & 1.15133400 & $\mathrm{C}$ & -4.21094900 & 0.54075400 & 0.39800800 \\
\hline $\mathrm{H}$ & 2.23714300 & 0.60370000 & 2.33767200 & $\mathrm{H}$ & -5.02060700 & 1.27003100 & 0.29451100 \\
\hline C & -0.44528000 & 3.03095400 & 1.86119100 & $\mathrm{H}$ & -4.56494200 & -0.25380800 & 1.06396400 \\
\hline
\end{tabular}




\begin{tabular}{|c|c|c|c|c|c|c|c|}
\hline $\mathrm{H}$ & -3.36860500 & 1.03563600 & 0.88976900 & $\mathrm{H}$ & 3.34431900 & -3.27073600 & -0.35610100 \\
\hline $\mathrm{C}$ & -1.48720700 & -3.03294700 & -2.81775700 & $\mathrm{C}$ & 3.31284600 & -0.61976500 & -2.02208200 \\
\hline $\mathrm{H}$ & -1.80508400 & -3.87744400 & -2.19749900 & $\mathrm{H}$ & 2.67167600 & -0.86865000 & -2.87524200 \\
\hline $\mathrm{H}$ & -1.51999000 & -3.36138100 & -3.86114600 & $\mathrm{H}$ & 3.40330600 & 0.47023500 & -2.03367900 \\
\hline $\mathrm{H}$ & -0.44546500 & -2.81536800 & -2.56620300 & $\mathrm{C}$ & 1.87657500 & 2.35127600 & 2.12206900 \\
\hline $\mathrm{C}$ & 5.56010900 & -0.16703700 & -0.45809400 & $\mathrm{H}$ & 2.73582500 & 1.67763000 & 2.20636900 \\
\hline $\mathrm{C}$ & 5.08258400 & -1.46879400 & -0.56806300 & $\mathrm{H}$ & 2.27017600 & 3.37054200 & 2.21057500 \\
\hline $\mathrm{C}$ & 3.77462500 & -1.70491200 & -0.98230500 & $\mathrm{C}$ & -0.06768400 & 3.48216800 & 0.30203800 \\
\hline $\mathrm{C}$ & 2.89910200 & -0.65008600 & -1.27816100 & $\mathrm{H}$ & -0.46272200 & 3.43012100 & -0.71891200 \\
\hline $\mathrm{C}$ & 3.40725900 & 0.65452200 & -1.18636300 & $\mathrm{H}$ & -0.90217700 & 3.20359000 & 0.95351500 \\
\hline $\mathrm{C}$ & 4.71359200 & 0.89289100 & -0.78474100 & $\mathrm{C}$ & -2.72867600 & -0.38344800 & 1.74665100 \\
\hline $\mathrm{H}$ & 6.57782300 & 0.02131400 & -0.13151500 & $\mathrm{H}$ & -3.30101900 & -0.19017400 & 0.83265200 \\
\hline $\mathrm{H}$ & 5.73087200 & -2.30825100 & -0.33378400 & $\mathrm{H}$ & -3.33300100 & -1.06342400 & 2.35882000 \\
\hline $\mathrm{H}$ & 3.41952100 & -2.72735700 & -1.08008400 & C & -2.06284500 & -2.75774300 & 0.49444400 \\
\hline $\mathrm{H}$ & 2.75070000 & 1.48805900 & -1.40285500 & $\mathrm{H}$ & -2.82131300 & -3.08590000 & 1.21489500 \\
\hline $\mathrm{H}$ & 5.07321500 & 1.91576200 & -0.71390500 & $\mathrm{H}$ & -2.60733900 & -2.40433500 & -0.38809300 \\
\hline $\mathrm{C}$ & 1.50769100 & -0.94779400 & -1.66429600 & $\mathrm{Rh}$ & 0.26158300 & 0.06205400 & -0.32410300 \\
\hline $\mathrm{H}$ & 1.28740200 & -2.00961200 & -1.69506800 & $\mathrm{H}$ & -1.05798000 & 0.95914800 & -0.52161200 \\
\hline C & 0.75454700 & -0.12642200 & -2.53626200 & C & 0.40487900 & 4.89866200 & 0.62102100 \\
\hline $\mathrm{H}$ & 0.10115900 & -0.62431900 & -3.24846900 & $\mathrm{H}$ & 0.77601800 & 4.98156000 & 1.64700200 \\
\hline \multirow[t]{8}{*}{$\mathrm{H}$} & 1.23926200 & 0.75213000 & -2.95444100 & $\mathrm{H}$ & 1.20592500 & 5.22089100 & -0.05138200 \\
\hline & & & & $\mathrm{H}$ & -0.41821200 & 5.61219300 & 0.51357500 \\
\hline & & & & C & 2.20421700 & 2.99845800 & -2.05162900 \\
\hline & & & & $\mathrm{H}$ & 1.75315000 & 2.08867900 & -2.46325800 \\
\hline & & & & $\mathrm{H}$ & 1.47756100 & 3.80970100 & -2.16613300 \\
\hline & & & & $\mathrm{H}$ & 3.08284000 & 3.25484400 & -2.65171100 \\
\hline & & & & C & 0.87713900 & 2.05650000 & 3.23623300 \\
\hline & 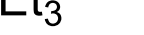 & & & $\mathrm{H}$ & 0.42957800 & 1.06872900 & 3.09083600 \\
\hline TS1' & & & & $\mathrm{H}$ & 1.35782200 & 2.08150500 & 4.21866800 \\
\hline$E=-1152.220302$ & $\mathrm{G}=-11$ & 51.536518 & & $\mathrm{H}$ & 0.06024700 & 2.78562000 & 3.24427600 \\
\hline C & 2.57407500 & 2.79138000 & -0.58364500 & C & 4.69019900 & -1.26481800 & -2.18214400 \\
\hline $\mathrm{H}$ & 2.92534900 & 3.72811000 & -0.13564100 & $\mathrm{H}$ & 5.12786000 & -1.00279800 & -3.15049500 \\
\hline $\mathrm{H}$ & 3.40227400 & 2.07844400 & -0.50167200 & $\mathrm{H}$ & 5.38578600 & -0.92735000 & -1.40840400 \\
\hline C & -0.49669900 & -1.93747900 & 2.77335300 & $\mathrm{H}$ & 4.64028700 & -2.35685100 & -2.12935400 \\
\hline $\mathrm{H}$ & -0.10054900 & -1.07510300 & 3.32130200 & C & 3.00983700 & -1.10233900 & 2.22962400 \\
\hline $\mathrm{H}$ & 0.38361500 & -2.52775100 & 2.49402100 & $\mathrm{H}$ & 3.71312300 & -0.81079900 & 3.01588900 \\
\hline $\mathrm{C}$ & 3.55825000 & -0.76276200 & 0.84522000 & $\mathrm{H}$ & 2.05966100 & -0.58796100 & 2.39852700 \\
\hline $\mathrm{H}$ & 3.84173900 & 0.29523400 & 0.80191900 & $\mathrm{H}$ & 2.82744700 & -2.17723500 & 2.33031400 \\
\hline $\mathrm{H}$ & 4.46529400 & -1.34190100 & 0.64016500 & C & 1.84711700 & -3.47859600 & -1.90820300 \\
\hline $\mathrm{P}$ & 1.16432400 & 2.08113800 & 0.41725800 & $\mathrm{H}$ & 1.67599900 & -4.55646700 & -1.83091400 \\
\hline $\mathrm{P}$ & -1.17944800 & -1.25360900 & 1.16997100 & $\mathrm{H}$ & 0.91221600 & -3.00834400 & -2.22446300 \\
\hline $\mathrm{P}$ & 2.30807500 & -1.04204800 & -0.50649600 & $\mathrm{H}$ & 2.58174200 & -3.31622800 & -2.70282800 \\
\hline C & 2.33339000 & -2.90659800 & -0.57518400 & C & -1.41780400 & -2.75677800 & 3.67586500 \\
\hline $\mathrm{H}$ & 1.68563600 & -3.24668300 & 0.23870500 & $\mathrm{H}$ & -0.88961000 & -3.07403900 & 4.58094500 \\
\hline
\end{tabular}




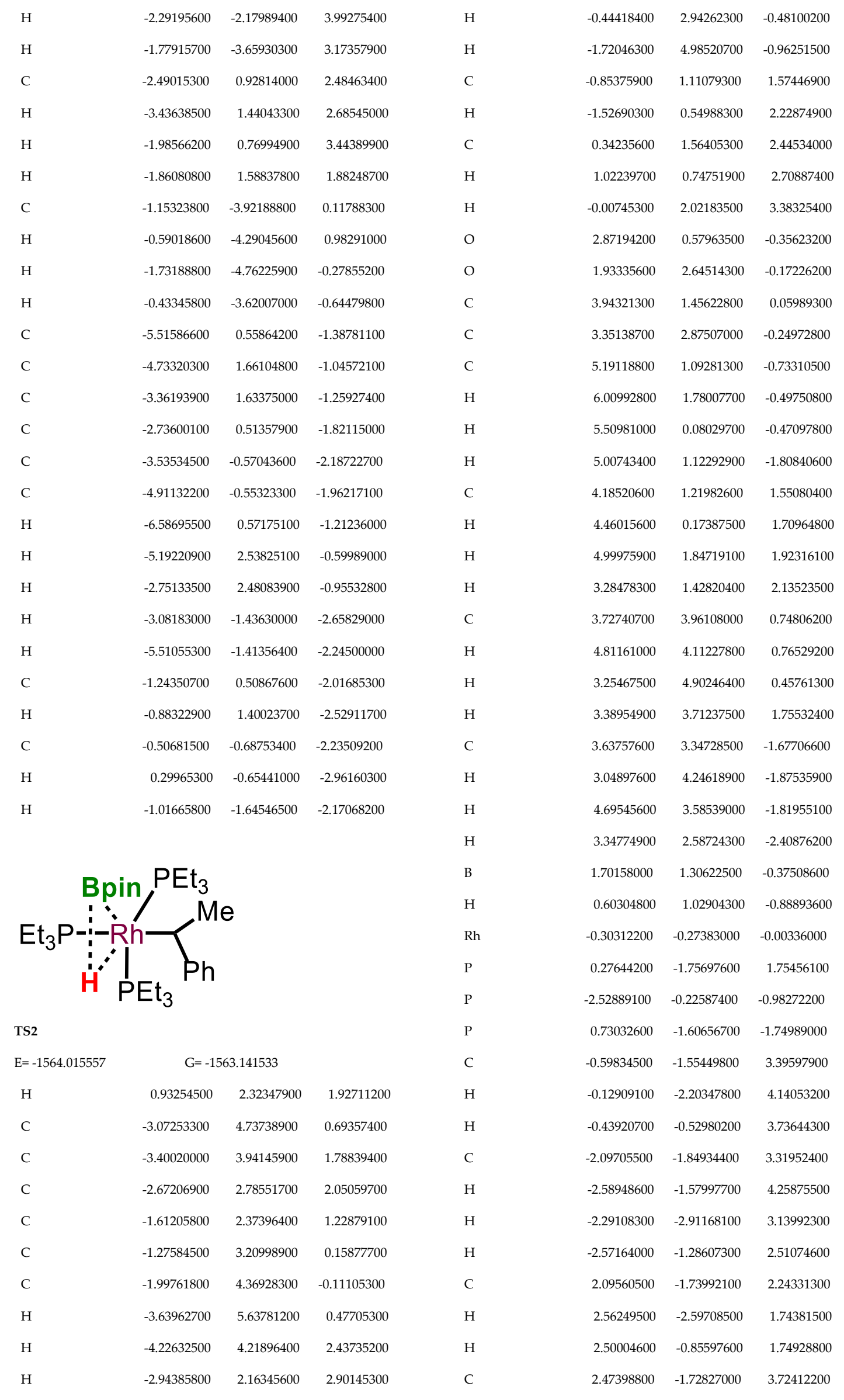




\begin{tabular}{|c|c|c|c|c|c|c|c|}
\hline $\mathrm{H}$ & 2.03001200 & -0.87324400 & 4.24327500 & $\mathrm{H}$ & 1.94701000 & -3.26299800 & -0.45690900 \\
\hline $\mathrm{H}$ & 3.56073900 & -1.65092900 & 3.83713700 & $\mathrm{C}$ & 3.05719700 & -3.29948500 & -2.29930300 \\
\hline $\mathrm{H}$ & 2.15150600 & -2.63576600 & 4.24286000 & $\mathrm{H}$ & 2.44654100 & -4.03144200 & -2.83769300 \\
\hline C & -0.00306700 & -3.59579200 & 1.52312300 & $\mathrm{H}$ & 3.88609600 & -3.84398600 & -1.83593500 \\
\hline $\mathrm{H}$ & -1.07657100 & -3.73349300 & 1.36584000 & $\mathrm{H}$ & 3.49063900 & -2.61773200 & -3.03560500 \\
\hline $\mathrm{H}$ & 0.46082700 & -3.87262700 & 0.57460900 & $\mathrm{C}$ & -2.79787700 & 0.43348100 & -2.71094700 \\
\hline C & 0.48277600 & -4.53792100 & 2.62471200 & $\mathrm{H}$ & -3.81393000 & 0.19046300 & -3.04436500 \\
\hline $\mathrm{H}$ & 0.27850100 & -5.57905300 & 2.35467000 & $\mathrm{H}$ & -2.11690200 & -0.12921300 & -3.35767100 \\
\hline $\mathrm{H}$ & -0.01732100 & -4.34545800 & 3.57829100 & & & & \\
\hline $\mathrm{H}$ & 1.56095800 & -4.44822200 & 2.78779300 & & & & \\
\hline C & -3.90414900 & 0.65166900 & -0.07703100 & & & & \\
\hline $\mathrm{H}$ & -3.80289700 & 0.38410100 & 0.98025300 & & & & \\
\hline $\mathrm{H}$ & -3.66173900 & 1.71476600 & -0.12364600 & & & & \\
\hline $\mathrm{C}$ & -5.34133000 & 0.43527900 & -0.54847300 & & & & \\
\hline $\mathrm{H}$ & -6.01726700 & 1.08497800 & 0.01634000 & TS2' & & & \\
\hline $\mathrm{H}$ & -5.68029600 & -0.59459500 & -0.40164800 & $E=-1564.026319$ & $G=-15$ & 63.153692 & \\
\hline $\mathrm{H}$ & -5.46637300 & 0.67963000 & -1.60823800 & $\mathrm{H}$ & -2.27951900 & -0.55619000 & -1.38497200 \\
\hline $\mathrm{C}$ & -3.33780900 & -1.88986900 & -1.24770000 & $\mathrm{C}$ & -6.15987200 & -2.48031100 & 0.65041700 \\
\hline $\mathrm{H}$ & -4.29095300 & -1.74167300 & -1.76763100 & $\mathrm{C}$ & -5.31872000 & -3.47146600 & 0.15451800 \\
\hline $\mathrm{H}$ & -2.70176500 & -2.46057100 & -1.92760700 & $\mathrm{C}$ & -4.04824500 & -3.14151300 & -0.31326900 \\
\hline C & -3.55354000 & -2.67161400 & 0.04277400 & $\mathrm{C}$ & -3.59525600 & -1.82156800 & -0.29459400 \\
\hline $\mathrm{H}$ & -2.60777800 & -2.78112700 & 0.57671300 & C & -4.44997900 & -0.83498900 & 0.21109500 \\
\hline $\mathrm{H}$ & -3.96065000 & -3.66800000 & -0.15646500 & $\mathrm{C}$ & -5.71906800 & -1.15771000 & 0.67733500 \\
\hline $\mathrm{H}$ & -4.24672700 & -2.15473200 & 0.71462400 & $\mathrm{H}$ & -7.15115500 & -2.73412100 & 1.01378100 \\
\hline C & -2.54337800 & 1.93186600 & -2.85648400 & $\mathrm{H}$ & -5.65279500 & -4.50486800 & 0.12829500 \\
\hline $\mathrm{H}$ & -2.56132500 & 2.23103700 & -3.90928700 & $\mathrm{H}$ & -3.39669400 & -3.92055500 & -0.70368700 \\
\hline $\mathrm{H}$ & -1.57422200 & 2.21103900 & -2.43485000 & $\mathrm{H}$ & -4.09266200 & 0.19194600 & 0.23844100 \\
\hline $\mathrm{H}$ & -3.29856600 & 2.51874100 & -2.32545200 & $\mathrm{H}$ & -6.37020400 & -0.37751900 & 1.06219100 \\
\hline $\mathrm{C}$ & -0.16344600 & -2.86586100 & -2.79648300 & $\mathrm{C}$ & -2.20677400 & -1.44852300 & -0.75693700 \\
\hline $\mathrm{H}$ & -1.03621000 & -2.35784600 & -3.22067400 & $\mathrm{H}$ & -1.81344000 & -2.23962300 & -1.40268200 \\
\hline $\mathrm{H}$ & 0.47623900 & -3.13927600 & -3.64344000 & $\mathrm{C}$ & -1.26591200 & -1.17757400 & 0.43118600 \\
\hline $\mathrm{C}$ & -0.59159700 & -4.12035700 & -2.03705000 & $\mathrm{H}$ & -1.22071300 & -2.08980200 & 1.03990600 \\
\hline $\mathrm{H}$ & 0.27930400 & -4.69821100 & -1.70987700 & $\mathrm{H}$ & -1.77833800 & -0.42893300 & 1.05048400 \\
\hline $\mathrm{H}$ & -1.20196100 & -4.77194700 & -2.66964000 & $\mathrm{O}$ & -2.09915700 & 1.79252700 & -0.34859800 \\
\hline $\mathrm{H}$ & -1.17570400 & -3.87102400 & -1.14703300 & $\mathrm{O}$ & -0.57970600 & 2.90480000 & -1.63354900 \\
\hline $\mathrm{C}$ & 1.51038400 & -0.59321000 & -3.11418100 & $\mathrm{C}$ & -2.69109800 & 3.08036500 & -0.60997400 \\
\hline $\mathrm{H}$ & 2.32636700 & -0.04035600 & -2.63879200 & $\mathrm{C}$ & -1.46812400 & 3.92471000 & -1.12762100 \\
\hline $\mathrm{H}$ & 1.96853800 & -1.28513100 & -3.82866500 & $\mathrm{C}$ & -3.32589700 & 3.59459700 & 0.67468800 \\
\hline C & 0.58434700 & 0.36318300 & -3.85799400 & $\mathrm{H}$ & -3.75170800 & 4.59133100 & 0.52169200 \\
\hline $\mathrm{H}$ & -0.18802700 & -0.17616600 & -4.41527700 & $\mathrm{H}$ & -4.13247500 & 2.92136300 & 0.97681300 \\
\hline $\mathrm{H}$ & 1.15084600 & 0.95880900 & -4.58089000 & $\mathrm{H}$ & -2.60309900 & 3.64341500 & 1.49099600 \\
\hline $\mathrm{H}$ & 0.08613900 & 1.04878300 & -3.16966800 & $\mathrm{C}$ & -3.76723100 & 2.85327400 & -1.67353600 \\
\hline C & 2.25512400 & -2.55596500 & -1.23021600 & $\mathrm{H}$ & -4.48252500 & 2.11515900 & -1.30163400 \\
\hline $\mathrm{H}$ & 2.87538400 & -1.80626600 & -0.72933800 & $\mathrm{H}$ & -4.30655300 & 3.77590400 & -1.90521900 \\
\hline
\end{tabular}




\begin{tabular}{|c|c|c|c|c|c|c|c|}
\hline $\mathrm{H}$ & -3.33221800 & 2.45909900 & -2.59616400 & $\mathrm{H}$ & 3.20341100 & -3.98117500 & -0.97916800 \\
\hline C & -1.79341500 & 4.88634100 & -2.26338600 & $\mathrm{H}$ & 3.63427800 & -2.28268000 & -0.87149300 \\
\hline $\mathrm{H}$ & -2.52821700 & 5.62924900 & -1.93767500 & $\mathrm{C}$ & 3.15918500 & -3.17690500 & 1.03398700 \\
\hline $\mathrm{H}$ & -0.88573500 & 5.41460100 & -2.56662600 & $\mathrm{H}$ & 2.79632000 & -2.28027700 & 1.54454800 \\
\hline $\mathrm{H}$ & -2.18713800 & 4.36282900 & -3.13548800 & $\mathrm{H}$ & 4.21685200 & -3.31663300 & 1.27822400 \\
\hline C & -0.72436500 & 4.67121000 & -0.02069200 & $\mathrm{H}$ & 2.60905700 & -4.03217000 & 1.43935300 \\
\hline $\mathrm{H}$ & 0.19856900 & 5.08772800 & -0.43377100 & $\mathrm{C}$ & -0.01959900 & -2.80721800 & -3.48768800 \\
\hline $\mathrm{H}$ & -1.32507700 & 5.49391000 & 0.37704300 & $\mathrm{H}$ & 0.10081900 & -2.95600900 & -4.56502100 \\
\hline $\mathrm{H}$ & -0.45844100 & 4.00258600 & 0.80001300 & $\mathrm{H}$ & -0.46245900 & -1.81919600 & -3.32557900 \\
\hline B & -0.95100800 & 1.69892100 & -1.09164700 & $\mathrm{H}$ & -0.74096600 & -3.54961200 & -3.12904500 \\
\hline $\mathrm{H}$ & -0.41856600 & 0.66412000 & -1.43411100 & $\mathrm{C}$ & 4.24580300 & 0.40201500 & -1.00717400 \\
\hline $\mathrm{Rh}$ & 0.63309000 & -0.39326900 & -0.07586200 & $\mathrm{H}$ & 4.25991900 & -0.47443200 & -1.66451300 \\
\hline $\mathrm{P}$ & 0.68335100 & 0.29362500 & 2.15853400 & $\mathrm{H}$ & 4.82260500 & 1.17899100 & -1.52193900 \\
\hline $\mathrm{P}$ & 1.22927500 & -2.50619400 & -0.94722900 & $\mathrm{C}$ & 4.88444500 & 0.06997900 & 0.33871500 \\
\hline $\mathrm{P}$ & 2.46462400 & 0.94272600 & -0.89634600 & $\mathrm{H}$ & 4.96220900 & 0.96278400 & 0.96876600 \\
\hline C & -0.15941000 & -0.83521000 & 3.37951500 & $\mathrm{H}$ & 5.89429200 & -0.33045000 & 0.20713400 \\
\hline $\mathrm{H}$ & -0.15395900 & -0.36631400 & 4.36795500 & $\mathrm{H}$ & 4.29424500 & -0.66975900 & 0.88560000 \\
\hline $\mathrm{H}$ & -1.20562300 & -0.91174400 & 3.07138400 & $\mathrm{C}$ & 2.23234700 & 1.54286800 & -2.64910000 \\
\hline C & 0.46141400 & -2.22781400 & 3.45661100 & $\mathrm{H}$ & 1.38602200 & 2.23522400 & -2.62490700 \\
\hline $\mathrm{H}$ & -0.15163600 & -2.89052500 & 4.07467300 & $\mathrm{H}$ & 3.11716000 & 2.12368500 & -2.93344500 \\
\hline $\mathrm{H}$ & 1.46452100 & -2.20094200 & 3.89494800 & $\mathrm{C}$ & 1.98127900 & 0.44831600 & -3.68027200 \\
\hline $\mathrm{H}$ & 0.54548800 & -2.66712000 & 2.46008000 & $\mathrm{H}$ & 2.82337300 & -0.24931000 & -3.74315100 \\
\hline C & -0.14144000 & 1.92971100 & 2.55205400 & $\mathrm{H}$ & 1.83980500 & 0.88192000 & -4.67517600 \\
\hline $\mathrm{H}$ & 0.60888200 & 2.69691500 & 2.32121000 & $\mathrm{H}$ & 1.08547800 & -0.12264800 & -3.42516900 \\
\hline $\mathrm{H}$ & -0.91611300 & 2.01231000 & 1.78387400 & $\mathrm{C}$ & 2.61263900 & 2.61034900 & -0.06185900 \\
\hline C & -0.77058000 & 2.18264000 & 3.92365000 & $\mathrm{H}$ & 1.61531600 & 3.04750400 & -0.17613100 \\
\hline $\mathrm{H}$ & -1.58999100 & 1.48435100 & 4.11474500 & $\mathrm{H}$ & 2.74284500 & 2.43098200 & 1.00832300 \\
\hline $\mathrm{H}$ & -1.18898000 & 3.19444500 & 3.96628300 & $\mathrm{C}$ & 3.67979100 & 3.58876300 & -0.55208900 \\
\hline $\mathrm{H}$ & -0.05388000 & 2.08755200 & 4.74292500 & $\mathrm{H}$ & 4.68973200 & 3.18857600 & -0.41820600 \\
\hline C & 2.36438000 & 0.42930100 & 2.96669600 & $\mathrm{H}$ & 3.62339000 & 4.52643900 & 0.01027800 \\
\hline $\mathrm{H}$ & 2.78827400 & -0.57902500 & 2.90869300 & $\mathrm{H}$ & 3.55289600 & 3.83503100 & -1.60962900 \\
\hline $\mathrm{H}$ & 2.99085900 & 1.03582900 & 2.30958300 & $\mathrm{C}$ & 1.31708500 & -2.93948800 & -2.76182700 \\
\hline C & 2.46183300 & 0.95405900 & 4.39856300 & $\mathrm{H}$ & 1.71917200 & -3.95185700 & -2.88245400 \\
\hline $\mathrm{H}$ & 3.49264900 & 0.88455100 & 4.76108700 & $\mathrm{H}$ & 2.05275000 & -2.25866700 & -3.19957700 \\
\hline $\mathrm{H}$ & 1.83371900 & 0.38241500 & 5.08833900 & & & & \\
\hline $\mathrm{H}$ & 2.16323500 & 2.00439100 & 4.46158500 & & & & \\
\hline C & 0.25080800 & -3.95863900 & -0.28926200 & & & & \\
\hline $\mathrm{H}$ & 0.31648800 & -3.88719500 & 0.80060700 & & & & \\
\hline $\mathrm{H}$ & -0.80052600 & -3.77519600 & -0.52370400 & & & & \\
\hline C & 0.64965400 & -5.36110600 & -0.74604400 & & & & \\
\hline $\mathrm{H}$ & 0.04310200 & -6.11327200 & -0.23166000 & & & & \\
\hline $\mathrm{H}$ & 1.69900800 & -5.58289500 & -0.52805700 & TS3 & & & \\
\hline $\mathrm{H}$ & 0.49572000 & -5.49869000 & -1.82050800 & $E=-1564.020401$ & $G=-15$ & 63.144793 & \\
\hline $\mathrm{C}$ & 2.95550000 & -3.04116500 & -0.47278100 & $\mathrm{H}$ & 100 & 1.87194400 & 1800 \\
\hline
\end{tabular}


$\begin{array}{lll}0.04841900 & 2.87260900 & -1.08983900\end{array}$

$\begin{array}{lll}-0.48375900 & 3.53700000 & 0.01824400\end{array}$

$\begin{array}{lll}-0.17963100 & 4.87235000 & 0.27561700\end{array}$

$0.90763700 \quad 6.62202900 \quad-0.36103800$

$1.87765900 \quad 5.47660900 \quad-2.34669800$

$1.33291400 \quad 3.11770900 \quad-2.79923800$

$\begin{array}{lll}-1.13044500 & 2.98712400 \quad 0.69031600\end{array}$

$\begin{array}{lll}-0.60464200 & 5.35561600 \quad 1.15126900\end{array}$

$-0.23744800 \quad 1.42265100 \quad-1.44909200$

$\begin{array}{lll}0.72984700 & 1.03964100 & -1.79790500\end{array}$

$\begin{array}{lll}-1.11443400 & 1.36974300 & -2.72040900\end{array}$

$\begin{array}{lll}-1.34605900 & 0.34319400 & -3.01175900\end{array}$

$\begin{array}{lll}-0.60685600 & 1.86864200 & -3.55750800\end{array}$

$-2.60473200 \quad-0.18461600 \quad-1.00787000$

$\begin{array}{lll}-2.32797700 & 1.25056600 & 0.73131100\end{array}$

$-3.91933000$

$-3.69861800$

0.11166400

1.37485600

$-0.54607600$

$-4.42213900-1.10945700$

0.36136700

$-5.45979900 \quad-0.98769900$

0.23134300

$-4.36422800 \quad-1.98864200$

0.55621400

$\begin{array}{lll}-3.80482400 & -1.29935600 & 1.11322900\end{array}$

$-4.82124800 \quad 0.34347100 \quad-1.75429700$

$-4.90238000 \quad-0.58158800 \quad-2.33254900$

$\begin{array}{lll}-5.82824800 & 0.63896400 & -1.44130100\end{array}$

$\begin{array}{lll}-4.41691800 & 1.11585400 & -2.41064800\end{array}$

$\begin{array}{lll}-3.88238700 & 2.70694900 & -0.37395800\end{array}$

$\begin{array}{lll}-4.92658600 & 2.87034800 & -0.65697800\end{array}$

$\begin{array}{lll}-3.57279100 & 3.52102200 & 0.28660600\end{array}$

$\begin{array}{lll}-3.26102300 & 2.76419700 \quad-1.26920900\end{array}$

$-4.54706800 \quad 1.40153000 \quad 1.63076600$

$\begin{array}{lll}-4.29500200 & 2.29036400 & 2.21571200\end{array}$

$\begin{array}{lll}-5.61367300 & 1.44599100 & 1.38670100\end{array}$

$\begin{array}{lll}-4.37151100 & 0.52664100 & 2.25899300\end{array}$

$\begin{array}{lll}-1.63063100 & 0.43950700 & -0.19798200\end{array}$

$\begin{array}{lll}0.05314300 & 0.88753900 & 1.21295200\end{array}$

$\begin{array}{lll}0.26426900 & -0.21310200 & 0.14882100\end{array}$

$0.43518700 \quad-1.93434200 \quad-1.61107900$

$\begin{array}{lll}2.65733300 & 0.45416000 & 0.74484900\end{array}$

$\begin{array}{lll}-0.34851400 & -1.55559100 & 1.90200000\end{array}$

$\begin{array}{lll}1.14959200 & -1.35328100 & -3.23174900\end{array}$

$1.03441000 \quad-2.13210400 \quad-3.99081400$

\begin{tabular}{|c|c|c|}
\hline 0.54289000 & -0.50461800 & -3.55780800 \\
\hline 2.61382100 & -0.93548900 & -3.12840100 \\
\hline 2.95190900 & -0.46967000 & -4.05863100 \\
\hline 3.26518200 & -1.79293700 & -2.93185400 \\
\hline 2.75756900 & -0.21851700 & -2.31629000 \\
\hline-1.18682600 & -2.71993400 & -2.12198100 \\
\hline-1.27690300 & -3.64149900 & -1.53433200 \\
\hline-1.95506200 & -2.03299200 & -1.76151900 \\
\hline-1.42367400 & -3.01634700 & -3.60370200 \\
\hline-1.40511800 & -2.09849600 & -4.19879700 \\
\hline-2.41042300 & -3.47094300 & -3.73803500 \\
\hline-0.68378500 & -3.70350300 & -4.02509400 \\
\hline 1.48612500 & -3.43471300 & -1.27111400 \\
\hline 2.46805400 & -3.06006300 & -0.96900500 \\
\hline 1.06569700 & -3.89714000 & -0.37300400 \\
\hline 1.62893400 & -4.48038100 & -2.37546300 \\
\hline 2.28716000 & -5.29236300 & -2.05070800 \\
\hline 2.05878900 & -4.05451100 & -3.28703800 \\
\hline 0.66293900 & -4.92333000 & -2.63395100 \\
\hline 3.42992600 & 1.79354000 & -0.29709400 \\
\hline 3.53762200 & 1.39448700 & -1.31155200 \\
\hline 2.66744000 & 2.57436000 & -0.37425600 \\
\hline 4.75781100 & 2.38586900 & 0.17383300 \\
\hline 5.06931100 & 3.19592900 & -0.49291900 \\
\hline 5.55922500 & 1.64065300 & 0.18231700 \\
\hline 4.68012700 & 2.80389200 & 1.18197900 \\
\hline 4.12857400 & -0.65526800 & 1.06959300 \\
\hline 4.89628200 & -0.07415200 & 1.59342400 \\
\hline 3.79342700 & -1.42668300 & 1.77134700 \\
\hline 4.73396200 & -1.29922800 & -0.17454300 \\
\hline 4.00384700 & -1.91627400 & -0.70211000 \\
\hline 5.58291700 & -1.93706000 & 0.08958200 \\
\hline 5.09119300 & -0.54440700 & -0.88273700 \\
\hline 1.92284300 & 2.65037600 & 2.44580700 \\
\hline 1.83041600 & 3.01046600 & 3.47521600 \\
\hline 0.92308600 & 2.59338700 & 2.01164900 \\
\hline 2.47712900 & 3.40292400 & 1.87714100 \\
\hline 0.93436100 & -2.30347000 & 3.03177300 \\
\hline 1.55702800 & -1.47470500 & 3.38445200 \\
\hline 0.43875900 & -2.71948400 & 3.91670600 \\
\hline 1.81154500 & -3.36052400 & 2.36512900 \\
\hline 1.24052700 & -4.26355400 & 2.12535500 \\
\hline 2.63371100 & -3.65892200 & 3.02273000 \\
\hline & 200 & 1.4 \\
\hline
\end{tabular}




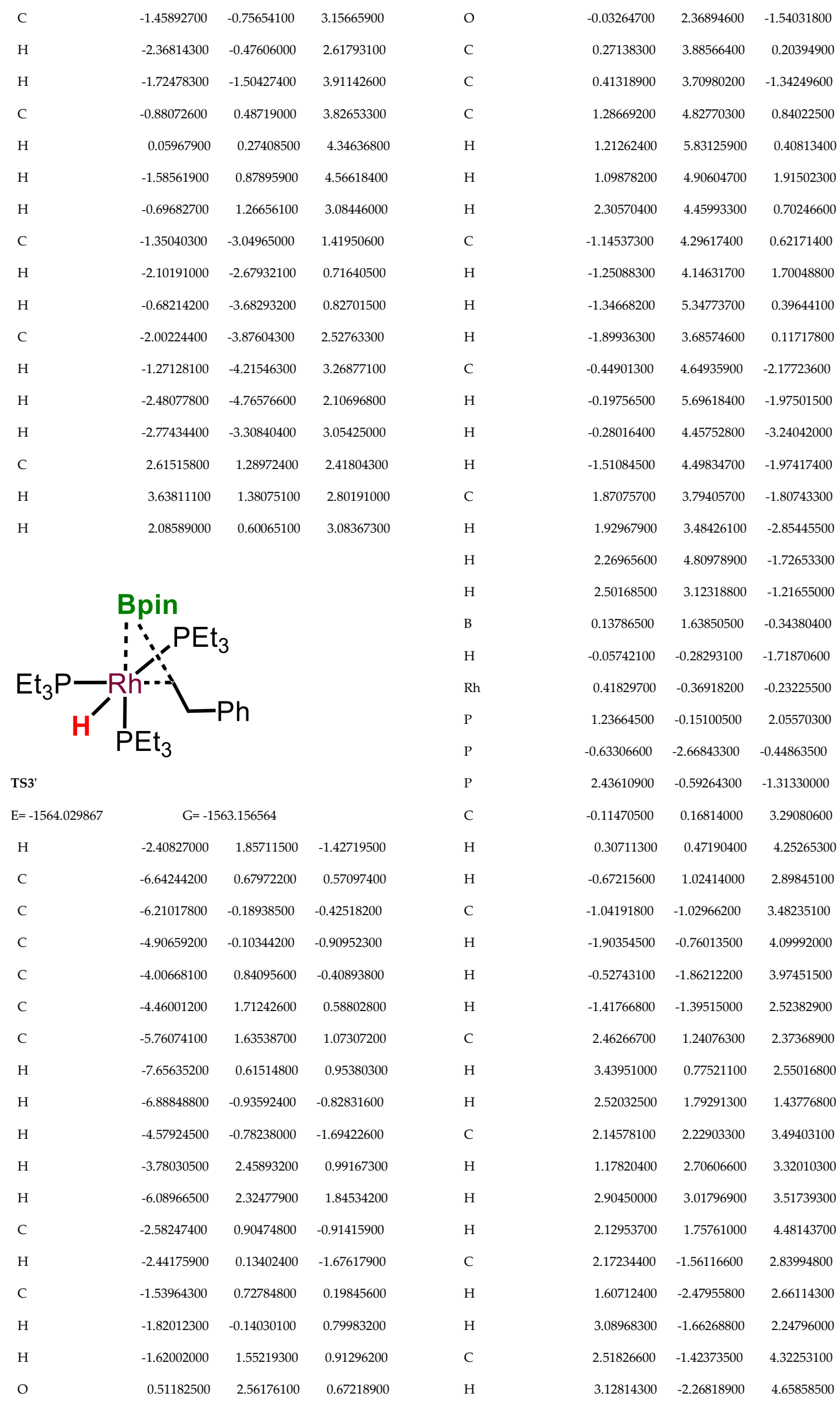




\begin{tabular}{|c|c|c|c|c|c|c|c|}
\hline $\mathrm{H}$ & 1.61893100 & -1.39928100 & 4.94523600 & & & & \\
\hline $\mathrm{H}$ & 3.08370700 & -0.50794300 & 4.52101200 & & & & \\
\hline$C$ & -2.42966500 & -2.71170700 & 0.04889600 & & & & \\
\hline $\mathrm{H}$ & -2.46082700 & -2.54456400 & 1.13081600 & & & & \\
\hline $\mathrm{H}$ & -2.89086600 & -1.82259900 & -0.38910400 & & & & \\
\hline $\mathrm{C}$ & -3.24435000 & -3.95515400 & -0.30556400 & & & & \\
\hline $\mathrm{H}$ & -4.27743500 & -3.83830300 & 0.03625200 & B11 & & & \\
\hline $\mathrm{H}$ & -2.84038100 & -4.85797700 & 0.16293200 & $E=-1638.966001$ & $\mathrm{G}=$ & 1638.358787 & \\
\hline $\mathrm{H}$ & -3.27384300 & -4.12951400 & -1.38500200 & $\mathrm{P}$ & -2.13452200 & 0.92287800 & 0.41382100 \\
\hline $\mathrm{C}$ & -0.03609900 & -4.29258800 & 0.26332400 & $\mathrm{P}$ & 2.25472400 & 0.97011000 & -0.35199200 \\
\hline $\mathrm{H}$ & -0.50116200 & -5.12597600 & -0.27614100 & $\mathrm{P}$ & -0.16243400 & -1.57302700 & -1.06880400 \\
\hline $\mathrm{H}$ & 1.03836900 & -4.35011900 & 0.06205500 & $\mathrm{C}$ & -3.08620800 & 2.45463700 & 0.01452600 \\
\hline $\mathrm{C}$ & -0.30357000 & -4.43608000 & 1.76227300 & $\mathrm{C}$ & -4.32961900 & 2.71054300 & 0.60447900 \\
\hline $\mathrm{H}$ & -0.00896200 & -3.53818000 & 2.31107400 & $\mathrm{H}$ & -4.75953000 & 1.98899800 & 1.29424400 \\
\hline $\mathrm{H}$ & 0.24416800 & -5.28474100 & 2.18214700 & $\mathrm{C}$ & -5.03020400 & 3.87205800 & 0.30229300 \\
\hline $\mathrm{H}$ & -1.36710300 & -4.59724800 & 1.96236900 & $\mathrm{H}$ & -5.99201600 & 4.06053100 & 0.76937600 \\
\hline C & -1.68412300 & -2.29168800 & -3.09055300 & C & -4.49933600 & 4.78937900 & -0.60346200 \\
\hline $\mathrm{H}$ & -1.61066900 & -2.56138700 & -4.14849500 & $\mathrm{H}$ & -5.04642700 & 5.69663800 & -0.84164800 \\
\hline $\mathrm{H}$ & -1.42613800 & -1.23410700 & -2.99137800 & $\mathrm{C}$ & -3.26920900 & 4.53909900 & -1.20234400 \\
\hline $\mathrm{H}$ & -2.73041800 & -2.40873700 & -2.79104200 & $\mathrm{H}$ & -2.85486300 & 5.24911500 & -1.91167400 \\
\hline $\mathrm{C}$ & 3.10994300 & -2.30028200 & -1.62767100 & $\mathrm{C}$ & -2.56441400 & 3.37688300 & -0.89338800 \\
\hline $\mathrm{H}$ & 2.30803500 & -2.86163300 & -2.11698400 & $\mathrm{H}$ & -1.59726000 & 3.17491600 & -1.34498800 \\
\hline $\mathrm{H}$ & 3.94173300 & -2.25226600 & -2.34038700 & C & -3.45172700 & -0.35865300 & 0.18294800 \\
\hline C & 3.52639800 & -3.01453500 & -0.34390100 & $\mathrm{C}$ & -3.48318500 & -1.50381700 & 0.98466600 \\
\hline $\mathrm{H}$ & 4.41487300 & -2.55666200 & 0.10369800 & $\mathrm{H}$ & -2.78554000 & -1.61898900 & 1.80850300 \\
\hline $\mathrm{H}$ & 3.75728900 & -4.06742000 & -0.53199900 & C & -4.38525300 & -2.53161000 & 0.72109500 \\
\hline $\mathrm{H}$ & 2.72157800 & -2.96695400 & 0.39576800 & $\mathrm{H}$ & -4.38050900 & -3.41723000 & 1.34901300 \\
\hline C & 2.44467700 & 0.13849700 & -3.01996400 & C & -5.27286200 & -2.42943700 & -0.34495800 \\
\hline $\mathrm{H}$ & 1.98953400 & 1.12629500 & -2.90283200 & $\mathrm{H}$ & -5.97615400 & -3.23047600 & -0.55076900 \\
\hline $\mathrm{H}$ & 3.47694300 & 0.27075600 & -3.36042700 & C & -5.25571500 & -1.28970500 & -1.14690500 \\
\hline $\mathrm{C}$ & 1.65901300 & -0.66768600 & -4.05246600 & $\mathrm{H}$ & -5.94782800 & -1.19855600 & -1.97854400 \\
\hline $\mathrm{H}$ & 2.11245100 & -1.64762400 & -4.23458000 & C & -4.35121700 & -0.26591000 & -0.88645500 \\
\hline $\mathrm{H}$ & 1.62752600 & -0.13613500 & -5.00791900 & $\mathrm{H}$ & -4.34277800 & 0.61584000 & -1.52094500 \\
\hline $\mathrm{H}$ & 0.62930400 & -0.82038100 & -3.72098200 & $\mathrm{C}$ & -2.03362600 & 1.01475300 & 2.25945500 \\
\hline $\mathrm{C}$ & 3.91148600 & 0.24200000 & -0.54217300 & $\mathrm{H}$ & -1.45274800 & 1.90526700 & 2.50734100 \\
\hline $\mathrm{H}$ & 3.63612600 & 1.29729600 & -0.44698400 & $\mathrm{H}$ & -1.49372600 & 0.14180700 & 2.63397800 \\
\hline $\mathrm{H}$ & 3.97197900 & -0.14033400 & 0.48147500 & $\mathrm{H}$ & -3.01861700 & 1.06832600 & 2.73071400 \\
\hline C & 5.27089200 & 0.10208400 & -1.22687200 & C & 2.74334800 & 0.14807200 & 1.22731100 \\
\hline $\mathrm{H}$ & 5.57813500 & -0.94446900 & -1.31361300 & C & 2.22615600 & 0.66767400 & 2.42253100 \\
\hline $\mathrm{H}$ & 6.04045800 & 0.62265200 & -0.64848400 & $\mathrm{H}$ & 1.64814800 & 1.58714200 & 2.39283500 \\
\hline $\mathrm{H}$ & 5.27203400 & 0.53454600 & -2.23091200 & C & 2.42308700 & 0.00480300 & 3.62707600 \\
\hline C & -0.75402800 & -3.16494200 & -2.24865800 & $\mathrm{H}$ & 2.01595100 & 0.42080000 & 4.54372200 \\
\hline $\mathrm{H}$ & -1.06306700 & -4.21452000 & -2.31724800 & C & 3.13370900 & -1.19587700 & 3.65680700 \\
\hline $\mathrm{H}$ & 0.26065400 & -3.10503400 & -2.65571100 & $\mathrm{H}$ & 3.27965900 & -1.71967200 & 4.59654100 \\
\hline
\end{tabular}




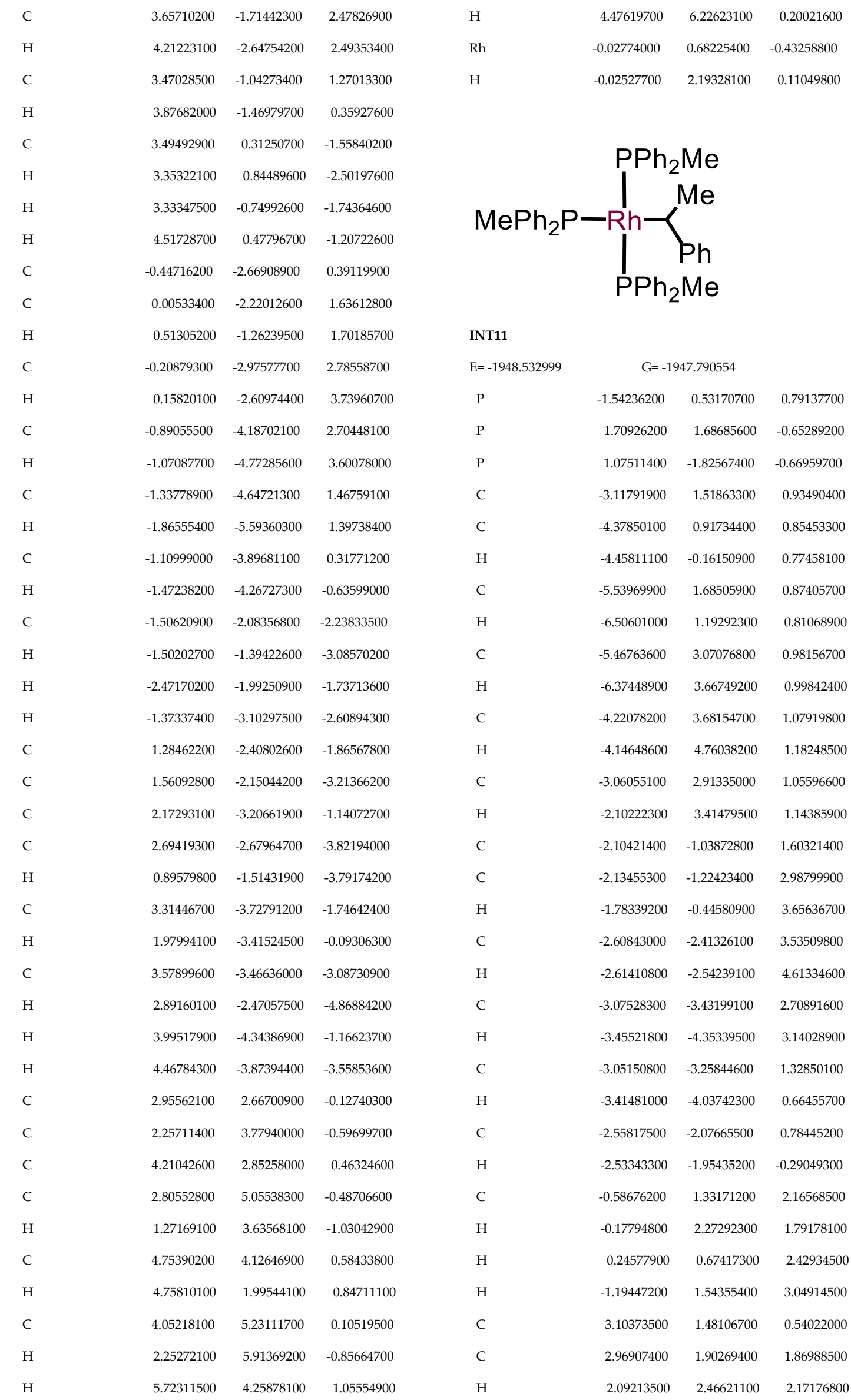




\begin{tabular}{|c|c|c|c|c|c|c|c|}
\hline $\mathrm{C}$ & 3.94246700 & 1.60159600 & 2.81545500 & $\mathrm{H}$ & 3.14950800 & 4.18886100 & 0.21532700 \\
\hline $\mathrm{H}$ & 3.81763700 & 1.93772800 & 3.84031100 & $\mathrm{C}$ & 0.36844800 & 6.11237700 & -0.08117000 \\
\hline C & 5.06838900 & 0.86594300 & 2.45028800 & $\mathrm{H}$ & -1.57933000 & 5.38633200 & -0.65256500 \\
\hline $\mathrm{H}$ & 5.82318900 & 0.61932600 & 3.19025700 & $\mathrm{H}$ & 2.41177700 & 6.52675600 & 0.45925000 \\
\hline $\mathrm{C}$ & 5.21888200 & 0.45227300 & 1.13115100 & $\mathrm{H}$ & 0.04597100 & 7.14302000 & 0.03029500 \\
\hline $\mathrm{H}$ & 6.08835700 & -0.12462900 & 0.83226800 & $\mathrm{Rh}$ & -0.02572900 & 0.12314000 & -1.09977300 \\
\hline C & 4.24777600 & 0.76008000 & 0.18160400 & $\mathrm{H}$ & -0.54828600 & 1.39110500 & -2.59107500 \\
\hline $\mathrm{H}$ & 4.38169000 & 0.40155300 & -0.83395600 & $\mathrm{C}$ & -5.21480300 & -2.09256800 & -2.16285400 \\
\hline $\mathrm{C}$ & 2.62806800 & 1.96386400 & -2.24059000 & C & -4.20769200 & -2.97530000 & -2.54252300 \\
\hline $\mathrm{H}$ & 1.90056500 & 2.28036500 & -2.99203600 & C & -2.90807600 & -2.51430000 & -2.73090800 \\
\hline $\mathrm{H}$ & 3.08931000 & 1.03595600 & -2.58097600 & $\mathrm{C}$ & -2.57114900 & -1.16882600 & -2.52079000 \\
\hline $\mathrm{H}$ & 3.39173700 & 2.73860500 & -2.12934800 & $\mathrm{C}$ & -3.60207800 & -0.29257000 & -2.15330300 \\
\hline $\mathrm{C}$ & 1.17374000 & -2.27874300 & 1.12096100 & $\mathrm{C}$ & -4.90424500 & -0.74614300 & -1.98264800 \\
\hline $\mathrm{C}$ & 1.91337200 & -1.46325600 & 1.98560800 & $\mathrm{H}$ & -6.23027200 & -2.44704000 & -2.01654100 \\
\hline $\mathrm{H}$ & 2.46587100 & -0.62071500 & 1.59014100 & $\mathrm{H}$ & -4.43414200 & -4.02556200 & -2.70332100 \\
\hline $\mathrm{C}$ & 1.96254900 & -1.72310200 & 3.34993400 & $\mathrm{H}$ & -2.13287100 & -3.21001900 & -3.04183500 \\
\hline $\mathrm{H}$ & 2.55159900 & -1.07513700 & 3.99219600 & $\mathrm{H}$ & -3.37086600 & 0.75112000 & -1.95796600 \\
\hline $\mathrm{C}$ & 1.26285300 & -2.80421200 & 3.88033000 & $\mathrm{H}$ & -5.67582500 & -0.04241500 & -1.68490400 \\
\hline $\mathrm{H}$ & 1.29217400 & -3.00570300 & 4.94681400 & C & -1.16216000 & -0.72031600 & -2.66677700 \\
\hline C & 0.52444400 & -3.62181100 & 3.03257300 & $\mathrm{H}$ & -0.58503800 & -1.50362800 & -3.16078000 \\
\hline $\mathrm{H}$ & -0.03629100 & -4.46003400 & 3.43338500 & $\mathrm{C}$ & -0.93983600 & 0.62020000 & -3.33366200 \\
\hline $\mathrm{C}$ & 0.48381300 & -3.36485100 & 1.66429100 & $\mathrm{H}$ & -0.20739300 & 0.58126900 & -4.14200400 \\
\hline $\mathrm{H}$ & -0.11825700 & -4.00845800 & 1.03386600 & $\mathrm{H}$ & -1.86185100 & 1.07951100 & -3.70146900 \\
\hline $\mathrm{C}$ & 0.38451200 & -3.38083700 & -1.39892400 & & & & \\
\hline $\mathrm{H}$ & 0.48560500 & -3.32171700 & -2.48490500 & & & & \\
\hline $\mathrm{H}$ & -0.67849300 & -3.45882700 & -1.15736200 & & & & \\
\hline $\mathrm{H}$ & 0.92122100 & -4.26413400 & -1.04390800 & & & & \\
\hline $\mathrm{C}$ & 2.83678900 & -2.02442000 & -1.22130900 & & & & \\
\hline C & 3.14032500 & -1.56839000 & -2.50899000 & & & & \\
\hline $\mathrm{C}$ & 3.85766300 & -2.58583600 & -0.45208000 & & & & \\
\hline $\mathrm{C}$ & 4.43356000 & -1.65457800 & -3.01363300 & INT11' & & & \\
\hline $\mathrm{H}$ & 2.34769800 & -1.11992300 & -3.10548700 & $E=-1948.544063$ & $G=-1$ & 47.805802 & \\
\hline $\mathrm{C}$ & 5.15762600 & -2.66064900 & -0.94926600 & $\mathrm{P}$ & 1.31720300 & 1.49322400 & -0.65223700 \\
\hline $\mathrm{H}$ & 3.64638200 & -2.94909900 & 0.54832500 & $\mathrm{P}$ & -2.00963400 & 0.50378000 & -0.80566000 \\
\hline $\mathrm{C}$ & 5.45103700 & -2.19131900 & -2.22600900 & $\mathrm{P}$ & -0.67306400 & -1.73577000 & 1.57039600 \\
\hline $\mathrm{H}$ & 4.64973400 & -1.29249600 & -4.01430400 & $\mathrm{C}$ & 0.65141000 & 2.97547800 & 0.24019600 \\
\hline $\mathrm{H}$ & 5.94360500 & -3.08645100 & -0.33252300 & $\mathrm{C}$ & 0.81855700 & 4.28161300 & -0.22446500 \\
\hline $\mathrm{H}$ & 6.46498000 & -2.24807700 & -2.60994000 & $\mathrm{H}$ & 1.35665100 & 4.47189600 & -1.14704200 \\
\hline $\mathrm{C}$ & 1.20303900 & 3.44835500 & -0.34991100 & $\mathrm{C}$ & 0.27198200 & 5.35684200 & 0.46999200 \\
\hline $\mathrm{C}$ & -0.12300400 & 3.80917500 & -0.59688500 & $\mathrm{H}$ & 0.39788700 & 6.36445000 & 0.08525700 \\
\hline C & 2.11170300 & 4.44592100 & 0.02339800 & $\mathrm{C}$ & -0.43653900 & 5.14137900 & 1.64908500 \\
\hline $\mathrm{C}$ & -0.54036000 & 5.13233700 & -0.46482800 & $\mathrm{H}$ & -0.86447900 & 5.98132600 & 2.18801000 \\
\hline $\mathrm{H}$ & -0.83808500 & 3.04014100 & -0.88038800 & C & -0.58823300 & 3.84500300 & 2.13455400 \\
\hline $\mathrm{C}$ & 1.69668300 & 5.76608100 & 0.16152200 & $\mathrm{H}$ & -1.12486800 & 3.66075700 & 3.06004600 \\
\hline
\end{tabular}




\begin{tabular}{|c|c|c|c|c|c|c|c|}
\hline $\mathrm{C}$ & -0.05145100 & 2.77013600 & 1.43164400 & $\mathrm{H}$ & 1.36941900 & -2.92457300 & 2.27312800 \\
\hline $\mathrm{H}$ & -0.19400900 & 1.75670800 & 1.79543200 & $\mathrm{H}$ & 0.21819000 & -3.99321600 & 1.46653900 \\
\hline $\mathrm{C}$ & 3.05559500 & 1.51525800 & -0.01204600 & $\mathrm{H}$ & -0.06753800 & -3.51471100 & 3.15894200 \\
\hline $\mathrm{C}$ & 4.18068700 & 1.67614200 & -0.82137200 & $\mathrm{C}$ & -0.56403200 & -0.58689500 & 3.01749100 \\
\hline $\mathrm{H}$ & 4.07287600 & 1.82188900 & -1.89131600 & C & 0.69614800 & -0.32619300 & 3.57121800 \\
\hline C & 5.46288100 & 1.63542200 & -0.27341300 & $\mathrm{C}$ & -1.65724500 & 0.15096200 & 3.48200800 \\
\hline $\mathrm{H}$ & 6.32632200 & 1.75450100 & -0.92053900 & $\mathrm{C}$ & 0.85783300 & 0.64281100 & 4.55540200 \\
\hline $\mathrm{C}$ & 5.63428300 & 1.43234100 & 1.09067700 & $\mathrm{H}$ & 1.56780000 & -0.86232600 & 3.20754500 \\
\hline $\mathrm{H}$ & 6.63172500 & 1.39649500 & 1.51743900 & C & -1.49697600 & 1.12024300 & 4.47001500 \\
\hline $\mathrm{C}$ & 4.51590600 & 1.26925300 & 1.90779900 & $\mathrm{H}$ & -2.64506900 & -0.02973900 & 3.07232300 \\
\hline $\mathrm{H}$ & 4.64059900 & 1.10680900 & 2.97418200 & $\mathrm{C}$ & -0.23907200 & 1.37271500 & 5.00798800 \\
\hline $\mathrm{C}$ & 3.24096900 & 1.30681400 & 1.35957300 & $\mathrm{H}$ & 1.84483600 & 0.83166200 & 4.96654200 \\
\hline $\mathrm{H}$ & 2.37344500 & 1.16586300 & 1.99847200 & $\mathrm{H}$ & -2.36146700 & 1.67790100 & 4.81826300 \\
\hline C & 1.52080500 & 2.11889300 & -2.37891500 & $\mathrm{H}$ & -0.11286700 & 2.13205200 & 5.77314700 \\
\hline $\mathrm{H}$ & 0.54083400 & 2.42957500 & -2.74705100 & $\mathrm{C}$ & -2.11492400 & 2.03032400 & -1.85474400 \\
\hline $\mathrm{H}$ & 1.87539000 & 1.29520400 & -3.00348100 & $\mathrm{C}$ & -2.23421600 & 3.29239000 & -1.26510100 \\
\hline $\mathrm{H}$ & 2.21922900 & 2.95547400 & -2.46321800 & C & -1.99600000 & 1.95236500 & -3.24730500 \\
\hline $\mathrm{C}$ & -2.79710500 & -0.72104000 & -1.94526400 & $\mathrm{C}$ & -2.23151700 & 4.44528900 & -2.04510700 \\
\hline C & -1.94768000 & -1.57369800 & -2.66073100 & $\mathrm{H}$ & -2.30664400 & 3.39175200 & -0.18638600 \\
\hline $\mathrm{H}$ & -0.87191300 & -1.48276400 & -2.51765500 & $\mathrm{C}$ & -1.99197600 & 3.10492700 & -4.02712400 \\
\hline C & -2.46727500 & -2.54541400 & -3.51071800 & $\mathrm{H}$ & -1.90633600 & 0.98367200 & -3.72923400 \\
\hline $\mathrm{H}$ & -1.79601500 & -3.20092700 & -4.05666400 & C & -2.10796600 & 4.35666200 & -3.42773600 \\
\hline $\mathrm{C}$ & -3.84632900 & -2.68341800 & -3.64820600 & $\mathrm{H}$ & -2.31464200 & 5.41385100 & -1.56232400 \\
\hline $\mathrm{H}$ & -4.25464300 & -3.44697200 & -4.30312800 & $\mathrm{H}$ & -1.89833900 & 3.02182900 & -5.10560100 \\
\hline $\mathrm{C}$ & -4.69956700 & -1.83745700 & -2.94409400 & $\mathrm{H}$ & -2.10343100 & 5.25642800 & -4.03529300 \\
\hline $\mathrm{H}$ & -5.77517400 & -1.94068700 & -3.05009900 & $\mathrm{Rh}$ & 0.07946100 & -0.39594900 & -0.16108100 \\
\hline C & -4.17789000 & -0.85760000 & -2.10366000 & $\mathrm{H}$ & 2.88665700 & -0.53771600 & -1.78446800 \\
\hline $\mathrm{H}$ & -4.85766200 & -0.20900900 & -1.56072000 & $\mathrm{C}$ & 5.96388800 & -4.19281900 & -1.46800700 \\
\hline C & -3.35256800 & 0.85623900 & 0.41855900 & C & 6.02910800 & -2.94480300 & -0.85026900 \\
\hline $\mathrm{H}$ & -2.94679700 & 1.52190600 & 1.18459800 & C & 4.93120200 & -2.09195100 & -0.87741100 \\
\hline $\mathrm{H}$ & -3.64086400 & -0.08490400 & 0.89447700 & $\mathrm{C}$ & 3.74844500 & -2.46511000 & -1.52565800 \\
\hline $\mathrm{H}$ & -4.22874500 & 1.32831100 & -0.03245200 & $\mathrm{C}$ & 3.69548700 & -3.71805100 & -2.13853900 \\
\hline $\mathrm{C}$ & -2.35700200 & -2.49162900 & 1.74084700 & $\mathrm{C}$ & 4.79183800 & -4.57743900 & -2.11164000 \\
\hline $\mathrm{C}$ & -3.00102700 & -2.92074800 & 0.57802700 & $\mathrm{H}$ & 6.82046200 & -4.86001600 & -1.44745900 \\
\hline $\mathrm{H}$ & -2.52777100 & -2.76367600 & -0.38482600 & $\mathrm{H}$ & 6.94033600 & -2.63519600 & -0.34617900 \\
\hline C & -4.24571700 & -3.54373800 & 0.63927600 & $\mathrm{H}$ & 4.98375300 & -1.12134400 & -0.39008700 \\
\hline $\mathrm{H}$ & -4.73088500 & -3.85677900 & -0.27989100 & $\mathrm{H}$ & 2.78186100 & -4.02272500 & -2.64406800 \\
\hline C & -4.85950000 & -3.75038600 & 1.86978200 & $\mathrm{H}$ & 4.72963100 & -5.54777800 & -2.59599500 \\
\hline $\mathrm{H}$ & -5.83248900 & -4.22925500 & 1.92196000 & C & 2.55077900 & -1.54299300 & -1.50612900 \\
\hline C & -4.21766000 & -3.34663800 & 3.03955300 & $\mathrm{H}$ & 1.83713600 & -1.85943800 & -2.27773500 \\
\hline $\mathrm{H}$ & -4.68711800 & -3.51514600 & 4.00398100 & $\mathrm{C}$ & 1.85844200 & -1.52354300 & -0.13247800 \\
\hline C & -2.97378600 & -2.72815700 & 2.97601600 & $\mathrm{H}$ & 1.63290600 & -2.57031300 & 0.10332900 \\
\hline $\mathrm{H}$ & -2.48260100 & -2.42045500 & 3.89474200 & $\mathrm{H}$ & 2.58261700 & -1.19082200 & 0.62471500 \\
\hline C & 0.31176000 & -3.17988800 & 2.19060300 & & & & \\
\hline
\end{tabular}




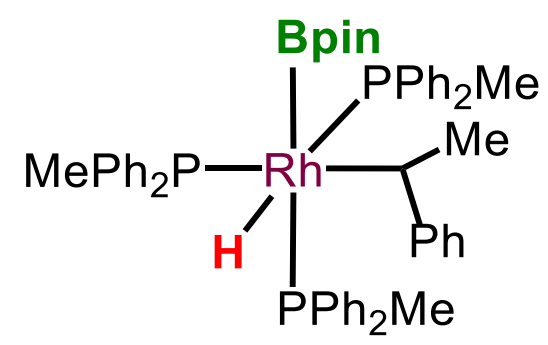

INT22

$E=-2360.322216$

$G=-2359.392376$

O

C

C

C

$\mathrm{H}$

$\mathrm{H}$

C

$\mathrm{H}$

$\mathrm{H}$

$\mathrm{H}$

C

$\mathrm{H}$

$\mathrm{H}$

C

$\mathrm{H}$

$\mathrm{H}$

$\mathrm{H}$

\begin{tabular}{|c|c|c|}
\hline 1.76315900 & 4.00685000 & -1.08287800 \\
\hline 1.27509700 & 1.23535800 & -1.32580800 \\
\hline .33588700 & 0.47883200 & 3.01312800 \\
\hline 3.04920800 & 1.76617200 & 1.14118900 \\
\hline 4.07403600 & 1.23589500 & -1.92883700 \\
\hline 5.37604600 & 1.72549900 & -1.83331700 \\
\hline 5.66823200 & 2.76644600 & -0.96067700 \\
\hline 4.65693800 & 3.29069800 & 75200 \\
\hline 0.56662000 & 2.91742300 & 7700 \\
\hline 0.436 & 3.43273300 & -2.96784600 \\
\hline-0.07170900 & 4.71199500 & 3.17661200 \\
\hline .43598700 & 5.50677500 & 04600 \\
\hline-0.29598800 & 5.01164900 & -0.80232200 \\
\hline 0.18900500 & 3.72528600 & -0.59695600 \\
\hline-2.15753300 & 0.72008600 & 37400 \\
\hline 2.19254800 & 0.89481700 & -2.44246600 \\
\hline-2.66052400 & 2.40828900 & -0.03684000 \\
\hline-2.40309600 & 2.76190300 & 1.28968300 \\
\hline-2.80497100 & 3.99708000 & 1.78793300 \\
\hline-3.46102700 & 4.90222300 & 0.95780200 \\
\hline-3.71778500 & 4.56150600 & -0.36753600 \\
\hline-3.32739600 & 3.31955600 & -0.85880400 \\
\hline-3.79157900 & .15015600 & -0.44 \\
\hline-3.98891300 & -1.32827300 & -1.17222900 \\
\hline-5.21817600 & -1.97449900 & -1.16319700 \\
\hline-6.27698900 & 1.45076700 & -0.42539600 \\
\hline-6.08867000 & -0.28254200 & 0.30338600 \\
\hline-4.85524800 & 0.36354700 & 0.29827200 \\
\hline 0.56204700 & 4.59898600 & 0.56727800 \\
\hline 1.340 & -3.37761700 & 41200 \\
\hline-0.41201000 & -3.57759100 & 66667700 \\
\hline 3.02318500 & -1.00109200 & 0.58318300 \\
\hline 5.14084400 & 59400 & 800 \\
\hline 5.30763000 & -3.81266200 & 2.87430300 \\
\hline 3.31947800 & -5.29428400 & -3.01587300 \\
\hline 1.20033600 & -4.63145500 & -1.95454000 \\
\hline-0.14608300 & .83228900 & 3.1137900 \\
\hline-1.80013900 & -2.69799800 & 4.72039100 \\
\hline-3.44917400 & -4.41649500 & -4.00727600 \\
\hline-3.41062500 & -5.25425600 & -1.66947500 \\
\hline-1.766 & -4.39195000 & -0.06873900 \\
\hline 1.9237 & 1.06497100 & -3.7233420 \\
\hline 1.778693 & -0.51594700 & -2.92750000 \\
\hline 0.31701 & 37216 & -3.387582 \\
\hline
\end{tabular}




\begin{tabular}{|c|c|c|c|c|c|c|c|}
\hline $\mathrm{H}$ & 3.87174300 & 0.43633400 & -2.63314600 & & 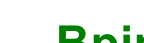 & & \\
\hline $\mathrm{H}$ & 6.15490000 & 1.30096800 & -2.45970100 & & & & \\
\hline $\mathrm{H}$ & 6.67659700 & 3.16357800 & -0.89820900 & & & & \\
\hline $\mathrm{H}$ & 4.87608400 & 4.09164600 & 0.54153700 & & & & \\
\hline $\mathrm{H}$ & 0.72137200 & 2.84002800 & -3.83050400 & & & & \\
\hline $\mathrm{H}$ & -0.17743400 & 5.08870000 & -4.18948300 & & & & \\
\hline $\mathrm{H}$ & -0.83535700 & 6.50283600 & -2.25766200 & INT22' & & & \\
\hline $\mathrm{H}$ & -0.59960400 & 5.60880300 & 0.05102300 & $E=-2360.331140$ & $G=-2$ & 359.403870 & \\
\hline $\mathrm{H}$ & 0.24885200 & 3.32903600 & 0.41085800 & $\mathrm{O}$ & -2.41851500 & -0.98324300 & -1.84724300 \\
\hline $\mathrm{H}$ & -3.19957400 & 1.10880400 & -2.80686700 & $\mathrm{O}$ & -0.94110800 & -2.66324600 & -1.49016600 \\
\hline $\mathrm{H}$ & -1.51421300 & 1.68779100 & -2.76066800 & $\mathrm{C}$ & -3.03291400 & -2.15870200 & -2.38918300 \\
\hline $\mathrm{H}$ & -1.87191100 & -0.05394500 & -2.87898800 & $\mathrm{C}$ & -1.80866900 & -3.11887100 & -2.53831400 \\
\hline $\mathrm{H}$ & -1.87391300 & 2.06158500 & 1.92478100 & $\mathrm{C}$ & -3.72956300 & -1.79413600 & -3.69415700 \\
\hline $\mathrm{H}$ & -2.60168200 & 4.25079300 & 2.82393600 & $\mathrm{H}$ & -4.11154400 & -2.69141400 & -4.19251200 \\
\hline $\mathrm{H}$ & -3.77181000 & 5.86905800 & 1.34241700 & $\mathrm{H}$ & -4.57347000 & -1.13267200 & -3.48124900 \\
\hline $\mathrm{H}$ & -4.22474100 & 5.26357000 & -1.02236900 & $\mathrm{H}$ & -3.05397900 & -1.27356200 & -4.37505600 \\
\hline $\mathrm{H}$ & -3.53915800 & 3.07525000 & -1.89400800 & C & -4.07111300 & -2.65163200 & -1.37883100 \\
\hline $\mathrm{H}$ & -3.18115400 & -1.74547600 & -1.76145800 & $\mathrm{H}$ & -4.74225000 & -1.82058300 & -1.13879000 \\
\hline $\mathrm{H}$ & -5.34337600 & -2.88810800 & -1.73687000 & $\mathrm{H}$ & -4.66414100 & -3.47722400 & -1.78398000 \\
\hline $\mathrm{H}$ & -7.24072500 & -1.95090400 & -0.41834300 & $\mathrm{H}$ & -3.59764900 & -2.98863200 & -0.45343100 \\
\hline $\mathrm{H}$ & -6.90283300 & 0.13038500 & 0.89106700 & C & -2.11099200 & -4.59494700 & -2.32118600 \\
\hline $\mathrm{H}$ & -4.72845600 & 1.26837100 & 0.88148900 & $\mathrm{H}$ & -2.83607000 & -4.95421900 & -3.05893100 \\
\hline $\mathrm{C}$ & 3.36671000 & 2.78278100 & -0.23388000 & $\mathrm{H}$ & -1.19264100 & -5.17807900 & -2.43396400 \\
\hline $\mathrm{H}$ & 2.60034000 & 3.18654100 & 0.41690400 & $\mathrm{H}$ & -2.50962900 & -4.77800600 & -1.32162400 \\
\hline $\mathrm{Rh}$ & 0.16114600 & 0.05926400 & 0.45980900 & C & -1.06331000 & -2.92433000 & -3.86126500 \\
\hline $\mathrm{H}$ & -0.60229500 & 1.47529500 & 3.44303600 & $\mathrm{H}$ & -0.10746000 & -3.45320000 & -3.81651400 \\
\hline C & -4.76218700 & -1.63588400 & 3.00476900 & $\mathrm{H}$ & -1.63588900 & -3.31120000 & -4.70947700 \\
\hline $\mathrm{C}$ & -3.92985800 & -2.35279000 & 2.14857300 & $\mathrm{H}$ & -0.85293900 & -1.86506300 & -4.03490500 \\
\hline C & -2.62829600 & -1.93121300 & 1.92570400 & B & -1.22411100 & -1.32059400 & -1.22210700 \\
\hline $\mathrm{C}$ & -2.07832300 & -0.80763900 & 2.56366700 & $\mathrm{H}$ & -0.10875200 & 0.38836300 & -1.65330600 \\
\hline $\mathrm{C}$ & -2.95120800 & -0.08061400 & 3.38785600 & $\mathrm{H}$ & 1.21475700 & -1.37103500 & -2.84283300 \\
\hline C & -4.26831200 & -0.48409400 & 3.60219400 & $\mathrm{C}$ & 4.86527300 & -4.49392100 & -2.83472200 \\
\hline $\mathrm{H}$ & -5.78588300 & -1.95407700 & 3.17478300 & $\mathrm{C}$ & 5.29485500 & -3.17084500 & -2.85980500 \\
\hline $\mathrm{H}$ & -4.30627600 & -3.22780600 & 1.62692400 & C & 4.38562100 & -2.13708100 & -2.64552600 \\
\hline $\mathrm{H}$ & -2.02069100 & -2.47582100 & 1.21469600 & $\mathrm{C}$ & 3.03736700 & -2.40109400 & -2.40102400 \\
\hline $\mathrm{H}$ & -2.59761200 & 0.80459000 & 3.90443400 & C & 2.61916800 & -3.73717200 & -2.37751400 \\
\hline $\mathrm{H}$ & -4.90503100 & 0.10700300 & 4.25501500 & $\mathrm{C}$ & 3.52079800 & -4.77302400 & -2.59391900 \\
\hline C & -0.60550400 & -0.49188700 & 2.45259400 & $\mathrm{H}$ & 5.57155800 & -5.30164500 & -3.00159500 \\
\hline $\mathrm{H}$ & -0.08883600 & -1.43824400 & 2.64316400 & $\mathrm{H}$ & 6.34026200 & -2.94251300 & -3.04751900 \\
\hline C & -0.16766100 & 0.47689600 & 3.56278600 & $\mathrm{H}$ & 4.72677400 & -1.10475100 & -2.67158500 \\
\hline $\mathrm{H}$ & 0.91048200 & 0.63628300 & 3.55661500 & $\mathrm{H}$ & 1.57183900 & -3.95287800 & -2.17714100 \\
\hline \multirow[t]{3}{*}{$\mathrm{H}$} & -0.45588500 & 0.10940300 & 4.55904300 & $\mathrm{H}$ & 3.17522900 & -5.80290200 & -2.57435500 \\
\hline & & & & C & 2.04527000 & -1.29286300 & -2.13242700 \\
\hline & & & & $\mathrm{H}$ & 2.53110200 & -0.33179300 & -2.32324300 \\
\hline
\end{tabular}


$1.49312200 \quad-1.36563100 \quad-0.70583400$

$\begin{array}{lll}2.32788200 & -1.25439200 & -0.00192300\end{array}$

$\begin{array}{lll}1.10823000 & -2.37522200 & -0.58543800\end{array}$

$\begin{array}{lll}-0.18778800 & -1.35641300 & 1.88084600\end{array}$

$\begin{array}{lll}0.25149600 & -3.13450600 \quad 1.63270200\end{array}$

$-1.85551200 \quad-1.62616200 \quad 2.64561400$

$\begin{array}{lll}-2.98296100 & -1.54954900 & 1.82643900\end{array}$

$\begin{array}{lll}-4.24798200 & -1.85431900 & 2.32363100\end{array}$

$-4.40370900 \quad-2.22275600 \quad 3.65535500$

$-3.28616200 \quad-2.29429800 \quad 4.48496400$

$\begin{array}{lll}-2.02136600 & -2.00693900 & 3.98260200\end{array}$

$\begin{array}{lll}0.81489900 & -0.93418100 & 3.37843500\end{array}$

$\begin{array}{lll}0.47250900 & 0.19530200 & 4.13323800\end{array}$

$\begin{array}{lll}1.22447800 & 0.57509600 & 5.23856000\end{array}$

$\begin{array}{lll}2.33966300 & -0.17383300 \quad 5.61400600\end{array}$

$\begin{array}{lll}2.68557200 & -1.30106800 & 4.87827100\end{array}$

$\begin{array}{lll}1.93153800 & -1.67607400 \quad 3.76735800\end{array}$

$\begin{array}{lll}-1.83944400 & 1.58290000 & 0.33825800\end{array}$

$-2.18397100$

$-3.56617600$

$-4.63423500$

$-5.91112600$

$-6.14071600$

$-5.08377200$

$-1.48876200$

$-1.75291100$

$-1.45317500$

$-0.89897400$

$-0.64677900$

$-0.93298800$

1.69859100

1.41058000

2.19751400

2.01457000

2.41476300

2.98404900

3.16099400

2.78205300

3.35305400

3.66684100

4.87773200

5.78735100

5.48292400

4.27944100
1.94332400

1.36475900

1.01896200

0.81912300

0.98231200

1.34367600

3.27989100

4.45226600

5.69894600

5.79587400

4.63902700

3.39353900

1.65911000

2.86120100

2.78849700

2.37284900

3.17365800

4.42006000

4.85165200

4.03739200

0.98405700

0.73842000

0.14553500

$-0.22214300$

0.01627200

0.61974000
2.12007700

$-0.30865300$

0.52098400

$-0.00316400$

$-1.36401100$

$-2.19792500$

$-0.31986400$

0.39736600

$-0.14394100$

$-1.41739200$

$-2.14590400$

$-1.59672400$

0.42558200

1.80568500

$-0.95838100$

$-2.27932200$

$-3.34486800$

$-3.10381300$

$-1.79191600$

$-0.72924600$

0.89753800

2.23652700

2.58324000

1.59781500

0.26027000

$-0.08519000$

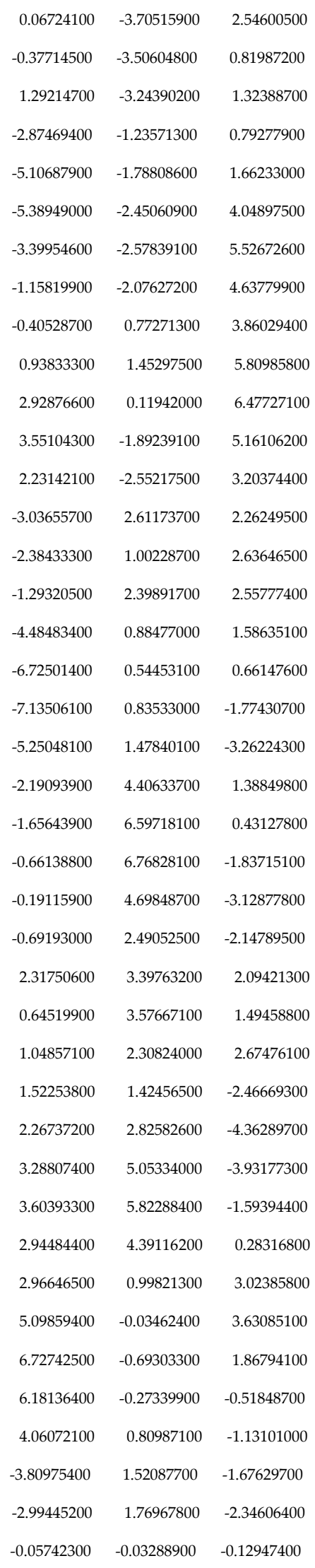




\section{$\mathrm{Ph} \quad \mathrm{PPh}_{2} \mathrm{Me}$

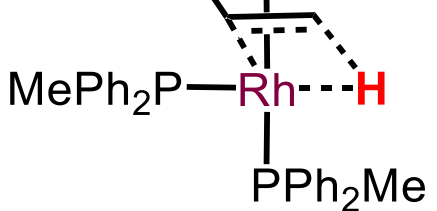

TS11

$E=-1948.520647$

\section{P}

P

$$
G=-1947.785688
$$

$$
2.15471200 \quad 0.18348900 \quad-1.45171000
$$$$
\begin{array}{lll}
-1.14669100 & 1.78445500 & 0.40660400
\end{array}
$$$$
0.13215800 \quad-1.59416500 \quad 0.83594600
$$$$
\begin{array}{lll}
3.42796500 & 0.97333000 & -0.36354700
\end{array}
$$$$
\begin{array}{lll}
4.75865800 & 1.17107600 & -0.75308700
\end{array}
$$$$
\begin{array}{lll}
5.09912700 & 0.81970000 & -1.72375700
\end{array}
$$$$
\begin{array}{lll}
5.66058100 & 1.79862800 & 0.09937100
\end{array}
$$$$
\begin{array}{lll}
6.68993200 & 1.94426900 & -0.21425600
\end{array}
$$$$
\begin{array}{lll}
5.24247800 & 2.24260900 & 1.35324600
\end{array}
$$$$
\begin{array}{lll}
5.94694000 & 2.73427000 & 2.01741500
\end{array}
$$$$
3.92262400 \quad 2.05596100 \quad 1.74854100
$$$$
\begin{array}{lll}
3.58746100 & 2.39408400 & 2.72374000
\end{array}
$$$$
\begin{array}{lll}
3.02386500 & 1.42535600 & 0.89319800
\end{array}
$$$$
1.99052200 \quad 1.27571600 \quad 1.19216600
$$$$
2.96267900 \quad-1.40810500 \quad-1.95505700
$$$$
2.31972300 \quad-2.13791700 \quad-2.96511700
$$$$
1.43525900 \quad-1.72380700 \quad-3.44340500
$$$$
\begin{array}{lll}
2.77714800 & -3.39205300 & -3.34948600
\end{array}
$$$$
2.26358000 \quad-3.93598500 \quad-4.13647700
$$$$
3.88434300 \quad-3.95409400 \quad-2.71601500
$$$$
\begin{array}{lll}
4.23878700 & -4.93854500 & -3.00486200
\end{array}
$$$$
\begin{array}{lll}
4.53076400 & -3.24375000 & -1.71025000
\end{array}
$$$$
5.39590700 \quad-3.67195600 \quad-1.21273000
$$$$
\begin{array}{lll}
4.07878400 & -1.97813500 & -1.33671700
\end{array}
$$$$
4.59627200 \quad-1.43632700 \quad-0.55116500
$$$$
2.41520600 \quad 1.14895600 \quad-3.02094400
$$$$
\begin{array}{lll}
2.07091800 & 2.17373000 & -2.86063100
\end{array}
$$$$
1.80530200 \quad 0.70934700 \quad-3.81427300
$$$$
\begin{array}{lll}
3.45799900 & 1.15981600 & -3.34719000
\end{array}
$$$$
\begin{array}{lll}
-2.90335300 & 2.08761600 & -0.08575400
\end{array}
$$$$
\begin{array}{lll}
-3.15545100 \quad 2.63629700 & -1.35065400
\end{array}
$$$$
\begin{array}{lll}
-2.32073900 & 2.96572000 & -1.96472900
\end{array}
$$$$
-4.45407000 \quad 2.76276500 \quad-1.82774800
$$$$
\begin{array}{lll}
-4.62868300 & 3.19236300 & -2.80948900
\end{array}
$$$$
\begin{array}{lll}
-5.53061100 & 2.33949600 & -1.04792500
\end{array}
$$

\begin{tabular}{|c|c|c|}
\hline .29351300 & 1.80308400 & 0.21278000 \\
\hline-6.12473300 & 1.47592700 & .83059900 \\
\hline-3.98999800 & .67925500 & 0.69153600 \\
\hline-3.82832000 & .24440300 & .67326100 \\
\hline 1.28503500 & .77105500 & 2.25562900 \\
\hline-0.27443400 & 1.86557300 & .66026400 \\
\hline-1.70819000 & 0.82209200 & .59424700 \\
\hline-1.89459600 & 2.59800000 & 2.63036700 \\
\hline-1.30618300 & 2.02706200 & 1.91324500 \\
\hline-2.56157300 & -1.50418300 & 60783800 \\
\hline-2.66063600 & -0.82974600 & .76392900 \\
\hline-3.67955400 & -1.85363100 & 2.36094400 \\
\hline-4.64972100 & -1.44673600 & .09300000 \\
\hline-3.54796300 & -2.72889500 & .43344600 \\
\hline-4.41710600 & -3.00473900 & 4.02314300 \\
\hline-2.29604800 & -3.25624600 & 3.74916000 \\
\hline-2.18909600 & -3.94030700 & 58561900 \\
\hline-1.18248100 & -2.90975300 & 2.99237400 \\
\hline-0.20881900 & -3.31869000 & 3.24915400 \\
\hline 0.57235800 & -3.26869900 & 0.18633900 \\
\hline 1.38894600 & -3.18955200 & .53477700 \\
\hline-0.30711900 & -3.65617400 & -0.32904300 \\
\hline 0.84668500 & -3.95674500 & 0.99010300 \\
\hline 1.46277300 & -1.34413100 & 2.09574900 \\
\hline 2.75624300 & -1.82063200 & 43400 \\
\hline 1.22157500 & -0.59548300 & 3.25238200 \\
\hline 3.78861600 & -1.53458200 & 2.75741300 \\
\hline 2.97032500 & -2.41194300 & .98469600 \\
\hline 2.25129900 & -0.30899400 & 4.14220000 \\
\hline 0.21725400 & -0.24604800 & 3.46741700 \\
\hline 3.54139100 & -0.77201300 & 3.89350500 \\
\hline 4.788 & -1.90714500 & .55718700 \\
\hline 2.04316900 & 0.27066500 & .0366270 \\
\hline 4.34589800 & -0.54337200 & 4.58538500 \\
\hline-0.4843 & 3.50998800 & 42900 \\
\hline 0.76237100 & 3.70330500 & 0.39695200 \\
\hline 1.19437500 & 4.63044700 & .64355100 \\
\hline 1.30323400 & 4.98053400 & 0.52871000 \\
\hline 1.31081000 & 2.83747700 & 0.74853 \\
\hline-0.66278800 & 90765100 & 0.50875600 \\
\hline-2.17827300 & 4.50387100 & 1.08810700 \\
\hline 0.59061300 & 6.08531800 & 0.07629000 \\
\hline 2.28015600 & 5.10708500 & -0.98534800 \\
\hline & 76746800 & 8577190 \\
\hline
\end{tabular}$$
-6.54526800 \quad 2.43354100 \quad-1.42193100
$$ 


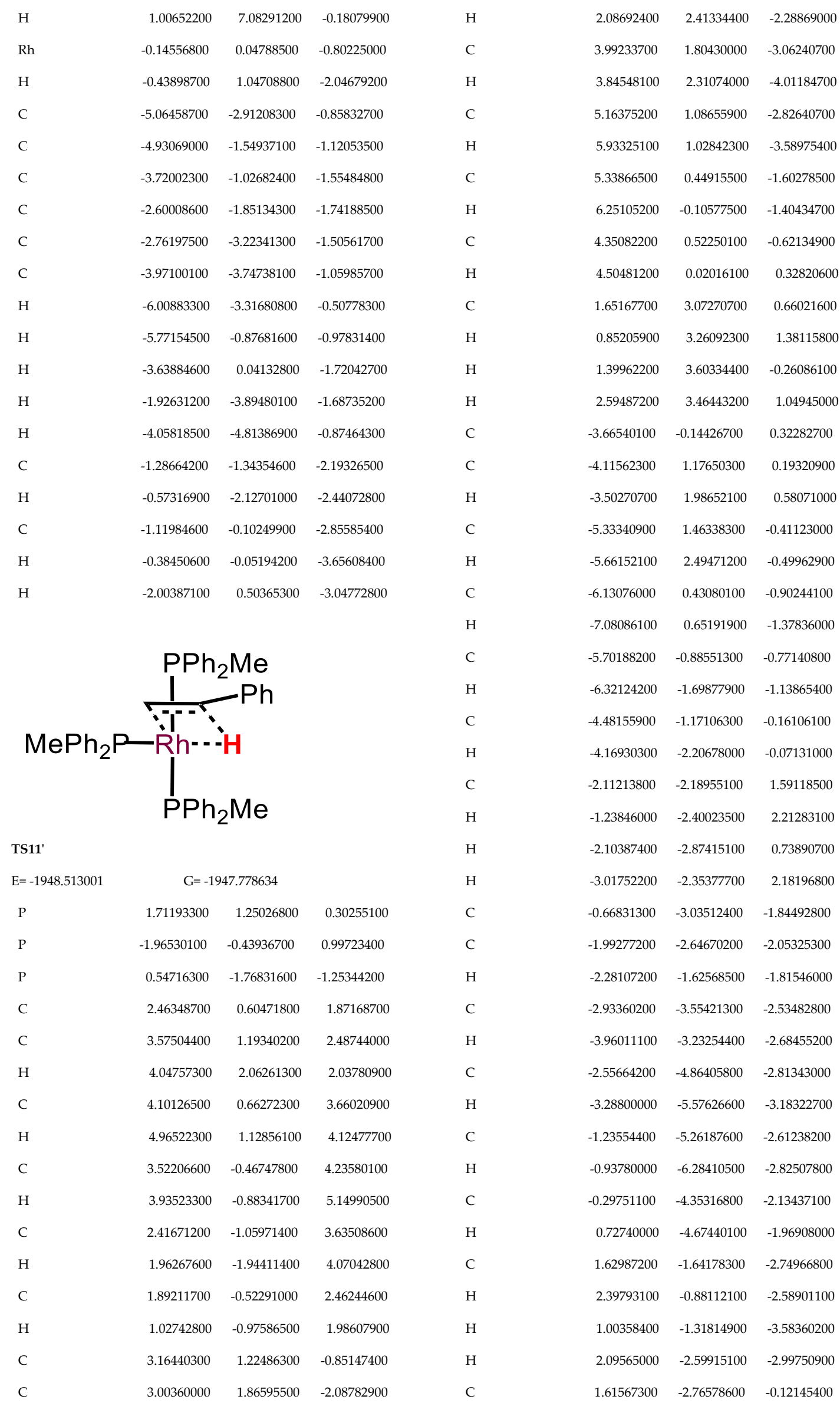




\begin{tabular}{|c|c|c|}
\hline 2.99820600 & -2.56448000 & -0.10441000 \\
\hline 1.05890100 & -3.64138500 & 0.81690500 \\
\hline 3.80033000 & -3.19926400 & 0.83919000 \\
\hline 3.45944100 & -1.89444900 & -0.82138700 \\
\hline 1.85928600 & -4.27992500 & 1.7583 \\
\hline-0.00815600 & -3.83655500 & 0.8056 \\
\hline 3.23369500 & -4.05445500 & 1.777848 \\
\hline 4.87112000 & -3.01931200 & 0.84085 \\
\hline 1.40815800 & -4.95898000 & 2.4757 \\
\hline 3.85797700 & -4.54657000 & 2.51695 \\
\hline-2.13621100 & 0.43432900 & 2.6278 \\
\hline-1.09489800 & 1.24084300 & 3.0836 \\
\hline-3.27578000 & 0.28972000 & 3.4284 \\
\hline-1.17585700 & 1.88067600 & 4.31903 \\
\hline-0.21488100 & 1.35724500 & 2.460918 \\
\hline-3.36424000 & 0.93154400 & 4.65804 \\
\hline-4.10788900 & -0.31693100 & 3.07967 \\
\hline-2.31096300 & 1.72755500 & 5.1073 \\
\hline-0.35031400 & 2.49772900 & 4.6605 \\
\hline-4.25661300 & 0.81478900 & 5.26562 \\
\hline-2.38016400 & 2.22756200 & 6.0687 \\
\hline-0.31732400 & 0.25325800 & -0.51379500 \\
\hline-1.07190000 & 1.68498000 & -0.34371 \\
\hline-0.08604900 & 5.97811200 & -1.805415 \\
\hline-0.94669200 & 5.48454300 & -0.825743 \\
\hline-1.33891700 & 4.15327900 & -0.850456 \\
\hline-0.89182200 & 3.28419800 & -1.85239400 \\
\hline-0.06255700 & 3.80030800 & -2.849547 \\
\hline 0.35016800 & 5.13180400 & -2.8183 \\
\hline 0.23428900 & 7.01485900 & -1.7808 \\
\hline-1.29982300 & 6.13677400 & $-0.0328 \mathrm{~s}$ \\
\hline-1.97778300 & 3.76538200 & -0.0607 \\
\hline 0.25875000 & 3.15839200 & -3.66361 \\
\hline 1.00952700 & 5.50662000 & -3.5953 \\
\hline-1.32772400 & 1.84398100 & -1.846249 \\
\hline-2.41005000 & 1.72841600 & -1.7665 \\
\hline-0.65248900 & 0.84414400 & -2.59560 \\
\hline-1.25392100 & 0.08839400 & -3.092 \\
\hline 0.28025700 & 1.08555100 & 3.0987 \\
\hline
\end{tabular}

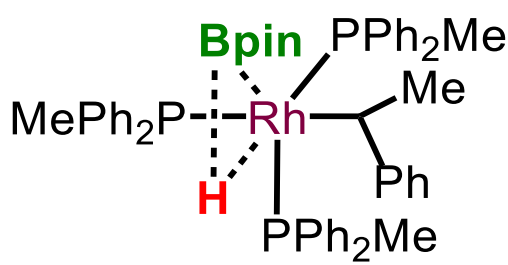

TS22

$E=-2360.315641$

$G=-2359.385251$

$\mathrm{O}$

$\mathrm{O}$

C

C 


\begin{tabular}{rrr}
1.36102000 & 1.45019700 & 2.75622700 \\
2.87465100 & -0.71456800 & 1.70945600 \\
3.95275900 & 0.00085200 & 2.23412000 \\
5.22209200 & -0.56724400 & 2.30507100 \\
5.43863800 & -1.86074900 & 1.84858000 \\
4.37126000 & -2.58304900 & 1.32073800 \\
0.39580100 & -1.29828000 & 2.82796400 \\
0.41243100 & -1.17898800 & 4.22112500 \\
-0.14162300 & -2.17183600 & 5.02371800 \\
-0.69845800 & -3.30912100 & 4.44462400 \\
-0.70286900 & -3.44517200 & 3.06074800 \\
-0.17089500 & -2.44156800 & 2.25766500 \\
\hline-2.20693100 & 0.47870200 & 1.03318000 \\
-2.10494700 & 1.10979300 & 2.78403800 \\
-2.96906800 & -1.19161700 & 1.29372300 \\
-2.96716500 & -2.09046400 & 0.22270700 \\
-3.53117900 & -3.35493100 & 0.35264500 \\
\hline & & \\
\hline
\end{tabular}

H

$\mathrm{H}$

C

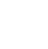

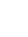

.

.

.

C

.

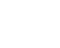

C

(

C

C

C

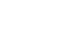

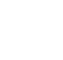

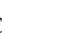

C

C

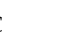

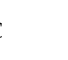

C

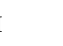

$\mathrm{H}$

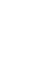

H

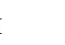

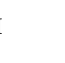

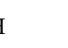

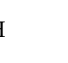

H

$6.42674900 \quad-2.30656800 \quad 1.90766100$

$\begin{array}{lll}4.52404300 & -3.60174100 & 0.97517700\end{array}$

$\begin{array}{lll}0.85089700 & -0.30591600 \quad 4.69363100\end{array}$

$\begin{array}{lll}-0.13198800 & -2.05737400 & 6.10350200\end{array}$

$\begin{array}{lll}-1.13047900 & -4.08311600 & 5.07158200\end{array}$

$\begin{array}{lll}-1.15257200 & -4.31633400 \quad 2.59580700\end{array}$

$\begin{array}{lll}-0.20725300 & -2.54943200 & 1.17927200\end{array}$

$\begin{array}{lll}-3.09441600 & 1.14895300 \quad 3.24469500\end{array}$

$\begin{array}{lll}-1.44590700 \quad 0.48992500 & 3.39344600\end{array}$

$\begin{array}{lll}-1.71728800 & 2.13142500 & 2.75239400\end{array}$

$-2.51864600 \quad-1.80421700 \quad-0.72138400$

$\begin{array}{lll}-3.52501600 & -4.02830600 & -0.49893000\end{array}$

$\begin{array}{lll}-4.53501600 & -4.73575100 & 1.66818800\end{array}$

$\begin{array}{lll}-4.53706500 & -3.15956600 & 3.58662300\end{array}$

$\begin{array}{lll}-3.55873300 & -0.92716800 \quad 3.35616300\end{array}$

$\begin{array}{lll}-2.60670600 & 3.32975300 & 0.61532000\end{array}$

$\begin{array}{lll}-4.55654700 \quad 4.75166000 & 0.16289900\end{array}$

$-6.80827700 \quad 3.73444100 \quad-0.11789800$

$\begin{array}{lll}-7.07537300 & 1.27449500 \quad 0.09689700\end{array}$

$\begin{array}{lll}-5.12640100 & -0.15059000 & 0.56225100\end{array}$

$3.10284500 \quad-2.01812600 \quad 1.25278800$

$2.28353500 \quad-2.60154700 \quad 0.84829000$

$\begin{array}{lll}-0.13027900 & 0.43409700 & -0.30867600\end{array}$

$\begin{array}{lll}-0.90060700 & 3.12464900 & -2.13441100\end{array}$

$\begin{array}{lll}-4.23372100 & -1.95699500 & -3.26676700\end{array}$

$\begin{array}{lll}-4.59266900 & -0.74507600 & -2.68923100\end{array}$

$\begin{array}{lll}-3.62482700 & 0.20503200 & -2.37110700\end{array}$

$\begin{array}{lll}-2.25998200 & -0.03376300 & -2.57916100\end{array}$

$-1.92781100 \quad-1.23781700 \quad-3.22736300$

$-2.88858800 \quad-2.18583400 \quad-3.55612100$

$\begin{array}{lll}-4.98688400 & -2.69863300 & -3.51404700\end{array}$

$\begin{array}{lll}-5.63688700 & -0.52635600 & -2.48305000\end{array}$

$-3.94954100 \quad 1.14574400 \quad-1.94684100$

$\begin{array}{lll}-0.88666400 & -1.42126700 & -3.47313600\end{array}$

$\begin{array}{lll}-2.58878800 & -3.10454800 & -4.05484000\end{array}$

$\begin{array}{lll}-1.18560700 & 0.94550300 & -2.18649800\end{array}$

$\begin{array}{lll}-0.37807400 & 0.79476100 & -2.90844000\end{array}$

$\begin{array}{lll}-1.68670900 & 2.38609800 & -2.30205700\end{array}$

$\begin{array}{lll}-2.11619400 & 2.57649200 & -3.29681400\end{array}$

$-2.46232600 \quad 2.61193400 \quad-1.57103600$ 


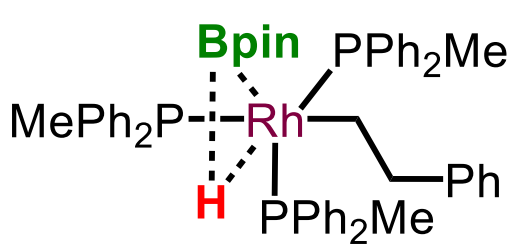

TS22'

$E=-2360.321823$

$\mathrm{O}$

O

C

C

C

$\mathrm{H}$

$\mathrm{H}$

$\mathrm{H}$

C

$\mathrm{H}$

$\mathrm{H}$

$\mathrm{H}$
$G=-2359.395664$

$\begin{array}{ccc}G=-2359.395664 & \\ -2.44942000 & -1.53560000 & -1.33574600 \\ -0.71941500 & -1.96712900 & -2.75323800 \\ -2.76625200 & -2.79163500 & -1.96726500 \\ -1.82072700 & -2.77666700 & -3.21460500\end{array}$

$\begin{array}{llll}-4.25670700 & -2.80311200 & -2.27597100\end{array}$

$\begin{array}{lll}-4.52919600 & -3.69958400 & -2.84233300\end{array}$

$\begin{array}{lll}-4.81973300 & -2.80493600 & -1.33844000\end{array}$

$-4.55291100 \quad-1.91645600 \quad-2.83833600$

$-2.42047200 \quad-3.90522100 \quad-0.98038700$

$\begin{array}{llll}-2.95414600 & -3.72969500 & -0.04206600\end{array}$

$\begin{array}{lll}-2.71423000 & -4.88523600 & -1.36689800\end{array}$

$\begin{array}{lll}-1.34689700 & -3.92083800 & -0.77316500\end{array}$

$\begin{array}{llll}-1.29255400 & -4.13855300 & -3.64096500\end{array}$

$\begin{array}{lll}-2.11851600 & -4.80600700 & -3.90703800\end{array}$

$\begin{array}{llll}-0.65093600 & -4.02547400 & -4.51915000\end{array}$

$-0.70283500 \quad-4.60471600 \quad-2.84970300$

$-2.43517200 \quad-2.04591300 \quad-4.41015100$

$-1.66033800 \quad-1.88417100 \quad-5.16367000$

$-3.24485700 \quad-2.62652100 \quad-4.86129000$

$\begin{array}{lll}-2.83077500 & -1.07083600 & -4.10982300\end{array}$

$-1.19980900 \quad-1.15476900 \quad-1.75239500$

$-0.65272500 \quad-0.05501300 \quad-1.59550400$

$\begin{array}{lll}1.56702600 & -1.23047200 & -2.35134300\end{array}$

$\begin{array}{lll}4.30816800 & -5.15000300 & -2.06093700\end{array}$

$\begin{array}{lll}4.83012900 & -4.17560300 & -1.21218100\end{array}$

$\begin{array}{lll}4.16599300 & -2.96441500 & -1.04233200\end{array}$

$2.97091000 \quad-2.70560400 \quad-1.72020300$

$\begin{array}{lll}2.45528200 & -3.69029000 & -2.56644500\end{array}$

$\begin{array}{llll}3.11626400 & -4.90342400 & -2.73770800\end{array}$

$\begin{array}{lll}4.82734100 & -6.09447900 & -2.19439000\end{array}$

$\begin{array}{llll}5.76158700 & -4.35914300 & -0.68382000\end{array}$

$\begin{array}{llll}4.57189000 & -2.20535300 & -0.37671100\end{array}$

$\begin{array}{lll}1.52339100 & -3.49002500 & -3.08869200\end{array}$

$\begin{array}{llll}2.70386000 & -5.65605600 & -3.40433400\end{array}$

$2.21569900 \quad-1.41983600 \quad-1.49080700$

$2.92925100 \quad-0.59369400 \quad-1.42891500$

$\begin{array}{lll}1.37616700 & -1.50428200 & -0.20961100\end{array}$

\begin{tabular}{|c|c|c|}
\hline 2.06004300 & -1.62725900 & 0.64175100 \\
\hline 0.80188700 & -2.43718300 & -0.28158400 \\
\hline-0.56128800 & -1.19181400 & 2.02834800 \\
\hline-0.35230300 & -3.03483300 & 2.01903000 \\
\hline-2.27553100 & -1.18275600 & 2.74062800 \\
\hline-3.32915500 & -1.31562600 & 1.82998900 \\
\hline-4.64293900 & -1.41642700 & 2.28026800 \\
\hline-4.92396100 & -1.36004000 & 3.64215000 \\
\hline-3.88176200 & -1.21497900 & 4.55454200 \\
\hline-2.56475800 & -1.13767800 & 4.10840100 \\
\hline 0.46555300 & -0.75625200 & 3.50936500 \\
\hline 0.34680700 & 0.52180100 & 4.07151400 \\
\hline 1.17159900 & 0.93086200 & 5.11211400 \\
\hline 2.14710200 & 0.06814400 & 5.61042700 \\
\hline 2.27297800 & -1.20534700 & 5.06832600 \\
\hline 1.43717300 & -1.61531800 & 4.02964500 \\
\hline-1.67217400 & 1.79380300 & 0.35969400 \\
\hline-2.21540400 & 2.33720100 & 2.03861500 \\
\hline-3.29939900 & 1.45488200 & -0.47376900 \\
\hline-4.47931000 & 1.25517800 & 0.24426300 \\
\hline-5.66990900 & 0.94090100 & -0.40890500 \\
\hline-5.70418200 & 0.83787700 & -1.79423500 \\
\hline-4.53675900 & 1.05324600 & -2.52365500 \\
\hline-1.27369300 & 3.41947500 & -0.44273700 \\
\hline-1.52764700 & 4.66471400 & 0.14101200 \\
\hline-1.17249400 & 5.84134400 & -0.51399400 \\
\hline-0.56971000 & 5.79302600 & -1.76767600 \\
\hline-0.32235000 & 4.56105500 & -2.36385000 \\
\hline-0.66609500 & 3.38886600 & -1.70078300 \\
\hline 1.84742200 & 1.57126600 & 0.18559600 \\
\hline 1.67773500 & 2.91074100 & 1.45914000 \\
\hline 2.37734200 & 2.53124100 & -1.31359200 \\
\hline 2.14881200 & 1.96547000 & -2.57129800 \\
\hline 2.53097700 & 2.62602100 & -3.73483500 \\
\hline 3.13659300 & 3.87780200 & -3.65716600 \\
\hline 3.36256000 & 4.45646000 & -2.41128600 \\
\hline 2.99375400 & 3.78378800 & -1.24979300 \\
\hline 3.48105500 & 0.87620300 & 0.72362200 \\
\hline 3.54825400 & 0.27562900 & 1.98465900 \\
\hline 4.72795300 & -0.30225100 & 2.43823000 \\
\hline 5.86514000 & -0.29472100 & 1.63339300 \\
\hline 5.811002 & 0.30236 & 0.3778 \\
\hline 4.62857 & 0.88552700 & -0.07355600 \\
\hline 55 & 452 & 005 \\
\hline
\end{tabular}




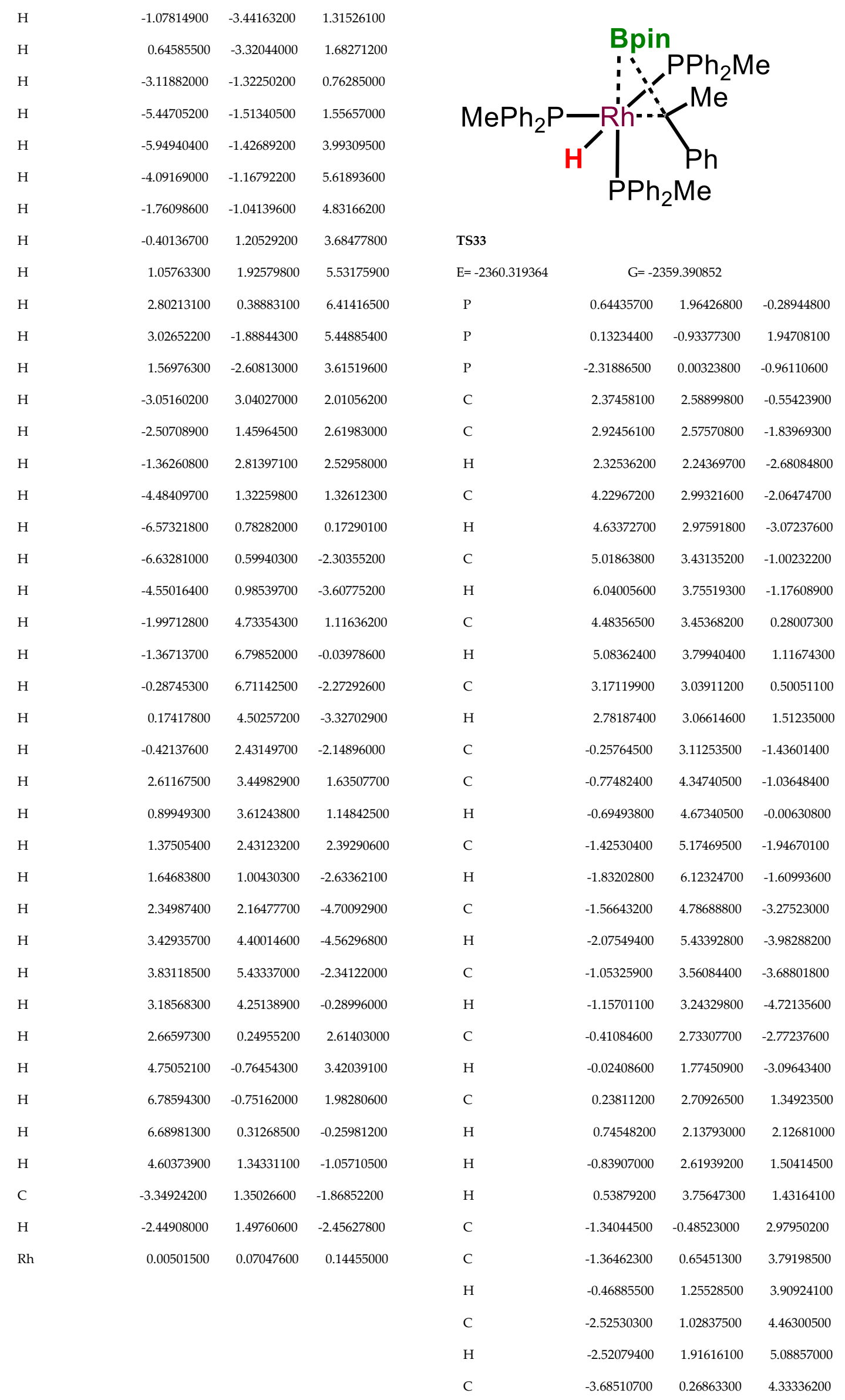




\begin{tabular}{|c|c|c|c|c|c|c|c|}
\hline $\mathrm{H}$ & -4.59246600 & 0.56200300 & 4.85195900 & $\mathrm{H}$ & 2.58474500 & -0.30851400 & 6.24988200 \\
\hline C & -3.67231300 & -0.86963500 & 3.53242600 & $\mathrm{H}$ & 4.46797400 & 0.93655200 & 5.21479400 \\
\hline $\mathrm{H}$ & -4.57071900 & -1.46524800 & 3.40860700 & $\mathrm{Rh}$ & 0.02825500 & -0.45398500 & -0.29426300 \\
\hline $\mathrm{C}$ & -2.51199200 & -1.24193800 & 2.86013500 & $\mathrm{H}$ & 0.11245400 & -0.09329100 & -3.52018400 \\
\hline $\mathrm{H}$ & -2.53917600 & -2.11369600 & 2.21380600 & $\mathrm{C}$ & -1.56659600 & -4.97505100 & -2.92615000 \\
\hline $\mathrm{C}$ & 0.25746400 & -2.74711200 & 2.27618900 & $\mathrm{C}$ & -0.65538400 & -4.97658400 & -1.86825600 \\
\hline $\mathrm{H}$ & 1.16393200 & -3.08800300 & 1.77168700 & $\mathrm{C}$ & 0.17839500 & -3.88876800 & -1.64991900 \\
\hline $\mathrm{H}$ & -0.59712000 & -3.25628100 & 1.82548200 & $\mathrm{C}$ & 0.13220200 & -2.75221200 & -2.47885500 \\
\hline $\mathrm{H}$ & 0.30204900 & -2.96465700 & 3.34596500 & $\mathrm{C}$ & -0.75255500 & -2.78953000 & -3.56131000 \\
\hline $\mathrm{C}$ & -3.15008600 & 1.55982400 & -0.41572600 & $\mathrm{C}$ & -1.60161500 & -3.87711700 & -3.77461500 \\
\hline $\mathrm{C}$ & -3.23471400 & 1.81960700 & 0.95755700 & $\mathrm{H}$ & -2.22699000 & -5.82097400 & -3.08943700 \\
\hline $\mathrm{H}$ & -2.84875000 & 1.09505900 & 1.66462100 & $\mathrm{H}$ & -0.59700300 & -5.83386700 & -1.20295700 \\
\hline $\mathrm{C}$ & -3.82191800 & 2.98706000 & 1.43017300 & $\mathrm{H}$ & 0.87676600 & -3.89360400 & -0.81991900 \\
\hline $\mathrm{H}$ & -3.88198300 & 3.15555600 & 2.50119100 & $\mathrm{H}$ & -0.78755300 & -1.96134000 & -4.26106600 \\
\hline C & -4.32841200 & 3.92575500 & 0.53349300 & $\mathrm{H}$ & -2.28899000 & -3.86018200 & -4.61568200 \\
\hline $\mathrm{H}$ & -4.78305800 & 4.84149300 & 0.89891200 & $\mathrm{C}$ & 1.05984000 & -1.58119700 & -2.23509600 \\
\hline $\mathrm{C}$ & -4.24628400 & 3.68046400 & -0.83237400 & $\mathrm{H}$ & 2.04846200 & -2.05639600 & -2.29422800 \\
\hline $\mathrm{H}$ & -4.62404400 & 4.40964000 & -1.54239700 & $\mathrm{C}$ & 1.09222100 & -0.54768300 & -3.35659200 \\
\hline $\mathrm{C}$ & -3.66536500 & 2.50563400 & -1.30422800 & $\mathrm{H}$ & 1.80525400 & 0.23736500 & -3.09790200 \\
\hline $\mathrm{H}$ & -3.59438500 & 2.35378100 & -2.37523600 & $\mathrm{H}$ & 1.41025400 & -0.99003200 & -4.31150500 \\
\hline$C$ & -2.75096800 & -0.04129900 & -2.75960800 & $\mathrm{O}$ & 3.02402000 & -0.37934900 & -0.81642600 \\
\hline $\mathrm{H}$ & -2.47015400 & -1.02702900 & -3.13201700 & $\mathrm{O}$ & 2.42066800 & -2.44447300 & -0.03698100 \\
\hline $\mathrm{H}$ & -2.18889600 & 0.71765800 & -3.30852100 & $\mathrm{C}$ & 4.24825500 & -1.10786900 & -0.77260200 \\
\hline $\mathrm{H}$ & -3.82310400 & 0.10436200 & -2.91556600 & $\mathrm{C}$ & 3.84801300 & -2.47787600 & -0.06986700 \\
\hline $\mathrm{C}$ & -3.50129200 & -1.29908200 & -0.37674700 & C & 5.27658900 & -0.27910100 & -0.00708800 \\
\hline $\mathrm{C}$ & -3.11245400 & -2.63315500 & -0.54206800 & $\mathrm{H}$ & 6.24817500 & -0.78350800 & 0.01503600 \\
\hline $\mathrm{C}$ & -4.74710500 & -1.02724400 & 0.19124300 & $\mathrm{H}$ & 5.39522800 & 0.68737400 & -0.50373800 \\
\hline C & -3.93968200 & -3.67130800 & -0.12997100 & $\mathrm{H}$ & 4.96232600 & -0.09286700 & 1.01928100 \\
\hline $\mathrm{H}$ & -2.15119600 & -2.86550800 & -0.98824200 & $\mathrm{C}$ & 4.73881800 & -1.25200300 & -2.21980800 \\
\hline C & -5.57145000 & -2.06788700 & 0.61539600 & $\mathrm{H}$ & 4.87502600 & -0.24843900 & -2.63257600 \\
\hline $\mathrm{H}$ & -5.07443700 & -0.00161800 & 0.32487800 & $\mathrm{H}$ & 5.69288800 & -1.78405900 & -2.27848000 \\
\hline $\mathrm{C}$ & -5.16899600 & -3.39131900 & 0.46351000 & $\mathrm{H}$ & 4.01755800 & -1.77358200 & -2.85399800 \\
\hline $\mathrm{H}$ & -3.61482900 & -4.69711800 & -0.27514900 & $\mathrm{C}$ & 4.26421900 & -3.72507100 & -0.85280000 \\
\hline $\mathrm{H}$ & -6.53203300 & -1.83992300 & 1.06814600 & $\mathrm{H}$ & 5.35248900 & -3.79551600 & -0.94798800 \\
\hline $\mathrm{H}$ & -5.81293900 & -4.19922500 & 0.79766600 & $\mathrm{H}$ & 3.90945000 & -4.61474300 & -0.32520500 \\
\hline C & 1.51964600 & -0.32583500 & 3.01398400 & $\mathrm{H}$ & 3.82460800 & -3.73777200 & -1.85268200 \\
\hline $\mathrm{C}$ & 2.59353600 & 0.35311900 & 2.43957400 & $\mathrm{C}$ & 4.33486000 & -2.61245200 & 1.37380400 \\
\hline $\mathrm{C}$ & 1.53470900 & -0.56415400 & 4.39457400 & $\mathrm{H}$ & 3.93622000 & -3.54094600 & 1.79334500 \\
\hline $\mathrm{C}$ & 3.64644400 & 0.81405800 & 3.22956900 & $\mathrm{H}$ & 5.42685000 & -2.65281300 & 1.43205600 \\
\hline $\mathrm{H}$ & 2.62223000 & 0.50233300 & 1.36389100 & $\mathrm{H}$ & 3.97824400 & -1.78883700 & 1.99392000 \\
\hline $\mathrm{C}$ & 2.58994900 & -0.11778600 & 5.18112400 & B & 1.93235400 & -1.24759400 & -0.61600600 \\
\hline $\mathrm{H}$ & 0.70504000 & -1.08645100 & 4.86287400 & $\mathrm{H}$ & -0.47994500 & -1.89179000 & -0.12020600 \\
\hline $\mathrm{C}$ & 3.64753900 & 0.58010300 & 4.59955700 & & & & \\
\hline $\mathrm{H}$ & 4.46624500 & 1.35196600 & 2.76194500 & & & & \\
\hline
\end{tabular}




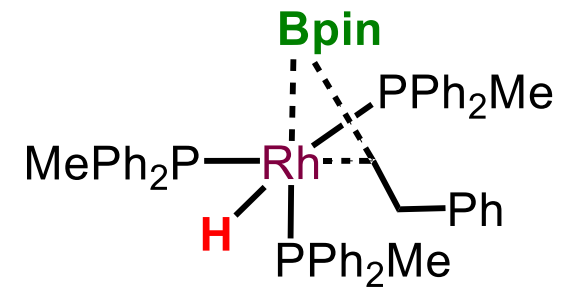

TS33'

$E=-2360.323968$

C

$\mathrm{H}$

$\mathrm{H}$

C

C

$\mathrm{H}$

$\mathrm{H}$

$$
G=-2359.394648
$$

$1.00552400 \quad 1.92869300$

$0.08307500 \quad-0.98289000 \quad 1.83184500$

$-2.32872600 \quad 0.51868000$

$2.83227700 \quad 2.19976000 \quad-0.51091600$

$\begin{array}{lll}3.43323500 & 1.93727100 & -1.74635400\end{array}$

$2.82716400 \quad 1.60007200 \quad-2.58135400$

$\begin{array}{lll}4.80045000 & 2.09817300 & -1.92328600\end{array}$

$\begin{array}{lll}5.24543100 & 1.89443000 & -2.89246500\end{array}$

$5.60066700 \quad 2.51516600 \quad-0.86029900$

$\begin{array}{lll}6.67026300 & 2.63968900 & -0.99735800\end{array}$

$\begin{array}{lll}5.01663300 & 2.77614100 \quad 0.37349600\end{array}$

$5.62737700 \quad 3.10724400 \quad 1.20839700$

$3.64093600 \quad 2.62455200 \quad 0.54405500$

$3.20974400 \quad 2.82817700 \quad 1.51859800$

$\begin{array}{lll}0.44273600 & 3.03503100 & -1.69895300\end{array}$

$\begin{array}{lll}0.37365500 & 4.42598800 & -1.58994500\end{array}$

$0.64286100 \quad 4.91628800 \quad-0.66049200$

$\begin{array}{lll}-0.06277500 & 5.20076800 & -2.65958700\end{array}$

$\begin{array}{lll}-0.12724000 & 6.27916000 & -2.55105600\end{array}$

$-0.41835700 \quad 4.59818000 \quad-3.86420600$

$\begin{array}{lll}-0.75748300 & 5.20464800 & -4.69827300\end{array}$

$\begin{array}{lll}-0.33054800 & 3.21526700 & -3.99359400\end{array}$

$\begin{array}{lll}-0.59821400 & 2.73423400 & -4.92966700\end{array}$

$\begin{array}{lll}0.08942700 & 2.44254700 & -2.91342200\end{array}$

$0.12506100 \quad 1.36077200 \quad-3.00605700$

$\begin{array}{lll}0.62925200 & 2.91759500 & 1.18667600\end{array}$

$\begin{array}{lll}0.96983000 & 2.34548700 & 2.05304700\end{array}$

$\begin{array}{lll}-0.45326100 & 3.03711900 \quad 1.26138800\end{array}$

$\begin{array}{lll}1.11338000 & 3.89706600 & 1.19878500\end{array}$

$\begin{array}{lll}-1.29261300 & -0.36925600 & 2.91980400\end{array}$

$\begin{array}{lll}-1.12943200 & 0.76080500 \quad 3.73002900\end{array}$

$\begin{array}{lll}-0.16481500 & 1.25493600 \quad 3.78143600\end{array}$

$-2.18422200 \quad 1.25456600 \quad 4.49141100$

$\begin{array}{lll}-2.03105300 & 2.12997100 \quad 5.11546800\end{array}$

$\begin{array}{lll}-3.42942700 & 0.63197200 & 4.45043700\end{array}$

$-4.25496400 \quad 1.01929500 \quad 5.03947300$

\begin{tabular}{|c|c|c|}
\hline-3.60649400 & -0.49079300 & 3.64703700 \\
\hline-4.57274100 & -0.98140500 & 3.59077800 \\
\hline-2.54758000 & -0.98656800 & 2.89103700 \\
\hline-2.72415400 & -1.84720900 & 2.25596500 \\
\hline-0.07792700 & -2.80853700 & 2.04696400 \\
\hline 0.68157400 & -3.26538000 & 1.41169100 \\
\hline-1.05231300 & -3.12151900 & 1.66538700 \\
\hline 0.03683200 & -3.12434700 & 3.08657900 \\
\hline-2.75061700 & 2.16723000 & -0.17919400 \\
\hline-2.95590300 & 2.28882800 & 1.20080400 \\
\hline-2.95817200 & 1.40200600 & 1.82254200 \\
\hline-3.16476500 & 3.53220400 & 1.78455700 \\
\hline-3.32422000 & 3.59463800 & 2.85662900 \\
\hline-3.15667200 & 4.68415200 & 0.99970300 \\
\hline-3.30986200 & 5.65773200 & 1.45507200 \\
\hline-2.95092600 & 4.57573300 & -0.37117000 \\
\hline-2.93401400 & 5.46430700 & -0.99483400 \\
\hline-2.75521600 & 3.32747900 & -0.95813900 \\
\hline-2.58180500 & 3.27554000 & -2.02820900 \\
\hline-2.99393400 & 0.70385100 & -2.62126400 \\
\hline-3.07543400 & -0.29633100 & -3.05222600 \\
\hline-2.32884300 & 1.30718200 & -3.24215200 \\
\hline-3.98787700 & 1.15883700 & -2.60021500 \\
\hline-3.68378700 & -0.57192200 & -0.24762000 \\
\hline-3.51049400 & -1.95765300 & -0.31889100 \\
\hline-4.89414800 & -0.07246700 & 0.24204700 \\
\hline-4.50947600 & -2.82289200 & 0.11614300 \\
\hline-2.58493700 & -2.36853900 & -0.70845500 \\
\hline-5.88695200 & -0.93733200 & 0.69473400 \\
\hline-5.06068700 & 0.99865200 & 0.29186500 \\
\hline-5.69551600 & -2.31526700 & 0.63976800 \\
\hline-4.34995100 & -3.89392300 & 0.04026800 \\
\hline-6.81277000 & -0.53001600 & 1.09012100 \\
\hline-6.47018500 & -2.98943900 & .99272600 \\
\hline 1.52496300 & -0.60148600 & 2.92915200 \\
\hline 2.63956400 & 0.03893200 & 2.39406300 \\
\hline 1.52761000 & -0.94163000 & 4.28869200 \\
\hline 3.73069700 & 0.36045300 & 3.20170800 \\
\hline 2.67190200 & 0.24156800 & 1.3282780 \\
\hline 2.61748200 & -0.63202100 & 5.09249300 \\
\hline 0.66189000 & -1.43083200 & 4.72665400 \\
\hline 3.72131900 & 0.02699700 & 4.55040000 \\
\hline 4.58886800 & 0.85865700 & $2.760010 c$ \\
\hline 2.605044 & 990 & .1451310 \\
\hline
\end{tabular}




\begin{tabular}{|c|c|c|c|}
\hline $\mathrm{H}$ & 4.57006600 & 0.27430800 & 5.18086700 \\
\hline $\mathrm{Rh}$ & -0.06013400 & -0.27082000 & -0.30506700 \\
\hline $\mathrm{O}$ & 2.97499000 & -0.84018800 & -0.77726500 \\
\hline $\mathrm{O}$ & 1.90707800 & -2.84404100 & -0.52487300 \\
\hline $\mathrm{C}$ & 4.02311000 & -1.79961000 & -0.70103200 \\
\hline $\mathrm{C}$ & 3.27873700 & -3.10771200 & -0.21791500 \\
\hline $\mathrm{C}$ & 5.10172900 & -1.28480800 & 0.24780400 \\
\hline $\mathrm{H}$ & 5.94810000 & -1.97904900 & 0.28369400 \\
\hline $\mathrm{H}$ & 5.45996300 & -0.31678700 & -0.11520600 \\
\hline $\mathrm{H}$ & 4.72421800 & -1.14836400 & 1.25985400 \\
\hline $\mathrm{C}$ & 4.64314300 & -1.91680200 & -2.10130200 \\
\hline $\mathrm{H}$ & 5.00602400 & -0.92822300 & -2.39588600 \\
\hline $\mathrm{H}$ & 5.48412000 & -2.61637400 & -2.11642700 \\
\hline $\mathrm{H}$ & 3.91639800 & -2.24173800 & -2.84927500 \\
\hline $\mathrm{C}$ & 3.68712100 & -4.37502400 & -0.96863600 \\
\hline $\mathrm{H}$ & 4.74740000 & -4.59995100 & -0.81285100 \\
\hline $\mathrm{H}$ & 3.10116300 & -5.22105000 & -0.59870500 \\
\hline $\mathrm{H}$ & 3.50180700 & -4.28209200 & -2.04029000 \\
\hline $\mathrm{C}$ & 3.40651600 & -3.36300900 & 1.28521100 \\
\hline $\mathrm{H}$ & 2.78756900 & -4.22300100 & 1.55810000 \\
\hline $\mathrm{H}$ & 4.44035500 & -3.59069400 & 1.56165700 \\
\hline $\mathrm{H}$ & 3.07174800 & -2.50465600 & 1.86966200 \\
\hline B & 1.73436000 & -1.49897300 & -0.94690900 \\
\hline $\mathrm{H}$ & -0.76688400 & -1.65000800 & -0.27752800 \\
\hline $\mathrm{H}$ & -0.84098600 & -0.66915100 & -3.18402300 \\
\hline $\mathrm{C}$ & -2.72545000 & -4.92930700 & -2.34692000 \\
\hline $\mathrm{C}$ & -1.48658000 & -4.81342000 & -1.72464600 \\
\hline $\mathrm{C}$ & -0.64103500 & -3.74202600 & -2.01156500 \\
\hline $\mathrm{C}$ & -1.02743900 & -2.75754600 & -2.92197800 \\
\hline $\mathrm{C}$ & -2.26606400 & -2.89958700 & -3.56052600 \\
\hline $\mathrm{C}$ & -3.11136900 & -3.96594900 & -3.27630100 \\
\hline $\mathrm{H}$ & -3.38283100 & -5.76240600 & -2.11615300 \\
\hline $\mathrm{H}$ & -1.16822200 & -5.56120200 & -1.00377000 \\
\hline $\mathrm{H}$ & 0.31331100 & -3.66484000 & -1.50407000 \\
\hline $\mathrm{H}$ & -2.57285400 & -2.15710400 & -4.29476100 \\
\hline $\mathrm{H}$ & -4.07240300 & -4.04264700 & -3.77569600 \\
\hline $\mathrm{C}$ & -0.18732000 & -1.54241600 & -3.25892100 \\
\hline $\mathrm{H}$ & 0.06117000 & -1.61539800 & -4.32865000 \\
\hline $\mathrm{C}$ & 1.12649200 & -1.25531000 & -2.50595800 \\
\hline $\mathrm{H}$ & 1.49410000 & -0.28467200 & -2.85256100 \\
\hline $\mathrm{H}$ & 1.86571700 & -1.96326600 & -2.90964200 \\
\hline
\end{tabular}

